Desenvolvimento de estratégias de captura de descontinuidades para leis de conservação e problemas relacionados em dinâmica dos fluidos

Giseli Aparecida Braz de Lima 

SERVIÇO DE PÓS-GRADUAÇÃO DO ICMC-USP

Data de Depósito:

Assinatura:

\title{
Desenvolvimento de estratégias de captura de descontinuidades para leis de conservação e problemas relacionados em dinâmica dos fluidos
}

\author{
Giseli Aparecida Braz de Lima
}

Orientador: Prof. Dr. Valdemir Garcia Ferreira

Dissertação apresentada ao Instituto de Ciências Matemáticas e de Computação - ICMC-USP, como parte dos requisitos para obtenção do título de Mestre em Ciências - Ciências de Computação e Matemática Computacional.

USP - São Carlos

Fevereirro/2010 

O homem se torna muitas vezes o que ele próprio acredita que é. Se insisto em repetir para mim mesmo que não posso fazer uma determinada coisa, é possível que acabe me tornando realmente incapaz de fazê-la. Ao contrário, se tenho a convicção de que posso fazê-la, certamente adquirirei a capacidade de realizá-la, mesmo que não a tenha no começo. 



\section{Agradecimentos}

Primeiramente a Deus pelo dom da vida e a Nossa Senhora Aparecida pela graça alcançada (o meu tão sonhado título de mestre).

Aos meus queridos pais, Francisco e Janete, pelo apoio, motivação, paciência e amor incondicional. Às minhas irmãs, Cintia e Magda, pelo carinho e paciência. Às minhas amadas avós, Madalena e Clara, pela motivação, apoio e orações.

Ao meu amigo e professor Dr. Valdemir Garcia Ferreira do ICMC-USP pela confiança e orientação.

À minha amiga e professora Dr. Magda Kimico Kaibara da UNESP-Bauru pela orientação em iniciação científica.

Aos amigos Rafael A. B. Queiroz, Italo Tasso, Fernando A. Kurokawa, Cássio Oishi, Fernando P. Martins, Rafael G. Cuenca, Larissa A. Petri e Pedro A. Cruz, pelo trabalho em equipe e toda ajuda.

Às minhas queridas amigas "irmãs" Laís, Letrícia e Josiane, pela paciência, motivação, apoio e carinho.

Ao meu querido amigo Alysson Naves pela paciência e ajuda.

A todos os professores do LCAD-ICMC pela motivação, apoio e ensinamentos.

Aos funcionários do ICMC-USP pela saudável amizade, em especial a Leonardo Martinussi pela paciência e prontidão.

À Fundação de Amparo e Pesquisa do Estado de São Paulo (FAPESP), pelo suporte financeiro concedido para realização do meu projeto de pesquisa.

Enfim, a todos que direta ou indiretamente contribuíram para a realização desse trabalho. 


\section{Resumo}

Esta dissertação trata da solução numérica de problemas em dinâmica dos fluidos usando dois novos esquemas upwind de alta resolução, denominados FDPUS-C1 (Five-Degree Polynomial Upwind Scheme of $C^{1}$ Class) e SDPUS-C1 (Six-Degree Polynomial Upwind Scheme of $C^{1}$ Class), para a discretização de termos convectivos lineares e não-lineares. Os esquemas são baseados nos critérios de estabilidade TVD (Total Variation Diminishing) e CBC (Convection Boundedness Criterion) e são implementados, nos contextos das metodologias de diferenças finitas e volumes finitos, no ambiente de simulação Freeflow (an integrated simulation system for Free surface Flow) para escoamentos incompressíveis 2D, 2D-1/2 e 3D, ou no código bem conhecido CLAWPACK (Conservation LAW PACKage) para problemas compressíveis 1D e 2D. Vários testes computacionais são feitos com o objetivo de verificar e validar os métodos numéricos contra outros esquemas upwind populares. Os novos esquemas são então aplicados na resolução de uma gama ampla de problemas em CFD (Computational Fluids Dynamics), tais como propagação de ondas de choque e escoamentos incompressíveis envolvendo superfícies livres móveis. Em particular, os resultados numéricos para leis de conservação hiperbólicas 2D e equações de Navier-Stokes incompressíveis 2D, 2D-1/2 e 3D demonstram que esses novos esquemas convectivos tipo upwind polinomiais funcionam muito bem.

Palavras-chave: Esquemas de alta resolução; Estratégias upwind; Discretização de termos convectivos; Escoamentos incompressíveis com superfícies livres; Equações de Navier-Stokes; Leis de conservação. 


\section{Abstract}

This dissertation deals with the numerical solution of fluid dynamics problems using two new high resolution upwind schemes, namely FDPUS-C1 and SDPUS-C1, for the discretization of the linear and non-linear convection terms. The schemes are based on TVD and CBC stability criteria and are implemented in the context of the finite difference and finite volume methodologies, either into the Freeflow code for 2D, 2D-1/2 and 3D incompressible flows or in the well-known CLAWPACK code for 1D eand 2D compressible flows. Several computational tests are performed to verify and validate the numerical methods against other popularly used upwind schemes. The new schemes are then applied to solve a wide range of problems in CFD, such as shock wave propagation and incompressible fluid flows involving moving free surfaces. In particular, the numerical results for $2 \mathrm{D}$ hyperbolic conservation laws and 2D, 2D-1/2 and 3D incompressible Navier-Stokes equations show that these new polynomial upwind convection schemes perform very well.

Key-words: High resolution schemes; Upwinding; Convection term discretization; Incompressible free surface flow; Navier-Stokes equations; Conservation laws. 


\section{Sumário}

1 Introdução 1

2 Formulação Matemática $\quad \mathbf{5}$

2.1 Leis de Conservação $1 \mathrm{D} \ldots \ldots \ldots \ldots$

2.1.1 Equação de Advecção . . . . . . . . . . . . . . . . . . . 5

2.1.2 Equação de Burgers com e sem Viscosidade . . . . . . . . . . . . . . 6

2.1.3 Equação de Buckley-Leverett . . . . . . . . . . . . . . . . . . 7

2.1 .4 Equações de Águas Rasas . . . . . . . . . . . . . . . . . . . . . . . 7

2.1.5 Equações de Euler . . . . . . . . . . . . . . . . . . . . . 8

2.2 Leis de Conservação 2D . . . . . . . . . . . . . . . . . . . . . 8

2.2.1 Equações de Águas Rasas . . . . . . . . . . . . . . . . . . . . . . . 9

2.2 .2 Equações de Euler . . . . . . . . . . . . . . . . . . . . . . 9

2.3 Equações Instantâneas de Navier-Stokes ． . . . . . . . . . . . . . . . . . . 10

2.4 Equações Médias de Reynolds . . . . . . . . . . . . . . . . . . . . . 11

2.5 Modelo Oldroyd-B . . . . . . . . . . . . . . . . . . . 13

2.6 Tema da Pesquisa . . . . . . . . . . . . . . . . . . . . . . . 14

3 Base Teórica de Esquemas Upwind de Alta Resolução NVD/TVD 15

3.1 Molécula Computacional de Três Pontos . . . . . . . . . . . . . . . . . 15

3.2 Variáveis Normalizadas . . . . . . . . . . . . . . . . . . . . 16

3.3 Diagrama de Variáveis Normalizadas . . . . . . . . . . . . . . . . . 16

3.4 Critério de Limitação CBC . . . . . . . . . . . . . . . . . . . . . . . . 18

3.5 Restrições TVD . . . . . . . . . . . . . . . . . . . 20

3.6 Limitadores de Fluxo . . . . . . . . . . . . . . . . . . . . . . . . 21

4 Desenvolvimento dos Novos Esquemas Upwind FDPUS-C1 e SDPUS-C1 25

4.1 O Esquema FDPUS-C1 . . . . . . . . . . . . . . . . . . 25

4.2 O Esquema SDPUS-C1 . . . . . . . . . . . . . . . . . . 29 
5 Modelagem Computacional $\mathbf{3 5}$

5.1 Discretização das Leis de conservação $1 \mathrm{D} \ldots$. . . . . . . . . . . . . . 35

5.1 .1 Equação de Advecção . . . . . . . . . . . . . . . . . . . 36

5.1 .2 Equação de Burgers . . . . . . . . . . . . . . . . . . 36

5.1 .3 Equação de Buckley-Leverett . . . . . . . . . . . . . . . . 36

5.1.4 Sistemas Hiperbólicos (Águas Rasas e Euler) . . . . . . . . . . . . . . 37

5.2 Discretização das Leis de Conservação 2D . . . . . . . . . . . . . . . . . 38

5.3 Discretização de Navier-Stokes 2D, 2D-1/2 e 3D . . . . . . . . . . . . 38

5.3.1 Algoritmo Base para Simulação de Escoamentos Incompressíveis com Superfícies Livres . . . . . . . . . . . . . . . . . . . . . . 44

$\begin{array}{lll}6 & \text { Resultados Numéricos 1D } & 47\end{array}$

6.1 Advecção de Escalares . . . . . . . . . . . . . . . . . . . . . . . . 47

6.2 Equação de Burgers sem e com Viscosidade . . . . . . . . . . . . . . . . 61

6.2.1 Equação de Burgers sem Viscosidade . . . . . . . . . . . . . . . 61

6.2.2 Equação de Burgers com Viscosidade . . . . . . . . . . . . . . . . . 71

6.3 Equação de Buckley-Leverett ． . . . . . . . . . . . . . . . . . . . . 73

6.4 Sistema Hiperbólico Águas Rasas . . . . . . . . . . . . . . . . . . . . 77

6.5 Sistema Hiperbólico Euler . . . . . . . . . . . . . . . . . . . . . . . 82

7 Resultados Numéricos 2D, 2D-1/2 e Aplicações 93

7.1 Leis de Conservação 2D . . . . . . . . . . . . . . . . . . . . . . 93

7.1 .1 Sistema Hiperbólico Águas Rasas . . . . . . . . . . . . . . . . . . . 93

7.1 .2 Sistema Hiperbólico Euler . . . . . . . . . . . . . . . . . 97

7.2 Escoamentos de Fluidos Incompressíveis Laminares 2D . . . . . . . . . . . . . 104

7.2.1 Colapso de uma Coluna fluido . . . . . . . . . . . . . . . . . . . 104

7.2 .2 Jato Livre sobre uma Superfície Rígida Impermeável . . . . . . . . . . 114

7.3 Escoamento de Fluido Incompressível Laminar 2D-1/2 . . . . . . . . . . . . . . 121

7.3.1 Jato Livre sobre uma Superfície Rígida Impermeável . . . . . . . . . . 121

7.3.2 Experimento de Taylor . . . . . . . . . . . . . . . . 125

7.4 Simulação de Escoamentos Viscoelásticos . . . . . . . . . . . . . . . 136

7.4.1 Escoamento em um Canal . . . . . . . . . . . . . . . . . . . . 136

7.4 .2 Inchamento do Extrudado . . . . . . . . . . . . . . . . . . . . . 139

7.5 Escoamento de Fluido Incompressível Turbulento . . . . . . . . . . . . . . . 143

7.5.1 Colapso de uma Coluna de Fluido . . . . . . . . . . . . . . . . . . . 143

8 Resultados Numéricos 3D $\quad 145$

8.1 Ressalto Hidráulico Circular . . . . . . . . . . . . . . . . . . . . . . . 145

8.2 Jatos Oscilantes . . . . . . . . . . . . . . . . . . . . 146 
9 Contribuições da Autora $\quad 153$

9.1 Artigos Submetidos em Periódicos . . . . . . . . . . . . . . . . . . 153

9.2 Artigos Publicados em Anais de Congresso . . . . . . . . . . . . . . . . . . 154

9.3 Artigos Submetidos para Publicação em Anais de Congresso . . . . . . . . . . . 156

9.4 Material Publicado em Curso de Curta Duração . . . . . . . . . . . . . . . . . 157

9.5 Material Submetido para Curso de Curta Duração . . . . . . . . . . . . . . . 157

9.6 Relatório Técnico Científico . . . . . . . . . . . . . . . . . . . 158

10 Conclusões e Trabalhos Futuros 159

A Demonstração da Propriedade TVD: Esquema FDPUS-C1 161

B Demonstração da Propriedade TVD: Esquema SDPUS-C1 $(\gamma=12) \quad 165$

$\begin{array}{ll}\text { C Princípio de Sweby e Diferenciabilidade } & 169\end{array}$

$\begin{array}{ll}\text { D CLAWPACK } & 173\end{array}$

$\begin{array}{ll}\text { Referências Bibliográficas } & 175\end{array}$ 


\section{Lista de Figuras}

3.1 Nós computacionais. Posições dos nós computacionais $D, R$ e $U$ conforme o sinal da velocidade $V_{f}$ (fluxo) de uma variável convectada. . . . . . . . . . . 16

3.2 Diagrama de variáveis normalizadas. . . . . . . . . . . . . . . . . . . 17

3.3 Região CBC em variáveis normalizadas. . . . . . . . . . . . . . . . . . . 19

3.4 Região TVD em variáveis normalizadas. . . . . . . . . . . . . . . . . . . 21

3.5 Representação esquemática dos gradientes consecutivos. . . . . . . . . . . . . 22

3.6 Diagrama de Sweby. . . . . . . . . . . . . . . . . . . . . . . . 23

4.1 Esquema FDPUS-C1 na região TVD. O esquema no plano $\hat{\phi}_{f} \perp \hat{\phi}_{U}$ (a) e seu respectivo limitador de fluxo no plano $\psi \perp r($ b) . . . . . . . . . . . . . . . . . . 29

4.2 Esquema SDPUS-C1 na região TVD. Esquema no plano $\hat{\phi}_{f} \perp \hat{\phi}_{U}$ (a) e seu respectivo limitador de fluxo no plano $\psi \perp r(\mathrm{~b})$, para $\gamma=4,6,8,10,12 \ldots \ldots 33$

5.1 Representação esquemática para se obter aproximações dos termos convectivos. Mostrando o ponto $A$ de discretização e seus vizinhos, as faces envolvidas $f$ e $g$ para a aproximação e a direção das velocidades $V_{f}$ e $V_{g}$ de convecção nas faces $f$ e $g$, respectivamente. . . . . . . . . . . . . . . . . . . .

6.1 Análise do parâmetro $\gamma$ para o esquema SDPUS-C1. Comparação entre a solução exata e os resultados numéricos para $\gamma=4,6,8,10,12$ (a); ampliação do pico (b); ampliação do degrau (c). Resultados numéricos para a equação de advecção, com condição inicial (6.1) e contorno de Direchlet homogênea. . . . . . . . . . . . . . . 49

6.2 Comparação dos esquemas FDPUS-C1 e SDPUS-C1 com WENO. Comparação entre a solução exata e os esquemas WENO, FDPUS-C1 (a) e SDPUS-C1 (c); confronto dos erros absolutos do esquema WENO com FDPUS-C1 (b) e SDPUS-C1 (d). Resultados numéricos para a equação de advecção, com condição inicial (6.2) e contorno de Direchlet homogênea. 
6.3 Análise da restrição TVD. Comparação entre a solução exata e os esquemas Lax-Wendroff (a) e FOU (c); comparação da TV exata com a TV numérica dos esquemas Lax-Wendroff (b) e FOU (d). Resultados numéricos para a equação de advecção, com condição inicial (6.4) e contorno de Direchlet homogênea.

6.4 Análise da restrição TVD. Comparação entre a solução exata e os esquemas ADBQUICKEST (a), FDPUS-C1 (c) e SDPUS-C1 (e); comparação da TV exata com as TVs numéricas dos esquemas ADBQUICKEST (b), FDPUS-C1 (d) e SDPUS-C1 (f). Resultados numéricos para a equação de advecção, com condição inicial (6.4) e contorno de Direchlet homogênea. . . . . . . . . . . . . . . . . . . . . . . . . . 54

6.5 Forma $\boldsymbol{W}$-Shape. Comparação entre a solução exata e os esquemas ADBQUICKEST (a), TOPUS (b), CUBISTA (c), VONOS (d), FDPUS-C1 (e) e SDPUS-C1 (f) para a equação de advecção, com condição inicial (6.13) e contorno de Direchlet homogênea. . 57

6.6 Aplicação interessante. Comparação entre a solução exata e os esquemas ADBQUICKEST (a), TOPUS (b), CUBISTA (c), VONOS (d), FDPUS-C1 (e) e SDPUS-C1 (f) para a equação de advecção, com condição inicial (6.14) e contorno de Direchlet homogênea. . . . . . . . . . . . . . . . . . . . . . .

6.7 Tempos longos de simulação. Comparação entre a solução exata e os esquemas ADBQUiCKEST (a), TOPUS (b), CUBISTA (c), VONOS (d), FDPUS-C1 (e) e SDPUS-C1 (f) para a equação de advecção, com condição inicial (6.15) e contorno de Direchlet homogênea. . . . . . . . . . . . . . . . . . . . . . . . .

6.8 Dados iniciais contínuos por partes. Comparação entre a solução exata e os esquemas ADBQUICKEST (a), TOPUS (b), FDPUS-C1 (c) e SDPUS-C1 (d); comparação das soluções numéricas (e). Resultados numéricos para a equação de Burgers sem viscosidade, com as condições inicial (6.16) e de contorno (6.17) . . . . . . . . . . . .

6.9 Problema de Platzman. i) Etapa pré-choque - Análise TVD. Comparação entre a TV semi-analítica e a TV numérica dos esquemas ADBQUICKEST (a), TOPUS (b), Superbee (c), FDPUS-C1 (d) e SDPUS-C1 (e) para a equação de Burgers sem viscosidade, com condição inicial (6.19) e com condição de Direchlet homogênea no contorno.

6.10 Problema de Platzman. i) Etapa pré-choque - Análise do erro absoluto. Erro absoluto (em escala logarítmica) dos esquemas ADBQUICKEST (a), TOPUS (b), Superbee (c), FDPUS-C1 (d) e SDPUS-C1 (e), para a equação de Burgers, sem viscosidade, com condição inicial (6.19) e condição de contorno de Direchlet homogênea. 66

6.11 Problema de Platzman. i) Etapa pré-choque - Caso transiente. Soluções numéricas dos esquemas FDPUS-C1 (a), SDPUSC1 (b) e solução semi-analítica (c) para a equação de Burgers sem viscosidade, com condição inicial dada por (6.19) e condição de contorno de Direchlet homogêneas, geradas em intervalos de tempo de 0.25 , com tempo final de simulação $t=1$. 
6.12 Problema de Platzman. ii) Etapa choque - Comparações. Comparação entre a solução semi-analítica e os esquemas ADBQUICKEST (a), TOPUS (c) e Superbee (e); erro absoluto (em escala logarítmica) dos esquemas ADBQUICKEST (b), TOPUS (d) e Superbee (f). Resultados numéricos para a equação de Burgers, sem viscosidade, com condições inicial (6.19) e de contorno Direchlet homogênea. . . . . . . . . . . . .

6.13 Problema de Platzman. ii) Etapa choque - Comparações. Comparação entre a solução semi-analítica e os esquemas FDPUS-C1 (a) e SDPUS-C1 (c); erros absolutos (em escala logarítmica) dos esquemas FDPUS-C1 (b) e SDPUS-C1 (d). Resultados numéricos para a equação de Burgers, sem viscosidade, com condição inicial (6.19) e condição de contorno Direchlet homogênea.

6.14 Problema de Platzman. iii) Etapa pós-choque - Comparações. Comparação entre a solução de referência e os esquemas ADBQUICKEST (a), TOPUS (b), Superbee (c), FDPUS-C1 (d) e SDPUS-C1 (e) para a equação de Burgers sem viscosidade, com condição inicial (6.19) e condição de contorno Direchlet homogênea. . . . . . . . . . .

6.15 Caso transiente. Resultados numéricos dos esquemas FDPUS-C1 (a) e SDPUS-C1 (b) para a equação de Burgers, com viscosidade, com condições inicial (6.21) e contorno (6.22), gerados em intervalos de tempo de 0.25 , com tempo final de simulação $t=3.25$.

6.16 Dado inicial constante por partes. Comparação entre a solução de referência e os esquemas ADBQUICKEST (a), TOPUS (b), FDPUS-C1 (c) e SDPUS-C1 (d); comparação das soluções numéricas (e); ampliação (f). Resultados numéricos para a equação de Buckley-Leverett, com condições inicial (6.16) e contorno (6.17). . . . . . .

6.17 Problema desafiador. Comparação entre a solução de referência e os esquemas ADBQUICKEST (a), TOPUS (b), FDPUS-C1 (c) e SDPUS-C1 (d); comparação das soluções numéricas (e); ampliação (f). Resultados numéricos para a equação de Buckley-Leverett, com condição inicial (6.23) e condição de Direchlet homogênea no contorno. . . . . . . . . . . . . . . . . . . . .

6.18 Problema $\boldsymbol{d a m}$-break. Comparação entre a solução de referência e os esquemas ADBQUICKEST (a), TOPUS (b), van Leer (c), FDPUS-C1 (d) e SDPUS-C1 (e); comparação das soluções numéricas (f). Resultados numéricos para o sistema hiperbólico de águas rasas (variável $h$ ), com condições iniciais (6.24)-(6.25) e extrapolação de ordem zero no contorno. . . . . . . . . . . . . . . . . . . . .

6.19 Problema dam-break. Comparação entre a solução de referência e os esquemas ADBQUICKEST (a), TOPUS (b), van Leer (c), FDPUS-C1 (d) e SDPUS-C1 (e); comparação das soluções numéricas (f). Resultados numéricos para o sistema hiperbólico de águas rasas (variável hu), com condições iniciais (6.24)-(6.25) e extrapolação de ordem zero no contorno. . . . . . . . . . . . . . . . . . . . . 
6.20 Problema dam-break. Comparação entre a solução de referência e os esquemas ADBQUICKEST (a), TOPUS (b), van Leer (c), FDPUS-C1 (d) e SDPUS-C1 (e); comparação das soluções numéricas (f). Resultados numéricos para o sistema hiperbólico de águas rasas (variável $u$ ), com condições iniciais (6.24)-(6.25) e extrapolação de

6.21 Problema dam-break. Comparação entre a solução de referência e os esquemas ADBQUICKEST (a), TOPUS (b), van Leer (c), FDPUS-C1 (d) e SDPUS-C1 (e); comparação das soluções numéricas (f). Resultados numéricos para o sistema hiperbólico de águas rasas (variável $F r$ ), com condições iniciais (6.24)-(6.25) e extrapolação de ordem zero no contorno. . . . . . . . . . . . . . . . . . . . . .

6.22 Condição inicial suave (hump). Comparação entre a solução de referência e os esquemas ADBQUICKEST (a), TOPUS (b), FDPUS-C1 (c) e SDPUS-C1 (d); comparação das soluções numéricas (e); ampliação (f). Soluções numéricas para as equações de Euler (variável $\rho$ ), com condições iniciais (6.26).

6.23 Tubo de choque de Sod. Comparação entre a solução de referência e os esquemas ADBQUICKEST (a), TOPUS (b), FDPUS-C1 (c) e SDPUS-C1 (d); comparação das soluções numéricas (e); ampliação (f). Soluções numéricas para as equações de Euler (para a variável $\rho$ ), com condições iniciais (6.27).

6.24 Tubo de choque de Sod. Comparação entre a solução de referência e os esquemas ADBQUICKEST (a), TOPUS (b), FDPUS-C1 (c) e SDPUS-C1 (d); comparação das soluções numéricas (e); ampliação (f). Soluções numéricas para as equações de Euler (para a variável $u$ ), com as condições iniciais (6.27).

6.25 Tubo de choque de Shu e Osher. Comparação entre a solução de referência e os esquemas ADBQUICKEST (a), TOPUS (b), FDPUS-C1 (c) e SDPUS-C1 (d); comparação das soluções numéricas (e); ampliação (f). Soluções numéricas para as equações de Euler (para variável $\rho$ ), com condições iniciais $(6.28) \ldots \ldots$. . . . . . . . . .

6.26 Ondas com fortes colisões. Comparação entre a solução de referência e os esquemas ADBQUICKEST (a), TOPUS (b), FDPUS-C1 (c) e SDPUS-C1 (d); comparação das soluções numéricas (e); ampliação (f). Soluções numéricas para as equações de Euler (para a variável $\rho$ ), com as condições iniciais (6.29), para $t=0.19 \ldots \ldots$. . . .

6.27 Ondas com fortes colisões. Comparação entre a solução de referência e os esquemas ADBQUICKEST (a), TOPUS (b), FDPUS-C1 (c) e SDPUS-C1 (d); comparação das soluções numéricas (e); ampliação (f). Soluções numéricas para as equações de Euler (para a variável $P$ ), com condições iniciais $(6.29)$, para $t=0.19 \ldots \ldots$. . . .

6.28 Ondas com fortes colisões. Comparação entre a solução de referência e os resultados esquemas ADBQUICKEST (a), TOPUS (b), FDPUS-C1 (c) e SDPUS-C1 (d); comparação das soluções numéricas (e); ampliação (f). Soluções para as equações de Euler (para a variável $\rho$ ), com as condições iniciais (6.29), para $t=0.38 . \quad \ldots$. . . . 91 
6.29 Ondas com fortes colisões. Comparação entre a solução de referência e os esquemas ADBQUICKEST (a), TOPUS (b), FDPUS-C1 (c) e SDPUS-C1 (d); comparação das soluções numéricas (e); ampliação (f). Soluções numéricas para as equações de Euler (para a variável $P$ ), com condições iniciais $(6.29)$, para $t=0.38 \ldots \ldots$. . . . . 92

7.1 Problema radial dam-break. Comportamento da onda $H$ antes $(t=0)$ e após $(t=0.25)$ o rompimento da barragem circular. . . . . . . . . . . . . . . .

7.2 Problema radial dam-break. Solução de referência (a) e resultados numéricos dos esquemas ADBQUICKEST (b), FDPUS-C1 (c) e SDPUS-C1 (d) para o contorno de $H$, no plano $x \perp y$ e $t=1.5$.

7.3 Problema radial dam-break. Comparação entre a solução de referência e os esquemas ADBQUICKEST (a), FDPUS-C1 (b) e SDPUS-C1 (c); comparação dos resultados numéricos (d). Resultados numéricos para o sistema de águas rasas, para a variação da profundidade $H$ em função da distância da origem $x(\operatorname{com} x \in[0,2.5])$. . . . . . 96

7.4 Problema radial dam-break. Solução de referência (a) e resultados numéricos dos esquemas ADBQUICKEST (b), FDPUS-C1 (c) e SDPUS-C1 (d), para o sistema de águas rasas com respeito a altura, em $t=1.5$.

7.5 Interação choque-choque. Diagrama esquemático do problema formulado pelas

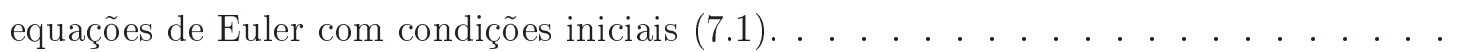

7.6 Interação choque-choque. Solução de referência (a) e resultados numéricos dos esquemas ADBQUICKEST (b), FDPUS-C1 (c) e SDPUS-C1 (d) para as equações de Euler, com condições iniciais (7.1), para o contorno de $\rho$ no plano $x \perp y$. . . . . . . . 100

7.7 Interação choque-choque. Comparação entre a solução de referência e os esquemas ADBQUICKEST (a), FDPUS-C1 (b) e SDPUS-C1 (c); comparação dos resultados numéricos (d). Resultados para as equações de Euler, com condições iniciais (7.1),

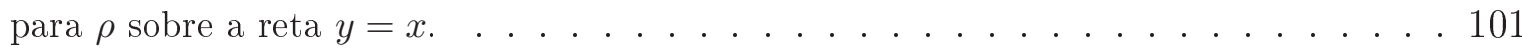

7.8 Interação choque-choque. Solução de referência (a) e resultados numéricos dos esquemas ADBQUICKEST (b), FDPUS-C1 (c) e SDPUS-C1 (d) para as equações de Euler, com condições iniciais $(7.1)$, para o contorno de $P$ no plano $x \perp y$. . . . . . . 102

7.9 Interação choque-choque. Comparação entre a solução de referência e os esquemas ADBQUICKEST (a), FDPUS-C1 (b) e SDPUS-C1 (c); comparação dos resultados numéricos (d). Resultados numéricos para as equações de Euler, com condições inicial (7.1), para $P$ sobre a reta $y=x \ldots \ldots \ldots$. . . . . . . . . . . 103

7.10 Colapso de fluido. Diagrama esquemático do problema Broken Dam. . . . . . . . . 104

7.11 Colapso de fluido (Martim e Moyce e Koshizuka e Oka). Comparação entre os dados experimentais e os esquemas FDPUS-C1 (a) e SDPUS-C1 (b) para $x_{\max }$ em função do tempo. . . . . . . . . . . . . . . . . . . . . 106

7.12 Colapso de fluido (Martim e Moyce). Comparação entre os dados experimentais e os esquemas FDPUS-C1 (a) e SDPUS-C1 (b) para $y_{\max }$ em função do tempo. . . . . 106 
7.13 Colapso de fluido (Martim e Moyce e Koshizuka e Oka). Resultados numéricos do esquema FDPUS-C1 para a evolução da superfície livre do fluido (campos de pressão

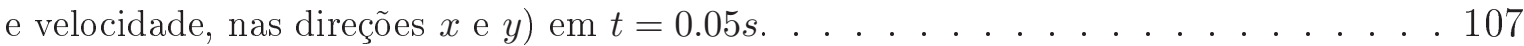

7.14 Colapso de fluido (Martim e Moyce e Koshizuka e Oka). Resultados numéricos do esquema FDPUS-C1 para a evolução da superfície livre do fluido (campos de pressão

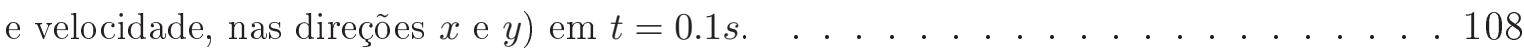

7.15 Colapso de fluido (Martim e Moyce e Koshizuka e Oka). Resultados numéricos do esquema FDPUS-C1 para a evolução da superfície livre do fluido (campos de pressão

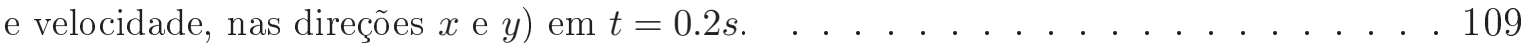

7.16 Colapso de fluido (Colagrossi e Landrini). Comparação entre os dados numéricos/teórico/experimental e os esquemas FDPUS-C1 (a) e SDPUS-C1 (b) para $x_{\max }$ em função do tempo. . . . . . . . . . . . . . . . . . . . . . . . . . . . 110

7.17 Colapso de fluido (Colagrossi e Landrini). Resultados numéricos do esquema SDPUS-C1 para a evolução da superfície livre do fluido (campos de pressão e velocidade, nas direções $x$ e $y$ ) em $t=0.05 s \ldots \ldots \ldots$. . . . . . . . . 111

7.18 Colapso de fluido (Colagrossi e Landrini). Resultados numéricos do esquema SDPUS-C1 para a evolução da superfície livre do fluido (campos de pressão e veloci-

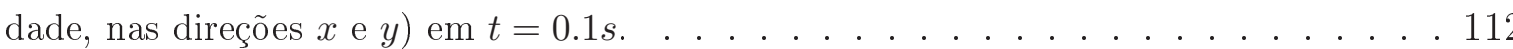

7.19 Colapso de fluido (Colagrossi e Landrini). Resultados numéricos do esquema SDPUS-C1 para a evolução da superfície livre do fluido (campos de pressão e veloci-

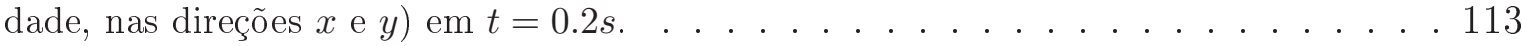

7.20 Jato livre sobre uma superfície rígida impermeável. Diagrama esquemático. . . 114

7.21 Jato livre a baixo número de Reynolds. Representação esquemática. . . . . . . . 115

7.22 Jato livre a baixo número de Reynolds. Comparação entre os dados (experimentais e numéricos) por Cruickshank e Munson e os esquemas FDPUS-C1 (a) e SDPUS-C1 (b) para os valores de $a(x) \ldots \ldots \ldots \ldots \ldots \ldots$

7.23 Jato livre a baixo número de Reynolds. Resultados numéricos do esquema FDPUS-C1 para a evolução da superfície livre do fluido (campos de pressão e velo-

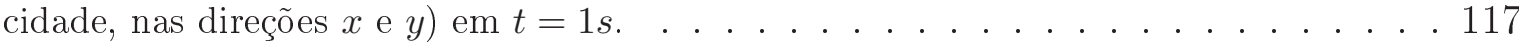

7.24 Jato livre a baixo número de Reynolds. Resultados numéricos do esquema FDPUS-C1 para a evolução da superfície livre do fluido (campos de pressão e velo-

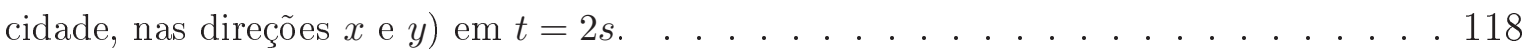

7.25 Jato livre a baixo número de Reynolds. Resultados numéricos do esquema FDPUS-C1 para a evolução da superfície livre do fluido (campos de pressão e velocidade, nas direções $x$ e $y$ ) em $t=6 s$.

7.26 Jato livre a alto número de Reynolds. Representação esquemática. 
7.27 Jato livre a alto número de Reynolds. Comparação entre a solução analítica de Watson e os esquemas FDPUS-C1 (a) e SDPUS-C1 (b), para a altura $H$ entre a superfície rígida e a superfície livre do fluido. . . . . . . . . . . . . . . . . . 121

7.28 Jato livre a alto número de Reynolds. Resultados numéricos do esquema SDPUS-C1 para a evolução da superfície livre do fluido (campos de pressão e velocidade, nas direções $x$ e $y$ ) em $t=0.1 s$.

7.29 Jato livre a alto número de Reynolds. Resultados numéricos do esquema SDPUS-C1 para a evolução da superfície livre do fluido (campos de pressão e velocidade, nas direções $x$ e $y$ ) em $t=0.2 s$.

7.30 Jato livre a alto número de Reynolds. Resultados numéricos do esquema SDPUS-C1 para a evolução da superfície livre do fluido (campos de pressão e velocidade, nas direções $x$ e $y)$ em $t=0.8 s$.

7.31 Jato livre, a baixo número de Reynolds. Comparação entre os dados (experimentais e numéricos) de Cruickshank e Munson e os esquemas SDPUS-C1 para os valores

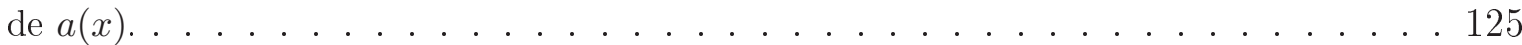

7.32 Jato livre, a baixo número de Reynolds. Resultados numéricos do esquema SDPUS-C1 para a evolução da superfície livre do fluido para os valores de número de Reynolds $R e=0.02211, R e=0.13459$ e $R e=0.63292 \ldots \ldots$. . . . . . . . . . 126

7.33 Experimento de Taylor. Diagrama esquemático de um jato vertical de fluido incidindo perpendicularmente sobre uma caixa contento fluido em repouso. . . . . . . . . 127

7.34 Experimento de Taylor $(\mathbf{R e}=\mathbf{1 0})$. Comparação entre os dados experimentais (a) e o esquema FDPUS-C1 (b); visão tridimensional (c). Resultados gerados para $R e=10$ ,nos tempos $t=0.25$ e $t=0.75$.

7.35 Experimento de Taylor $(\mathbf{R e}=\mathbf{1 0})$. Comparação entre os dados experimentais (a) e o esquema SDPUS-C1 (b); visão tridimensional (c). Resultados gerados para $R e=10$ ,nos tempos $t=0.25 \mathrm{~s}$ e $t=0.75 \mathrm{~s}$

7.36 Experimento de Taylor $(\mathbf{R e}=\mathbf{1 0})$. Resultados numéricos do esquema FDPUS-C1 (a); visão tridimensional (c). Resultados gerados para $R e=10$ para o tempo $t=10 \mathrm{~s}$. . 130

7.37 Experimento de Taylor $(\mathbf{R e}=\mathbf{1 0})$. Resultados numéricos do esquema FDPUS-C1, com respeito a evolução da superfície livre do fluido para os campos de pressão e velocidade (nas direções $x$ e $y$ ), no tempo $t=10 \mathrm{~s}$.

7.38 Experimento de Taylor $(\mathbf{R e}=\mathbf{2 0 0})$. Comparação entre os dados experimentais (a) e o esquema FDPUS-C1 (b), para $R e=200$, nos tempos $t=0.25 \mathrm{~s}$ e $t=0.75 \mathrm{~s} . \quad$. 132

7.39 Experimento de Taylor $(\mathbf{R e}=\mathbf{2 0 0})$. Comparação entre os dados experimentais (a) e o esquema SDPUS-C1 (b), para $R e=200$, nos tempos $t=0.25 \mathrm{~s}$ e $t=0.75 \mathrm{~s}$. . 133

7.40 Experimento de Taylor $(\mathbf{R e}=\mathbf{2 0 0})$. Estrutura completa (a); estrutura com corte (b) capturada pelo esquema SDPUS-C1, a $R e=200$ e no tempo $t=10 \mathrm{~s}$. 
7.41 Experimento de Taylor $(\mathbf{R e}=\mathbf{2 0 0})$. Resultados numéricos do esquema SDPUS-C1, com respeito a evolução da superfície livre do fluido para os campos de pressão e velo-

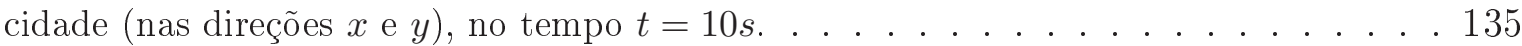

7.42 Escoamento em um canal. Diagrama esquemático de um canal de placas paralelas. 136

7.43 Escoamento em um canal de placas paralelas. Comparação entre os perfis exatos e os numéricos (esquema SDPUS-C1) para $u, T_{x x}=T_{11}$ e $T_{x y}=T_{12} \ldots \ldots$. . . 138

7.44 Inchamento do Extrudado. Representação esquemática. . . . . . . . . . . . . . . 139

7.45 Inchamento do Extrudado. Comparação entre a solução analítica (para $S_{r}$ em função de $W e$ ) e o esquema SDPUS-C1 para $t=9 s, t=13 \mathrm{~s}$ e estado estacionário. . . 140

7.46 Inchamento do Extrudado. Soluções numéricas do esquema SDPUS-C1, para o inchamento do extrudado de um fluido Oldroyd-B, com $W e=0.6$, em $t=0.13 \mathrm{~s}$, para

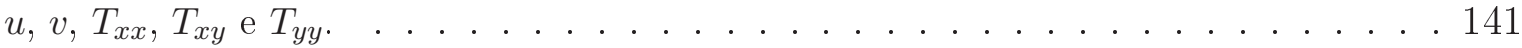

7.47 Inchamento do Extrudado. Soluções numéricas do esquema SDPUS-C1, para o inchamento do extrudado de um fluido Oldroyd-B, com $W e=0.6$, no estado estacionário,

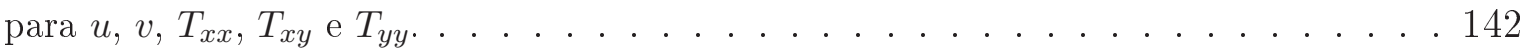

7.48 Colapso de uma coluna fluido em regime turbulento. Comparação entre os dados experimentais e o esquema FDPUS-C1 para $x_{\max }$ em função do tempo. . . . . . 143

7.49 Colapso de uma coluna de fluido em regime turbulento. Resultados numéricos do esquema FDPUS-C1 para a evolução da superfície livre do fluido para o campo de pressão em $t=0.2 s$.

8.1 Ressalto hidráulico circular. Fenômeno de ressalto hidráulico. . . . . . . . . . . . 146

8.2 Ressalto hidráulico circular. Comparação entre o experimento de Ellegard (a) e os esquemas FDPUS-C1 (b) e SDPUS-C1 (c) . . . . . . . . . . . . . . . . . . . . 147

8.3 Ressalto hidráulico circular. Resultados numéricos do esquema SDPUS-C1 para os campos de pressão e velocidade (nas direções $x$ e $z$ ) no plano $x \perp z(y=0.5)$. . . 148

8.4 Jatos oscilantes. Fenômeno de jato oscilante. . . . . . . . . . . . . . . . . . . . 149

8.5 Jatos oscilantes. Resultados numéricos do esquema FDPUS-C1, nos tempos $t=0.2 s$,

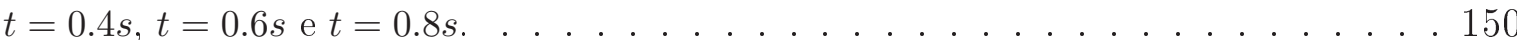

8.6 Jatos oscilantes. Resultados numéricos do esquema SDPUS-C1, nos tempos $t=0.2 \mathrm{~s}$,

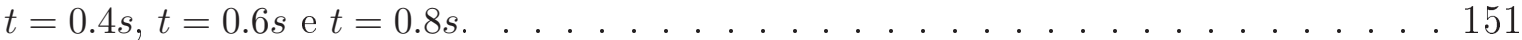

8.7 Jatos oscilantes. Resultados numéricos do esquema SDPUS-C1, nos tempos $t=0.2 \mathrm{~s}$ e e $t=0.6 s$, para os campos de pressão e velocidade (nas direções $x, y$ e $z$.). . . . . . 152

A.1 Esquema FDPUS-C1 - Análise TVD. Função $h=\hat{\phi}_{f}-\hat{\phi}_{U} \ldots \ldots$. . . . . . . . 162

A.2 Esquema FDPUS-C1 - Análise TVD. Função $g=\hat{\phi}_{f}-2 \hat{\phi}_{U} \ldots \ldots$. . . . . . . 162

A.3 Esquema FDPUS-C1 - Análise TVD. Função $s=\hat{\phi}_{f}-1 \ldots \ldots$. . . . . . . . 163

B.1 Esquema SDPUS-C1 - Análise TVD. Função $h=\hat{\phi}_{f}-\hat{\phi}_{U} \ldots \ldots \ldots$. . . . . . . 166

B.2 Esquema SDPUS-C1 - Análise TVD. Função $g=\hat{\phi}_{f}-2 \hat{\phi}_{U}$. . . . . . . . . . . . 166 
B.3 Esquema SDPUS-C1 - Análise TVD. Função $s=\hat{\phi}_{f}-1 \ldots \ldots 7$ 


\section{Lista de Tabelas}

6.1 Ordem de convergência. Erros nas normas $L_{1}, L_{2}$ e $L_{\infty}$ e estimativas da ordem de convergência para os esquemas ADBQUICKEST, TOPUS, CUBISTA, VONOS, FDPUS-C1 e SDPUS-C1. Dados para a equação de adveç̧ão, com condição inicial (6.5) e com condição de Dirichelet homogênea no contorno. . . . . . . . . . . . . . . 56

6.2 Problema de Platzman. i) Etapa pré-choque - Ordem de convergência. Erros nas normas $L_{1}, L_{2}$ e $L_{\infty}$ e estimativas da ordem de convergência para os esquemas ADBQUICKEST, TOPUS, Superbee, FDPUS-C1 e SDPUS-C1 para a equação de Burgers, sem viscosidade, com condição inicial (6.19) e com condição de Dirichelet homogênea no contorno. . . . . . . . . . . . . . . . . . . 67

7.1 Escoamento em um canal de placas paralelas. Erros relativos na norma $L_{2}$ e estimativa da ordem de convergência para o esquema SDPUS-C1, para os perfis $u, T_{11}$

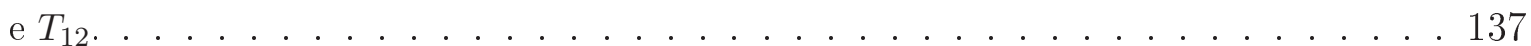

7.2 Inchamento do Extrudado. Massa numérica calculada pela vazão do injetor e erros relativos cometidos pelo esquema SDPUS-C1. . . . . . . . . . . . . . . 140 
CAPÍTULO

\section{1}

\section{Introdução}

A solução numérica de problemas de escoamentos de fluidos tem surgido como uma alternativa viável para ambos os estudos experimental e analítico. Com o objetivo de fazer as simulações desses problemas mais aceitáveis e confiáveis, existe uma demanda crescente para o desenvolvimento, análise e implementação de um esquema convectivo upwind não linear que resolva fenômenos complexos e ofereça simplicidade, precisão, robustez e versatilidade. Um tal esquema é, particularmente, importante quando se deseja simular escoamentos incompressíveis transientes, a altos valores do número de Reynolds e com superfícies livres móveis, por uma modelagem completa 3D (tridimensional) que é usualmente cara do ponto de vista computacional.

Na literatura especializada existe uma variedade de esquemas para aproximar termos convectivos, tais como os esquemas clássicos de primeira ordem upwind FOU (First-Order Upwinding) [16] (ou o seu relacionado híbrido HDS (Hybrid Differencing Scheme) [61]), e Godunov [44]. Entretanto, esses esquemas são geralmente inadequados para aplicações envolvendo longos tempos de simulação (a menos que se use malhas extremamente finas), principalmente porque extremos podem ser suavizados e a dissipação numérica pode se tornar dominante. Para contornar essa situação, tem sido usado, devido a falta intrínseca de dissipação numérica, esquemas convencionais, tais como diferenças centradas, o esquema SOU (Second Order Upwind) [74] e QUICK (Quadratic-Upstream interpolation for Convective Kinematics) [41], ou o seu relacionado QUICKEST (QUICK with Estimated Streamline Terms) [42], para citar apenas alguns. No entanto, sob condições severas de convecção, esses esquemas inevitavelmente geram oscilações não físicas e instabilidades em regiões onde as variáveis convectadas apresentam descontinuidades (ou choques).

Para superar os defeitos apresentados pelos esquemas mencionados anteriormente, um número considerável de esquemas upwind monotônicos de alta resolução tem aparecido, como 
por exemplo o esquema SHARP (Simple High-Accuracy Resolution Program) [43], o esquema SMART (Sharp and Monotonic Algorithm for Realistic Tranport) [28], o esquema VONOS (Variable-Order Non-Oscillatory Scheme) [73], o esquema WACEB (Weighted-Average Coefficient Ensuring Boundedness) [60], o esquema CUBISTA (Convergent and Universally Bounded Interpolation Scheme for the Treatment of Advection) [2], uma versão limitada do esquema QUICKEST denominada ADBQUICKEST (Adaptative Bounded QUICKEST) [27], e mais recentemente o esquema TOPUS (Third-Order Polynomial Upwind Scheme) [52, 26]. Além disso, de um ponto de vista "mais compressível", pode-se adicionar a essa lista o esquema pioneiro MUSCL (Monotone Upstream Scheme for Consevation Laws) originalmente proposto por van Leer [72] e os seus limitadores associados desenvolvidos por van Leer [70], van Albada [69] (e as suas variações), Osher [50], Superbee [4], MC (Monotonized Central - difference limiter) [71], entre outros. O objetivo principal desses esquemas é recuperar soluções suaves daquelas contaminadas por oscilações e, ao mesmo tempo, melhorar a ordem de convergência. É importante observar também que esses esquemas (alguns deles pelo menos), embora funcionem bem em alguns problemas, não podem ser limitados em determinadas situações tais como fenômenos envolvendo choques em escoamentos compressível (ver, por exemplo, [38] e [45]) e/ou a simulação de escoamentos incompressíveis viscoelásticos com modelos constitutivos hiperbólicos (ver, por exemplo, [79]). Lin e Chieng [46] e Lin e Lin [45], por exemplo, observaram que os esquemas SHARP e SMART, embora preservem alta precisão, produzem níveis altos de ruídos (oscilações) no caso dos problemas 1D (unidimensional) de tubo de choque. Alves et al. [2], usando esquemas upwind de alta ordem, rodaram uma série de testes para simular escoamentos viscoelásticos e observaram que os cálculos apresentaram dificuldades de convergência, com o refinamento da malha, e com a forte tendência em oscilar.

Não se pode esquecer, ainda, que há uma outra classe de esquemas de alta resolução de sucesso consagrado na captura de choques, isto é, o esquema ENO (Essentially Non-Oscillatory) [35] e o seu relacionado WENO (Weight ENO) [6]. Entretanto, em comparação com os esquemas mencionados anteriormente, a implementação do esquema ENO é difícil. Além disso, até o presente, os esquemas tipo ENO são os métodos de maior sucesso na captura de choque, entretanto eles também apresentam problemas, a saber: i) muito julgamento é necessário para selecionar ou construir uma molécula computacional adequada; ii) em muitas aplicações, segunda ordem é suficiente (ver, por exemplo, [7]); e iii) os esquemas ENO tem sido bastante usados na simulação da turbulência com LES (Large Eddy Simulation), onde tem-se mostrado frequentemente dissipativo.

Portanto, a necessidade de um esquema upwind de alta resolução que seja simples, eficiente e robusto, para aproximar termos convectivos (lineares e não lineares) de leis de conservação hiperbólicas gerais e equações relacionadas em dinâmica dos fluidos, continua a estimular a comunidade científica em CFD e esta é a principal motivação para desenvolvimento de esquemas upwind neste trabalho de mestrado. Outra motivação importante do estudo é o interesse em 
desenvolver uma técnica numérica que seja igualmente aplicável em problemas de escoamento de fluidos transientes ou estacionários, bem como compressíveis e incompressíveis. Também, como motivação, o desenvolvimento desses novos esquemas permitirá ao ambiente de simulação Freeflow [12] simular problemas de escoamentos incompressíveis com superfícies livres móveis 3D muito mais complexos tais como os viscoelásticos com os efeitos da turbulência.

Este trabalho de mestrado tem como principais objetivos desenvolver, analisar e implementar esquemas upwind de alta resolução para aproximação de termos convectivos (lineares e não lineares) de leis de conservação hiperbólicas e problemas relacionados em dinâmica dos fluidos. Objetiva-se também investigar a validade e receptividade desses esquemas em resolver uma gama ampla de problemas em CFD. Em particular, dois novos esquemas upwind polinomiais de alta resolução, denominados FDPUS-C1 (Five-Degree Polynomial Upwind Scheme of $C^{1}$ Class) e SDPUS-C1 (Six-Degree Polynomial Upwind Scheme of $C^{1}$ Class), são apresentados. Para a derivação desses novos esquemas são aplicados os conceitos de variáveis normalizadas de Leonard [42], o critério de limitação CBC (Convection Boundedness Criterion) de Gaskell e Lau [28] e as restrições TVD (Total Variation Diminishing) de Harten [30]. Uma variedade de testes computacionais 1D são realizados para verificar os métodos numéricos propostos contra outros esquemas popularmente utilizados. Os esquemas FDPUS-C1 e SDPUS-C1 são então aplicados na resolução de uma variedade de problemas em dinâmica dos fluidos, tais como propagação de descontinuidades 1D e 2D (bidimensional) e escoamentos incompressíveis envolvendo superfícies livres móveis 2D, 2D-1/2 (caso laminar em coordenadas cilíndricas) e 3D. Em particular, os resultados numéricos obtidos para os problemas transientes simulados demonstram que os esquemas FDPUS-C1 e SDPUS-C1 funcionam muito bem.

A ênfase do presente trabalho de mestrado é examinar a utilidade e efetividade dos novos esquemas FDPUS-C1 e SDPUS-C1 em leis de conservação hiperbólicas gerais e problemas relacionados em dinâmica dos fluidos. As contribuições desse trabalho são: i) a descrição de dois novos esquemas upwind de alta resolução TVD polinomiais; ii) a avaliação desses novos esquemas acoplados a uma variedade de equações de conservação; iii) a comparação com outros esquemas bem conhecidos na literatura na solução de problemas formulados pelas equações de advecção, Burgers, Buckley-Leverett, águas rasas e Euler; e iv) dados de simulações computacionais de problemas altamente complexos tais como, sistemas hiperbólicos 2D de águas rasas e Euler e escoamentos incompressíveis com superfície livres móveis 2D, 2D-1/2 e 3D.

A presente dissertação está organizada da seguinte maneira: o próximo capítulo contém uma síntese das EDPs (Equações Diferenciais Parciais) básicas consideradas nessa pesquisa; o capítulo 3 é reservado para descrição da base teórica necessária para o desenvolvimento de esquemas upwind de alta resolução para aproximação de termos convectivos; a derivação dos dois novos esquemas FDPUS-C1 e SDPUS-C1 está apresentada no capítulo 4; o capítulo 5 contempla a discretização das EDPS básicas consideradas nessa pesquisa; no capítulo 6 são apresentados os resultados numéricos $1 \mathrm{D}$, bem como a verificação/validação e análise do de- 
sempenho dos novos esquemas na resolução de leis de conservação 1D; os resultados numéricos $2 \mathrm{D}$ para leis de conservação $2 \mathrm{D}$ e $2 \mathrm{D}-1 / 2$ de escoamentos de fluidos incompressíveis com superfícies livres móveis laminares, turbulentos e viscoelásticos são apresentados no capítulo 7; no capítulo 8 estão os resultados numéricos 3D para escoamentos de fluidos incompressíveis com superfícies livres móveis laminares; no capítulo 9 são listadas as contribuições do autor para a comunidade científica em CFD; as principais conclusões do trabalho são delineadas no capítulo 10; no apêndice A demonstra-se a propriedade TVD para o esquema FDPUS-C1; no apêndice B demonstra-se a propriedade TVD para o esquema SDPUS-C1 $(\gamma=12)$; no apêndice C confrontam-se o princípio de monotonicidade de Sweby com a condição $\hat{\phi}_{f}^{\prime}(0)=1$; e, por fim, no apêndice D apresenta-se uma síntese do algoritmo de resolução aplicado pelo software CLAWPACK. 


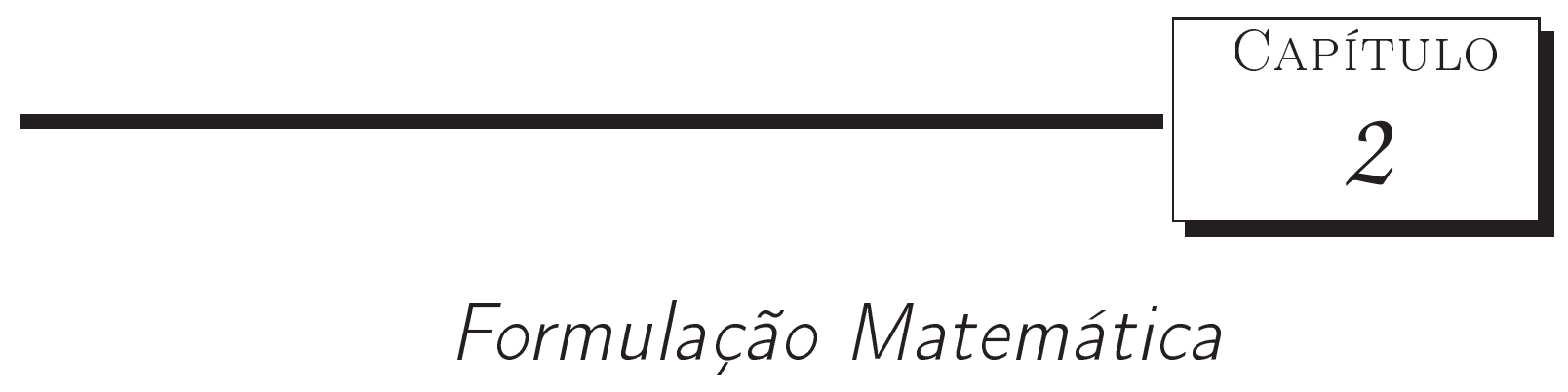

Neste capítulo são apresentadas as EDPs básicas, mais as condições iniciais e de contorno associadas, consideradas neste trabalho de mestrado.

\subsection{Leis de Conservação 1D}

Muitos problemas em ciência e engenharia envolvem quantidades que se conservam e que conduzem a certos tipos de EDPs denominadas leis de conservação hiperbólicas. Essas leis são geralmente não lineares e dependentes do tempo. No caso 1D são definidas por

$$
\frac{\partial \phi}{\partial t}+\frac{\partial F(\phi)}{\partial x}=0
$$

em que $\phi=\phi(x, t): \mathbb{R} \times \mathbb{R} \rightarrow \mathbb{R}^{m}$ é um vetor m-dimensional de quantidades conservadas e $F(\phi)=F(\phi(x, t)): \mathbb{R}^{m} \rightarrow \mathbb{R}^{m}$ é denominada função fluxo. Nesta seção são apresentados casos particulares das leis (2.1), a saber: equação de advecção de escalares (ou simplesmente equação de advecção); equações não lineares de Burgers (com ou sem viscosidade) e Bucley-Leverett; e os sistemas hiperbólicos águas rasas e Euler da dinâmica dos gases. Essas leis são definidas em domínios fechados $\left(x \in\left[x_{L}, x_{R}\right]\right)$ e suplementadas com condições iniciais e de contorno.

\subsubsection{Equação de Advecção}

A equação linear de advecção é o representante mais simples das leis (2.1) (veja, por exemplo, [44]), porém apresenta dificuldades semelhantes à aquelas encontradas em sistemas mais complexos. O modelo é formulado por (2.1), em que o vetor das variáveis conservadas e a função fluxo são dados, respectivamente, por

$$
\phi=u
$$


$\mathrm{e}$

$$
F(u)=a u,
$$

em que $a$ é a velocidade de convecção (por todo o texto considerar-se-á $a=1$ ).

As condições iniciais e de contorno consideradas são

$$
u(x, 0)=u_{0}(x)
$$

$\mathrm{e}$

$$
u\left(x_{L}, t\right)=u_{L}, \quad u\left(x_{R}, t\right)=u_{R},
$$

com $u_{L}$ e $u_{R}$ constantes. A solução exata para esse modelo é dada por

$$
u(x, t)=u_{0}(x-a t)
$$

que é uma cópia da perfil inicial deslocado para direita (ou esquerda se $a<0$ ).

\subsubsection{Equação de Burgers com e sem Viscosidade}

A equação de Burgers 1D (também conhecida como equação de Burgers sem viscosidade) é o representante não linear mais simples das leis (2.1), em que o vetor de variáveis conservadas e a função fluxo convexa $\left(F^{\prime \prime}(u)>0\right.$ ou $\left.F^{\prime \prime}(u)<0, \forall u\right)$ são dados, respectivamente, por

$$
\phi=u
$$

e

$$
F(u)=\frac{1}{2} u^{2}
$$

No caso da equação de Burgers com viscosidade, o vetor de variáveis conservadas e função fluxo permanecem os mesmos que no caso sem viscosidade e o lado direito da lei (2.1) (não nulo) contém o termo viscoso $\nu \frac{\partial^{2} u}{\partial x^{2}}$, em que $\nu>0$ é o coeficiente de viscosidade. Em resumo, esse modelo é expresso por

$$
\frac{\partial u}{\partial t}+\frac{\partial}{\partial x}\left(\frac{u^{2}}{2}\right)=\nu \frac{\partial^{2} u}{\partial x^{2}}
$$

A equação (2.9) tem sido introduzida na literatura como um modelo bastante simplificado de equações para a captura de características interessantes em dinâmica dos gases, tais como, efeitos de advecção (não lineares) e efeitos viscosos (lineares). Essa equação pode ser usada também como um modelo simplificado para o entendimento da formação do choque e da turbulência nos fluidos (ver, por exemplo, Karpman [36]). Quando $\nu>0$, o termo da viscosidade conduz às soluções suaves e a energia do sistema dissipa suavemente. Entretanto, para $\nu \rightarrow 0$, descontinuidades (choques) podem aparecer na solução, mesmo no caso de condições iniciais suaves. Essa equação é muito utilizada também para avaliações de métodos numéricos, uma 
vez que possui características comuns com as equações de Navier-Stokes devido a sua não linearidade. Neste trabalho, a equação de Burgers (com e sem viscosidade) são suplementadas com condições iniciais e de contorno dadas, respectivamente, por

$$
u(x, 0)=u_{0}(x)
$$

e

$$
u\left(x_{L}, t\right)=f\left(x_{L}, t\right), \quad u\left(x_{R}, t\right)=g\left(x_{R}, t\right)
$$

\subsubsection{Equação de Buckley-Leverett}

A equação de Buckley-Leverett é não linear e possui função fluxo não-convexa. Essa equação modela escoamentos (em meios porosos) de dois fluidos imiscíveis tais como água e óleo [1]. Nesse caso, o vetor das variáveis conservadas e a função fluxo são dados, respectivamente, por

$$
\phi=u
$$

e

$$
F(u)=\frac{u^{2}}{u^{2}+\frac{1}{4}(1-u)^{2}} .
$$

Esse modelo é suplementado com as condições iniciais e de contorno

$$
u(x, 0)=u_{0}(x)
$$

e

$$
u\left(x_{L}, t\right)=f\left(x_{L}, t\right), \quad u\left(x_{R}, t\right)=g\left(x_{R}, t\right)
$$

\subsubsection{Equações de Águas Rasas}

As equações de águas rasas modelam em geral o movimento hidrostático de um fluido incompressível com superfície livre. Para derivar o sistema hiperbólico considera-se o fluido em um canal com comprimento unitário, assume-se que a velocidade vertical é desprezível e considera-se que a velocidade horizontal $u$ é aproximadamente constante em toda secção transversal do canal. Esse sistema hiperbólico (ver, por exemplo, LeVeque [44]) possui o vetor das variáveis conservadas e a função fluxo como

$$
\phi=[h, \quad h u]^{T}
$$

e

$$
F(\phi)=\left[h u, \quad h u^{2}+\frac{1}{2} g h^{2}\right]^{T}
$$


em que $h, h u$ e $g$ são, respectivamente, a profundidade do fluido no canal, a vazão e a constante gravitacional. Esse sistema hiperbólico é suplementado com as condições iniciais

$$
u(x, 0)=u_{0}(x) \quad \text { e } \quad h(x, 0)=h_{0}(x)
$$

e com extrapolação de ordem zero no contorno (ver LeVeque [44]).

\subsubsection{Equações de Euler}

As equações de Euler constituem um sistema não linear de equações hiperbólicas e modelam, por exemplo, o problema do tubo de choque (um problema de Riemann). Este problema tem sido usado na literatura com frequência para investigar vários fenômenos físicos, tais como reações químicas, efeitos de ondas de choque e aerotermodinâmica de veículos supersônicos/hipersônicos. Nesse caso, o vetor das variáveis conservadas e a função fluxo são dados, respectivamente, por

$$
\phi=\left[\begin{array}{lll}
\rho, & \rho u, & E
\end{array}\right]^{T}
$$

e

$$
F(\phi)=\left[\rho u, \quad \rho u^{2}+P, \quad u(E+P)\right]^{T},
$$

Para fechar o sistema de equações, considera-se a equação de gás ideal

$$
P=(\gamma-1)\left(E-\frac{1}{2} \rho u^{2}\right)
$$

em que $\gamma=1.4$ é a razão do calor específico (Harten [31]). As condições inicias são

$$
\phi(x, 0)=\phi_{0}(x)= \begin{cases}{\left[\rho_{L}, u_{L}, P_{L}\right]^{T},} & x \leq x_{0} \\ {\left[\rho_{R}, u_{R}, P_{R}\right]^{T},} & x>x_{0}\end{cases}
$$

e as condições de contorno (ver detalhes em Toro [68])

$$
\phi\left(x_{L}, t\right)=\phi_{L}(t)=\left[\rho_{L}(t), u_{L}(t), P_{L}(t)\right]^{T} \text { e } \phi\left(x_{R}, t\right)=\phi_{R}(t)=\left[\rho_{R}(t), u_{R}(t), P_{R}(t)\right]^{T}
$$

\subsection{Leis de Conservação 2D}

Para problemas 2D, a leis de conservação são dadas por

$$
\frac{\partial \phi}{\partial t}+\frac{\partial F(\phi)}{\partial x}+\frac{\partial G(\phi)}{\partial y}=0
$$

em que $\phi=\phi(x, y, t): \mathbb{R}^{2} \times \mathbb{R} \rightarrow \mathbb{R}^{m}$ é o vetor m-dimensional de variáveis conservadas e $F(\phi)=F(\phi(x, y, t)), \quad G(\phi)=G(\phi(x, y, t)): \mathbb{R}^{m} \rightarrow \mathbb{R}^{m}$ são as funções fluxo, com $x \in\left[x_{L}, x_{R}\right]$ 
e $y \in\left[y_{B}, y_{T}\right]$. Neste trabalho considerar-se-ão os sistemas de águas rasas e Euler.

\subsubsection{Equações de Águas Rasas}

O sistema não linear hiperbólico de equações de águas rasas (ver LeVeque [44]) possui o vetor das variáveis conservadas e as funções fluxo dados, respectivamente, por

$$
\begin{aligned}
& \phi=\left[\begin{array}{lll}
h, & h u, & h v
\end{array}\right]^{T}, \\
& F(\phi)=\left[h u, \quad h u^{2}+\frac{1}{2} g h^{2}, \quad h u v\right]^{T}
\end{aligned}
$$

e

$$
G(\phi)=\left[h v, \quad h u v, \quad h v^{2}+\frac{1}{2} g h^{2}\right]^{T}
$$

em que $h,[u, v]^{T}$, $[h u, h v]^{T}$ e $g$ são a altura, o vetor velocidade, o vetor vazão e a constante gravitacional, respectivamente. Esse sistema hiperbólico é suplementado com as condições iniciais

$$
u(x, 0)=u_{0}(x), \quad v(x, 0)=v_{0}(x), \quad \text { e } \quad h(x, 0)=h_{0}(x) .
$$

As condições de contorno são dadas pela extrapolação de ordem zero (ver LeVeque [44]).

\subsubsection{Equações de Euler}

O sistema não linear hiperbólico de equações de Euler da dinâmica dos gases possui o vetor das variáveis conservadas e funções fluxo dados, respectivamente, por

$$
\begin{gathered}
\phi=\left[\begin{array}{cccc}
\rho, & \rho u, & \rho v, & E
\end{array}\right]^{T}, \\
F(\phi)=\left[\rho u, \quad \rho u^{2}+P, \quad \rho u v, \quad(E+P) u\right]^{T}
\end{gathered}
$$

e

$$
G(\phi)=\left[\rho v, \quad \rho u v, \quad \rho v^{2}+P, \quad(E+P) v\right]^{T},
$$

em que as variáveis $\rho,[u, v]^{T},[\rho u, \rho v]^{T}, E, P$ são a massa específica, o vetor velocidade, o vetor quantidade de movimento, a energia total e a pressão, respectivamente. Para fechar o sistema (2.27)-(2.29), é considerada a equação do gás ideal

$$
P=(\gamma-1)\left(E-\frac{1}{2} \rho\left(u^{2}+v^{2}\right)\right)
$$

com $\gamma=1.4$ a razão do calor específico (Harten [31]). Esse sistema hiperbólico é suplementado com as condições iniciais

$$
\phi(x, 0)=\phi_{0}(x)= \begin{cases}{\left[\rho_{L}, u_{L}, v_{L}, P_{L}\right]^{T},} & x \leq x_{0}, \\ {\left[\rho_{R}, u_{R}, v_{R}, P_{R}\right]^{T},} & x>x_{0}\end{cases}
$$


e com extrapolação de ordem zero no contorno (ver LeVeque [44]).

\subsection{Equações Instantâneas de Navier-Stokes}

As equações instantâneas de Navier-Stokes modelam problemas de escoamentos de fluidos incompressíveis em ambos os regimes laminar e turbulento. No caso em que o fluido é considerado um meio homogêneo, a massa específica $\rho(x, t)=\rho_{0}$ das partículas não varia durante o seu movimento e as propriedades de transporte são constantes, as equações matemáticas das leis físicas de conservação, consideradas para a simulação do regime laminar, são as equações instantâneas de Navier-Stokes e continuidade que na forma conservativa para os casos 2D e 2D-1/2 (simetria radial) são escritas

$$
\begin{aligned}
\frac{\partial u}{\partial t}+\frac{1}{r^{\alpha}} \frac{\partial\left(r^{\alpha} u u\right)}{\partial r}+\frac{\partial(u v)}{\partial z} & =-\frac{\partial P}{\partial r}+\frac{1}{R e} \frac{\partial}{\partial z}\left(\frac{\partial u}{\partial z}-\frac{\partial v}{\partial r}\right)+\frac{1}{F r^{2}} g_{r} \\
\frac{\partial v}{\partial t}+\frac{1}{r^{\alpha}} \frac{\partial\left(r^{\alpha} v u\right)}{\partial r}+\frac{\partial(v v)}{\partial z} & =-\frac{\partial P}{\partial z}+\frac{1}{R e} \frac{1}{r^{\alpha}} \frac{\partial}{\partial r}\left(r^{\alpha}\left(\frac{\partial u}{\partial z}-\frac{\partial v}{\partial r}\right)\right)+\frac{1}{F r^{2}} g_{z} \\
\frac{1}{r^{\alpha}} \frac{\partial\left(r^{\alpha} u\right)}{\partial r}+\frac{\partial v}{\partial z} & =0
\end{aligned}
$$

em que t é o tempo, $u=u(r, z, t)$ e $v=v(r, z, t)$ são, respectivamente, as componentes do vetor velocidade nas direções $r$ e $z ; g=\left(g_{r}, g_{z}\right)^{T}$ é a aceleração gravitacional e $P$ é a pressão cinética (pressão dividida pela massa específica). Os parâmetros adimensionais $R e=\frac{U_{0} L_{0}}{\nu} \mathrm{e}$ $F r=\frac{U_{0}}{\sqrt{L_{0} / g}}$ correspondem, respectivamente, aos números de Reynolds e Froud, com $\nu$ representando o coeficiente de viscosidade cinemática molecular do fluido dada por $\nu=\frac{\mu}{\rho}$ ( $\mu$ é o coeficiente de viscosidade dinâmica), $U_{0}$ e $L_{0}$ são, respectivamente, as escalas de velocidade e comprimento característicos. O parâmetro $\alpha$ nessas equações é utilizado para definir o sistema de coordenadas: no caso em que $\alpha=0$, coordenadas cartesianas são usadas, em que $r$ é interpretado como $x$ e $z$ como $y$; e no caso $\alpha=1$ coordenadas cilíndricas são empregadas.

No caso 3D, as equações instantâneas de Navier-Stokes e continuidade, em coordenadas cartesianas, assumem as formas

$$
\begin{gathered}
\frac{\partial u_{i}}{\partial t}+\frac{\partial\left(u_{i} u_{j}\right)}{\partial x_{j}}=-\frac{\partial P}{\partial x_{i}}+\frac{1}{R_{e}} \frac{\partial}{\partial x_{j}}\left(\frac{\partial u_{i}}{\partial x_{j}}\right)+\frac{1}{F_{r}^{2}} g_{i}, \quad i=1,2,3 \\
\frac{\partial u_{i}}{\partial x_{i}}=0 .
\end{gathered}
$$

As equações de Navier-Stokes (em coordenadas cartesianas e cilíndricas) são suplementadas com condições iniciais (para mais detalhes, ver Tomé e Mckee [67]) e condições de contorno, as quais são descritas como segue:

- Na entrada e saída de fluido: na entrada de fluido (injetor) as condições de contorno prescritas 
são

$$
u_{n}=U_{0}, \quad u_{t}=0
$$

em que $u_{n}$ é a velocidade normal ao contorno e $u_{t}$ é a velocidade tangencial ao contorno. Na saída de fluido (ejetor) as condições são dadas por

$$
\frac{\partial u_{n}}{\partial n}=0, \quad \frac{\partial u_{t}}{\partial u_{n}}=0
$$

- No contorno rígido: dois casos são considerados, a saber, a condição de contorno sem escorregamento (no-slip) dada por

$$
u_{t}=0, \quad u_{n}=0
$$

e com escorregamento (free-slip) dada por

$$
u_{n}=0, \frac{u_{t}}{u_{n}}=0
$$

- Na superfície livre:

$$
\begin{aligned}
n \cdot(\sigma \cdot n) & =P_{e x t}, \\
m_{1} \cdot(\sigma \cdot n) & =0 \\
m_{2} \cdot(\sigma \cdot n) & =0,
\end{aligned}
$$

em que $P_{\text {ext }}$ é a pressão externa (atmosférica local) assumida zero neste trabalho. No caso 3D $m_{1}=\left(m_{1 x}, m_{1 y}, m_{1 z}\right)$ e $m_{2}=\left(m_{2 x}, m_{2 y}, m_{2 z}\right)$ são os vetores tangentes à superfície livre, e $n=\left(n_{x}, n_{y}, n_{z}\right)$ é o vetor unitário normal exterior à superfície. No caso $2 \mathrm{D}, m_{1}=\left(m_{1 x}, m_{1 y}\right)$ e $n=\left(n_{x}, n_{y}\right)$, e no caso 2D-1/2 $m_{1}=\left(-n_{z}, 0, n_{r}\right)$ e $n=\left(n_{r}, 0, n_{z}\right) . \sigma$ é o tensor de tensões total dado, para $2 \mathrm{D}$ e $3 \mathrm{D}$, por

$$
\sigma=-P I+2 \mu\left(\nabla u+(\nabla u)^{T}\right)
$$

e para $2 \mathrm{D}-1 / 2$ por

$$
\sigma=-P I+\frac{1}{R e}\left(\nabla u+(\nabla u)^{T}\right)
$$

$I$, em (2.44) e (2.45), é o tensor identidade.

\subsection{Equações Médias de Reynolds}

Para simulação dos efeitos da turbulência são consideradas as equações médias de Reynolds

$$
\frac{\partial \bar{u}_{i}}{\partial t}+\frac{\partial\left(\bar{u}_{i} \bar{u}_{j}\right)}{\partial x_{j}}=-\frac{\partial \bar{P}}{\partial x_{i}}+\frac{1}{R e} \frac{\partial}{\partial x_{j}}\left(\frac{\partial \bar{u}_{i}}{\partial x_{j}}\right)+\frac{1}{F r^{2}} g_{i}-\frac{1}{R e} \frac{\overline{u_{i} u_{j}}}{\partial x_{j}}, \quad i=1,2
$$




$$
\frac{\partial \bar{u}_{i}}{\partial x_{i}}=0
$$

em que $\bar{u}_{i}$ é a i-ésima componente da velocidade média, e $\bar{P}$ é a pressão média. O termo $\overline{u_{i} u_{j}}$ é o tensor das tensões de Reynolds que será modelado neste trabalho pela aproximação de Boussinesq [9]

$$
\overline{u_{i} u_{j}}=-\nu_{t} D_{i j}+\frac{2}{3} \kappa \delta_{i j}, \quad i=1,2,
$$

em que

$$
\begin{aligned}
\nu_{t} & =C_{\mu} \frac{\kappa^{2}}{\varepsilon} \mathrm{e} \\
D_{i j} & =\frac{\partial \bar{u}_{i}}{\partial x_{j}}+\frac{\partial \overline{u_{j}}}{\partial x_{i}},
\end{aligned}
$$

sendo $\kappa$ a energia cinética da turbulência, $\delta_{i j}$ é o operador delta de Kronecker, $\nu_{t}$ é a viscosidade turbulenta local, $C_{\mu}$ é uma constante empírica e $\varepsilon$ é a dissipação de energia cinética $\kappa$ definida por

$$
\varepsilon=\nu\left(\overline{\frac{\partial u_{i}}{\partial x_{j}}}\right)^{2} .
$$

A determinação das variáveis $\kappa$ e $\varepsilon$ será feita pela modelagem $\kappa-\varepsilon$ padrão, isto é, pela resolução das EDPs de transporte

$$
\begin{gathered}
\frac{\partial \kappa}{\partial t}+\frac{\partial\left(\kappa u_{j}\right)}{\partial x_{j}}=\frac{1}{R e} \frac{\partial}{\partial x_{j}}\left[\left(1+\frac{\nu_{t}}{\sigma_{\kappa}}\right) \frac{\partial \kappa}{\partial x_{j}}\right]+\nu_{t} D_{i j} \frac{\partial u_{i}}{\partial x_{j}}-\varepsilon, \\
\frac{\partial \varepsilon}{\partial t}+\frac{\partial\left(\varepsilon u_{j}\right)}{\partial x_{j}}=\frac{1}{R e} \frac{\partial}{\partial x_{j}}\left[\left(1+\frac{\nu_{t}}{\sigma_{\varepsilon}}\right) \frac{\partial \varepsilon}{\partial x_{j}}\right]+\frac{\varepsilon}{\kappa}\left(C_{1 \varepsilon} \nu_{t} D_{i j} \frac{\partial u_{i}}{\partial x_{j}}-C_{2 \varepsilon} \varepsilon\right),
\end{gathered}
$$

em que $\sigma_{\kappa}=1.0$ e $\sigma_{\varepsilon}=1.3$ são os coeficientes de difusão turbulentos, $C_{1 \varepsilon}=1.44, C_{2 \varepsilon}=1.92 \mathrm{e}$ $C_{\mu}=0.09$ são constantes empíricas.

As condições iniciais e de contorno adotadas neste trabalho para as variáveis médias velocidade e pressão são as mesmas descritas na seção 2.3. As condições iniciais e de contorno para as variáveis turbulentas são descritas na sequência.

As condições iniciais são (ver Brandi [10])

$$
\kappa=0.08 R e, \quad \varepsilon=\frac{100 \kappa}{3} \sqrt{\frac{\kappa}{R e}}
$$

e as condições de contorno são as seguintes:

- Na entrada e na saída de fluido: as condições no injetor coincidem com às impostas para $\kappa$ e $\varepsilon$ em (2.54), e no ejetor essas variáveis turbulentas são calculadas pela aplicação da condição 
de Neumann

$$
\frac{\partial \kappa}{\partial n}=0, \quad \frac{\partial \varepsilon}{\partial n}=0
$$

- Na superfície rígida: próximo ao contorno rígido é aplicada uma modificação das leis clássicas de parede (ver [21], [59] e [10]);

- Na superfície livre do fluido:

$$
\begin{aligned}
P+\frac{2}{3 R e} \kappa-\frac{2}{R e}\left(1+\nu_{t}\right)\left[\frac{\partial u}{\partial x} n_{x}^{2}+\frac{\partial v}{\partial y} n_{y}^{2}+\left(\frac{\partial u}{\partial y}+\frac{\partial v}{\partial x}\right) n_{x} n_{y}\right] & =0 \\
\frac{1}{R e}\left(1+\nu_{t}\right)\left[2 \frac{\partial u}{\partial x} m_{x} n_{x}+2 \frac{\partial v}{\partial y} m_{y} n_{y}+\left(\frac{\partial u}{\partial y}+\frac{\partial v}{\partial x}\right)\left(m_{x} n_{y}+m_{y} n_{x}\right)\right] & =0 .
\end{aligned}
$$

\subsection{Modelo Oldroyd-B}

Segundo a formulação EVSS (Elastic Viscous Split Stress) [54], o problema 2D de escoamento de um fluido incompressível viscoelástico tipo Oldroyd-B (ver, por exemplo, Bird et al. [8]) é modelado por

$$
\begin{gathered}
\frac{\partial u_{i}}{\partial t}+\frac{\partial\left(u_{i} u_{j}\right)}{\partial x_{j}}=-\frac{\partial P}{\partial x_{i}}+\frac{\beta}{R e} \frac{\partial}{\partial x_{j}}\left(\frac{\partial u_{i}}{\partial x_{j}}\right)+\frac{\partial T_{i j}}{\partial x_{j}}+\frac{1}{F r^{2}} g_{i}, \quad i=1,2 \\
T_{i j}+W e\left[\frac{\partial T_{i j}}{\partial t}+\frac{\partial\left(u_{k} T_{i j}\right)}{\partial x_{k}}+T_{i k} \frac{\partial u_{j}}{\partial x_{k}}+T_{j k} \frac{\partial u_{i}}{\partial x_{k}}\right]=\frac{2(1-\beta)}{R e}\left(\frac{\partial u_{i}}{\partial x_{j}}+\frac{\partial u_{j}}{\partial x_{i}}\right) \\
\frac{\partial u_{j}}{\partial x_{j}}=0
\end{gathered}
$$

em que $T_{i j}$ é o tensor extra tensão, We é o número de Weissenberg dado por

$$
W e=\frac{\lambda_{1} U_{0}}{L}
$$

$\beta$ é a razão de retardamento dada por

$$
\beta=\frac{\lambda_{2}}{\lambda_{1}}
$$

$\lambda_{1}$, em (2.61) e (2.62), é o tempo de relaxação e $\lambda_{2}$, em (2.62), é o tempo de retardamento.

As condições de contorno adotadas para o campo velocidade são iguais àquelas mencionadas na seção 2.3. A condição inicial para as componentes do tensor $T_{i j}$ são consideradas nulas $\left(T_{i j}=0\right)$ e as condições de contorno para as componentes do tensor $T_{i j}$ são definidas como seguem:

- Na entrada e saída de fluido: no injetor são consideradas

$$
T_{11}=T_{12}=T_{22}=0
$$


e no ejetor de fluido

$$
\frac{\partial T_{11}}{\partial n}=\frac{\partial T_{12}}{\partial n}=\frac{\partial T_{22}}{\partial n}
$$

- Na superfície rígida: as condições são derivadas da aplicação da condição no-slip (ver [48]), isto é, no contorno rígido paralelo ao eixo x consideram-se

$$
\begin{aligned}
T_{11}^{\left(t+\delta_{t}\right)} & =\exp \left(-\frac{\delta_{t}}{W e} t\right) T_{11}^{(t)}+\delta_{t}\left[\frac{\partial u}{\partial y} \exp \left(-\frac{\delta_{t}}{W e}\right) T_{12}+\frac{\partial u}{\partial y} T_{12}\right], \\
T_{12}^{\left(t+\delta_{t}\right)} & =\exp \left(-\frac{\delta_{t}}{W e} t\right) T_{12}^{(t)}+\frac{(1-\beta)}{R e} \frac{\partial u}{\partial y}\left[1-\exp \left(-\frac{\delta_{t}}{W e}\right)\right], \\
T_{22} & =0,
\end{aligned}
$$

e no contorno rígido paralelo ao eixo y aplicam-se

$$
\begin{aligned}
T_{22}^{\left(t+\delta_{t}\right)} & =\exp \left(-\frac{\delta_{t}}{W e}\right) T_{22}^{(t)}+\delta_{t}\left[\frac{\partial v}{\partial x} \exp \left(-\frac{\delta_{t}}{W e}\right) T_{12}+\frac{\partial v}{\partial x} T_{12}\right], \\
T_{12}^{\left(t+\delta_{t}\right)} & =\exp \left(-\frac{\delta_{t}}{W e}\right) T_{12}^{(t)}+\frac{(1-\beta)}{R e} \frac{\partial v}{\partial x}\left[1-\exp \left(-\frac{\delta_{t}}{W e}\right)\right], \\
T_{11} & =0
\end{aligned}
$$

em que $\delta_{t}$ é o espaçamento temporal;

- Na superfície livre:

$$
\begin{gathered}
P-\frac{2 \beta}{R e}\left[\frac{\partial u}{\partial x} n_{x}^{2}+\frac{\partial v}{\partial y} n_{y}^{2}+\left(\frac{\partial u}{\partial y}+\frac{\partial v}{\partial x}\right) n_{x} n_{y}\right]+T_{11} n_{x}^{2}+2 T_{12} n_{x} n_{y}+T_{22} n_{y}^{2}=0, \\
\frac{2 \beta}{R e}\left[\left(\frac{\partial v}{\partial y}-\frac{\partial u}{\partial x}\right) n_{x} n_{y}+\left(\frac{\partial u}{\partial y}-\frac{\partial v}{\partial x}\right)\left(n_{x}^{2}-n_{y}^{2}\right)\right]+\left(T_{22}-T_{11}\right) n_{x} n_{y}+T_{12}\left(n_{x}^{2}-n_{y}^{2}\right)=0 .
\end{gathered}
$$

\subsection{Tema da Pesquisa}

O tema central deste trabalho de mestrado é o desenvolvimento, a análise e a implementação de esquemas de captura de choque tipo upwind de alta resolução para termos convectivos (lineares ou não lineares) presentes nas leis de conservação (2.1) e (2.23) e nas equações de Navier-Stokes (2.32), (2.33), (2.35), e também para os modelo $\kappa-\varepsilon$ em (2.46), (2.52) e (2.53) e o modelo Oldroyd-B em (2.58) e (2.59). 


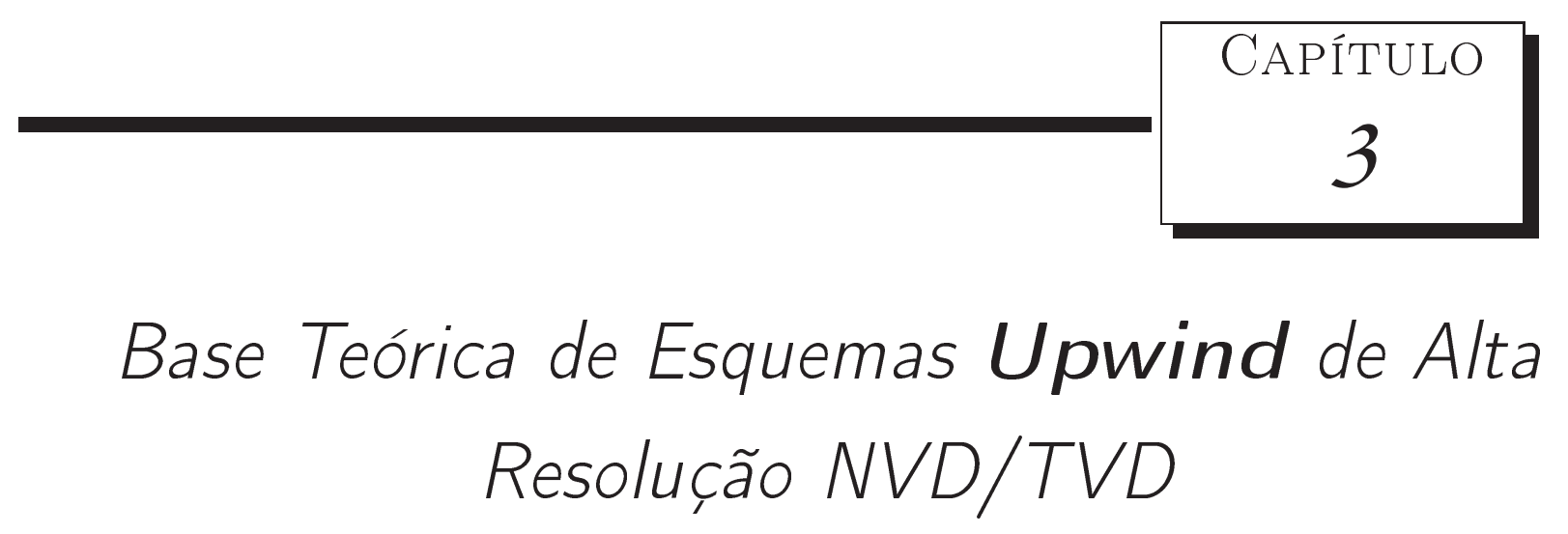

Neste capítulo são apresentadas as principais ferramentas necessárias para o desenvolvimento de esquemas de captura de choque, tipo upwind de alta resolução, nos contextos de variáveis normalizadas de Leonard [42], do critério de limitação CBC de Gaskell e Lau [28] e das restrições TVD de Harten [30]. Ainda neste capítulo, apresenta-se os principais conceitos para a determinação de limitadores de fluxo.

\subsection{Molécula Computacional de Três Pontos}

Para se derivar um esquema upwind de alta ordem para os termos convectivos, nos contextos de variáveis normalizadas, do critério de limitação CBC e das restrições TVD, é considerada uma molécula computacional envolvendo três pontos: $D$ (Downstream), $U$ (Upstream) e $R$ (Remote-upstream), que são, respectivamente, as posições a jusante, a montante e a mais a montante da interface $f$ entre duas células computacionais. A escolha dessas posições $D, U$ e $R$ depende do sinal da velocidade média $V_{f}$ de uma variável convectada $\phi$ em $f, \phi_{f}$. Essas definições estão ilustradas na Figura 3.1.

Qualquer esquema de convecção do tipo upwind, que utilize somente os valores de $\phi$ nos pontos $D, U$ e $R$ para avaliar $\phi_{f}$, pode ser representado na forma funcional

$$
\phi_{f}=\phi_{f}\left(\phi_{D}, \phi_{U}, \phi_{R}\right) .
$$



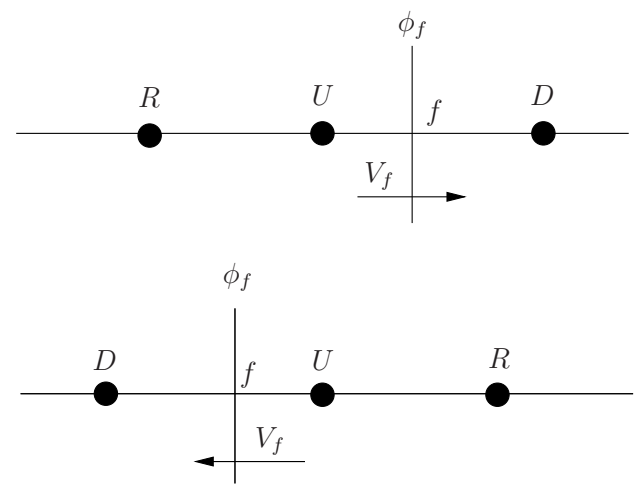

Figura 3.1: Nós computacionais. Posições dos nós computacionais $D, R$ e $U$ conforme o sinal da velocidade $V_{f}$ (fluxo) de uma variável convectada.

\subsection{Variáveis Normalizadas}

Seja $\phi$ a variação de um escalar na direção normal a uma face $f$. Para facilitar a análise, a variável original $\phi$, numa posição arbitrária, é transformada em variáveis normalizadas de Leonard [42] por

$$
\left.\hat{\phi}_{[}\right]=\frac{\left.\phi_{[}\right]-\phi_{R}}{\phi_{D}-\phi_{R}}
$$

em que $\phi_{D}$ e $\phi_{R}$ são, respectivamente, os valores não-normalizados da propriedade $\phi$ nos pontos $D$ e $R$.

A partir da definição (3.2) tem-se

$$
\hat{\phi}_{R}=0 \text { e } \hat{\phi}_{D}=1
$$

e, se $\hat{\phi}_{U}=0, \phi_{U}=\phi_{R}$ e se $\hat{\phi}_{U}=1, \phi_{U}=\phi_{D}$. Assim, qualquer esquemas upwind de alta resolução, escrito na forma (3.1), pode ser reescrito de forma funcional simplificada como

$$
\hat{\phi}_{f}=\hat{\phi}_{f}\left(\hat{\phi}_{U}\right)
$$

\subsection{Diagrama de Variáveis Normalizadas}

O diagrama de variáveis normalizadas (NVD - Normalized Variable Diagram) proposto por Leonard [42] tem como base a definição (3.2) e a relação funcional (3.3). Essa relação funcional é visualizada no plano $\hat{\phi}_{f} \perp \hat{\phi}_{U}$ e é crucial para a análise do esquema. Por exemplo, na Figura 3.2 são apresentados, no contexto NVD, os esquemas QUICK de Leonard [41], o esquema FOU de Spalding [61] e o esquema de diferenças central de segunda ordem clássica (ver, por exemplo, 
Cunha [19]). Esses esquemas em variáveis normalizadas são, respectivamente, dados por

$$
\begin{aligned}
& \hat{\phi}_{f}=0.75+0.75\left(\hat{\phi}_{U}-0.5\right) \\
& \hat{\phi}_{f}=\hat{\phi}_{U} \\
& \hat{\phi}_{f}=0.75+0.5\left(\hat{\phi}_{U}-0.5\right) .
\end{aligned}
$$

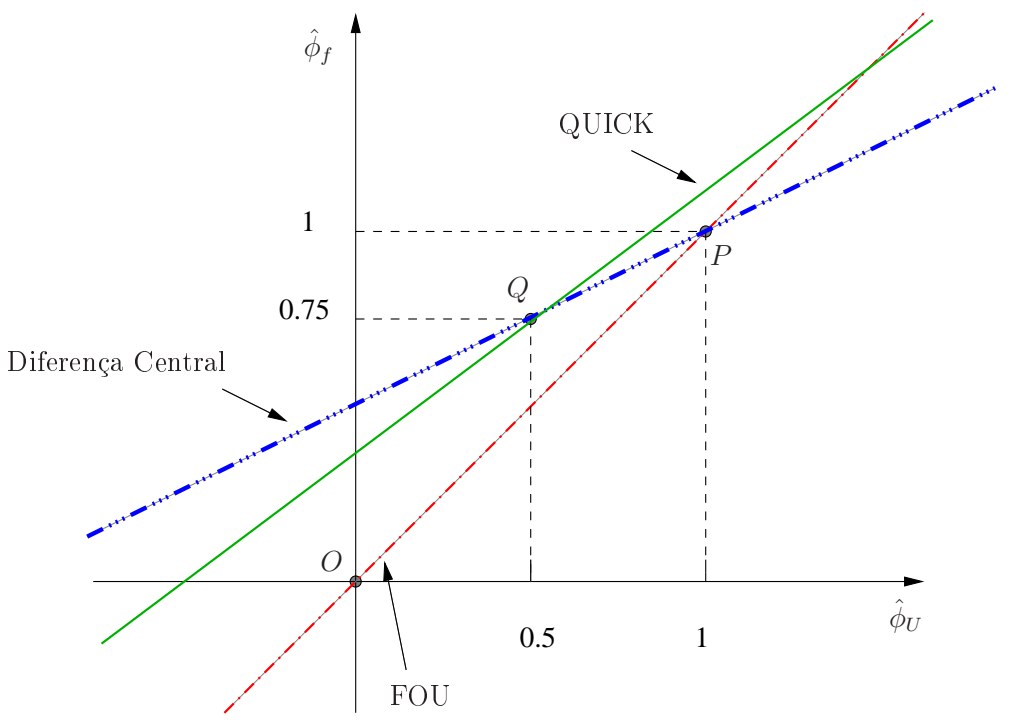

Figura 3.2: Diagrama de variáveis normalizadas.

Neste contexto, estudos de Leonard [42] mostram que os esquemas (definidos pela relação funcional (3.3)) que possuem intersecção com o segundo quadrante do plano $\hat{\phi}_{f} \perp \hat{\phi}_{U}$ podem produzir oscilações numéricas (não físicas) e os que interceptam o quarto quadrante são artificialmente difusivos. Diante disso, Leonard [42] introduziu uma condição necessária para um esquema ser livre de oscilações e difusão numéricas: a relação funcional deve estar definida na origem $O=(0,0)$. Ainda mais, segundo o próprio Leonard, experimentos numéricos mostram que os esquemas em NVD que interceptam a reta $\hat{\phi}_{U}=1$ acima de $\hat{\phi}_{f}=1$ geram soluções oscilatórias e aqueles que interceptam a reta $\hat{\phi}_{U}=1$ abaixo de $\hat{\phi}_{f}=1$ injetam difusão numérica. Assim, Leonard introduziu uma segunda condição necessária para um esquema ser livre de oscilações e difusão numérica: a relação funcional deve estar definida em $P=(1,1)$. Leonard (ver também Waterson e Deconink [75] ou Lin e Chieng [46]) também provou que para se ter um esquema de segunda ordem de precisão é necessário e suficiente que ele, dado pela relação funcional (3.3), esteja definido no ponto $Q=\left(\frac{1}{2}, \frac{3}{4}\right)$; e, além disso, para alcançar terceira ordem ele deve ter inclinação $\frac{3}{4}$ nesse mesmo ponto. Em síntese, para se derivar um esquema upwind no contexto NVD, não linear, monôtonico (livre de oscilações) e que atinja até terceira ordem, as seguintes condições sobre $\hat{\phi}_{f}=\hat{\phi}_{f}\left(\hat{\phi}_{U}\right)$ (definida em $0 \leq \hat{\phi}_{U} \leq 1$ ) devem ser impostas:

- passar por $P=(1,1)$ (condição necessária);

- passar por $O=(0,0)$ (condição necessária); 
- passar por $Q=\left(\frac{1}{2}, \frac{3}{4}\right)$ (condição necessária e suficiente para segunda ordem de precisão);

- passar por $Q$ com inclinação de $\frac{3}{4}$ (condição necessária e suficiente para terceira ordem de precisão).

Leonard ainda aconselha que para $\hat{\phi}_{U}$ menores que 0 e maiores que 1 , os esquemas devem ser estendidos de maneira contínua pelo esquema FOU. Lin e Chieng [46] aconselham também que a relação funcional (3.3) seja continuamente diferenciável em todo o domínio de $\hat{\phi}_{U}$, pois segundo esses autores se este fato ocorrer, então os problemas de convergência em malhas grosseiras são evitados.

\subsection{Critério de Limitação CBC}

Em busca por esquemas upwind limitados, isto é, aqueles cujos resultados numéricos não ultrapassam os valores máximos e mínimos inerentes ao processo físico (e portanto não geram oscilações), Gaskell e Lau [28] introduziram o critério de limitação denominado CBC que, segundo Waterson e Deconink [75], é a referência mais aceita sobre critérios de limitação usando variáveis normalizadas. Essencialmente, uma solução é limitada se o seu valor em um ponto computacional é limitado pelos valores da solução nos pontos vizinhos e esta é a base do tratamento dos termos convectivos em variáveis normalizadas. Dentro deste contexto, Gaskell e Lau propuseram que um esquema definido por uma função continua, ou continua por partes, $\hat{\phi}_{f}=\hat{\phi}_{f}\left(\hat{\phi}_{U}\right)$ é limitado se as seguintes condições são satisfeitas:

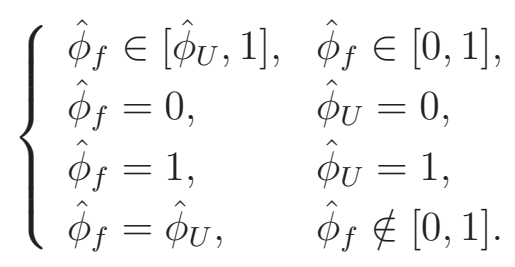

A Figura 3.3 ilustra a região CBC definida por (3.4). Segundo Gaskell e Lau, o gráfico que define o esquema limitado $\hat{\phi}_{f}\left(\hat{\phi}_{U}\right)$ deve estar inteiramente contido nessa região (a recíproca é verdadeira).

Abaixo estão alguns exemplos de esquemas que satisfazem o critério de limitação CBC. Os mesmos são apresentados em variáveis não normalizadas (forma aplicada na implementação).

- ADBQUICKEST [27]:

$$
\hat{\phi}_{f}= \begin{cases}(2-|\theta|) \hat{\phi}_{U}, & 0<\hat{\phi}_{U}<a, \\ \hat{\phi}_{U}+\frac{1}{2}(1-|\theta|)\left(1-\hat{\phi}_{U}\right)-\frac{1}{6}\left(1-\theta^{2}\right)\left(1-\hat{\phi}_{U}\right), & a \leq \hat{\phi}_{U} \leq b, \\ 1-|\theta|+|\theta| \hat{\phi}_{U}, & b<\hat{\phi}_{U}<1, \\ \hat{\phi}_{U}, & \hat{\phi}_{U} \notin[0,1],\end{cases}
$$




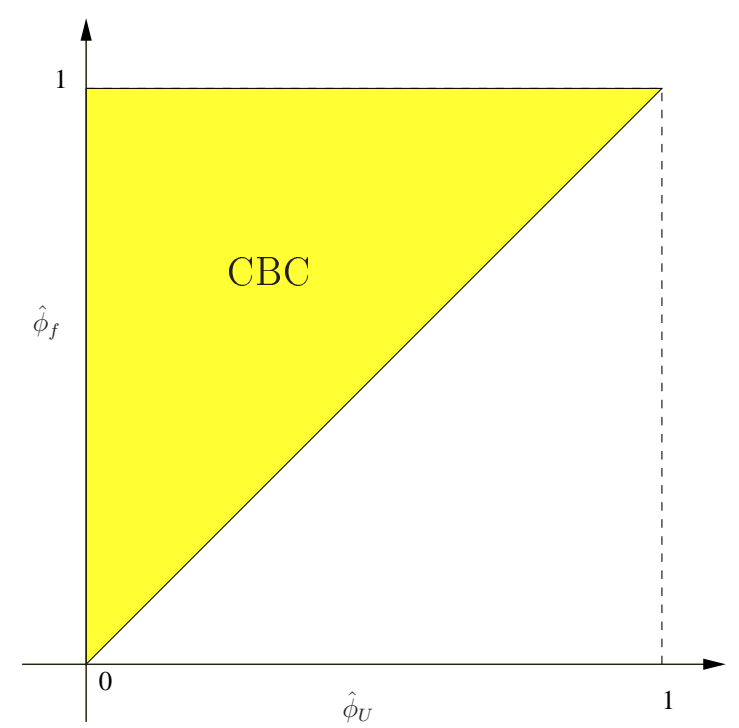

Figura 3.3: Região CBC em variáveis normalizadas.

com

$$
a=\frac{2-3|\theta|+\theta^{2}}{7-9|\theta|+2 \theta^{2}} \quad \text { e } b=\frac{-4+3|\theta|+\theta^{2}}{-5+3|\theta|+2 \theta^{2}}
$$

em que $\theta$ é o número de Courant;

- TOPUS [52]:

$$
\hat{\phi}_{f}= \begin{cases}\alpha \hat{\phi}_{U}^{4}+(-2 \alpha+1) \hat{\phi}_{U}^{3}+\left(\frac{5 \alpha-10}{4}\right) \hat{\phi}_{U}^{2}+\left(\frac{-\alpha+10}{4}\right) \hat{\phi}_{U}, & 0 \leq \phi_{U} \leq 1, \\ \hat{\phi}_{U}, & \hat{\phi}_{U} \notin[0,1],\end{cases}
$$

em que $\alpha \in[0,2]$. Em todo esse texto emprega-se $\alpha=2$ (ver Queiroz [52]);

- CUBISTA [2]:

$$
\hat{\phi}_{f}= \begin{cases}\frac{7}{4} \hat{\phi}_{U}, & 0 \leq \hat{\phi}_{U}<\frac{3}{8} \\ \frac{1}{8}\left(3+6 \hat{\phi}_{U}\right), & \frac{3}{8} \leq \hat{\phi}_{U}<\frac{3}{4} \\ \frac{1}{4}\left(3+\hat{\phi}_{U}\right), & \frac{3}{4} \leq \hat{\phi}_{U} \leq 1 \\ \hat{\phi}_{U}, & \hat{\phi}_{U}, \notin[0,1]\end{cases}
$$

- VONOS [73]:

$$
\hat{\phi}_{f}= \begin{cases}10 \hat{\phi}_{U}, & 0 \leq \hat{\phi}_{U}<\frac{3}{74}, \\ \frac{1}{8}\left(3+6 \hat{\phi}_{U}\right), & \frac{3}{74} \leq \hat{\phi}_{U}<\frac{1}{2} \\ \frac{3}{2} \hat{\phi}_{U}, & \frac{1}{2} \leq \hat{\phi}_{U}<\frac{2}{3} \\ 1, & \frac{2}{3} \leq \hat{\phi}_{U} \leq 1, \\ \hat{\phi}_{U}, & \hat{\phi}_{U} \notin[0,1] ;\end{cases}
$$


- Superbee [4]:

$$
\hat{\phi}_{f}= \begin{cases}2 \hat{\phi}_{U}, & 0 \leq \hat{\phi}_{U}<\frac{1}{3}, \\ \frac{1}{2}\left(1+\hat{\phi}_{U}\right), & \frac{1}{3} \leq \hat{\phi}_{U} \leq \frac{1}{2} \\ \frac{3}{2} \hat{\phi}_{U}, & \frac{1}{2} \leq \hat{\phi}_{U}<\frac{2}{3}, \\ 1, & \frac{2}{3} \leq \hat{\phi}_{U} \leq 1, \\ \hat{\phi}_{U}, & \hat{\phi}_{U} \notin[0,1] .\end{cases}
$$

Apesar do critério CBC de Gaskell e Lau [28] tratar o problema de estabilidade adequadamente, ele não garante convergência da solução numérica. Para convergência, as restrições TVD de Harten [30] devem ser satisfeitas.

\subsection{Restrições TVD}

As restrições TVD se traduzem em uma ferramenta matemática rigorosa introduzida por Harten [30] que combina monotonicidade e alta ordem de precisão. Os métodos TVD, além de gerarem soluções numéricas convergentes e fisicamente aceitáveis, são conservativos (ver LeVeque [44]). Formalmente, dada a sequência de aproximações discretas

$$
\phi(t)=\phi_{i}(t)_{i \in \mathbb{Z}}
$$

a variação total (TV - Total Variation) da solução discreta, no nível de tempo $t$, é definida por

$$
T V(\phi(t))=\sum_{i \in \mathbb{Z}}\left|\phi_{i+1}(t)-\phi_{i}(t)\right|
$$

Considerando o esquema de diferenças explícito envolvendo $(2 k+1)$ pontos da forma

$$
\phi_{i}^{n+1}=H\left(\phi_{i-k}^{n}, \ldots, \phi_{i+k}^{n}\right), \forall n \geq 0, i \in \mathbb{Z}
$$

onde $H: \mathbb{R}^{2 k+1} \longrightarrow \mathbb{R}$ é uma função contínua e $\phi_{i}^{n}$ uma aproximação da solução exata nos pontos de malha (uniforme) $\left(x_{i}, t_{n}\right)$, com $x_{i}=i \delta_{x}, t_{n}=n \delta_{t}$, sendo $\delta_{x}$ o passo espacial e $\delta_{t}$ a marcha no tempo, tem-se por definição:

Definição 1 (Harten [32]) O esquema (3.12) é TVD se para todo conjunto de dados $\phi^{n}$, os valores $\phi^{n+1}$ computados satisfazem

$$
T V\left(\phi^{n+1}\right) \leq T V\left(\phi^{n}\right)
$$

Teorema 1 (Harten [32]) (ver também LeVeque [44] e Toro [68]) Métodos TVD são convergentes para problemas de valor inicial em leis de conservação. 
Com as considerações e conceitos introduzidos por Harten [30, 32], Sweby [62] definiu (no contexto NVD) o conjunto de restrições

$$
\begin{cases}\hat{\phi}_{f} \in\left[\hat{\phi}_{U}, 2 \hat{\phi}_{U}\right] \text { e } \hat{\phi}_{f} \leq 1, & \hat{\phi}_{U} \in[0,1], \\ \hat{\phi}_{f}=\hat{\phi}_{U}, & \hat{\phi}_{U} \notin[0,1]\end{cases}
$$

as quais deram origem a região TVD, ilustrada na Figura 3.4. Segundo Sweby, o gráfico que define o esquema $\hat{\phi}_{f}=\hat{\phi}_{f}\left(\hat{\phi}_{U}\right)$ deve estar inteiramente contido nessa região para que o mesmo seja TVD (a recíproca também é verdadeira).

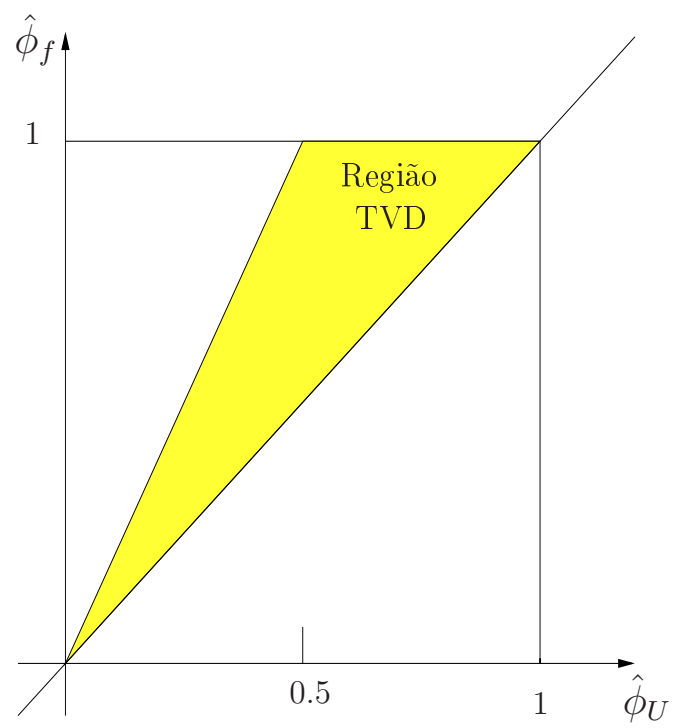

Figura 3.4: Região TVD em variáveis normalizadas.

\subsection{Limitadores de Fluxo}

Uma vez derivado um esquema upwind, embasado nas condições de Leonard, no critério CBC e na restrição TVD, o limitador de fluxo correspondente é determinado reescrevendo-se o esquema da seguinte forma (ver Sweby [62] e Waterson e Deconinck [75]):

$$
\hat{\phi}_{f}=\hat{\phi}_{U}+\frac{1}{2} \psi(r)\left(1-\hat{\phi}_{U}\right)
$$

em que $\psi(r)$ é o limitador de fluxo, que determina o nível de antidifusividade, e $r$ é a razão de dois gradientes consecutivos (um sensor que detecta variação nos gradientes - ver Figura 3.5) dado por

$$
r=\frac{\left(\frac{\partial \phi}{\partial x}\right)_{g}}{\left(\frac{\partial \phi}{\partial x}\right)_{f}}
$$


o qual em malhas uniformes torna-se

$$
r=\frac{\phi_{U}-\phi_{R}}{\phi_{D}-\phi_{U}}
$$

e sua normalização fica

$$
r=\frac{\hat{\phi}_{U}}{1-\hat{\phi}_{U}} .
$$

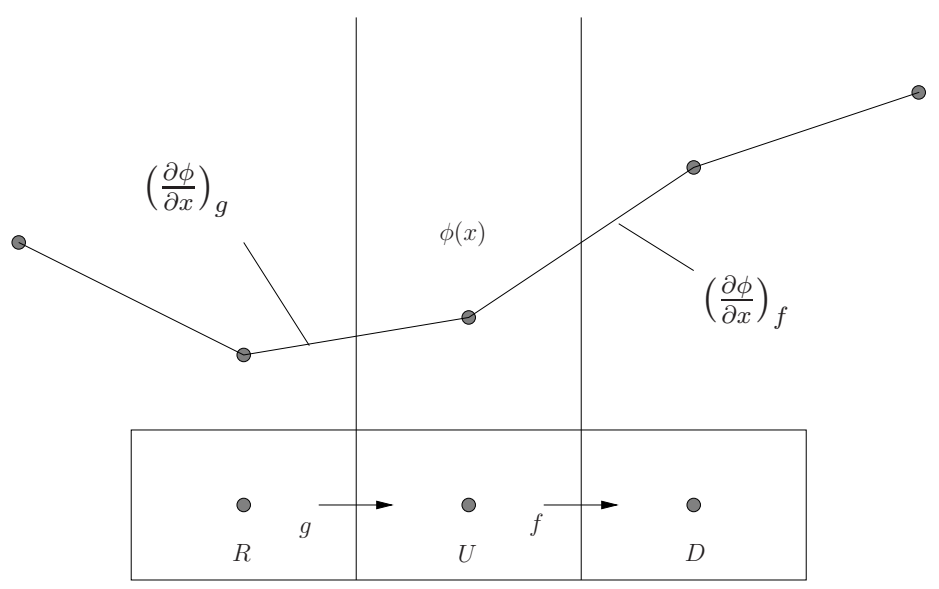

Figura 3.5: Representação esquemática dos gradientes consecutivos.

Segundo Waterson e Deconinck [75], um esquema convectivo de alta precisão deve ter preservação da linearidade, ou seja, o esquema deve respeitar exatamente a variação linear da solução, isso é satisfeito quando

$$
\psi(1)=1
$$

Segundo Sweby [62], em malhas uniformes, a condição (3.19) é necessária para que o esquema atinja segunda ordem de precisão. De acordo com Zijlema [80], a condição sobre o limitador de fluxo

$$
\psi^{\prime}(1)=\frac{1}{4}
$$

é necessária e suficiente para que um esquema atinja terceira ordem de precisão. Zijlema afirma ainda que se o limitador $\psi(r)$ é uma função suave de $r$, então o esquema apresenta melhor comportamento, com respeito à convergência, durante o processo numérico.

A restrição TVD pode ser imposta também para o desenvolvimento dos limitadores de fluxo. Para tanto, Sweby [62] transformou os conceitos introduzidos por Harten [30] em um conjunto de limitações para o comportamento das funções limitadores de fluxo, definidas por 


$$
\begin{cases}\psi(r)=0, & r \leq 0 \\ 0 \leq \psi(r) \leq \min \{2,2 r\}, & r>0 .\end{cases}
$$

Essas condições sintetizam a região TVD no plano $\psi \perp r$ (também conhecida como diagrama de Sweby), ilustrada na Figura 3.6. Segundo Sweby, um esquema é TVD se o seu limitador de fluxo $\psi(r)$ esta contido nesta região (a reciproca é verdadeira).

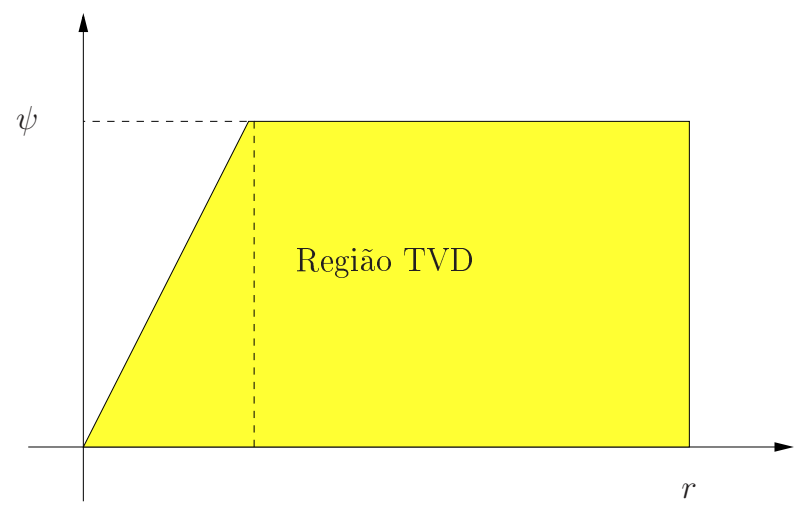

Figura 3.6: Diagrama de Sweby.

Sweby [62] também introduziu uma condição suficiente, porém não necessária, para que um esquema seja monotônico. Esta condição é definida por

$$
\text { se } r \longrightarrow 0, \text { então } \psi^{\prime}(r)=2
$$

e é conhecida como o princípio de monotonicidade de Sweby.

Segundo Waterson e Deconinck [75], se um esquema upwind possui relação funcional $\hat{\phi}_{f}=$ $\hat{\phi}_{f}\left(\hat{\phi}_{U}\right)$, em $[0,1]$, definida por um polinômio de grau $n$, então seu limitador de fluxo correspondente $\psi(r)=\psi\left(\frac{\hat{\phi}_{U}}{1-\hat{\phi}_{U}}\right)$, denotado neste trabalho por $\Psi=\Psi\left(\hat{\phi}_{U}\right)$, será definido por um polinômio $\Psi\left(\hat{\phi}_{U}\right)$ de grau $n-1$. Esta idéia é essencial para a determinação do limitador de fluxo após o desenvolvimento do esquema polinomial em variáveis normalizadas.

A vantagem de se trabalhar com funções polinomiais é que elas são suaves e apresentam melhor comportamento convergente em malhas grosseiras (ver Zijlema [80]). Isto justifica o desenvolvimento e aplicação dos esquemas polinomiais FDPUS-C1 e SDPUS-C1 objeto desse trabalho de mestrado.

Abaixo estão alguns exemplos de limitadores de fluxo presentes na literatura. - ADBQUICKEST [27]:

$$
\psi(r)=\max \left\{0, \quad \min \left[2 r, \frac{2+\theta^{2}-3 \theta+\left(1-\theta^{2}\right) r}{3-3 \theta}, 2\right]\right\}
$$


- TOPUS [52]:

$$
\psi(r)=\max \left\{0, \frac{0.5(|r|+r)\left(6 r^{2}+2 r\right)}{(1+|r|)^{3}}\right\}
$$

- van Leer [70]:

$$
\psi(r)=\frac{r+|r|}{1+|r|}
$$

- Superbee [4]:

$$
\psi(r)=\max \{0, \quad \min (1,2 r), \quad \min (2, r)\}
$$

- MC [71]:

$$
\psi(r)=\max \left\{0, \quad \min \left(1, \frac{1+r}{2}, 2,2 r\right)\right\}
$$




\section{Desenvolvimento dos Novos Esquemas Upwind FDPUS-C1 e SDPUS-C1}

Neste capítulo apresenta-se o desenvolvimento dos esquemas upwind de alta ordem originais FDPUS-C1 e SDPUS-C1.

\subsection{O Esquema FDPUS-C1}

Para a derivação do novo esquema upwind polinomial FDPUS-C1, para cálculo dos fluxos numéricos nas interfaces das células computacionais, considera-se parte de um polinômio de grau cinco, definido em $\hat{\phi}_{U} \in[0,1]$, dado por

$$
\hat{\phi}_{f}\left(\hat{\phi}_{U}\right)=\sum_{l=0}^{5} a_{l} \hat{\phi}_{U}^{l},
$$

e como recomendado por Leonard [42], para $\hat{\phi}_{U} \notin[0,1]$, considera-se o esquema FOU [61], dado por

$$
\hat{\phi}_{f}=\hat{\phi}_{U}
$$

Derivando o polinômio (4.1) em relação a $\hat{\phi}_{U}$, obtém -se

$$
\hat{\phi}_{f}^{\prime}\left(\hat{\phi}_{U}\right)=\sum_{l=1}^{5} l a_{l} \hat{\phi}_{U}^{l-1}
$$

A determinação dos coeficientes $a_{l}, l=0, \cdots, 5$, em (4.1) é feita impondo-se as condições de Leonard [42] para monotonicidade e terceira ordem de precisão, como apresentadas na seção 3.3 do capítulo 3 , isto é, 
- impondo-se $\hat{\phi}_{f}(0)=0$ acarreta

$$
a_{0}=0
$$

- impondo-se $\hat{\phi}_{f}(1)=1$ acarreta

$$
a_{5}+a_{4}+a_{3}+a_{2}+a_{1}+a_{0}=1 ;
$$

- impondo-se $\hat{\phi}_{f}\left(\frac{1}{2}\right)=\frac{3}{4}$ acarreta

$$
\begin{aligned}
a_{5}\left(\frac{1}{2}\right)^{5}+a_{4}\left(\frac{1}{2}\right)^{4}+a_{3}\left(\frac{1}{2}\right)^{3}+a_{2}\left(\frac{1}{2}\right)^{2}+a_{1}\left(\frac{1}{2}\right)+a_{0} & =\frac{3}{4} \text { ou } \\
a_{5}+2 a_{4}+4 a_{3}+8 a_{2}+16 a_{1}+32 a_{0} & =24
\end{aligned}
$$

- impondo-se $\hat{\phi}_{f}^{\prime}\left(\frac{1}{2}\right)=\frac{3}{4}$ acarreta

$$
\begin{aligned}
5 a_{5}\left(\frac{1}{2}\right)^{4}+4 a_{4}\left(\frac{1}{2}\right)^{3}+3 a_{3}\left(\frac{1}{2}\right)^{2}+2 a_{2}\left(\frac{1}{2}\right)+a_{1} & =\frac{3}{4} \text { ou } \\
5 a_{5}+8 a_{4}+12 a_{3}+16 a_{2}+16 a_{1} & =12 .
\end{aligned}
$$

Para fechar o sistema (4.4) - (4.7), são consideradas as condições para que a expressão global do esquema torne-se continuamente derivável ( $\hat{\phi}_{f}$ é de classe $C^{1}\left(\hat{\phi}_{U}\right)$, com $\hat{\phi}_{U} \in \mathbb{R}$ ), ou seja, $\hat{\phi}_{f}$ seja derivável em todo $\hat{\phi}_{U} \in \mathbb{R}$ com derivada contínua, ou seja,

- impondo-se $\hat{\phi}_{f}^{\prime}(0)=1$ acarreta

$$
a_{1}=1
$$

- impondo-se $\hat{\phi}_{f}^{\prime}(1)=1$ acarreta

$$
5 a_{5}+4 a_{4}+3 a_{3}+2 a_{2}+a_{1}=1 .
$$

Segundo Lin e Chieng [46], se $\hat{\phi}_{f}$ é de classe $C^{1}\left(\hat{\phi}_{U}\right)$, então os problemas de convergência em malhas grosseiras são praticamente evitados.

Da resolução do sistema (4.4) - (4.9), os coeficientes $a_{l}$ são dados por

$$
a_{0}=0, \quad a_{1}=1, \quad a_{2}=6, \quad a_{3}=-16, \quad a_{4}=14 \text { e } a_{5}=-4 .
$$

Substituindo os coeficientes $a_{l}$ em (4.1) e considerando (4.2), obtém-se uma nova formulação em variáveis normalizadas definida por

$$
\hat{\phi}_{f}= \begin{cases}-4 \hat{\phi}_{U}^{5}+14 \hat{\phi}_{U}^{4}-16 \hat{\phi}_{U}^{3}+6 \hat{\phi}_{U}^{2}+\hat{\phi}_{U}, & \hat{\phi}_{U} \in[0,1], \\ \hat{\phi}_{U}, & \hat{\phi}_{U} \notin[0,1] .\end{cases}
$$


Essa nova estratégia upwind denomina-se FDPUS-C1 (Five-Degree Polynomial Upwind Scheme of $C^{1}$ Class), uma vez que sua relação funcional, em [0,1], é parte de um polinômio de grau cinco e sua expressão global $\hat{\phi}_{f}\left(\hat{\phi}_{U}\right) \in C^{1}\left(\hat{\phi}_{U}\right)$, com $\hat{\phi}_{U} \in \mathbb{R}$. Aplicando a definição de variáveis normalizadas (ver (3.2)) em (4.11), obtém-se o esquema FDPUS-C1 em variáveis não normalizadas dado por

$$
\phi_{f}= \begin{cases}\phi_{R}+\left(\phi_{D}-\phi_{R}\right)\left[-4 \hat{\phi}_{U}^{5}+14 \hat{\phi}_{U}^{4}-16 \hat{\phi}_{U}^{3}+6 \hat{\phi}_{U}^{2}+\hat{\phi}_{U}\right], & \hat{\phi}_{U} \in[0,1], \\ \phi_{U}, & \hat{\phi}_{U} \notin[0,1],\end{cases}
$$

em que

$$
\hat{\phi}_{U}=\frac{\phi_{U}-\phi_{R}}{\phi_{D}-\phi_{R}}
$$

O limitador de fluxo correspondente $\psi(r)$ para o esquema FDPUS-C1 é obtido reescrevendo (4.11) na formulação (3.15). Para isso, comparam-se as equações (4.11) e (3.15) para obter $\psi(r)=0$ (uma vez que $\hat{\phi}_{f}=\hat{\phi}_{U}$ ) para $r<0$ (devido a definição de $r$ em (3.18) e por $\left.\hat{\phi}_{U} \notin[0,1]\right)$. Para $\hat{\phi}_{U} \in[0,1]$, considera-se que o limitador $\Psi=\Psi\left(\hat{\phi}_{U}\right)$ é um polinômio de grau 4 (lembrando que o esquema upwind em variáveis normalizadas é definido por um polinômio de grau 5) definido por

$$
\Psi\left(\hat{\phi}_{U}\right)=\sum_{m=0}^{4} \alpha_{m} \hat{\phi}_{U}^{m}
$$

Substituindo esse polinômio na definição (3.15), obtém-se

$$
\begin{aligned}
\hat{\phi}_{f} & =\hat{\phi}_{U}+\frac{1}{2}\left(\alpha_{4} \hat{\phi}_{U}^{4}+\alpha_{3} \hat{\phi}_{U}^{3}+\alpha_{2} \hat{\phi}_{U}^{2}+\alpha_{1} \hat{\phi}_{U}+\alpha_{0}\right)\left(1-\hat{\phi}_{U}\right) \\
& =\frac{-\alpha_{4}}{2} \hat{\phi}_{U}^{5}+\frac{\left(\alpha_{4}-\alpha_{3}\right)}{2} \hat{\phi}_{U}^{4}+\frac{\left(\alpha_{3}-\alpha_{2}\right)}{2} \hat{\phi}_{U}^{3}+\frac{\left(\alpha_{2}-\alpha_{1}\right)}{2} \hat{\phi}_{U}^{2}+\left[\frac{\left(\alpha_{1}-\alpha_{0}\right)}{2}+1\right] \hat{\phi}_{U}+\alpha_{0}
\end{aligned}
$$

Igualando as expressões (4.15) e (4.11), para $\hat{\phi}_{U} \in[0,1]$, determinam-se os coeficientes $\alpha_{m}$, dados por

$$
\alpha_{0}=0, \quad \alpha_{1}=0, \quad \alpha_{2}=6, \quad \alpha_{3}=-10, \quad \text { e } \alpha_{4}=4 .
$$

Substituindo esses coeficientes em (4.14), define-se o polinômio $\Psi\left(\hat{\phi}_{U}\right)$ por

$$
\Psi\left(\hat{\phi}_{U}\right)=8 \hat{\phi}_{U}^{4}-20 \hat{\phi}_{U}^{3}+12 \hat{\phi}_{U}^{2}
$$


Da definição (3.18), a expressão (4.17) pode ser reescrita como

$$
\begin{aligned}
\Psi\left(\hat{\phi}_{U}\right) & =8\left(\frac{r}{1+r}\right)^{4}-20\left(\frac{r}{1+r}\right)^{3}+12\left(\frac{r}{1+r}\right)^{2} \\
& =\frac{8 r^{4}-20 r^{3}(1+r)+12 r^{2}(1+r)^{2}}{(1+r)^{4}} \\
& =\frac{4 r^{3}+12 r^{2}}{(1+r)^{4}}=\psi(r) .
\end{aligned}
$$

Em resumo, o limitador de fluxo do esquema FDPUS-C1 é dado por

$$
\psi(r)= \begin{cases}\frac{4 r^{3}+12 r^{2}}{(1+r)^{4}}, & r \geq 0, \\ 0, & r<0 .\end{cases}
$$

Com o objetivo de se evitar condicionais na implementação computacional, reescreve-se as sentenças (4.18), para qualquer $r$, na forma

$$
\psi(r)=\frac{0.5(|r|+r)\left(4 r^{2}+12 r\right)}{(1+|r|)^{4}} .
$$

Na notação mais usual (ver LeVeque [44]), o limitador de fluxo FDPUS-C1 é escrito como

$$
\psi(r)=\max \left\{0, \frac{0.5(|r|+r)\left(4 r^{2}+12 r\right)}{(1+|r|)^{4}}\right\} .
$$

Nota-se que o esquema FDPUS-C1 é monotônico e de segunda ordem, já que o seu limitador de fluxo (4.18) satisfaz, para $r \geq 0$, a condição (3.19). De fato, pois

$$
\psi(1)=\frac{4+12}{2^{4}}=1
$$

Derivando a expressão (4.18), para $r \geq 0$, tem-se

$$
\psi^{\prime}(r)=\frac{\left(12 r^{2}+24 r\right)(1+r)^{4}-\left(4 r^{3}+12 r^{2}\right) 4(1+r)^{3}}{(1+r)^{8}} .
$$

Constata-se também que o esquema FDPUS-C1 pode atingir terceira ordem de precisão, já que o seu limitador de fluxo (4.18) satisfaz, para $r \geq 0$, a condição (3.20). De fato, pois

$$
\begin{aligned}
\psi^{\prime}(1) & =\frac{(12+24) 2^{4}-4(4+12) 2^{3}}{2^{8}} \\
& =\frac{1}{4} .
\end{aligned}
$$


É importante observar que o limitador de fluxo do esquema FDPUS-C1 (4.18) não satisfaz o princípio de Sweby, definido por (3.22), pois ele é de classe $C^{1}\left(\hat{\phi}_{U}\right)$, com $\hat{\phi}_{U} \in \mathbb{R}$, e portanto satisfaz a condição $\hat{\phi}_{f}^{\prime}(0)=1$. Esta última condição, por sua vez, não pode ser satisfeita simultaneamente com esse princípio de monotonicidade (ver apêndice C). Ainda mais, quando se compara os resultados obtidos com o esquema FDPUS-C1 com aqueles obtidos com o esquema TOPUS de Queiroz [52] - que satisfaz o princípio de motonicidade de Sweby - observa-se, para leis de conservação 1D, que o primeiro fornece, em geral, melhores resultados. Isto pode ser constatado no capítulo 6.

Em resumo, o esquema polinomial FDPUS-C1 pode atingir terceira ordem, é TVD (ver demonstração no apêndice A e Figura 4.1-(a)), consequentemente, é CBC. O seu limitador de fluxo está contido na região TVD (ver Figura 4.1-(b)), satisfaz a condição necessária para segunda ordem $(\psi(1)=1)$ e a condição necessária e suficiente para terceira ordem $\left(\psi^{\prime}(1)=\frac{1}{4}\right)$.

(a)

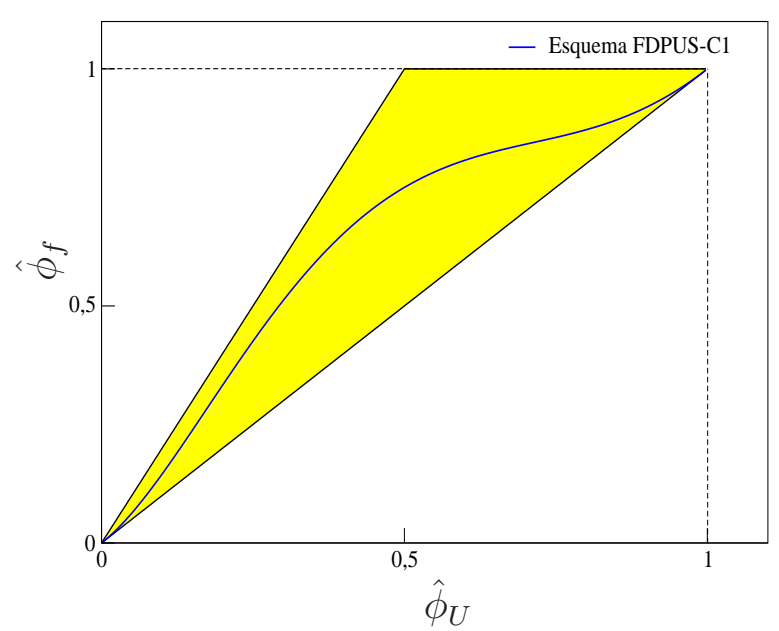

(b)

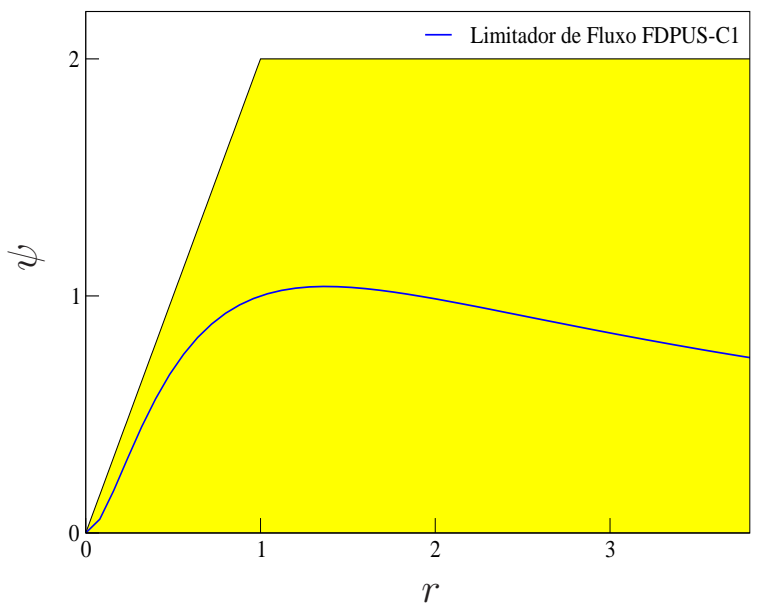

Figura 4.1: Esquema FDPUS-C1 na região TVD. O esquema no plano $\hat{\phi}_{f} \perp \hat{\phi}_{U}$ (a) e seu respectivo limitador de fluxo no plano $\psi \perp r$ (b).

\subsection{O Esquema SDPUS-C1}

Objetivando a derivação de uma classe de novos esquemas upwind com as mesmas características e propriedades do esquema FDPUS-C1 e que seja flexível, isto é o usuário está livre para escolher um esquema TVD polinomial apropriado ao seu problema, apresenta-se nesta seção o desenvolvimento da classe de esquema SDPUS-C1 (neste texto referida como esquema SDPUS-C1). Para tanto, considera-se parte de um polinômio de grau seis, para $\hat{\phi}_{U} \in[0,1]$, dado por

$$
\hat{\phi}_{f}\left(\hat{\phi}_{U}\right)=\sum_{q=0}^{6} b_{q} \hat{\phi}_{U}^{q}
$$


e o esquema FOU, dado por (4.2), para $\hat{\phi}_{U} \notin[0,1]$. Derivando o polinômio (4.22), obtém-se

$$
\hat{\phi}_{f}\left(\hat{\phi}_{U}\right)=\sum_{q=1}^{6} q b_{q} \hat{\phi}_{U}^{q-1} .
$$

Mantendo o coeficiente $b_{2}=\gamma$ como um parâmetro livre, os demais coeficientes em (4.22) são determinados por meio das condições de Leonard [42], apresentadas na seção 3.3 do capítulo 3, isto é,

- impondo-se $\hat{\phi}_{f}(0)=0$ obtém-se

$$
b_{0}=0
$$

- impondo-se $\hat{\phi}_{f}(1)=1$ obtém-se

$$
b_{6}+b_{5}+b_{4}+b_{3}+\gamma+b_{1}+b_{0}=1
$$

- impondo-se $\hat{\phi}_{f}\left(\frac{1}{2}\right)=\frac{3}{4}$ obtém-se

$$
\begin{aligned}
b_{6}\left(\frac{1}{2}\right)^{6}+b_{5}\left(\frac{1}{2}\right)^{5}+b_{4}\left(\frac{1}{2}\right)^{4}+b_{3}\left(\frac{1}{2}\right)^{3}+\gamma\left(\frac{1}{2}\right)^{2}+b_{1}\left(\frac{1}{2}\right)+b_{0} & =\frac{3}{4}, \quad \text { ou } \\
b_{6}+2 b_{5}+4 b_{4}+8 b_{3}+16 \gamma+32 b_{1}+64 b_{0} & =48
\end{aligned}
$$

- impondo-se $\hat{\phi}_{f}^{\prime}\left(\frac{1}{2}\right)=\frac{3}{4}$ obtém-se

$$
\begin{aligned}
6 b_{6}\left(\frac{1}{2}\right)^{5}+5 b_{5}\left(\frac{1}{2}\right)^{4}+4 b_{4}\left(\frac{1}{2}\right)^{3}+3 b_{3}\left(\frac{1}{2}\right)^{2}+2 \gamma\left(\frac{1}{2}\right)^{1}+b_{1} & =\frac{3}{4}, \text { ou } \\
3 b_{6}+5 b_{5}+8 b_{4}+12 b_{3}+16 \gamma+16 b_{1} & =12 .
\end{aligned}
$$

Para fechar o sistema $(4.24)$ - (4.27), consideram-se as condições para que $\hat{\phi}_{f}=\hat{\phi}_{f}\left(\hat{\phi}_{U}\right)$ seja de classe $C^{1}\left(\hat{\phi}_{U}\right)$, com $\hat{\phi}_{U} \in \mathbb{R}$, ou seja,

- impondo-se $\hat{\phi}_{f}^{\prime}(0)=1$ obtém-se

$$
b_{1}=1
$$

- impondo-se $\hat{\phi}_{f}^{\prime}(1)=1$ obtém-se

$$
6 b_{6}+5 b_{5}+4 b_{4}+3 b_{3}+2 \gamma+b_{1}=1 \text {. }
$$

Resolvendo o sistema formado por (4.24) - (4.29), obtém-se os coeficientes em função de $\gamma$

$$
b_{0}=0, b_{1}=1, b_{2}=\gamma, b_{3}=(20-6 \gamma), b_{4}=(-64+13 \gamma), b_{5}=(68-12 \gamma) \text { e } b_{6}=(-24+4 \gamma)
$$

Substituindo esses coeficientes em (4.22) e considerando (4.2), obtém-se a nova classe de esque- 
mas em variáveis normalizadas dada por

$$
\hat{\phi}_{f}= \begin{cases}(-24+4 \gamma) \hat{\phi}_{U}^{6}+(68-12 \gamma) \hat{\phi}_{U}^{5}+(-64+13 \gamma) \hat{\phi}_{U}^{4}+(20-6 \gamma) \hat{\phi}_{U}^{3}+\gamma \hat{\phi}_{U}^{2}+\hat{\phi}_{U}, & \hat{\phi}_{U} \in[0,1], \\ \hat{\phi}_{U}, & \hat{\phi}_{U} \notin[0,1],\end{cases}
$$

a qual é denominada SDPUS-C1 (Six-Degree Polynomial Upwind Scheme of $C^{1}$ Class), uma vez que, para qualquer $\gamma$, a relação funcional (4.31), em $[0,1]$, é parte de um polinômio de grau seis e sua expressão global $\hat{\phi}_{f}=\hat{\phi}_{f}\left(\hat{\phi}_{U}\right) \in C^{1}\left(\hat{\phi}_{U}\right)$, para $\hat{\phi}_{U} \in \mathbb{R}$. Aplicando a definição de variáveis normalizadas (ver equação (3.2)) em (4.31), obtém-se o esquema SDPUS-C1 em variáveis não normalizadas

$$
\phi_{f}=\left\{\begin{array}{rr}
\phi_{R}+\left(\phi_{D}-\phi_{R}\right)\left[(-24+4 \gamma) \hat{\phi}_{U}^{6}+(68-12 \gamma) \hat{\phi}_{U}^{5}+(-64+13 \gamma) \hat{\phi}_{U}^{4}\right. \\
\left.-(20-6 \gamma) \hat{\phi}_{U}^{3}+\gamma \hat{\phi}_{U}^{2}+\hat{\phi}_{U}\right], & \hat{\phi}_{U} \in[0,1], \\
\phi_{U}, & \hat{\phi}_{U} \notin[0,1] .
\end{array}\right.
$$

O limitador de fluxo correspondente para o esquema SDPUS-C1 é obtido reescrevendo (4.31) na formulação (3.15). Comparando as equações (4.31) e (3.15), obtém-se $\psi(r)=0$ (uma vez que $\hat{\phi}_{f}=\hat{\phi}_{U}$ ) para $r<0$ (devido a definição de $r$ em $(3.18)$ e $\hat{\phi}_{U} \notin[0,1]$ ). Para $\hat{\phi}_{U} \in[0,1]$ considera-se, para o limitador de fluxo, um polinômio de grau 5 (lembrando que o esquema SDPUS-C1 em variáveis normalizadas é definido por um polinômio de grau 6) definido por

$$
\Psi\left(\hat{\phi}_{U}\right)=\sum_{s=0}^{5} \varrho_{s} \hat{\phi}_{U}^{s} .
$$

Substituindo esse polinômio na formulação (3.15), obtém-se

$$
\begin{aligned}
\hat{\phi}_{f} & =\hat{\phi}_{U}+\frac{1}{2}\left(\varrho_{5} \hat{\phi}_{U}^{5}+\varrho_{4} \hat{\phi}_{U}^{4}+\varrho_{3} \hat{\phi}_{U}^{3}+\varrho_{2} \hat{\phi}_{U}^{2}+\varrho_{1} \hat{\phi}_{U}+\varrho_{0}\right)\left(1-\hat{\phi}_{U}\right) \\
& =\frac{-\varrho_{5}}{2} \hat{\phi}_{U}^{6}+\frac{\left(\varrho_{5}-\varrho_{4}\right)}{2} \hat{\phi}_{U}^{5}+\frac{\left(\varrho_{4}-\varrho_{3}\right)}{2} \hat{\phi}_{U}^{4}+\frac{\left(\varrho_{3}-\varrho_{2}\right)}{2} \hat{\phi}_{U}^{3}+\frac{\left(\varrho_{2}-\varrho_{1}\right)}{2} \hat{\phi}_{U}^{2}+\left[\frac{\left(\varrho_{1}-\varrho_{0}\right)}{2}+1\right] \hat{\phi}_{U}+\varrho_{0} .
\end{aligned}
$$

Igualando (4.34) e (4.31), para $\hat{\phi}_{U} \in[0,1]$, determinam-se os coeficientes $\varrho_{s}(s=0, \cdots, 5)$ dados em função de $\gamma$ como

$$
\varrho_{0}=0, \quad \varrho_{1}=0, \quad \varrho_{2}=2 \gamma, \quad \varrho_{3}=(40-10 \gamma), \quad \varrho_{4}=(-88+16 \gamma) \mathrm{e} \quad \varrho_{5}=(48-8 \gamma) .
$$

Substituindo esses coeficientes em (4.33), obtém-se o limitador de fluxo $\Psi\left(\hat{\phi}_{U}\right)$ para a classe de 
esquemas SDPUS-C1

$$
\Psi\left(\hat{\phi}_{U}\right)=(48-8 \gamma) \hat{\phi}^{5}+(-88+16 \gamma) \hat{\phi}_{U}^{4}+(40-10 \gamma) \hat{\phi}_{U}^{3}+2 \gamma \hat{\phi}_{U}^{2}
$$

Da definição (3.18), a expressão (4.35) é reescrita por

$$
\begin{aligned}
\Psi\left(\hat{\phi}_{U}\right) & =(48-8 \gamma)\left(\frac{r}{1+r}\right)^{5}+(-88+16 \gamma)\left(\frac{r}{1+r}\right)^{4}+(40-10 \gamma)\left(\frac{r}{1+r}\right)^{3}+2 \gamma\left(\frac{r}{1+r}\right)^{2} \\
& =\frac{(28-8 \gamma) r^{5}+(-88+16 \gamma) r^{4}(1+r)+(40-10 \gamma) r^{3}(1+r)^{2}+2 \gamma r^{2}(1+r)^{3}}{(1+r)^{5}} \\
& =\frac{(-8+2 \gamma) r^{4}+(40-4 \gamma) r^{3}+2 \gamma r^{2}}{(1+r)^{5}}=\psi(r) .
\end{aligned}
$$

Em resumo, o limitador de fluxo do esquema SDPUS-C1 é dado por

$$
\psi(r)= \begin{cases}\frac{(-8+2 \gamma) r^{4}+(40-4 \gamma) r^{3}+2 \gamma r^{2}}{(1+r)^{5}}, & r \geq 0, \\ 0, & r<0 .\end{cases}
$$

Portanto, da mesma forma como foi feito para o esquema FDPUS-C1, reescrevem-se as sentenças (4.36), com o objetivo de se evitar condicionais na implementação computacional, como

$$
\psi(r)=\frac{0.5(|r|+r)\left((-8+2 \gamma) r^{3}+(40-4 \gamma) r^{2}+2 \gamma r\right.}{(1+|r|)^{5}}
$$

ou na notação mais usual (ver LeVeque [44]) como

$$
\psi(r)=\max \left\{0, \frac{0.5(|r|+r)\left((-8+2 \gamma) r^{3}+(40-4 \gamma) r^{2}+2 \gamma r\right.}{(1+|r|)^{5}}\right\} .
$$

Nota-se que o esquema SDPUS-C1 é monotônico e de segunda ordem, pois o seu limitador de fluxo (4.36) satisfaz, para $r \geq 0$ e $\forall \gamma$, a condição (3.19). De fato, pois

$$
\psi(1)=\frac{-8+2 \gamma+40-4 \gamma+2 \gamma}{2^{5}}=1
$$

Derivando a expressão (4.36), para $r \geq 0$, tem-se

$$
\psi^{\prime}(r)=\frac{\left[4(-8+2 \gamma) r^{3}+3(40-4 \gamma) r^{2}+4 \gamma r\right](1+r)^{5}-5\left[(-8+2 \gamma) r^{4}+(40-4 \gamma) r^{3}+2 \gamma r^{2}\right](1+r)^{4}}{(1+r)^{10}}
$$

Constata-se que o esquema SDPUS-C1 pode atingir terceira ordem, uma vez que o seu limitador de fluxo (4.36) satisfaz, para $r \geq 0$ e $\forall \gamma$, a condição (3.20). De fato, considerando essa definição 
e a expressão (4.39), tem-se

$$
\begin{aligned}
\psi^{\prime}(1) & =\frac{[4(-8+2 \gamma)+3(40-4 \gamma)+4 \gamma] 2^{5}-5[(-8+2 \gamma)+(40-4 \gamma)+2 \gamma] 2^{4}}{2^{10}} \\
& =\frac{1}{4} .
\end{aligned}
$$

Novamente, como já era de se esperar, o limitador de fluxo do esquema SDPUS-C1 (4.36) não satisfaz o princípio de Sweby, definido por (3.22), uma vez que a condição $\hat{\phi}_{f}^{\prime}(0)=1$ é satisfeita; assim a condição (3.22) não pode ser simultaneamente satisfeita (ver apêndice C).

Em resumo, o esquema upwind polinomial SDPUS-C1 pode atingir terceira ordem, e em particular ele é TVD para $\gamma \in[4,12]$. A Figura 4.2-(a) ilustra o esquema SDPUS-C1 para alguns valores de $\gamma$ no plano $\hat{\phi}_{f} \perp \hat{\phi}_{U}$ e a Figura 4.2-(b) mostra os correspondente limitadores no plano $\psi \perp r$. Neste trabalho de mestrado é considerado $\gamma=12$ (no apêndice B está demonstrado que o esquema SDPUS-C1, com $\gamma=12$, é TVD). Considerando o caso $\gamma=6$ em (4.31), obtém-se o esquema FDPUS-C1 (4.11).

(a)
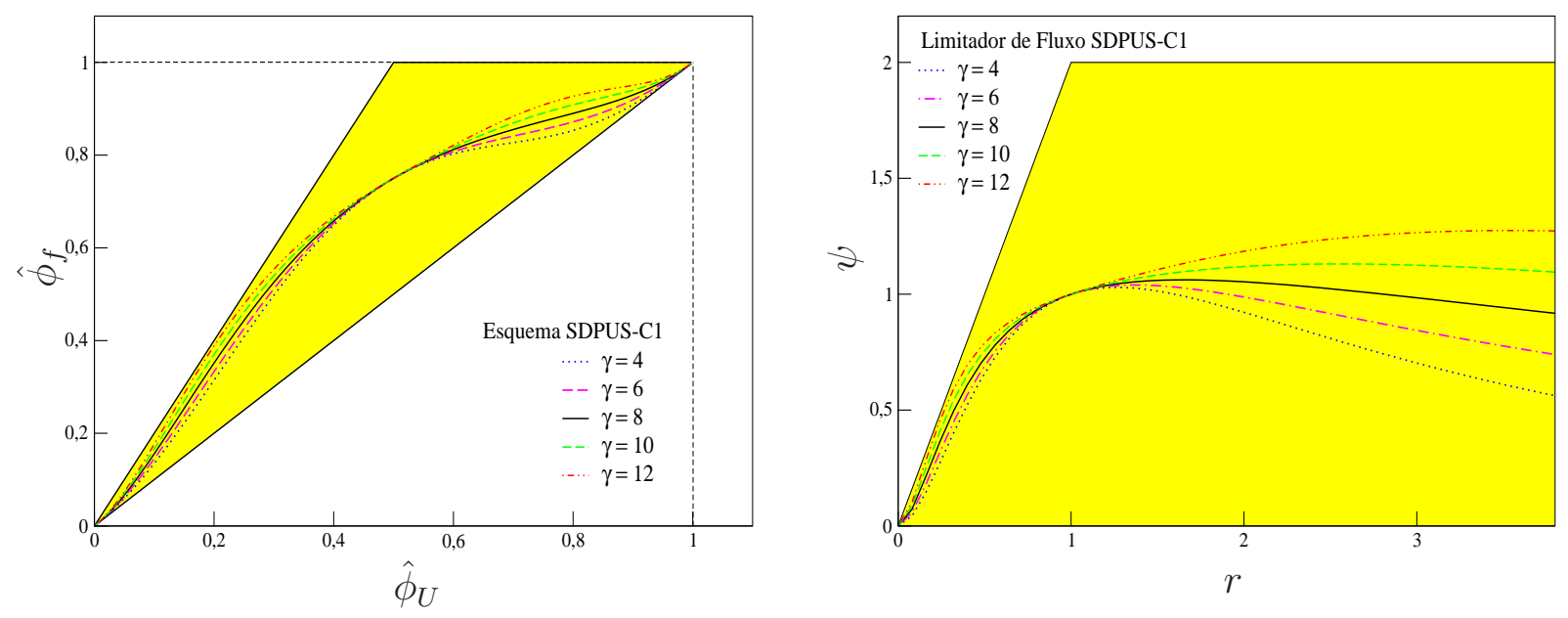

Figura 4.2: Esquema SDPUS-C1 na região TVD. Esquema no plano $\hat{\phi}_{f} \perp \hat{\phi}_{U}$ (a) e seu respectivo limitador de fluxo no plano $\psi \perp r$ (b), para $\gamma=4,6,8,10,12$. 


\section{Modelagem Computacional}

Neste capítulo discute-se a discretização dos termos convectivos das leis de conservação 1D e 2D definidas, respectivamente, por (2.1) e (2.23); das equações instantâneas de Navier-Stokes (2.32), (2.33) e (2.35); para o modelo $\kappa-\varepsilon$ em (2.46), (2.52) e (2.53); e o modelo Oldroyd-B em (2.58) e (2.59). Apresenta-se também o algoritmo base para simulação de problemas de escoamento de fluidos incompressíveis com superfícies livres móveis sem modelagem dos efeitos de turbulência e viscoelásticos.

Em todas as equações estudadas neste trabalho, a marcha no tempo é feita pelo método de Euler explícito [5]. Para os termos difusivos e gradientes de pressão, aplica-se a diferenciação centrada de segunda ordem. Os termos convectivos das leis de conservação 1D são aproximados pelos esquemas FDPUS-C1 e SDPUS-C1 mais aqueles apresentados nas seções 3.4 e 3.6 do capítulo 3. Os termos convectivos das leis de conservação 2D são aproximados pelos esquemas ADBQUICKEST, FDPUS-C1 e SDPUS-C1. Já, os termos das equações de Navier-Stokes 2D, 2D-1/2 e 3D são aproximados apenas por FDPUS-C1 e SDPUS-C1.

\subsection{Discretização das Leis de conservação 1D}

As leis de conservação 1D (2.1) (para advecção de escalares, Burgers, Buckley-Leverett, águas rasas e Euler) são aproximadas, no contexto do método de diferenças finitas, pelo método numérico

$$
\phi_{i, j+1}=\phi_{i, j}-\frac{\delta_{t}}{\delta_{x}}\left(F(\phi)_{i+\frac{1}{2}, j}-F(\phi)_{i-\frac{1}{2}, j}\right)
$$

em que $\phi_{i, j}=\phi\left(i \delta_{x}, j \delta_{t}\right)$ é a solução numérica no ponto de malha $(i, j) \equiv\left(i \delta_{x}, j \delta_{t}\right)$. sendo $\delta_{x}$ e $\delta_{t}$ os espaçamentos da malha (uniforme) nas direções $x$ e $t$, respectivamente. Os termos $F(\phi)_{i+\frac{1}{2}, j}$ e $F(\phi)_{i-\frac{1}{2}, j}$ são os fluxos numéricos nas interfaces $f=i+\frac{1}{2}$ e $g=i-\frac{1}{2}$ das células computacionais 
(ver Figura 3.1). Esses fluxos numéricos são estimados (interpolados) por esquemas upwind de alta resolução.

\subsubsection{Equação de Advecção}

Na equação de advecção a variável conservada e função fluxo são dados, respectivamente, por $(2.2)$ e (2.3). O fluxo é aproximado no ponto $(i, j)$ da malha pela combinação $F(\phi)_{i+\frac{1}{2}, j}-$ $F(\phi)_{i-\frac{1}{2}, j}$ e então aproximada por

$$
F(\phi)_{i+\frac{1}{2}, j}-F(\phi)_{i-\frac{1}{2}, j}=\left.(a u)\right|_{i+\frac{1}{2}, j}-\left.(a u)\right|_{i-\frac{1}{2}, j}=a_{i+\frac{1}{2}, j} \cdot u_{i+\frac{1}{2}, j}-a_{i-\frac{1}{2}, j} \cdot u_{i-\frac{1}{2}, j} \cdot
$$

em que a velocidade convectiva $a$ (aqui considerada igual a 1) é constante para todo ponto do domínio e a variável convectada $u$ é aproximada nas faces $f\left(u_{i+\frac{1}{2}, j}=u_{f}\right)$ e $g\left(u_{i-\frac{1}{2}, j}=u_{g}\right)$ pelos esquemas upwind de alta resolução presentes na literatura e apresentados na seção 3.4, do capítulo 3, bem como pelos novos esquemas FDPUS-C1 e SDPUS-C1 apresentados no capítulo 4 . O método numérico explícito (5.1) - (5.2) é implementado em um código originalmente desenvolvido pela autora, sendo que a estabilidade é regida pela condição CFL (Courant-Friedrichs-Lewy), isto é a marcha no tempo é selecionada de modo que CFL $=\theta=\frac{a \delta_{t}}{\delta_{x}} \leq 1$.

\subsubsection{Equação de Burgers}

A equação de Burgers possui variável conservada e função fluxo dados, respectivamente, por $(2.7)$ e (2.8). Assim, o termo $F(\phi)_{i+\frac{1}{2}, j}-F(\phi)_{i-\frac{1}{2}, j}$ é aproximado por

$$
F(\phi)_{i+\frac{1}{2}, j}-F(\phi)_{i-\frac{1}{2}, j}=\left.\left(\frac{u^{2}}{2}\right)\right|_{i+\frac{1}{2}, j}-\left.\left(\frac{u^{2}}{2}\right)\right|_{i-\frac{1}{2}, j}=\frac{1}{2}\left(\bar{u}_{i+\frac{1}{2}, j} \cdot u_{i+\frac{1}{2}, j}-\bar{u}_{i-\frac{1}{2}, j} \cdot u_{i-\frac{1}{2}, j}\right)
$$

com as velocidades de transporte da variável $u$ dadas pela médias

$$
\bar{u}_{i-\frac{1}{2}, j}=\frac{1}{2}\left(u_{i, j}+u_{i-1, j}\right) \quad \text { e } \quad \bar{u}_{i+\frac{1}{2}, j}=\frac{1}{2}\left(u_{i+1, j}+u_{i, j}\right)
$$

e a variável convectada $u$ nas faces $f\left(u_{i+\frac{1}{2}, j}=u_{f}\right)$ e $g\left(u_{i-\frac{1}{2}, j}=u_{g}\right)$ são calculadas pelos esquemas upwind de alta resolução apresentados na seção 3.4, do capítulo 3, bem como pelos novos esquemas FDPUS-C1 e SDPUS-C1 apresentados no capítulo 4. O código computacional empregado para a solução da equação de Burgers é de autoria da autora. Nesse caso, a estabilidade é também regida pela condição $\theta=\frac{\delta_{t}}{\delta_{x}} \leq 1$.

\subsubsection{Equação de Buckley-Leverett}

A equação de Buckley-Leverett possui variável conservada e função fluxo dados, respectivamente, por (2.12) e (2.13). Diferentemente das aproximações (5.2) e (5.3), em que os esquemas são aplicados nas variáveis conservadas isto é se $\phi$ é uma variável conservada, então $\phi_{f}=\phi_{f}\left(\phi_{U}, \phi_{D}, \phi_{R}\right)$, o cálculo da combinação $F(\phi)_{i+\frac{1}{2}, j}-F(\phi)_{i-\frac{1}{2}, j}$, para a equação de 
Buckley-Leverett, é feito pela aplicação direta dos esquemas na função fluxo (2.13) isto é

$$
F(\phi)_{i+\frac{1}{2}, j}-F(\phi)_{i-\frac{1}{2}, j}=F_{f}\left(F\left(u_{U}\right), F\left(u_{D}\right), F\left(u_{R}\right)\right)-F_{g}\left(F\left(u_{U}\right), F\left(u_{D}\right), F\left(u_{R}\right)\right)
$$

em que $F\left(u_{U}\right), F\left(u_{D}\right)$ e $F\left(u_{R}\right)$ são os valores da função fluxo (2.13) avaliada em $u_{U}, u_{D}$ e $u_{R}$, respectivamente. As várias sentenças matemáticas que definem os esquemas, antes condicionadas aos intervalos segundo o valor normalizado $\hat{\phi}_{U}$, passam agora a serem condicionadas segundo à normalização

$$
\hat{F}\left(u_{U}\right)=\frac{F\left(u_{U}\right)-F\left(u_{R}\right)}{F\left(u_{D}\right)-F\left(u_{R}\right)}
$$

As posições $D, U$ e $R$ são definidas agora de acordo com o sinal das velocidades

$$
\widetilde{u}_{i+\frac{1}{2}, j}= \begin{cases}\frac{\delta_{t}}{\delta_{x}} \frac{\left.F(u)\right|_{i+1, j}-\left.F(u)\right|_{i, j}}{u_{i+1, j}-u_{i, j}}, & u_{i+1, j} \neq u_{i, j}, \\ \left.\frac{\delta_{t}}{\delta_{x}} F^{\prime}(u)\right|_{i, j}, & u_{i+1, j}=u_{i, j}\end{cases}
$$

e

$$
\widetilde{u}_{i-\frac{1}{2}, j}= \begin{cases}\frac{\delta_{t}}{\delta_{x}} \frac{\left.F(u)\right|_{i, j}-\left.F(u)\right|_{i-1, j}}{u_{i, j}-u_{i-1, j}}, & u_{i, j} \neq u_{i-1, j} \\ \left.\frac{\delta_{t}}{\delta_{x}} F^{\prime}(u)\right|_{i-1, j}, & u_{i, j}=u_{i-1, j}\end{cases}
$$

em que

$$
F^{\prime}(u)=\frac{8 u-8 u^{2}}{\left(5 u^{2}-2 u+1\right)^{2}}
$$

A equação de Buckley-Leverett também é simulada em um código desenvolvido pela autora e a estabilidade é também regida pela condição $\theta=\frac{\delta_{t}}{\delta_{x}} \leq 1$.

\subsubsection{Sistemas Hiperbólicos (Águas Rasas e Euler)}

O sistema hiperbólico águas rasas possui o vetor de variáveis conservadas e o vetor função fluxo dados, respectivamente, por (2.16) e (2.17), e o sistema de equações de Euler possui o vetor das variáveis conservadas e função fluxo dados, respectivamente, por (2.18) e (2.19). As simulações desses sistemas hiperbólicos são feitas no pacote computacional CLAWPACK (Conservation LAW PACKage) de LeVeque [44] equipado com os limitadores de fluxo dos esquemas ADBQUICKEST, TOPUS, van Leer, FDPUS-C1 e SDPUS-C1. Em síntese, este pacote computacional resolve leis de conservação gerais $1 \mathrm{D}, 2 \mathrm{D}$ e $3 \mathrm{D}$ por meio do método dos volumes finitos. As soluções para essas leis podem ser computadas usando o método de 
primeira ordem de Godunov [44] ou por sua variante de segunda ordem de precisão proposto por LeVeque [44] (Godunov de primeira ordem com termo de correção). Os detalhes sobre o método de solução utilizado no programa CLAWPACK estão descritos no apêndice D.

\subsection{Discretização das Leis de Conservação 2D}

As simulações dos sistemas hiperbólicos 2D águas rasas (em que o vetor das variáveis conservadas e funções fluxo são dados, respectivamente, por (2.24), (2.25) e (2.26)) e de Euler (em que o vetor das variáveis conservadas e funções fluxo são dados, respectivamente, por (2.27), (2.28) e (2.29)) são feitas também utilizando-se o CLAWPACK equipado com os limitadores de fluxo para os esquemas ADBQUICKEST, FDPUS-C1 e SDPUS-C1. Para mais detalhes sobre o método de solução utilizado no CLAWPACK ver o apêndice D.

\subsection{Discretização de Navier-Stokes 2D, 2D-1/2 e 3D}

A discretização das equações de Navier-Stokes 2D, 2D-1/2 e 3D (com ou sem modelagem), para a simulação de escoamentos incompressíveis com superfícies livres móveis, é feita no contexto do método de diferença finitas. Nesse caso, utiliza-se uma malha deslocada (staggered grid) [3], onde as velocidades são calculadas nas faces das células computacionais e escalares tais como pressão, energia cinética da turbulência, entre outros, são calculados no centro dessas células. O sistema discreto resultante é implementado no ambiente de simulação Freeflow de Castelo et al. [12]. Este ambiente computacional soluciona as equações de transporte e conservação de massa baseado na metodologia GENSMAC (Generalized-Simplified-Marker-and-Cell) [67] que é uma variante do método de projeção. A discussão sobre a discretização dos termos convectivos presentes nessas equações são como segue.

Por simplicidade e sem perda de generalidade, considera-se um representante típico dos termos convectivos das equações (2.32), (2.33), (2.35), (2.46), (2.52), (2.53), (2.58) e (2.59) e que pode ser colocado e avaliado num ponto $A$ da malha como

$$
\left.\frac{\partial\left(u_{j} \phi\right)}{\partial x_{j}}\right|_{A}=\left.\frac{\partial(u \phi)}{\partial x}\right|_{A}+\left.\frac{\partial(v \phi)}{\partial y}\right|_{A}
$$

em que $\phi$ é a variável convectada, por exemplo $u, v, w, k$, etc; $u$ e $v$ são as velocidade de convecção; e o ponto $A$ em (5.10) é a posição em que o termo convectivo é aproximado.

Para exemplificar a discretização dos termos convectivos, considera-se a variável $\phi=u \mathrm{em}$ (5.10) transportada com velocidade $v_{f}$ na direção $y$ (ver Figura 5.1). Nesse caso, $A=\left(i+\frac{1}{2}, j\right)$, $f=j+\frac{1}{2}, g=j-\frac{1}{2}$, e o segundo termo da lado direito de (5.10) é aproximado por

$$
\left.\frac{\partial(v u)}{\partial y}\right|_{i+\frac{1}{2}, j}=\frac{\left(v_{f} \cdot u_{i+\frac{1}{2}, j+\frac{1}{2}}-v_{g} \cdot u_{i+\frac{1}{2}, j-\frac{1}{2}}\right)}{\delta y}
$$




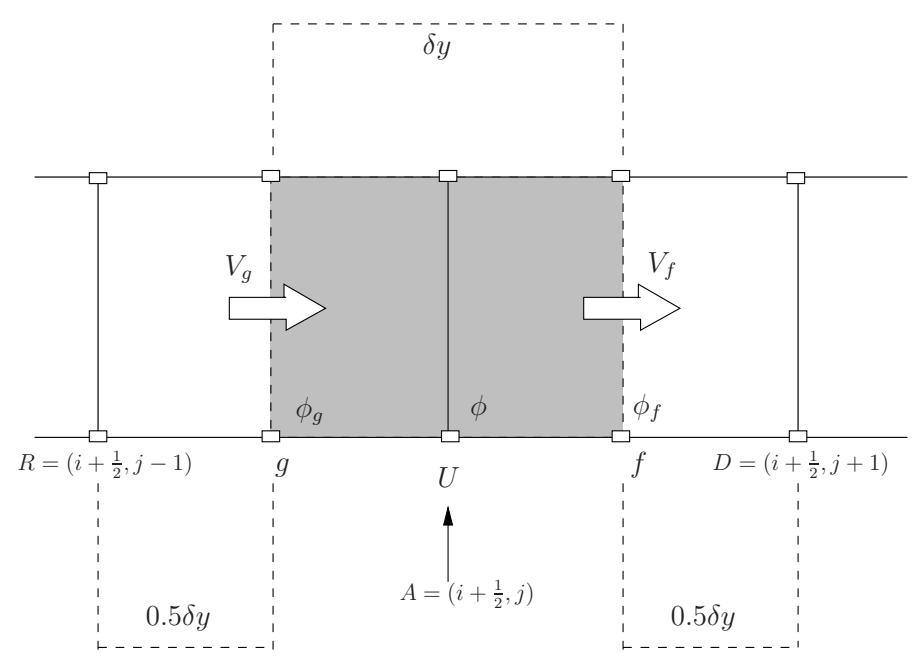

Figura 5.1: Representação esquemática para se obter aproximações dos termos convectivos. Mostrando o ponto $A$ de discretização e seus vizinhos, as faces envolvidas $f$ e $g$ para a aproximação e a direção das velocidades $V_{f}$ e $V_{g}$ de convecção nas faces $f$ e $g$, respectivamente.

em que $v_{f}$ e $v_{g}$ são aproximados, respectivamente, pelas médias

$$
\begin{aligned}
& v_{f}=v_{i+\frac{1}{2}, j+\frac{1}{2}}=\frac{\left(v_{i+1, j+\frac{1}{2}}+v_{i, j+\frac{1}{2}}\right)}{2}, \\
& v_{g}=v_{i+\frac{1}{2}, j-\frac{1}{2}}=\frac{\left(v_{i+1, j-\frac{1}{2}}+v_{i, j-\frac{1}{2}}\right)}{2} .
\end{aligned}
$$

Uma aproximação dos termos $u_{i+\frac{1}{2}, j+\frac{1}{2}}$ e $u_{i+\frac{1}{2}, j-\frac{1}{2}}$ é feita usando-se os esquemas FDPUS-C1 (4.12) e SDPUS-C1 (4.32) ( com $\gamma \in[4,12])$ em variáveis não normalizadas. Para tanto, os valores da propriedade transportada $u$ nas posições $\left(i+\frac{1}{2}, j+\frac{1}{2}\right)$ e $\left(i+\frac{1}{2}, j-\frac{1}{2}\right)$ são obtidos utilizando-se os pontos vizinhos $D, R$ e $U$, os quais são definidos de acordo com a direção das velocidades de convecção (sinais de $v_{f}$ e $v_{g}$ ). Em síntese, tem-se:

- Aproximações para $u_{i+\frac{1}{2}, j+\frac{1}{2}}$ quando $v_{f} \geq 0$ : nesse caso as posições $D, R$ e $U$ assumem, respectivamente, os valores

$$
D=\left(i+\frac{1}{2}, j+1\right), \quad R=\left(i+\frac{1}{2}, j-1\right) \text { e } U=\left(i+\frac{1}{2}, j\right)
$$

e o termo $u_{i+\frac{1}{2}, j+\frac{1}{2}}$ é aproximado por

- Esquema FDPUS-C1:

$$
u_{i+\frac{1}{2}, j+\frac{1}{2}}= \begin{cases}u_{i+\frac{1}{2}, j-1}+\left(u_{i+\frac{1}{2}, j+1}-u_{i+\frac{1}{2}, j-1}\right)\left(-4 \hat{u}_{U}^{5}+14 \hat{u}_{U}^{4}-16 \hat{u}_{U}^{3}+6 \hat{u}_{U}^{2}+\hat{u}_{U}\right), & \hat{u}_{U} \in[0,1], \\ u_{i+\frac{1}{2}, j}, & \hat{u}_{U} \notin[0,1],\end{cases}
$$


- Esquema SDPUS-C1:

$u_{i+\frac{1}{2}, j+\frac{1}{2}}= \begin{cases}u_{i+\frac{1}{2}, j-1}+\left(u_{i+\frac{1}{2}, j+1}-u_{i+\frac{1}{2}, j-1}\right)\left[(4 \gamma-24) \hat{u}_{U}^{6}+(68-12 \gamma) \hat{u}_{U}^{5}+\right. \\ \left.(13 \gamma-64) \hat{u}_{U}^{4}+(20-6 \gamma) \hat{u}_{U}^{3}+\gamma \hat{u}_{U}^{2}+\hat{u}_{U}\right], & \hat{u}_{U} \in[0,1], \\ u_{i+\frac{1}{2}, j}, & \hat{u}_{U} \notin[0,1],\end{cases}$

em que

$$
\hat{u}_{U}=\hat{u}_{i+\frac{1}{2}, j}=\frac{u_{i+\frac{1}{2}, j}-u_{i+\frac{1}{2}, j-1}}{u_{i+\frac{1}{2}, j+1}-u_{i+\frac{1}{2}, j-1}}
$$

- Aproximações para $u_{i+\frac{1}{2}, j+\frac{1}{2}}$ quando $v_{f}<0$ : nesse caso as posições $D, R$ e $U$ assumem, respectivamente, os valores

$$
D=\left(i+\frac{1}{2}, j\right), \quad R=\left(i+\frac{1}{2}, j+2\right) \text { e } U=\left(i+\frac{1}{2}, j+1\right)
$$

e, assim, o termo $u_{i+\frac{1}{2}, j+\frac{1}{2}}$ é aproximado por:

- Esquema FDPUS-C1:

$$
u_{i+\frac{1}{2}, j+\frac{1}{2}}= \begin{cases}u_{i+\frac{1}{2}, j+2}+\left(u_{i+\frac{1}{2}, j}-u_{i+\frac{1}{2}, j+2}\right)\left(-4 \hat{u}_{U}^{5}+14 \hat{u}_{U}^{4}-16 \hat{u}_{U}^{3}+6 \hat{u}_{U}^{2}+\hat{u}_{U}\right), & \hat{u}_{U} \in[0,1], \\ u_{i+\frac{1}{2}, j+1}, & \hat{u}_{U} \notin[0,1],\end{cases}
$$

- Esquema SDPUS-C1:

$$
\begin{gathered}
u_{i+\frac{1}{2}, j+\frac{1}{2}}=\left\{\begin{array}{rr}
u_{i+\frac{1}{2}, j+2}+\left(u_{i+\frac{1}{2}, j}-u_{i+\frac{1}{2}, j+2}\right)\left[(4 \gamma-24) \hat{u}_{U}^{6}+(68-12 \gamma) \hat{u}_{U}^{5}+\right. \\
\left.(13 \gamma-64) \hat{u}_{U}^{4}+(20-6 \gamma) \hat{u}_{U}^{3}+\gamma \hat{u}_{U}^{2}+\hat{u}_{U}\right], & \hat{u}_{U} \in[0,1], \\
u_{i+\frac{1}{2}, j+1}, & \hat{u}_{U} \notin[0,1],
\end{array}\right. \\
\hat{u}_{U}=\hat{u}_{i+\frac{1}{2}, j+1}=\frac{u_{i+\frac{1}{2}, j+1}-u_{i+\frac{1}{2}, j+2}}{u_{i+\frac{1}{2}, j}-u_{i+\frac{1}{2}, j+2}}
\end{gathered}
$$

- Aproximações para $u_{i+\frac{1}{2}, j-\frac{1}{2}}$ quando $v_{g} \geq 0$ : nesse caso as posições $D, R$ e $U$ assumem, respectivamente, os valores

$$
D=\left(i+\frac{1}{2}, j\right), \quad R=\left(i+\frac{1}{2}, j-2\right) \text { e } U=\left(i+\frac{1}{2}, j-1\right)
$$

e, assim, o termo $u_{i+\frac{1}{2}, j-\frac{1}{2}}$ é aproximado por: 
- Esquema FDPUS-C1:

$$
u_{i+\frac{1}{2}, j-\frac{1}{2}}= \begin{cases}u_{i+\frac{1}{2}, j-2}+\left(u_{i+\frac{1}{2}, j}-u_{i+\frac{1}{2}, j-2}\right)\left(-4 \hat{u}_{U}^{5}+14 \hat{u}_{U}^{4}-16 \hat{u}_{U}^{3}+6 \hat{u}_{U}^{2}+\hat{u}_{U}\right), & \hat{u}_{U} \in[0,1], \\ u_{i+\frac{1}{2}, j-1}, & \hat{u}_{U} \notin[0,1],\end{cases}
$$

- Esquema SDPUS-C1:

$$
\begin{gathered}
u_{i+\frac{1}{2}, j-\frac{1}{2}}=\left\{\begin{array}{cc}
u_{i+\frac{1}{2}, j-2}+\left(u_{i+\frac{1}{2}, j}-u_{i+\frac{1}{2}, j-2}\right)\left[(4 \gamma-24) \hat{u}_{U}^{6}+(68-12 \gamma) \hat{u}_{U}^{5}+\right. \\
\left.(13 \gamma-64) \hat{u}_{U}^{4}+(20-6 \gamma) \hat{u}_{U}^{3}+\gamma \hat{u}_{U}^{2}+\hat{u}_{U}\right], & \hat{u}_{U} \in[0,1], \\
u_{i+\frac{1}{2}, j-1}, & \hat{u}_{U} \notin[0,1],
\end{array}\right. \\
\hat{u}_{U}=\hat{u}_{i+\frac{1}{2}, j-1}=\frac{u_{i+\frac{1}{2}, j-1}-u_{i+\frac{1}{2}, j-2}}{u_{i+\frac{1}{2}, j}-u_{i+\frac{1}{2}, j-2}}
\end{gathered}
$$

- Aproximações para $u_{i+\frac{1}{2}, j+\frac{1}{2}}$ quando $v_{g}<0$ : nesse caso as posições $D, R$ e $U$ assumem, respectivamente, os valores

$$
D=\left(i+\frac{1}{2}, j-1\right), \quad R=\left(i+\frac{1}{2}, j+1\right) \text { e } U=\left(i+\frac{1}{2}, j\right)
$$

- Esquema FDPUS-C1:

$$
u_{i+\frac{1}{2}, j-\frac{1}{2}}= \begin{cases}u_{i+\frac{1}{2}, j+1}+\left(u_{i+\frac{1}{2}, j-1}-u_{i+\frac{1}{2}, j+1}\right)\left(-4 \hat{u}_{U}^{5}+14 \hat{u}_{U}^{4}-16 \hat{u}_{U}^{3}+6 \hat{u}_{U}^{2}+\hat{u}_{U}\right), & \hat{u}_{U} \in[0,1], \\ u_{i+\frac{1}{2}, j}, & \hat{u}_{U} \notin[0,1],\end{cases}
$$

- Esquema SDPUS-C1:

$u_{i+\frac{1}{2}, j-\frac{1}{2}}= \begin{cases}u_{i+\frac{1}{2}, j+1}+\left(u_{i+\frac{1}{2}, j-1}-u_{i+\frac{1}{2}, j+1}\right)\left[(4 \gamma-24) \hat{u}_{U}^{6}+(68-12 \gamma) \hat{u}_{U}^{5}+\right. \\ \left.(13 \gamma-64) \hat{u}_{U}^{4}+(20-6 \gamma) \hat{u}_{U}^{3}+\gamma \hat{u}_{U}^{2}+\hat{u}_{U}\right], & \hat{u}_{U} \in[0,1], \\ u_{i+\frac{1}{2}, j}, & \hat{u}_{U} \notin[0,1],\end{cases}$

$$
\hat{u}_{U}=\hat{u}_{i+\frac{1}{2}, j}=\frac{u_{i+\frac{1}{2}, j}-u_{i+\frac{1}{2}, j+1}}{u_{i+\frac{1}{2}, j-1}-u_{i+\frac{1}{2}, j+1}} .
$$

Considera-se agora o caso em que a variável $\phi=\kappa$ (a energia cinética média da turbulência) é transportada na direção $x$ com velocidade $u$, isto é o primeiro termo da equação (5.10). Nesse caso, a aproximação para a derivada convectiva correspondente é dada por

$$
\left.\frac{\partial(u \kappa)}{\partial x}\right|_{A}=\frac{\left.(u \kappa)\right|_{f}-\left.(u \kappa)\right|_{g}}{\delta_{x}}
$$


Nesse caso, $A=(i, j), f=i+\frac{1}{2}$ e $g=i-\frac{1}{2}$, e assim a equação (5.14) torna-se

$$
\left.\frac{\partial(\kappa u)}{\partial x}\right|_{(i, j)}=\frac{u_{i+\frac{1}{2}, j} \cdot \kappa_{i+\frac{1}{2}, j}-u_{i-\frac{1}{2}, k} \cdot \kappa_{i-\frac{1}{2}, j}}{\delta_{x}}
$$

em que as velocidade $u_{f}$ e $u_{g}$ são aproximadas por médias, ou seja

$$
\begin{aligned}
& u_{f}=u_{i+\frac{1}{2}, j+\frac{1}{2}}=\frac{u_{i+\frac{1}{2}, j+1}+u_{i+\frac{1}{2}, j}}{2} \\
& u_{g}=u_{i-\frac{1}{2}, j+\frac{1}{2}}=\frac{u_{i-\frac{1}{2}, j+1}+u_{i-\frac{1}{2}, j}}{2} .
\end{aligned}
$$

A variável turbulenta $\kappa$ nas posições $\left(i+\frac{1}{2}, j\right)$ e $\left(i-\frac{1}{2}, j\right)$ é aproximada por meio dos esquemas FDPUS-C1 e SDPUS-C1 (para $\gamma \in[4,12]$ ) como segue:

- Aproximações para $\kappa_{i+\frac{1}{2}, j}$ quando $u_{f} \geq 0$ : nesse caso as posições $D, R$ e $U$ assumem, respectivamente, os valores

$$
D=(i+1, j), \quad R=(i-1, j) \text { e } U=(i, j)
$$

e o termo $\kappa_{i+\frac{1}{2}, j}$ é aproximado por:

- Esquema FDPUS-C1:

$$
\kappa_{i+\frac{1}{2}, j}= \begin{cases}\kappa_{i-1, j}+\left(\kappa_{i+1, j}-\kappa_{i-1, j}\right)\left(-4 \hat{\kappa}_{U}^{5}+14 \hat{\kappa}_{U}^{4}-16 \hat{\kappa}_{U}^{3}+6 \hat{\kappa}_{U}^{2}+\hat{\kappa}_{U}\right), & \hat{\kappa}_{U} \in[0,1], \\ \kappa_{i, j}, & \hat{\kappa}_{U} \notin[0,1],\end{cases}
$$

- Esquema SDPUS-C1:

$$
\begin{gathered}
\kappa_{i+\frac{1}{2}, j}=\left\{\begin{array}{cc}
\kappa_{i-1, j}+\left(\kappa_{i+1, j}-\kappa_{i-1, j}\right)\left[(4 \gamma-24) \hat{\kappa}_{U}^{6}+(68-12 \gamma) \hat{\kappa}_{U}^{5}+\right. & \\
\left.(13 \gamma-64) \hat{\kappa}_{U}^{4}+(20-6 \gamma) \hat{\kappa}_{U}^{3}+\gamma \hat{\kappa}_{U}^{2}+\hat{\kappa}_{U}\right], & \hat{\kappa}_{U} \in[0,1], \\
\kappa_{i, j}, & \hat{\kappa}_{U} \notin[0,1],
\end{array}\right. \\
\hat{\kappa}_{U}=\hat{\kappa}_{i, j}=\frac{\kappa_{i, j}-\kappa_{i-1, j}}{\kappa_{i+1, j}-\kappa_{i-1, j}} ;
\end{gathered}
$$

- Aproximações para $\kappa_{i+\frac{1}{2}, j}$ quando $u_{f}<0$ : nesse caso as posições $D, R$ e $U$ assumem, respectivamente, os valores

$$
D=(i, j), \quad R=(i+2, j) \text { e } U=(i+1, j)
$$

e o termo $\kappa_{i+\frac{1}{2}, j}$ é aproximado por: 
- Esquema FDPUS-C1:

$$
\kappa_{i+\frac{1}{2}, j}= \begin{cases}\kappa_{i+2, j}+\left(\kappa_{i, j}-\kappa_{i+2, j}\right)\left(-4 \hat{\kappa}_{U}^{5}+14 \hat{\kappa}_{U}^{4}-16 \hat{\kappa}_{U}^{3}+6 \hat{\kappa}_{U}^{2}+\hat{\kappa}_{U}\right), & \hat{\kappa}_{U} \in[0,1], \\ \kappa_{i+1, j}, & \hat{\kappa}_{U} \notin[0,1],\end{cases}
$$

- Esquema SDPUS-C1:

$$
\kappa_{i+\frac{1}{2}, j}=\left\{\begin{array}{rr}
\kappa_{i+2, j}+\left(\kappa_{i, j}-\kappa_{i+2, j}\right)\left[(4 \gamma-24) \hat{\kappa}_{U}^{6}+(68-12 \gamma) \hat{\kappa}_{U}^{5}+\right. \\
\left.(13 \gamma-64) \hat{\kappa}_{U}^{4}+(20-6 \gamma) \hat{\kappa}_{U}^{3}+\gamma \hat{\kappa}_{U}^{2}+\hat{\kappa}_{U}\right], & \hat{\kappa}_{U} \in[0,1], \\
\kappa_{i+1, j}, & \hat{\kappa}_{U} \notin[0,1],
\end{array}\right.
$$

$$
\hat{\kappa}_{U}=\hat{\kappa}_{i+1, j}=\frac{\kappa_{i+1, j}-\kappa_{i+2, j}}{\kappa_{i, j}-\kappa_{i+2, j}}
$$

- Aproximações para $\kappa_{i-\frac{1}{2}, j}$ quando $u_{g} \geq 0$ : nesse caso as posições $D, R$ e $U$ assumem, respectivamente, os valores

$$
D=(i, j), \quad R=(i-2, j) \text { e } U=(i-1, j)
$$

e o termo $\kappa_{i-\frac{1}{2}, j}$ é aproximado por:

- Esquema FDPUS-C1:

$$
\kappa_{i-\frac{1}{2}, j}= \begin{cases}\kappa_{i-2, j}+\left(\kappa_{i, j}-\kappa_{i-2, j}\right)\left(-4 \hat{\kappa}_{U}^{5}+14 \hat{\kappa}_{U}^{4}-16 \hat{\kappa}_{U}^{3}+6 \hat{\kappa}_{U}^{2}+\hat{\kappa}_{U}\right), & \hat{\kappa}_{U} \in[0,1], \\ \kappa_{i-1, j}, & \hat{\kappa}_{U} \notin[0,1],\end{cases}
$$

- Esquema SDPUS-C1:

$$
\begin{gathered}
\kappa_{i-\frac{1}{2}, j}=\left\{\begin{array}{rr}
\kappa_{i-2, j}+\left(\kappa_{i, j}-\kappa_{i-2, j}\right)\left[(4 \gamma-24) \hat{\kappa}_{U}^{6}+(68-12 \gamma) \hat{\kappa}_{U}^{5}+\right. & \\
\left.(13 \gamma-64) \hat{\kappa}_{U}^{4}+(20-6 \gamma) \hat{\kappa}_{U}^{3}+\gamma \hat{\kappa}_{U}^{2}+\hat{\kappa}_{U}\right], & \hat{\kappa}_{U} \in[0,1], \\
\kappa_{i-1, j}, & \hat{\kappa}_{U} \notin[0,1],
\end{array}\right. \\
\hat{\kappa}_{U}=\hat{\kappa}_{i-1, j}=\frac{\kappa_{i-1, j}-\kappa_{i-2, j}}{\kappa_{i, j}-\kappa_{i-2, j}} ;
\end{gathered}
$$

- Aproximações para $\kappa_{i-\frac{1}{2}, j}$ quando $u_{g}<0$ : nesse caso as posições $D, R$ e $U$ assumem, respectivamente, os valores

$$
D=(i-1, j), \quad R=(i+1, j) \text { e } U=(i, j)
$$

e o termo $\kappa_{i-\frac{1}{2}, j}$ é aproximado por: 
- Esquema FDPUS-C1:

$$
\kappa_{i-\frac{1}{2}, j}= \begin{cases}\kappa_{i+1, j}+\left(\kappa_{i-1, j}-\kappa_{i+1, j}\right)\left(-4 \hat{\kappa}_{U}^{5}+14 \hat{\kappa}_{U}^{4}-16 \hat{\kappa}_{U}^{3}+6 \hat{\kappa}_{U}^{2}+\hat{\kappa}_{U}\right), & \hat{\kappa}_{U} \in[0,1], \\ \kappa_{i, j}, & \hat{\kappa}_{U} \notin[0,1],\end{cases}
$$

- Esquema SDPUS-C1:

$$
\kappa_{i-\frac{1}{2}, j}=\left\{\begin{array}{rr}
\kappa_{i+1, j}+\left(\kappa_{i-1, j}-\kappa_{i+1, j}\right)\left[(4 \gamma-24) \hat{\kappa}_{U}^{6}+(68-12 \gamma) \hat{\kappa}_{U}^{5}+\right. & \\
\left.(13 \gamma-64) \hat{\kappa}_{U}^{4}+(20-6 \gamma) \hat{\kappa}_{U}^{3}+\gamma \hat{\kappa}_{U}^{2}+\hat{\kappa}_{U}\right], & \hat{\kappa}_{U} \in[0,1], \\
\kappa_{i, j}, & \hat{\kappa}_{U} \notin[0,1],
\end{array}\right.
$$

$$
\hat{\kappa}_{U}=\hat{\kappa}_{i, j}=\frac{\kappa_{i, j}-\kappa_{i+1, j}}{\kappa_{i-1, j}-\kappa_{i+1, j}} .
$$

É importante observar que os esquemas FDPUS-C1 e SDPUS-C1 fazem uso de três pontos vizinhos $(D, U$ e $R)$ para estimar uma variável convectada. Assim para o cálculo próximo às fronteiras usa-se o esquema FOU [61] de primeira ordem quando não for possível utilizar esses três pontos.

\subsubsection{Algoritmo Base para Simulação de Escoamentos Incompressíveis com Superfícies Livres}

Nesta subseção apresenta-se o algoritmo base para a simulação de escoamentos incompressíveis com superfícies livres móveis sem envolver efeitos de turbulência e viscoelásticos. Os algoritmos completos para a simulação envolvendo efeitos de turbulência e viscoelásticos podem ser encontrados em Kurokawa [40] e Martins [48]. O algoritmo base é explicado como segue. Admite-se que num dado instante de tempo $t_{n}$, as variáveis dependentes são conhecidas e as condições de contorno associadas estão especificadas. Um ciclo computacional consiste em atualizar as variáveis discretas, a partir do tempo inicial $t_{0}$ (ou um tempo anterior $t_{n}$ ) no tempo $t_{1}=t_{0}+\delta_{t}\left(\right.$ ou $\left.t_{n+1}=t_{n}+\delta_{t}\right)$, utilizando-se uma série de passos inter-relacionados, baseados no método da projeção de Chorin [13]. Para tanto, considera-se que no tempo $t_{0}$ (ou $t_{n}$ ) as condições iniciais e de contorno são conhecidas para o campo de velocidade e pressão [67]. O campo de velocidade em $t_{1}=t_{0}+\delta_{t}$ (ou $t_{n+1}=t_{n}+\delta_{t}$ ) é calculado pela sequência de passos:

- Passo 1: Atualizam-se as condições de contorno nas regiões de entrada, na saída de fluido e nas paredes rígidas. Na superfície livre, o campo de velocidade é calculado por meio das equações (2.41) e (2.42), e a pressão é determinada por meio da equação (2.43) [67];

- Passo 2: Calcula-se o campo de velocidade intermediário $\widetilde{u}_{i}$ explicitamente por meio de

$$
\widetilde{u}_{i}=u_{i}+\delta_{t}\left\{-\frac{\partial\left(u_{i} u_{j}\right)}{\partial x_{j}}-\frac{\partial \widetilde{P}}{\partial x_{i}}+\frac{1}{R e} \frac{\partial}{\partial x_{j}}\left(\frac{\partial u_{i}}{\partial x_{j}}\right)+\frac{1}{F r^{2}} g_{i}\right\}, \quad i=1,2,3,
$$


em que $\widetilde{P}$ é uma pressão arbitrária (tentativa);

- Passo 3: Resolve-se a equação de Poisson para a potencial auxiliar $\varphi$ por meio de

$$
\frac{\partial}{\partial x_{i}}\left(\frac{\partial \varphi}{\partial x_{i}}\right)=\left(\frac{\partial \widetilde{u}_{i}}{\partial x_{i}}\right), \quad i=1,2,3
$$

com a condição homogênea de Dirichlet na superfície livre e saída e a condição homogênea de Neumann em contornos rígidos e entrada. O sistema de equações lineares (5.15) é resolvido pelo método dos gradientes conjugados [34];

- Passo 4: Atualiza-se o campo de velocidade por (ver Denaro [20])

$$
u_{i}=\widetilde{u}_{i}-\frac{\partial \varphi}{\partial x_{i}}, \quad i=1,2,3
$$

- Passo 5: Determina-se a pressão por (para detalhes veja Ferreira [25])

$$
P=\widetilde{P}+\frac{\varphi}{\delta_{t}}
$$

- Passo 6: Definem-se as novas posições das partículas marcadoras que representam o fluido por meio da resolução do sistema de EDOs (Equações Diferenciais Ordinárias)

$$
\frac{d x_{i}}{d t}=u_{i}, \quad i=1,2,3
$$

pelo método de Euler explícito [5]. Essas partículas permitem a visualização do escoamento e a orientação da superfície livre.

Por fim, volta-se ao Passo 1 e dá-se início ao próximo ciclo computacional. 
CAPÍTULO

\section{Resultados Numéricos 1D}

Este capítulo tem como objetivo demonstrar/analisar o comportamento, a validade, a flexibilidade e a robustez dos esquemas FDPUS-C1 e SDPUS-C1. Para isso, resolvem-se vários casos de problemas lineares e não lineares de leis de conservação 1D, tais como: i) equação de advecção de escalares; ii) equações não lineares de Burgers e Buckley-Leverett; e iii) sistemas hiperbólicos de águas rasas e Euler. As soluções numéricas geradas pelos novos esquemas FDPUS-C1 e SDPUS-C1 e por esquemas presentes na literatura (ver seções 3.4 e 3.6 do capítulo 3) são comparadas com as soluções exatas (quando existentes) ou de referência. Ainda, neste capítulo, avalia-se o valor do parâmetro livre $\gamma$ para o esquema SDPUS-C1, confronta-se, para o problema de advecção de escalares, os resultados numéricos obtidos com os esquemas FDPUS-C1 e SDPUS-C1 com os do esquema WENO (ver Balsara e Shu [6]) de terceira ordem, determina-se a ordem de convergência, e analisa-se a restrição TVD para alguns dos esquemas anteriormente citados.

\subsection{Advecção de Escalares}

Problemas de advecção de escalares são formulados pela lei de conservação 1D (2.1), com a variável conservada e fluxo numérico dados, respectivamente, por (2.2) e (2.3). A equação de advecção é suplementada com diversas condições iniciais e, em todos os casos considerados, aplica-se a condição de Dirichlet homogênea no contorno. As soluções numéricas obtidas com os esquemas FDPUS-C1 e SDPUS-C1 são comparadas com as soluções exatas dadas por (2.6) e com as aproximações geradas pelos esquemas ADBQUICKEST, TOPUS $(\alpha=2)$, CUBISTA e VONOS. Para análise da restrição TVD também são considerados os esquemas FOU e Lax-Wendroff [19]. Além disso, os novos esquemas FDPUS-C1 e SDPUS-C1 são comparados com o esquema WENO de terceira ordem. A maioria desses resultados podem ser encontrados 
em anais de eventos e periódicos (ver o capítulo 9).

Caso 1 - Análise da parâmetro $\gamma$ para o esquema SDPUS-C1. Neste caso analisa-se o parâmetro $\gamma$ para o esquema SDPUS-C1. Para isso considera-se a equação de advecção definida em $x \in[0,2]$ e suplementada com a condição inicial

$$
u_{0}(x)=\left\{\begin{array}{lr}
\exp ^{-\log 50\left(\frac{\mathrm{x}-0.1}{0.05}\right)^{2}}, & 0 \leq x \leq 0.2 \\
1, & 0.4<x<0.6 \\
0, & 0.6 \leq x \leq 2
\end{array}\right.
$$

Para a simulação desse problema são consideradas 300 células computacionais, número de Courant $\theta=\frac{\delta t}{\delta x}=0.5$ e tempo final de simulação $t=0.5$. Os resultados numéricos estão disponíveis na Figura 6.1, onde Fig. 6.1-(a) mostra uma comparação entre a solução exata e os resultados obtidos com o esquema SDPUS-C1 para $\gamma=4,6,8,10,12$. Ainda, nesta mesma figura apresenta-se uma ampliação do pico (ver Fig. 6.1-(b)) e da descontinuidade (degrau) (ver Fig. 6.1-(c)) presentes na solução. Por essa figura, constata-se que o melhor resultado é obtido para o caso em que $\gamma=12$. Devido a este fato, os demais resultados numéricos obtidos com o esquema SDPUS-C1, apresentados nesse capítulo, são gerados para $\gamma=12$ (ver também apêndice B).

Caso 2 - Comparação dos esquemas FDPUS-C1 e SDPUS-C1 com WENO. O objetivo deste caso é comparar o desempenho dos novos esquemas FDPUS-C1 e SDPUS-C1 $(\gamma=12)$ com o esquema WENO de terceira ordem (para detalhes sobre esse esquema, ver Balsara e Shu [6]). Para isso, considera-se a equação de advecção, definida em $x \in[-1,1]$ e suplementada com a condição inicial dada por

$$
u_{0}(x)=\left\{\begin{array}{l}
1, \quad-\frac{1}{5} \leq x \leq \frac{1}{5} \\
0, \quad \text { caso contrário. }
\end{array}\right.
$$

Para simulação desse caso são considerados uma malha de 500 células computacionais, $\theta=0.5$ e $t=2.8$. Na Figura 6.2, à esquerda, apresenta-se a comparação da solução exata com os resultados numéricos obtidos com os esquemas WENO de terceira ordem, FDPUS-C1 (Fig. 6.2-(a)) e SDPUS-C1 (Fig. 6.2-(c)). À direita, dessa mesma figura, estão os erros absolutos dados, para cada ponto da malha, por

$$
E_{A}(x)=\left|u_{\text {numérica }}(x)-u_{\text {exata }}(x)\right|
$$

cometidos pelos esquemas WENO, FDPUS-C1 (Fig. 6.2-(b)) e SDPUS-C1 (Fig. 6.2-(d)) e plotados no plano $E_{A}(x) \perp x$. Por essa figura, vê-se que os resultados obtidos com os esquemas FDPUS-C1 e SDPUS-C1 são melhores e isso mostra que os novos esquemas FDPUS-C1 e SDPUS-C1 são competitivos com o esquema consagrado WENO de terceira ordem.

Caso 3 - Análise da restrição TVD. Neste caso, analisa-se a restrição TVD para o esquema 
(a)

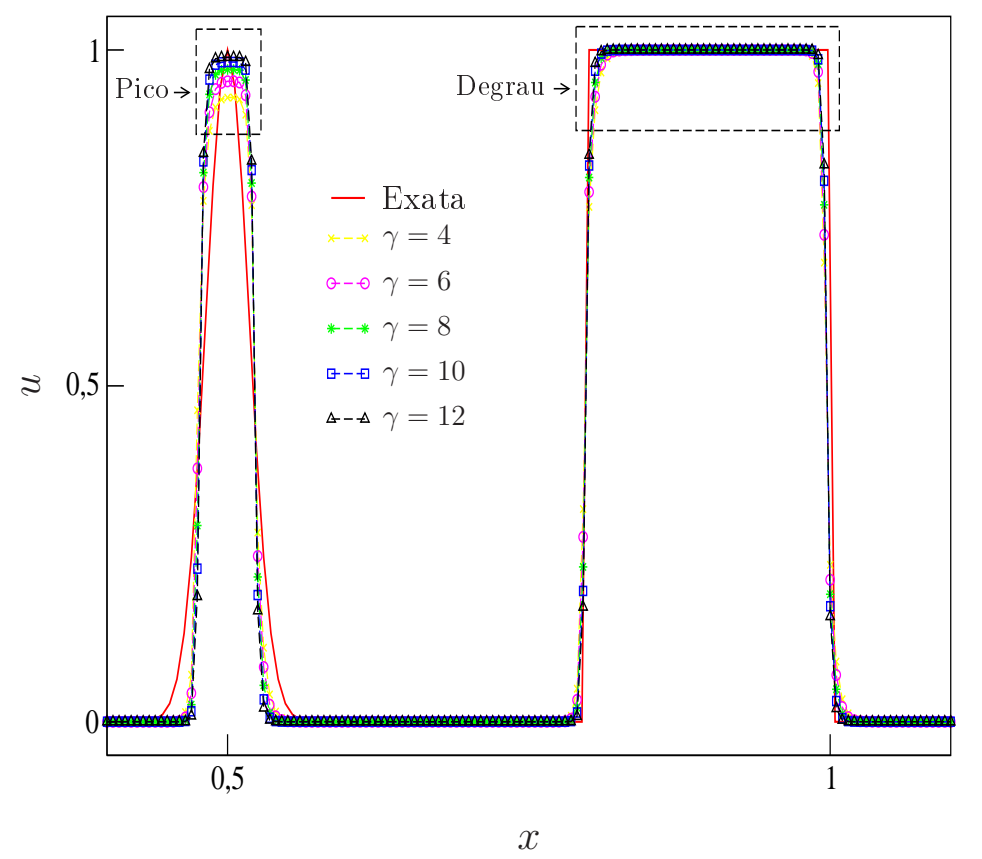

(b) - Pico

(c) - Degrau
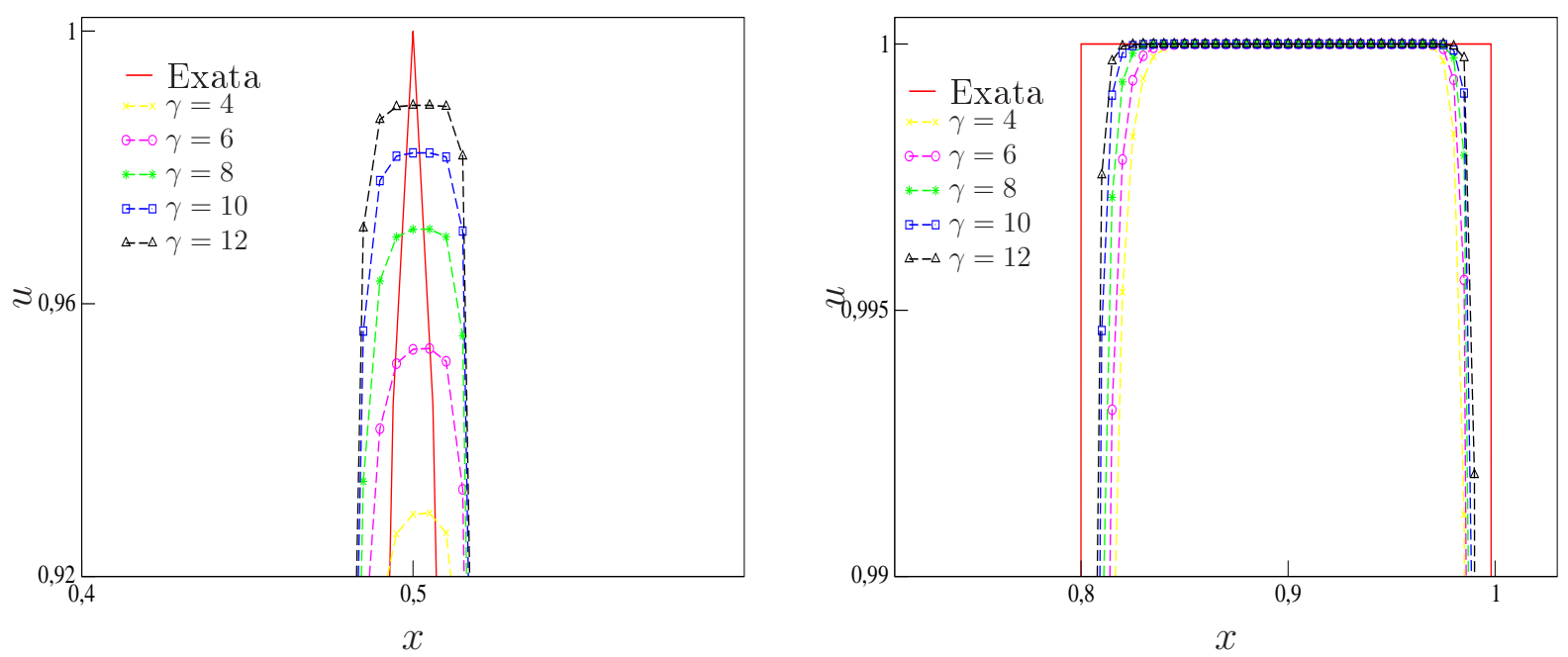

Figura 6.1: Análise do parâmetro $\gamma$ para o esquema SDPUS-C1. Comparação entre a solução exata e os resultados numéricos para $\gamma=4,6,8,10,12$ (a); ampliação do pico (b); ampliação do degrau (c). Resultados numéricos para a equação de advecção, com condição inicial (6.1) e contorno de Direchlet homogênea. 
(a)

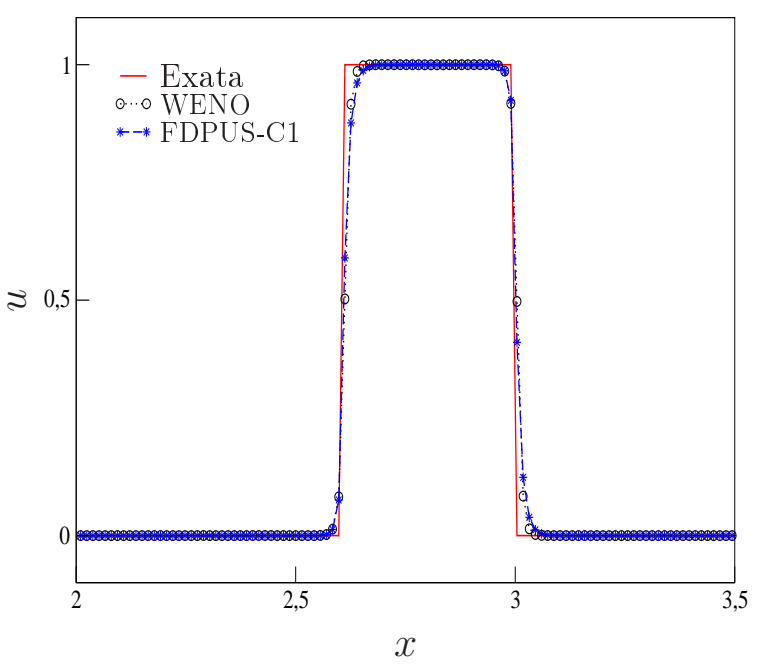

(c)

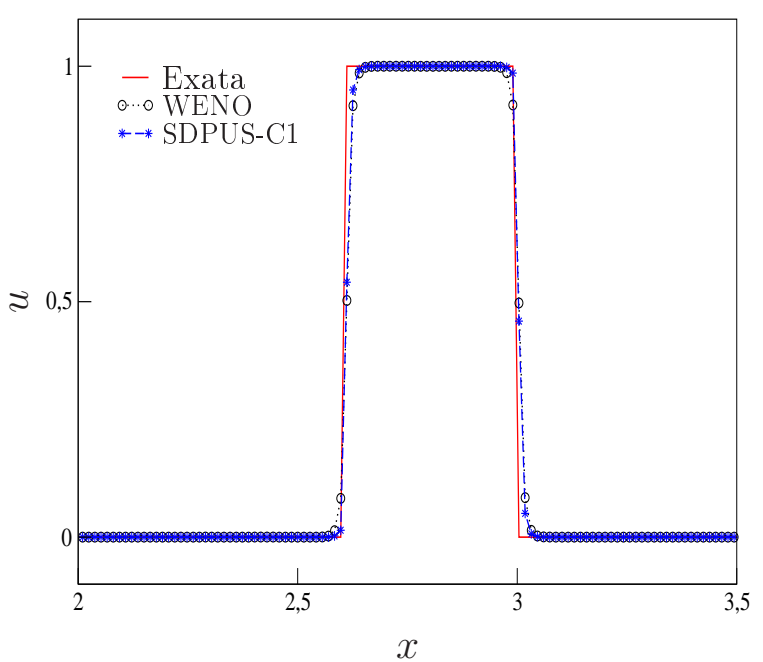

(b)

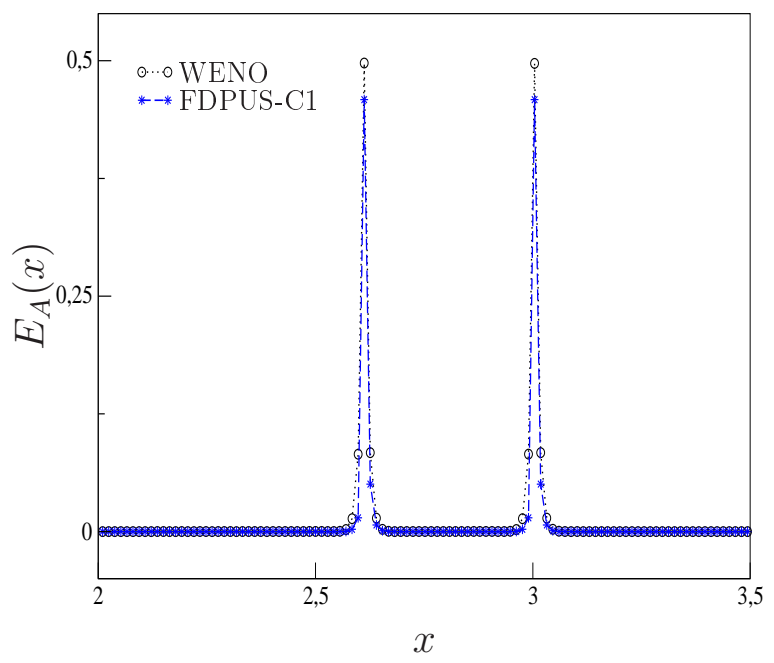

(d)

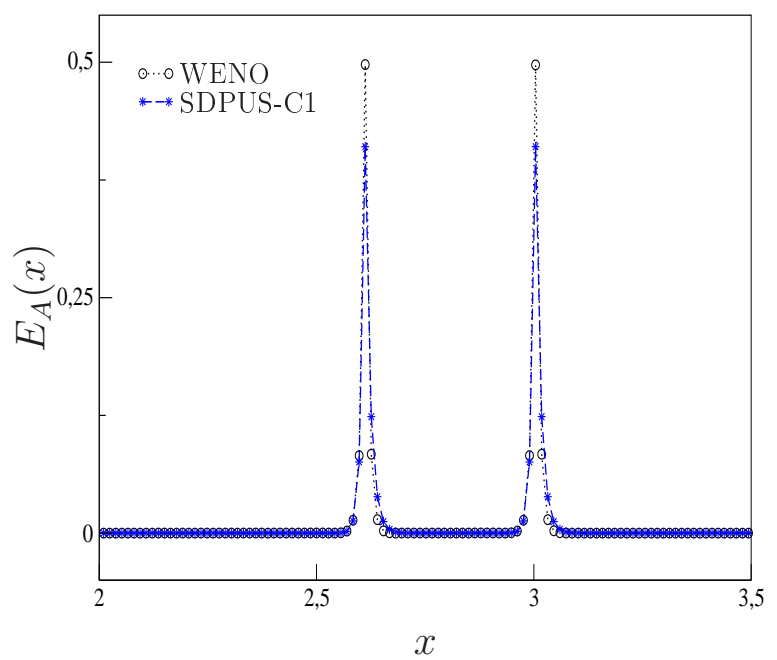

Figura 6.2: Comparação dos esquemas FDPUS-C1 e SDPUS-C1 com WENO. Comparação entre a solução exata e os esquemas WENO, FDPUS-C1 (a) e SDPUS-C1 (c); confronto dos erros absolutos do esquema WENO com FDPUS-C1 (b) e SDPUS-C1 (d). Resultados numéricos para a equação de advecção, com condição inicial (6.2) e contorno de Direchlet homogênea. 
de primeira ordem FOU, de segunda ordem Lax-Wendroff e os esquemas upwind de alta ordem ADBQUICKEST, FDPUS-C1 e SDPUS-C1 $(\gamma=12)$. Para isso, considera-se a equação de advecção definida em $x \in[-1,5]$ e suplementada com a condição inicial

$$
u_{0}(x)=\left\{\begin{array}{l}
1, \quad-\frac{1}{3} \leq x \leq \frac{1}{3} \\
0, \quad \text { caso contrário. }
\end{array}\right.
$$

Para essa análise, calcula-se a TV, dada por (3.11), a cada passo no tempo, em 100, 200 e 400 células computacionais, $\operatorname{com} \theta=0.5$ e $t=4$. Os resultados são, então, plotados no plano $T V(u(t)) \perp t$ e cada esquema é analisado quanto ao seu comportamento, ou seja, para que o mesmo satisfaça a restrição TVD, sua TV (função $T V=T V(u(t))$ ) deve ser constante, constante por parte ou decrescente (ver definição (3.13)). É interessante também detectar se essa função está convergindo para a TV exata (função constante dada pela reta $T V(u(t))=2$ ).

Como complementação, compara-se a solução exata com os resultados numéricos gerados pelos os esquemas Lax-Wendroff, FOU, ADBQUICKEST, FDPUS-C1 e SDPUS-C1. Salienta-se que esses resultados numéricos são apresentados para $x \in[3,5]$, uma vez que para o tempo considerado $(t=4)$ a condição inicial é transportada para esse intervalo (ver definição (2.6)).

Na Figura 6.3 (em que N representa o número de células computacionais) estão apresentadas, à esquerda, as comparações entre a solução exata e as soluções numéricas geradas pelos esquemas Lax-Wendroff (Fig. 6.3-(a)) e FOU (Fig. 6.3-(c)). À direita, dessa mesma figura, estão ilustradas as comparações entre a TV exata e as obtidas com os esquemas Lax-Wendroff (Fig. 6.3-(b)) e FOU (Fig. 6.3-(d)). Como já era de se esperar (ver por exemplo Cunha [19] e Thomas [65]), por essa figura, constata-se que o esquema Lax-Wendroff é dispersivo (ver Fig. 6.3-(a)), ou seja, apresenta oscilações não físicas, além disso, esse esquema não é TVD (ver Fig. 6.3-(b)), uma vez que a TV numérica não satisfaz (3.13) e não converge para TV exata. Também, por essa mesma figura, observa-se que o esquema FOU é dissipativo, ou seja, as soluções numéricas são suavizadas próximo as descontinuidades (ver Fig. 6.3-(c)) e que o mesmo satisfaz a restrição TVD, pois a TV tem um comportamento decrescente, e conforme refina-se a malha, a mesma converge para TV exata.

Na Figura 6.4 são apresentados os resultados para os esquemas de alta resolução que satisfazem a restrição TVD. À esquerda dessa figura são ilustradas as comparações entre a solução exata e os resultados numéricos gerados pelos esquemas ADBQUICKEST (Fig. 6.4-(a)), FDPUS-C1 (Fig. 6.4-(c)) e SDPUS-C1 (Fig. 6.4-(e)). À direita, compara-se a TV exata com as TVs dos esquemas ADBQUICKEST (Fig. 6.4-(b)), FDPUS-C1 (Fig. 6.4-(d)) e SDPUS-C1 (Fig. 6.4-(f)). Salienta-se que para ilustração dos resultados obtidos para TVs, a malha de 400 células computacionais é desconsiderada, pois a TV numérica determinada para os esquemas upwind de alta ordem (ADBQUICKEST, FDPUS-C1 e SDPUS-C1) converge para TV exata (função constante dada pela reta $T V(u(t))=2$ ) com 200 células computacionais. Observa-se por essa figura que as aproximações são satisfatórias e que os esquemas satisfazem à restrição 
(a)

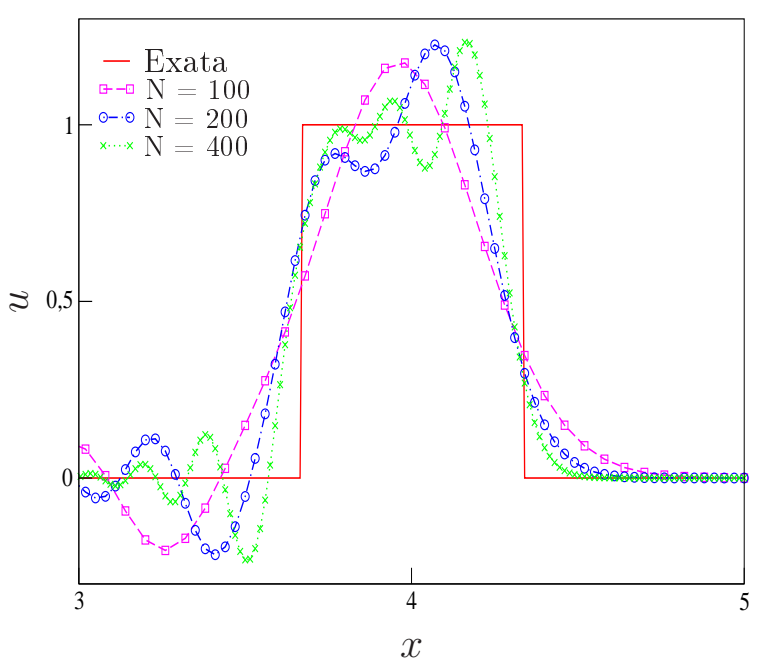

(c)

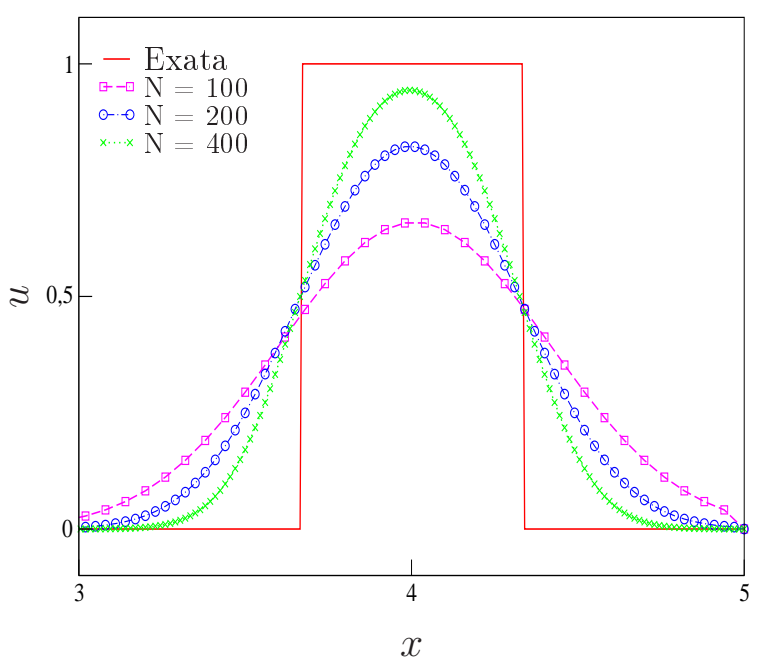

(b)

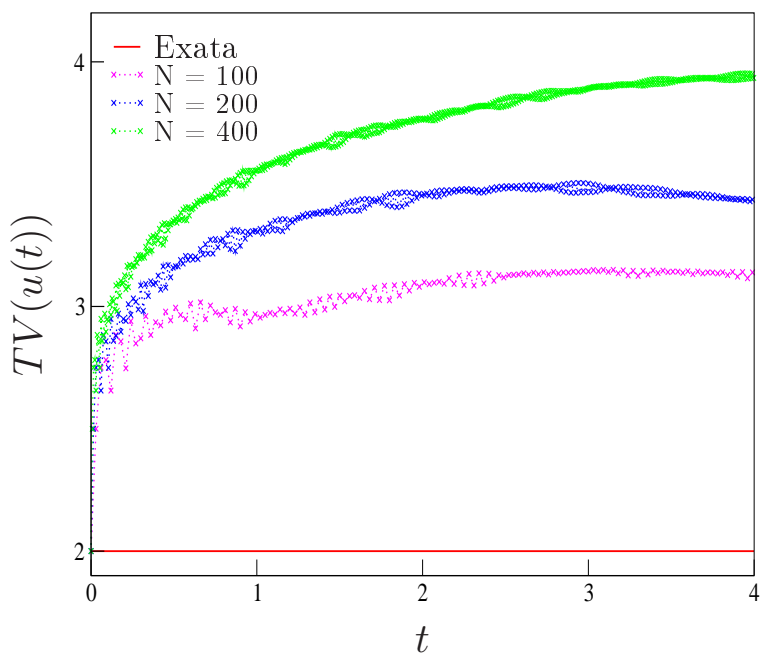

(d)

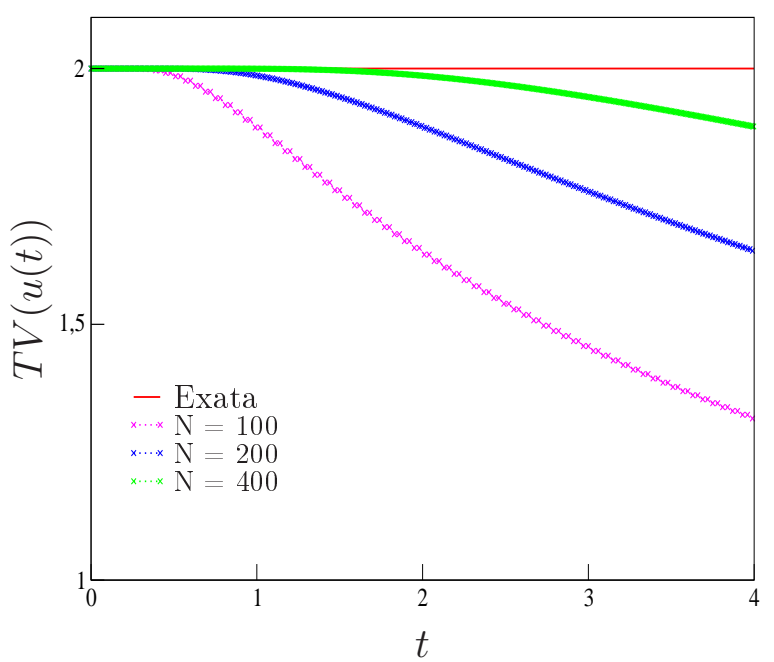

Figura 6.3: Análise da restrição TVD. Comparação entre a solução exata e os esquemas Lax-Wendroff (a) e FOU (c); comparação da TV exata com a TV numérica dos esquemas Lax-Wendroff (b) e FOU (d). Resultados numéricos para a equação de advecção, com condição inicial (6.4) e contorno de Direchlet homogênea. 
TVD, uma vez que as TVs convergem para TV exata $(\mathrm{TV}=2)$ e apresentam um comportamento decrescente ou constantes. Em particular, observa-se, por essa mesma figura, que os resultados obtidos com os esquemas FDPUS-C1 e SDPUS-C1 são melhores que os gerados pelo esquema ADBQUICKEST.

Caso 4 - Ordem de convergência. Este caso tem como propósito verificar a precisão dos esquemas ADBQUICKEST, TOPUS, CUBISTA, VONOS, FDPUS-C1 e SDPUS-C1 $(\gamma=12)$ em regiões suaves. Para tanto, considera-se a equação de advecção definida no domínio $[-\pi, \pi]$ e suplementada com a condição inicial suave (Herrmann et al. [33])

$$
u_{0}(x)=\operatorname{sen}(x)
$$

Por meio dos resultados numéricos obtidos para este problema, são determinados, para cada método, os erros relativos (entre as soluções exata e a numérica) e uma estimativa para a ordem de convergência. Os erros relativos $E_{h}$ entre as soluções exata e numérica (ambas geradas em uma malha de espaçamento $h$ ), nas normas $L_{1}, L_{2}$ e $L_{\infty}$, são definidos, respectivamente por

$$
\begin{aligned}
\left\|E_{h}\right\|_{1} & =\frac{\sum_{i=1}^{N}\left|u_{i, \text { exata }}-u_{i, \text { numérica }}\right|}{\sum_{i=1}^{N}\left|u_{i, \text { exata }}\right|}, \\
\left\|E_{h}\right\|_{2} & =\sqrt{\frac{\sum_{i=1}^{N}\left(u_{i, \text { exata }}-u_{i, \text { numérica }}\right)^{2}}{\sum_{i=1}^{N}\left(u_{i, \text { exata }}\right)^{2}}}, \\
\left\|E_{h}\right\|_{\infty} & =\frac{\max _{1 \leq i \leq N}\left|u_{i, \text { exata }}-u_{i, \text { numérica }}\right|}{\max _{1 \leq i \leq N}\left|u_{i, \text { exata }}\right|} .
\end{aligned}
$$

Uma estimativa para o erro $\left\|E_{h}\right\|_{k}$, com $k=1,2, \infty$, para as aproximações numéricas é dada da seguinte forma (ver Thomas [65])

$$
\left\|E_{h}\right\|_{k} \approx C h^{p}
$$

em que $C$ é uma constante dependente dos dados do problema e $p$ é uma estimativa para ordem de convergência.

Para o cálculo de $p$, estima-se o erro relativo $\left\|E_{\frac{h}{2}}\right\|_{k}$

$$
\left\|E_{\frac{h}{2}}\right\|_{k} \approx C\left(\frac{h}{2}\right)^{p}
$$

em seguida, divide-se (6.9) por (6.10) obtendo-se

$$
\frac{\left\|E_{h}\right\|_{k}}{\left\|E_{\frac{h}{2}}\right\|_{k}} \approx \frac{h^{p}}{\left(\frac{h}{2}\right)^{p}}=2^{p}
$$


(a)

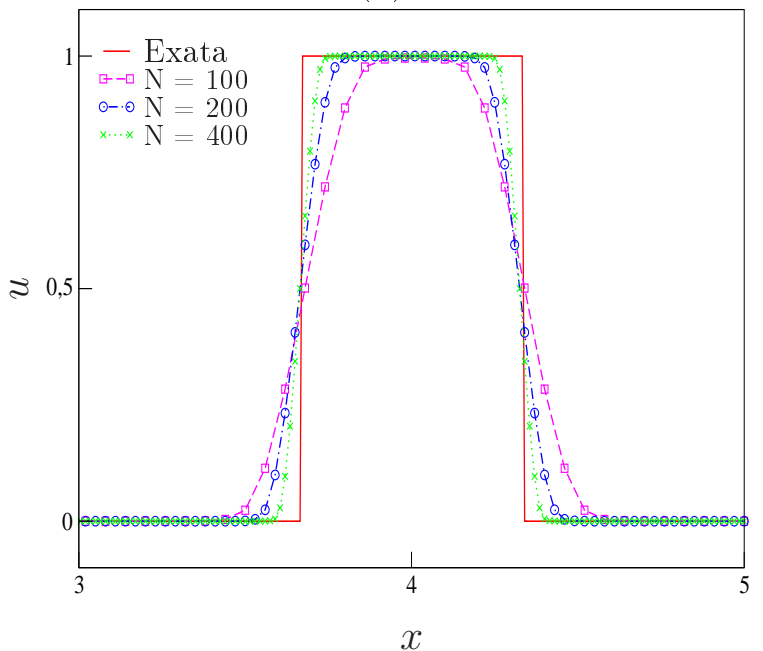

(c)

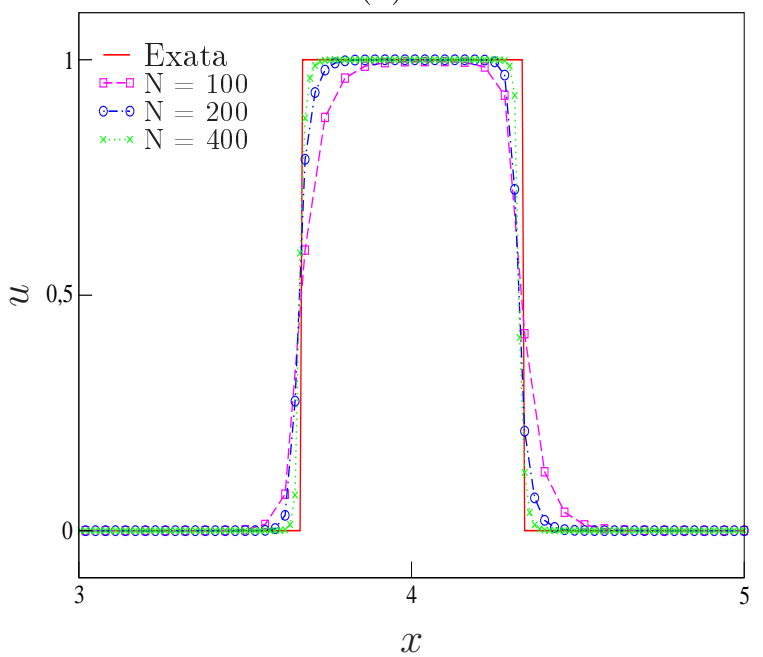

(e)

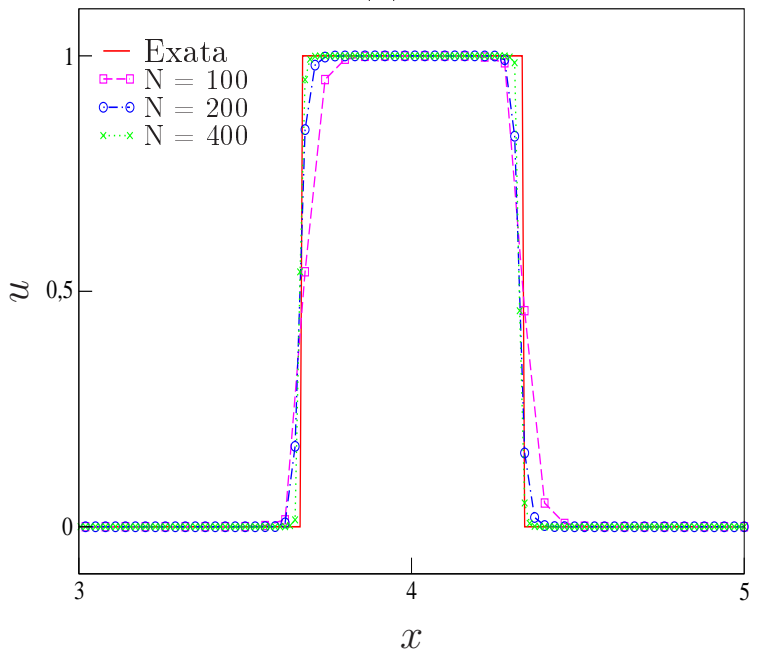

(b)

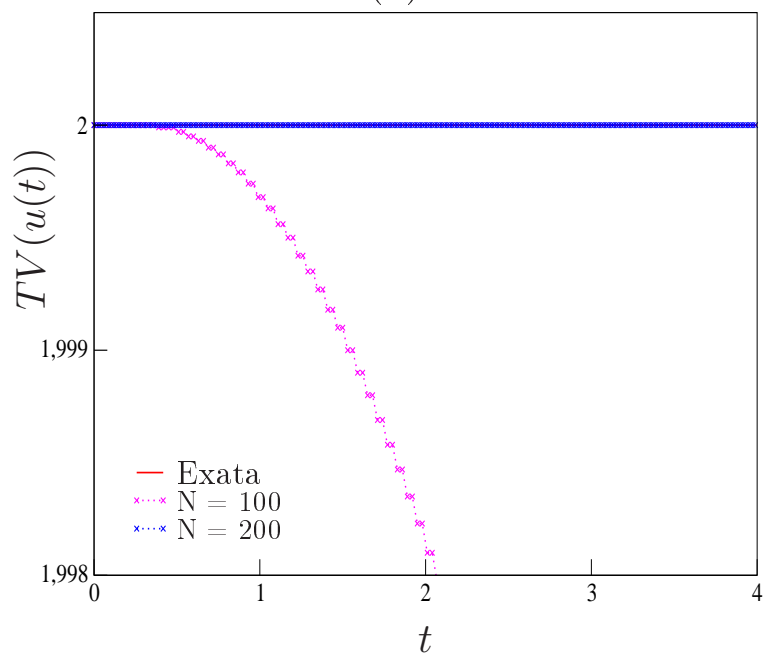

(d)

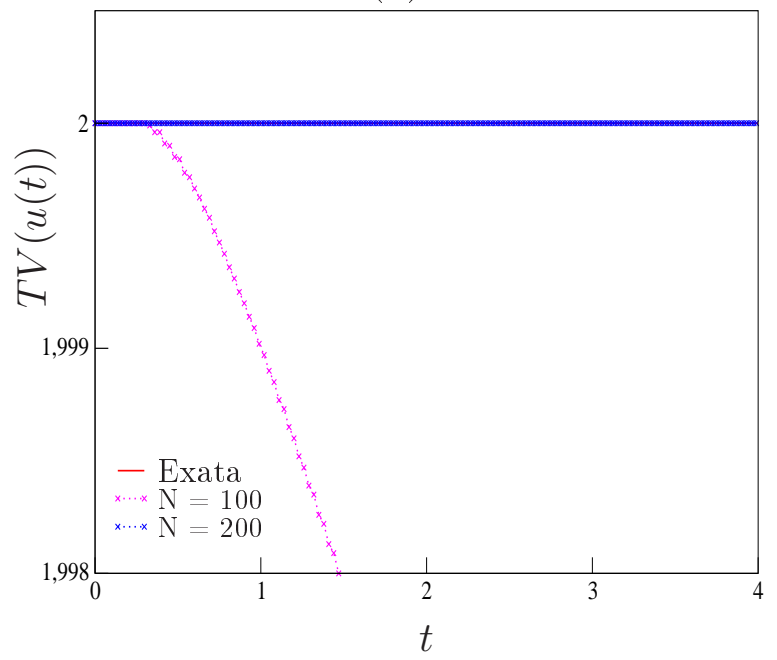

(f)

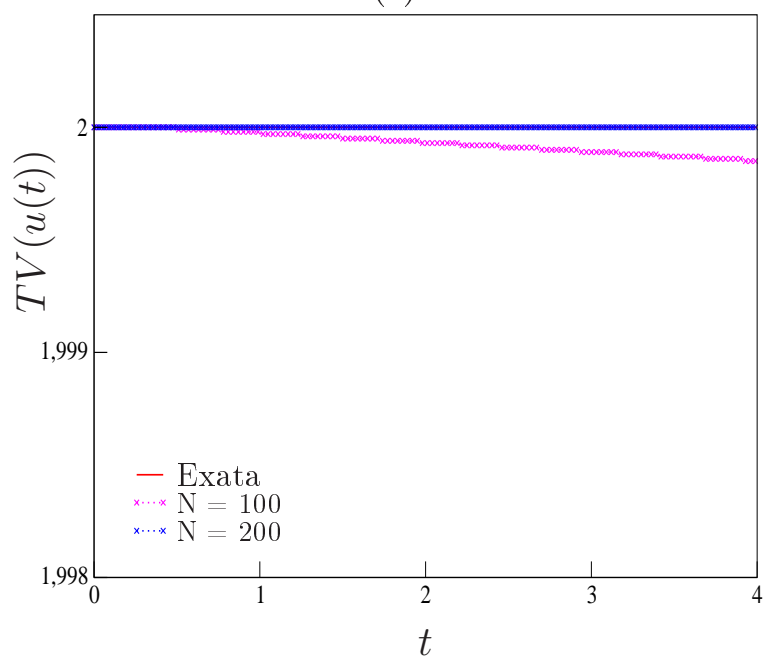

Figura 6.4: Análise da restrição TVD. Comparação entre a solução exata e os esquemas ADBQUICKEST (a), FDPUS-C1 (c) e SDPUS-C1 (e); comparação da TV exata com as TVs numéricas dos esquemas ADBQUICKEST (b), FDPUS-C1 (d) e SDPUS-C1 (f). Resultados numéricos para a equação de advecção, com condição inicial (6.4) e contorno de Direchlet homogênea. 
e aplica-se log em ambos os membros de (6.11) obtendo-se, assim,

$$
p \approx\left(\log \frac{\left\|E_{h}\right\|_{k}}{\left\|E_{\frac{h}{2}}\right\|_{k}}\right) / \log 2 .
$$

Os resultados numéricos são obtidos a partir de cinco malhas (com 20, 40, 80, 160 e 320 células computacionais), $\operatorname{com} \theta=0.5$ e $t=1$. Determinam-se os erros relativos por meio das definições (6.6),(6.7) e (6.8) e as estimativas para a ordem de convergência por meio de (6.12). Esses dados estão apresentados na Tabela 6.1. Por essa tabela consta-se que o esquema VONOS apresenta a estimativa mais baixa para ordem de convergência; os esquemas CUBISTA, TOPUS e FDPUS-C1 apresentam resultados semelhantes; e os esquemas ADBQUICKEST e SDPUS-C1 apresentam os melhores resultados.

Caso 5 - Forma $\boldsymbol{W}$-shape. Neste caso é apresentado o problema proposto por Wei e Gu [77] (a famosa forma $W$-shape) que corresponde à equação de advecção, suplementada com a condição inicial

$$
u_{0}(x)=\left\{\begin{array}{lr}
1, & 0 \leq x \leq 0.2, \\
4 x-\frac{3}{5}, & 0.2<x \leq 0.4 \\
-4 x+\frac{13}{5}, & 0.4<x \leq 0.6 \\
1, & 0.6<x \leq 0.8 \\
0, & \text { caso contrário. }
\end{array}\right.
$$

A solução deste problema apresenta descontinuidades severas que são difíceis de resolver (ver Toro [68], Wei e Gu [77]). Para simulação são considerados uma malha uniforme de 400 células computacionais, $\theta=0.3$ e $t=0.1$. Na Figura 6.5 estão apresentadas a solução exata e as soluções numéricas obtidas com os esquemas ADBQUICKEST (Fig. 6.5-(a)), TOPUS (Fig. 6.5-(b)), CUBISTA (Fig. 6.5-(c)), VONOS (Fig. 6.5-(d)), FDPUS-C1 (Fig. 6.5-(e)) e SDPUS-C1 (Fig. 6.5-(f)). Pode ser observado por esta figura que os dados numéricos gerados por esses esquemas, exceto àquele pelo esquema VONOS, apresentam boa concordância, uma vez que os vales e os picos são capturados satisfatoriamente e são livres de oscilações.

Caso 6 - Aplicação interessante. Neste caso a equação de advecção é definida em [-1,1] e suplementada com a condição inicial dada por

$$
u_{0}(x)=\left\{\begin{array}{lr}
-\operatorname{sen}(\pi x)-0.5 x^{3}, & -1 \leq x<0 \\
-\operatorname{sen}(\pi x)-0.5 x^{3}+1, & 0 \leq x \leq 1 .
\end{array}\right.
$$

Para a simulação são considerados uma malha com 300 células computacionais, $t=0.1 \mathrm{e}$ $\theta=0.5$. A Figura 6.6 mostra a comparação entre a solução exata e as soluções numéricas geradas com os esquemas ADBQUICKEST (Fig. 6.6-(a)), TOPUS (Fig. 6.6-(b)), CUBISTA (Fig. 6.6-(c)), VONOS (Fig. 6.6-(d)), FDPUS-C1 (Fig. 6.6-(e)) e SDPUS-C1 (Fig. 6.6-(f)). 


\begin{tabular}{|c|c|c|c|c|c|c|c|}
\hline Esquema & $\mathrm{N}$ & $\overline{\mid E_{h} \|_{1}}$ & $\mathrm{p}$ & $\overline{\mid E_{h} \|_{2}}$ & $\mathrm{p}$ & $\mid E_{h} \|_{\infty}$ & $\mathrm{p}$ \\
\hline \multirow[t]{5}{*}{ ADBQUICKEST } & 20 & $0.274 \times 10^{-1}$ & - & $0.318 \times 10^{-1}$ & - & $0.529 \times 10^{-1}$ & 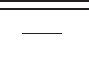 \\
\hline & 40 & $0.800 \times 10^{-2}$ & 1.78 & $0.112 \times 10^{-1}$ & 1.51 & $0.258 \times 10^{-1}$ & 1.09 \\
\hline & 80 & $0.252 \times 10^{-2}$ & 1.67 & $0.453 \times 10^{-2}$ & 1.31 & $0.120 \times 10^{-1}$ & 1.05 \\
\hline & 160 & $0.795 \times 10^{-3}$ & 1.66 & $0.182 \times 10^{-2}$ & 1.32 & $0.623 \times 10^{-2}$ & 0.95 \\
\hline & 320 & $0.247 \times 10^{-3}$ & 1.69 & $0.739 \times 10^{-3}$ & 1.30 & $0.292 \times 10^{-2}$ & 1.09 \\
\hline \multirow[t]{5}{*}{ "TOPUS } & 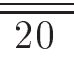 & $0.435 \times 10^{-1}$ & $\overline{-}$ & $0.455 \times 10^{-1}$ & - & $\overline{0.607 \times 10^{-1}}$ & - \\
\hline & 40 & $0.143 \times 10^{-1}$ & 1.61 & $0.171 \times 10^{-1}$ & 1.41 & $0.310 \times 10^{-1}$ & 0.97 \\
\hline & 80 & $0.465 \times 10^{-2}$ & 1.62 & $0.692 \times 10^{-2}$ & 1.30 & $0.182 \times 10^{-1}$ & 0.77 \\
\hline & 160 & $0.148 \times 10^{-2}$ & 1.65 & $0.282 \times 10^{-2}$ & 1.29 & $0.113 \times 10^{-1}$ & 0.68 \\
\hline & 320 & $0.564 \times 10^{-3}$ & 1.39 & $0.131 \times 10^{-2}$ & 1.11 & $0.877 \times 10^{-2}$ & 0.37 \\
\hline \multirow[t]{5}{*}{ CUBISTA } & 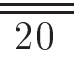 & 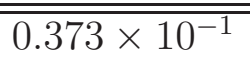 & - & $0.411 \times 10^{-1}$ & - & $\overline{0.646 \times 10^{-1}}$ & - \\
\hline & 40 & $0.115 \times 10^{-1}$ & 1.70 & $0.154 \times 10^{-1}$ & 1.42 & $0.332 \times 10^{-1}$ & 0.94 \\
\hline & 80 & $0.388 \times 10^{-2}$ & 1.58 & $0.646 \times 10^{-2}$ & 1.25 & $0.180 \times 10^{-1}$ & 0.88 \\
\hline & 160 & $0.123 \times 10^{-2}$ & 1.65 & $0.260 \times 10^{-2}$ & 1.31 & $0.909 \times 10^{-2}$ & 0.99 \\
\hline & 320 & $0.490 \times 10^{-3}$ & 1.69 & $0.200 \times 10^{-2}$ & 1.24 & $0.446 \times 10^{-2}$ & 1.03 \\
\hline \multirow[t]{5}{*}{ VONOS } & 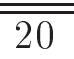 & 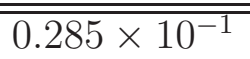 & - & $0.334 \times 10^{-1}$ & - & $0.494 \times 10^{-1}$ & - \\
\hline & 40 & $0.115 \times 10^{-1}$ & 1.31 & $0.152 \times 10^{-1}$ & 1.13 & $0.332 \times 10^{-1}$ & 0.57 \\
\hline & 80 & $0.337 \times 10^{-2}$ & 1.64 & $0.598 \times 10^{-2}$ & 1.35 & $0.169 \times 10^{-1}$ & 0.98 \\
\hline & 160 & $0.101 \times 10^{-2}$ & 1.87 & $0.219 \times 10^{-2}$ & 1.45 & $0.957 \times 10^{-2}$ & 0.82 \\
\hline & 320 & $0.394 \times 10^{-3}$ & 1.36 & $0.891 \times 10^{-3}$ & 1.30 & $0.501 \times 10^{-2}$ & 0.93 \\
\hline \multirow[t]{5}{*}{ FDPUS-C1 } & 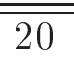 & $0.451 \times 10^{-1}$ & $\overline{-}$ & $0.487 \times 10^{-1}$ & 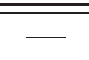 & $\overline{0.793 \times 10^{-1}}$ & - \\
\hline & 40 & $0.144 \times 10^{-1}$ & 1.62 & $0.181 \times 10^{-1}$ & 1.42 & $0.393 \times 10^{-1}$ & 1.05 \\
\hline & 80 & $0.573 \times 10^{-2}$ & 1.35 & $0.799 \times 10^{-2}$ & 1.18 & $0.202 \times 10^{-1}$ & 0.96 \\
\hline & 160 & $0.222 \times 10^{-2}$ & 1.37 & $0.329 \times 10^{-2}$ & 1.28 & $0.100 \times 10^{-2}$ & 1.10 \\
\hline & 320 & $0.865 \times 10^{-3}$ & 1.36 & $0.143 \times 10^{-2}$ & 1.21 & $0.443 \times 10^{-2}$ & 1.05 \\
\hline \multirow[t]{5}{*}{ "SDPUS-C1 } & 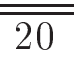 & 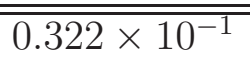 & $\overline{-}$ & 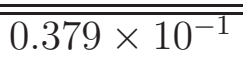 & $\overline{-}$ & $\overline{0.609 \times 10^{-1}}$ & - \\
\hline & 40 & $0.944 \times 10^{-1}$ & 1.77 & $0.129 \times 10^{-1}$ & 1.56 & $0.303 \times 10^{-1}$ & 1.01 \\
\hline & 80 & $0.307 \times 10^{-2}$ & 1.62 & $0.509 \times 10^{-2}$ & 1.34 & $0.138 \times 10^{-1}$ & 1.13 \\
\hline & 160 & $0.105 \times 10^{-2}$ & 1.54 & $0.212 \times 10^{-2}$ & 1.26 & $0.701 \times 10^{-2}$ & 0.98 \\
\hline & 320 & $0.425 \times 10^{-3}$ & 1.31 & $0.896 \times 10^{-3}$ & 1.25 & $0.354 \times 10^{-2}$ & 0.99 \\
\hline
\end{tabular}

Tabela 6.1: Ordem de convergência. Erros nas normas $L_{1}, L_{2}$ e $L_{\infty}$ e estimativas da ordem de convergência para os esquemas ADBQUICKEST, TOPUS, CUBISTA, VONOS, FDPUS-C1 e SDPUS-C1. Dados para a equação de advecção, com condição inicial (6.5) e com condição de Dirichelet homogênea no contorno. 
(a)

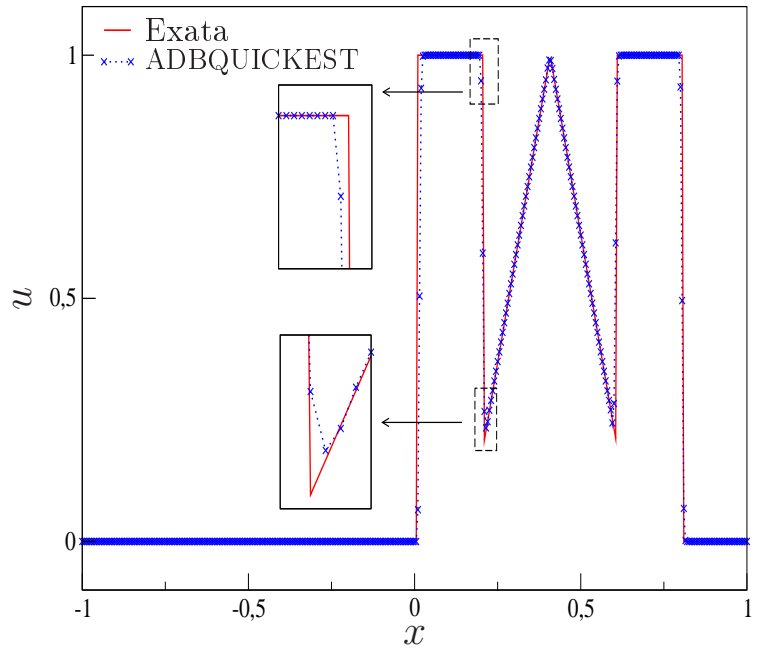

(c)

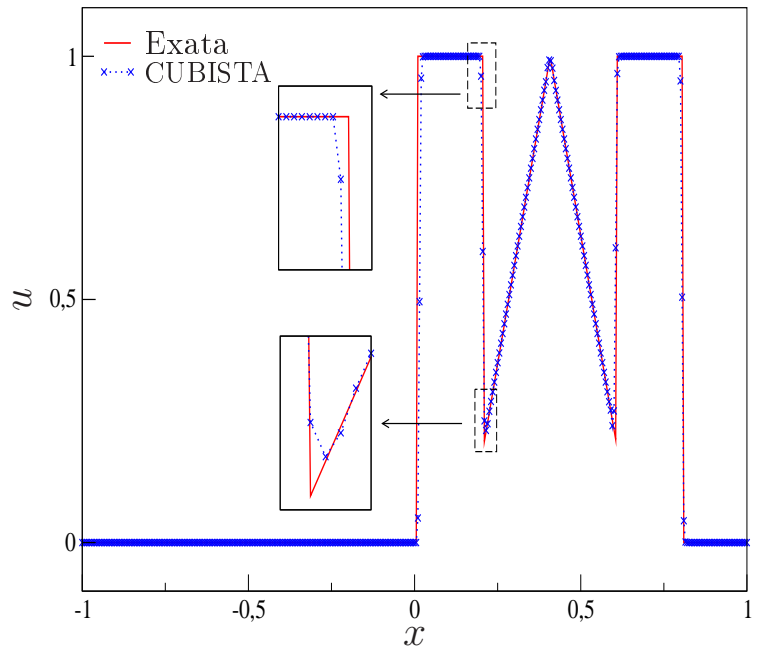

(e)

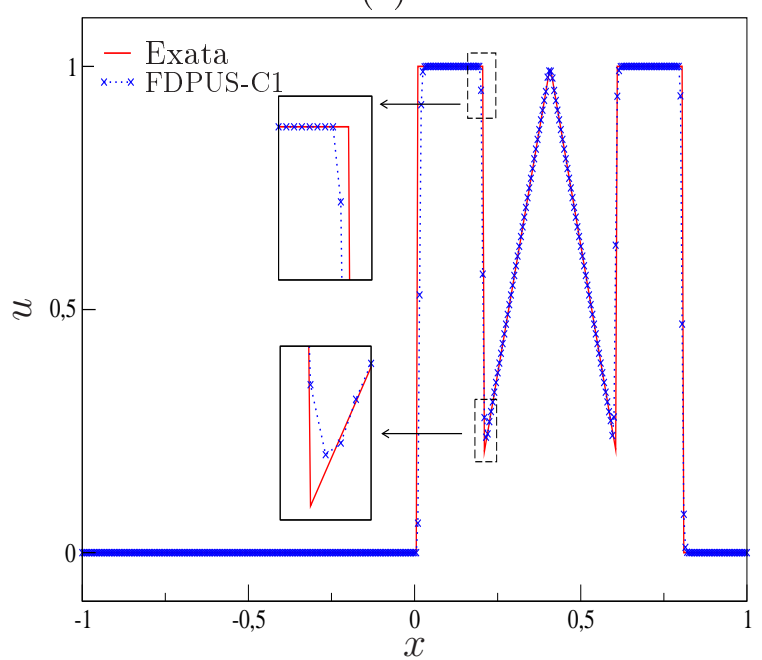

(b)

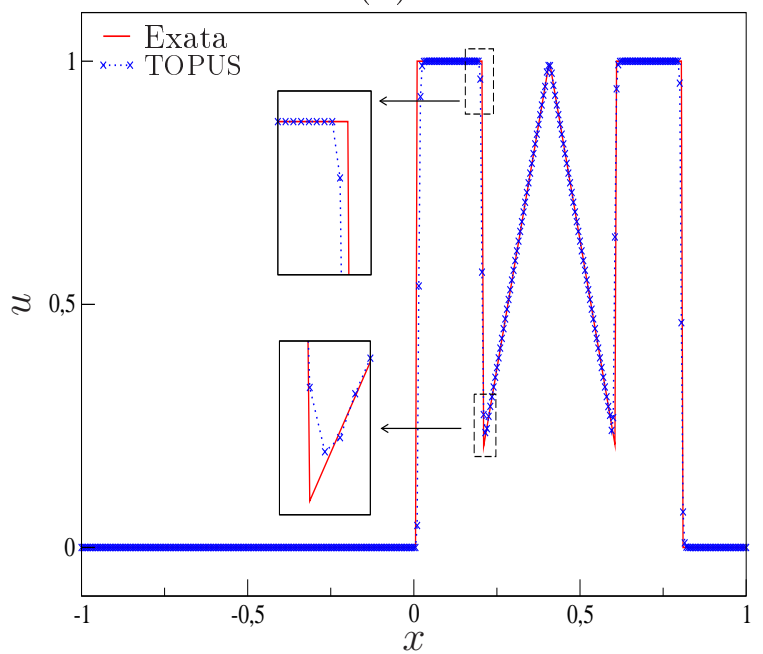

(d)

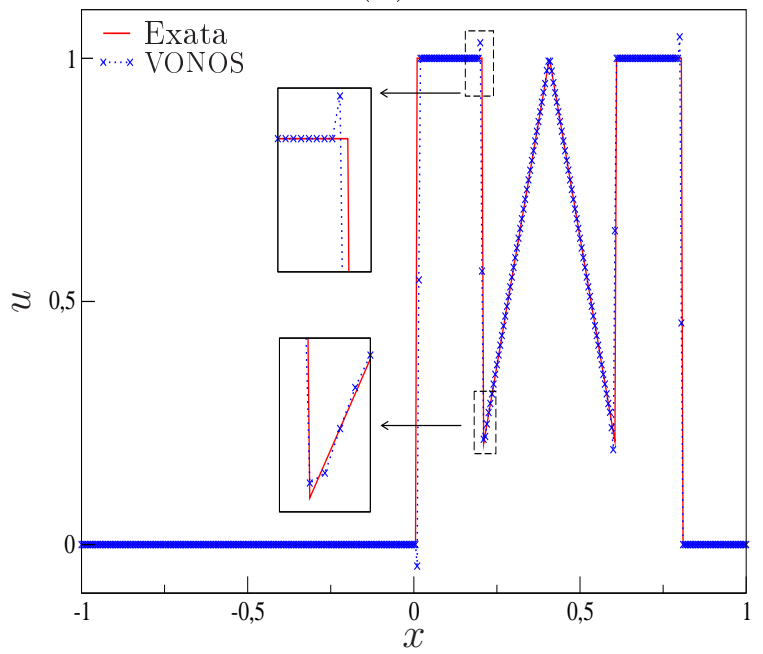

(f)

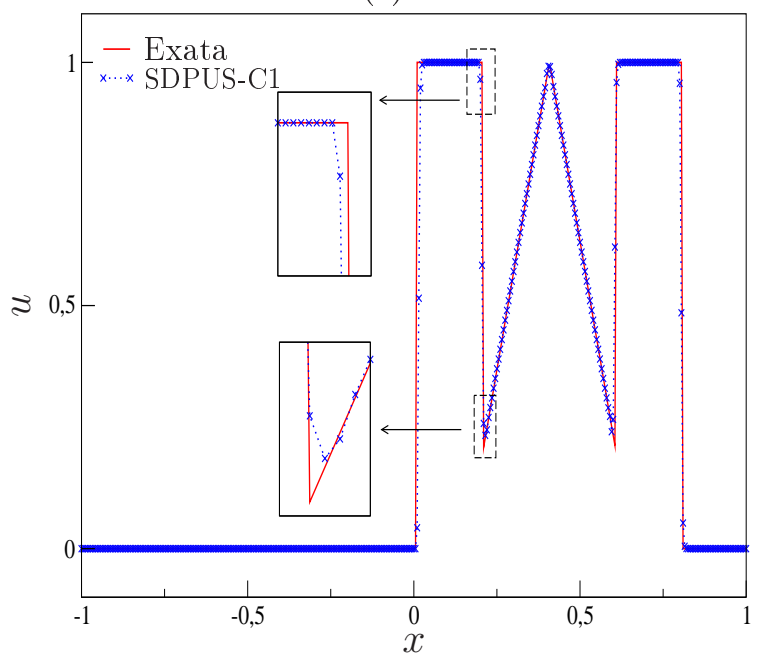

Figura 6.5: Forma $\boldsymbol{W}$-Shape. Comparação entre a solução exata e os esquemas ADBQUICKEST (a), TOPUS (b), CUBISTA (c), VONOS (d), FDPUS-C1 (e) e SDPUS-C1 (f) para a equação de advecção, com condição inicial (6.13) e contorno de Direchlet homogênea. 
Observa-se por esta figura que o esquema VONOS apresenta oscilações e o esquema ADBQUICKEST fornece o melhor resultado. Os novos esquemas FDPUS-C1 e SDPUS-C1 possuem comportamento semelhante aos esquemas CUBISTA e TOPUS e apresentam resultados satisfatórios.

Caso 7 - Tempos longos de simulação. Neste caso, a habilidade dos esquemas ADBQUICKEST, TOPUS, CUBISTA, VONOS, FDPUS-C1 e SDPUS-C1 é avaliada para tempos longos de simulação. Problemas desse tipo são importantes em muitas aplicações práticas, tais como aeroacústica e LES (Large Eddy Simulation) (ver, por exemplo, Ekaterinaris [22]). Neste caso, considera-se a equação de advecção, definida em $x \in[-55,245]$ e suplementada com a condição inicial

$$
u_{0}(x)=\cos (0,75|x|) \mathrm{e}^{-0,1|x|} .
$$

Os resultados numéricos são gerados em uma malha com 9000 células computacionais, em $t=200$ e $\theta=0.5$. Na Figura 6.7 estão apresentados os resultados numéricos (considerando $x \in$ $[145,255]$, uma vez que para $t=200$ a solução é deslocada para esse intervalo), para os esquemas ADBQUICKEST (Fig. 6.7-(a)), TOPUS (Fig. 6.7-(b)), CUBISTA (Fig. 6.7-(c)), VONOS (Fig. 6.7-(d)), FDPUS-C1 (Fig. 6.7-(e)) e SDPUS-C1 (Fig. 6.7-(f)). Por essa figura, constata-se que os resultados numéricos estão em boa concordância com a solução exata. Pode-se observar que os esquemas ADBQUICKEST, TOPUS, CUBISTA e os novos esquemas FDPUS-C1 e SDPUS-C1 são capazes de capturar a solução para longos tempos de iteração, ao contrário do que acontece com o esquema VONOS que exibe oscilações "brutais". 
(a)

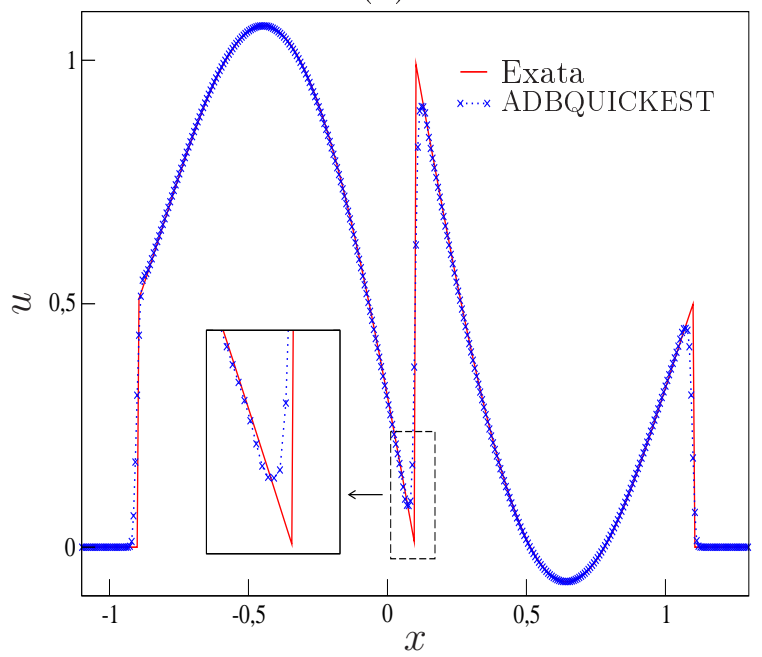

(c)

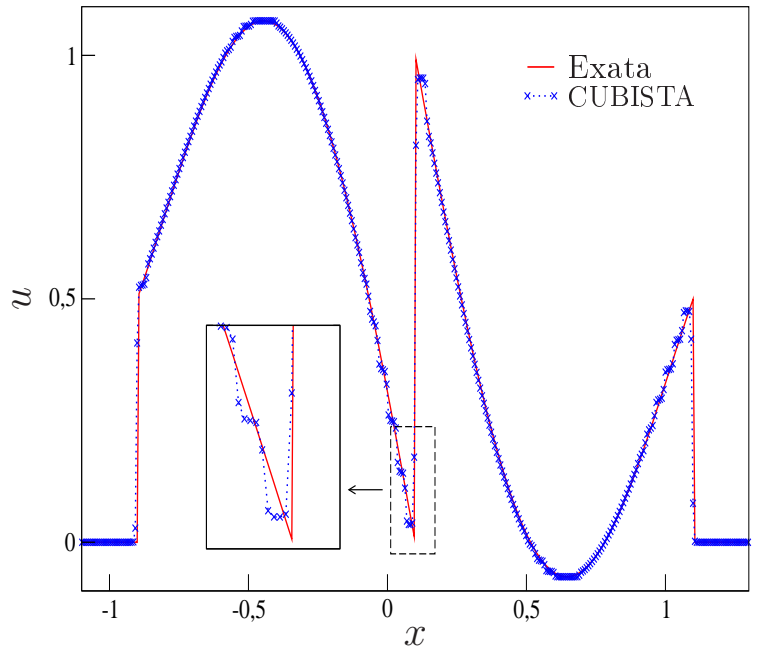

(e)

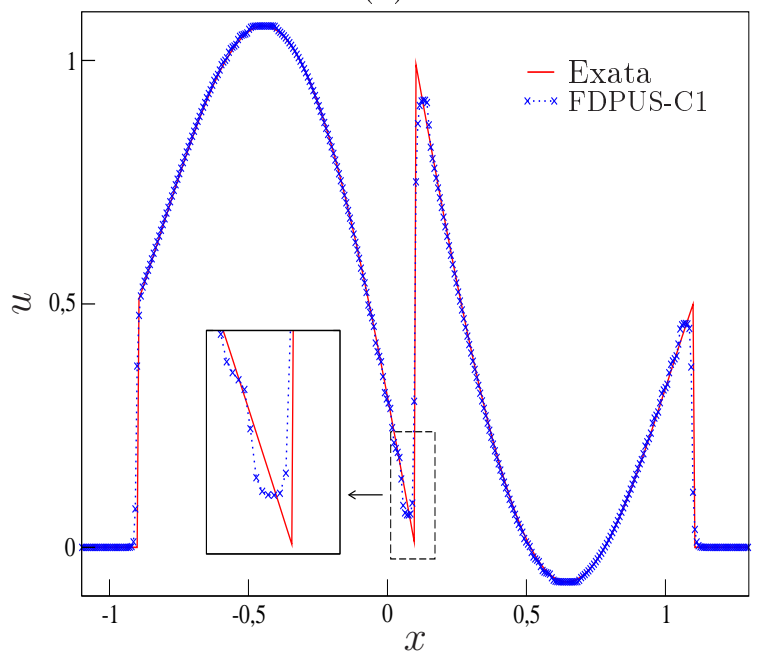

(b)

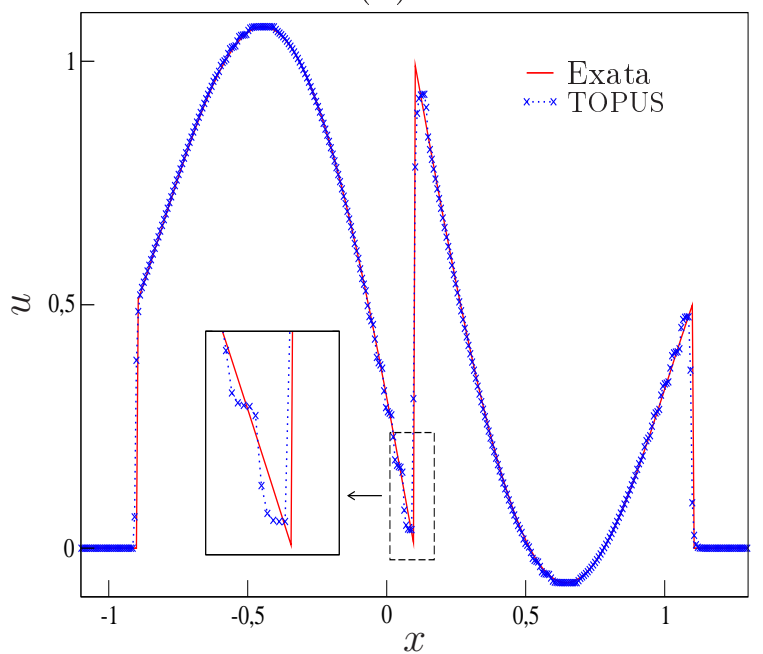

(d)

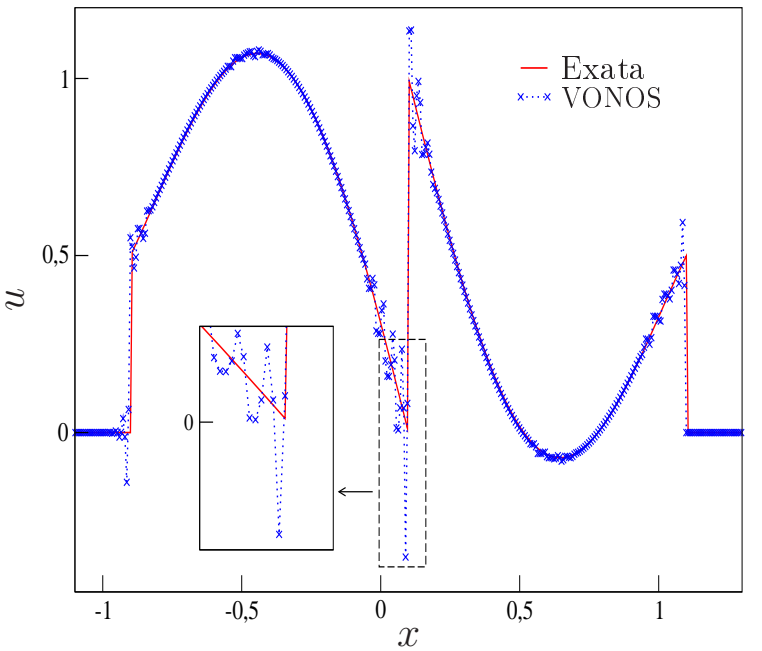

(f)

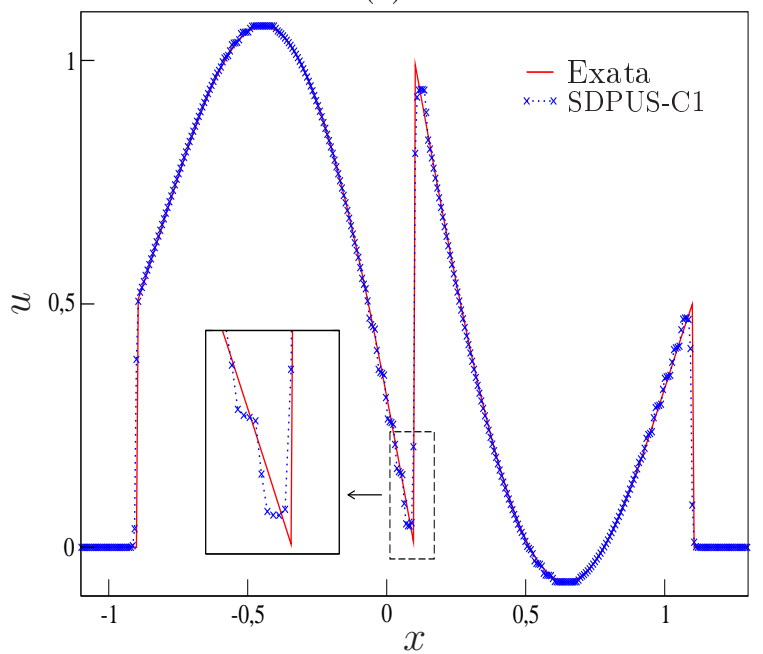

Figura 6.6: Aplicação interessante. Comparação entre a solução exata e os esquemas ADBQUICKEST (a), TOPUS (b), CUBISTA (c), VONOS (d), FDPUS-C1 (e) e SDPUS-C1 (f) para a equação de advecção, com condição inicial (6.14) e contorno de Direchlet homogênea. 
(a)

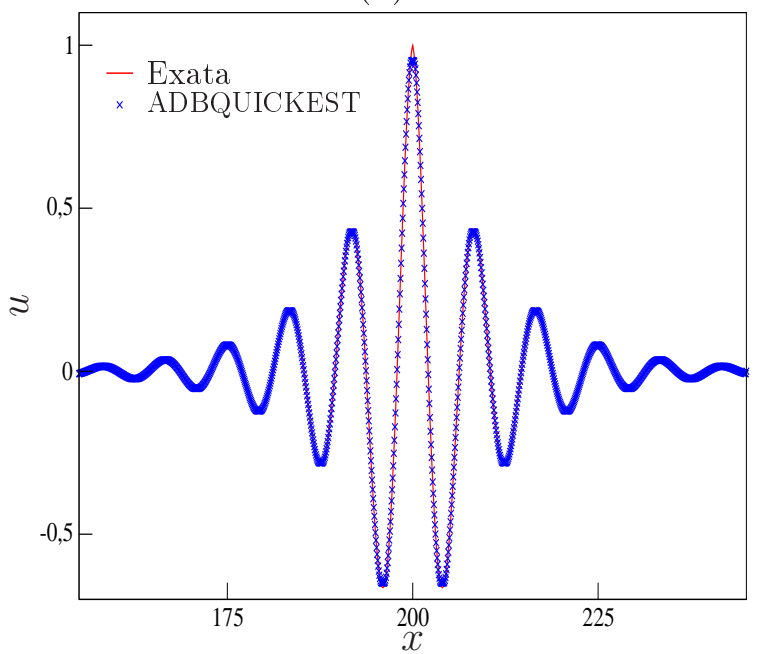

(c)

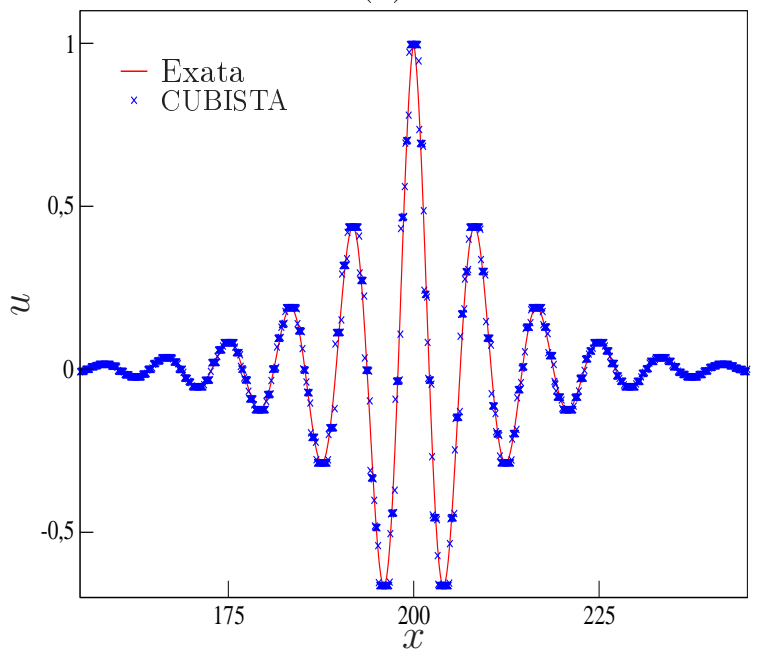

(e)

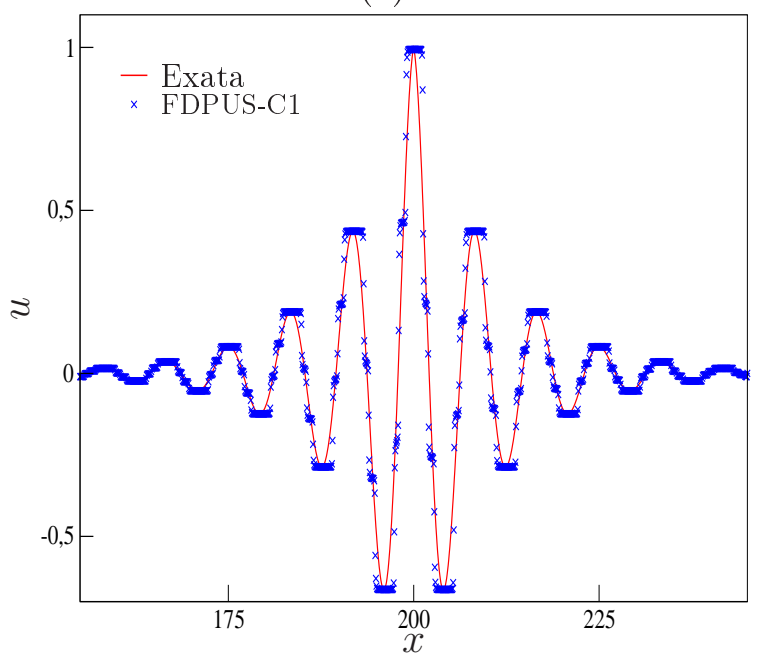

(b)

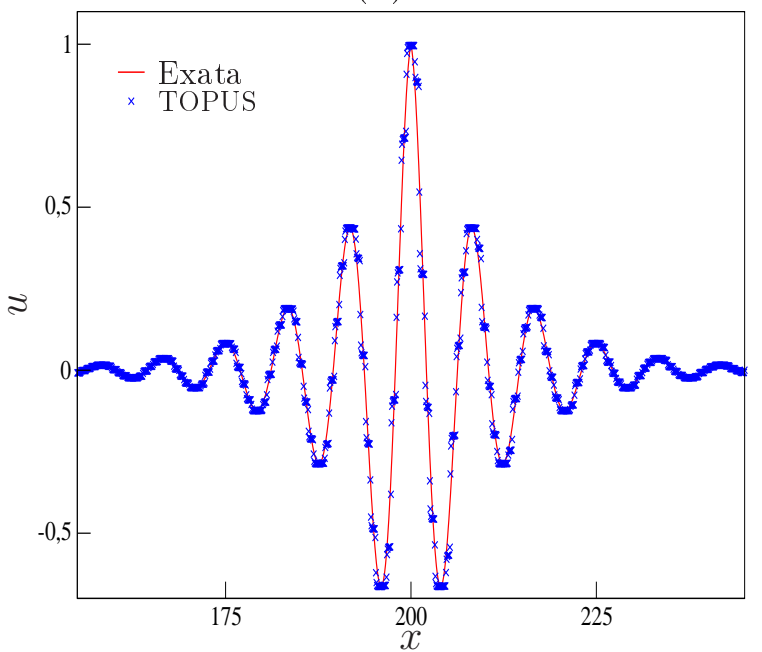

(d)

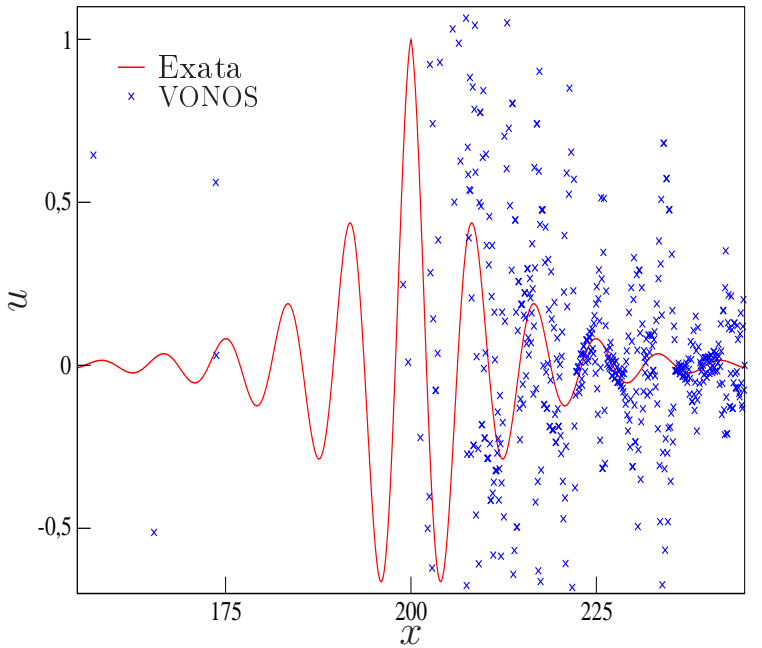

(f)

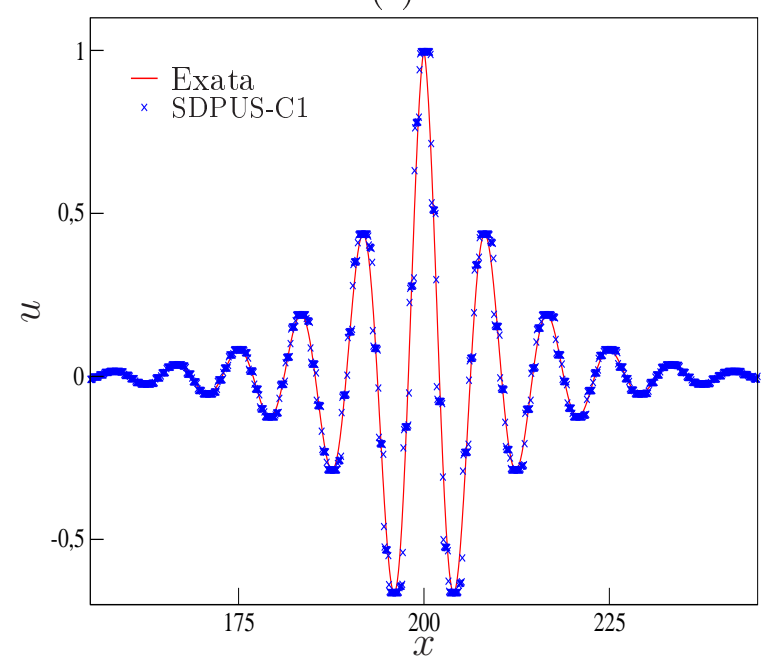

Figura 6.7: Tempos longos de simulação. Comparação entre a solução exata e os esquemas ADBQUICKEST (a), TOPUS (b), CUBISTA (c), VONOS (d), FDPUS-C1 (e) e SDPUS-C1 (f) para a equação de advecção, com condição inicial (6.15) e contorno de Direchlet homogênea. 


\subsection{Equação de Burgers sem e com Viscosidade}

A equação não linear de Burgers com viscosidade é formulada pela lei de conservação 1D (2.1), com variável conservada e fluxo numérico dados respectivamente por (2.7) e (2.8). As soluções numéricas, obtidas para essa equação, geradas com os novos esquemas FDPUS-C1 e SDPUS-C1 são comparadas com as soluções exatas e com os resultados numéricos obtidos pelos esquemas ADBQUICKEST, TOPUS e Superbee. Também analisa-se a restrição TVD e estimam-se as ordens de convergência. Ainda, neste capítulo, apresentam-se os resultados numéricos dos esquemas FDPUS-C1 e SDPUS-C1 para a equação de Burgers com viscosidade, dada por (2.9). Esses resultados podem ser encontrados em anais de eventos e periódicos publicados pela autora (ver o capítulo 9).

\subsubsection{Equação de Burgers sem Viscosidade}

Nesta subseção apresentam-se os resultados obtidos para equação de Burgers sem viscosidade.

Caso 1 - Dados iniciais contínuos por partes. Neste caso a equação de Burgers é definida em $x \in[0,0.6]$, suplementada com a condição inicial

$$
u_{0}(x)= \begin{cases}\frac{1}{2}, & x=0 \\ 0, & x>0\end{cases}
$$

e com condições de contorno dadas por

$$
u(0, t)=\frac{1}{2} \quad \text { e } \quad u(0.6, t)=0 .
$$

Este problema é interessante uma vez que possui solução exata dada por

$$
u(x, t)= \begin{cases}\frac{1}{2}, & x<\frac{1}{4} t, \\ 0, & x>\frac{1}{4} t .\end{cases}
$$

As soluções numéricas são geradas em uma malha com 60 células computacionais, $t=0.9$ e $\theta=0.9$. A Figura 6.8 mostra a comparação da solução exata com os resultados numéricos gerados pelos esquemas ADBQUICKEST (Fig. 6.8-(a)), TOPUS (Fig. 6.8-(b)), FDPUS-C1 (Fig. 6.8-(c)) e SDPUS-C1 (Fig. 6.8-(d)). Em Fig. 6.8-(e) confrontam-se as soluções numéricas obtidas com os esquemas anteriormente citados. Por essas figuras observa-se que os resultados gerados pelos esquemas estão em boa concordância com a solução exata. Constata-se ainda que o esquema SDPUS-C1 oferece o melhor resultado (ver Fig. 6.8-(e)).

Caso 2 - Problema de Platzman. Neste caso a equação de Burgers é definida em $x \in[0,2 \pi]$, 
(a)

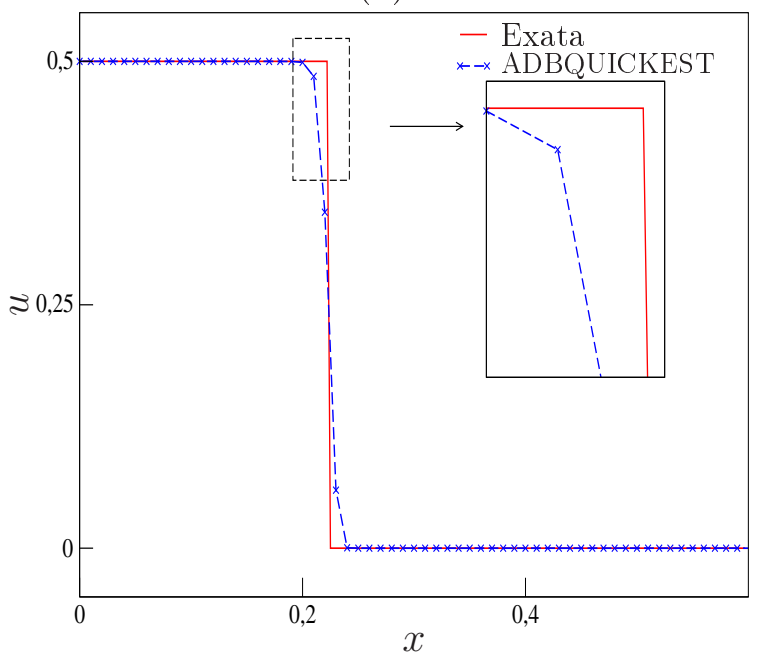

(c)

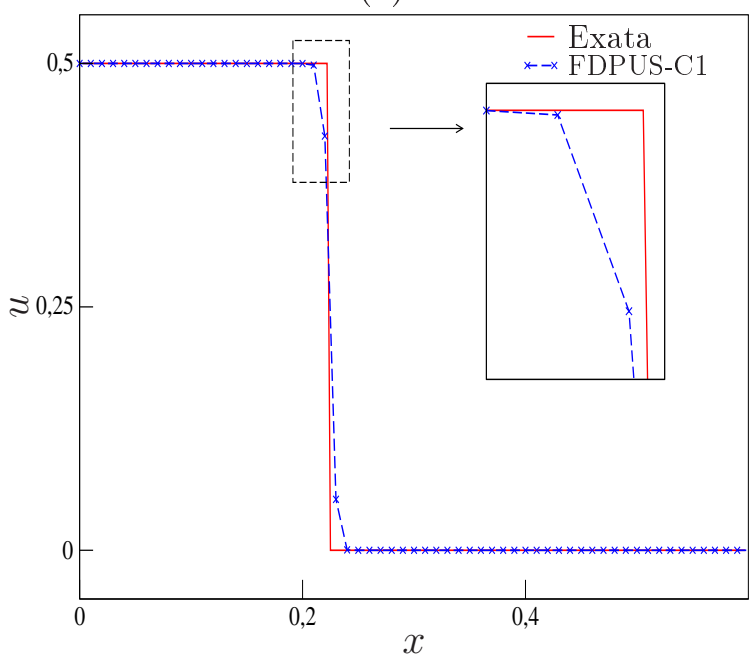

(b)

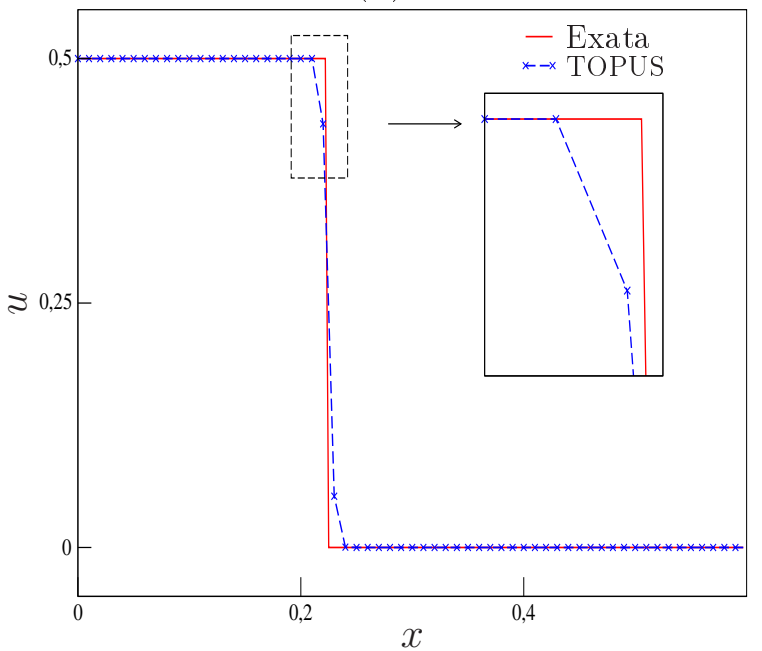

(d)

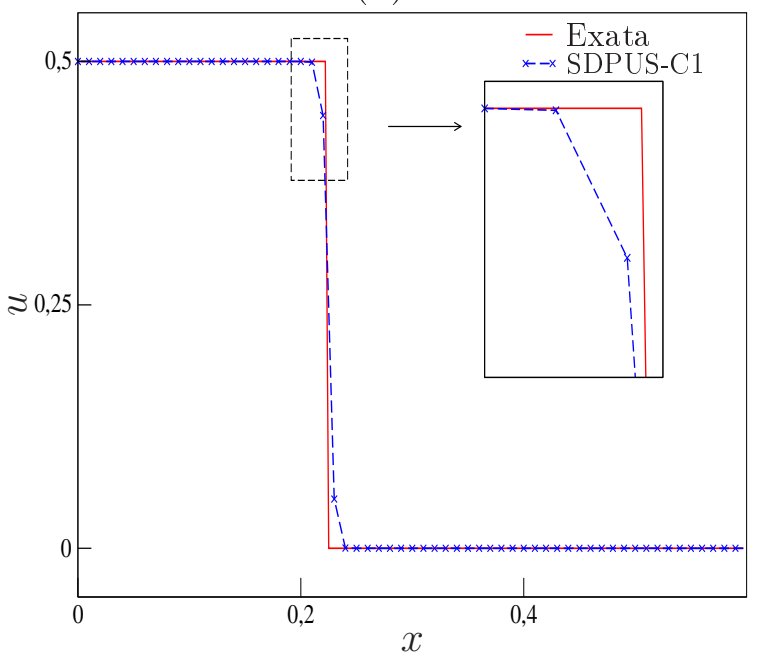

(e)

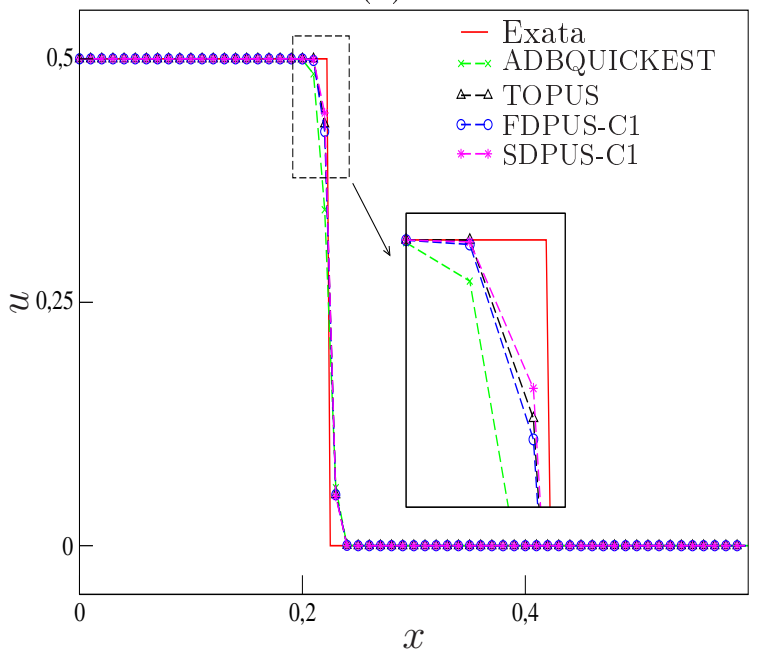

Figura 6.8: Dados iniciais contínuos por partes. Comparação entre a solução exata e os esquemas ADBQUICKEST (a), TOPUS (b), FDPUS-C1 (c) e SDPUS-C1 (d); comparação das soluções numéricas (e). Resultados numéricos para a equação de Burgers sem viscosidade, com as condições inicial (6.16) e de contorno (6.17). 
suplementada com a condição inicial dada por

$$
u(x, 0)=\operatorname{sen}(x)
$$

e com condições de contorno de Dirichlet homogêneas. Este caso é interessante para análise de desempenho dos esquemas, uma vez que possui solução exata dada por (ver Platzman [51])

$$
u(x, t)=-2 \sum_{m} \frac{J_{m}(-m t)}{m t} \operatorname{sen}(m x),
$$

em que $J_{m}$ é a função de Bessel. Esta solução é válida até $t=1$, uma vez que o choque ocorre nesse tempo. Para comparação com os resultados numéricos, até $t=1$ considera-se um solução semi-analítica em que a série em (6.20) é truncada para $m=200$. Após o choque $(t=1)$ considera-se uma solução de referência gerada pelo esquema FOU.

Para análise são consideradas três etapas, denominadas: i) pré-choque (antes de $t=1$ ); ii) choque (em $t=1)$; e iii) pós-choque (depois $t=1$ ). As soluções numéricas, obtidas nas três etapas, são geradas com os esquemas ADBQUICKEST, TOPUS, Superbee, FDPUS-C1 e SDPUS-C1.

i) Etapa pré-choque. Nesta etapa, os esquemas são analisados de quatro formas diferentes, a saber: análise da restrição TVD; análise de erro absoluto; cálculo do erro relativo e estimativas para ordem de convergência; e por fim, verificação da transição da etapa pré-choque para etapa choque (caso transiente).

- Análise da restrição TVD. Esta etapa se inicia com a avaliação dos esquemas ADBQUICKEST, TOPUS, Superbee, FDPUS-C1 e SDPUS-C1 quanto a restrição TVD. Para tanto, calcula-se a TV, dada por (3.11), a cada passo no tempo, plotam-se os resultados numéricos no plano $T V(u(t)) \perp t$ e, por fim, faz-se a análise do comportamento dos esquemas de forma análoga como a feita para a equação de advecção (Caso 3 - Análise TVD). Para isso, consideram-se 100, 200, 400 e 800 células computacionais e $t=0.25$ (antes do choque). Na Figura 6.9 são comparadas a TV gerada pela solução semi-analítica (função constante dada pela reta $T V(u(t))=4$ ) e as TVs derivadas dos esquemas ADBQUICKEST (Fig. 6.9-(a)), TOPUS (Fig. 6.9-(b)) Superbee (Fig. 6.9-(c)), FDPUS-C1 (Fig. 6.9-(d)) e SDPUS-C1 (Fig. 6.9-(e)). Por essa figura, nota-se que todos os esquemas são TVD, embora os resultados para as malha 100, 200 e 400 não satisfaçam a definição para restrição TVD (3.13), na malha 800 as TVs (calculadas para os esquemas anteriormente citados) tende a TV semi-analítica com comportamento decrescente.

- Análise de erro absoluto. A Figura 6.10 mostra a distribuições de erro absoluto ( em função de $x$, dado por (6.3) e plotado no plano $\log E_{A}(x) \perp \log x$ ), cometidos pelos esquemas ADBQUICKEST (Fig. 6.10-(a)), TOPUS (Fig. 6.10-(b)), Superbee (Fig. 6.10-(c)), FDPUS-C1 (Fig. 6.10-(d)) e SDPUS-C1 (Fig. 6.10-(e)). Para simulação são considerados uma malha com 
(a)

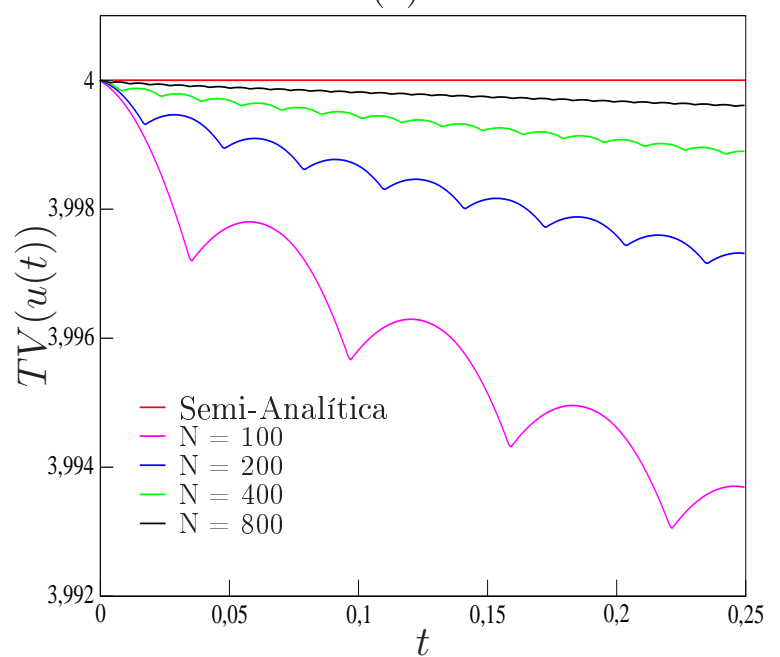

(c)

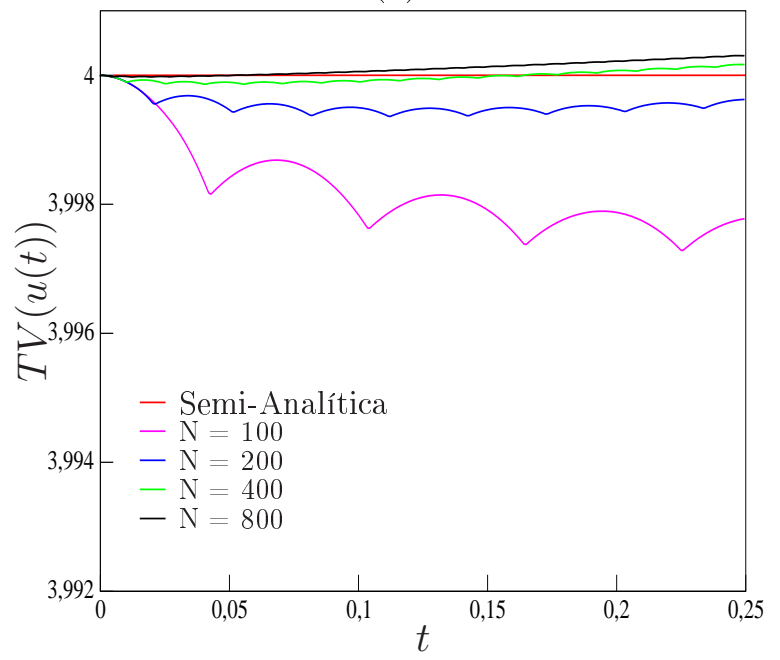

(b)

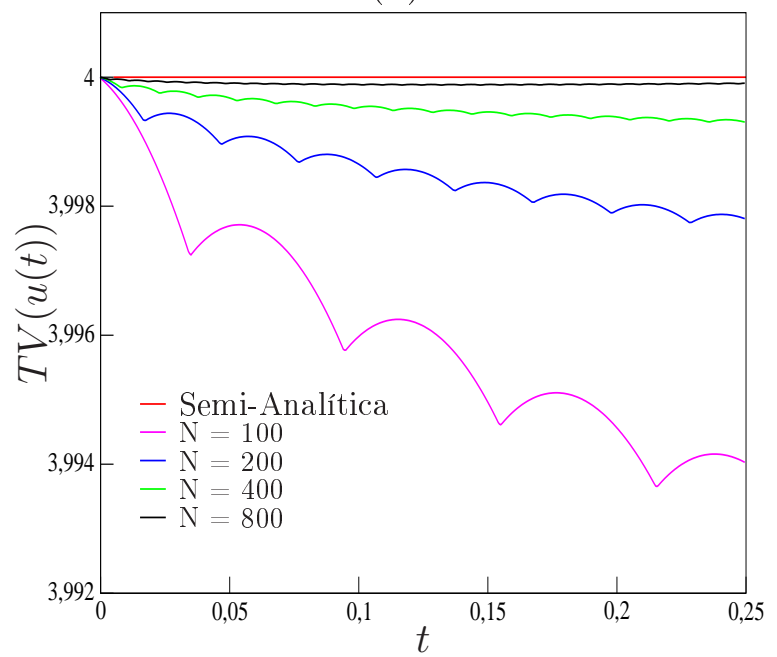

(d)

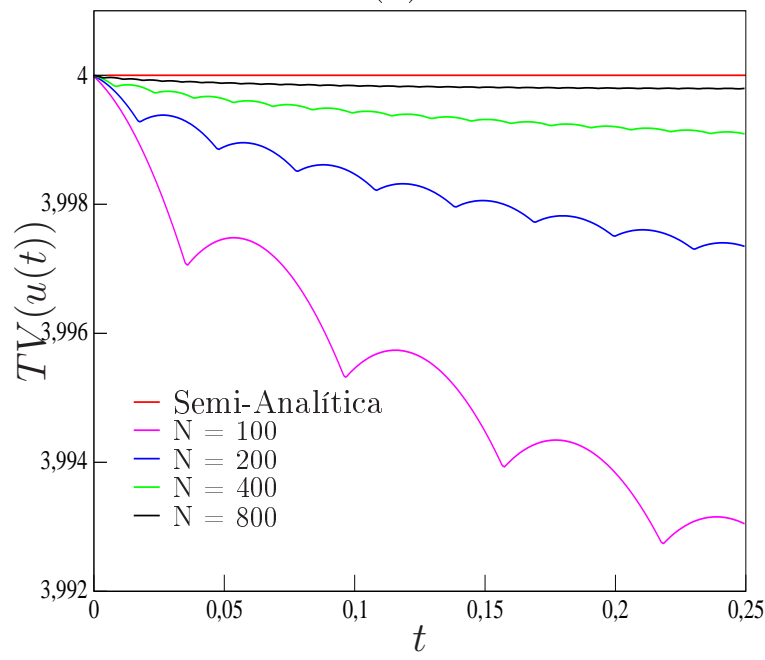

(e)

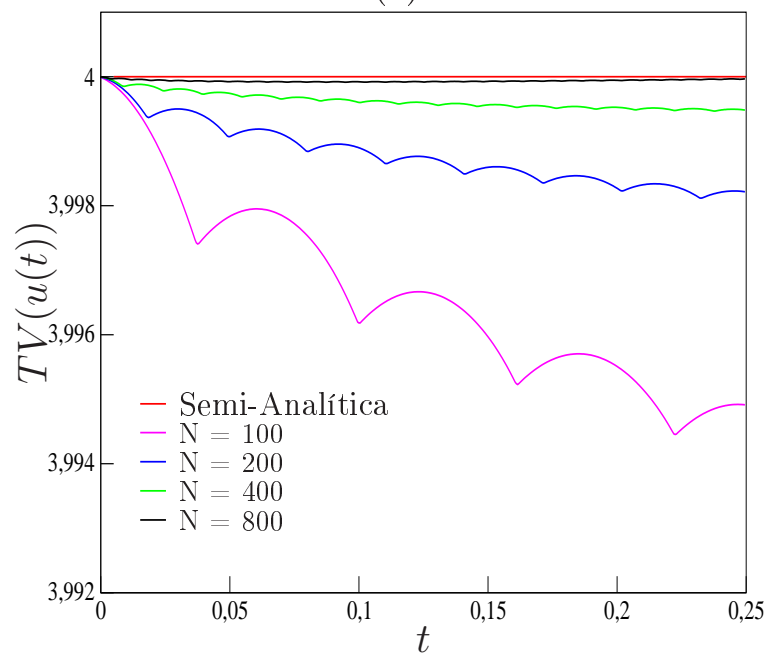

Figura 6.9: Problema de Platzman. i) Etapa pré-choque - Análise TVD. Comparação entre a TV semi-analítica e a TV numérica dos esquemas ADBQUICKEST (a), TOPUS (b), Superbee (c), FDPUS-C1 (d) e SDPUS-C1 (e) para a equação de Burgers sem viscosidade, com condição inicial (6.19) e com condição de Direchlet homogênea no contorno. 
400 células computacionais, $\theta=0.3$ e $t=0.25$. Por essa figura, constata-se que um limitante superior para o erro é menor que $10^{-3}$, o que implica boa concordância entre as soluções semi-analítica e as calculadas pelos esquemas anteriormente citados.

- Ordem de convergência. Agora, calcula-se o erro relativo $\left\|E_{h}\right\|_{k}(k=1,2, \infty)$, dado por (6.6), (6.7) e (6.8), e estima-se a ordem de convergência $p$, definida por (6.12), para os esquemas ADBQUICKEST, TOPUS, Superbee, FDPUS-C1 e SDPUS-C1. Os resultados numéricos são obtidos em malhas com 10, 20, 40, 80 e 160 células computacionais, $t=0.25$ (antes do choque) e $\theta=0.3$ e estão apresentados na Tabela 6.2. Por essa tabela, constata-se que os esquemas TOPUS e Superbee possuem desempenho semelhante; os esquemas ADBQUICKEST, FDPUS-C1 e SDPUS-C1 apresentam dados similares. Por essa mesma tabela, vê-se que o esquema SDPUS-C1 oferece os melhores resultados.

- Caso transiente. Para ilustrar o comportamento da solução na transição da etapa pré-choque para a etapa choque, consideram-se os novos esquemas FDPUS-C1 e SDPUS-C1, uma malha de 400 células computacionais e $\theta=0.5$. Os resultados numéricos são gerados em intervalos de tempo de 0.25, com tempo final de simulação $t=1$. Na Figura 6.11 apresentam-se as soluções numéricas geradas com os esquemas FDPUS-C1 (Fig. 6.11-(a)), SDPUS-C1 (Fig. 6.11-(b)) e solução semi-analítica (Fig. 6.11-(c)), dada por (6.20), com $m=200$. Por essa figura constata-se que os esquemas FDPUS-C1 e SDPUS-C1 capturam muito bem a solução.

ii) Etapa choque. Uma das características dos problemas formulados pela equação de Burgers é que, mesmo considerando condições iniciais suaves (como é o caso deste problema), com o passar do tempo irá ocorrer o choque. Neste caso simulado, com condição inicial suave dada por (6.19), o choque ocorre para $t=1$. Considerando esse tempo, uma malha com 400 células computacionais e $\theta=0.3$, na Figura 6.12, à esquerda, apresentam-se a solução exata, dada por (6.20), e os resultados numéricos gerados com os esquemas ADBQUICKEST (Fig. 6.12-(a)), TOPUS (Fig. 6.12-(c)), Superbee (Fig. 6.12-(e)). Ainda nesta figura, caso à direita, apresenta-se a distribuição do erro absoluto (dado por (6.3) e considerado em escala logarítmica), para os esquemas ADBQUICKEST (Fig. 6.12-(b)), TOPUS (Fig. 6.12-(d)), Superbee (Fig. 6.12 - (f)).

Na Figura 6.13, à esquerda, comparam-se a solução semi-analítica com os resultados numéricos gerados com os esquemas FDPUS-C1 (Fig. 6.13-(a)) e SDPUS-C1 (Fig. 6.13-(c)). À direita dessa mesma figura, apresenta-se a distribuição do erro absoluto (definido por (6.3) e plotado no plano $\left.\log E_{A}(x) \perp \log x\right)$ gerada pelos esquemas FDPUS-C1 (Fig. 6.13-(b)) e SDPUS-C1 (Fig. 6.13-(d)). Por essas figuras, 6.12 e 6.13, constata-se que as soluções numéricas estão em ótima concordância com a solução semi-analítica. Também verifica-se que um limitante superior para o erro absoluto, cometido pelos esquemas, é menor que $10^{-2}$, o que implica em boa concordância entre as soluções semi-analítica e numérica. Além disso, pode-se observar que os esquemas possuem desempenho similar.

iii) Etapa pós-choque. Nesta etapa analisa-se o comportamento das soluções (de referência 
(a)

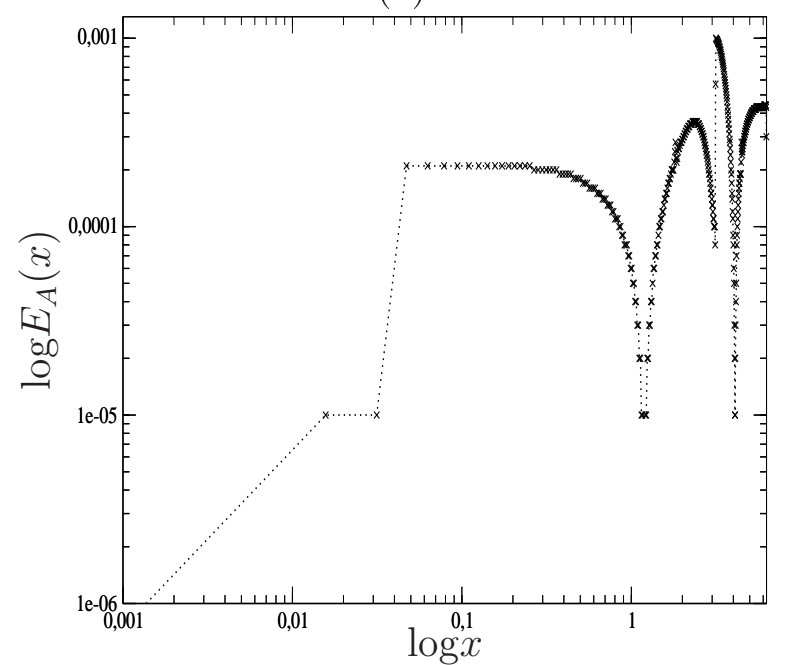

(c)

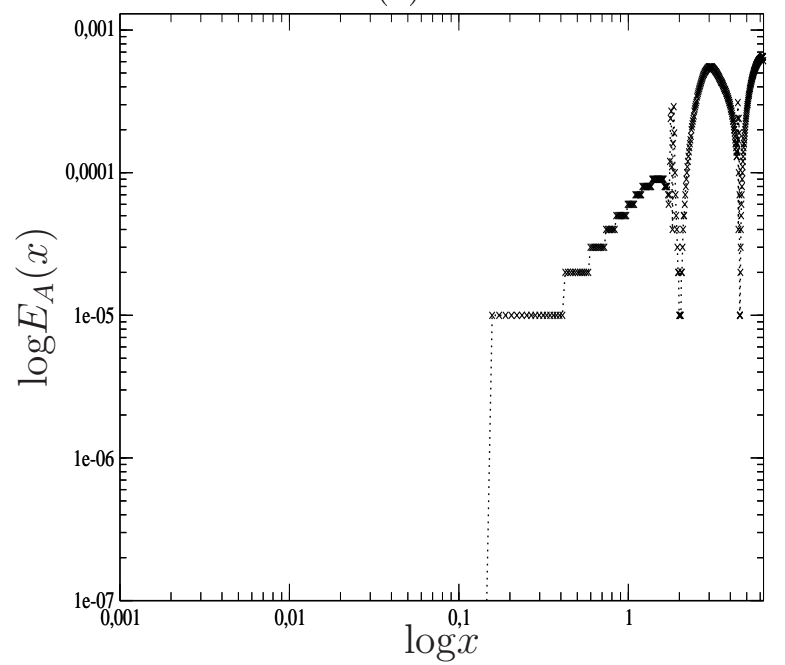

(b)

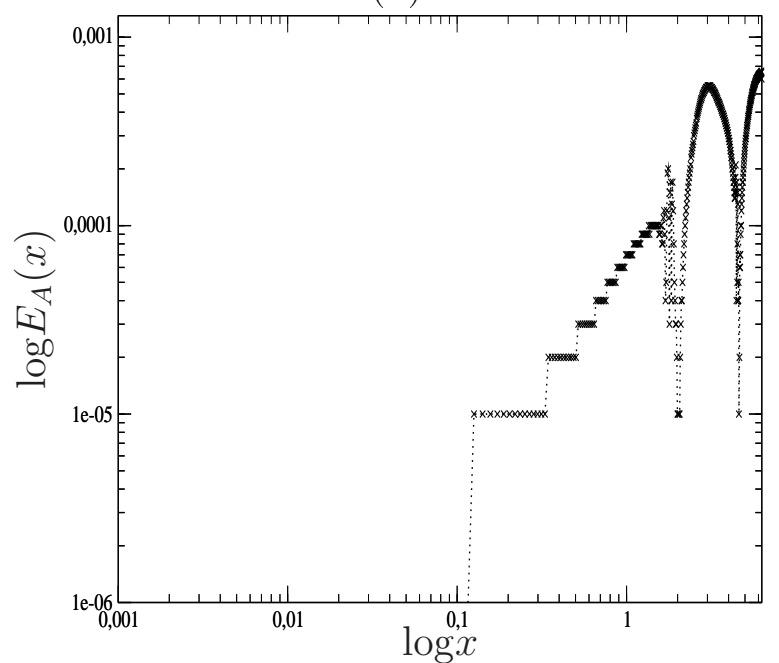

(d)

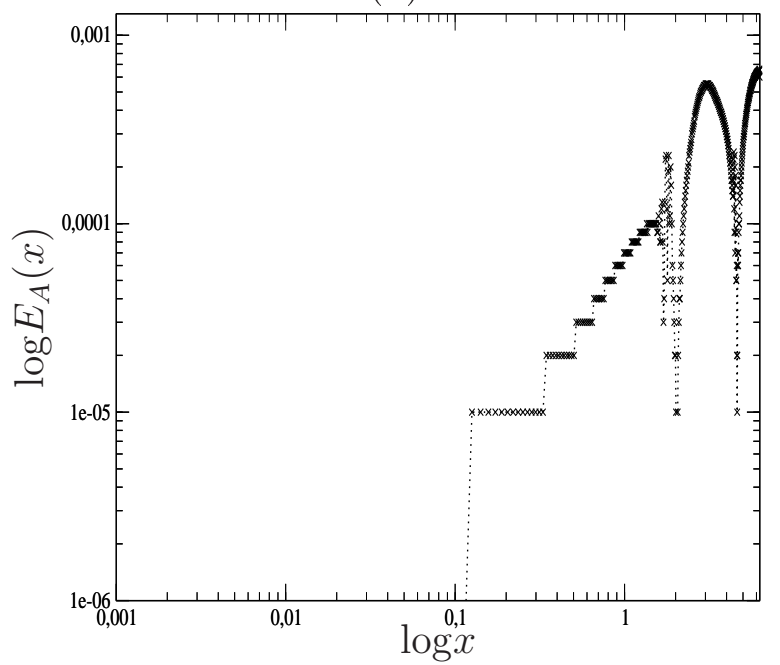

(e)

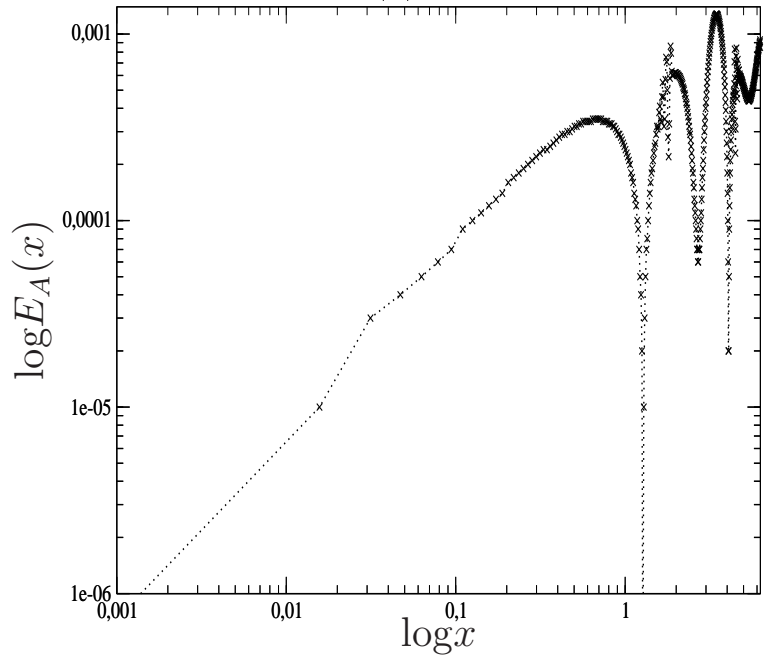

Figura 6.10: Problema de Platzman. i) Etapa pré-choque - Análise do erro absoluto. Erro absoluto (em escala logarítmica) dos esquemas ADBQUICKEST (a), TOPUS (b), Superbee (c), FDPUS-C1 (d) e SDPUS-C1 (e), para a equação de Burgers, sem viscosidade, com condição inicial (6.19) e condição de contorno de Direchlet homogênea. 


\begin{tabular}{l|ccccccc}
\hline Esquema & $\mathrm{N}$ & $\left\|E_{h}\right\|_{1}$ & $\mathrm{p}$ & $\left\|E_{h}\right\|_{2}$ & $\mathrm{p}$ & $\left\|E_{h}\right\|_{\infty}$ & $\mathrm{p}$ \\
\hline \hline ADBQUICKEST & 10 & $0.149 \times 10^{-1}$ & - & $0.152 \times 10^{-1}$ & - & $0.178 \times 10^{-1}$ & - \\
& 20 & $0.406 \times 10^{-2}$ & 1.87 & $0.424 \times 10^{-2}$ & 1.84 & $0.553 \times 10^{-2}$ & 1.68 \\
& 40 & $0.137 \times 10^{-2}$ & 1.56 & $0.157 \times 10^{-2}$ & 1.43 & $0.246 \times 10^{-2}$ & 1.17 \\
& 80 & $0.601 \times 10^{-3}$ & 1.19 & $0.631 \times 10^{-3}$ & 1.31 & $0.100 \times 10^{-2}$ & 1.29 \\
& 160 & $0.300 \times 10^{-3}$ & 1.01 & $0.301 \times 10^{-3}$ & 1.06 & $0.504 \times 10^{-3}$ & 0.99 \\
\hline \hline TOPUS & 10 & $0.187 \times 10^{-1}$ & - & $0.207 \times 10^{-1}$ & - & $0.246 \times 10^{-1}$ & - \\
& 20 & $0.650 \times 10^{-2}$ & 1.52 & $0.718 \times 10^{-2}$ & 1.52 & $0.974 \times 10^{-2}$ & 1.33 \\
& 40 & $0.303 \times 10^{-2}$ & 1.09 & $0.335 \times 10^{-2}$ & 1.09 & $0.485 \times 10^{-2}$ & 1.00 \\
& 80 & $0.148 \times 10^{-2}$ & 1.03 & $0.166 \times 10^{-2}$ & 1.01 & $0.283 \times 10^{-2}$ & 0.77 \\
& 160 & $0.739 \times 10^{-3}$ & 1.00 & $0.904 \times 10^{-3}$ & 0.87 & $0.150 \times 10^{-2}$ & 0.89 \\
\hline \hline Superbee & 10 & $0.195 \times 10^{-1}$ & - & $0.333 \times 10^{-1}$ & - & $0.359 \times 10^{-1}$ & - \\
& 20 & $0.727 \times 10^{-2}$ & 1.42 & $0.111 \times 10^{-1}$ & 1.57 & $0.173 \times 10^{-2}$ & 1.04 \\
& 40 & $0.305 \times 10^{-2}$ & 1.25 & $0.499 \times 10^{-2}$ & 1.15 & $0.801 \times 10^{-2}$ & 1.11 \\
& 80 & $0.127 \times 10^{-2}$ & 1.26 & $0.206 \times 10^{-2}$ & 1.27 & $0.320 \times 10^{-2}$ & 1.32 \\
& 160 & $0.681 \times 10^{-3}$ & 0.89 & $0.909 \times 10^{-3}$ & 1.17 & $0.159 \times 10^{-2}$ & 1.01 \\
\hline \hline FDPUS-C1 & 10 & $0.199 \times 10^{-1}$ & - & $0.240 \times 10^{-1}$ & - & $0.244 \times 10^{-1}$ & - \\
& 20 & $0.541 \times 10^{-2}$ & 1.87 & $0.703 \times 10^{-2}$ & 1.82 & $0.736 \times 10^{-2}$ & 1.72 \\
& 40 & $0.209 \times 10^{-2}$ & 1.36 & $0.302 \times 10^{-2}$ & 1.21 & $0.336 \times 10^{-2}$ & 1.13 \\
& 80 & $0.100 \times 10^{-2}$ & 1.06 & $0.140 \times 10^{-2}$ & 1.11 & $0.161 \times 10^{-2}$ & 1.05 \\
& 160 & $0.502 \times 10^{-3}$ & 0.99 & $0.705 \times 10^{-3}$ & 0.99 & $0.817 \times 10^{-3}$ & 0.89 \\
\hline \hline SDPUS-C1 & 10 & $0.177 \times 10^{-1}$ & - & $0.200 \times 10^{-1}$ & - & $0.258 \times 10^{-1}$ & - \\
& 20 & $0.477 \times 10^{-2}$ & 1.89 & $0.557 \times 10^{-2}$ & 1.85 & $0.802 \times 10^{-2}$ & 1.68 \\
& 40 & $0.158 \times 10^{-2}$ & 1.59 & $0.201 \times 10^{-2}$ & 1.47 & $0.343 \times 10^{-2}$ & 1.22 \\
& 80 & $0.657 \times 10^{-3}$ & 1.26 & $0.785 \times 10^{-3}$ & 1.35 & $0.160 \times 10^{-2}$ & 1.10 \\
& 160 & $0.303 \times 10^{-3}$ & 1.11 & $0.353 \times 10^{-3}$ & 1.15 & $0.820 \times 10^{-3}$ & 0.96 \\
\hline \hline
\end{tabular}

Tabela 6.2: Problema de Platzman. i) Etapa pré-choque - Ordem de convergência. Erros nas normas $L_{1}, L_{2}$ e $L_{\infty}$ e estimativas da ordem de convergência para os esquemas ADBQUICKEST, TOPUS, Superbee, FDPUS-C1 e SDPUS-C1 para a equação de Burgers, sem viscosidade, com condição inicial (6.19) e com condição de Dirichelet homogênea no contorno. 
(a)

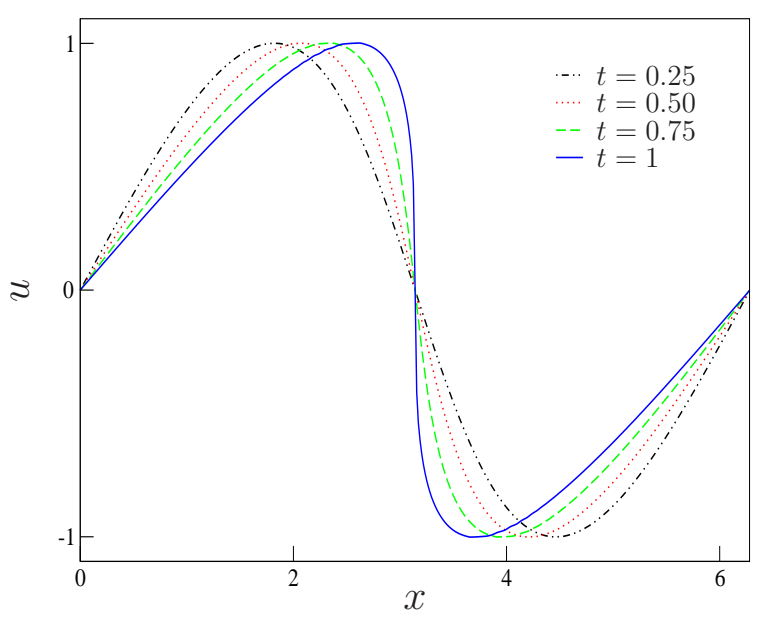

(b)

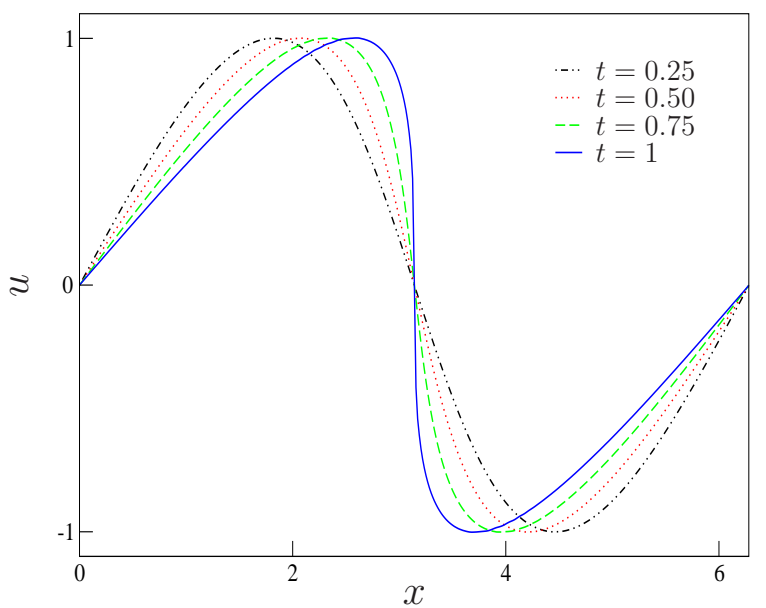

(c)

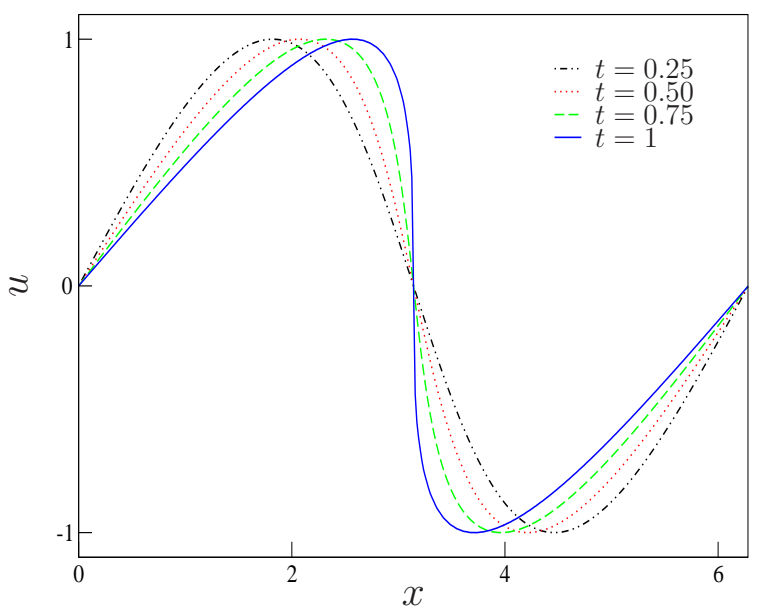

Figura 6.11: Problema de Platzman. i) Etapa pré-choque - Caso transiente. Soluções numéricas dos esquemas FDPUS-C1 (a), SDPUSC1 (b) e solução semi-analítica (c) para a equação de Burgers sem viscosidade, com condição inicial dada por (6.19) e condição de contorno de Direchlet homogêneas, geradas em intervalos de tempo de 0.25 , com tempo final de simulação $t=1$. 
(a)

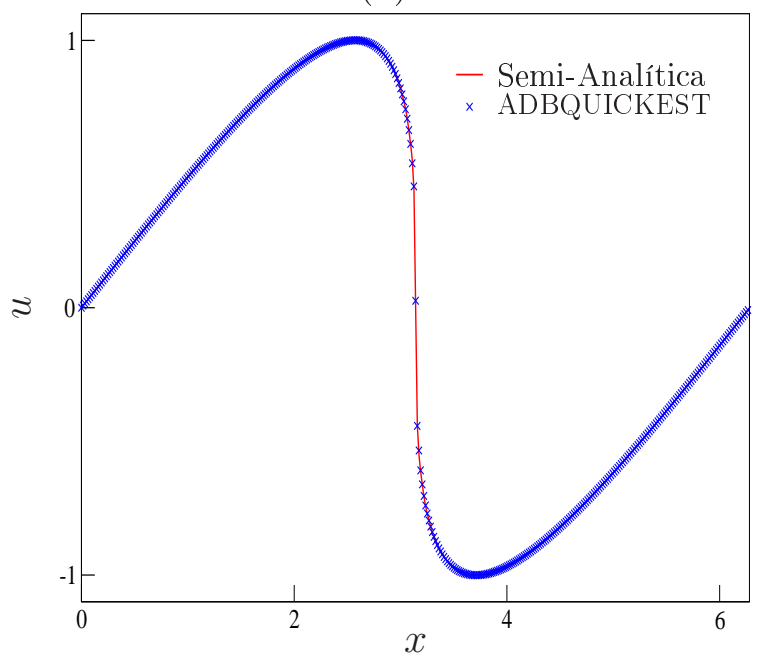

(c)

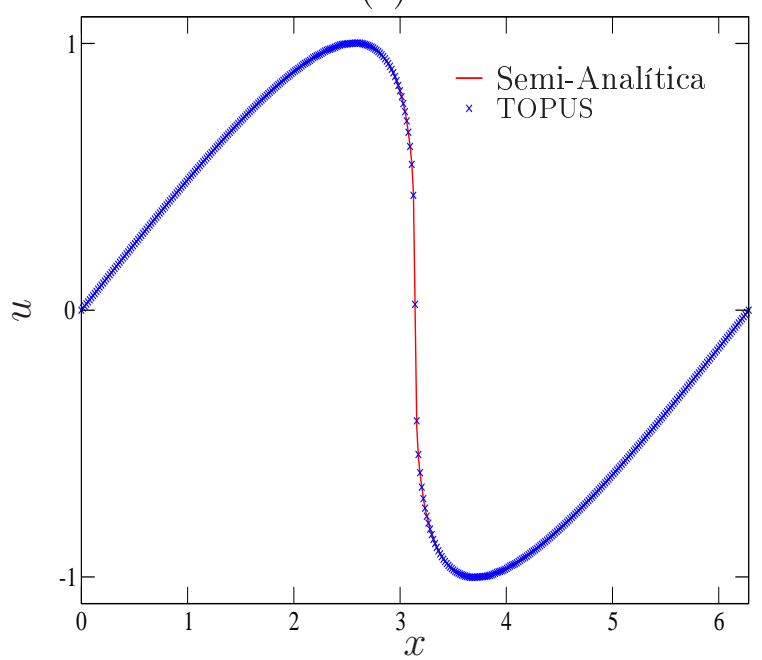

(e)

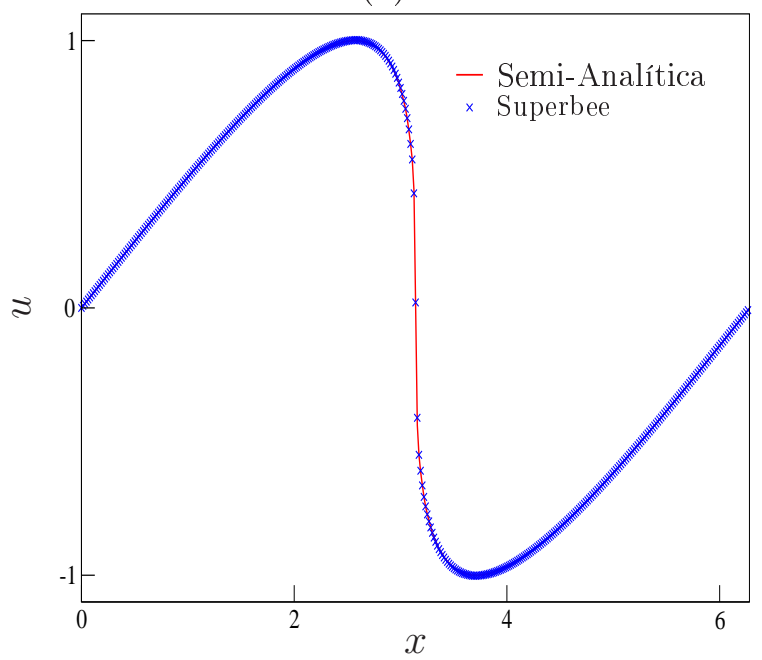

(b)

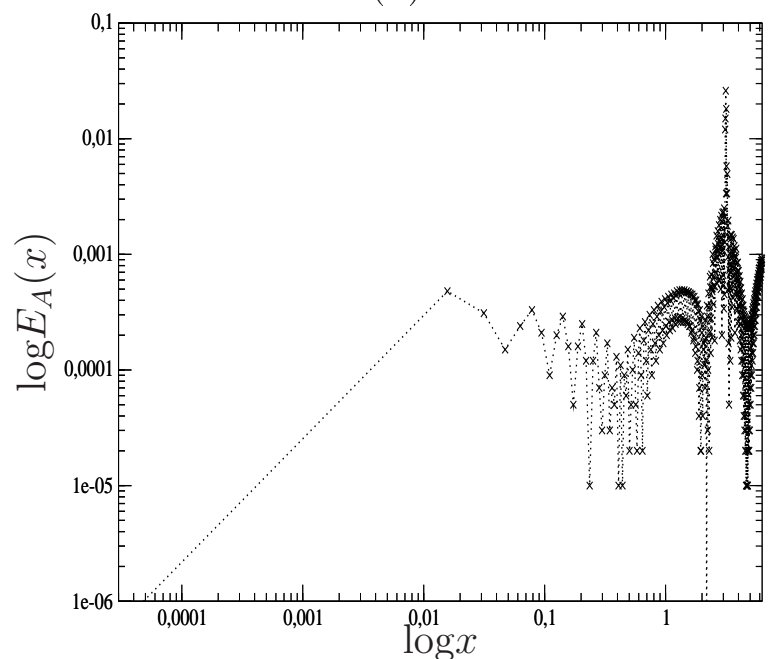

(d)

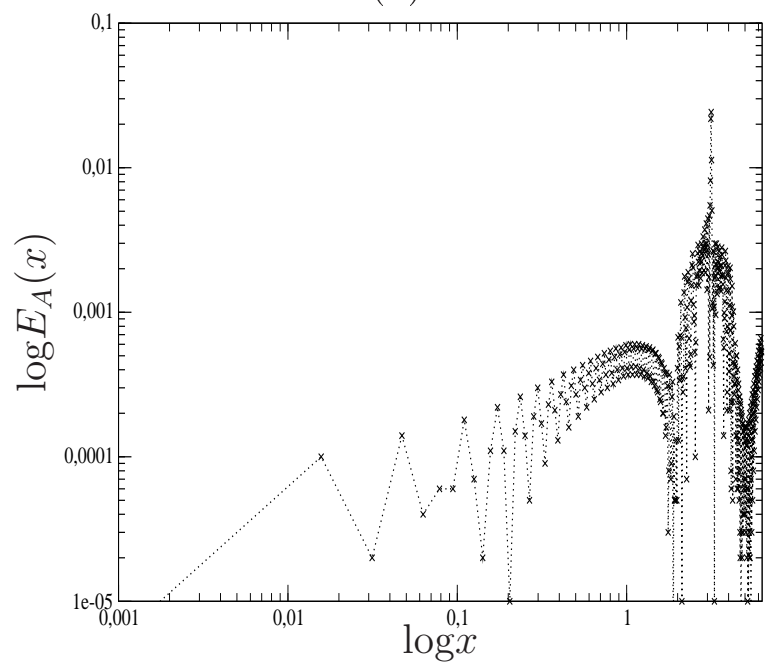

(f)

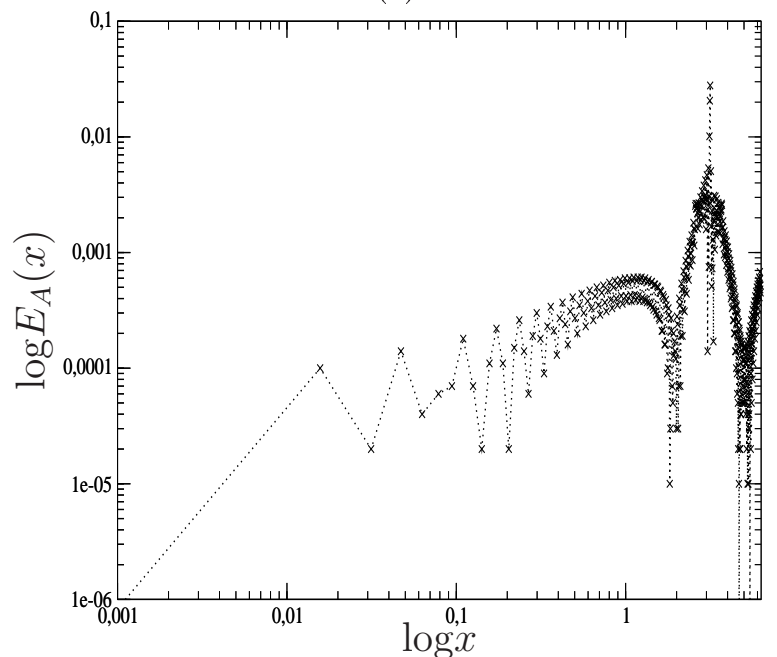

Figura 6.12: Problema de Platzman. ii) Etapa choque - Comparações. Comparação entre a solução semi-analítica e os esquemas ADBQUICKEST (a), TOPUS (c) e Superbee (e); erro absoluto (em escala logarítmica) dos esquemas ADBQUICKEST (b), TOPUS (d) e Superbee (f). Resultados numéricos para a equação de Burgers, sem viscosidade, com condições inicial (6.19) e de contorno Direchlet homogênea. 
(a)

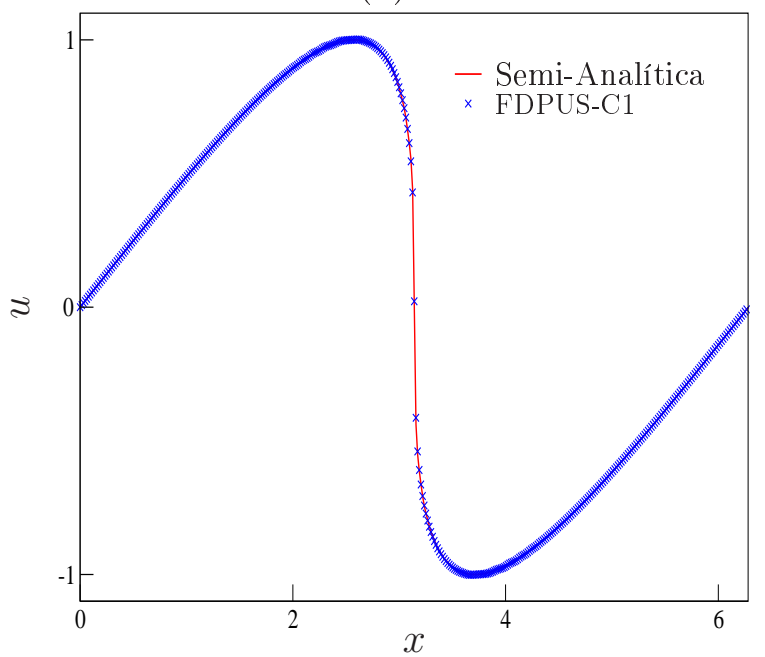

(c)

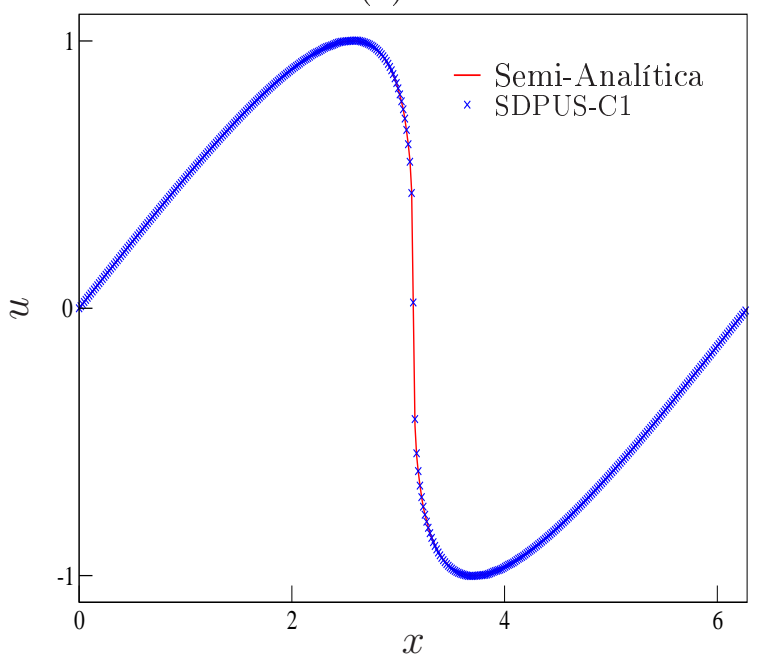

(b)

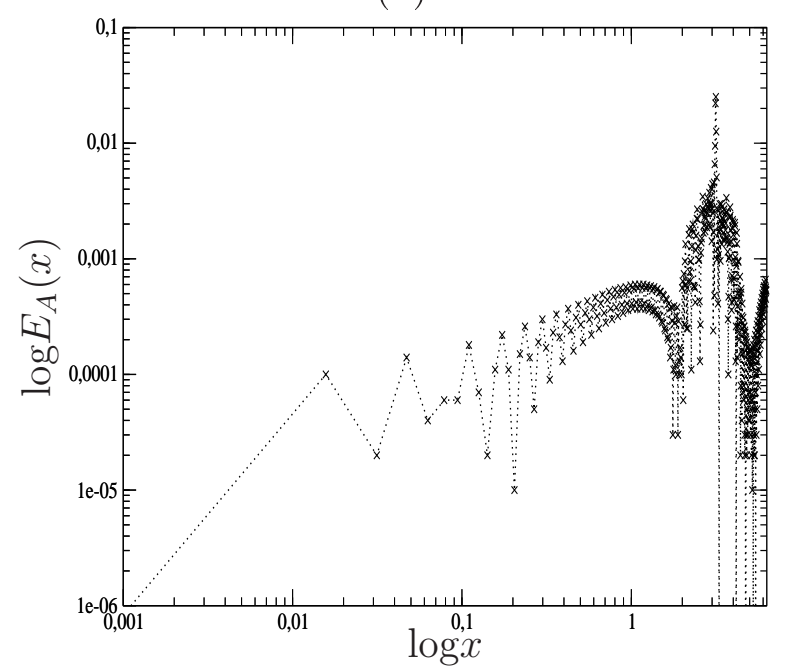

(d)

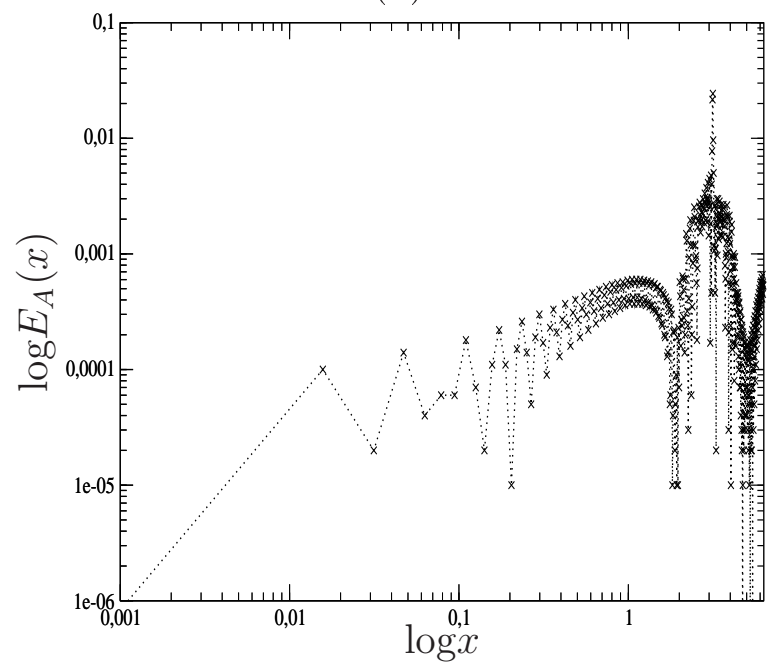

Figura 6.13: Problema de Platzman. ii) Etapa choque - Comparações. Comparação entre a solução semi-analítica e os esquemas FDPUS-C1 (a) e SDPUS-C1 (c); erros absolutos (em escala logarítmica) dos esquemas FDPUS-C1 (b) e SDPUS-C1 (d). Resultados numéricos para a equação de Burgers, sem viscosidade, com condição inicial (6.19) e condição de contorno Direchlet homogênea. 
e numérica) em $t=2$, ou seja, no tempo após ocorrência do choque. Para $t=2$ a solução exata dada por (6.20) não é mais válida, assim, uma solução de referência é gerada com o esquema FOU em 4000 células computacionais e $\theta=0.5$. As soluções numéricas são calculadas em 400 células computacionais, considerando-se $\theta=0.3$. A Figura 6.14 mostra a solução de referência e os resultados numéricos gerados com os esquemas ADBQUICKEST (Fig. 6.14-(a)), TOPUS (Fig. 6.14-(b)), Superbee (Fig. 6.14-(c)), FDPUS-C1 (Fig. 6.14-(d)) e SDPUS-C1 (Fig. 6.14-(e)). Por essa figura constata-se que as soluções numéricas estão em ótima concordância com a solução de referência. Vale salientar, que esses resultados mostram que os novos esquemas FDPUS-C1 e SDPUS-C1 são ferramentas eficientes na captura de choques.

\subsubsection{Equação de Burgers com Viscosidade}

Nesta subseção apresentam-se os resultados obtidos para a equação de Burgers com viscosidade.

Caso 1 - Caso transiente. Neste caso, considera-se a equação de Burgers com viscosidade (2.9) definida em $x \in[0,2 \pi]$, suplementada com a condição inicial

$$
u_{0}(x)=1+\cos (x)
$$

e com as condições de contorno

$$
u(0, t)=1+\cos (t) \text { e } u(1, t)=\cos (t)+\cos (2 \pi) .
$$

Para a simulação deste problema consideram-se 10000 células computacionais, $\theta=0.5$ e coeficiente de viscosidade $\nu=0.001$. Os resultados numéricos são gerados em intervalos de tempo de 0.25 , com tempo final de simulação $t=3.25$. A Figura 6.15 ilustra esses resultados e, a partir da mesma, pode-se observar que os resultados obtidos pelos métodos FDPUS-C1 (Fig. 6.15-(a)) e SDPUS-C1 (Fig. 6.15-(b)) estão em boa concordância com os resultados numéricos de Fatkullin e Hesthaven [24], ou seja, com o passar do tempo a solução numérica é propagada para direita com o decaimento da amplitude e, consequentemente, a formação de choque em regiões que apresentam gradientes elevados. 
(a)

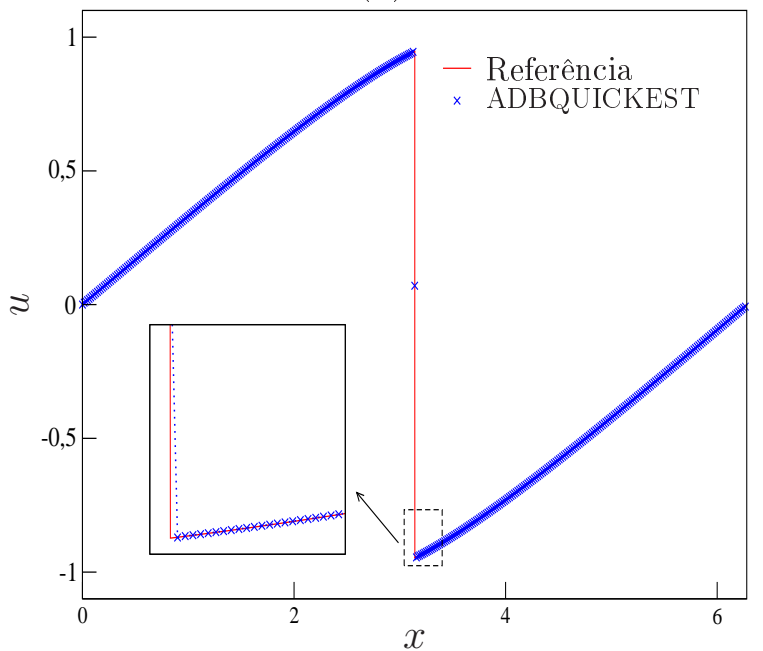

(c)

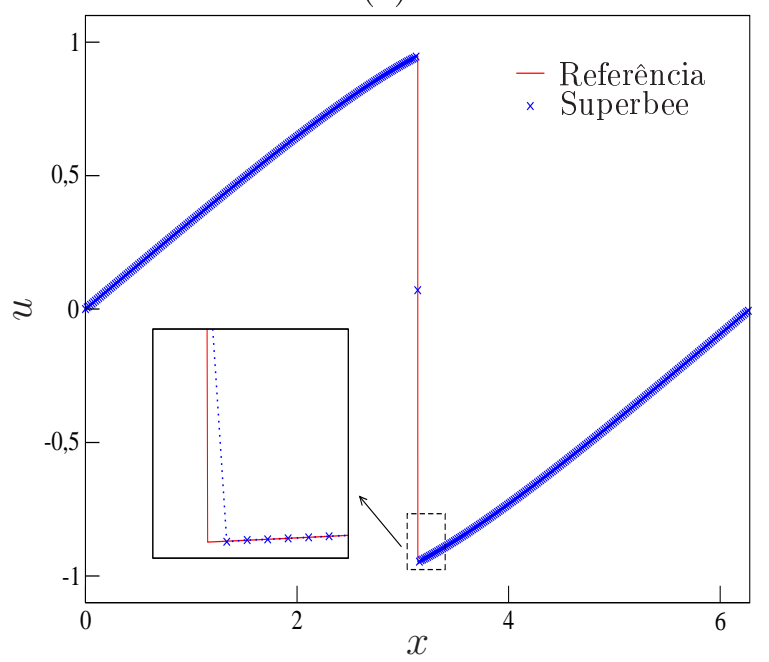

(b)

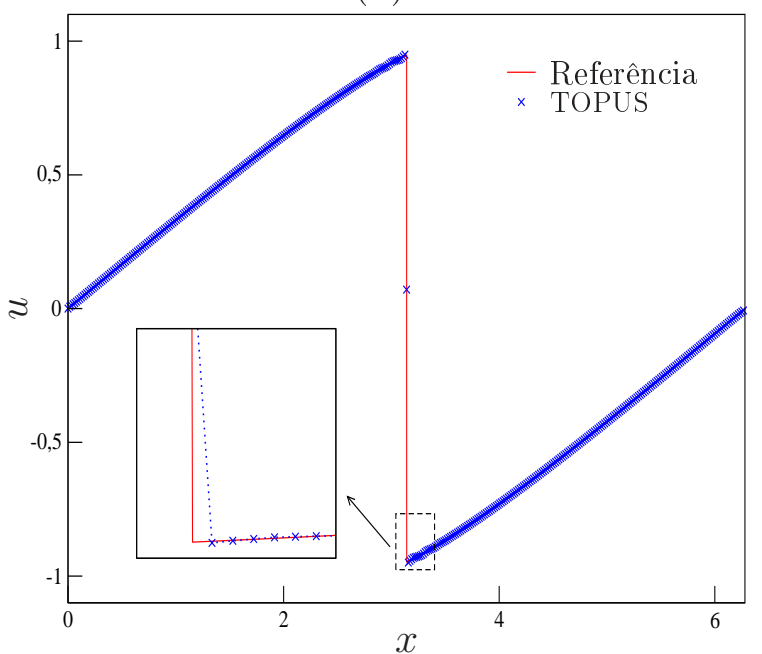

(d)

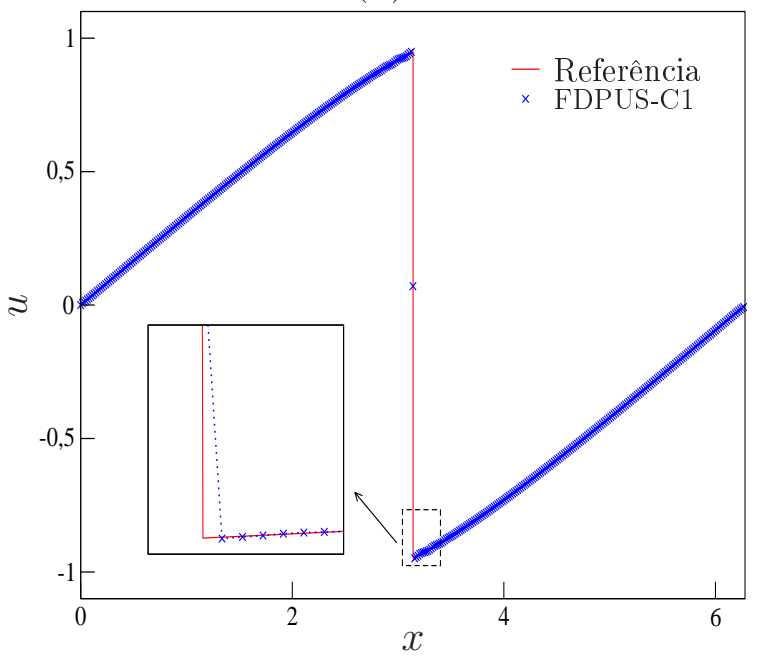

(e)

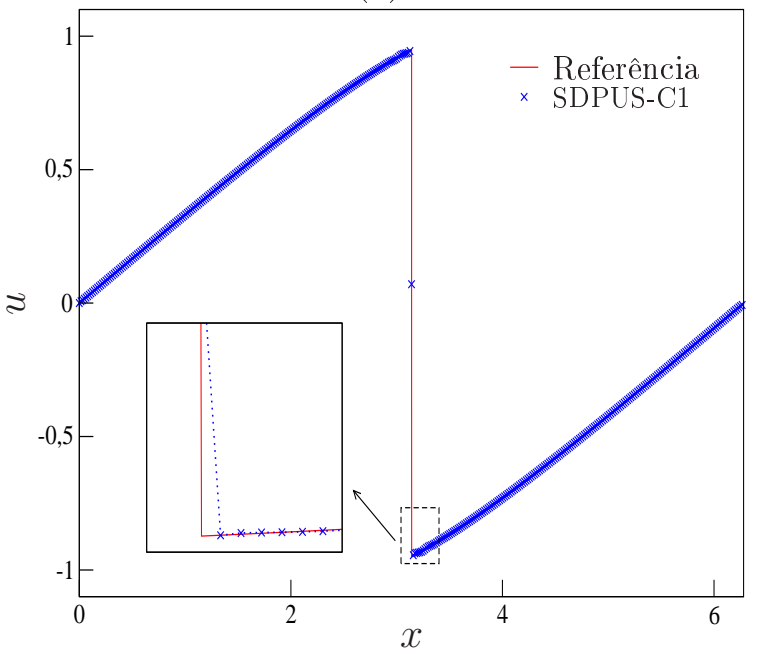

Figura 6.14: Problema de Platzman. iii) Etapa pós-choque - Comparações. Comparação entre a solução de referência e os esquemas ADBQUICKEST (a), TOPUS (b), Superbee (c), FDPUS-C1 (d) e SDPUS-C1 (e) para a equação de Burgers sem viscosidade, com condição inicial (6.19) e condição de contorno Direchlet homogênea. 
(a)

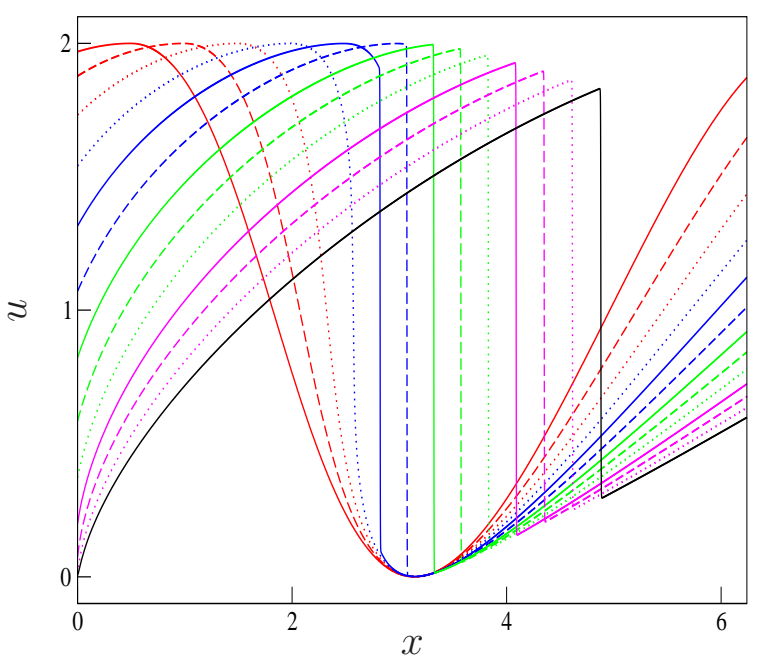

(b)

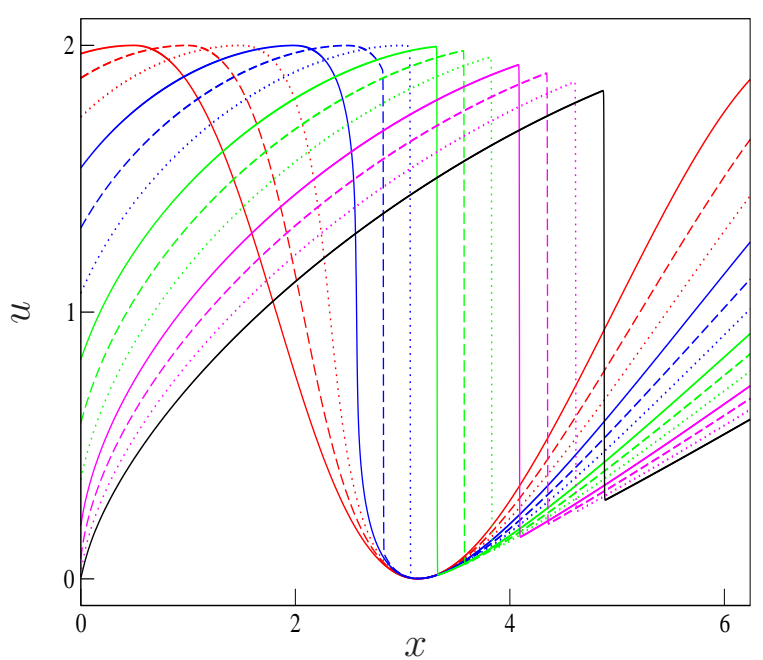

Figura 6.15: Caso transiente. Resultados numéricos dos esquemas FDPUS-C1 (a) e SDPUS-C1 (b) para a equação de Burgers, com viscosidade, com condições inicial (6.21) e contorno (6.22), gerados em intervalos de tempo de 0.25 , com tempo final de simulação $t=3.25$.

\subsection{Equação de Buckley-Leverett}

A equação não linear de Buckley-Leverett é formulada pela lei de conservação 1D (2.1), com variável conservada e fluxo numérico dados, respectivamente, por (2.12) e (2.13). As soluções numéricas obtidas para essa equação são geradas com os esquemas ADBQUICKEST, TOPUS, FDPUS-C1 e SDPUS-C1 e são comparadas com as soluções de referência.

Caso 1 - Dado inicial constante por partes. Neste caso a equação de Buckley-Leverett é definida no domínio [0,0.6], suplementada com condição inicial dada por (6.16) e contorno definido por (6.17). As soluções numéricas são obtidas com os esquemas ADBQUICKEST, TOPUS, FDPUS-C1 e SDPUS-C1, em uma malha de 500 células computacionais. A solução de referência é gerada pelo esquema FOU em 5000 células computacionais. Ambas soluções, de referência e numérica, são obtidas para $t=0.3, \operatorname{com} \theta=0.5$. A Figura 6.16 mostra a comparação entre a solução de referência e os resultados numéricos gerados com os esquemas ADBQUICKEST (Fig. 6.16-(a)), TOPUS (Fig. 6.16-(b)), FDPUS-C1 (Fig. 6.16-(c)) e SDPUS-C1 (Fig. 6.16-(d)). Para uma melhor análise, em Fig. 6.16-(e) apresenta-se a comparação das soluções, obtidas pelos esquemas anteriormente citados, e ainda, em Fig. 6.16-(f), apresenta-se uma ampliação da área demarcada em Fig. 6.16-(e). Observa-se por essa figura que as soluções, obtidas por tais esquemas, estão em boa concordância com a solução de referência. Vale salientar, que por essa mesma figura, constata-se que o esquema SDPUS-C1 apresenta melhor desempenho em relação aos demais esquemas.

Caso2 - Problema desafiador. Neste caso a equação de Buckley-Leverett é definida no 
(a)

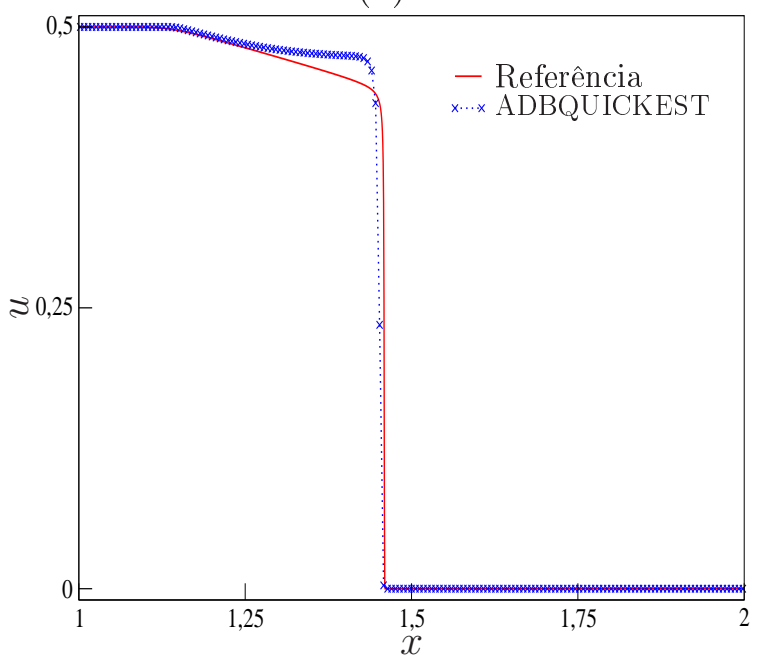

(c)

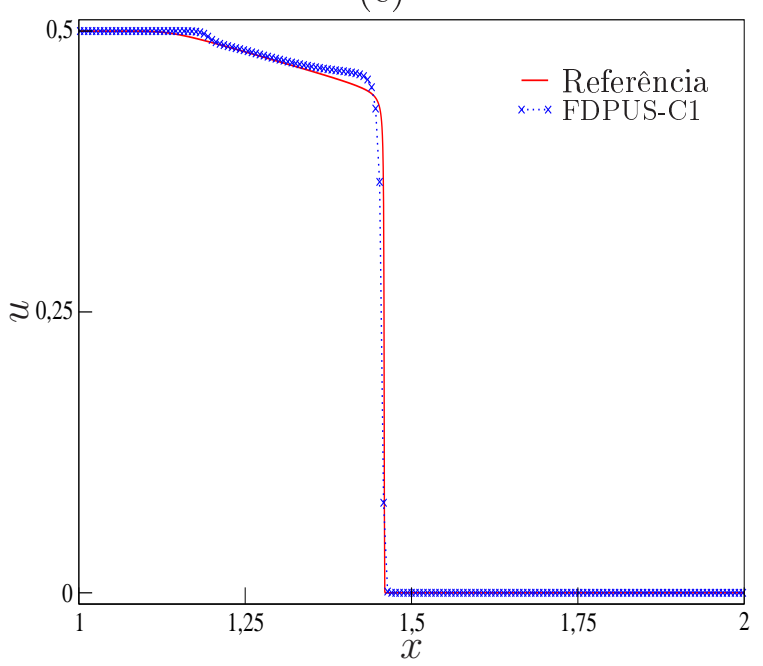

(e)

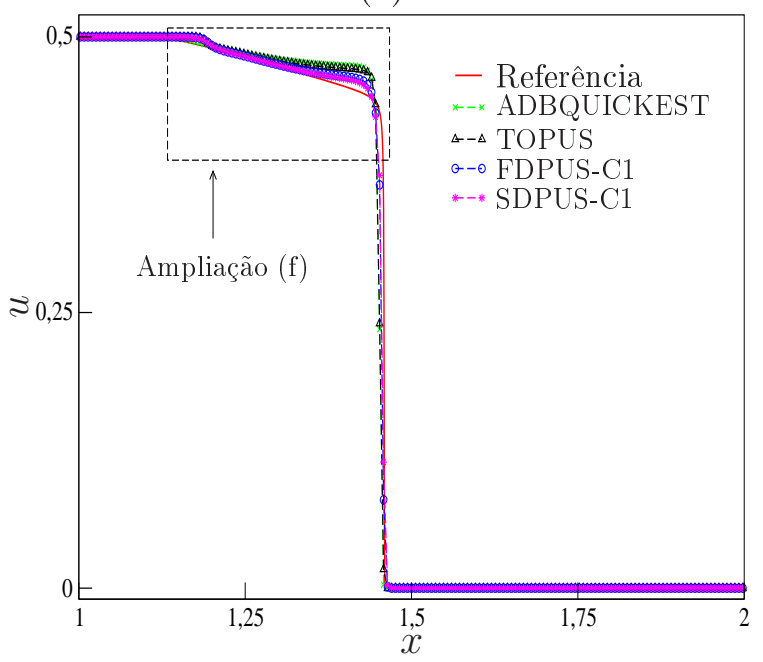

(b)

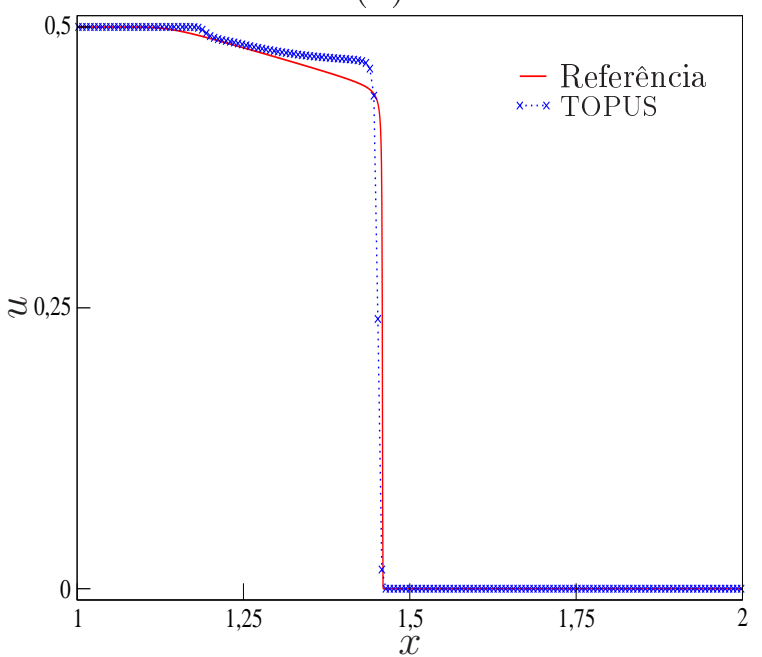

(d)

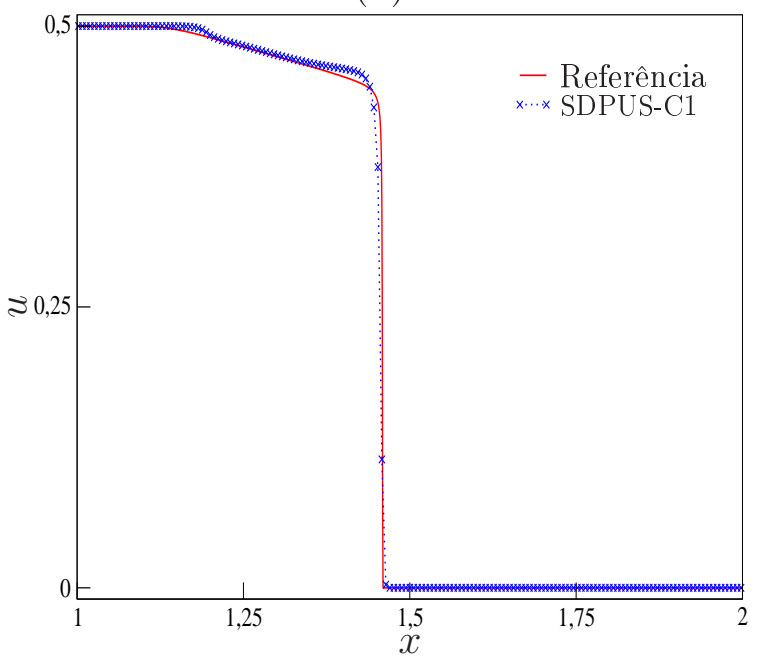

Ampliação (f)

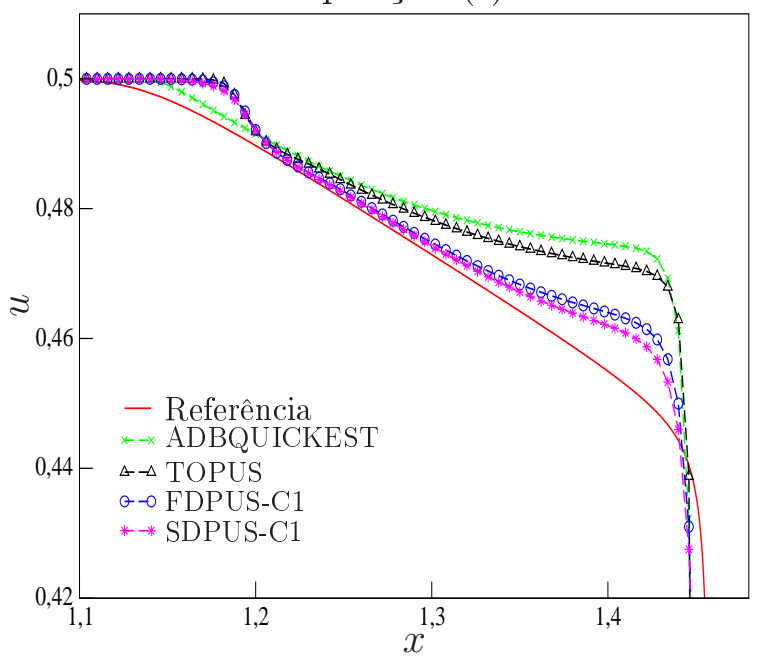

Figura 6.16: Dado inicial constante por partes. Comparação entre a solução de referência e os esquemas ADBQUICKEST (a), TOPUS (b), FDPUS-C1 (c) e SDPUS-C1 (d); comparação das soluções numéricas (e); ampliação (f). Resultados numéricos para a equação de Buckley-Leverett, com condições inicial (6.16) e contorno (6.17). 
domínio $[-1,1]$, suplementada com a condição inicial dada por

$$
u_{0}(x)= \begin{cases}1, & -0.5 \leq x \leq 0 \\ 0, & \text { caso contrário }\end{cases}
$$

e com condições de Direchlet homogênea no contorno.

Para simulação são considerados uma malha de 800 células computacionais, $\theta=0.3 \mathrm{e}$ $t=0.4$. A solução de referência é obtida com o esquema FOU em uma malha de 8000 células computacionais e $\theta=0.5$. Na Figura 6.17 apresentam-se a solução de referência e os resultados numéricos dos esquemas ADBQUICKEST (Fig. 6.17-(a)), TOPUS (Fig. 6.17-(b)), FDPUS-C1 (Fig.6.17-(c)) e SDPUS-C1 (Fig. 6.17-(d)). Nessa figura, faz-se também uma comparação das soluções numéricas obtidas com os esquemas anteriormente citados (Fig. 6.17-(e)) e em Fig. 6.17-(f) apresenta-se a ampliação da área demarcada em Fig. 6.17-(e). De forma geral, pode-se ver que os resultados numéricos são satisfatórios. Salienta-se (ver Fig. 6.17-(f)) que o esquema FDPUS-C1 apresenta o melhor resultado. 
(a)

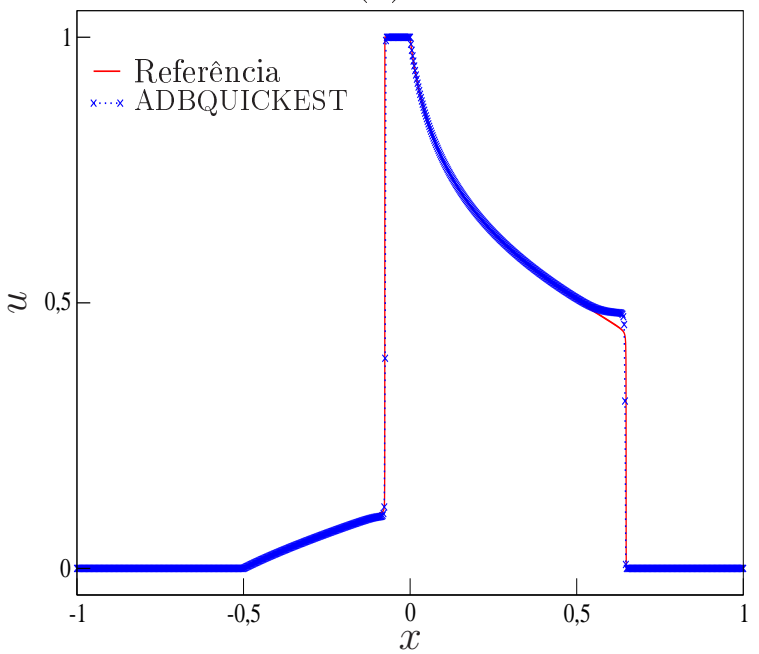

(c)

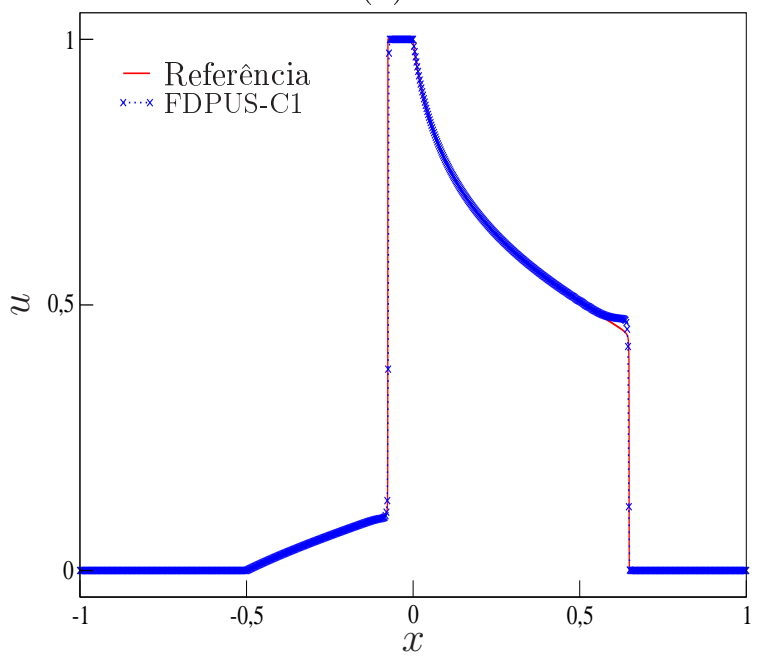

(e)

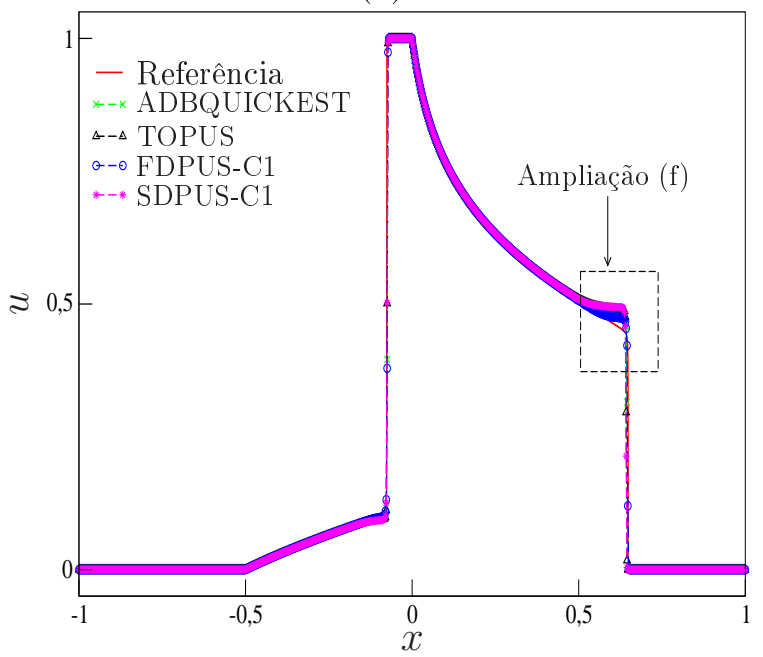

(b)

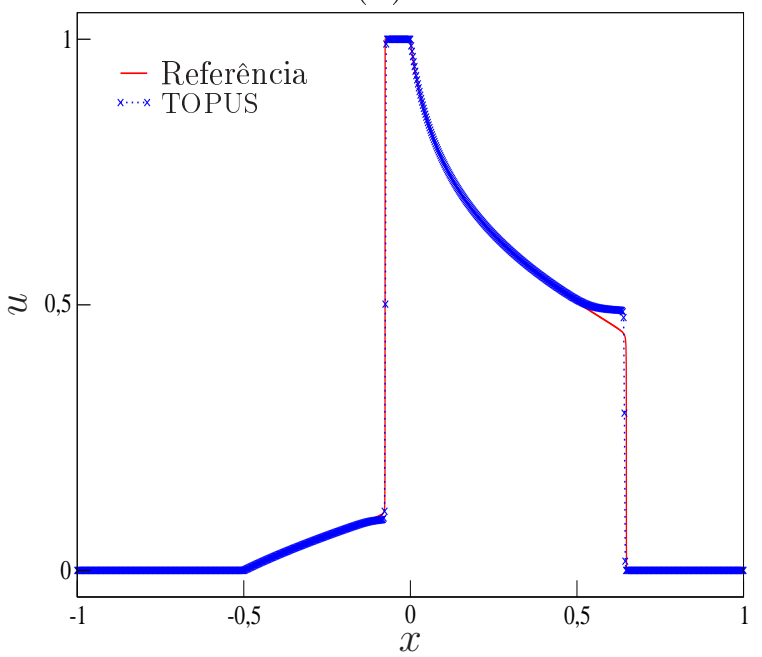

(d)

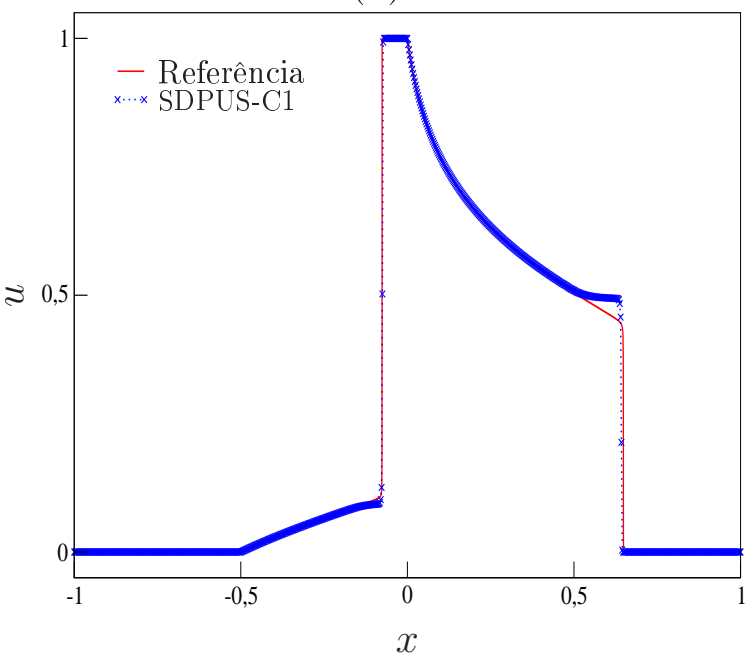

Ampliação (f)

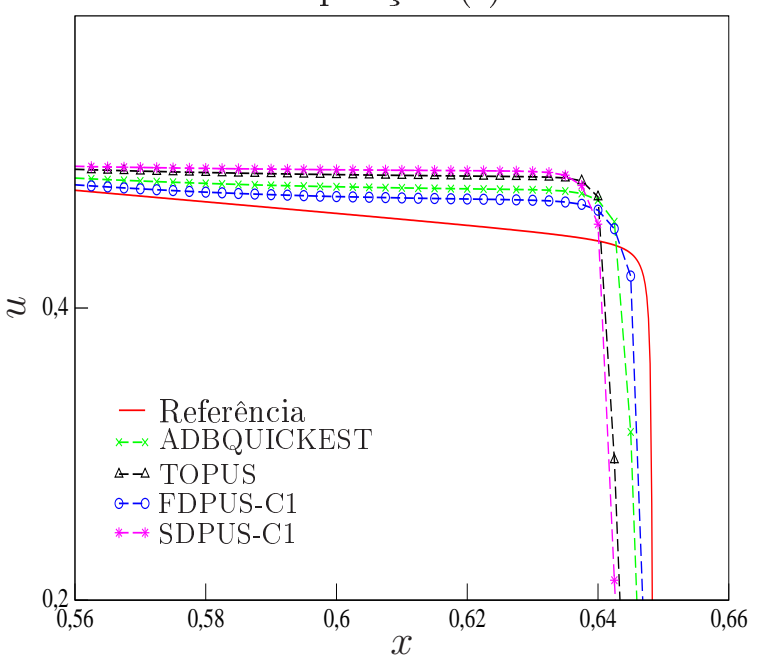

Figura 6.17: Problema desafiador. Comparação entre a solução de referência e os esquemas ADBQUICKEST (a), TOPUS (b), FDPUS-C1 (c) e SDPUS-C1 (d); comparação das soluções numéricas (e); ampliação (f). Resultados numéricos para a equação de Buckley-Leverett, com condição inicial (6.23) e condição de Direchlet homogênea no contorno. 


\subsection{Sistema Hiperbólico Águas Rasas}

O sistema hiperbólico de águas rasas é formulado pela lei de conservação 1D (2.1), com o vetor das variáveis conservadas e função fluxo dados, respectivamente, por (2.16) e (2.17). As soluções, para esse sistema, são calculadas por meio do pacote computacional CLAWPACK, equipado com os limitadores de fluxo dos esquemas ADBQUICKEST, TOPUS, FDPUS-C1 e SDPUS-C1. Para mais detalhes sobre esse software ver LeVeque [44] e apêndice D.

Caso 1 - Problema dam-break. Neste caso o sistema hiperbólico de águas rasas é definido em $x \in[-5,5]$. No início da simulação uma barragem que divide o domínio em duas partes, a saber, o reservatório a esquerda e um canal de fuga a direita, é rompida. Esse problema é conhecido na literatura por dam-break problem e as condições iniciais impostas para $h$, dados constantes por partes (do reservatório e do canal de fuga, separados por uma descontinuidade em $x=0)$, são dadas por

$$
h_{0}(x)= \begin{cases}3, & x \leq 0, \\ 1, & x>0,\end{cases}
$$

com velocidade inicial nula, ou seja,

$$
u_{0}(x)=0
$$

e com extrapolação de ordem zero no contorno (ver LeVeque [44]). A barreira é então instantaneamente removida e a solução é computada.

As soluções numéricas são geradas pelo método de segunda ordem (Godunov de primeira ordem com termo de correção, ver LeVeque [44] ), em que os limitadores de fluxo dos esquemas ADBQUICKEST, TOPUS, FDPUS-C1 e SDPUS-C1, mais o limitador van Leer (ferramenta disponível no pacote computacional CLAWPACK) são aplicados ao termo de correção. Para simulação são considerados $t=2, \theta=0.9$ e uma malha uniforme com 200 células computacionais. A solução de referência é calculada em 1000 células computacionais, pelo esquema de primeira ordem de Godunov (ver LeVeque [44]), $\operatorname{com} \theta=0.5$.

Nas Figuras 6.18, 6.19, 6.20 e 6.21 apresentam-se as soluções de referência e os resultados numéricos obtidos para $h$ (profundidade), $h u$ (vazão), $u$ (velocidade) e $F r=\frac{u}{\sqrt{g h}}$ (número de Froud). Nessas figuras, os resultados para os esquemas ADBQUICKEST, TOPUS, van Leer, FDPUS-C1 e SDPUS-C1 são apresentados, respectivamente, em (a), (b), (c), (d) e (e). Ainda nessas figuras, comparam-se as soluções numéricas geradas por esses esquemas, e essa comparação é mostrada em (f). Por essas figuras, vê-se que os resultados numéricos são satisfatórios e estão próximos da solução de referência. Em Fig. 6.18-(f), Fig. 6.19-(f), Fig. 6.20-(f) e Fig. 6.21-(f) observa-se, com mais cuidado, que os esquemas TOPUS e van Leer sobrestimam os valores da solução próximo as descontinuidades, os demais esquemas subestimam os valores nessas regiões. Em geral, pode-se afirmar que o esquema SDPUS-C1 apresenta o melhor resultado. 
(a)

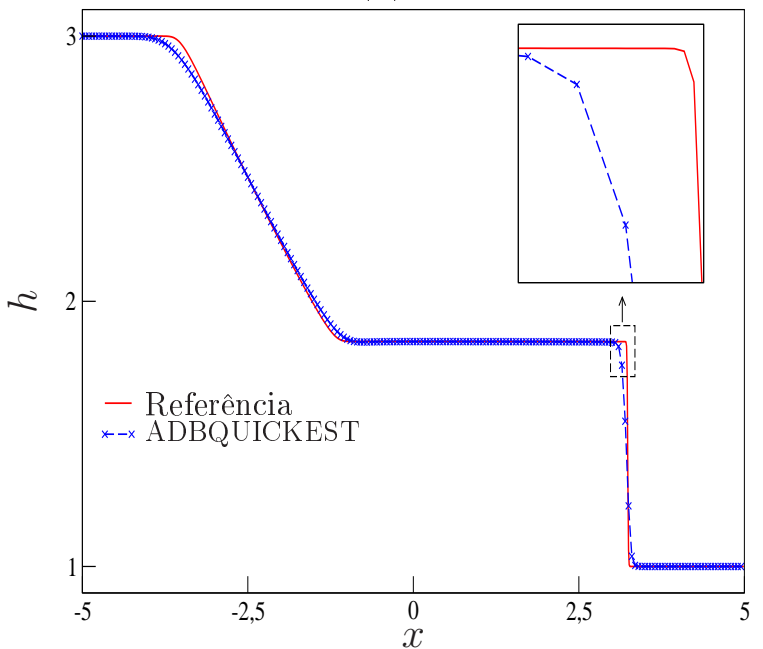

(c)

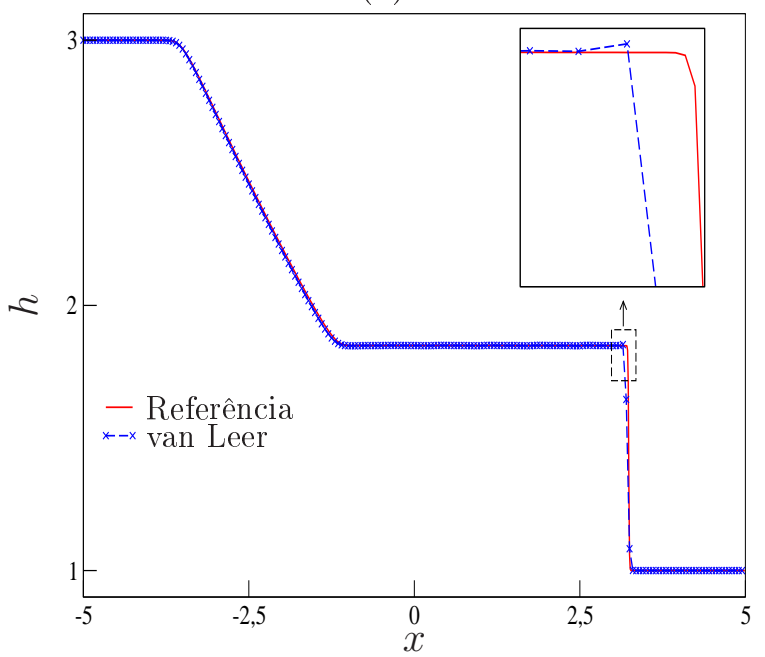

(e)

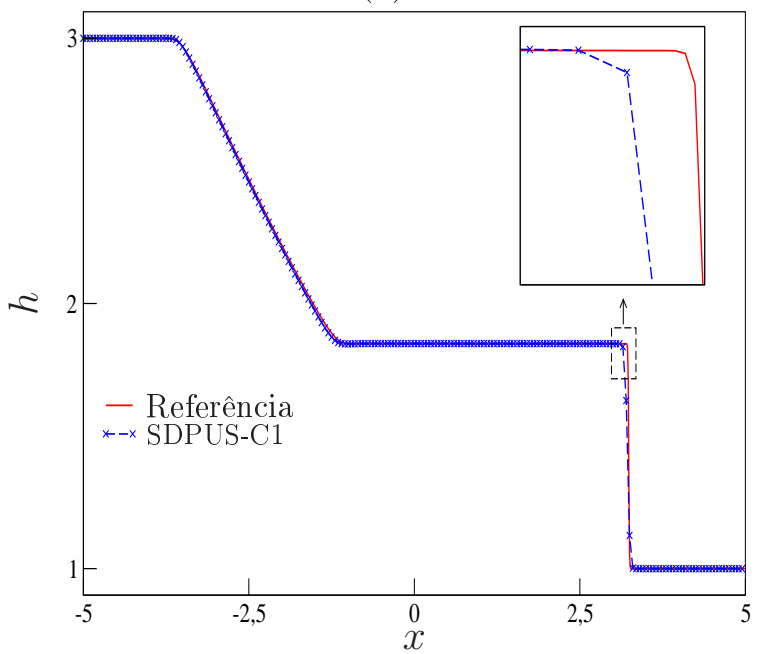

(b)

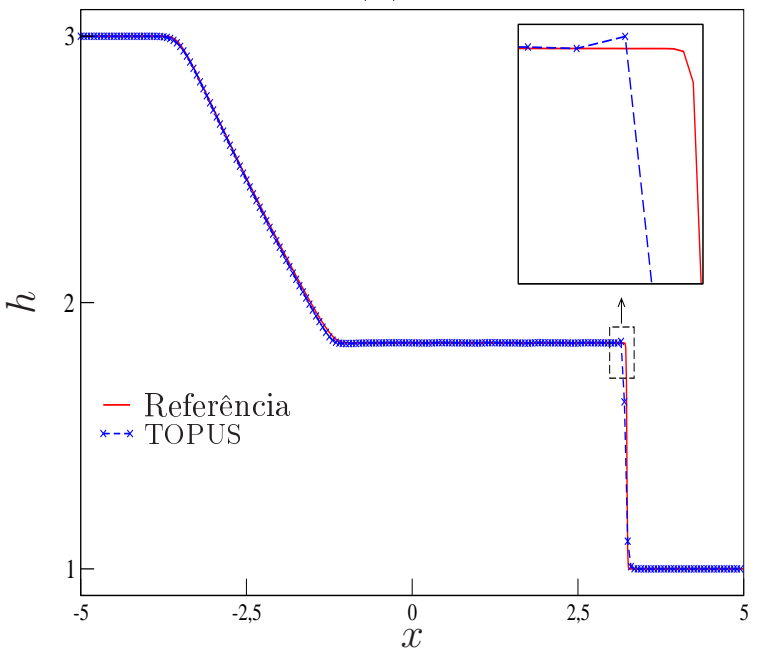

(d)

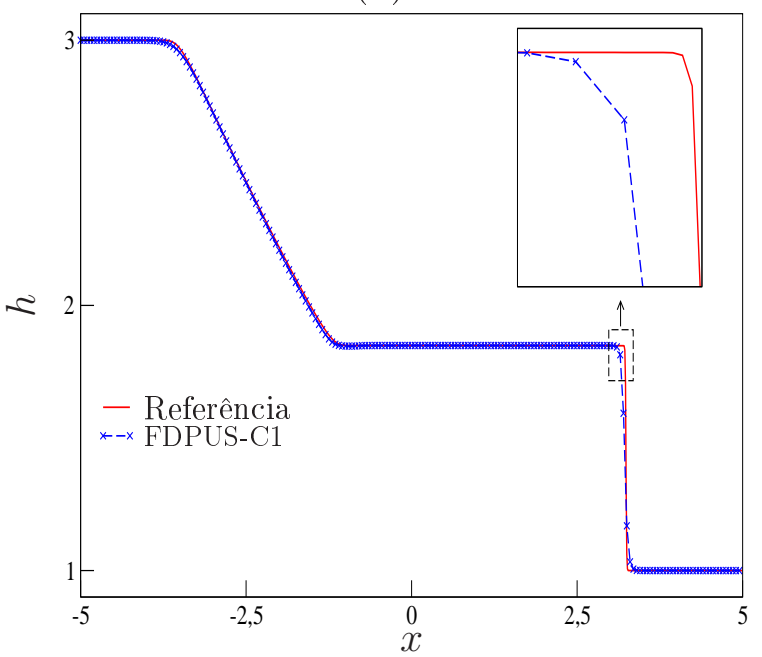

(f)

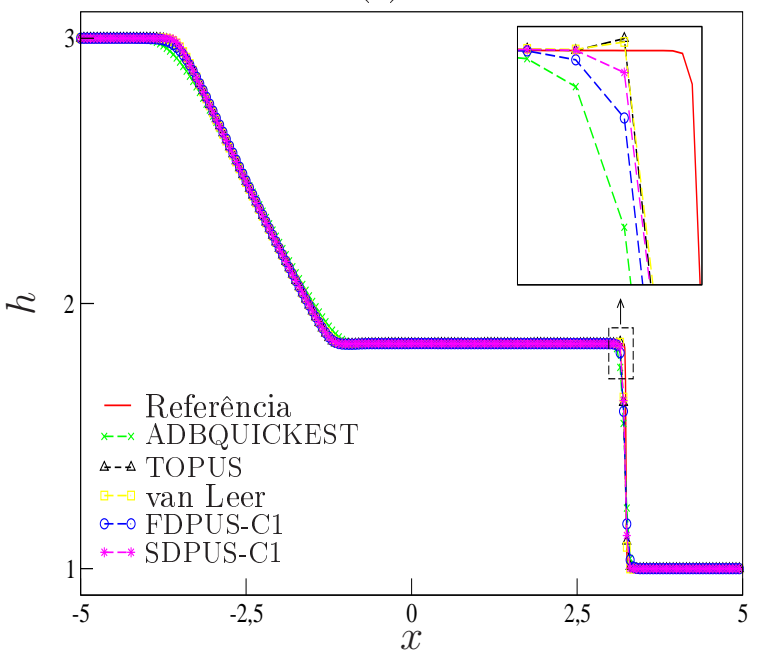

Figura 6.18: Problema $\boldsymbol{d a m}$-break. Comparação entre a solução de referência e os esquemas ADBQUICKEST (a), TOPUS (b), van Leer (c), FDPUS-C1 (d) e SDPUS-C1 (e); comparação das soluções numéricas (f). Resultados numéricos para o sistema hiperbólico de águas rasas (variável $h$ ), com condições iniciais (6.24)-(6.25) e extrapolação de ordem zero no contorno. 
(a)

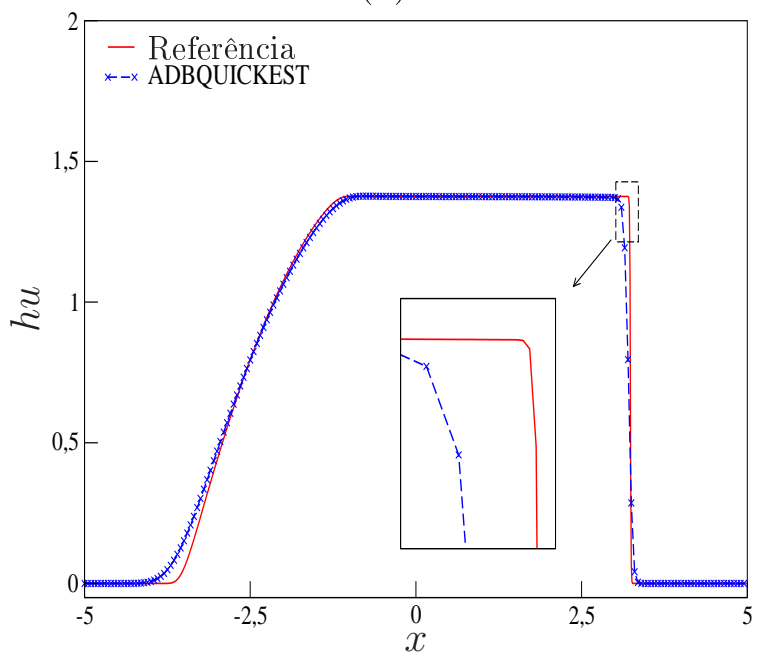

(c)

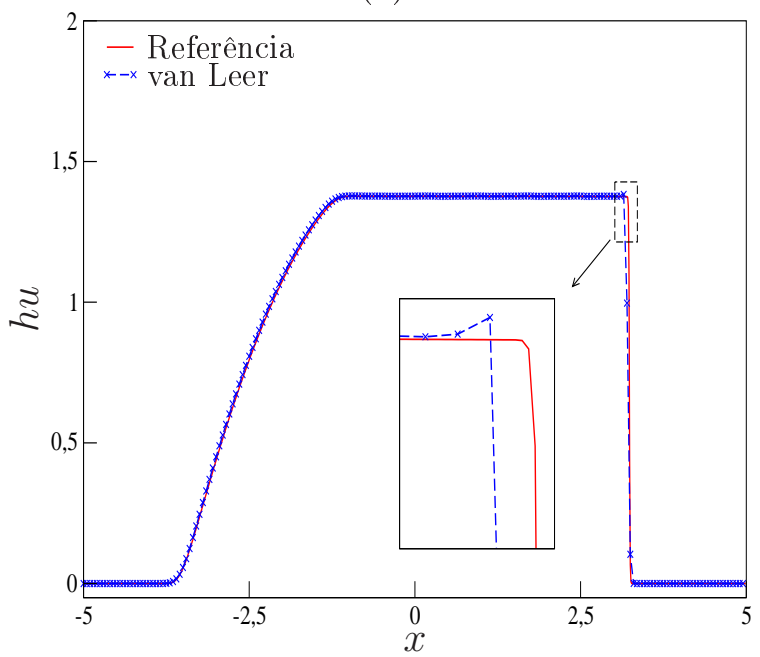

(e)

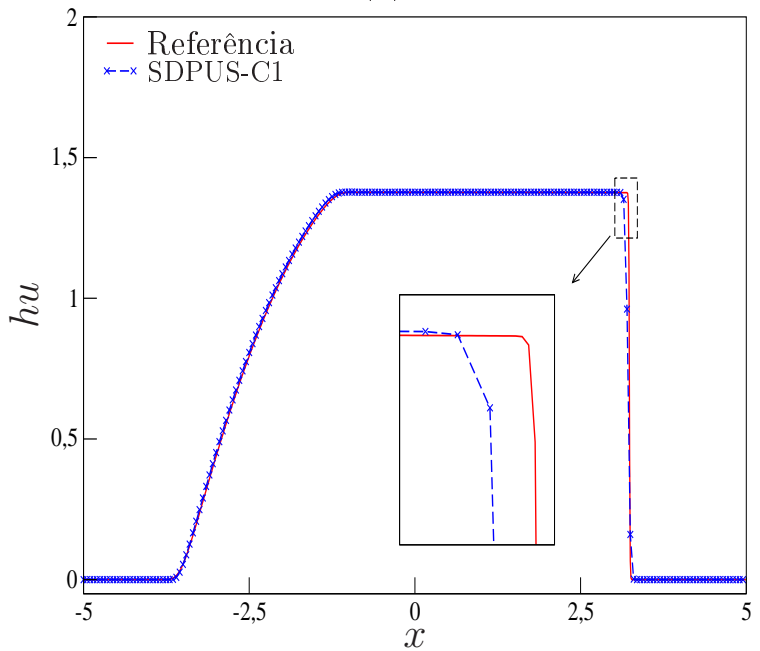

(b)

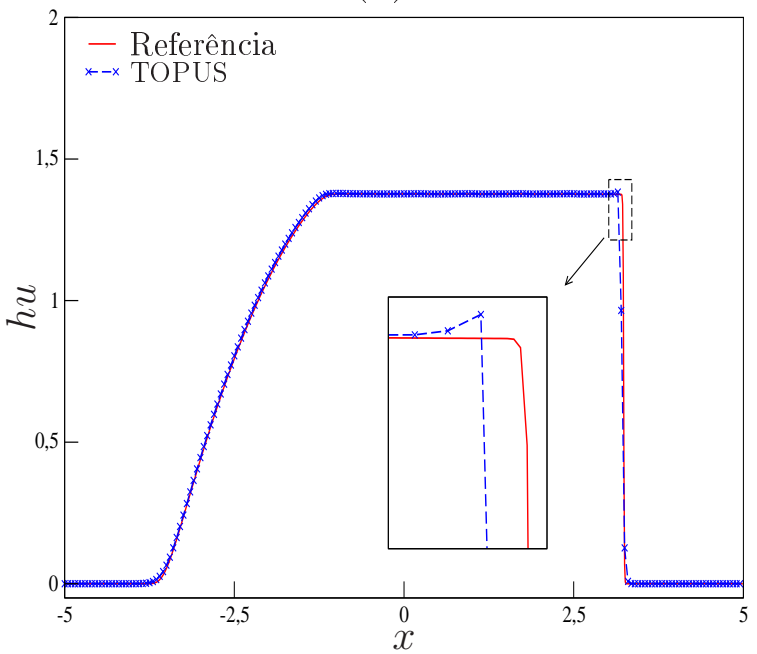

(d)

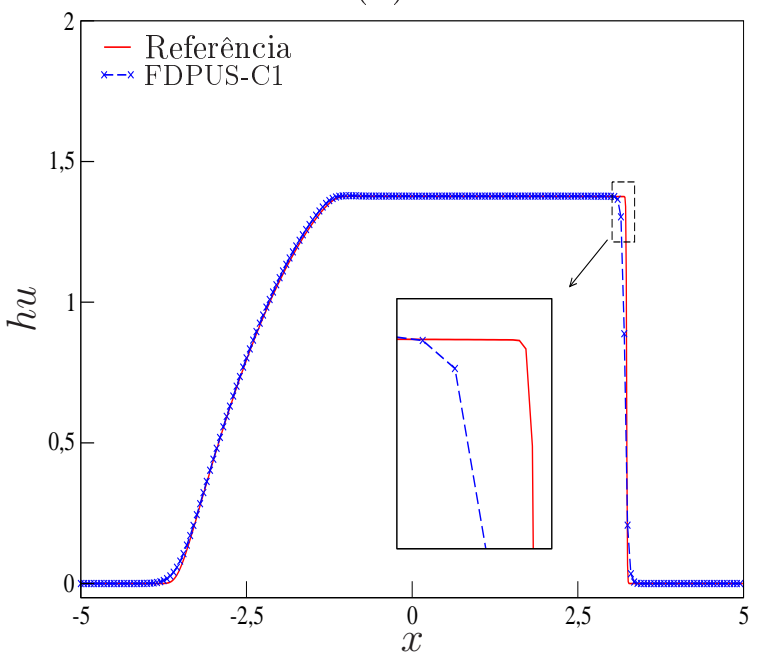

(f)

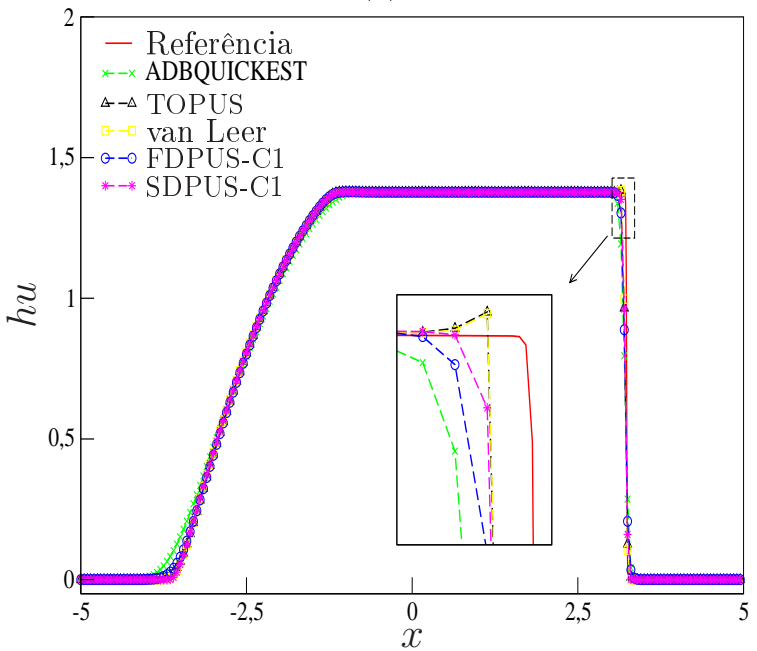

Figura 6.19: Problema dam-break. Comparação entre a solução de referência e os esquemas ADBQUICKEST (a), TOPUS (b), van Leer (c), FDPUS-C1 (d) e SDPUS-C1 (e); comparação das soluções numéricas (f). Resultados numéricos para o sistema hiperbólico de águas rasas (variável hu), com condições iniciais (6.24)-(6.25) e extrapolação de ordem zero no contorno. 
(a)

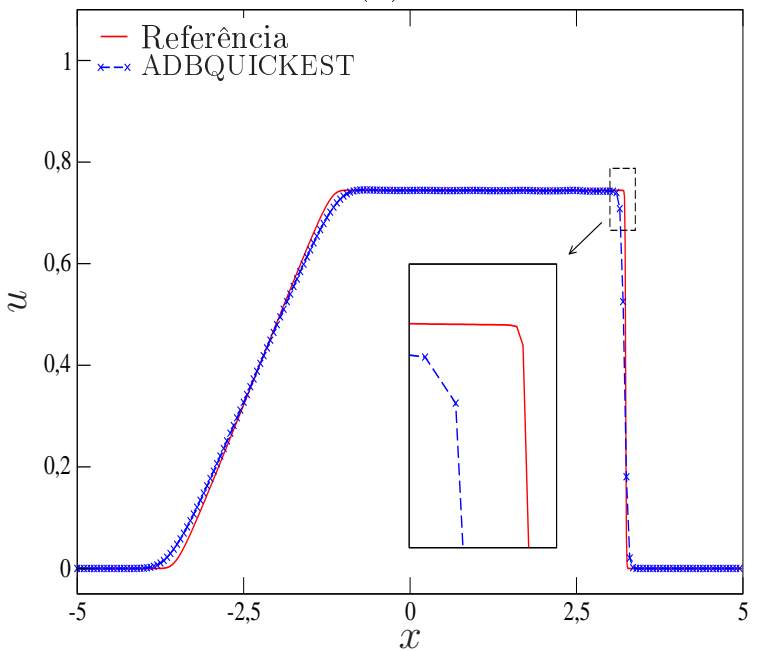

(c)

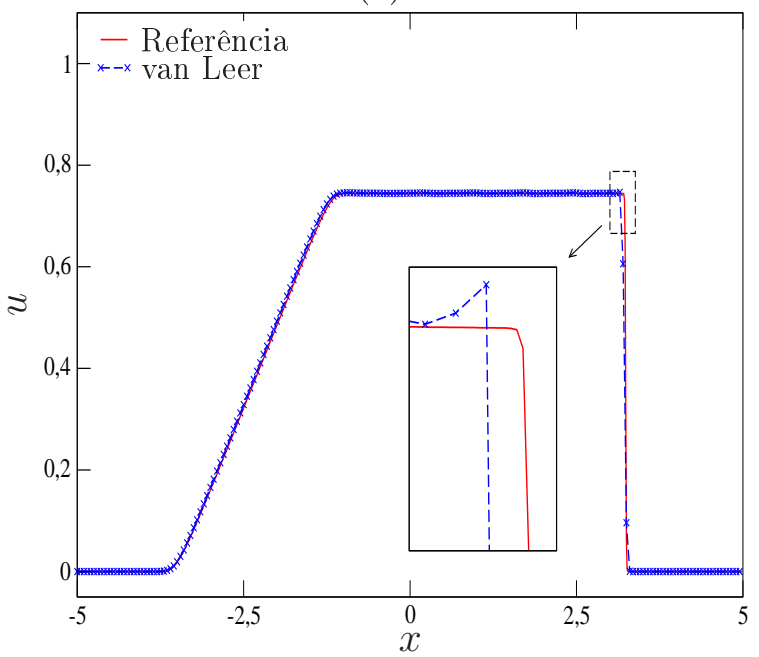

(e)

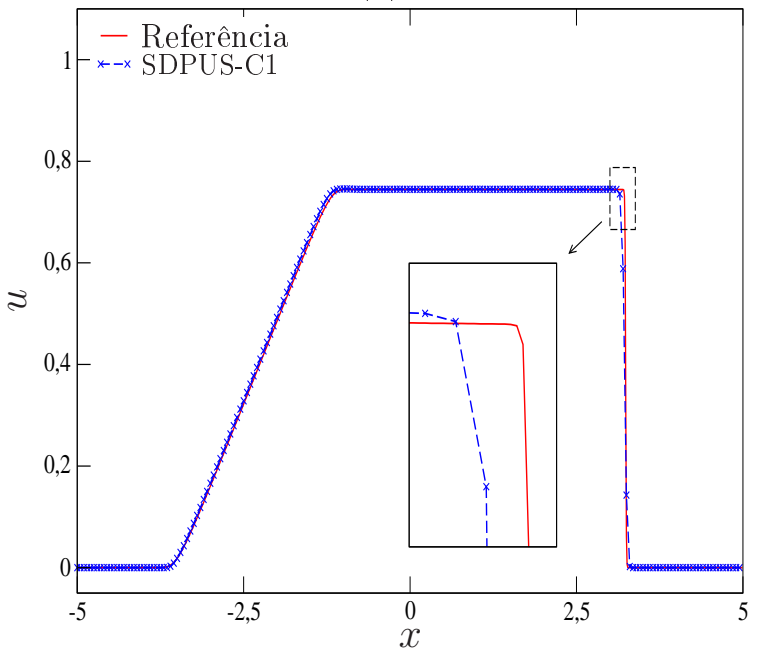

(b)

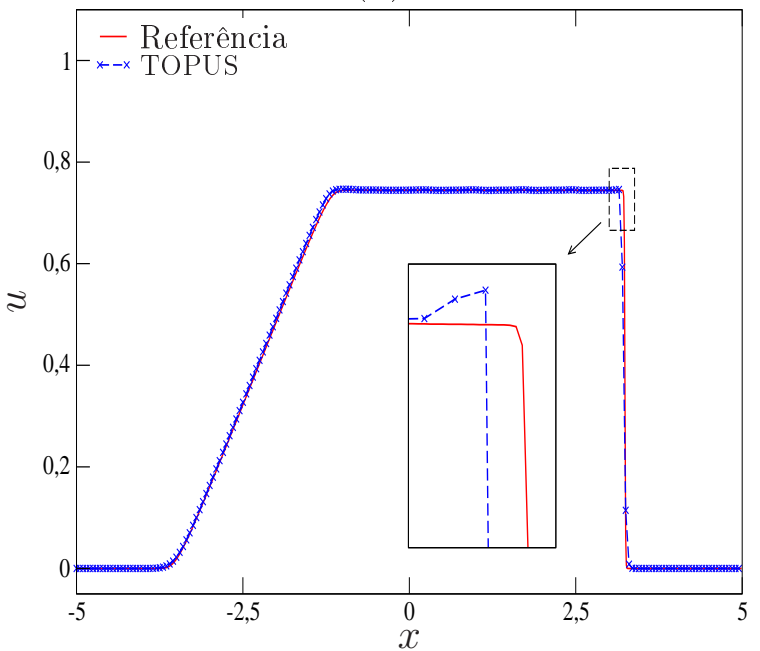

(d)

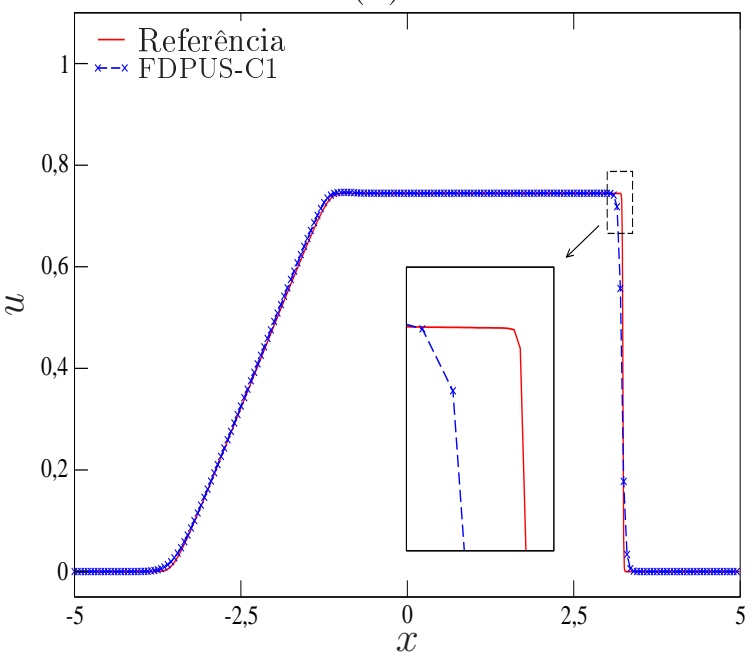

(f)

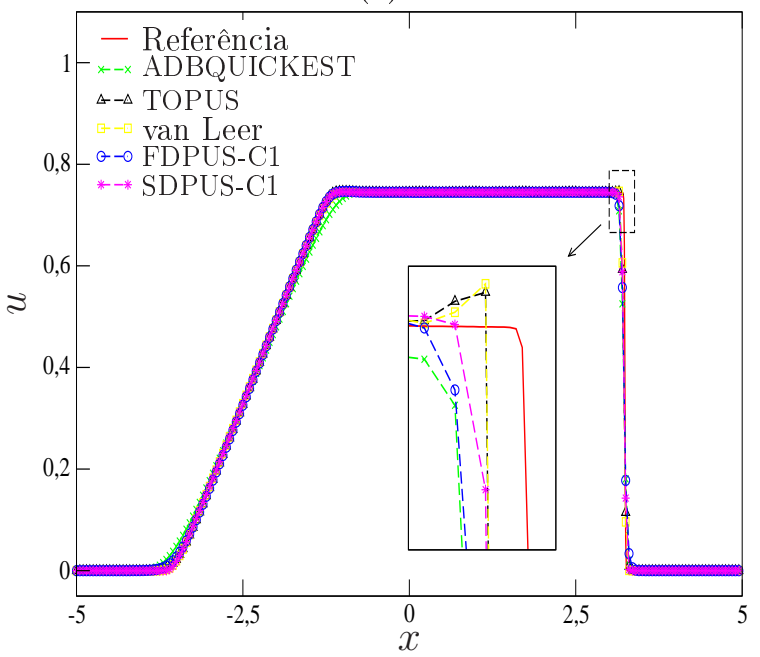

Figura 6.20: Problema dam-break. Comparação entre a solução de referência e os esquemas ADBQUICKEST (a), TOPUS (b), van Leer (c), FDPUS-C1 (d) e SDPUS-C1 (e); comparação das soluções numéricas (f). Resultados numéricos para o sistema hiperbólico de águas rasas (variável $u$ ), com condições iniciais (6.24)-(6.25) e extrapolação de ordem zero no contorno. 
(a)

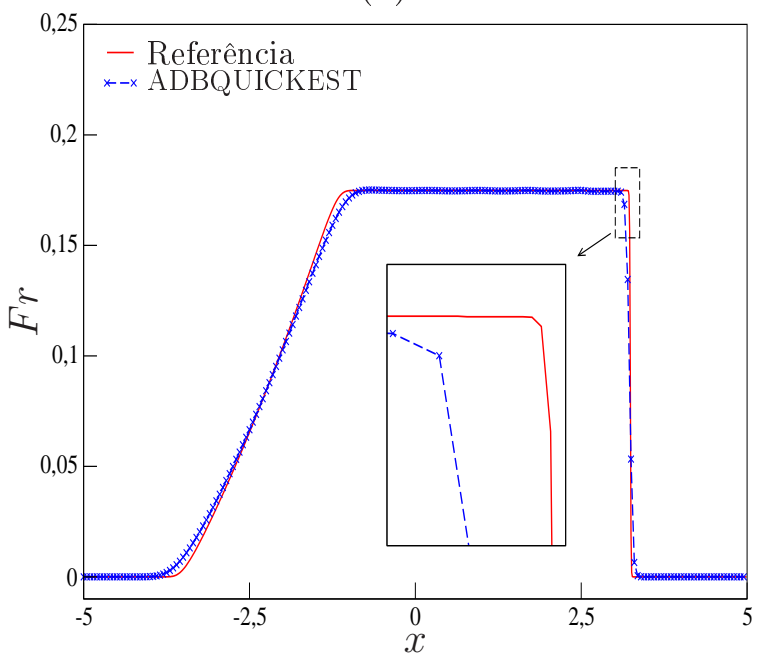

(c)

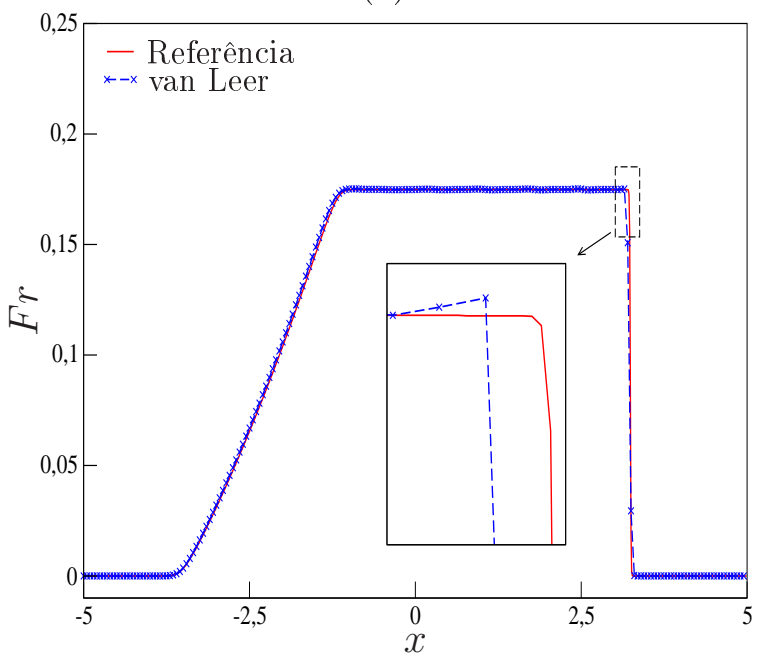

(e)

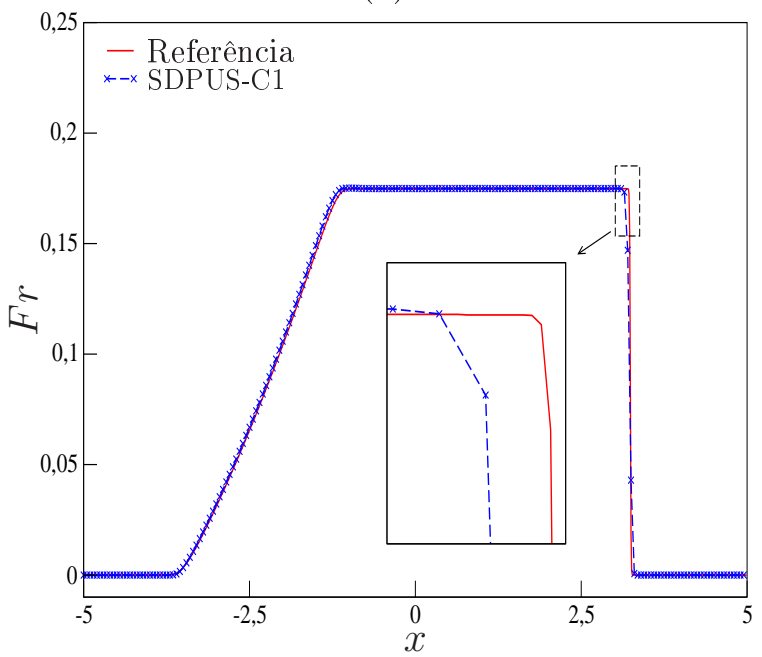

(b)

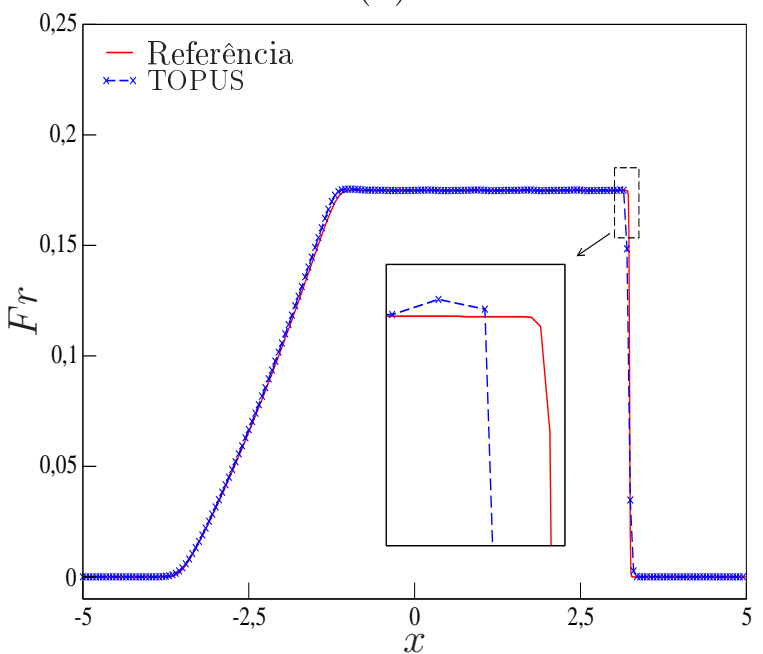

(d)

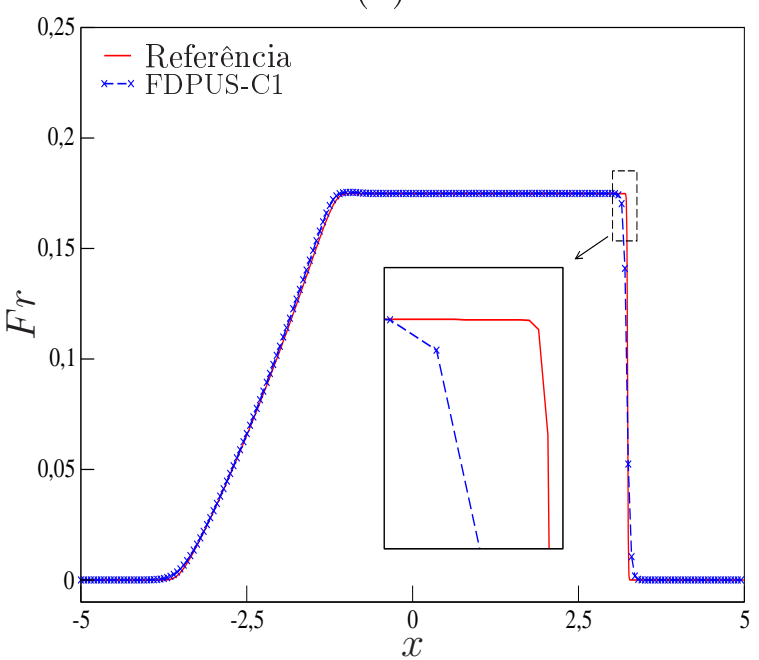

(f)

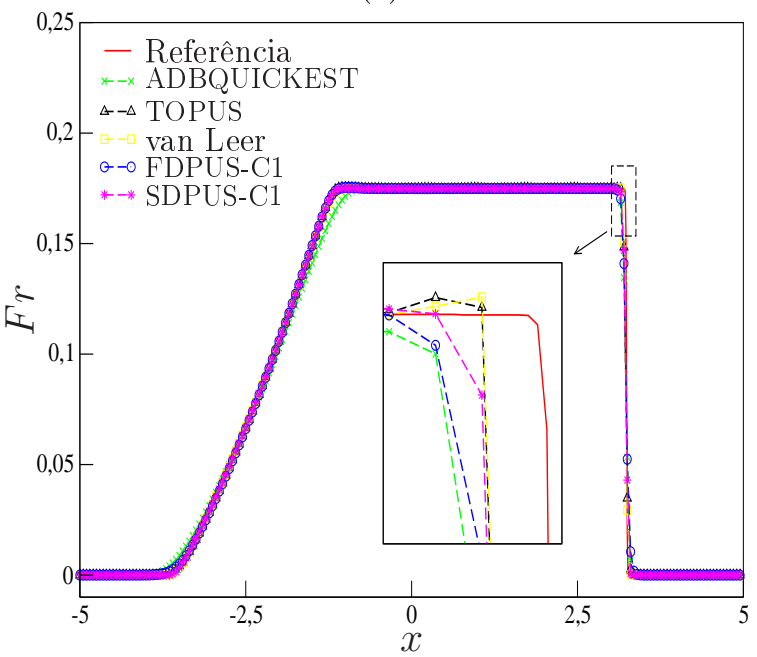

Figura 6.21: Problema dam-break. Comparação entre a solução de referência e os esquemas ADBQUICKEST (a), TOPUS (b), van Leer (c), FDPUS-C1 (d) e SDPUS-C1 (e); comparação das soluções numéricas (f). Resultados numéricos para o sistema hiperbólico de águas rasas (variável $\mathrm{Fr}$ ), com condições iniciais (6.24)-(6.25) e extrapolação de ordem zero no contorno. 


\subsection{Sistema Hiperbólico Euler}

As equações de Euler são formuladas pela lei de conservação 1D (2.1), com vetor de variáveis conservadas e função fluxo dados, respectivamente, por (2.18) e (2.19). As soluções numéricas são geradas no pacote computacional CLAWPACK equipado com os limitadores de fluxo dos esquemas ADBQUICKEST, TOPUS, FDPUS-C1 e SDPUS-C1. As soluções numéricas são comparadas com as soluções de referência propostas no próprio software. Também se comparam os resultados numéricos obtidos pelos quatro esquemas.

Caso 1 - Condição inicial suave (hump). Neste caso, as equações de Euler são definidas em $x \in[-1.5,1.5]$ e suplementadas com condições iniciais (suaves para densidade e pressão, com velocidade nula) dadas por

$$
\left[\rho_{0}, \quad u_{0}, \quad P_{0}\right]^{T}=\left[1+0.5 \exp \left\{-80(-0.15+x)^{2}\right\}, \quad 0, \quad 1+0.5 \exp \left\{-80(-0.15+x)^{2}\right\} 0\right]^{T}(.6 .26)
$$

A solução de referência para este problema de Riemann é calculada em uma malha com 1500 células computacionais, pelo método de Godunov de primeira ordem (para mais detalhes, ver LeVeque [44]), com $\theta=0.5$. As soluções numéricas são computadas pelo método de Godunov com termo de correção, aplicando-se os limitadores de fluxo dos esquemas ADBQUICKEST, TOPUS, FDPUS-C1 e SDPUS-C1, em uma malha com 500 células computacionais e $\theta=0.8$. Ambas soluções, de referência e numérica, são geradas para $t=1$.

A Figura 6.22 mostra a solução de referência e os resultados numéricos obtidos com os esquemas ADBQUICKEST (Fig. 6.22-(a)), TOPUS (Fig. 6.22-(b)), FDPUS-C1 (Fig. 6.22-(c)) e SDPUS-C1 (Fig. 6.22-(d)). Como complementação, apresenta-se uma comparação entre as soluções numéricas (Fig. 6.22-(e)), geradas pelos esquemas anteriormente citados, em que a área demarcada em Fig. 6.22-(e) é ampliada em Fig. 6.22-(f). Por meio dessa figura, observa-se que os resultados numéricos, obtidos pelos esquemas anteriormente citados, são semelhantes e estão em boa concordância com a solução de referência.

Caso 2 - Tubo de choque de Sod. Este problema de Riemann foi proposto por Sod [58] e consiste em uma rarefação à esquerda, um contacto e um choque à direita. As condições iniciais são dadas por

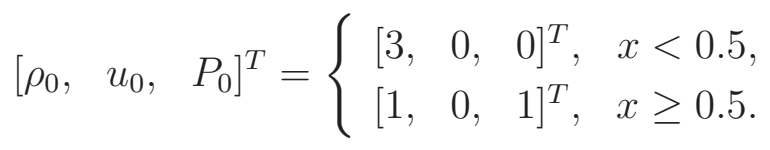

A solução de referência é calculada em uma malha com 1500 células computacionais, pelo método de Godunov com termo de correção, em que considera-se o limitador de fluxo MC (ver LeVeque [44]), $\operatorname{com} \theta=0.5$. As soluções numéricas são obtidas em 500 células computacionais, $\operatorname{com} \theta=0.8$. Ambas soluções, de referência e numérica, são calculadas para $\rho$ (ver Figura 6.23) e $u$ (ver Figura 6.24), em $t=0.1$. 
(a)

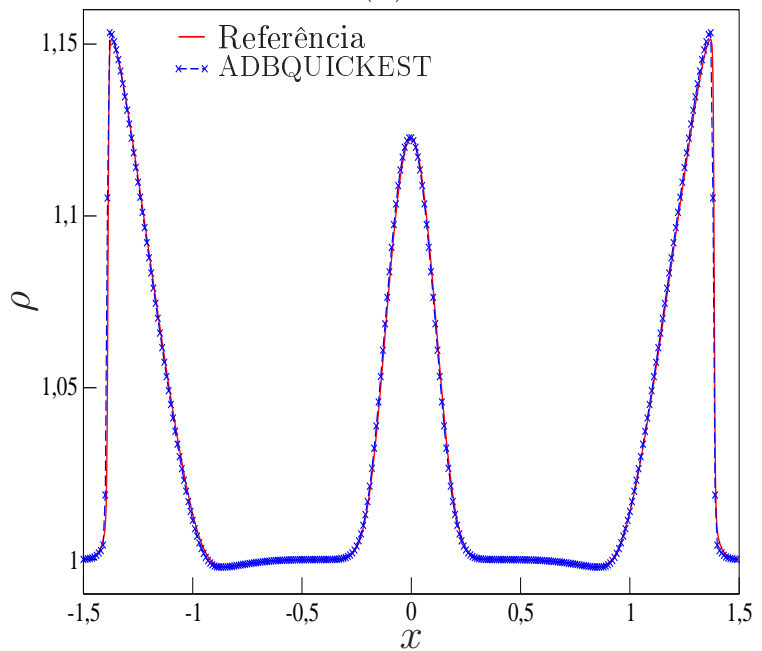

(c)

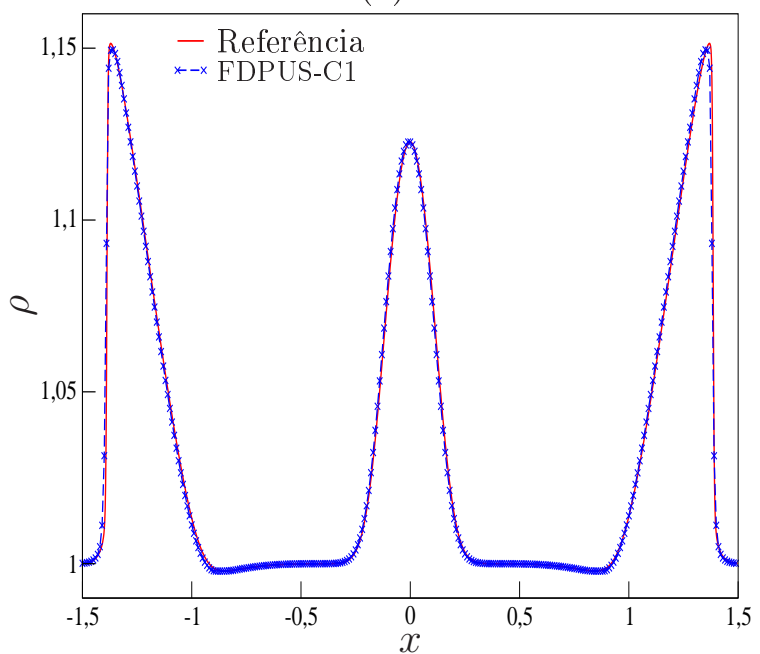

(e)

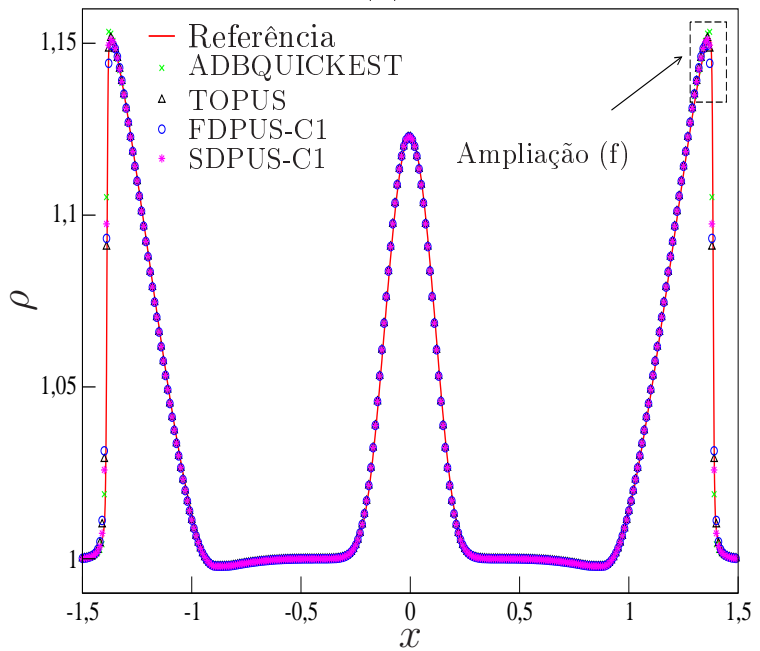

(b)

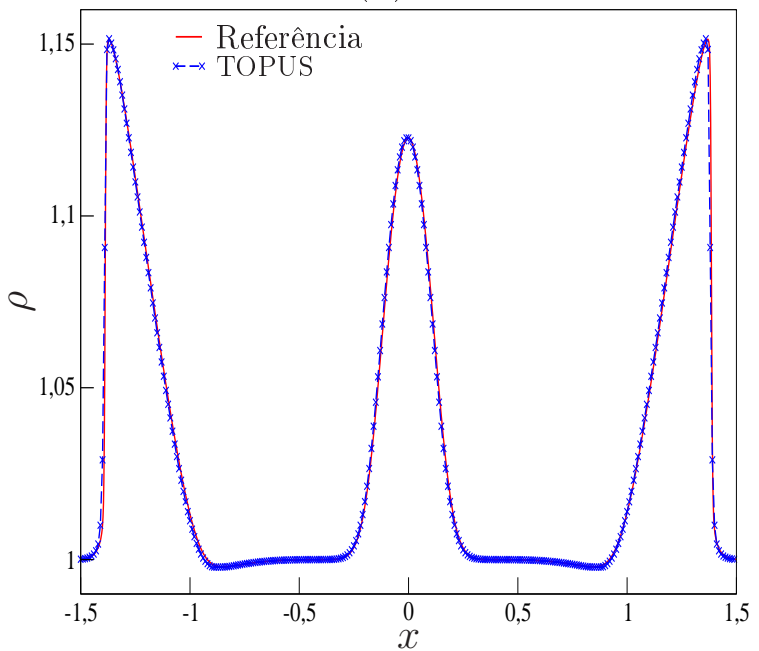

(d)

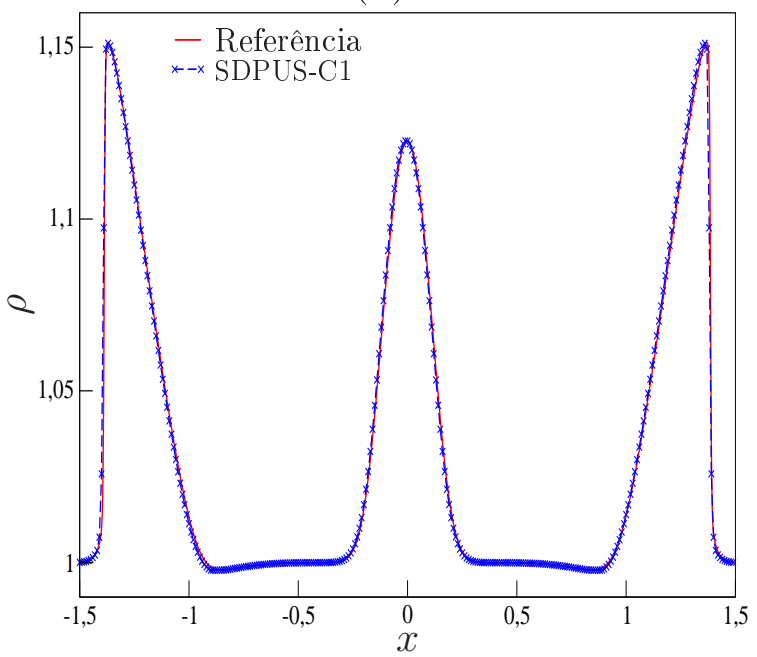

Ampliação (f)

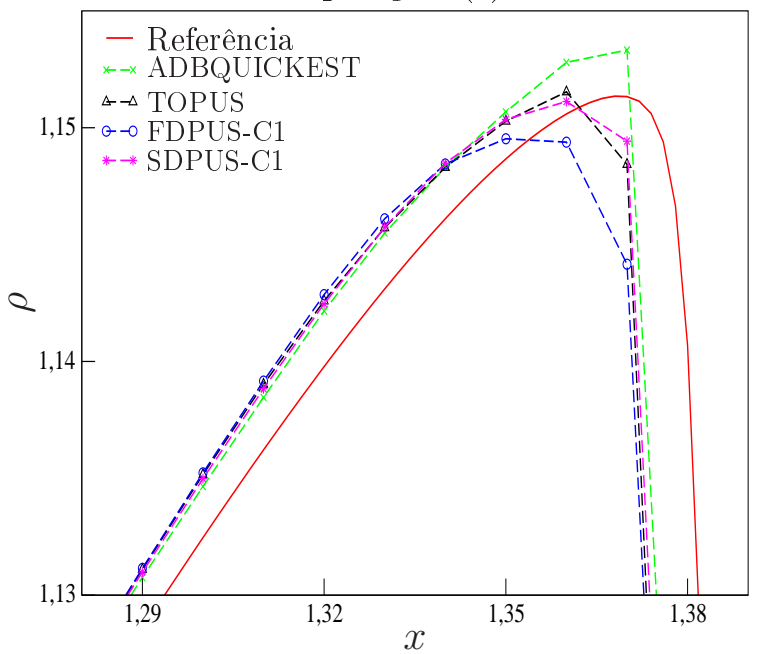

Figura 6.22: Condição inicial suave (hump). Comparação entre a solução de referência e os esquemas ADBQUICKEST (a), TOPUS (b), FDPUS-C1 (c) e SDPUS-C1 (d); comparação das soluções numéricas (e); ampliação (f). Soluções numéricas para as equações de Euler (variável $\rho$ ), com condições iniciais (6.26). 
Nas Figuras 6.23 e 6.24, apresenta-se a solução de referência e os resultados numéricos obtidos com os esquemas ADBQUICKEST (Fig. 6.23-(a) e Fig. 6.24-(a)), TOPUS (Fig. 6.23-(b) e Fig. 6.24-(b)), FDPUS-C1 (Fig. 6.23-(c) e Fig. 6.24-(c)) e SDPUS-C1 (Fig. 6.23-(d) e Fig. 6.24-(d)). Ainda nessas figuras, faz-se uma comparação entre os resultados numéricos (Fig. 6.23-(e) e Fig. 6.24-(e)). Para uma melhor compreensão, em Fig. 6.23-(f) e Fig. 6.24-(f) apresentam-se as ampliações das regiões demarcadas em Fig. 6.23-(e) e Fig. 6.24-(e). Em geral, vê-se que os resultados numéricos estão em ótima concordância com a solução de referência. Salienta-se que os novos esquemas FDPUS-C1 e SDPUS-C1 tiveram desempenho semelhante aos esquemas ADBQUICKEST e TOPUS.

Caso 3 - Tubo de choque de Shu e Osher. Este problema foi proposto por Shu e Osher [57] e simula uma onda acústica interagindo com uma pertubação na densidade tipo senóide. Para formulação do mesmo, o sistema hiperbólico de Euler é definido em $x \in[-5,5]$ e suplementado com as condições iniciais

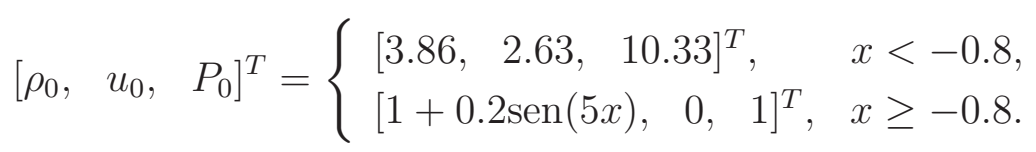

Nesse caso, a solução de referência é calculada em uma malha com 1600 células computacionais, pelo método de Godunov com termo de correção empregando-se o limitado de fluxo Superbee (ver LeVeque [44]) e $\theta=0.5$. Na Figura 6.25, comparam-se a solução de referência e os resultados numéricos gerados em uma malha computacional com 800 células, para $t=2 \mathrm{e}$ $\theta=0.8$, com os esquemas ADQUICKEST (Fig. 6.25-(a)), TOPUS (Fig. 6.25-(b)), FDPUS-C1 (Fig. 6.25-(c)) e SDPUS-C1 (Fig. 6.25-(d)). Nesta figura, as soluções numéricas também são comparadas e a área demarcada em Fig. 6.25-(e) é ampliada em Fig. 6.25-(f). Por essa figura observa-se que os resultados numéricos são satisfatórios.

Caso 4 - Ondas com fortes colisões. Neste caso, considera-se o problema desafiador de Riemann, sugerido por Wood e Colella [78], conhecido na literatura por Woodward-Colella colliding blast wave problem e que envolve múltiplas interações de choques fortes. As equações de Euler são definidas em $x \in[0,1]$ e suplementadas com as condições iniciais dadas por

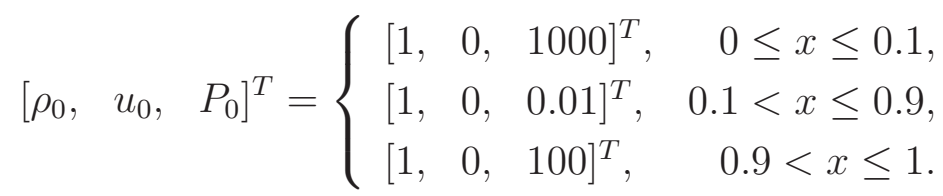

A solução de referência é gerada em uma malha com 2000 células computacionais usando o método de Godunov, com termo de correção, com o limitador de fluxo MC (ver LeVeque [44]), $\operatorname{com} \theta=0.5$. As soluções numéricas são obtidas em malhas uniformes de 1000 células computacionais, com $\theta=0.8$, também pelo método de Godunov, com termo de correção, em que são aplicados os limitadores de fluxo dos esquemas ADBQUICKEST, TOPUS, FDPUS-C1 
(a)

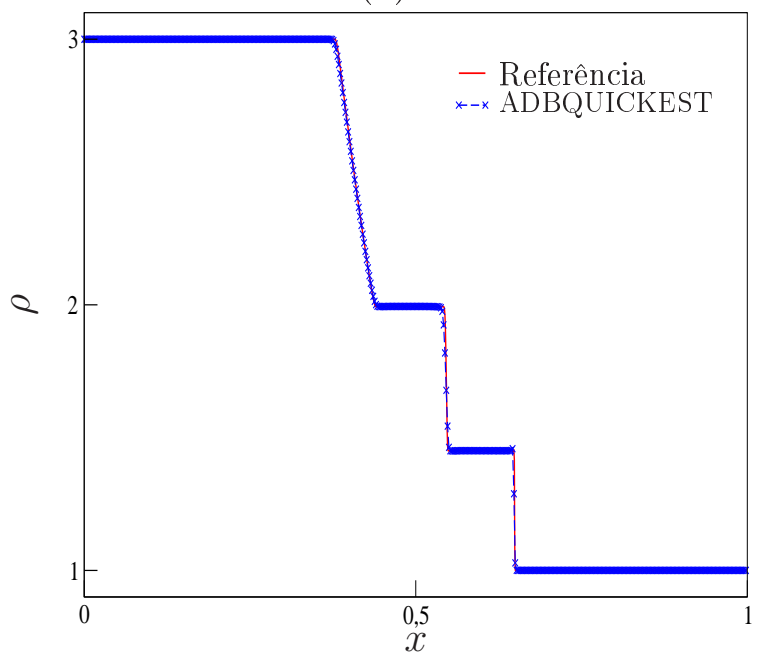

(c)

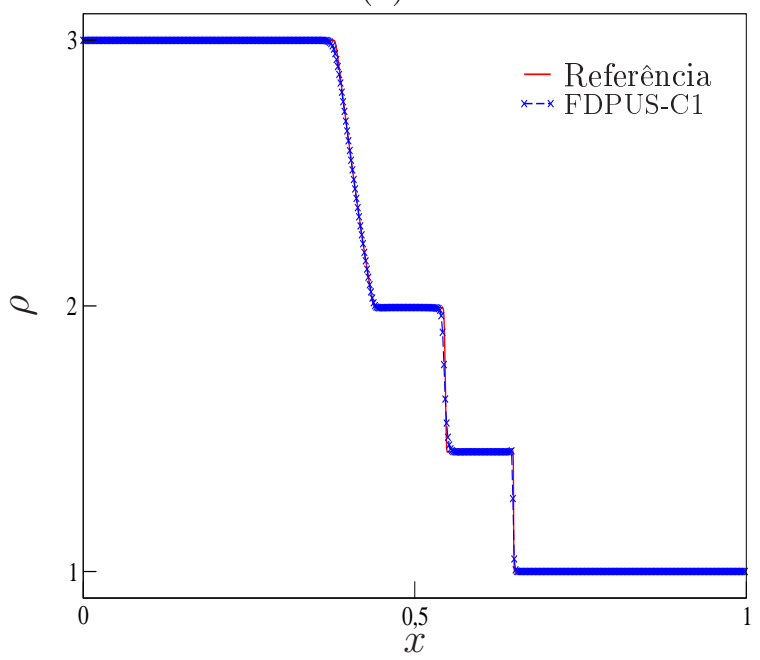

(e)

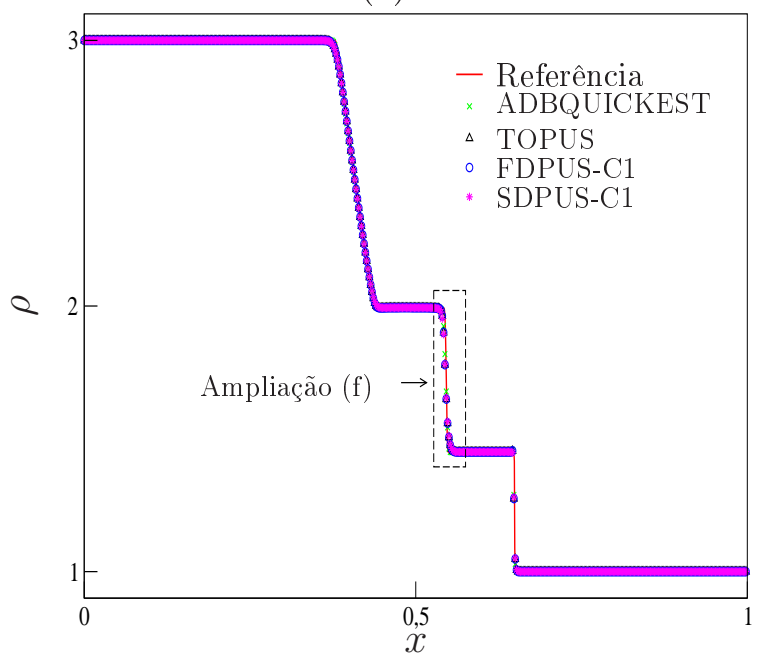

(b)

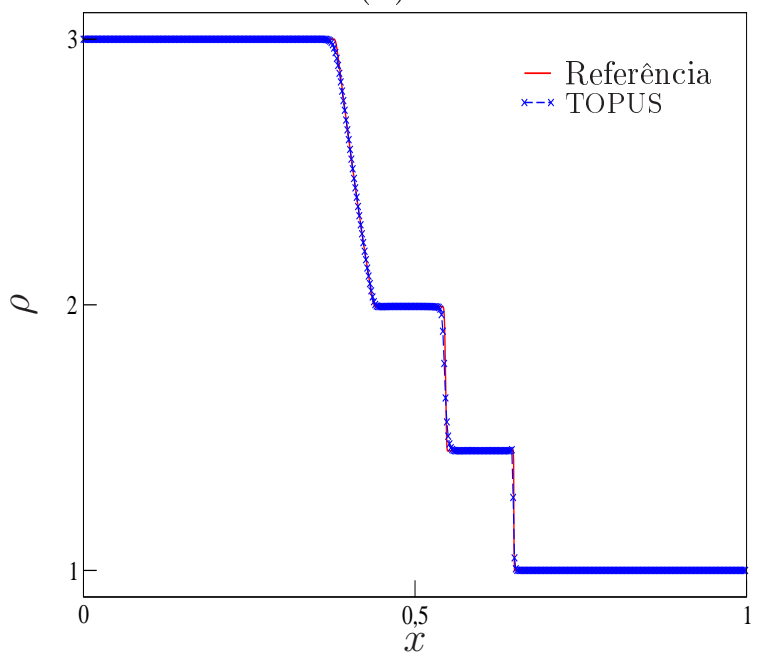

(d)

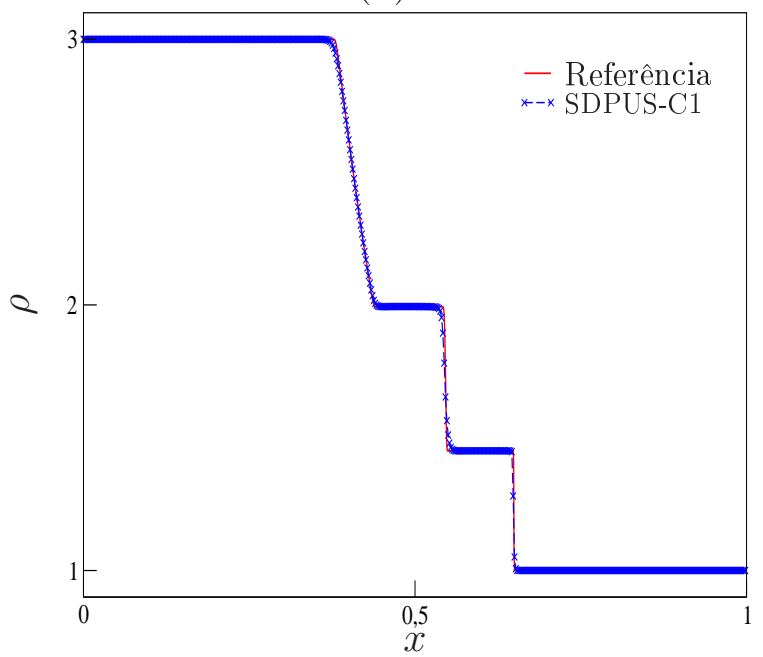

Ampliação (f)

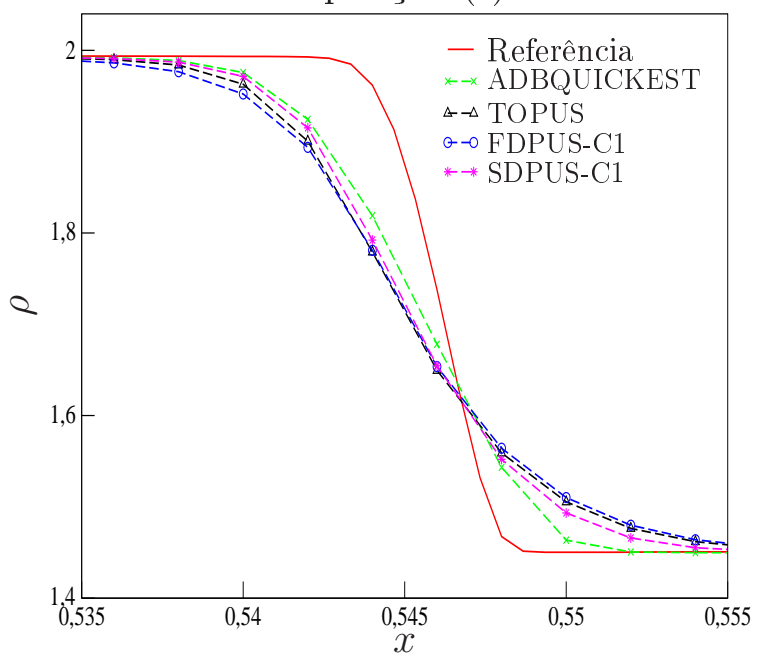

Figura 6.23: Tubo de choque de Sod. Comparação entre a solução de referência e os esquemas ADBQUICKEST (a), TOPUS (b), FDPUS-C1 (c) e SDPUS-C1 (d); comparação das soluções numéricas (e); ampliação (f). Soluções numéricas para as equações de Euler (para a variável $\rho$ ), com condições iniciais (6.27). 
(a)

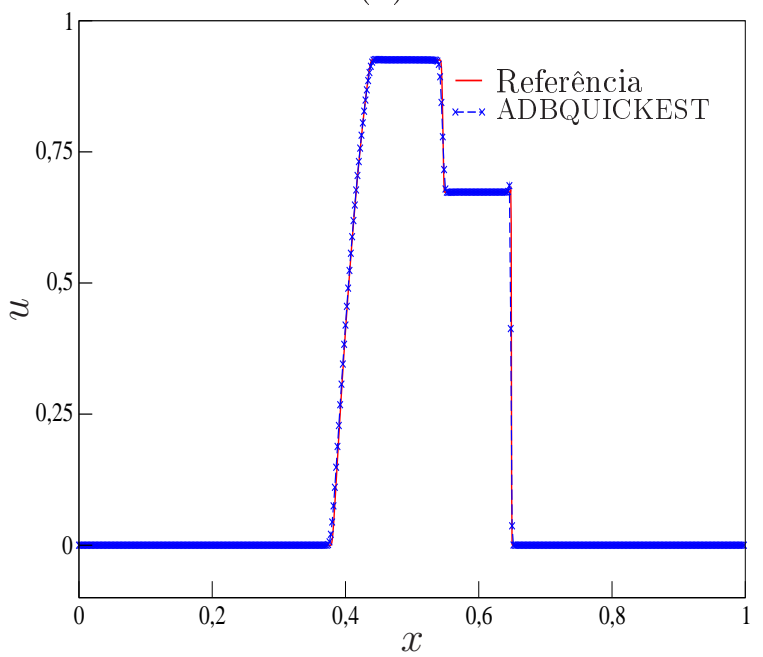

(c)

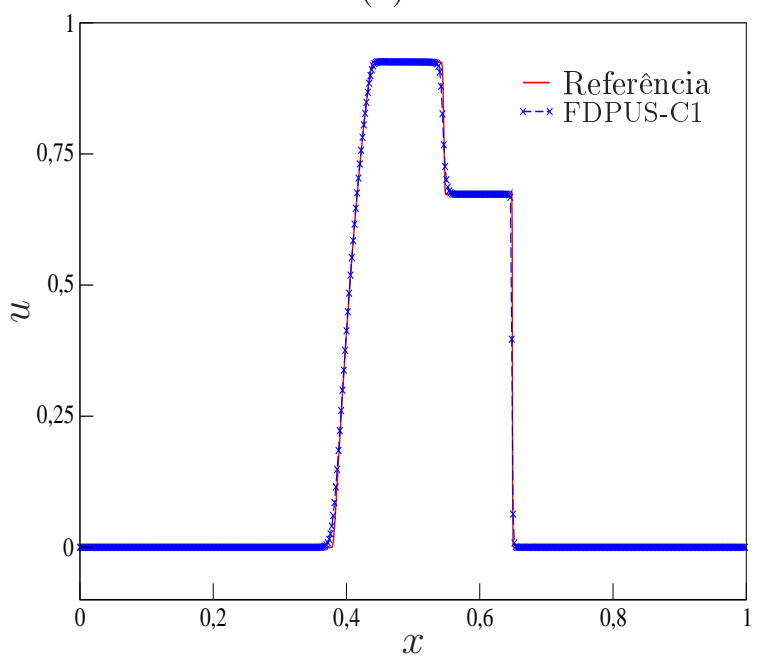

(e)

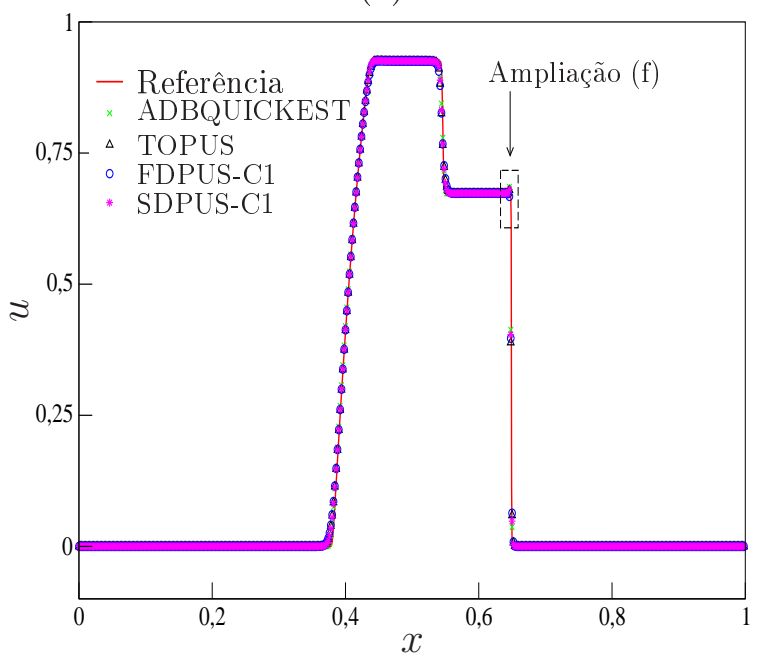

(b)

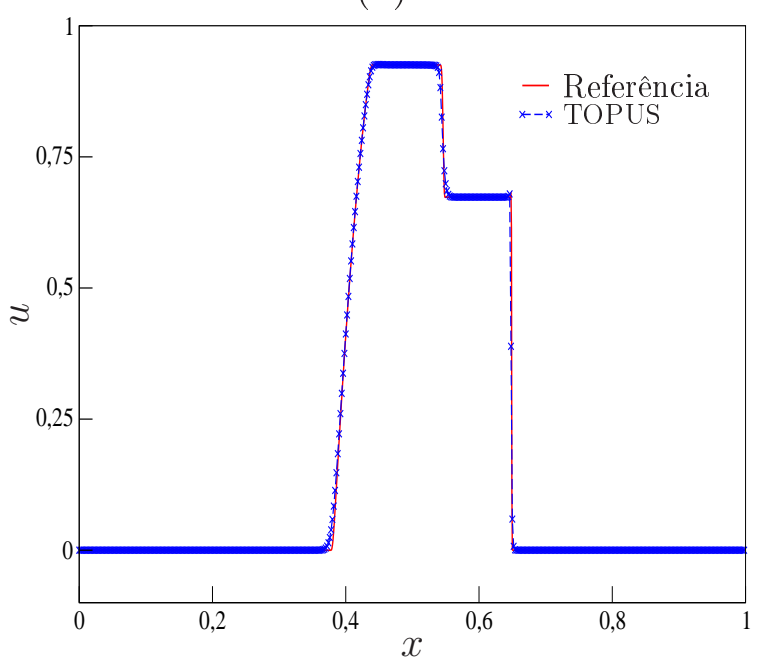

(d)

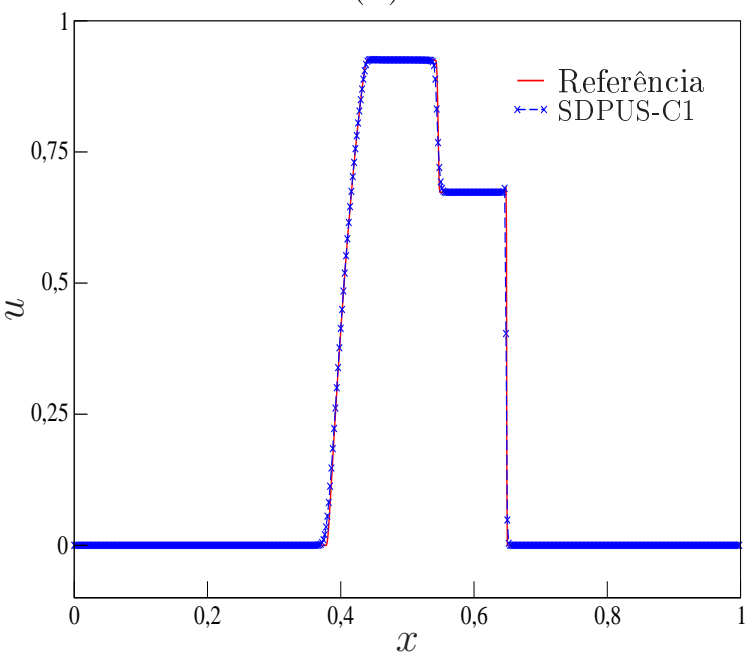

Ampliação (f)

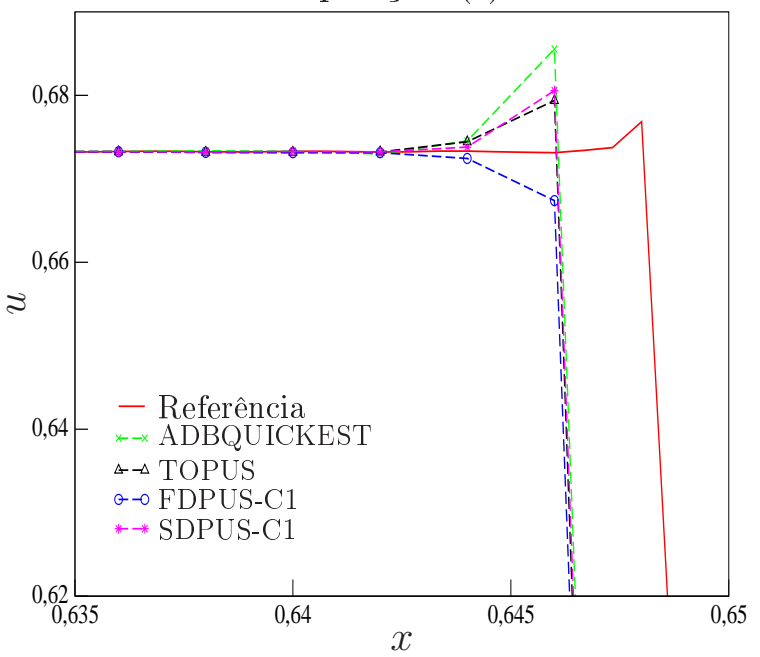

Figura 6.24: Tubo de choque de Sod. Comparação entre a solução de referência e os esquemas ADBQUICKEST (a), TOPUS (b), FDPUS-C1 (c) e SDPUS-C1 (d); comparação das soluções numéricas (e); ampliação (f). Soluções numéricas para as equações de Euler (para a variável u), com as condições iniciais (6.27). 
(a)

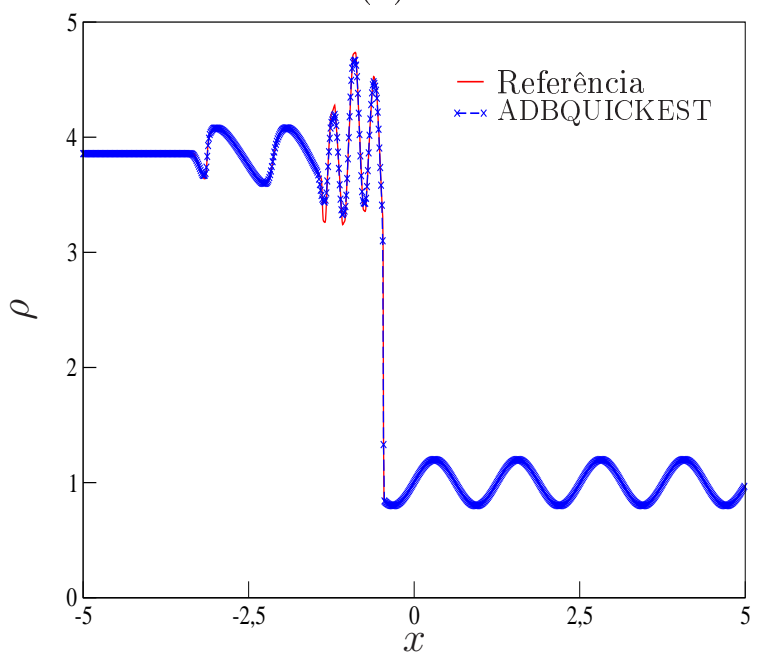

(c)

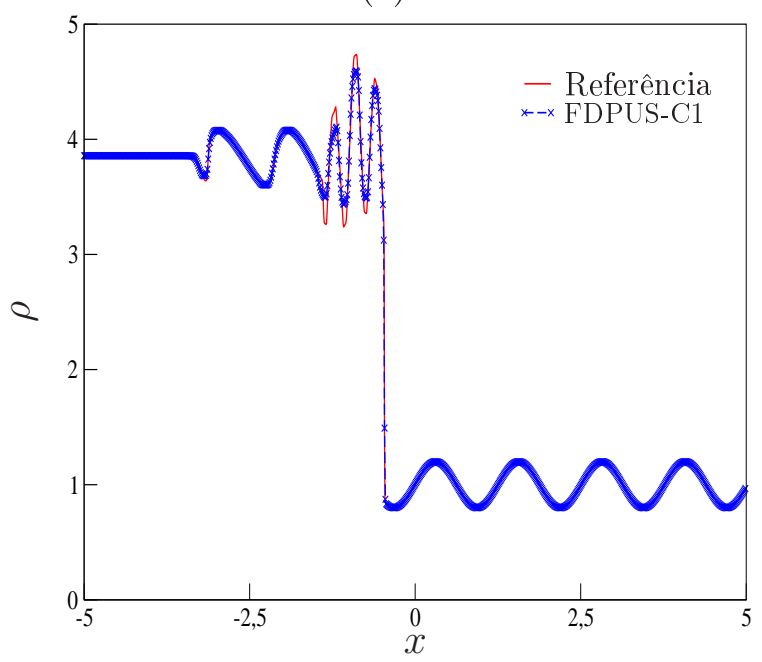

(e)

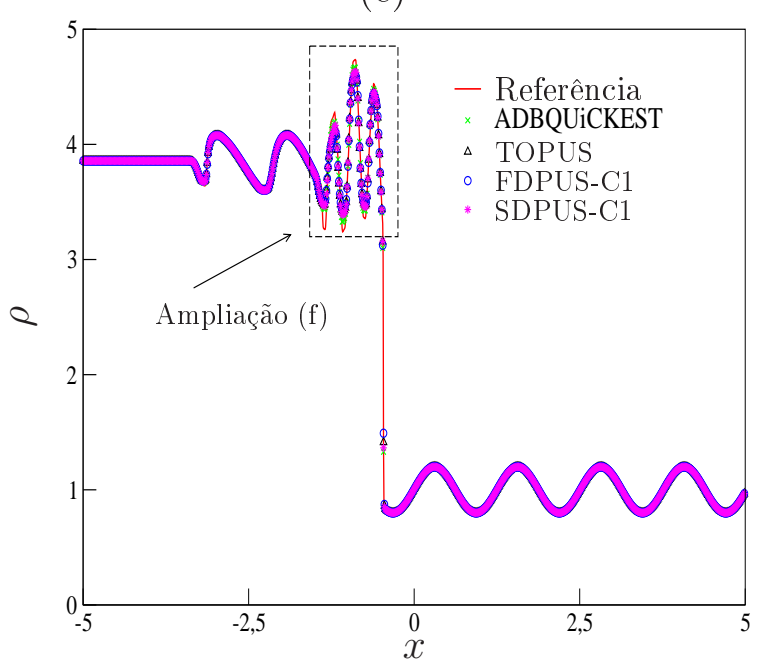

(b)

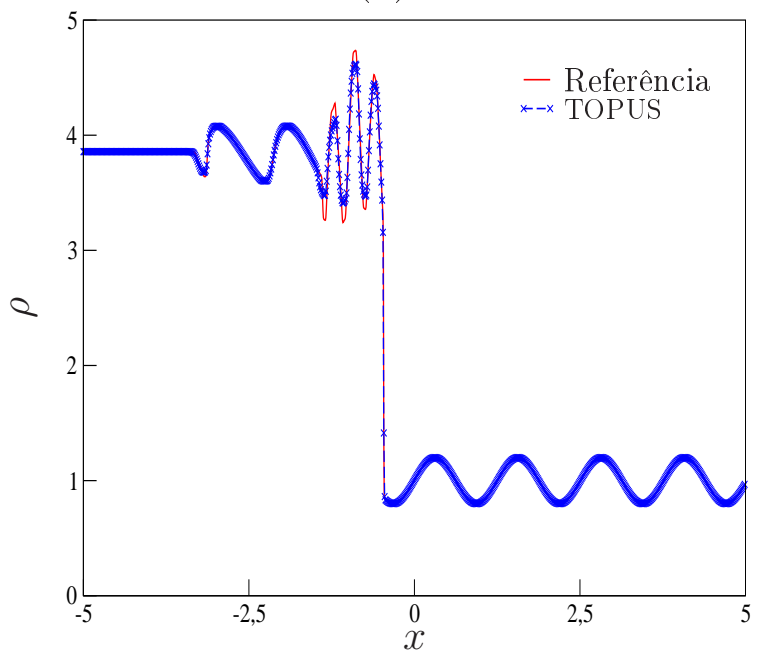

(d)

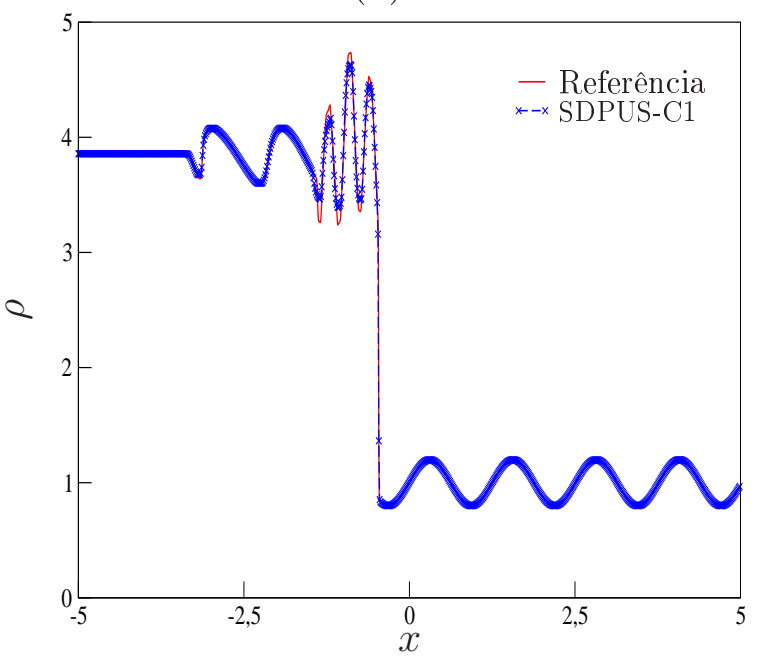

Ampliação (f)

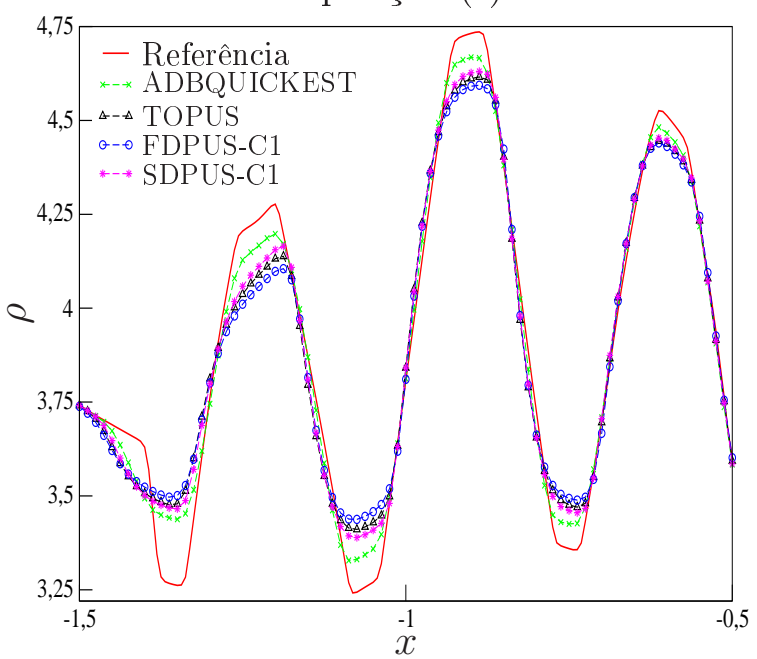

Figura 6.25: Tubo de choque de Shu e Osher. Comparação entre a solução de referência e os esquemas ADBQUICKEST (a), TOPUS (b), FDPUS-C1 (c) e SDPUS-C1 (d); comparação das soluções numéricas (e); ampliação (f). Soluções numéricas para as equações de Euler (para variável $\rho$ ), com condições iniciais (6.28). 
e SDPUS-C1. Ambas soluções, de referência e numéricas, são calculadas nos tempos $t=0.19$ e $t=0.38$ para densidade $\rho$ e pressão $P$.

As Figuras 6.26 e 6.28 mostram os resultados obtidos para densidade nos tempos $t=0.19$ e $t=0.38$, respectivamente. As Figuras 6.27 (para $t=0.19$ ) e 6.29 (para $t=0.38$ ) trazem os resultados para pressão. Nessas figuras, apresentam-se os resultados numéricos dos esquemas ADBQUICKEST, TOPUS, FDPUS-C1 e SDPUS-C1, respectivamente, em (a), (b), (c) e (d). Ainda nessas figuras, em (e), apresenta-se uma comparação entre os resultados numéricos e, em (f), amplia-se a área demarcada em (e). Observa-se, por essas figuras, que os esquemas anteriormente citados oferecem resultados satisfatórios. 
(a)

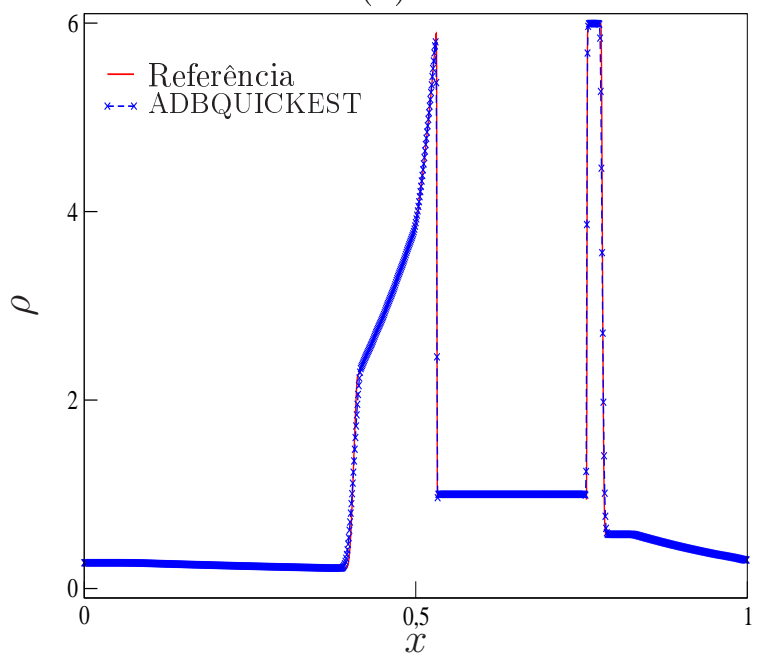

(c)

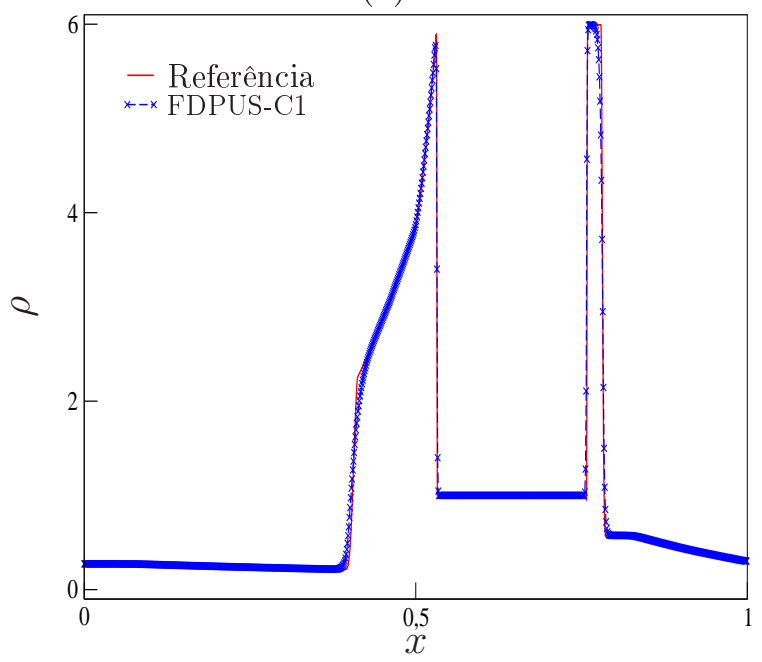

(e)

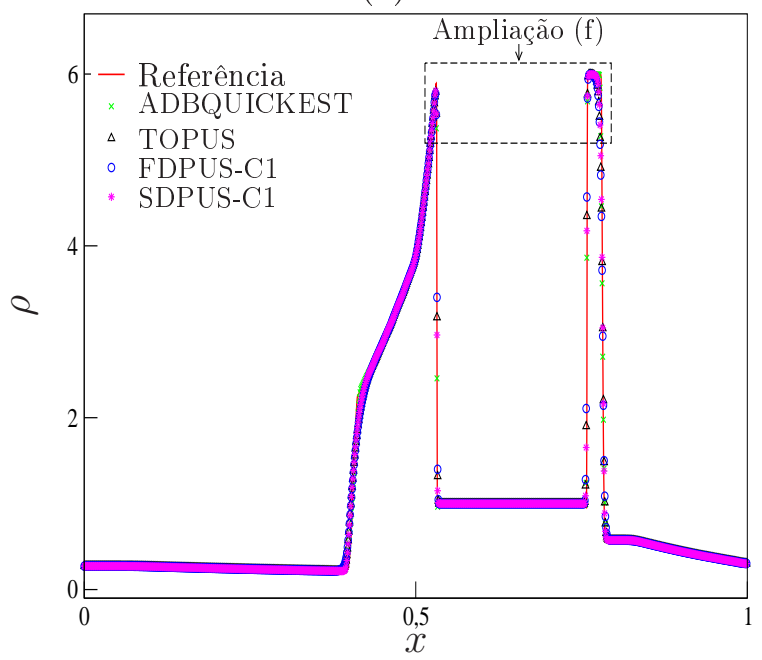

(b)

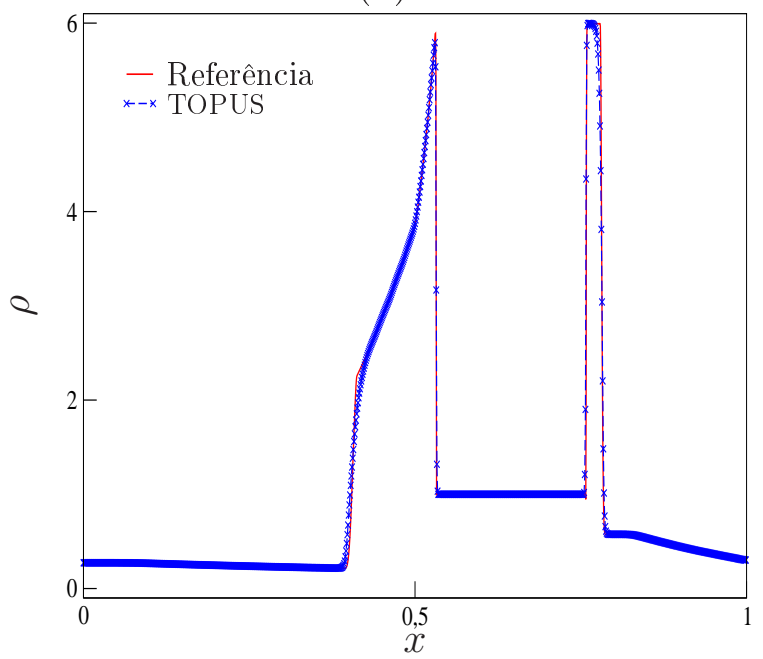

(d)

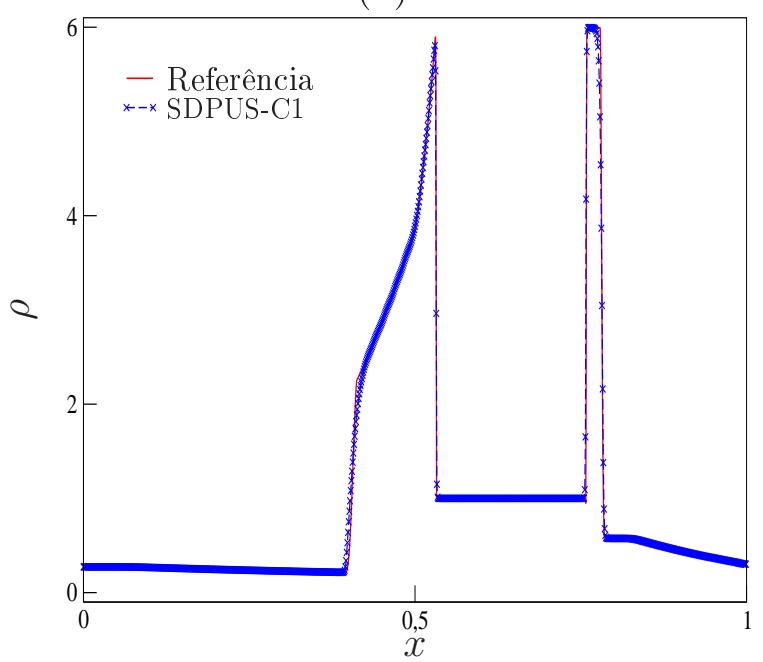

Ampliação (f)

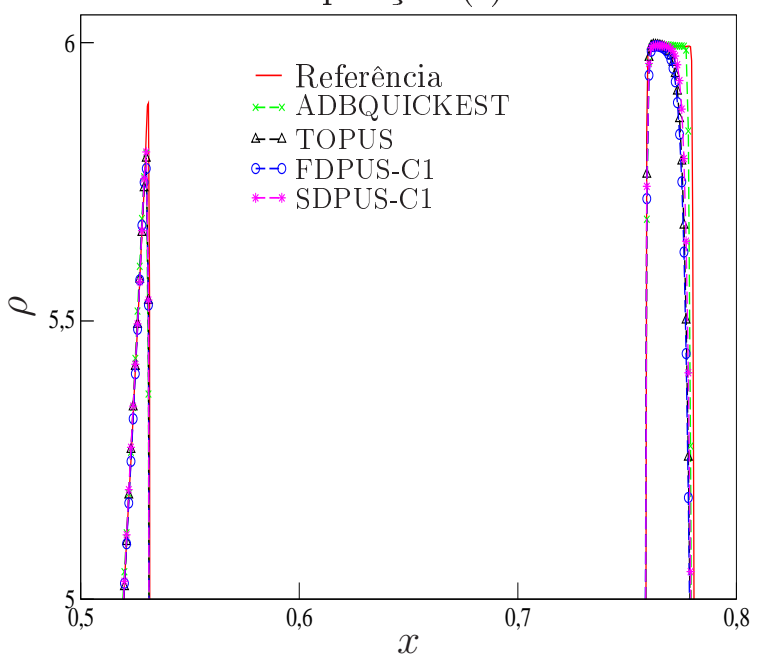

Figura 6.26: Ondas com fortes colisões. Comparação entre a solução de referência e os esquemas ADBQUICKEST (a), TOPUS (b), FDPUS-C1 (c) e SDPUS-C1 (d); comparação das soluções numéricas (e); ampliação (f). Soluções numéricas para as equações de Euler (para a variável $\rho$ ), com as condições iniciais (6.29), para $t=0.19$. 
(a)

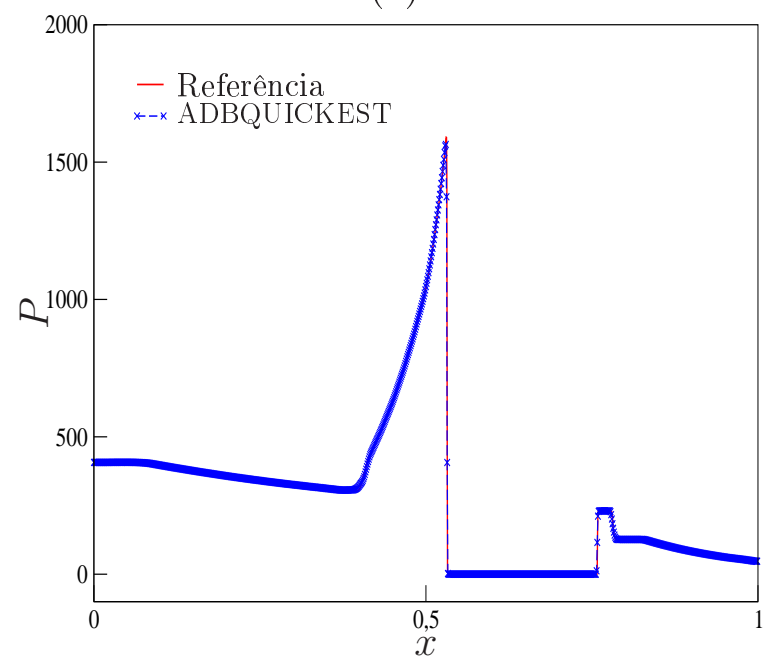

(c)

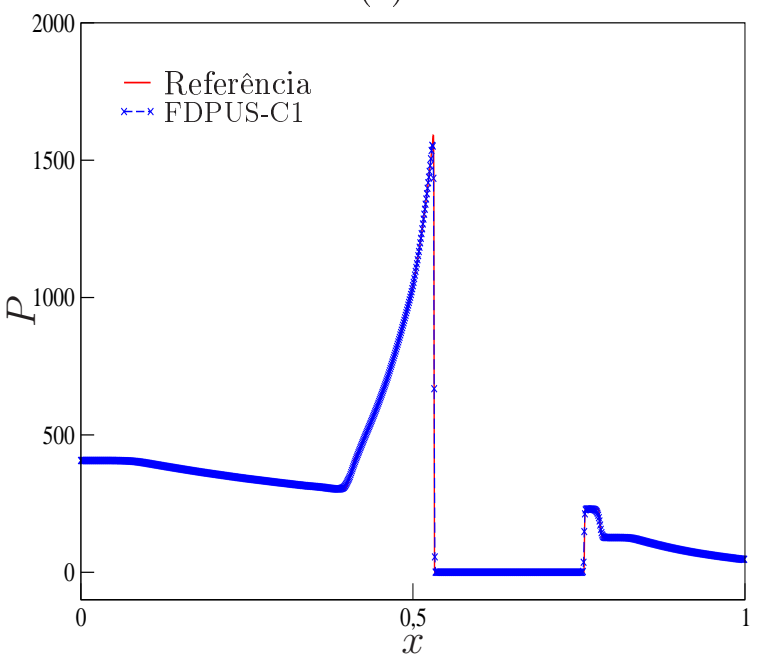

(e)

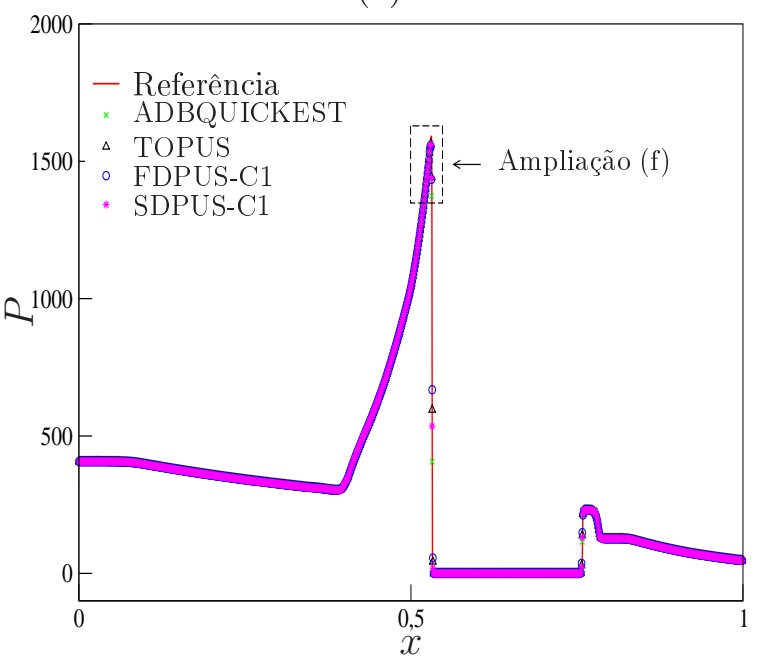

(b)

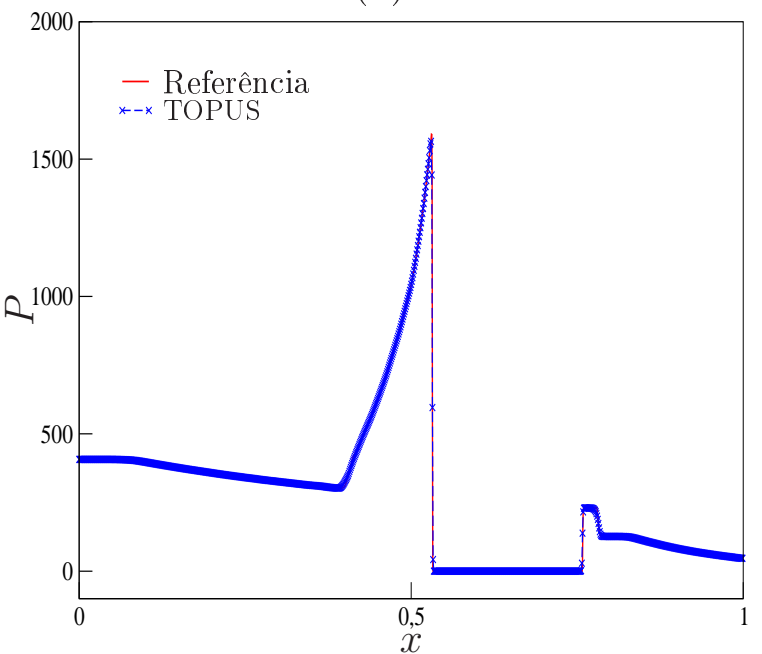

(d)

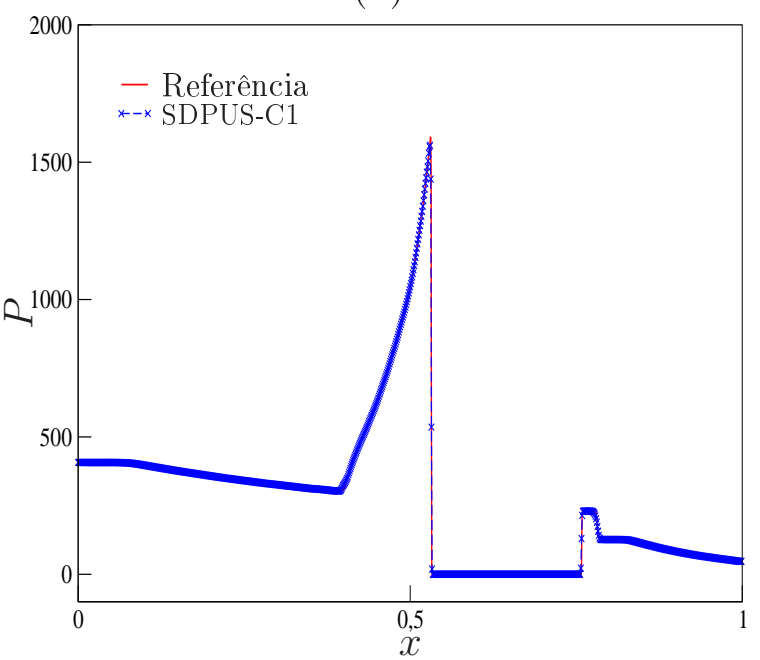

Ampliação (f)

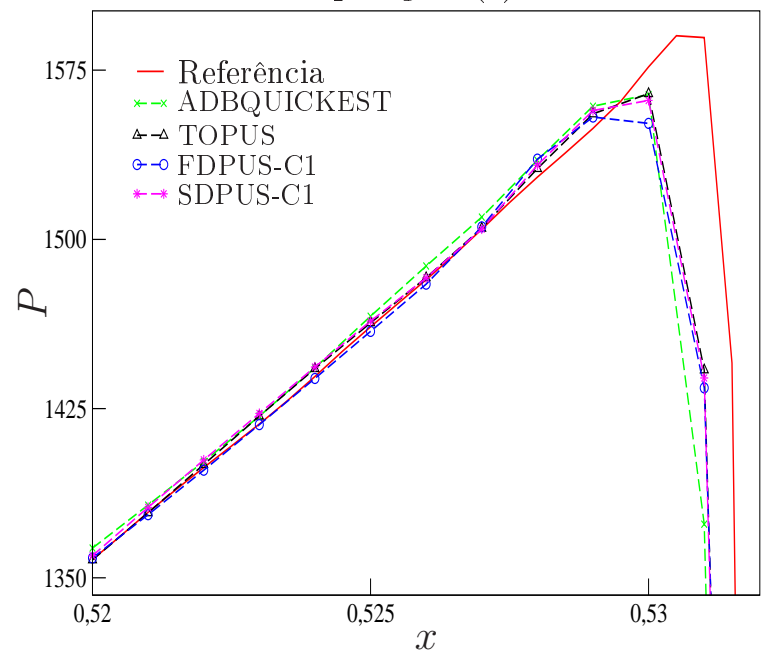

Figura 6.27: Ondas com fortes colisões. Comparação entre a solução de referência e os esquemas ADBQUICKEST (a), TOPUS (b), FDPUS-C1 (c) e SDPUS-C1 (d); comparação das soluções numéricas (e); ampliação (f). Soluções numéricas para as equações de Euler (para a variável $P$ ), com condições iniciais (6.29), para $t=0.19$. 
(a)

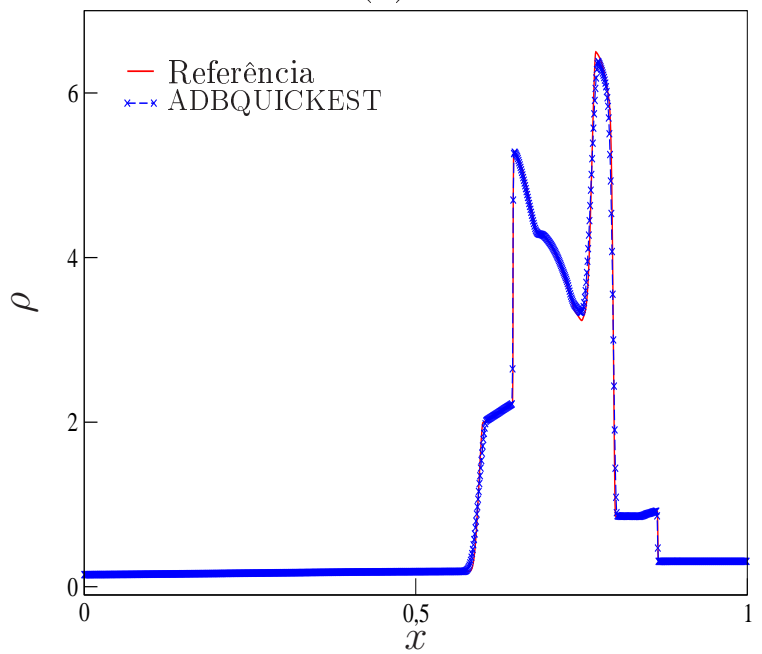

(c)

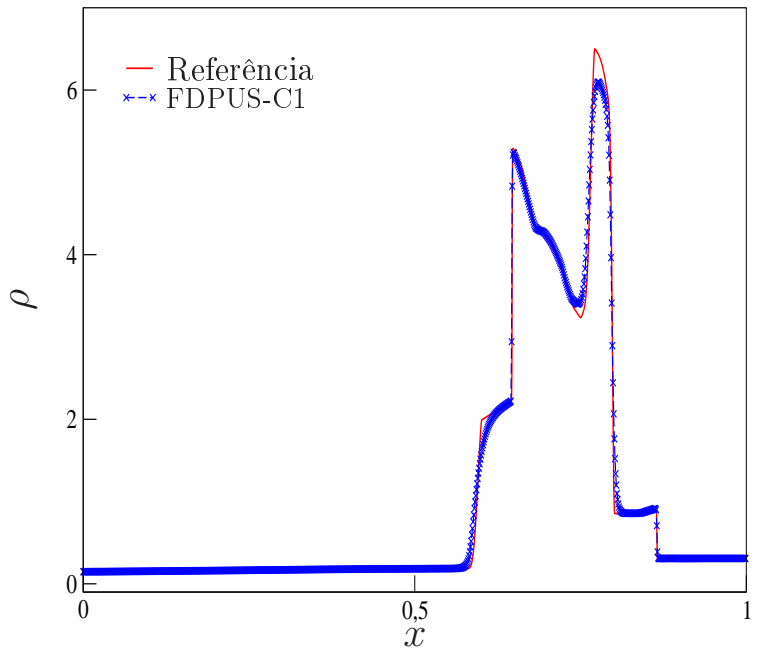

(e)

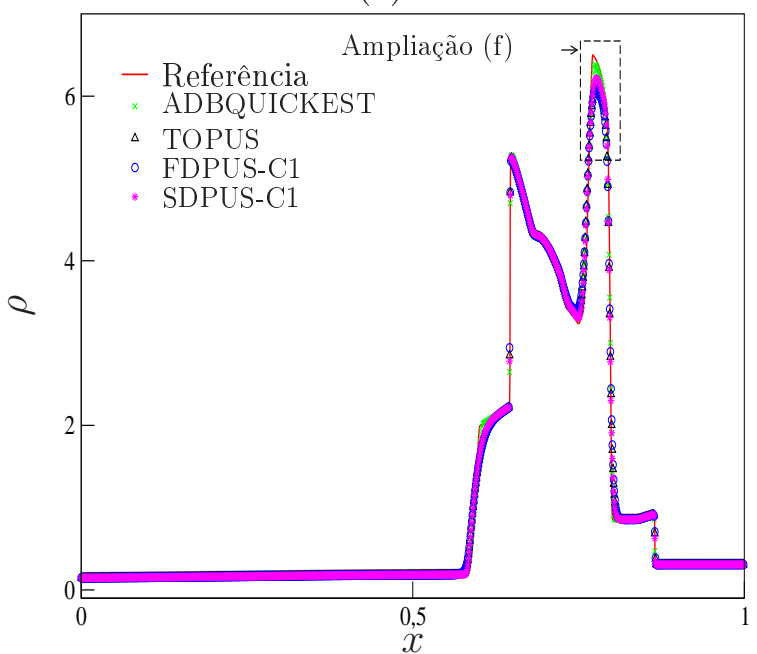

(b)

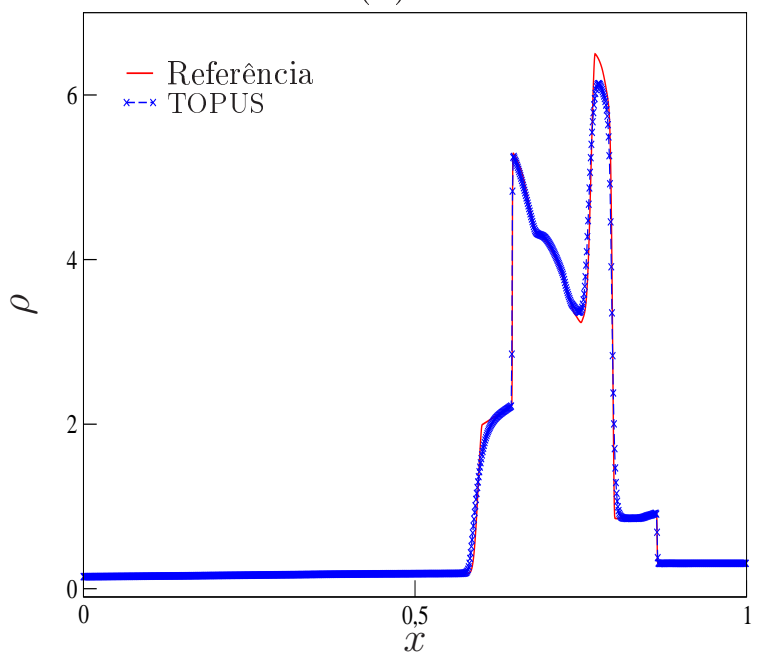

(d)

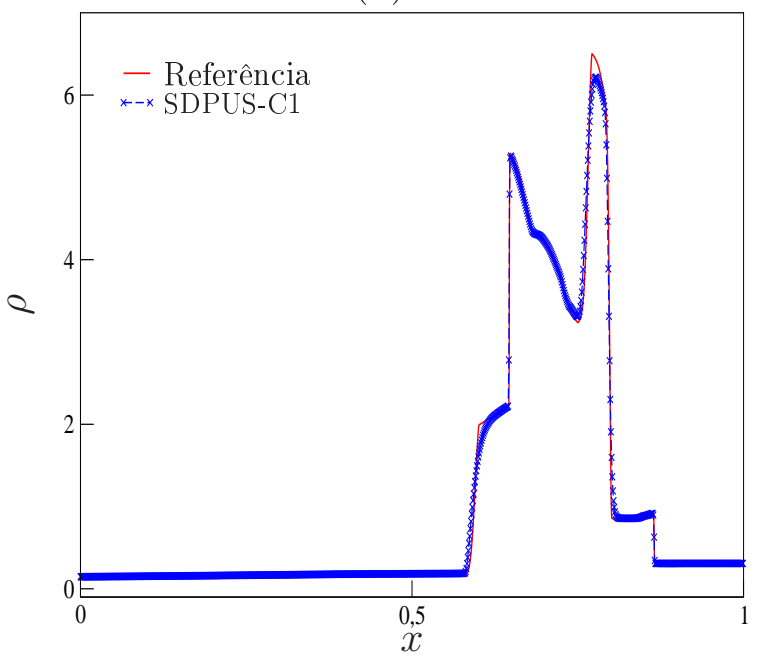

Ampliação (f)

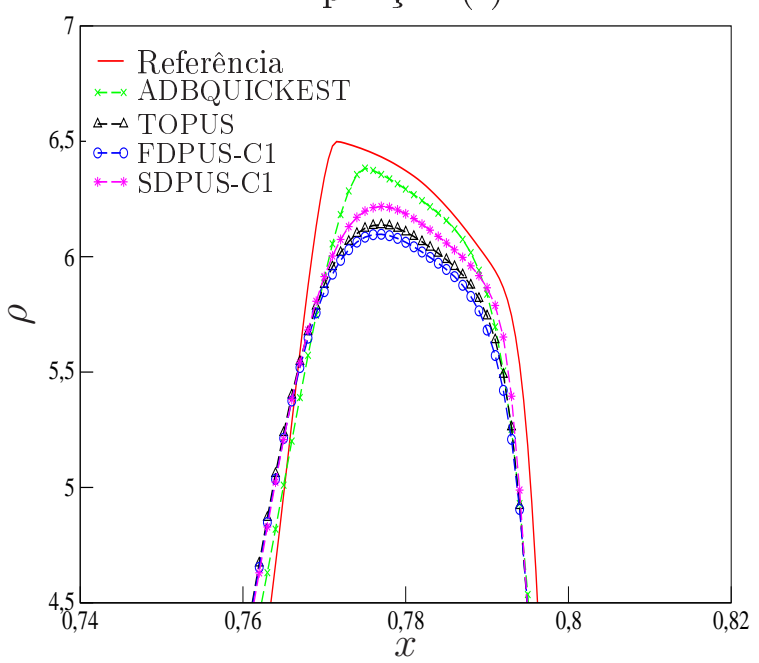

Figura 6.28: Ondas com fortes colisões. Comparação entre a solução de referência e os resultados esquemas ADBQUICKEST (a), TOPUS (b), FDPUS-C1 (c) e SDPUS-C1 (d); comparação das soluções numéricas (e); ampliação (f). Soluções para as equações de Euler (para a variável $\rho$ ), com as condições iniciais (6.29), para $t=0.38$. 
(a)

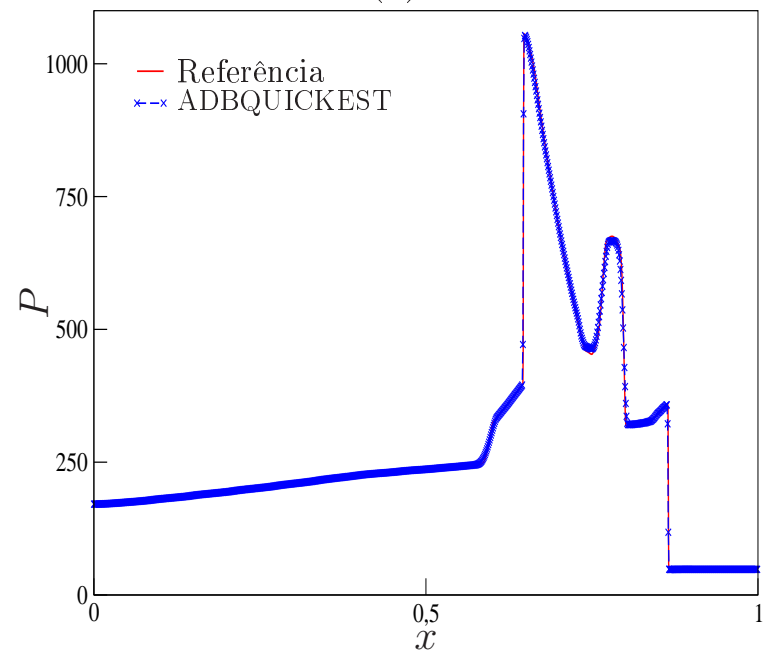

(c)

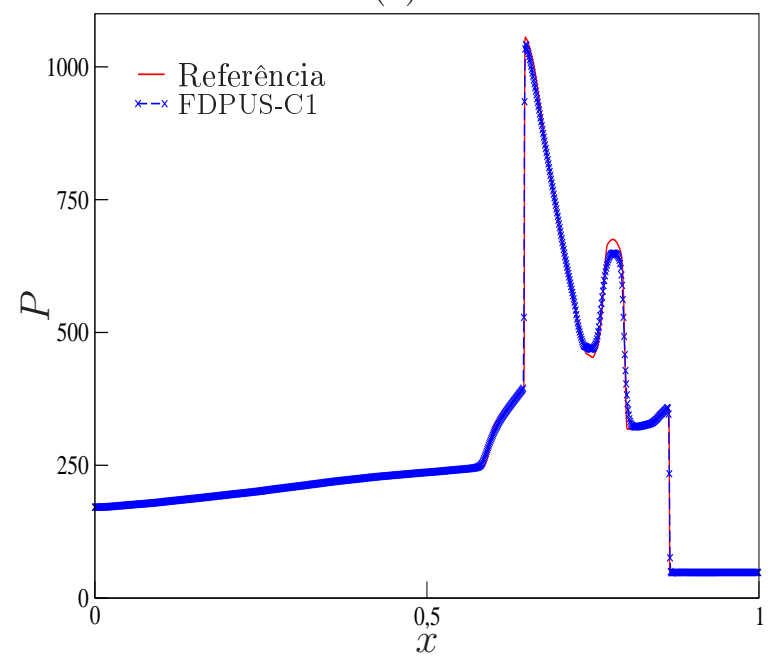

(e)

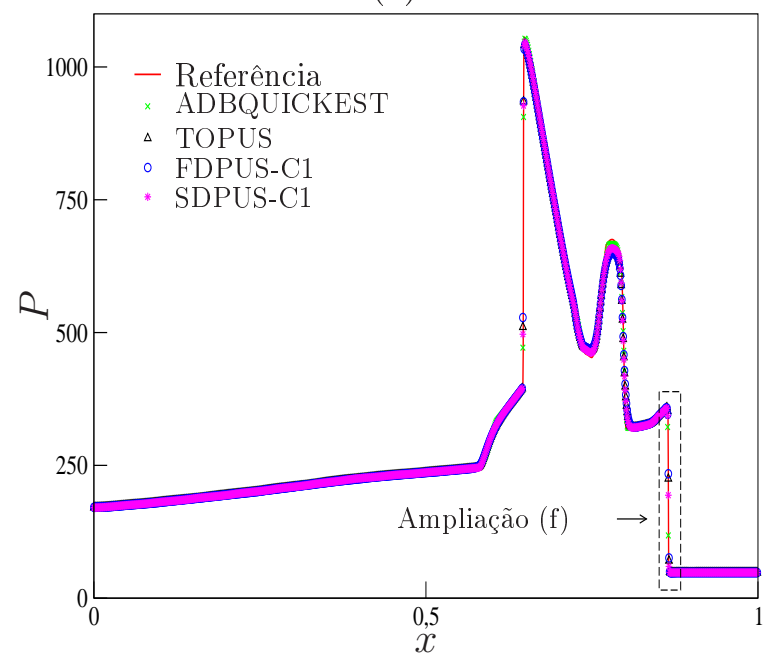

(b)

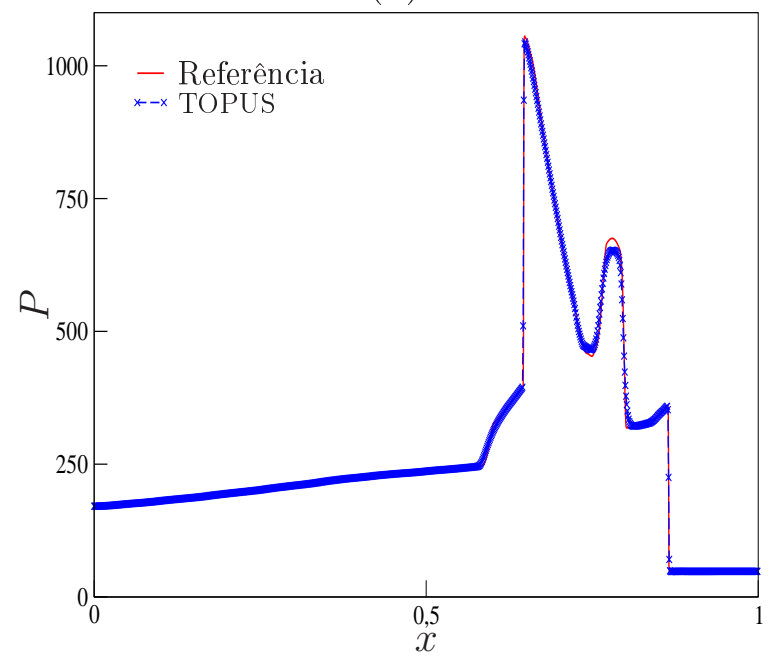

(d)

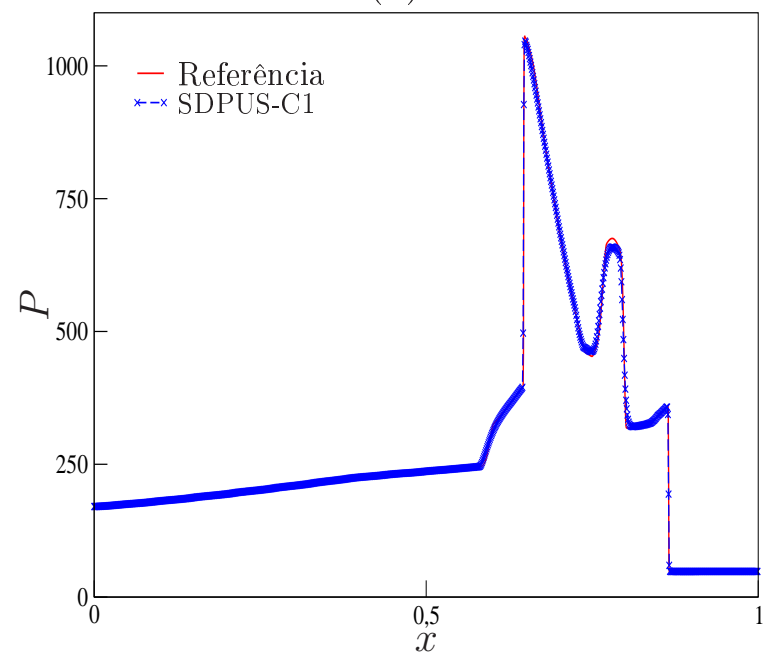

Ampliação (f)

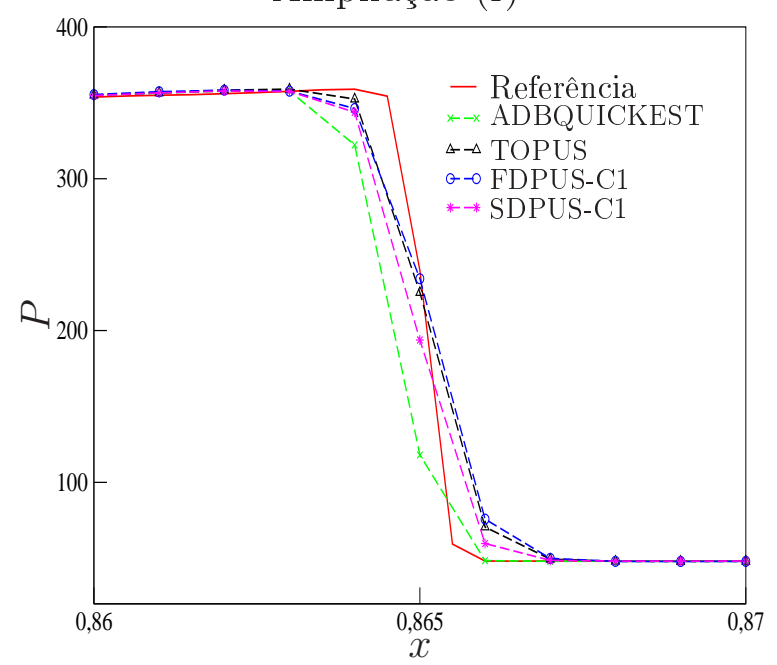

Figura 6.29: Ondas com fortes colisões. Comparação entre a solução de referência e os esquemas ADBQUICKEST (a), TOPUS (b), FDPUS-C1 (c) e SDPUS-C1 (d); comparação das soluções numéricas (e); ampliação (f). Soluções numéricas para as equações de Euler (para a variável $P$ ), com condições iniciais (6.29), para $t=0.38$. 


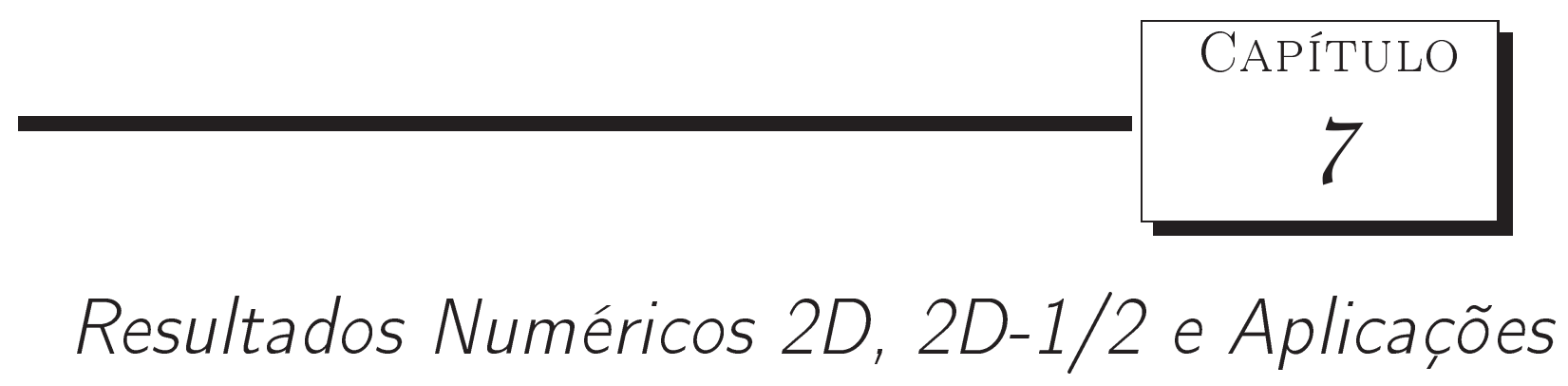

Este capítulo objetiva a investigação do desempenho dos esquemas FDPUS-C1 e SDPUS-C1 quando aplicados na resolução de problemas complexos bidimensionais. Para tanto, resolvem-se leis de conservação (águas rasas e Euler) e apresentam-se os resultados numéricos de escoamentos de fluidos incompressíveis com superfície livres móveis modelados pelas equações de Navier-Stokes. No caso de leis de conservação, os resultados numéricos são confrontados com soluções de referência. No caso das simulações usando-se as equações de Navier-Stokes, são apresentadas comparações qualitativas e quantitativas.

\subsection{Leis de Conservação 2D}

Nesta seção, apresentam-se os resultados numéricos obtidos para os sistemas hiperbólicos de águas rasas e Euler. As soluções numéricas são calculadas no pacote computacional CLAWPACK, equipado com os limitadores de fluxo dos esquemas ADBQUICKEST, FDPUS-C1 e SDPUS-C1. Para mais detalhes sobre esse software ver LeVeque [44] e apêndice D.

\subsubsection{Sistema Hiperbólico Águas Rasas}

O sistema hiperbólico águas rasas é formulado pela lei de conservação 2D (2.23), possui vetor das variáveis conservadas dado por (2.24) e funções fluxos dadas por (2.25) e (2.26). Seguem os resultados numéricos para um dos diversos casos que são formulados por esse sistema.

Caso 1- Problema radial dam-break. Neste caso o sistema hiperbólico de águas rasas é definido no domínio $[-2.5,2.5] \times[-2.5,2.5]$. Inicialmente a barragem circular que divide o domínio em duas partes (à de dentro e à de fora da barragem) é removida. Nesse instante, uma onda de choque translada radialmente, enquanto uma onda de rarefação move-se para o interior. Esse problema é conhecido na literatura por radial-dam e é ilustrado na Figura 7.1 
para os tempos $t=0$ (antes do rompimento da barragem) e $t=0.25$ (após o rompimento da barragem), em que inicialmente considera-se $H=2$ a profundidade dentro da barragem e $h=1$ fora da barragem. Esse problema é similar ao problema dam-break unidimensional considerado na seção 6.4 .
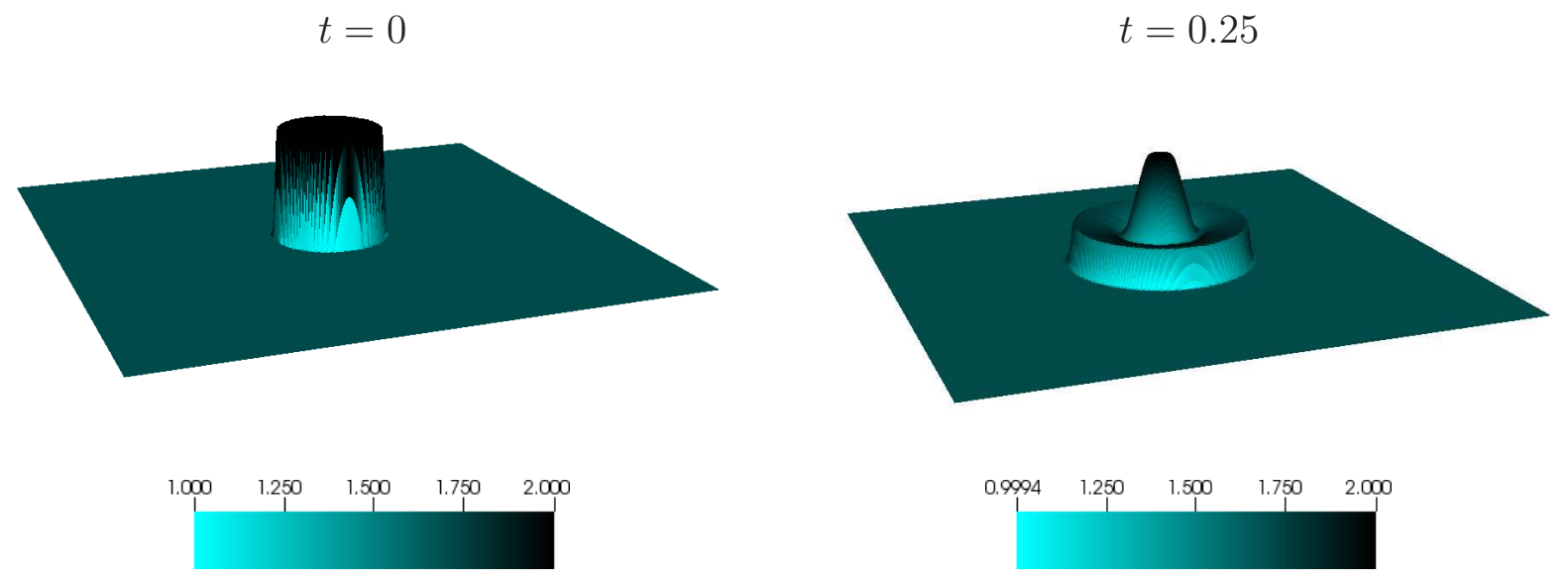

Figura 7.1: Problema radial dam-break. Comportamento da onda $H$ antes $(t=0)$ e após $(t=$ $0.25)$ o rompimento da barragem circular.

Para simulação são considerados os mesmo dados iniciais empregados para ilustração desse problema, ou seja, $H=2$ e $h=1$. A solução de referência é calculada em uma malha com $250 \times 250$ células computacionais, pelo método de Godunov com termo de correção, com limitador de fluxo $\mathrm{MC}$ e $\operatorname{com} \theta=0.5$. As soluções numéricas são também geradas por esse mesmo método, porém utilizando-se os limitadores de fluxo dos esquemas ADBQUICKEST, FDPUS-C1 e SDPUS-C1 em um malha com $125 \times 125$ células computacionais e $\theta=0.8$.

Na Figura 7.2 são apresentados a solução de referência (Fig. 7.2-(a)), e os resultados numéricos obtidos com os esquemas ADBQUICKEST (Fig. 7.2-(b)), FDPUS-C1 (Fig. 7.2-(c)) e SDPUS-C1 (Fig. 7.2-(d)) para o contorno de $H$, no plano $x \perp y$ e no tempo $t=1.5$. Por essa figura, observa-se que os resultados numéricos estão em boa concordância com a solução de referência.

Para complementar a análise, considera-se a variação da profundidade em função da distância a partir da origem, ou seja, calcula-se $H$ na reta $y=0$ (para $x \in[0,2.5]$ ). A Figura 7.3 mostra esses dados, em que é apresentada uma comparação entre a solução de referência e os resultados numéricos gerados com os esquemas ADBQUICKEST (Fig. 7.3-(a)), FDPUS-C1 (Fig. 7.3-(b)) e SDPUS-C (Fig. 7.3-(c)). Também, nesta mesma figura, confrontam-se as soluções numéricas (Fig. 7.3-(d)). De forma geral constata-se, por essa figura, que as soluções numéricas (geradas pelos esquemas anteriormente citados) são similares e estão em boa concordância com a solução de referência.

Como ilustração, na Figura 7.4 apresentam-se as soluções calculadas para a altura em 3D, ou seja, considera-se o plano $x \perp y$, então o valor da altura, em cada ponto do plano, é definido 
(a)

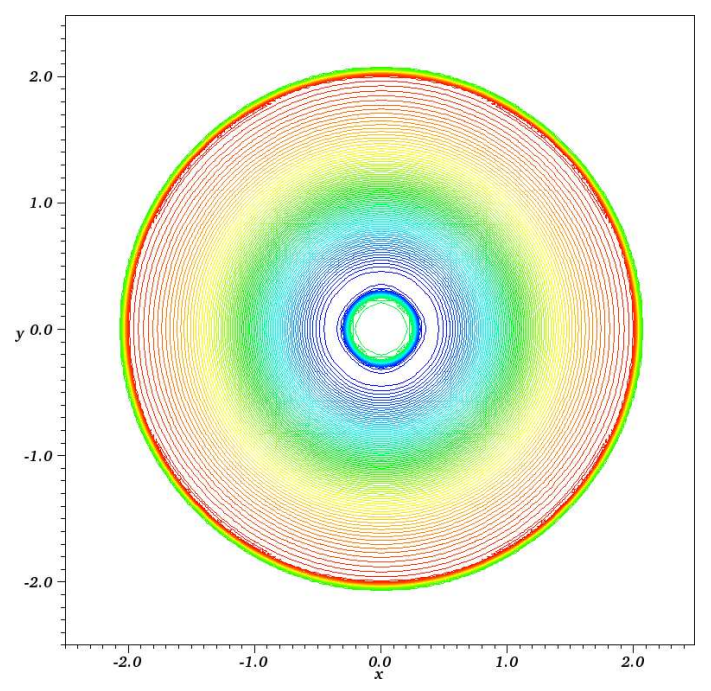

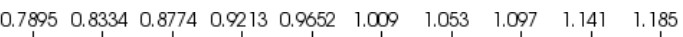

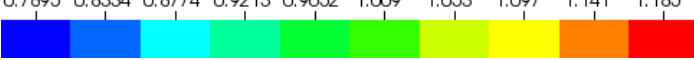

(c)

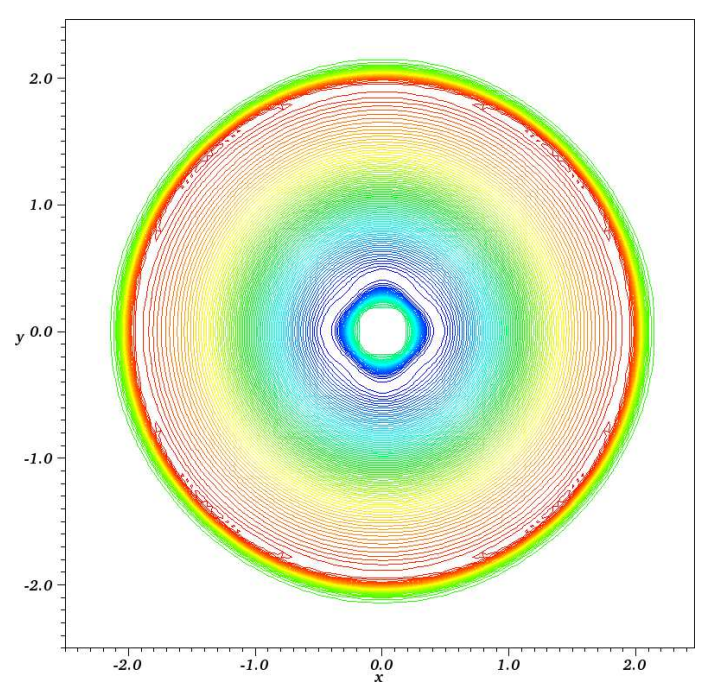

$\begin{array}{llllllllll}0.7929 & 0.8353 & 0.8778 & 0.9202 & 0.9627 & 1.005 & 1.048 & 1.090 & 1.132 & 1.175\end{array}$ (b)

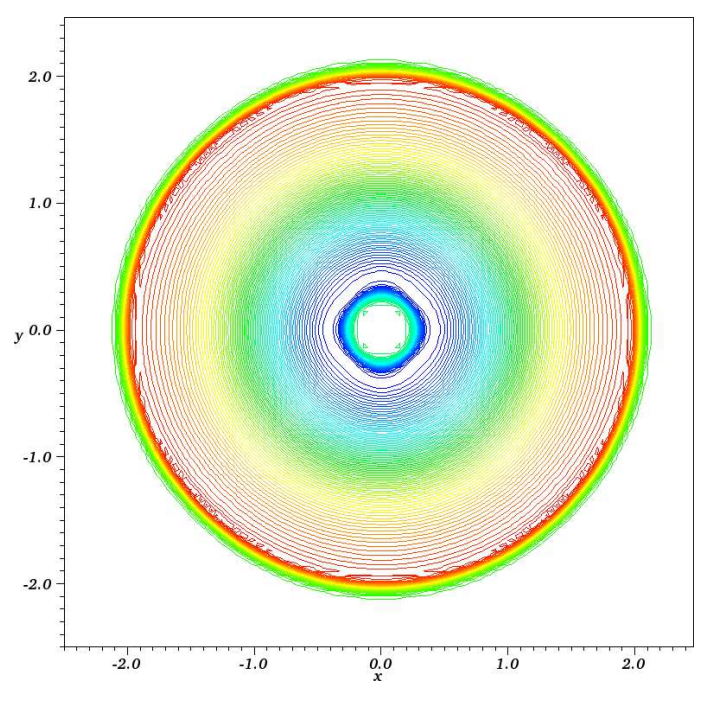

$\begin{array}{llllllllll}0.7924 & 0.8356 & 0.8789 & 0.9221 & 0.9653 & 1.009 & 1.052 & 1.095 & 1.138 & 1.181\end{array}$

(d)

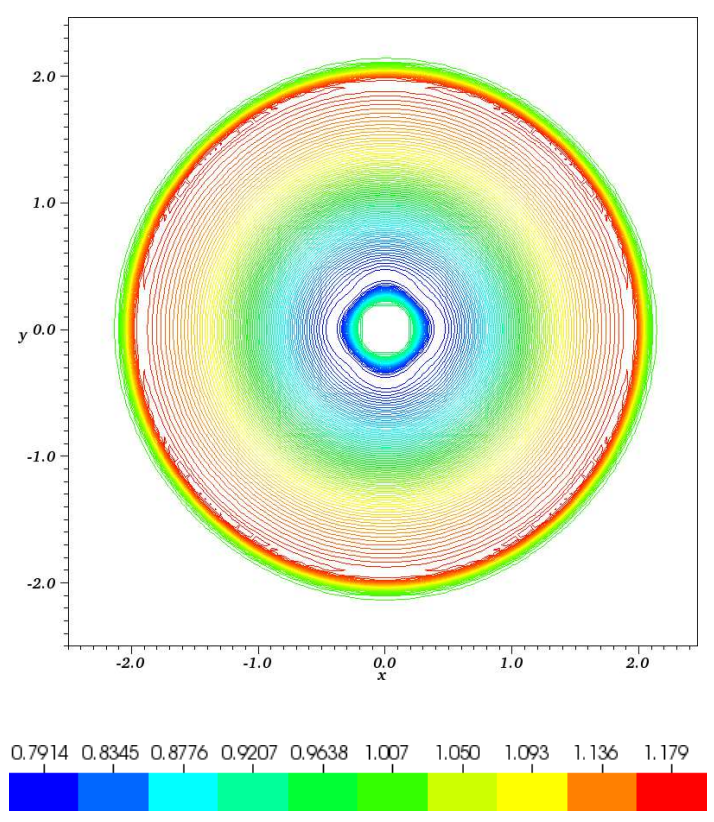

Figura 7.2: Problema radial dam-break. Solução de referência (a) e resultados numéricos dos esquemas ADBQUICKEST (b), FDPUS-C1 (c) e SDPUS-C1 (d) para o contorno de $H$, no plano $x \perp y$ e $t=1.5$. 
(a)

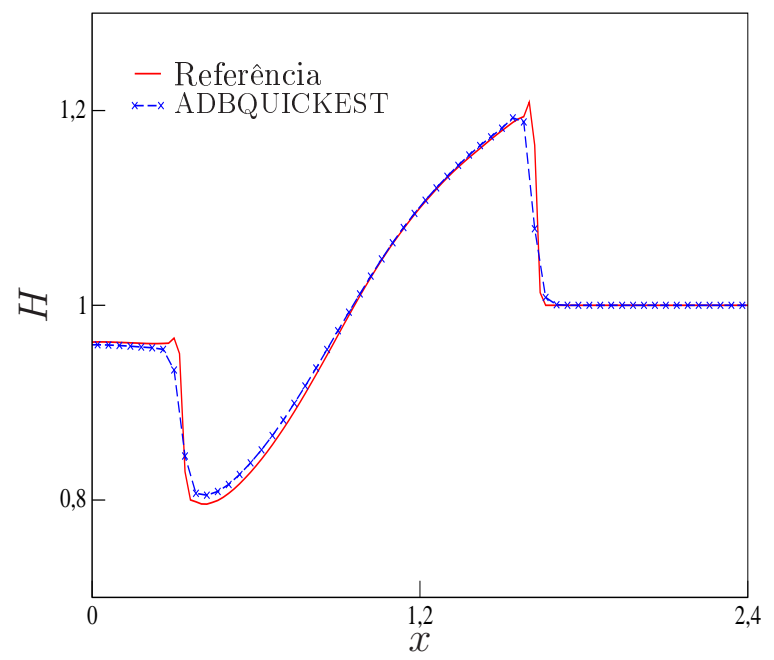

(c)

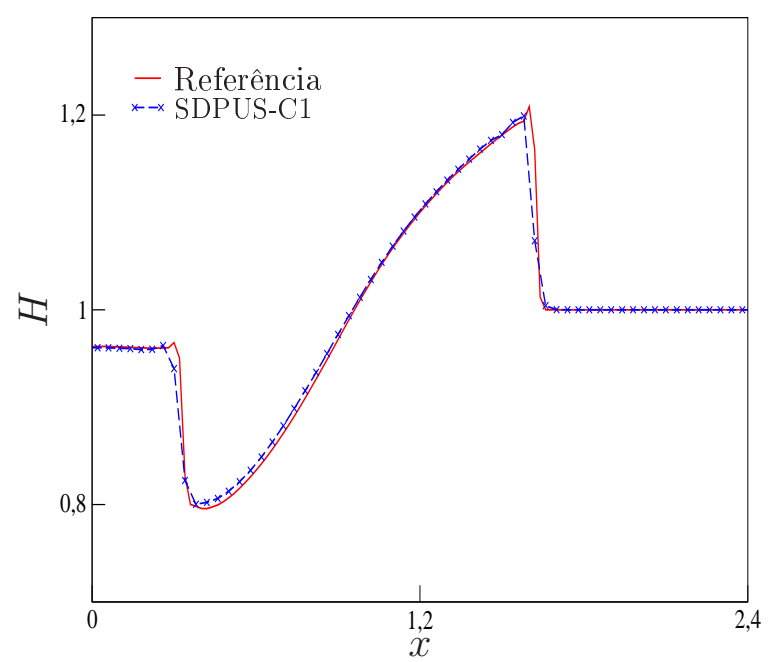

(b)

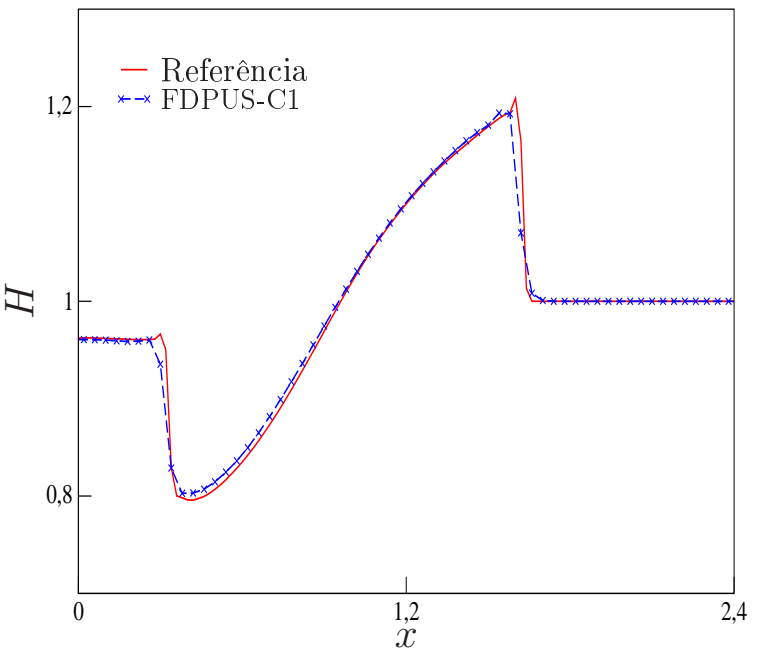

(d)

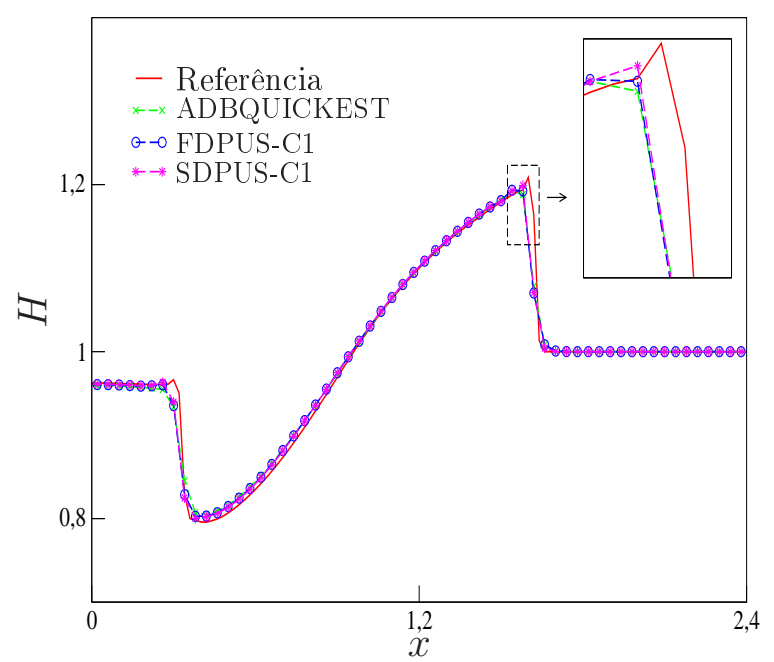

Figura 7.3: Problema radial dam-break. Comparação entre a solução de referência e os esquemas ADBQUICKEST (a), FDPUS-C1 (b) e SDPUS-C1 (c); comparação dos resultados numéricos (d). Resultados numéricos para o sistema de águas rasas, para a variação da profundidade $H$ em função da distância da origem $x(\operatorname{com} x \in[0,2.5])$. 
no eixo z. Nesta figura estão esboçados a solução de referência (Fig. 7.4-(a)) e os resultados numéricos obtidos com os esquemas ADBQUICKEST (Fig. 7.4-(b)), FDPUS-C1 (Fig. 7.4-(c)) e SDPUS-C1 (Fig. 7.4-(d)) e pode ser observado que os resultados numéricos estão em boa concordância com a solução de referência.

(a)
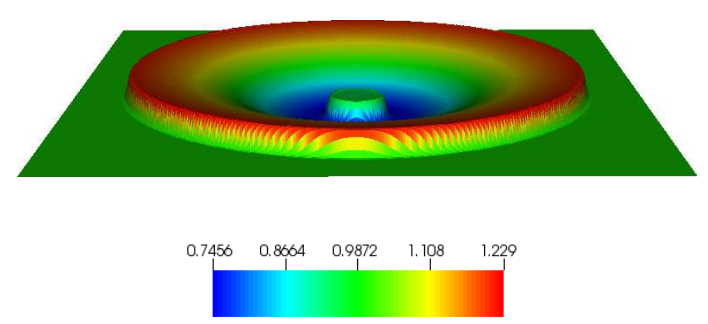

(c)

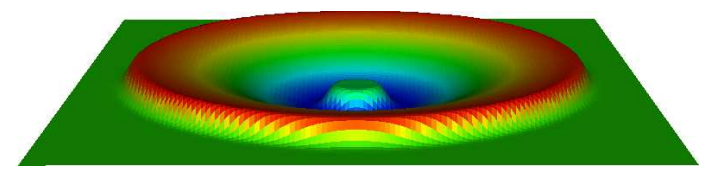

\begin{tabular}{|ccccc}
0.7505 & 0.8672 & 0.9839 & 1.101 & 1.217 \\
\hline & & & &
\end{tabular} (b)
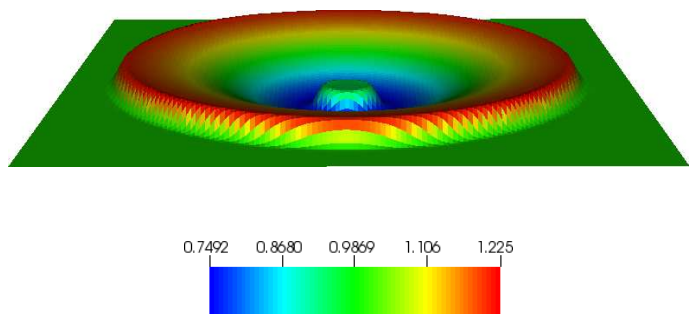

(d)
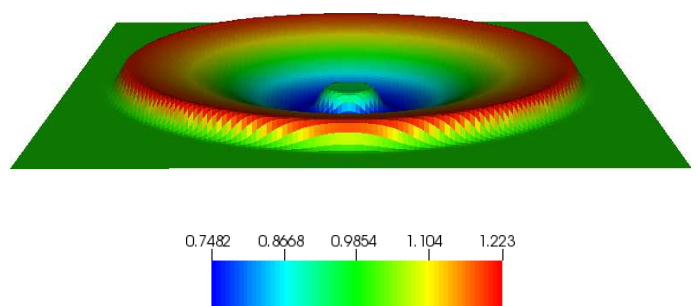

Figura 7.4: Problema radial dam-break. Solução de referência (a) e resultados numéricos dos esquemas ADBQUICKEST (b), FDPUS-C1 (c) e SDPUS-C1 (d), para o sistema de águas rasas com respeito a altura, em $t=1.5$.

\subsubsection{Sistema Hiperbólico Euler}

O sistema hiperbólico Euler é formulado pela lei de conservação 2D (2.23), possui vetor das variáveis conservadas dado por (2.27) e funções fluxo dadas por (2.28) e (2.29). Seguem os resultados numéricos para um dos diversos casos que são formulados por esse sistema.

Caso 1 - Interação choque-choque. Neste caso os esquemas são testados na captura de choques em uma situação multidimensional. O problema consiste na interação de dois choques oblíquos com dois choques normais, em que todas as descontinuidades se movem contrário a velocidade do pré-choque, como descrito na Figura 7.5. Tendo com referência essa figura, as equações de Euler são definidas no retângulo $[0,1] \times[0,1]$ e suplementadas com as condições 
iniciais dadas por

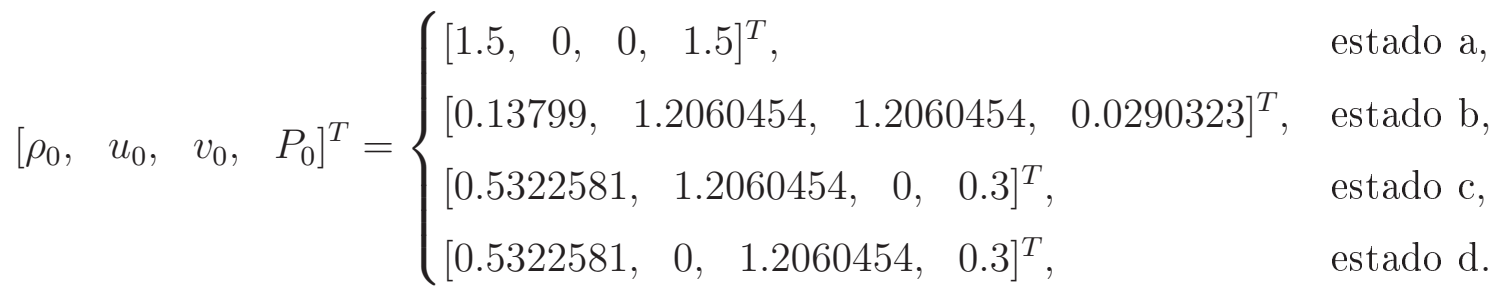

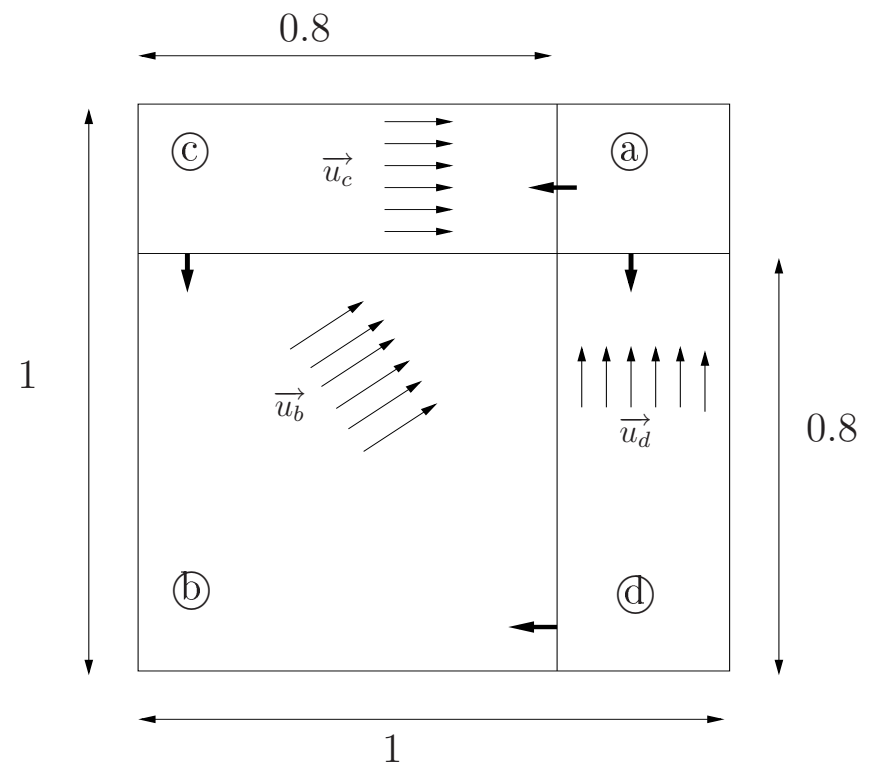

Figura 7.5: Interação choque-choque. Diagrama esquemático do problema formulado pelas equações de Euler com condições iniciais (7.1).

A solução de referência é calculada pelo método Godunov com termo de correção, aplicando-se o limitador de fluxo MC, em uma malha uniforme de $200 \times 200$ células computacionais, com $\theta=0.5$. Os resultados numéricos são gerados também por esse mesmo método em uma malha com $200 \times 200$ células computacionais e $\theta=0.8$.

Na Figura 7.6 apresentam-se a solução de referência (Fig. 7.6-(a)) e os resultados numéricos obtidos com os esquemas ADBQUICKEST (Fig. 7.6-(b)), FDPUS-C1 (Fig. 7.6-(c)), SDPUS-C1 (Fig. 7.6-(d)), para o contorno da densidade, no plano $x \perp y$ e em $t=0.8$. Na Figura 7.7, os valores de $\rho$ são considerados sobre a reta $y=x$, e plotados em relação a $x$, no plano $x \perp \rho$. Nessa mesma figura, são apresentados a solução de referência e os resultados numéricos gerados pelos esquemas ADBQUICKEST (Fig. 7.7-(a)), FDPUS-C1 (Fig. 7.7-(b)), SDPUS-C1 (Fig. 7.7-(c)) e a comparação das soluções numéricas (Fig. 7.7-(d)).

Assim como feito para densidade, na Figura 7.8 apresenta-se a solução de referência (Fig. 7.8-(a)) e os resultados numéricos obtidos com os esquemas ADBQUICKEST (Fig. 7.8-(b)), FDPUS-C1 (Fig. 7.8-(c)), SDPUS-C1 (Fig. 7.8-(d)) para o contorno da pressão. Na Figura 7.9 , consideram-se os valores da pressão sobre a reta $y=x$ e comparam-se a solução de refe- 
rência com os resultados numéricos gerados pelos esquemas ADBQUICKEST (Fig. 7.9-(a)), FDPUS-C1 (Fig. 7.9-(b)), SDPUS-C1 (Fig. 7.9-(c)), além do confronto entre as soluções numéricas (Fig. 7.9-(d)).

De forma geral, pode ser observado por essas figuras que os resultados numéricos estão em boa concordância com a solução de referência e com os resultados obtidos por Ricchiuto et al. [55]. Vale salientar que o esquema SDPUS-C1 apresenta melhor desempenho em relação aos outros esquemas. 
(a)

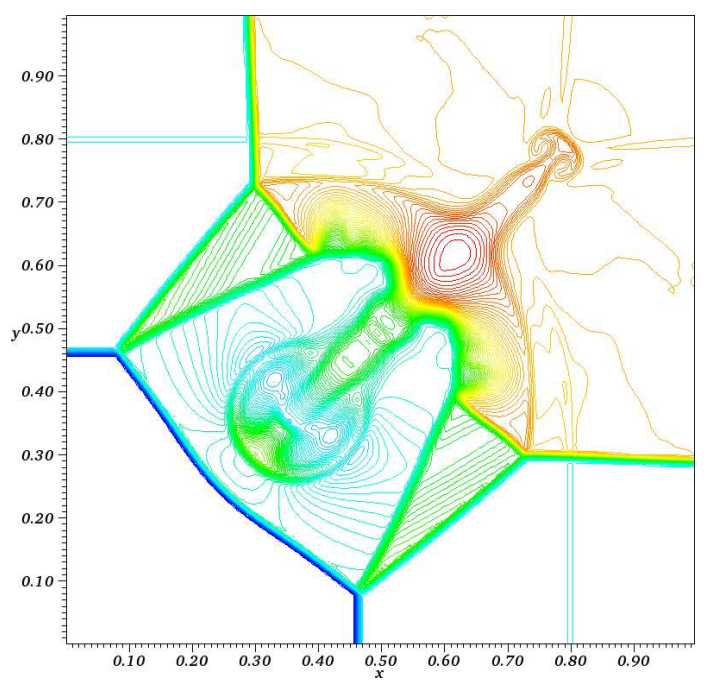

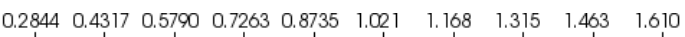

(c)

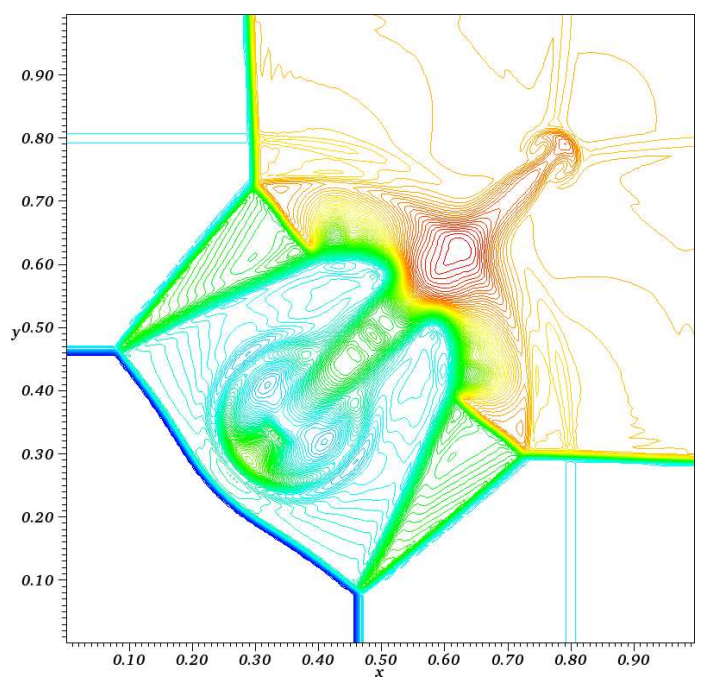

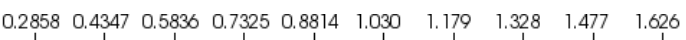

(b)

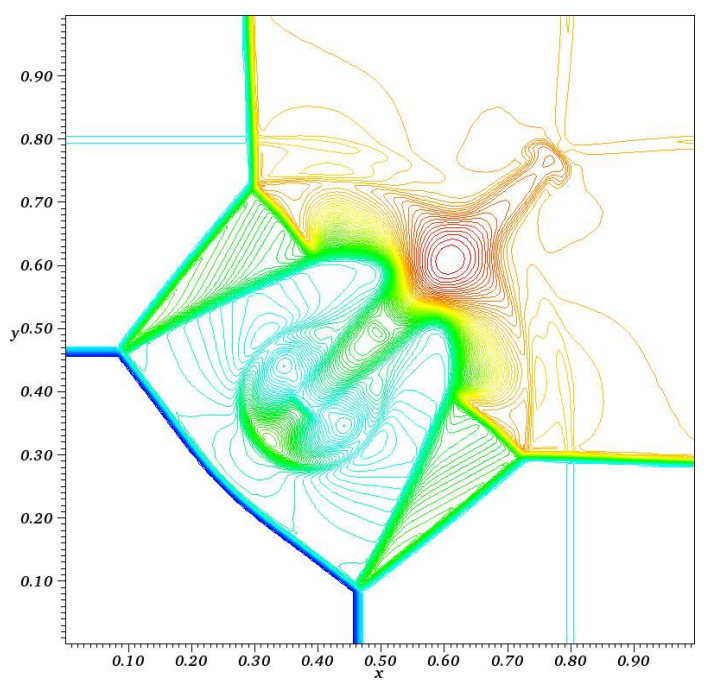

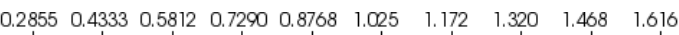

(d)

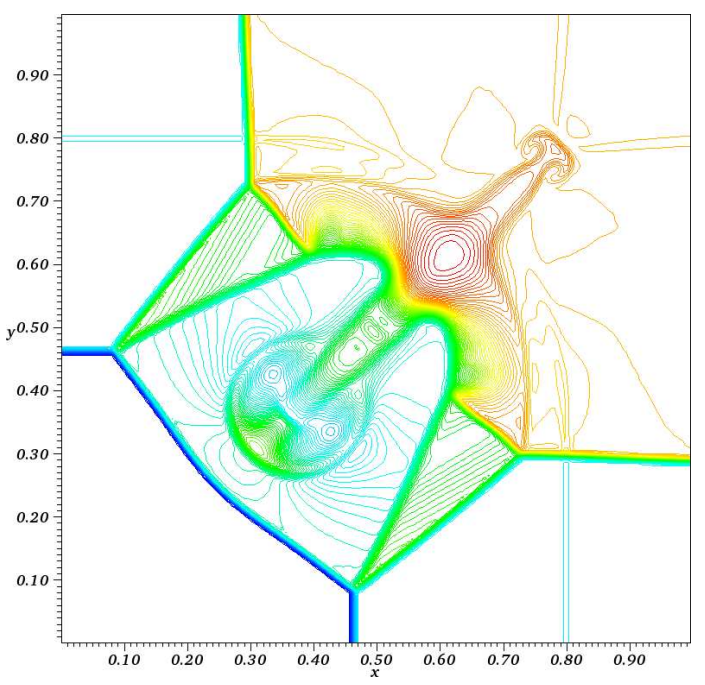

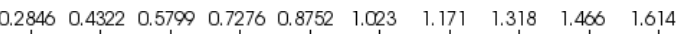

Figura 7.6: Interação choque-choque. Solução de referência (a) e resultados numéricos dos esquemas ADBQUICKEST (b), FDPUS-C1 (c) e SDPUS-C1 (d) para as equações de Euler, com condições iniciais (7.1), para o contorno de $\rho$ no plano $x \perp y$. 
(a)

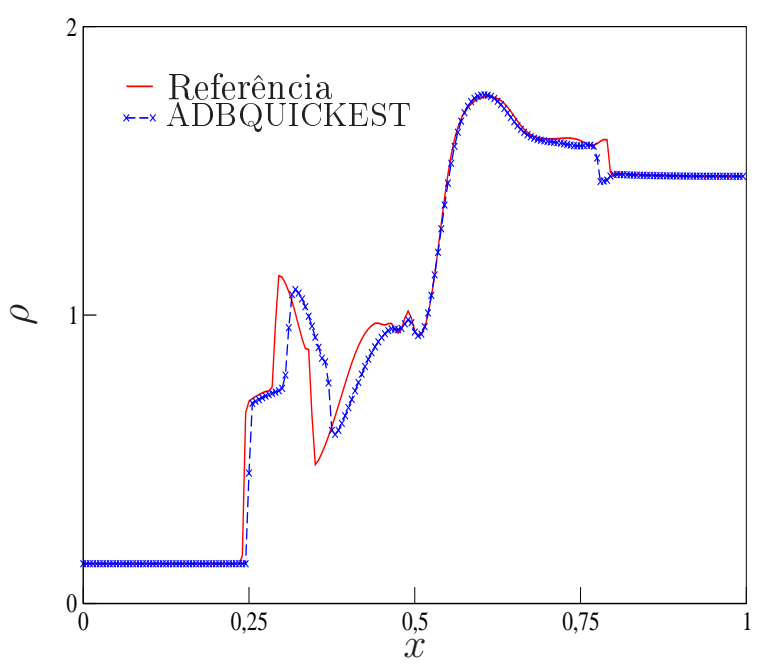

(c)

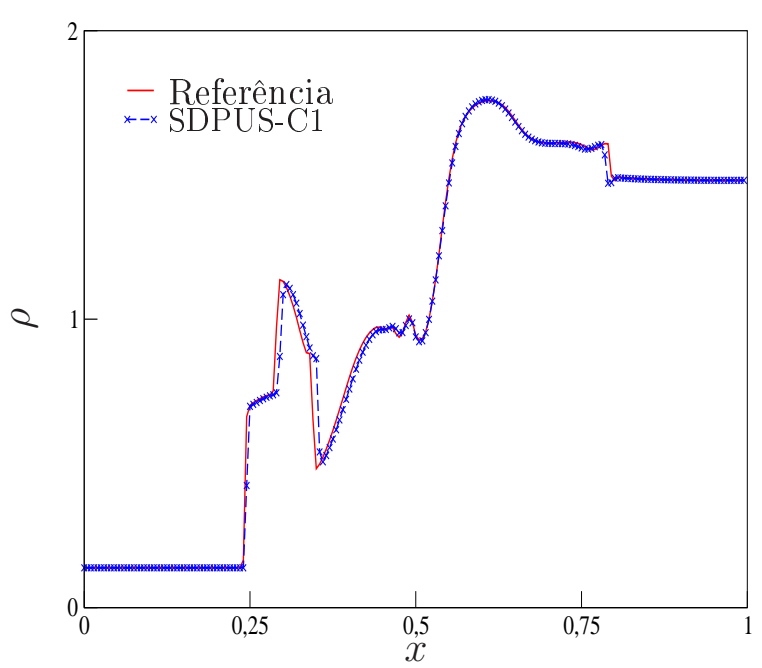

(b)

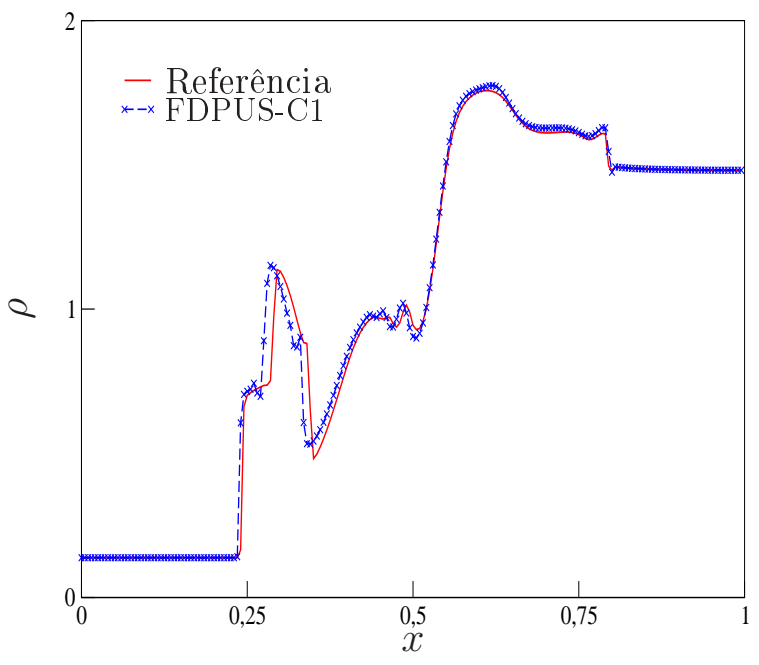

(d)

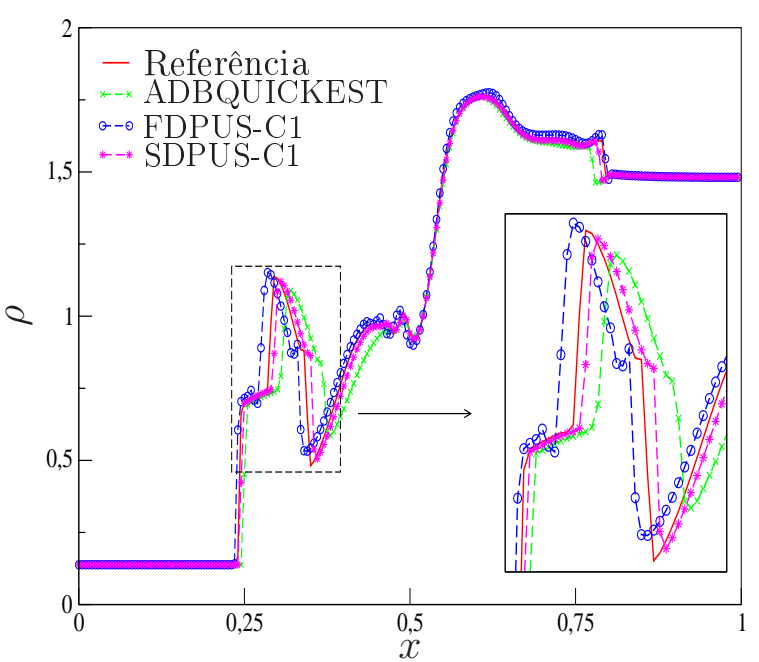

Figura 7.7: Interação choque-choque. Comparação entre a solução de referência e os esquemas ADBQUICKEST (a), FDPUS-C1 (b) e SDPUS-C1 (c); comparação dos resultados numéricos (d). Resultados para as equações de Euler, com condições iniciais (7.1), para $\rho$ sobre a reta $y=x$. 
(a)

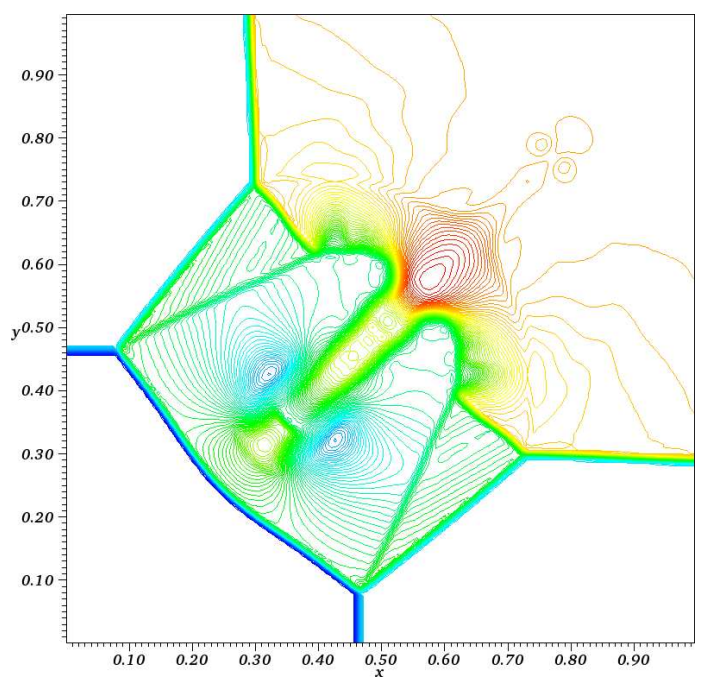

$\begin{array}{llllllllll}0.6424 & 1.014 & 1.386 & 1.758 & 2.130 & 2.502 & 2.874 & 3.246 & 3.618 & 3.990\end{array}$

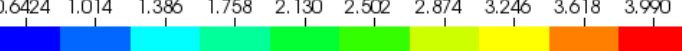

(c)

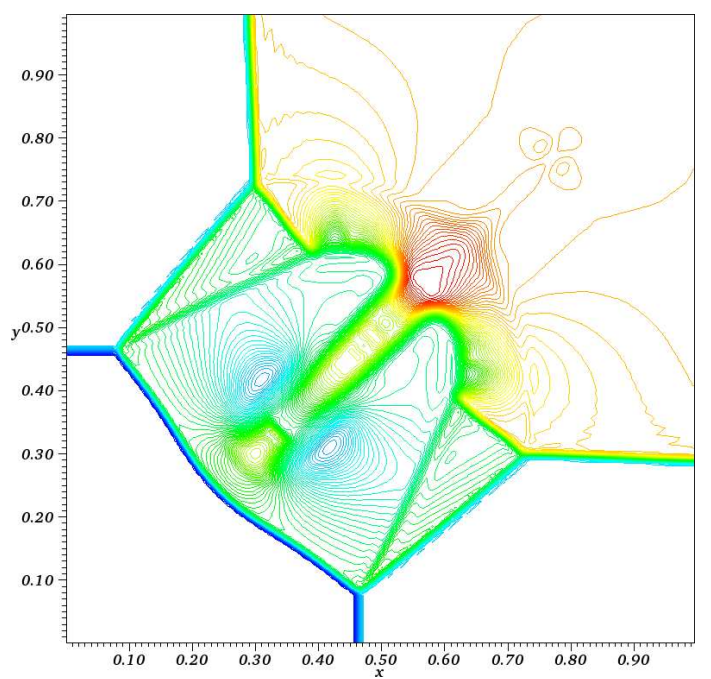

$\begin{array}{llllllllll}0.6434 & 1.016 & 1.389 & 1.763 & 2.136 & 2.509 & 2.882 & 3.255 & 3.628 & 4.001\end{array}$ (b)

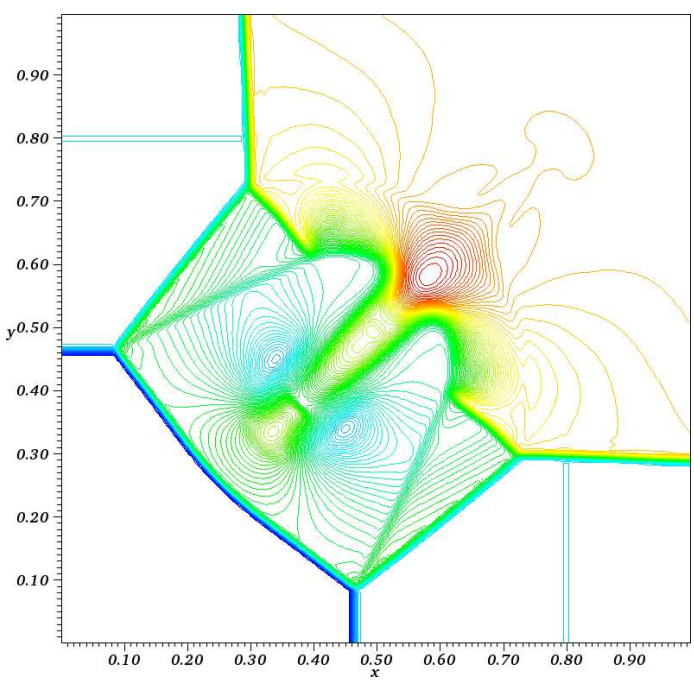

$\begin{array}{llllllllll}0.6473 & 1.022 & 1.397 & 1.772 & 2.147 & 2.522 & 2.897 & 3.272 & 3.647 & 4.022\end{array}$

(d)

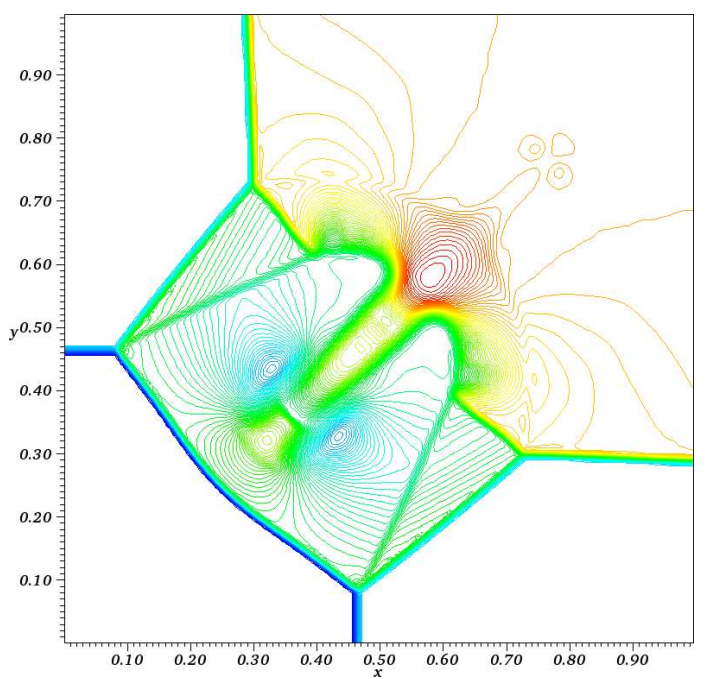

$\begin{array}{llllllllll}0.6431 & 1.016 & 1.389 & 1.761 & 2.134 & 2.507 & 2.880 & 3.252 & 3.625 & 3.998\end{array}$

Figura 7.8: Interação choque-choque. Solução de referência (a) e resultados numéricos dos esquemas ADBQUICKEST (b), FDPUS-C1 (c) e SDPUS-C1 (d) para as equações de Euler, com condições iniciais (7.1), para o contorno de $P$ no plano $x \perp y$. 
(a)

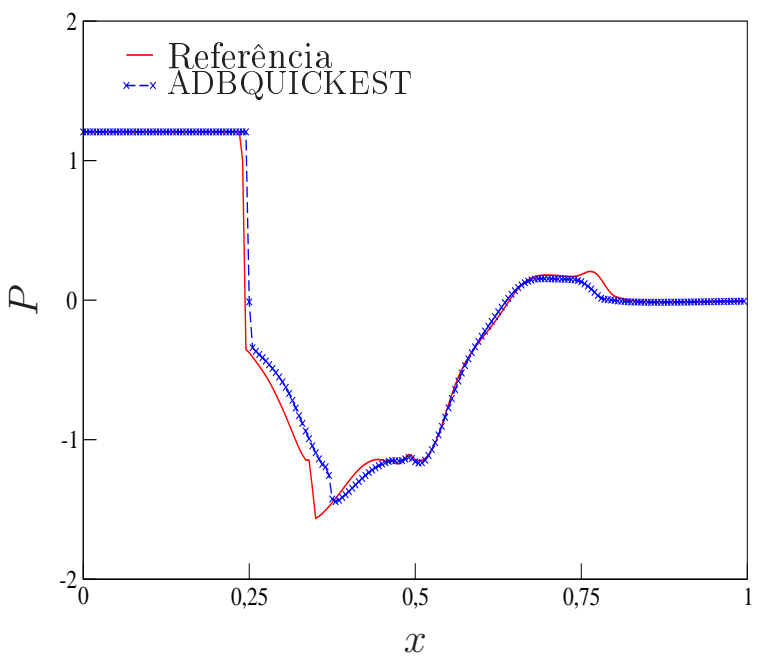

(c)

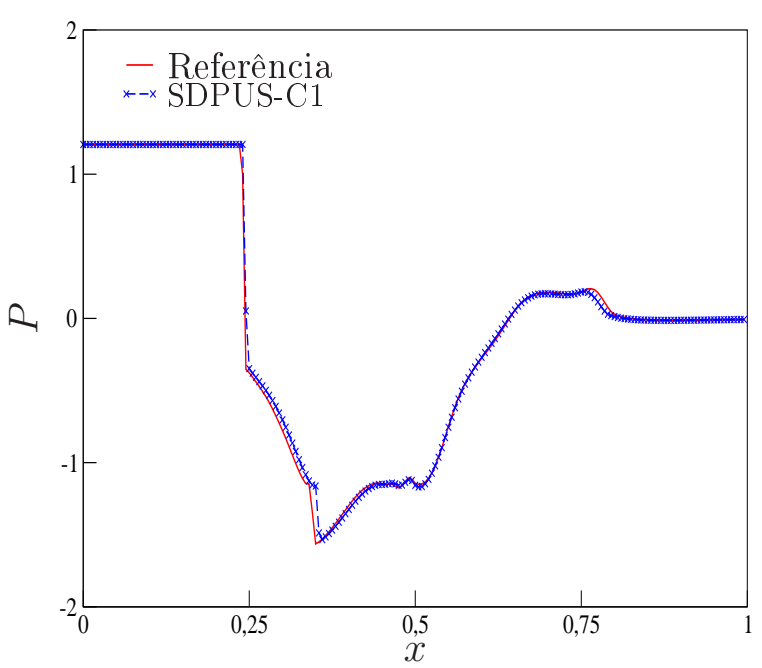

(b)

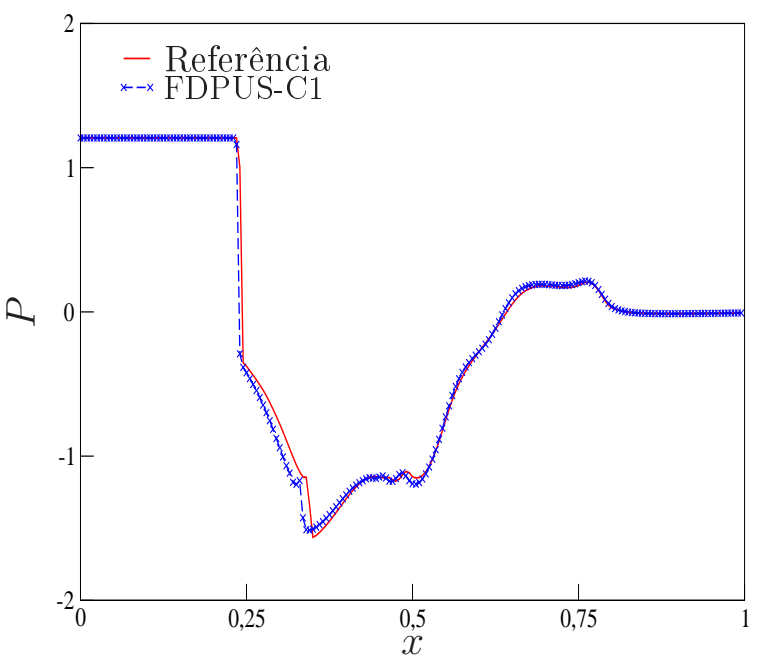

(d)

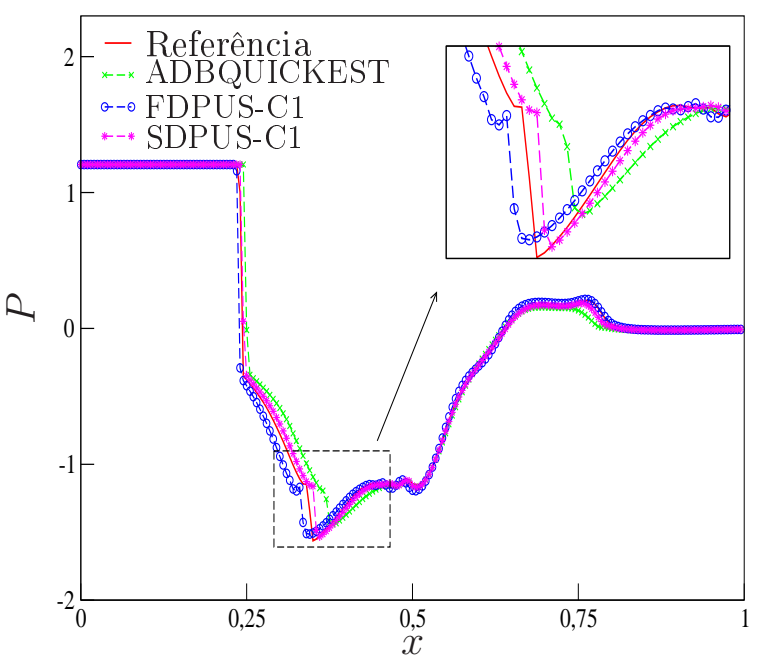

Figura 7.9: Interação choque-choque. Comparação entre a solução de referência e os esquemas ADBQUICKEST (a), FDPUS-C1 (b) e SDPUS-C1 (c); comparação dos resultados numéricos (d). Resultados numéricos para as equações de Euler, com condições inicial (7.1), para $P$ sobre a reta $y=x$. 


\subsection{Escoamentos de Fluidos Incompressíveis Laminares 2D}

Nesta seção são apresentados os resultados numéricos de simulações de escoamentos de fluidos incompressíveis laminares com superfícies livres móveis em 2D. Esses resultados são obtidos com os esquemas FDPUS-C1 e SDPUS-C1, implementados no ambiente de simulação Freeflow 2D de Castelo et al. [12].

\subsubsection{Colapso de uma Coluna fluido}

Os esquemas FDPUS-C1 e SDPUS-C1 são validados na simulação do problema do colapso de uma coluna de fluido. Esse tipo de escoamento com superfície livre é conhecido na literatura como broken dam e foi originalmente estudado por Martim e Moyce [47] que investigaram experimentalmente o espalhamento horizontal (posição frontal do fluido) e a taxa de decaimento (altura) da coluna de fluido com o tempo. Mais tarde, Koshizuka e Oka [37] repetiram o experimento de Martim e Moyce para validar um código semi-implícito de simulação. Recentemente, Colagrossi e Landrini[14] apresentaram dados experimentais, numéricos e teóricos para esse escoamento. Esse problema tem sido usado com frequência na literatura para a validação de métodos numéricos (ver, por exemplo, Kurokawa [40] e Queiroz [52]).

Em resumo, o problema consiste de uma coluna de fluido em equilíbrio hidrostático confinada entre paredes impermeáveis fixas (ver Figura 7.10) e sujeita à ação da gravidade. A fim de comparar os resultados numéricos com os dados dos autores acima mencionados, considera-se a condição de contorno free-slip aplicada nas paredes rígidas.

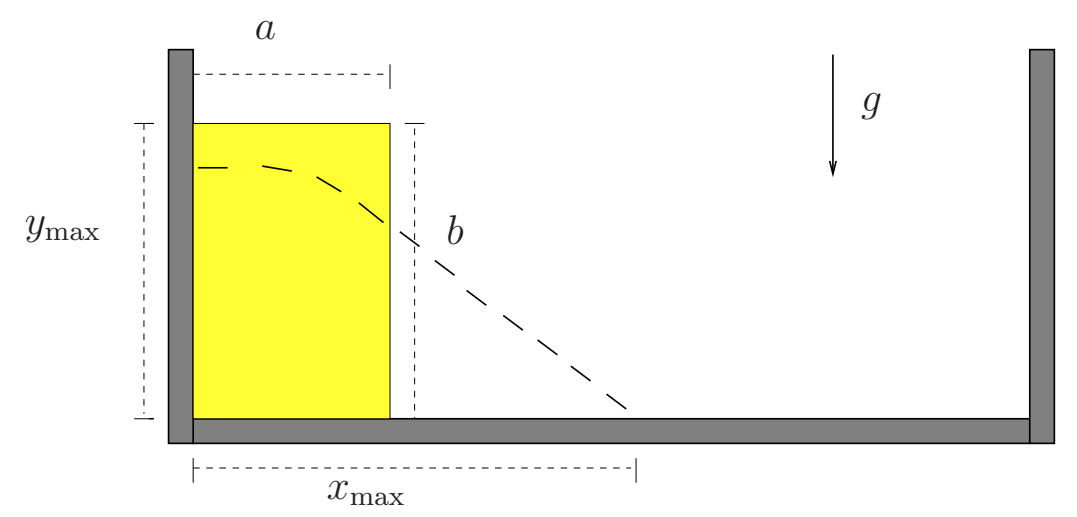

Figura 7.10: Colapso de fluido. Diagrama esquemático do problema Broken Dam.

Para esse problema são apresentados dois casos de comparação, a saber: i) entre os resultados numéricos para o espalhamento horizontal $\left(x_{\max }\right)$ e taxa de decaimento do fluido $\left(y_{\max }\right)$ , em vários tempos, e os dados de Martim e Moyce e Koshizuka e Oka; e ii) entre os resultados numéricos (para espalhamento horizontal do fluido, em vários tempos) e os dados numéricos/experimental/teórico de Colagrossi e Landrini. 
Caso 1 - Colapso de fluido (Martim e Moyce e Koshizuka e Oka). Nesse caso, a coluna de fluido é um retângulo com dimensões $a=0.05 m$ e $b=0.1 m$ (ver Fig. 7.10). Para a simulação, são utilizadas as malhas $150 \times 75,300 \times 150$ e $600 \times 300$ e os demais dados:

- Dimensão do domínio: $0.3 m \times 0.15 m$;

- Constante gravitacional: $g=9.81 \mathrm{~m} / \mathrm{s}^{2}$;

- Escala de comprimento: $L=b=0.01 m$;

- Escala de velocidade: $V_{0}=\sqrt{g \cdot L}=0.99045 \mathrm{~m} / \mathrm{s}$;

- Coeficiente de viscosidade cinemática: $\nu=10^{-6} \mathrm{~m}^{2} / \mathrm{s}$;

- Número de Reynolds: $R e=\frac{V_{0} \cdot L}{\nu}=99045.44$;

- Número de Froud: $F r=\frac{V_{0}}{\sqrt{g \cdot L}}=1$.

A Figura 7.11 mostra a comparação dos dados experimentais de Martim e Moyce [47] e Koshizuka e Oka [37] com os resultados numéricos gerados com os esquemas FDPUS-C1 (Fig. 7.11-(a)) e SDPUS-C1 (Fig. 7.11-(b)) para a posição frontal do escoamento do fluido $\left(x_{\max }\right) \mathrm{em}$ função do tempo. Na Figura 7.12, comparam-se os dados experimentais de Martim e Moyce [47], para o decaimento do fluido $\left(y_{\max }\right)$ em função do tempo, com os resultados numéricos gerados com os esquemas FDPUS-C1 (Fig. 7.12-(a)) e SDPUS-C1 (Fig. 7.12-(b)), nas três malhas. Em ambas figuras, Fig. 7.11 e Fig. 7.12, são apresentadas as soluções numéricas geradas nas três malhas, anteriormente mencionadas, em $t=0.2 \mathrm{~s}$. Por essas figuras, constata-se que os resultados numéricos estão em boa concordância com os dados experimentais.

Apenas como ilustração, nas Figuras 7.13, 7.14 e 7.15 são apresentados os resultados numéricos gerados pelo esquema FDPUS-C1, com respeito a evolução da superfície livre do fluido, para os campos de pressão e velocidade (nas direções $x$ e $y$ ), nos tempos $t=0.05 s, t=0.1 \mathrm{~s}$ e $t=0.2 s$, respectivamente. Os resultados numéricos obtidos com o esquema SDPUS-C1 são omitidos nesse texto pois apresentam muita similaridade com os resultados apresentados para o esquema FDPUS-C1.

Caso 2 - Colapso de fluido (Colagrossi e Landrini). Nesse caso, a coluna de fluido é um quadrado, com lados $a=b=0.057 m$ (ver Figura 7.10). Para simulação é utilizada uma malha com $1000 \times 200$ células computacionais e os seguintes dados: 
(a)

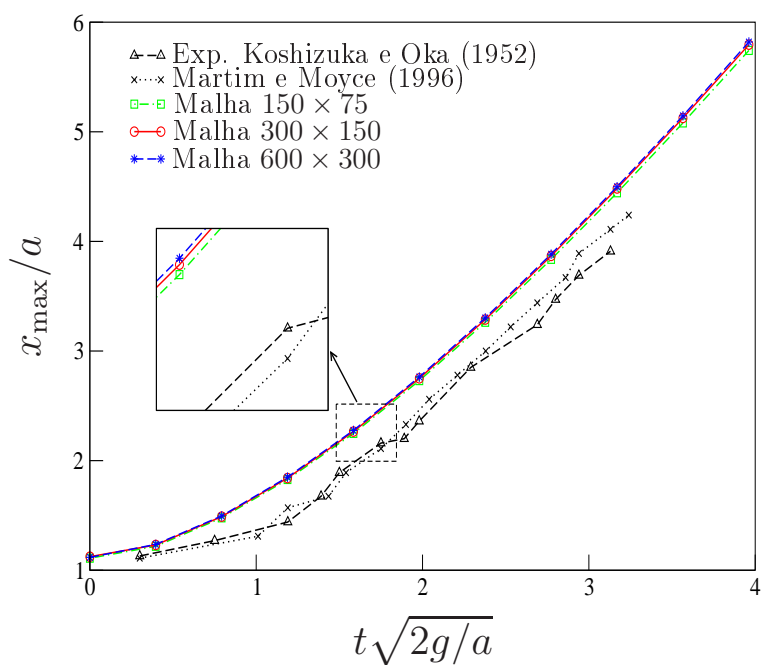

(b)

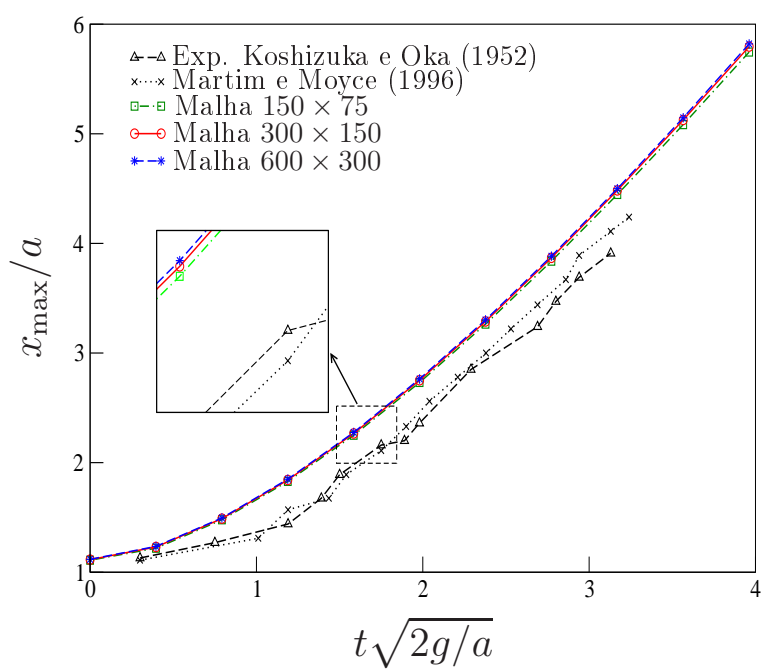

Figura 7.11: Colapso de fluido (Martim e Moyce e Koshizuka e Oka). Comparação entre os dados experimentais e os esquemas FDPUS-C1 (a) e SDPUS-C1 (b) para $x_{\max }$ em função do tempo.

(a)

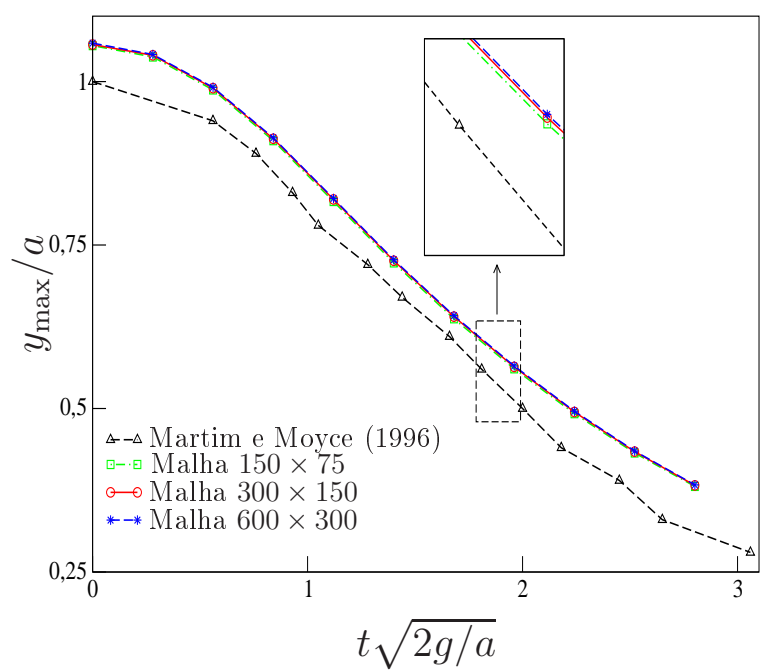

(b)

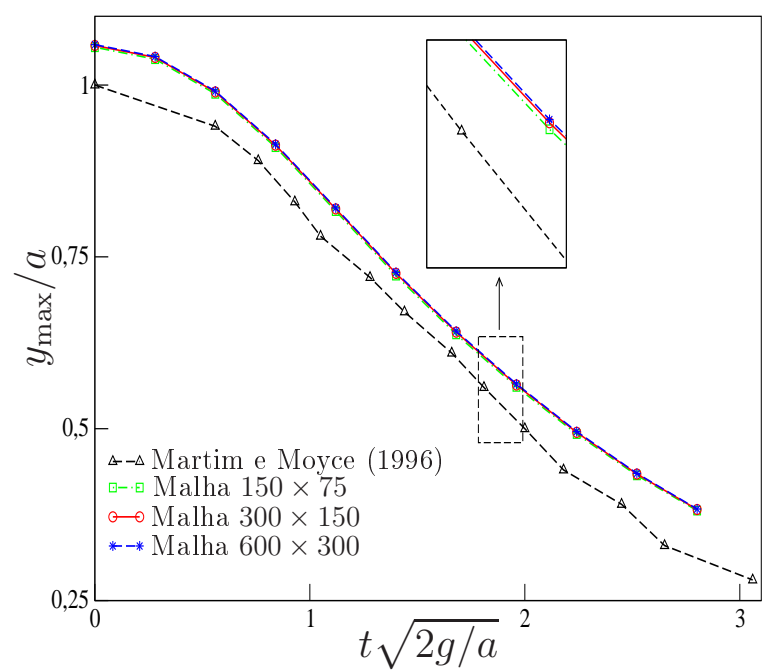

Figura 7.12: Colapso de fluido (Martim e Moyce). Comparação entre os dados experimentais e os esquemas FDPUS-C1 (a) e SDPUS-C1 (b) para $y_{\max }$ em função do tempo. 


\section{Campo de pressão}
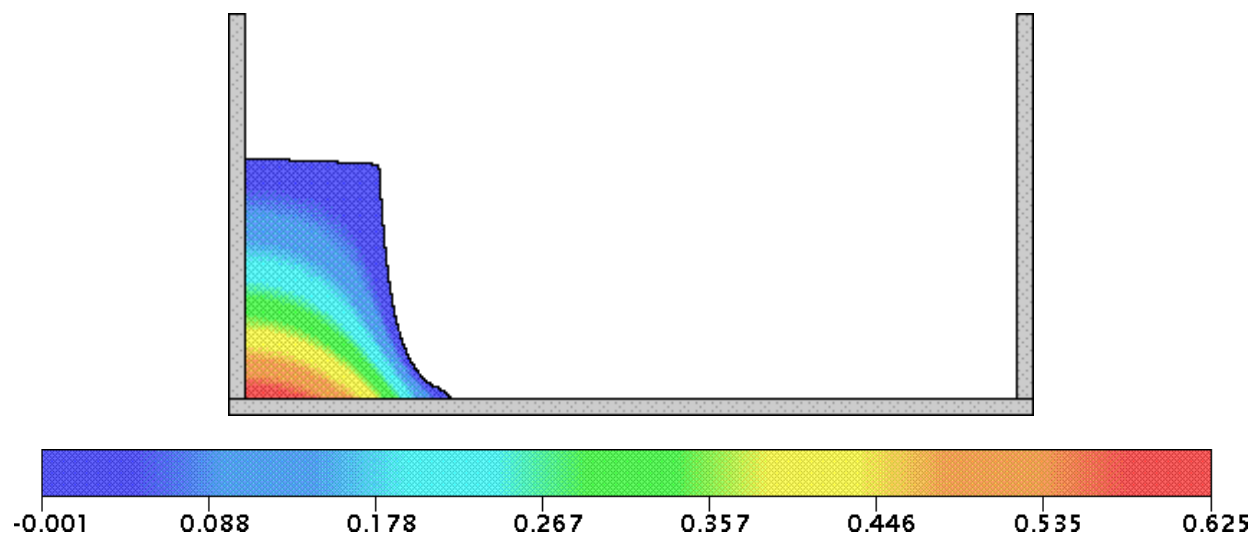

Campo de velocidade na direção $\mathrm{x}$
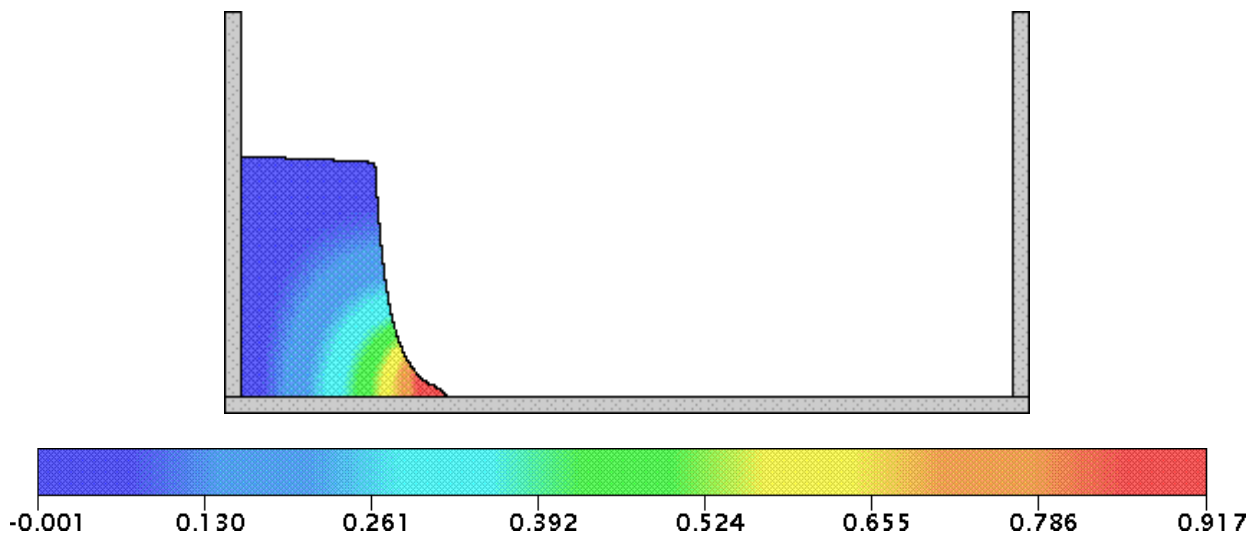

Campo de velocidade na direção y
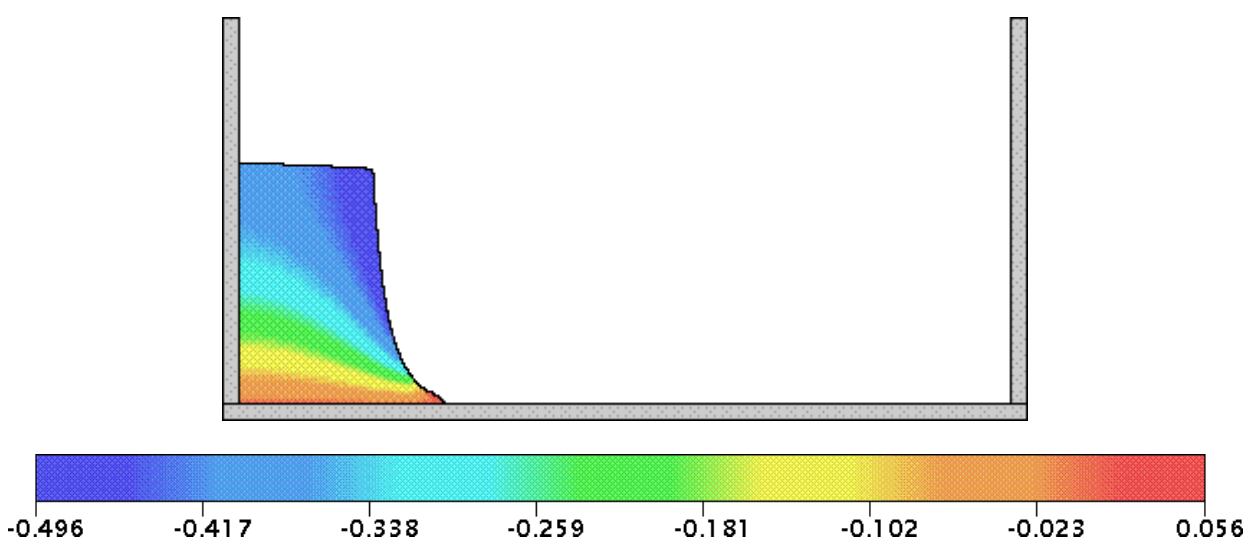

Figura 7.13: Colapso de fluido (Martim e Moyce e Koshizuka e Oka). Resultados numéricos do esquema FDPUS-C1 para a evolução da superfície livre do fluido (campos de pressão e velocidade, nas direções $x$ e $y$ ) em $t=0.05 s$. 


\section{Campo de pressão}
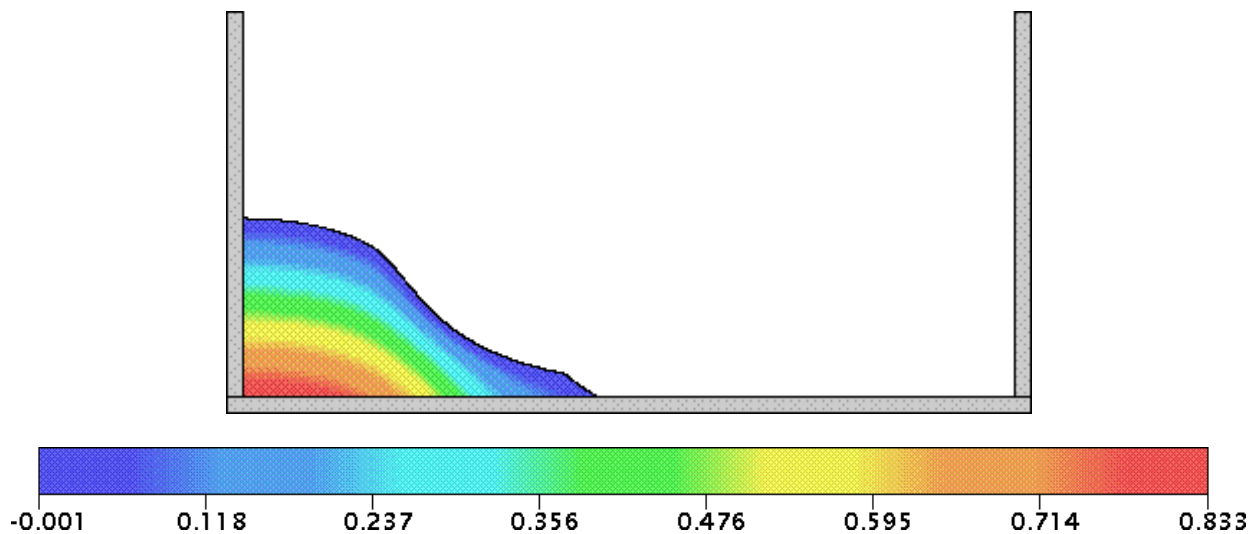

Campo de velocidade na direção $\mathrm{x}$
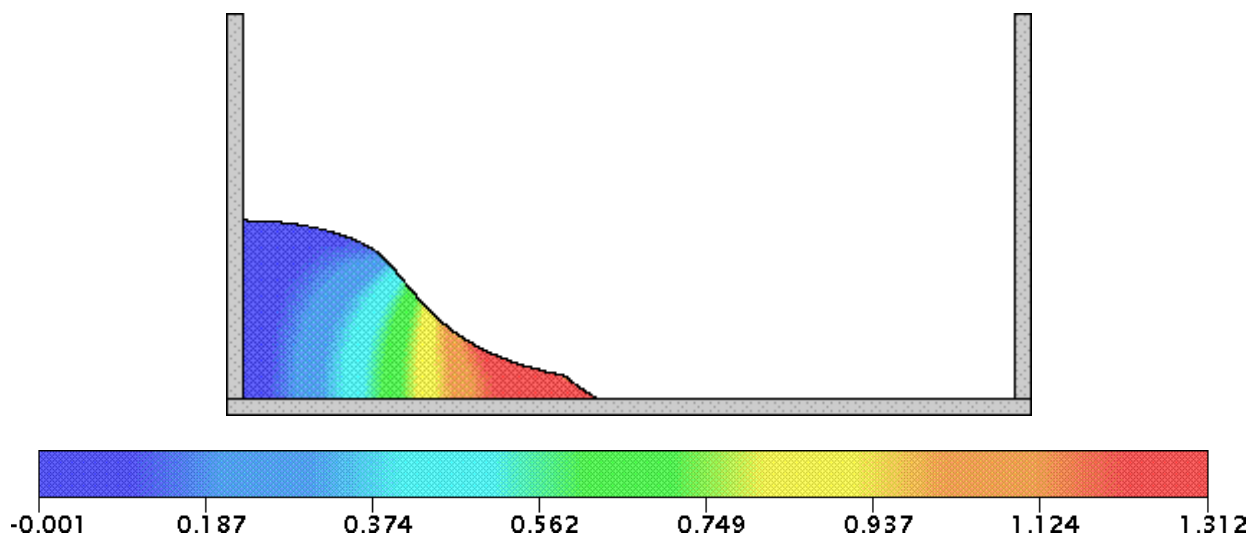

Campo de velocidade na direção y
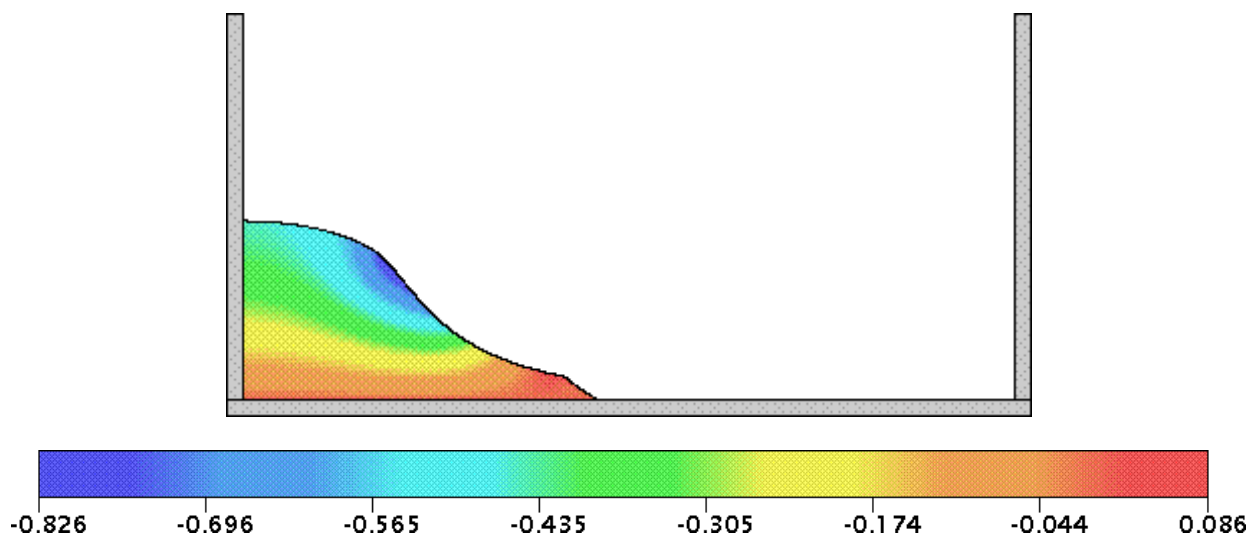

Figura 7.14: Colapso de fluido (Martim e Moyce e Koshizuka e Oka). Resultados numéricos do esquema FDPUS-C1 para a evolução da superfície livre do fluido (campos de pressão e velocidade, nas direções $x$ e $y$ ) em $t=0.1 s$. 


\section{Campo de pressão}
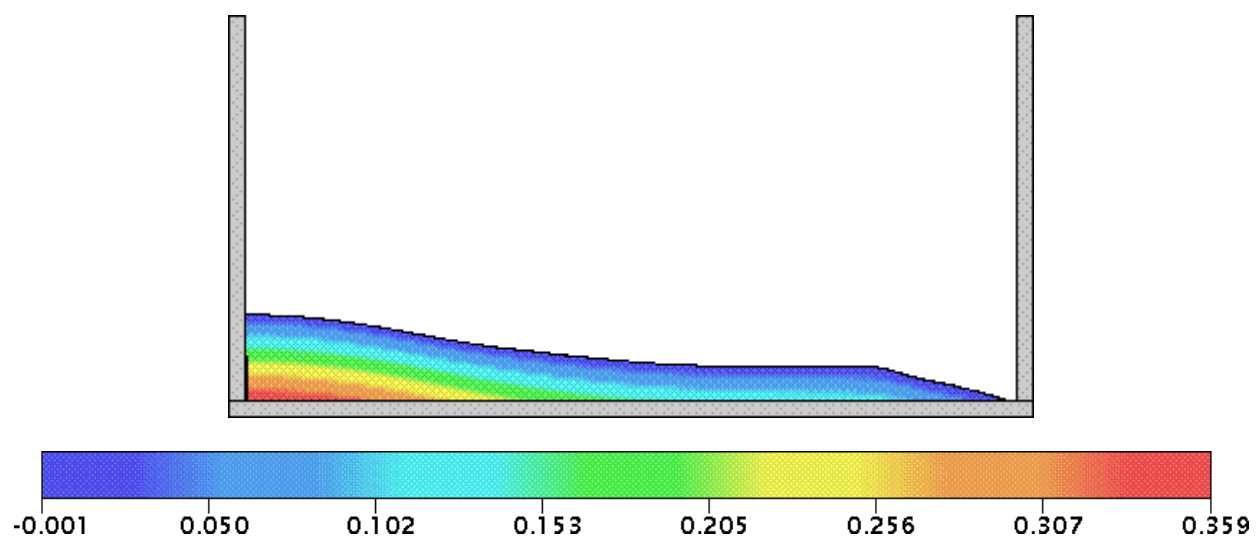

Campo de velocidade na direção $\mathrm{x}$
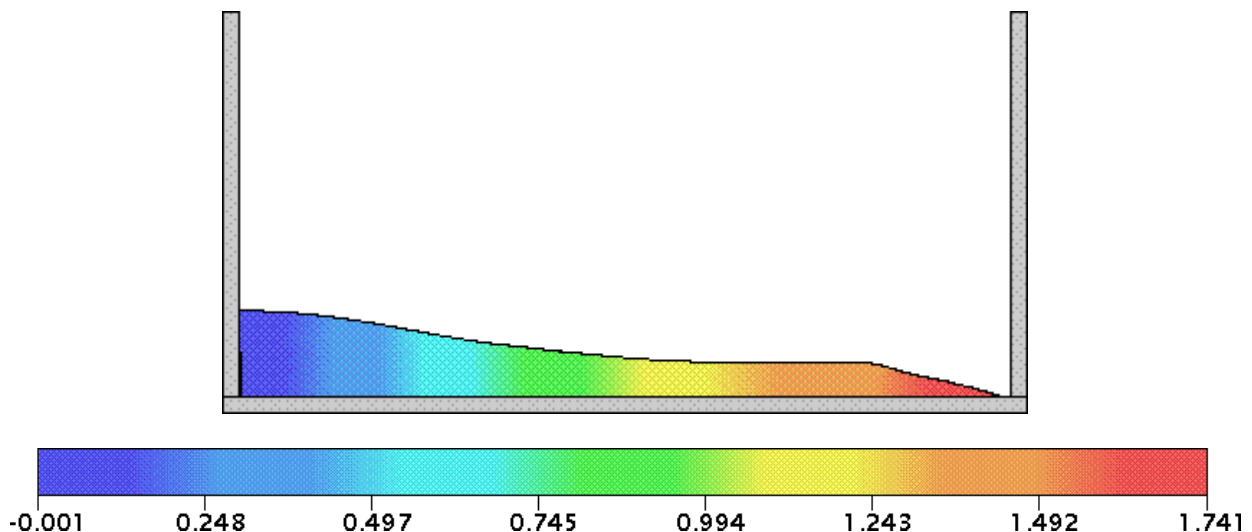

Campo de velocidade na direção y
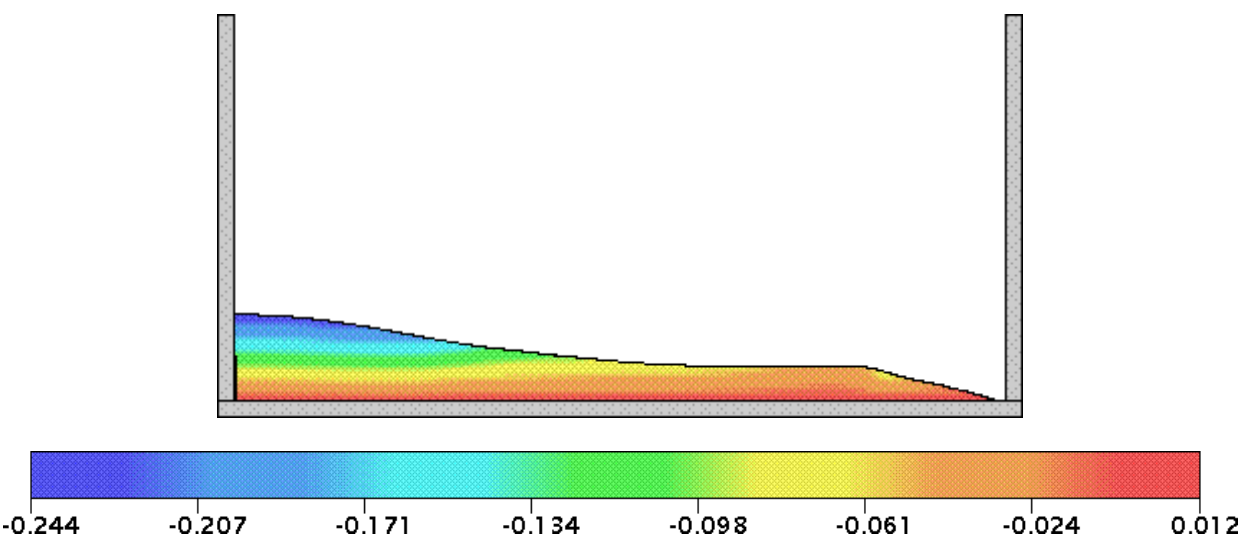

Figura 7.15: Colapso de fluido (Martim e Moyce e Koshizuka e Oka). Resultados numéricos do esquema FDPUS-C1 para a evolução da superfície livre do fluido (campos de pressão e velocidade, nas direções $x$ e $y$ ) em $t=0.2 s$. 
- Dimensão do domínio: $0.5 m \times 0.1 m$;

- Constante gravitacional: $g=9.81 \mathrm{~m} / \mathrm{s}^{2}$;

- Escala de comprimento: $L=a=0.057 m$;

- Escala de velocidade: $V_{0}=\sqrt{g \cdot L}=0.74778 \mathrm{~m} / \mathrm{s}$;

- Coeficiente de viscosidade cinemática: $\nu=10^{-6} \mathrm{~m}^{2} / \mathrm{s}$;

- Número de Reynolds: $R e=\frac{V_{0} \cdot L}{\nu}=42623.27$;

- Número de Froud: $\operatorname{Fr}=\frac{V_{0}}{\sqrt{g \cdot L}}=1$.

A Figura 7.16 mostra a comparação entre os dados numérico/experimental/teórico apresentados por Colagrossi e Landrini [14] e os resultados numéricos gerados pelos esquemas FDPUS-C1 (Fig. 7.16-(a)) e SDPUS-C1 (Fig. 7.16-(b)) para a posição frontal do movimento do fluido $\left(x_{\max }\right)$, em função do tempo, para o tempo final $t=0.2 s$. Os dados numéricos apresentados por Colagrossi e Landrini [14] são gerados com as formulações SPH (Smoothed Particle Hydordynamics method) de Colagrossi e Landrini [14], BEM (Bondary Element Method) de Greco et.al [29], Level Set de Colicchio et al. [15]; o dado experimental é de Martim e Moyce [47], e o dado teórico de Ritter [56]. Por essa figura, observa-se que os resultados numéricos obtidos com os esquemas FDPUS-C1 e SDPUS-C1 estão em ótima concordância com o dados apresentados por Colagrossi e Landrini.

(a)

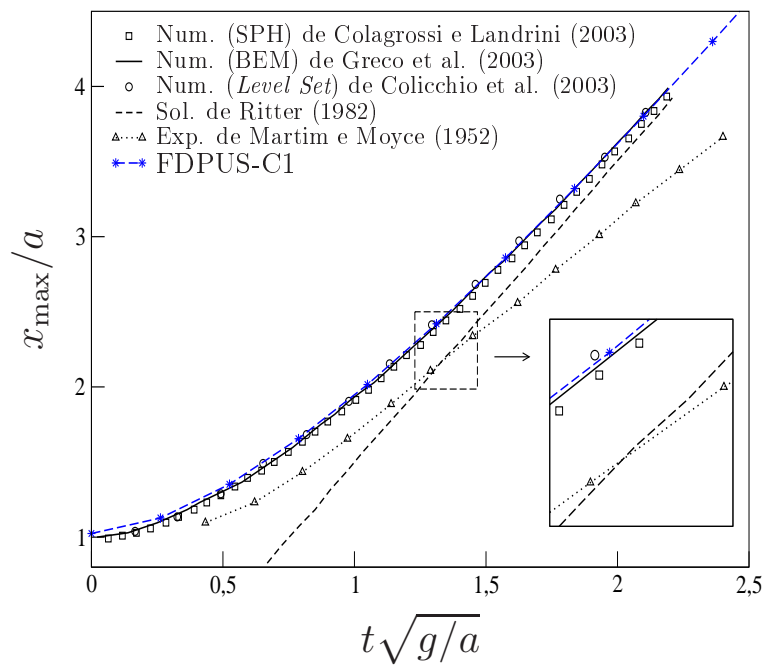

(b)

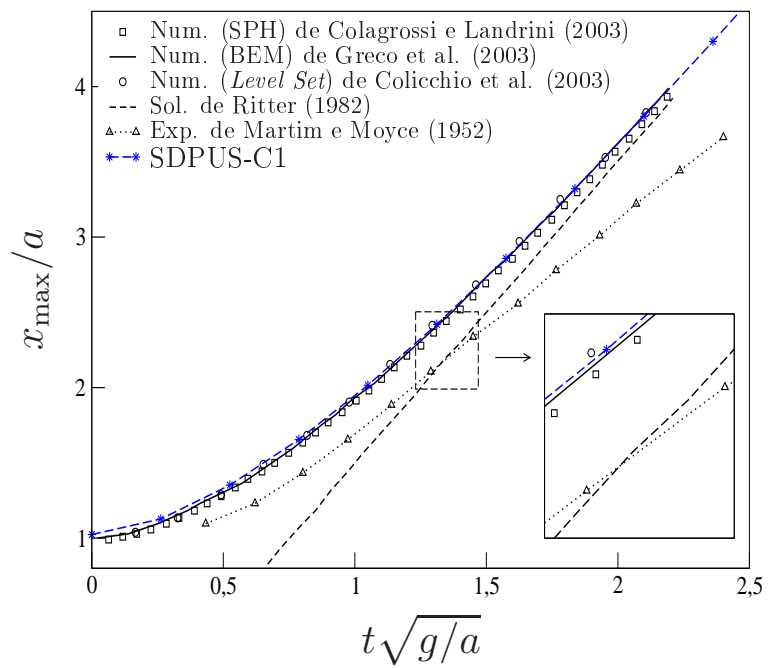

Figura 7.16: Colapso de fluido (Colagrossi e Landrini). Comparação entre os dados numéricos/teórico/experimental e os esquemas FDPUS-C1 (a) e SDPUS-C1 (b) para $x_{\max }$ em função do tempo.

Para ilustração desse caso, consideram-se os resultados obtidos com o esquema SDPUS-C1. 
Assim, nas Figuras 7.17, 7.18 e 7.19 são apresentados, respectivamente, os resultados numéricos gerados por esse esquema (com respeito a evolução da superfície livre do fluido, para os campos de pressão e velocidade, nas direções $x$ e $y$ ), para os tempos $t=0.05 s, t=0.1 s$ e $t=0.2 s$. Os resultados numéricos obtidos com o esquema FDPUS-C1 são omitidos devido a similaridade com os resultados gerados pelo esquema SDPUS-C1.

\section{Campo de pressão}

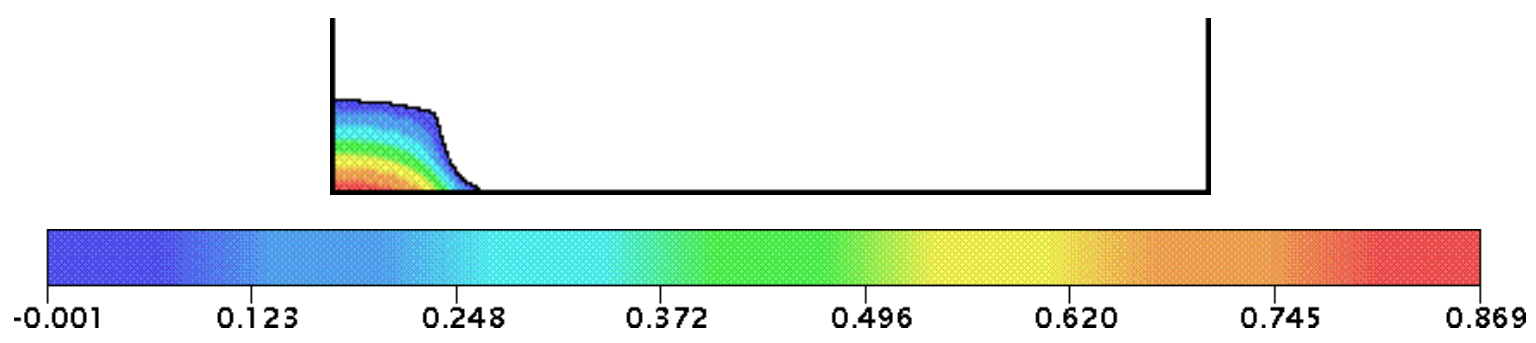

\section{Campo de velocidade na direção $\mathrm{x}$}
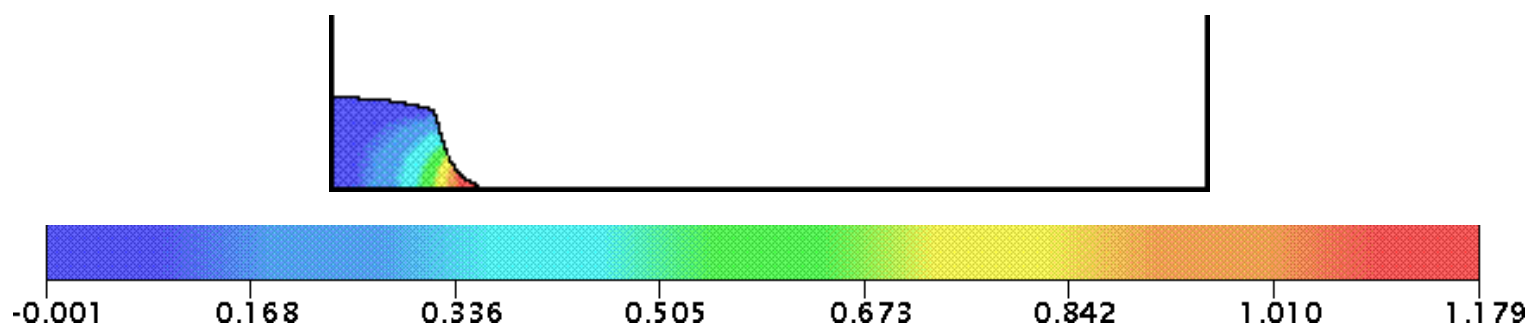

\section{Campo de velocidade na direção y}
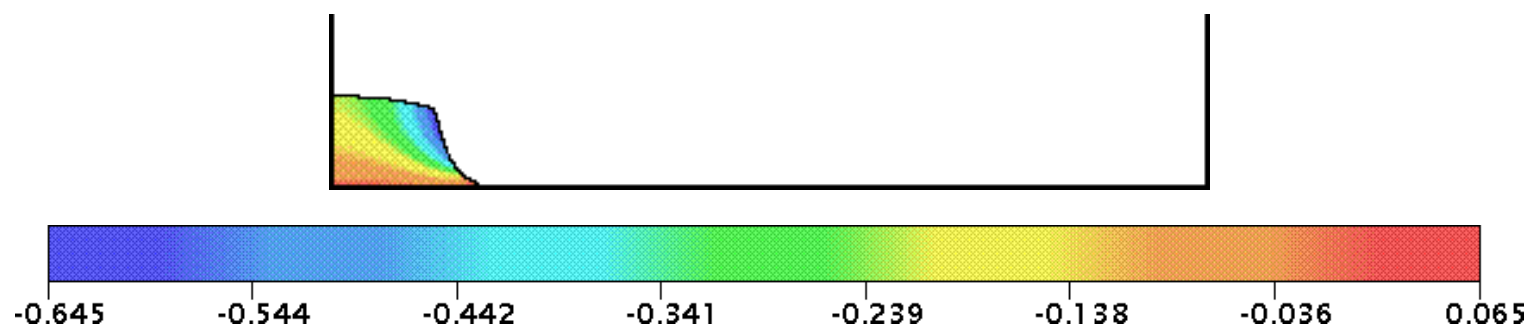

Figura 7.17: Colapso de fluido (Colagrossi e Landrini). Resultados numéricos do esquema SDPUS-C1 para a evolução da superfície livre do fluido (campos de pressão e velocidade, nas direções $x$ e $y)$ em $t=0.05 s$. 


\section{Campo de pressão}

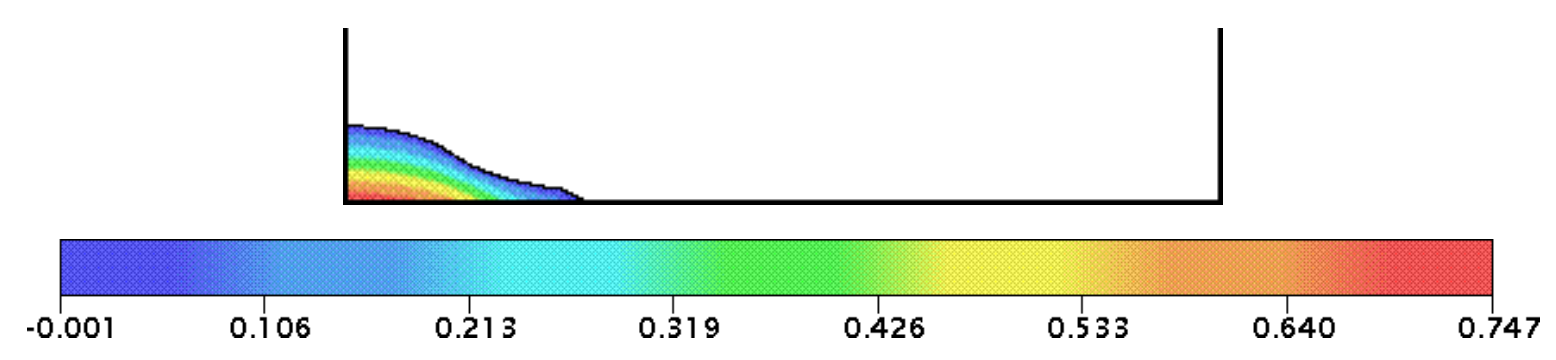

Campo de velocidade na direção $\mathrm{x}$

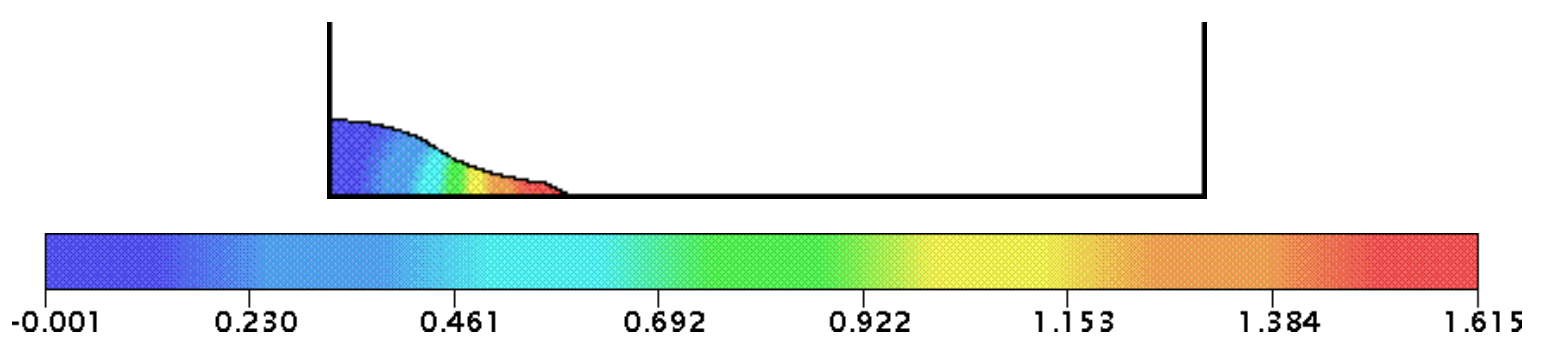

Campo de velocidade na direção y

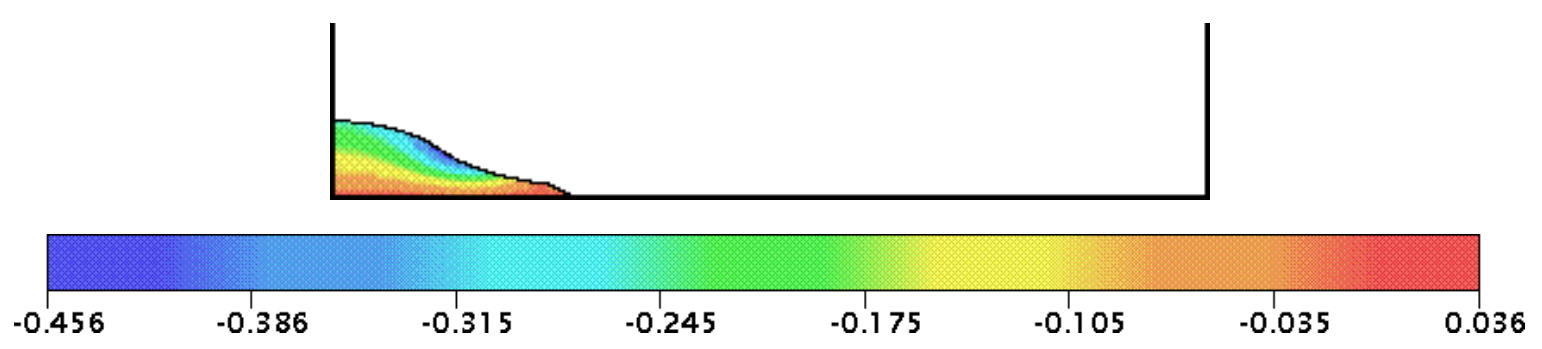

Figura 7.18: Colapso de fluido (Colagrossi e Landrini). Resultados numéricos do esquema SDPUS-C1 para a evolução da superfície livre do fluido (campos de pressão e velocidade, nas direções $x$ e $y)$ em $t=0.1 s$. 
Campo de pressão

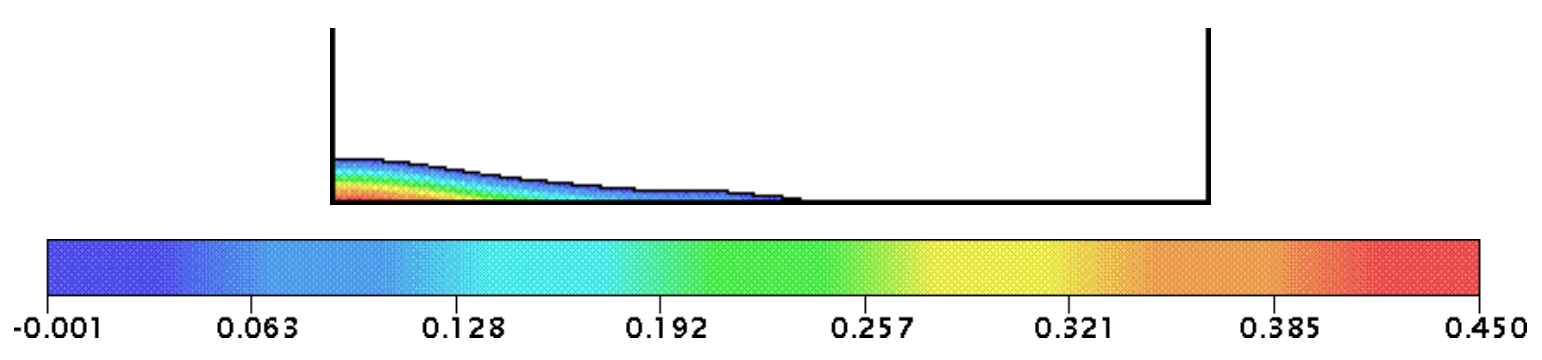

Campo de velocidade na direção $\mathrm{x}$

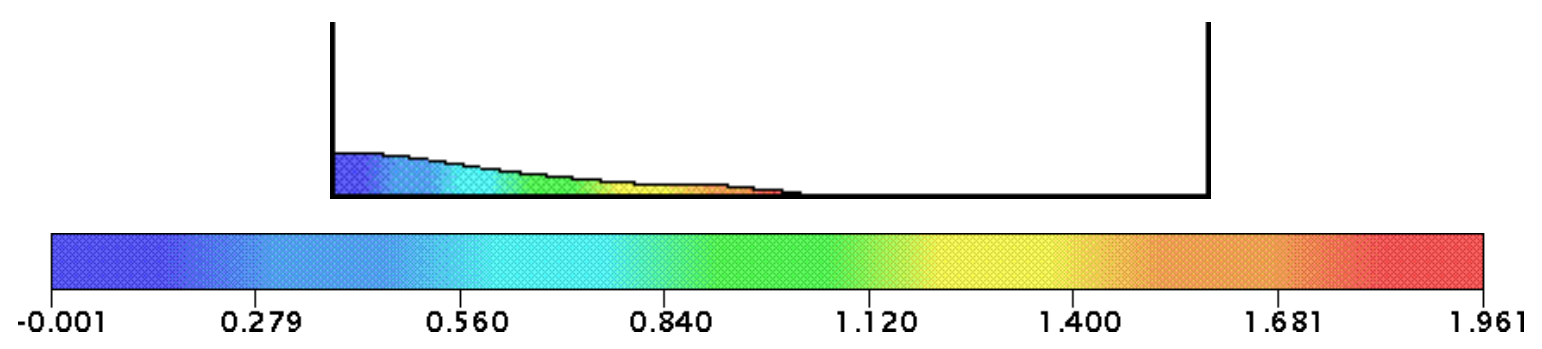

Campo de velocidade na direção y

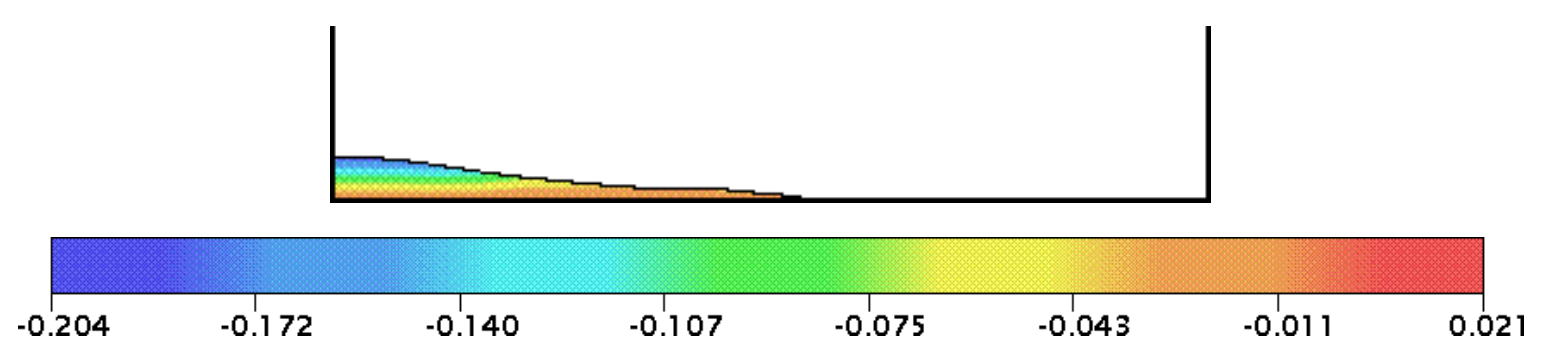

Figura 7.19: Colapso de fluido (Colagrossi e Landrini). Resultados numéricos do esquema SDPUS-C1 para a evolução da superfície livre do fluido (campos de pressão e velocidade, nas direções $x$ e $y)$ em $t=0.2 s$. 


\subsubsection{Jato Livre sobre uma Superfície Rígida Impermeável}

Nesta seção os esquemas FDPUS-C1 e SDPUS-C1 são aplicados na resolução do problema de escoamento de um jato livre, em regime laminar, incidindo perpendicularmente a uma superfície rígida impermeável e sob efeito do campo gravitacional (ver Figura 7.20). O problema é simulado em dois regimes: para baixos valores do número de Reynolds e outro a alto valor número de Reynolds. No caso de alto valor do número de Reynolds, os resultados numéricos são comparados com a solução analítica proposta por Watson [76] e para baixos valores do número de Reynolds, as soluções numéricas são comparadas com dados teóricos e experimentais apresentados por Cruickshank e Munson [18]. Nos dois casos é adotada a condição no-sleep nas paredes.

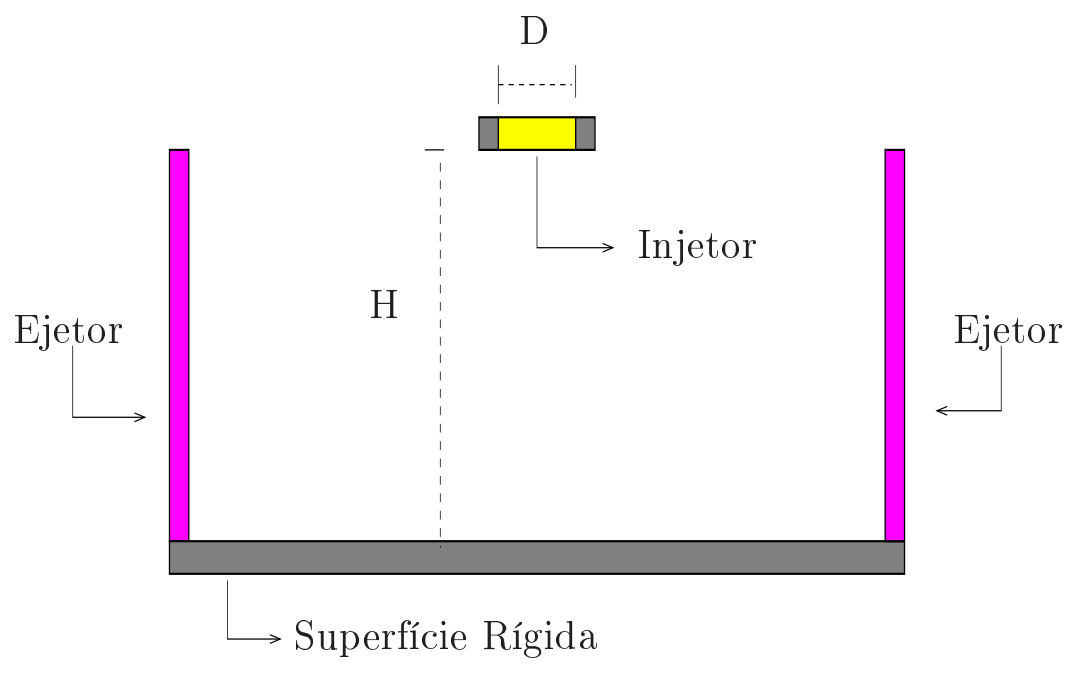

Figura 7.20: Jato livre sobre uma superfície rígida impermeável. Diagrama esquemático.

Caso 1 - Jato livre a baixos números de Reynolds. Neste caso, os resultados numéricos para os valores do raio do jato do fluido $a=a(x)$ com relação a altura $x$ (ver Figura 7.21), gerados pelos esquemas FDPUS-C1 e SDPUS-C1, são comparados com os dados apresentados por Cruickshank e Munson [18].

Para simulação do problema, considera-se uma malha com $800 \times 110$ células computacionais e os seguintes dados: 


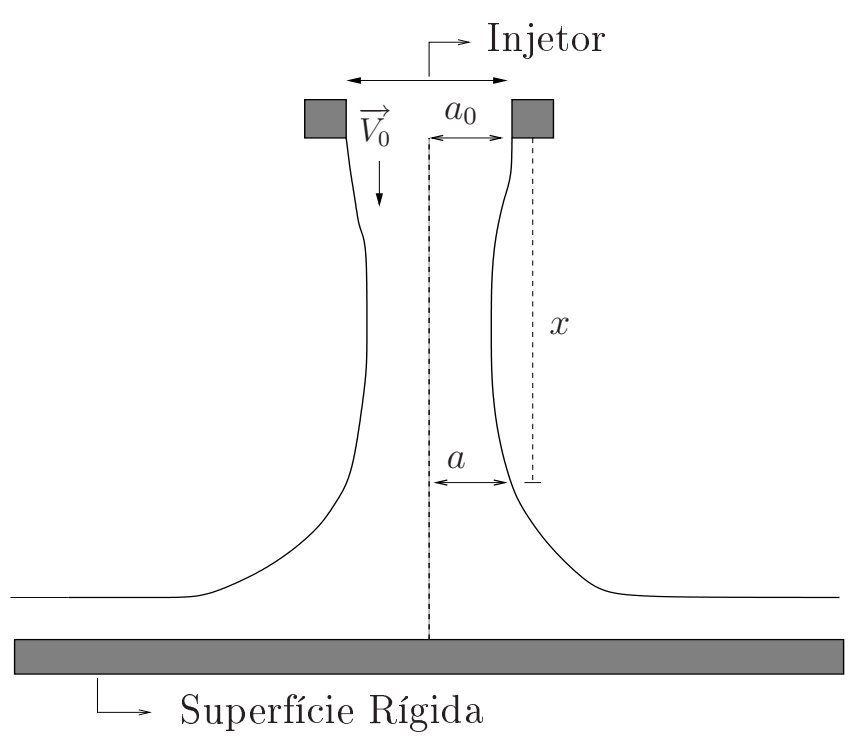

Figura 7.21: Jato livre a baixo número de Reynolds. Representação esquemática.

- Domínio: $0.224 m \times 0.0308 m$;

- Constante gravitacional: $g=9.81 \mathrm{~m} / \mathrm{s}^{2}$;

- $\quad$ Escala de comprimento: $L=a_{0}=0.00476 m$;

- Distância do injetor até a superfície rígida: $H=0.028 \mathrm{~m} / \mathrm{s}$;

- Coeficiente de viscosidade cinemática: $\nu=0.00551 \mathrm{~m}^{2} / \mathrm{s}$;

- Vazão $Q$, número de Reynolds $R e=\frac{Q}{\nu a_{0}}$, velocidade de injeção (escala) $V_{0}=\frac{R e \nu}{a_{0}}$ :

$-Q=16.60 \times 10^{-6} \mathrm{~m}^{3} / \mathrm{s}, \quad R e=\frac{Q}{\nu a_{0}}=0.63292, \quad V_{0}=\frac{R e \nu}{a_{0}}=0.73264 \mathrm{~m} / \mathrm{s} ;$

$-Q=3.53 \times 10^{-6} \mathrm{~m}^{3} / \mathrm{s}, \quad R e=\frac{Q}{\nu a_{0}}=0.13459, \quad V_{0}=\frac{R e \nu}{a_{0}}=0.15579 \mathrm{~m} / \mathrm{s} ;$

$-Q=0.58 \times 10^{-6} \mathrm{~m}^{3} / \mathrm{s}, \quad R e=\frac{Q}{\nu a_{0}}=0.02211, \quad V_{0}=\frac{R e \nu}{a_{0}}=0.02559 \mathrm{~m} / \mathrm{s}$.

Na Figura 7.22 apresenta-se a comparação entre os dados experimentais e teóricos, apresentados por Cruickshank e Munson [18], e os resultados numéricos gerados pelos esquemas FDPUS-C1 (Fig. 7.22-(a)) e SDPUS-C1 (Fig. 7.22-(b)), para $a=a(x)$ em função da altura $x$. Por essa figura, pode ser observado que as soluções numéricas estão em concordância com os dados experimentais e teóricos. Entretanto, surpreendentemente, no caso em que a vazão é $3.53 \times 10^{-6} m^{3}(R e=0.13459)$ os dados apresentam discrepâncias.

Apenas como ilustração, na Figura 7.23, 7.24 e 7.25 são apresentados, respectivamente, os resultados numéricos nos tempos $t=1 s, t=2 s$ e $t=8 s$, gerados pelo esquema FDPUS-C1, para o número de Reynolds $R e=0.02211$, com respeito a evolução da superfície livre do fluido, para os campos de pressão e velocidade (nas direções $x$ e $y$ ). Os resultados obtidos com o esquema SDPUS-C1 são omitidos, para esse problema, devido a similaridade com os resultados 
(a)

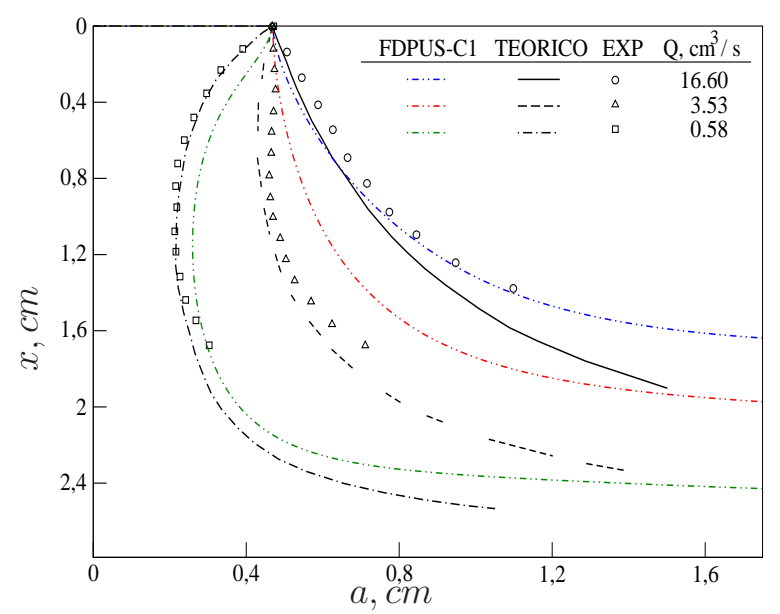

(b)

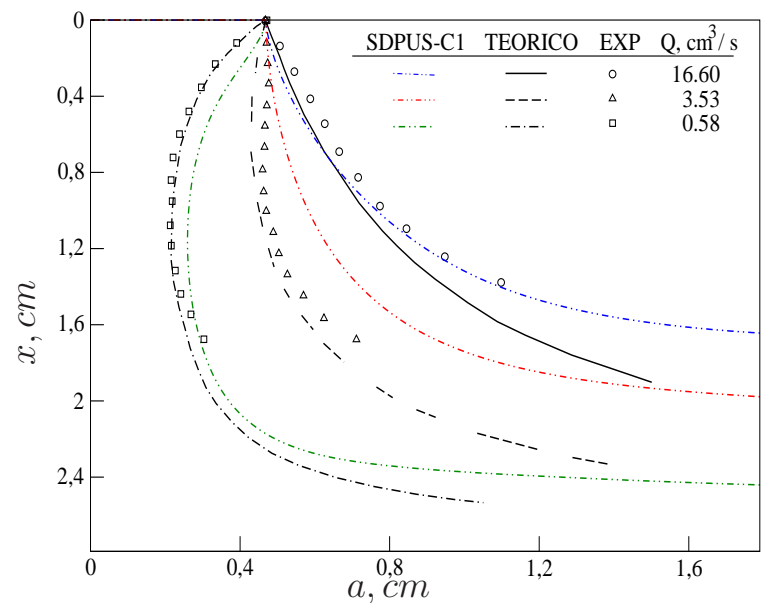

Figura 7.22: Jato livre a baixo número de Reynolds. Comparação entre os dados (experimentais e numéricos) por Cruickshank e Munson e os esquemas FDPUS-C1 (a) e SDPUS-C1 (b) para os valores de $a(x)$.

apresentados nessas figuras.

Caso 2 - Jato livre a alto número de Reynolds. Neste caso, a alto valor do número de Reynolds, os resultados numéricos gerados pelos esquemas FDPUS-C1 e SDPUS-C1 são verificados para a altura $H$ entre a superfície rígia e a superfície livre do fluido (ver Figura 7.26). Para essa verificação, os resultados numéricos são confrontados com a solução analítica proposta por Watson [76]. Desprezando os efeitos de tensão superficial, após o escoamento atingir o estado estacionário, Watson deduziu a seguinte relação explícita para a altura $H$

$$
H(x)= \begin{cases}\frac{\pi}{\sqrt{3}} \frac{\nu(x+l)}{Q} & x \geq x_{0} \\ a+\left(\frac{1-2 \pi}{3 \sqrt{3} c^{2}}\right) \delta(x) & x<x_{0}\end{cases}
$$

em que $Q$ é a vazão definida por $Q=a U, a=L / 2$,

$$
\begin{aligned}
x_{0} & =\left(\frac{3 \sqrt{3} c(\pi-c) \sqrt{3}}{2 \pi^{2}}\right) a R e, \\
l & =\left(\frac{3 \sqrt{3} c(2 \sqrt{3} c-\pi)}{2 \pi^{2}}\right) a R e
\end{aligned}
$$

$\mathrm{e}$

$$
\delta^{2}(x)=\frac{3 \sqrt{3} c^{3}}{2(\pi-c \sqrt{3})} \frac{\nu x}{U},
$$


Campo de pressão

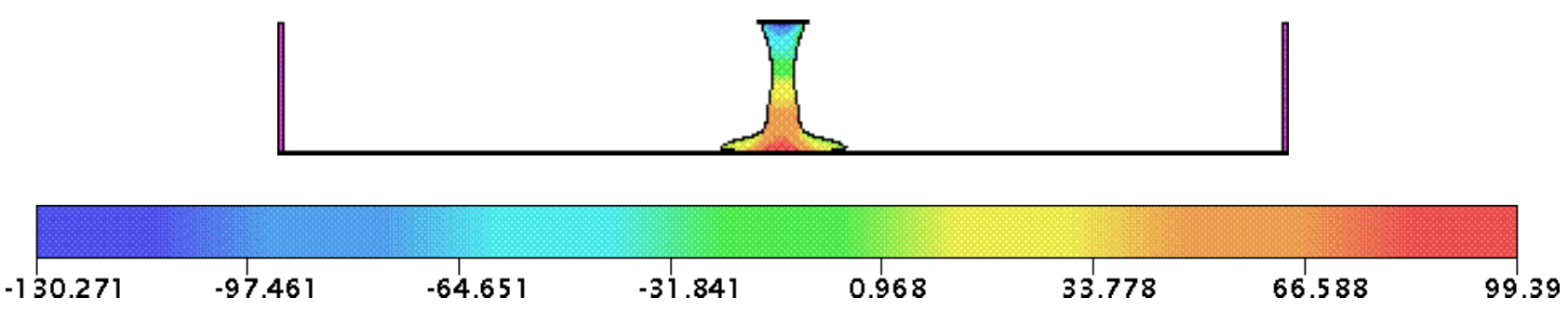

Campo de velocidade na direção $\mathrm{x}$

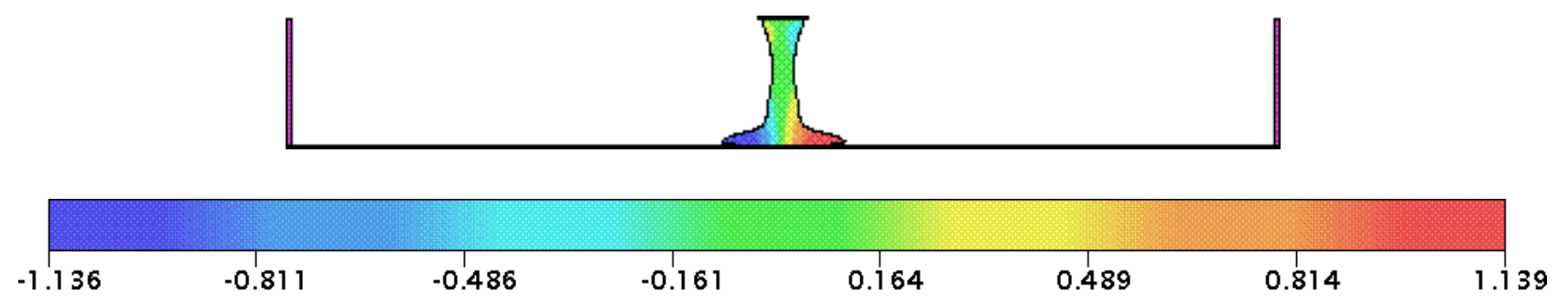

Campo de velocidade na direção y

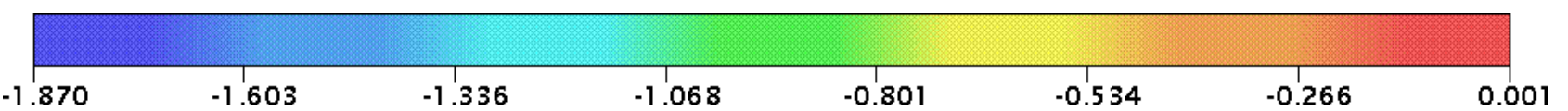

Figura 7.23: Jato livre a baixo número de Reynolds. Resultados numéricos do esquema FDPUS-C1 para a evolução da superfície livre do fluido (campos de pressão e velocidade, nas direções $x$ e $y)$ em $t=1 s$. 


\section{Campo de pressão}

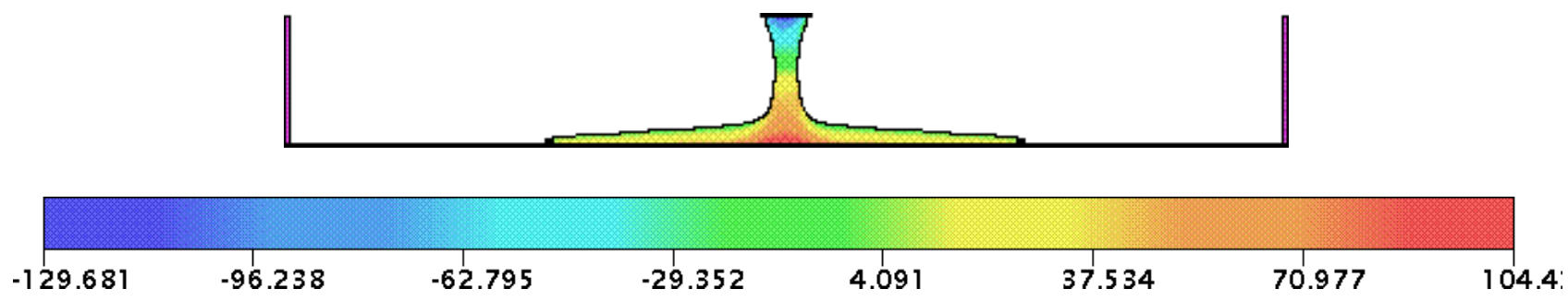

Campo de velocidade na direção $\mathrm{x}$

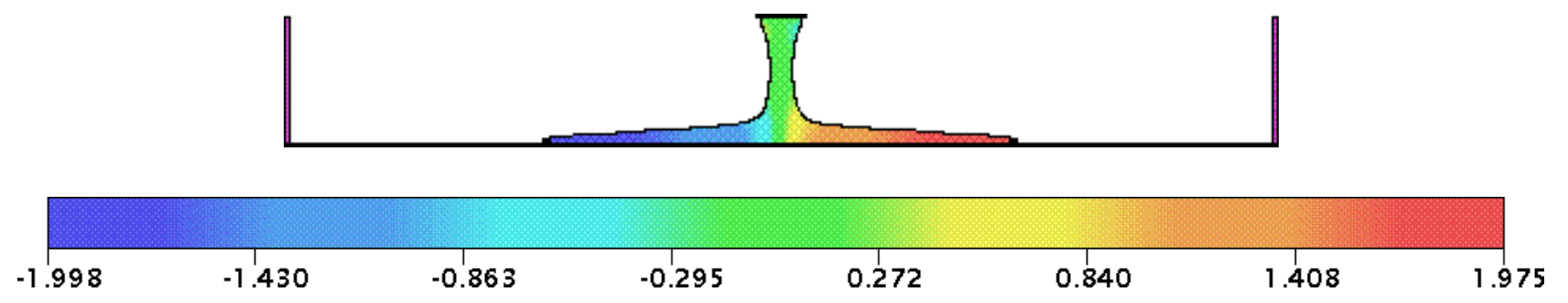

Campo de velocidade na direção y

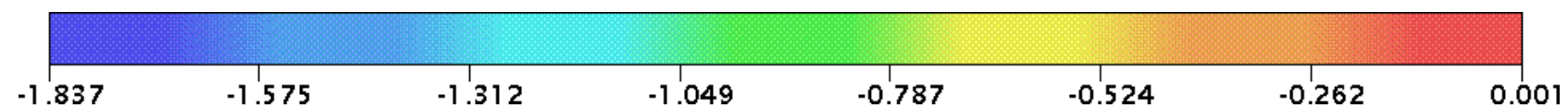

Figura 7.24: Jato livre a baixo número de Reynolds. Resultados numéricos do esquema FDPUS-C1 para a evolução da superfície livre do fluido (campos de pressão e velocidade, nas direções $x$ e $y)$ em $t=2 s$. 
Campo de pressão

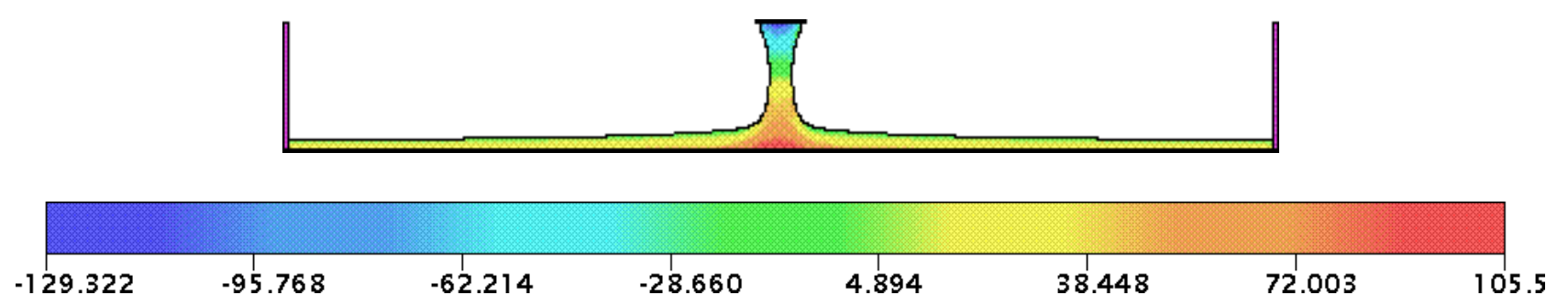

Campo de velocidade na direção $\mathrm{x}$

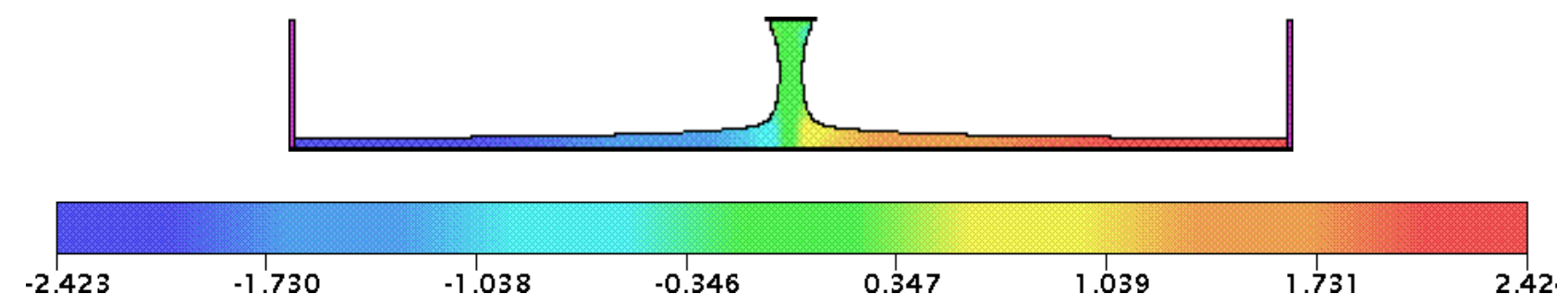

Campo de velocidade na direção y
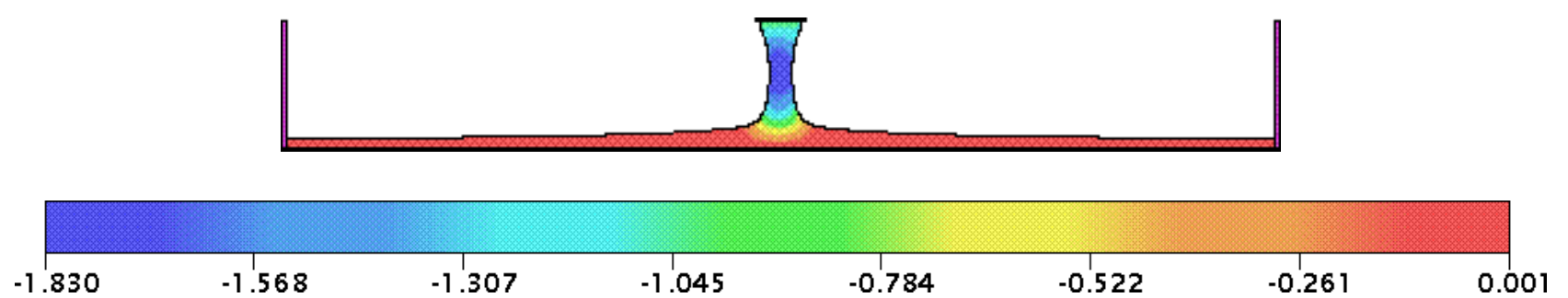

Figura 7.25: Jato livre a baixo número de Reynolds. Resultados numéricos do esquema FDPUS-C1 para a evolução da superfície livre do fluido (campos de pressão e velocidade, nas direções $x$ e $y)$ em $t=6 s$. 
nas quais, $c$ é uma constante dada por $c=1.402$ e $U$ é a velocidade de injeção. Para mais detalhes sobre a descrição dessa solução ver [76].

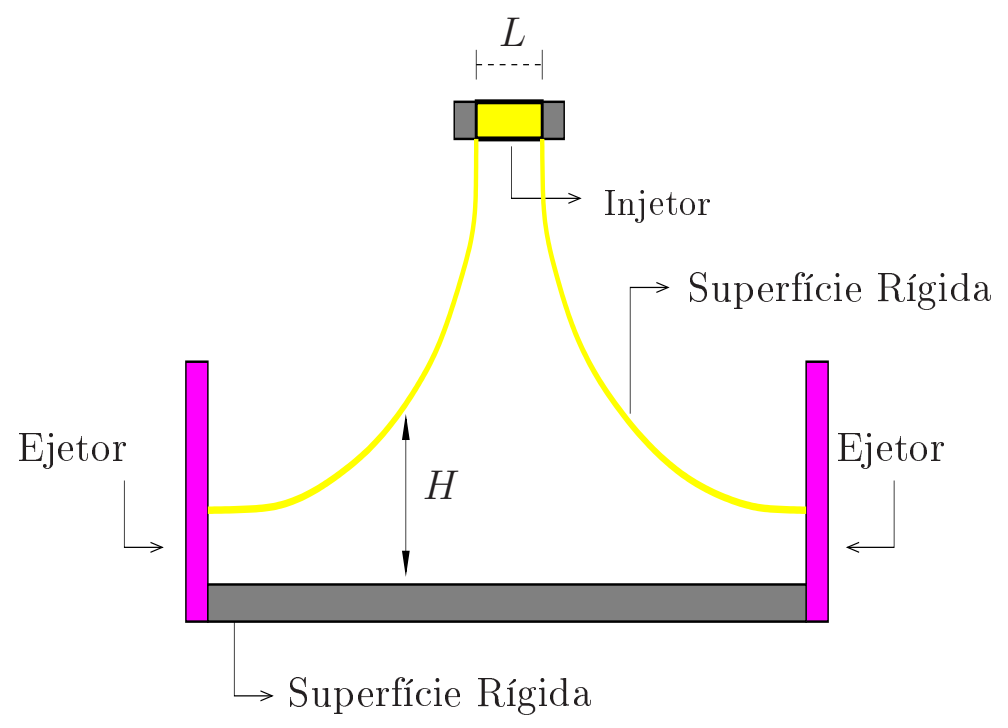

Figura 7.26: Jato livre a alto número de Reynolds. Representação esquemática.

Para simulação do problema, consideram-se quatro malhas de $100 \times 10,250 \times 25,500 \times 50$ e $1000 \times 100$ células computacionais e os seguintes dados:

- Dimensão do domínio: $0.1 m \times 0.025 m$;

- Constante gravitacional: $g=9.81 \mathrm{~m} / \mathrm{s}^{2}$;

- Escala de comprimento: $L=2 a=0.004 m$;

- Escala de velocidade: $V_{0}=\sqrt{g \cdot L}=1 \mathrm{~m} / \mathrm{s}$;

- Coeficiente de viscosidade cinemática: $\nu=2 \times 10^{-6} \mathrm{~m}^{2} / \mathrm{s}$;

- Número de Reynolds: $R e=\frac{V_{0} \cdot L}{\nu}=2000$;

- Número de Froud: $F r=\frac{V_{0}}{\sqrt{g \cdot L}}=5.04781$.

Na Figura 7.27 apresenta-se a comparação da solução analítica de Watson com os resultados numéricos gerados pelos esquemas FDPUS-C1 (Fig. 7.27-(a)) e SDPUS-C1 (Fig. 7.27-(b)), nas quatro malhas consideradas. Vê-se por essa figura que os resultados numéricos gerados pelos esquemas FDPUS-C1 e SDPUS-C1 estão em boa concordância com a solução analítica de Watson.

Como ilustração, nas Figuras 7.28, 7.29 e 7.30 são apresentados, respectivamente, os resultados numéricos nos tempos $t=0.1 s, t=0.2 s$ e $t=0.8 s$, gerados pelo esquema SDPUS-C1, com respeito a evolução da superfície livre do fluido para os campos de pressão e velocidade (nas direções $x$ e $y$ ). Os resultados numéricos gerados pelo esquema FDPUS-C1 são similares aos apresentados nessas figuras, portanto não são esboçados. 
(a)

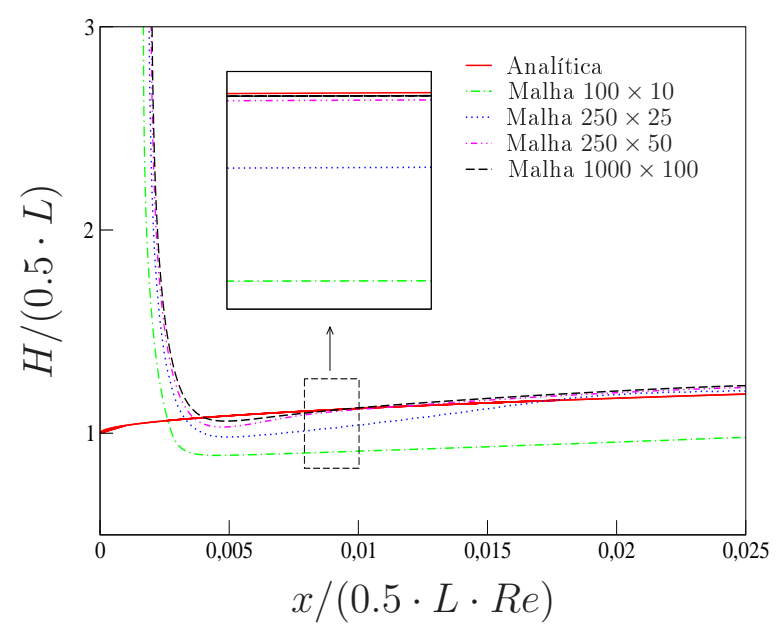

(b)

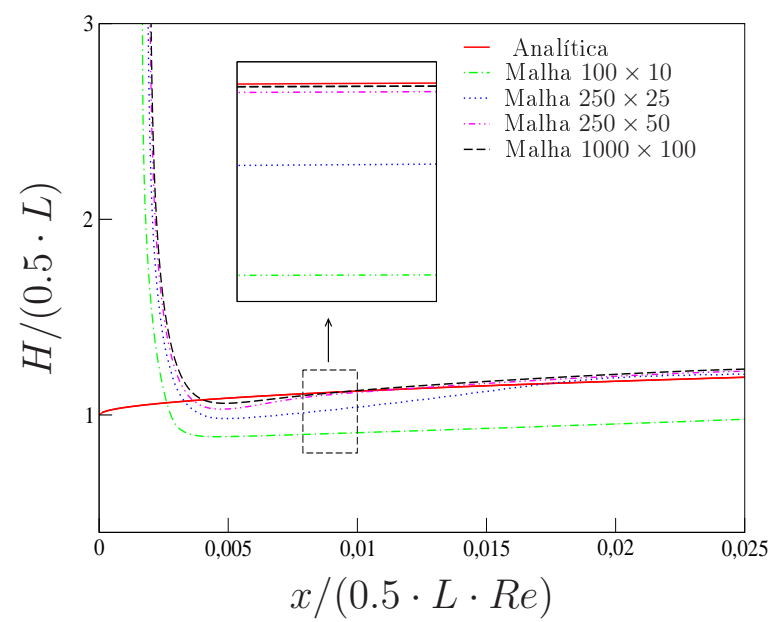

Figura 7.27: Jato livre a alto número de Reynolds. Comparação entre a solução analítica de Watson e os esquemas FDPUS-C1 (a) e SDPUS-C1 (b), para a altura $H$ entre a superfície rígida e a superfície livre do fluido.

\subsection{Escoamento de Fluido Incompressível Laminar 2D-1/2}

Nesta seção são apresentados os resultados numéricos de simulações de escoamentos de fluidos incompressíveis laminares, considerados em coordenadas cilíndricas (neste texto denominada por 2D-1/2). Esses resultados são gerados no código Freeflow 2D de Castelo et al. [11] (ver também Oliveira [49]) para problemas com simetria radial, em que os esquemas FDPUS-C1 e SDPUS-C1 são implementados.

\subsubsection{Jato Livre sobre uma Superfície Rígida Impermeável}

O esquema SDPUS-C1 é agora verificado/validado na simulação do problema de um jato livre sob à ação da gravidade, a baixo valores do número de Reynolds e sobre uma superfície rígida impermeável. O problema está descrito na subseção 7.2.2. Os resultados obtidos com o esquema SDPUS-C1 para valores do raio do jato $a=a(x)$ com relação a altura $x$ (ver Figura 7.21) são comparados com os resultados teóricos e experimentais de Cruickshank e Munson [18]. Os resultados obtidos com o esquema FDPUS-C1 são omitidos, para esse problema, uma vez que há similaridade com os resultados apresentados pelo esquema SDPUS-C1.

Caso 1 - Jato livre a baixo número de Reynolds. Os dados utilizados para a simulação deste caso são os mesmo daqueles utilizados no Caso 1 - Jato livre a baixos números de Reynolds, apresentados na subseção 7.2.2.

Na Figura 7.31 apresenta-se a comparação dos dados experimentais e teóricos de Cruickshank e Munson [18] com os resultados numéricos gerados pelo esquema SDPUS-C1. Por essa figura, 


\section{Campo de pressão}

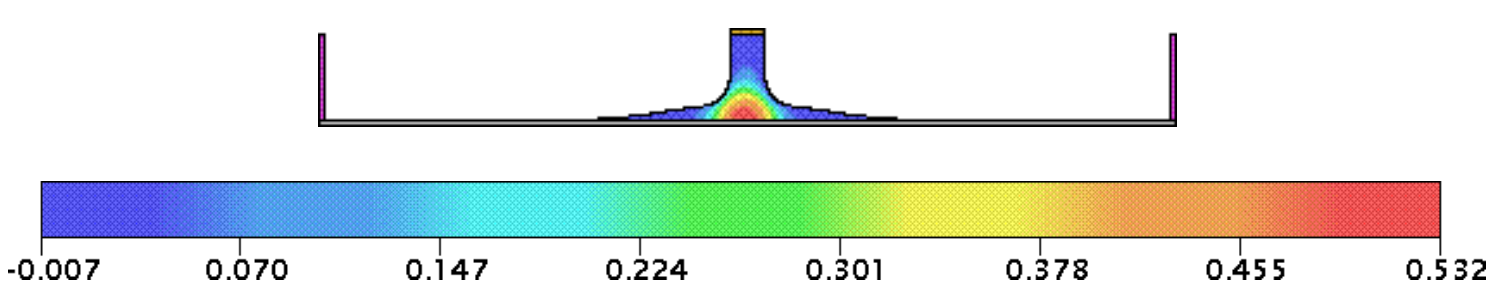

Campo de velocidade na direção $\mathrm{x}$

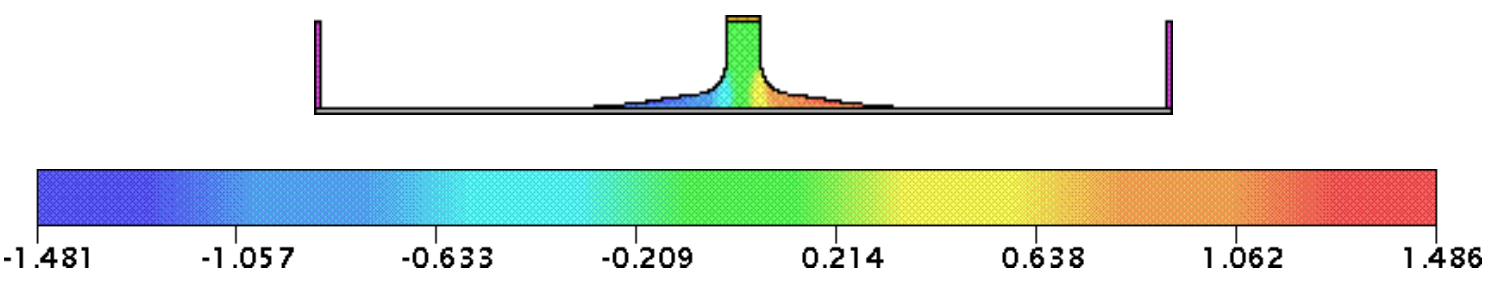

Campo de velocidade na direção y

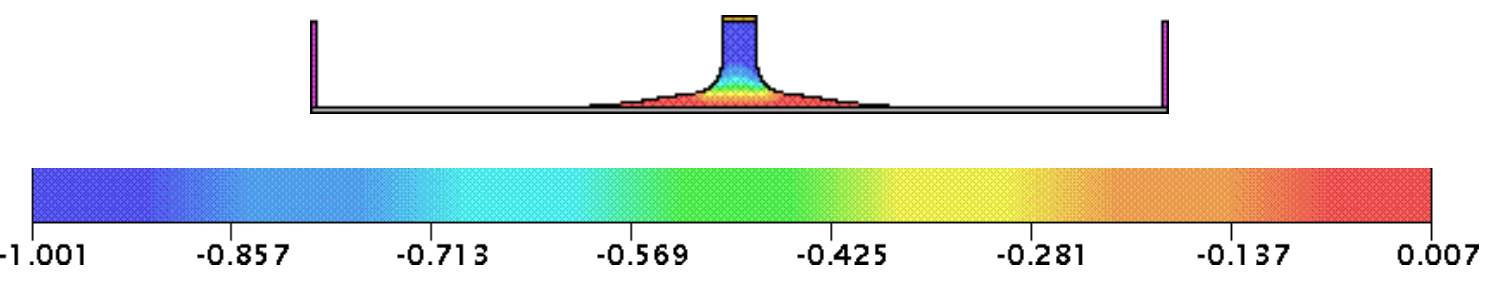

Figura 7.28: Jato livre a alto número de Reynolds. Resultados numéricos do esquema SDPUS-C1 para a evolução da superfície livre do fluido (campos de pressão e velocidade, nas direções $x$ e $y$ ) em $t=0.1 s$. 


\section{Campo de pressão}

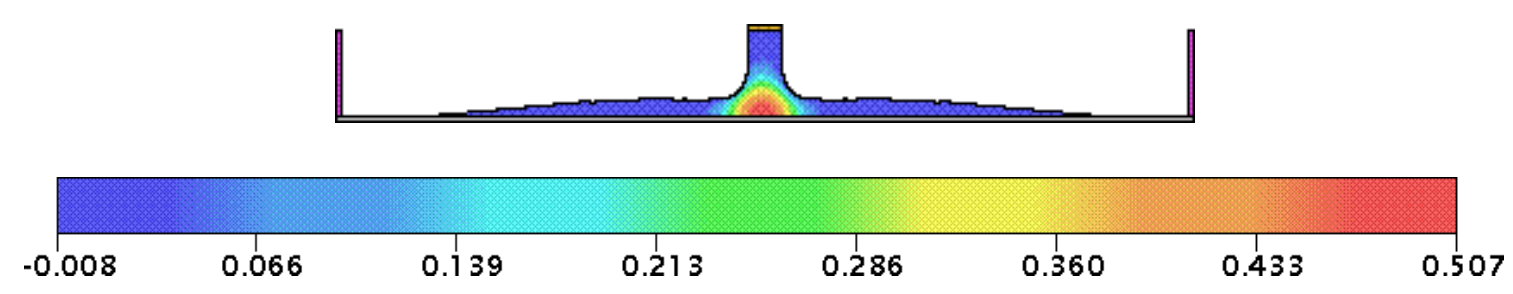

Campo de velocidade na direção $\mathrm{x}$

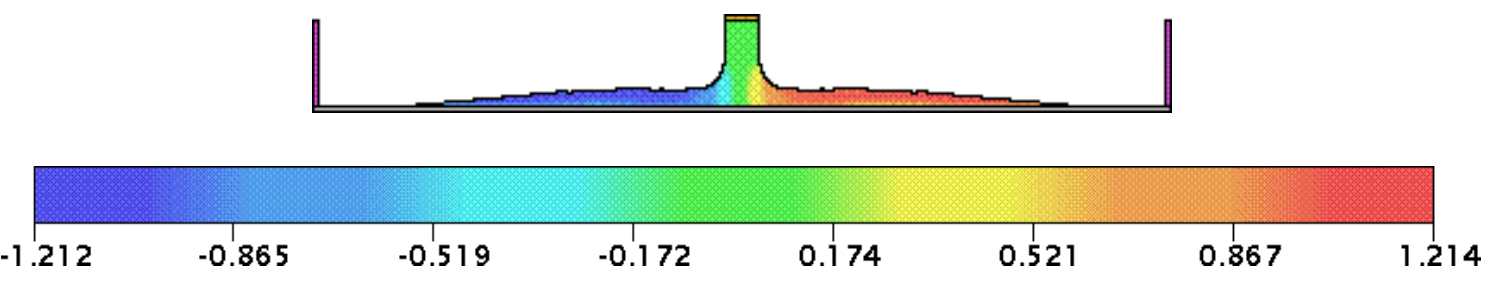

Campo de velocidade na direção y

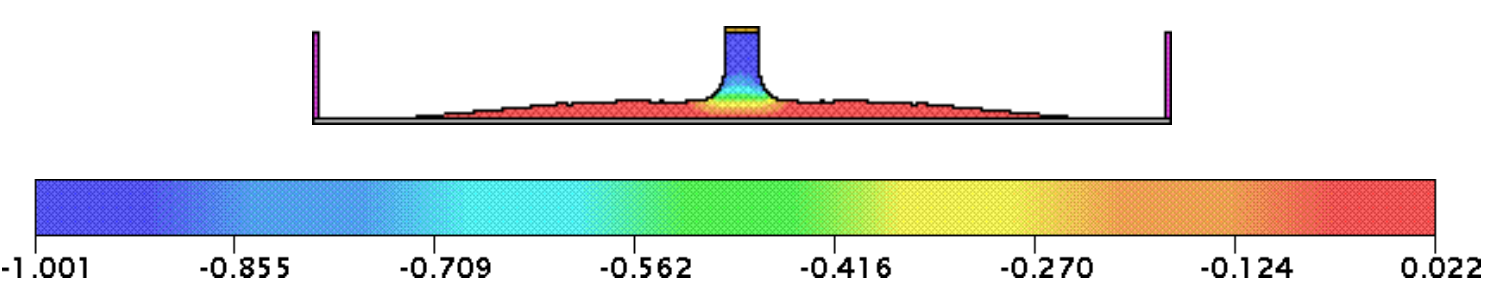

Figura 7.29: Jato livre a alto número de Reynolds. Resultados numéricos do esquema SDPUS-C1 para a evolução da superfície livre do fluido (campos de pressão e velocidade, nas direções $x$ e $y$ ) em $t=0.2 s$. 


\section{Campo de pressão}

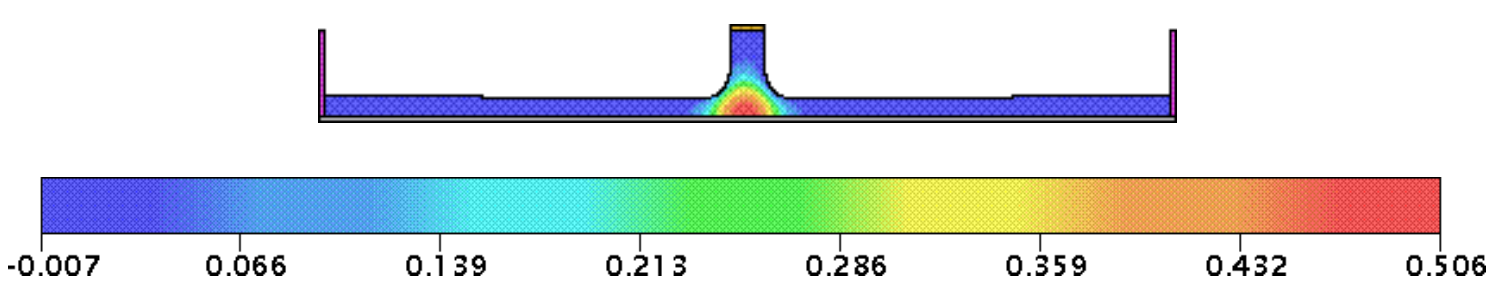

Campo de velocidade na direção $\mathrm{x}$

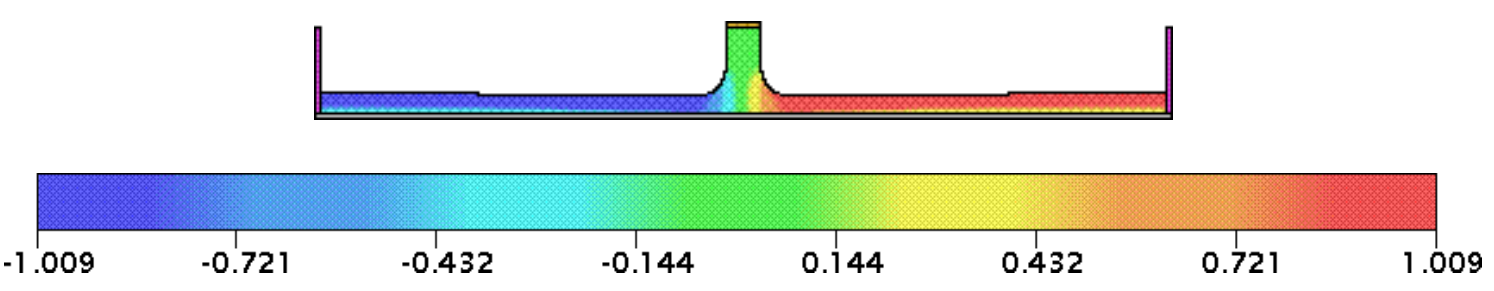

Campo de velocidade na direção y

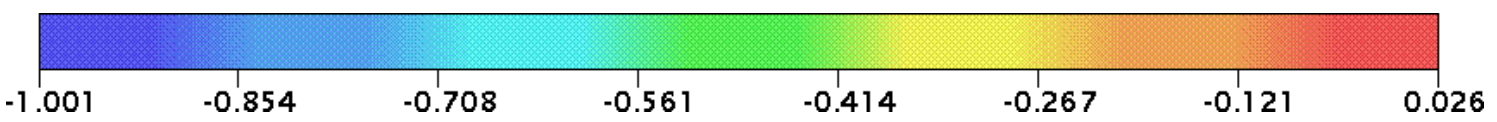

Figura 7.30: Jato livre a alto número de Reynolds. Resultados numéricos do esquema SDPUS-C1 para a evolução da superfície livre do fluido (campos de pressão e velocidade, nas direções $x$ e $y$ ) em $t=0.8 s$. 
pode-se observar que as soluções numéricas, geradas neste caso de coordenadas cilíndricas, estão em boa concordância com os dados experimentais e teóricos. Em particular, diferentemente do Caso 1 - Jato livre a baixos números de Reynolds, apresentado na subseção 7.2.2 simulado em coordenadas cartesianas 2D, os resultados numéricos obtidos da simulação do problema para vazão é $3.53 \times 10^{-6} m^{3}(R e=0.13459)$, neste sistema de coordenadas, mostrou estar muito mais consistentes com os dados de Cruickshank e Munson.

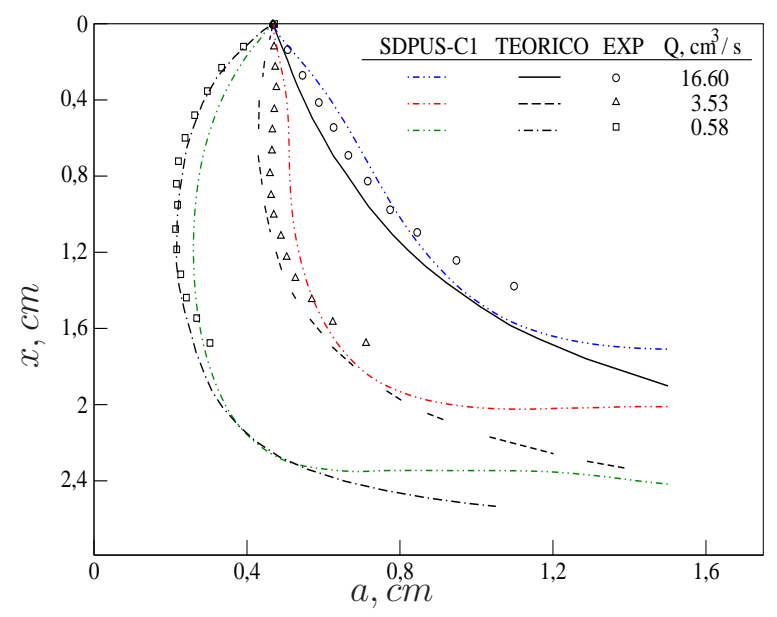

Figura 7.31: Jato livre, a baixo número de Reynolds. Comparação entre os dados (experimentais e numéricos) de Cruickshank e Munson e os esquemas SDPUS-C1 para os valores de $a(x)$.

Apenas como ilustração, na Figura 7.32 são apresentados os resultados numéricos gerados pelo esquema SDPUS-C1, para os valores de número de Reynolds $R e=0.02211$ ( $Q=0.58 \times$ $\left.10^{-6} \mathrm{~m}^{3} / \mathrm{s}\right), R e=0.13459$ e $R e=0.63292\left(Q=16.60 \times 10^{-6} \mathrm{~m}^{3} / \mathrm{s}\right)$ com respeito a evolução da superfície livre do fluido.

\subsubsection{Experimento de Taylor}

Os esquemas FDPUS-C1 e SDPUS-C1 são empregados na resolução do problema de um jato vertical de fluido incidindo perpendicularmente sobre um recipiente contendo o mesmo fluido em repouso, como ilustrado Figura 7.33. Esse experimento foi realizado por Taylor [64] e os dados obtidos por ele são utilizados para a validação qualitativa dos esquemas FDPUS-C1 e SDPUS-C1.

Este problema é simulado para dois valores do número de Reynolds: $R e=10$ e $R e=200$. Em ambos os casos, os resultados numéricos são comparados visualmente com os experimentos de Taylor. Para isso, considera-se um recipiente cilíndrico de raio $r_{r}=0.06 \mathrm{~m}$ e altura $h_{r}=0.17 \mathrm{~m}$ e uma porção de fluido interna a esse recipiente e com altura $h_{f}=0.16 \mathrm{~m}$. O injetor é posicionado a $0.1 \mathrm{~m}$ do fluido em repouso, ou seja, a $0.17 \mathrm{~m}$ da superfície rígida, com altura $h_{i}=0.03 m$ e raio $r_{i}=0.002 m$ (ver Figura 7.33). 
$\operatorname{Re}=0.02211$

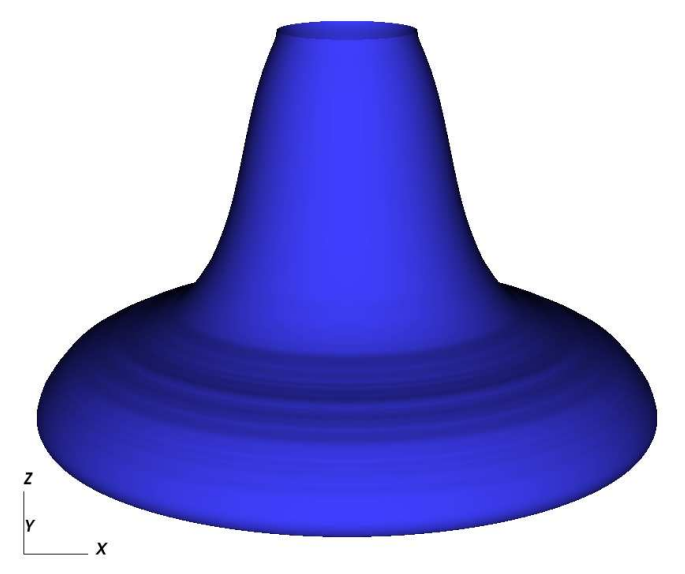

$\operatorname{Re}=0.13459$

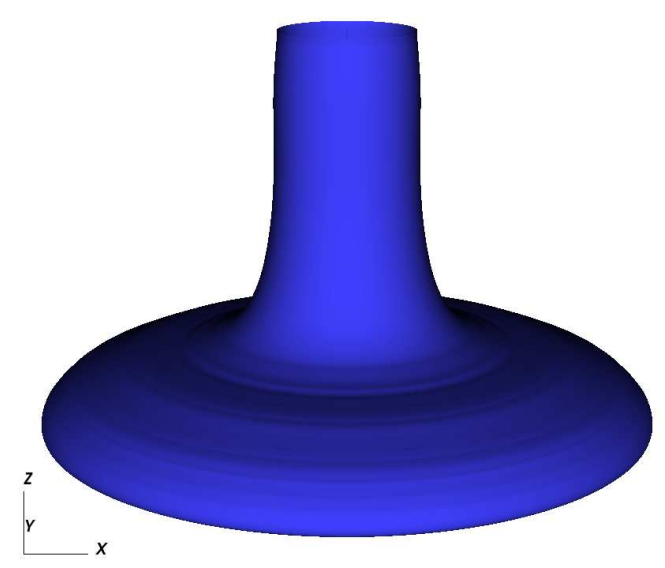

$\operatorname{Re}=0.63292$

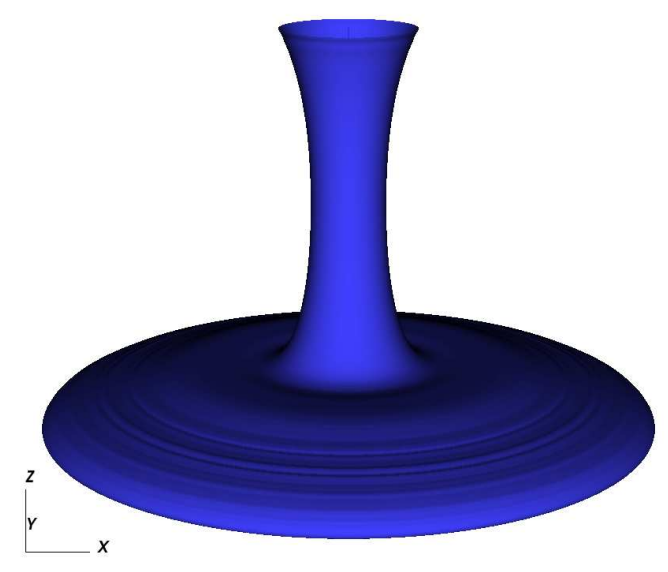

Figura 7.32: Jato livre, a baixo número de Reynolds. Resultados numéricos do esquema SDPUS-C1 para a evolução da superfície livre do fluido para os valores de número de Reynolds $R e=0.02211, R e=0.13459$ e $R e=0.63292$. 


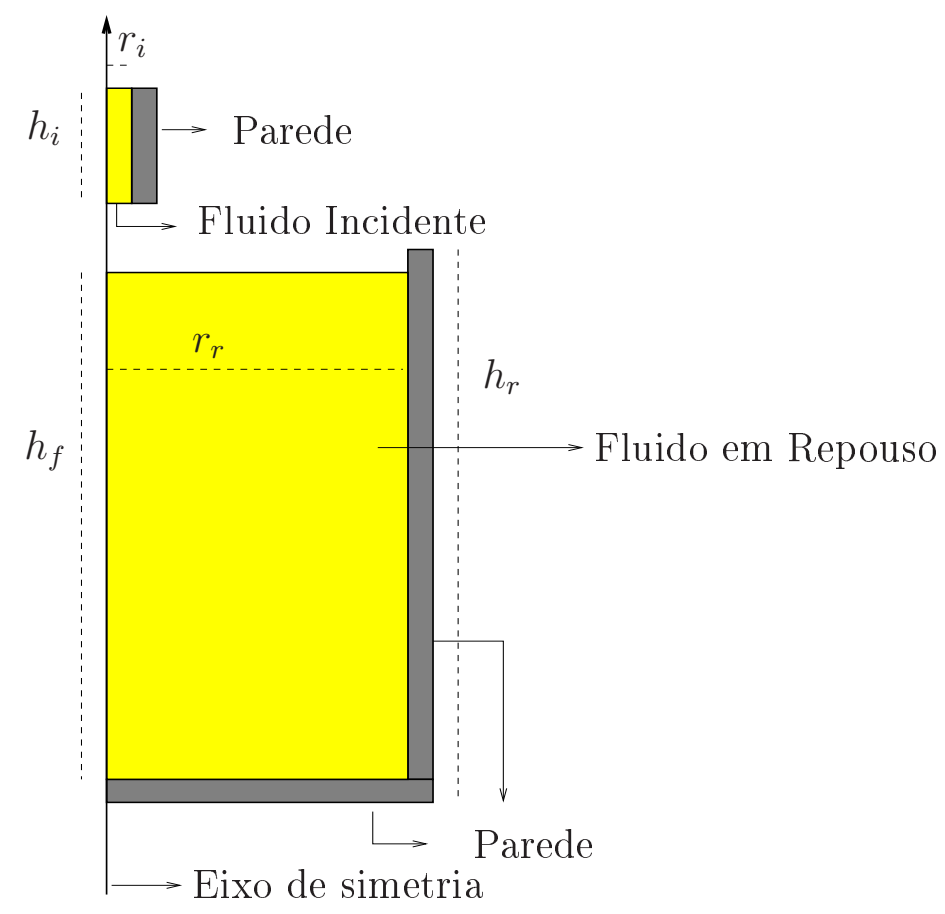

Figura 7.33: Experimento de Taylor. Diagrama esquemático de um jato vertical de fluido incidindo perpendicularmente sobre uma caixa contento fluido em repouso.

Caso 1 - Experimento de Taylor $(\mathrm{Re}=10)$. Neste caso, considera-se uma malha de $123 \times 403$ células computacionais e os seguintes dados para simulação:

- Dimensão do domínio: $0.0615 m \times 0.2015 m$;

- Constante gravitacional: $g=9.81 \mathrm{~m} / \mathrm{s}^{2}$;

- Escala de comprimento: $L=2 r_{i}=0.004 m$;

- Escala de velocidade: $V_{0}=0.5 \mathrm{~m} / \mathrm{s}$;

- Coeficiente de viscosidade cinemática: $\nu=2 \times 10^{-4} \mathrm{~m}^{2} / \mathrm{s}$;

- Número de Reynolds: $R e=\frac{V_{0} \cdot L}{\nu}=10$;

- Número de Froud: $F r=\frac{V_{0}}{\sqrt{g \cdot L}}=2.52409$.

A Figura 7.34 mostra a comparação entre o experimento de Taylor (Fig. 7.34-(a)) e os resultados numéricos obtidos com o esquema FDPUS-C1 (Fig. 7.34-(b)), para $R e=10$, nos tempos $t=0.25 \mathrm{~s}$ e $t=0.75 \mathrm{~s}$. Ainda nesta figura (Fig. 7.34-(c)) apresenta-se uma visão tridimensional do resultado numérico, ou seja, o plano de solução ilustrado na Figura 7.33 é rotacionado em torno do eixo de simetria. Por essa figura, constata-se que o esquema FDPUS-C1 captura bem a estrutura apresentada no experimento de Taylor.

Assim como apresentado para o esquema FDPUS-C1, na Figura 7.35 apresenta-se a comparação do experimento de Taylor (Fig. 7.35-(a)) com os resultados numéricos obtidos com o 


$$
\mathrm{t}=0.25 \mathrm{~s}
$$

(a)

(b)

(c)
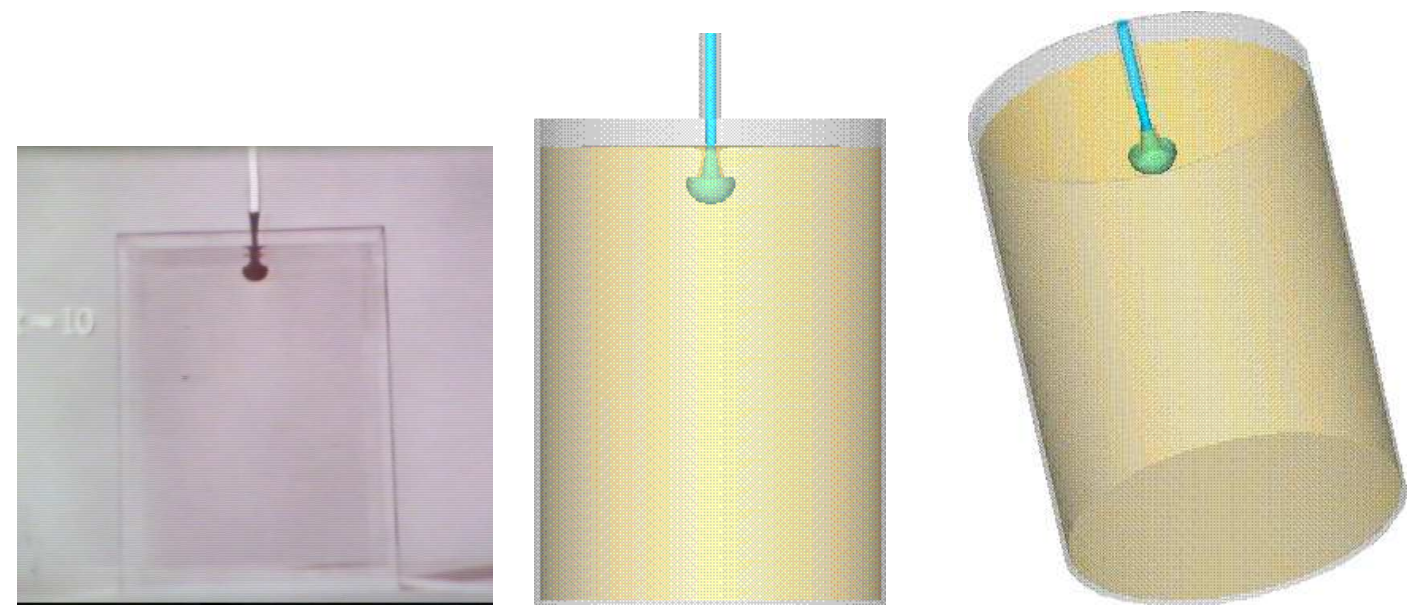

\section{$\mathrm{t}=0.75 \mathrm{~s}$}

(a)

(b)

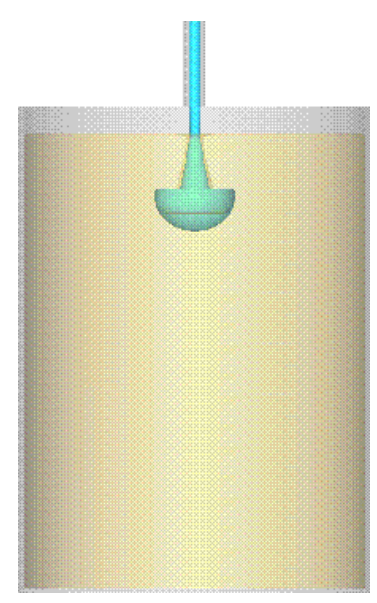

(c)

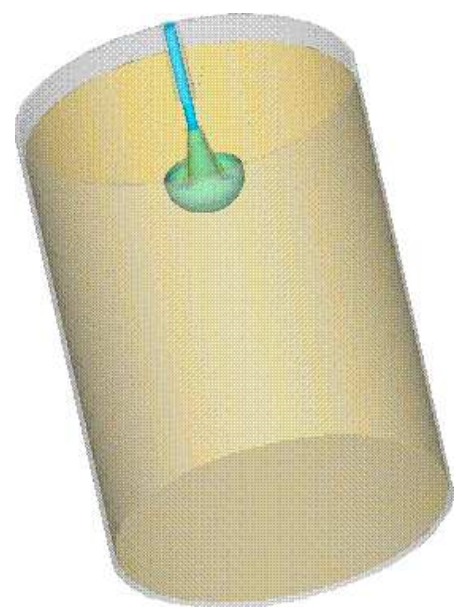

Figura 7.34: Experimento de Taylor $(\mathbf{R e}=\mathbf{1 0})$. Comparação entre os dados experimentais (a) e o esquema FDPUS-C1 (b); visão tridimensional (c). Resultados gerados para $R e=10$,nos tempos $t=0.25$ e $t=0.75$. 
esquema SDPUS-C1 (Fig. 7.35-(b)), para $R e=10$, nos tempos $t=0.25 \mathrm{~s}$ e $t=0.75 \mathrm{~s}$ e uma visão tridimensional do resultado numérico (Fig. 7.35-(c)). Por essa figura, constata-se também que o esquema SDPUS-C1 soluciona bem a estrutura apresentada no experimento de Taylor.

$\mathrm{t}=0.25 \mathrm{~s}$

(a)

(b)

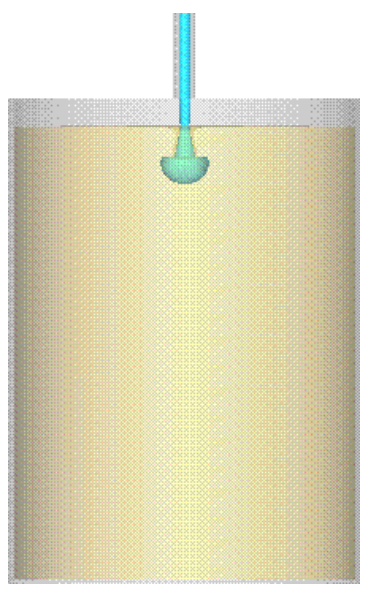

$\mathrm{t}=0.75 \mathrm{~s}$

(a)

(b)

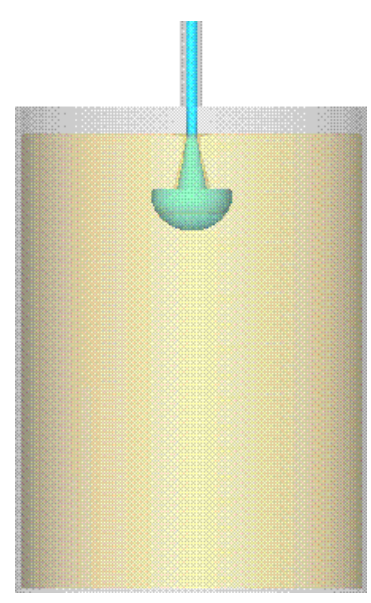

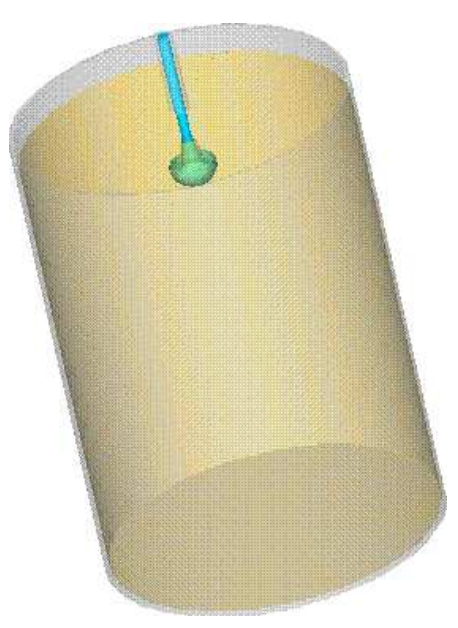

(c)

(c)

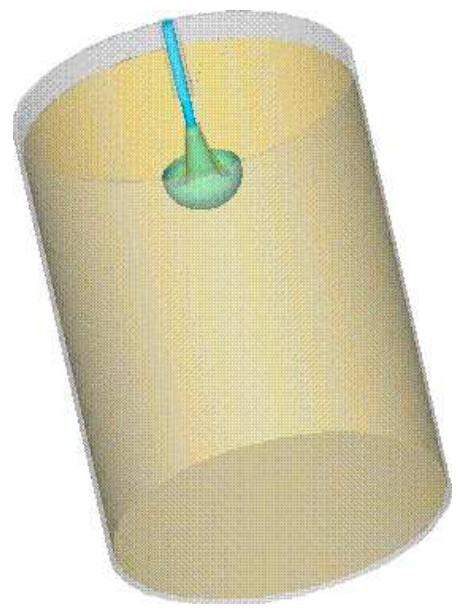

Figura 7.35: Experimento de Taylor $(\mathbf{R e}=\mathbf{1 0})$. Comparação entre os dados experimentais (a) e o esquema SDPUS-C1 (b); visão tridimensional (c). Resultados gerados para $R e=10$,nos tempos $t=0.25 \mathrm{~s}$ e $t=0.75 \mathrm{~s}$.

Como ilustração, apresenta-se a simulação do problema no tempo $t=10 \mathrm{~s}$. Na Figura 7.36, 
apresenta-se a estrutura tipo guada-chuva simulada pelo esquema FDPUS-C1 (Fig. 7.36-(a)). Nessa mesma figura, apresenta-se também uma visão 3D dessa estrutura (Fig. 7.36-(b)). Para complementação, na Figura 7.37, ilustram-se os campos de pressão e velocidade (nas direções $z$ e $r$ ). Os resultados numéricos gerados pelo esquema SDPUS-C1 são similares aos apresentados nessas figuras, portanto são omitidos nesse texto.

$$
\mathrm{t}=10 \mathrm{~s}
$$

(a)

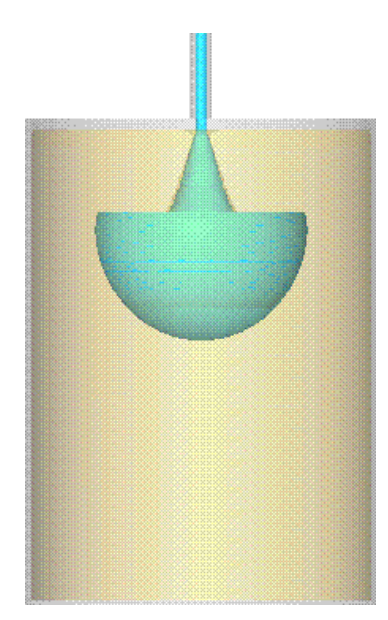

(b)

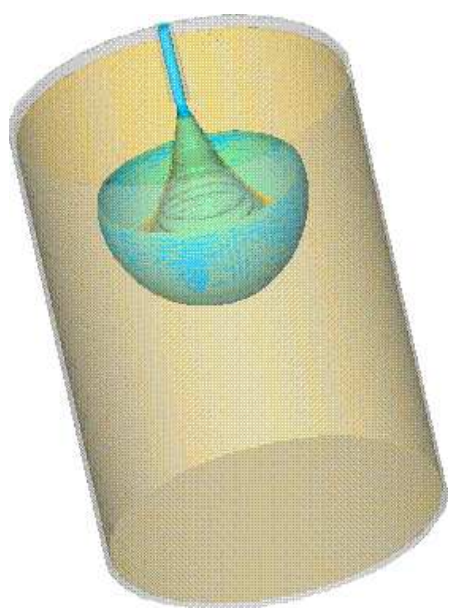

Figura 7.36: Experimento de Taylor $(\mathbf{R e}=\mathbf{1 0})$. Resultados numéricos do esquema FDPUS-C1 (a); visão tridimensional (c). Resultados gerados para $R e=10$ para o tempo $t=10 \mathrm{~s}$.

Caso 2 - Experimento de Taylor com $(\mathrm{Re}=200)$. Neste caso, considera-se uma malha de $123 \times 403$ células computacionais e os seguintes dados para simulação:

- Dimensão do domínio: $0.0615 m \times 0.2015 m$;

- Constante gravitacional: $g=9.81 \mathrm{~m} / \mathrm{s}^{2}$;

- Escala de comprimento: $L=2 r_{i}=0.004 m$;

- Escala de velocidade: $V_{0}=0.5 \mathrm{~m} / \mathrm{s}$;

- Coeficiente de viscosidade cinemática: $\nu=10^{-5} \mathrm{~m}^{2} / \mathrm{s}$;

- Número de Reynolds: $R e=\frac{V_{0} \cdot L}{\nu}=200$;

- Número de Froud: $\operatorname{Fr}=\frac{V_{0}}{\sqrt{g \cdot L}}=2.52409$.

A Figura 7.38 mostra a comparação entre os experimentos de Taylor (Fig. 7.38-(a) e as simulações numéricas obtidas com o esquema FDPUS-C1 (Fig. 7.38-(b)) a $R e=200$, nos tempos $t=0.75 \mathrm{~s}$ e $t=2.5 \mathrm{~s}$. Na Figura 7.39 apresenta-se a comparação entre os experimentos de Taylor (Fig. 7.39-(a)) e os resultados numéricos obtidos com o esquema SDPUS-C1 (Fig. 
Campo de pressão

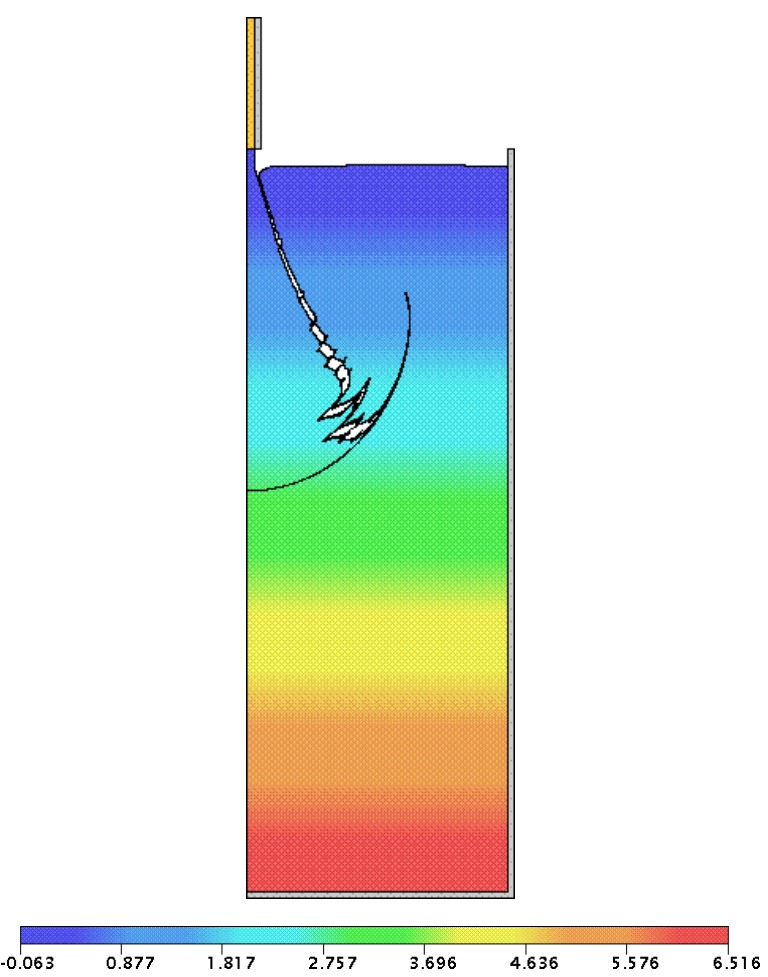

Campo de velocidade na direção $r \quad$ Campo de velocidade na direção $y$
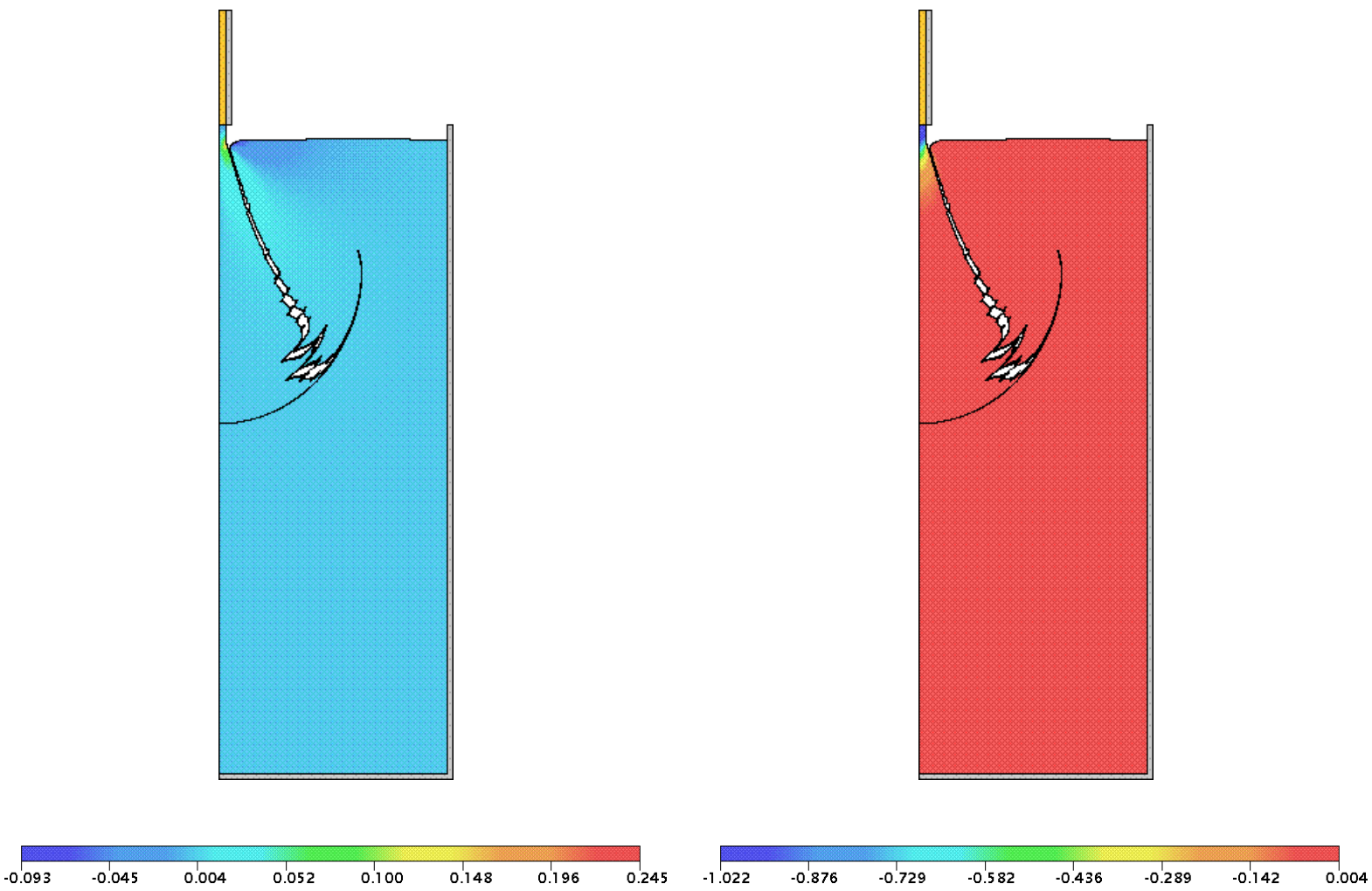

Figura 7.37: Experimento de Taylor $(\mathbf{R e}=\mathbf{1 0})$. Resultados numéricos do esquema FDPUS-C1, com respeito a evolução da superfície livre do fluido para os campos de pressão e velocidade (nas direções $x$ e $y$ ), no tempo $t=10 \mathrm{~s}$. 
7.39-(b)) a $R e=200$, nos tempos $t=0.75 \mathrm{~s}$ e $t=2.5 \mathrm{~s}$. Em particular, para $t=2.5 \mathrm{~s}$, o jato alcança o fundo da caixa formando um estrutura toroidal. Como pode ser observado por essas figuras os resultados obtidos com os esquemas FDPUS-C1 e SDPUS-C1 são bastante coerentes com os dados experimentais de Taylor.

$$
\mathrm{t}=0.25 \mathrm{~s}
$$

(a)

(b)
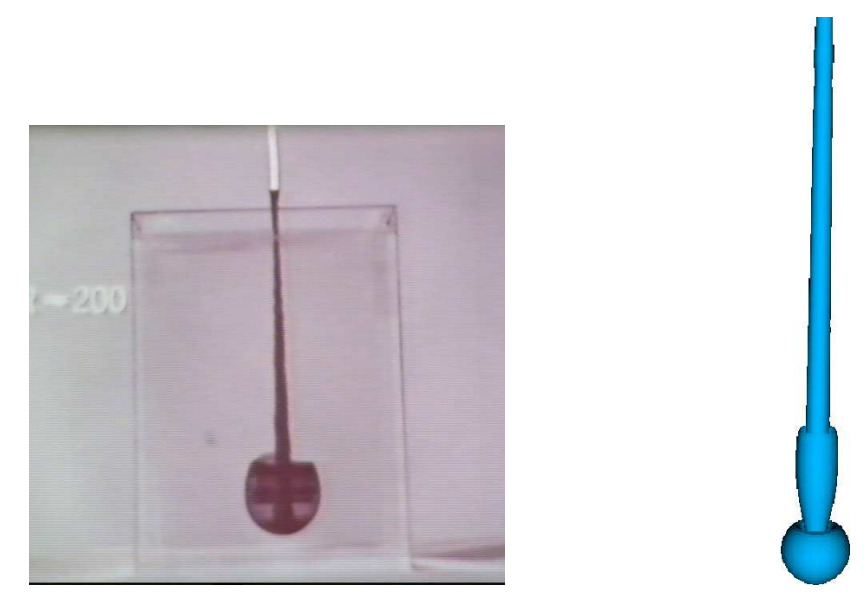

$\mathbf{t}=0.75 \mathrm{~s}$

(a)

(b)
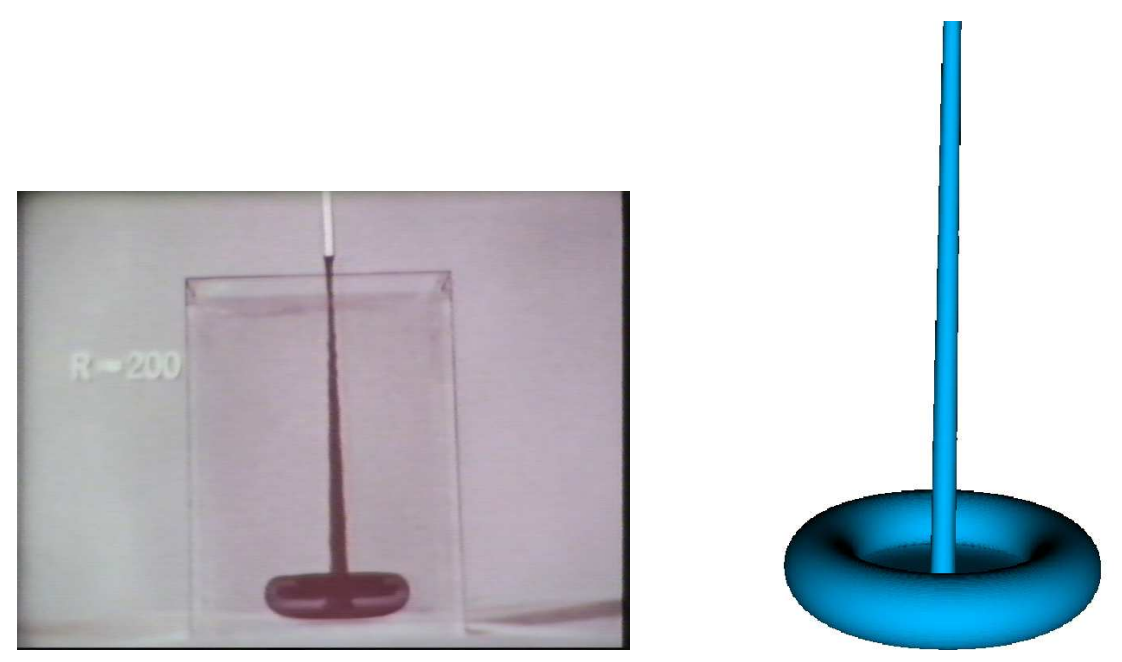

Figura 7.38: Experimento de Taylor $(\mathbf{R e}=\mathbf{2 0 0})$. Comparação entre os dados experimentais (a) e o esquema FDPUS-C1 (b), para $R e=200$, nos tempos $t=0.25 \mathrm{~s}$ e $t=0.75 \mathrm{~s}$. 
$\mathrm{t}=0.25 \mathrm{~s}$

(a)

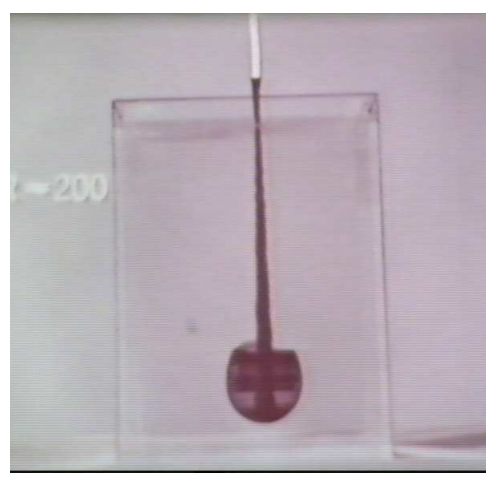

$\mathbf{t}=0.75 \mathrm{~s}$

(a)

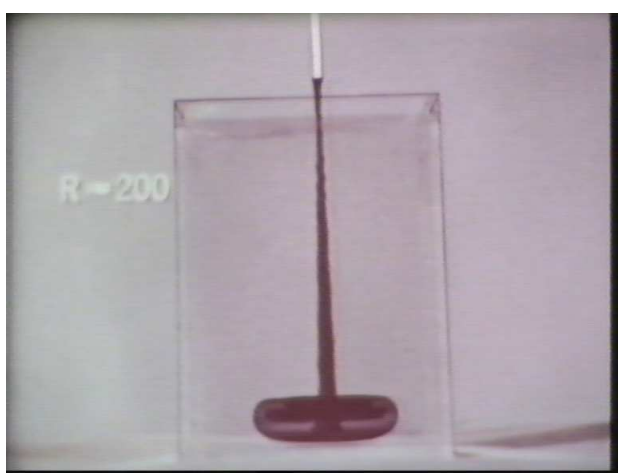

(b)

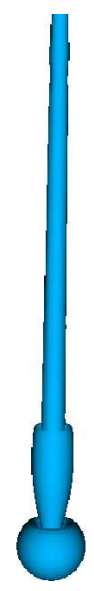

(b)

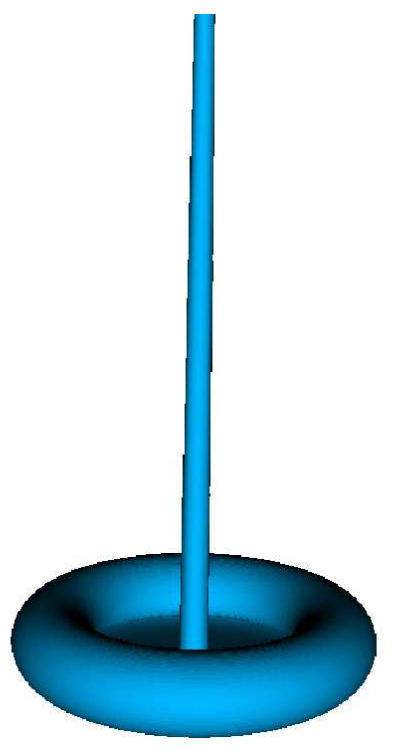

Figura 7.39: Experimento de Taylor $(\mathbf{R e}=\mathbf{2 0 0})$. Comparação entre os dados experimentais (a) e o esquema SDPUS-C1 (b), para $R e=200$, nos tempos $t=0.25 \mathrm{~s}$ e $t=0.75 \mathrm{~s}$. 
Como ilustração, considera-se a simulação do problema para $t=10 \mathrm{~s}$, tempo este que acontece a formação da estrutura vortical principal, com pode ser observado na Figura 7.40. Nessa figura, apresenta-se a estrutura completa simulada com o esquema SDPUS-C1 (Fig. 7.40-(a)). Apresenta-se também, nessa mesma figura, essa estrutura com um corte (Fig. 7.40-(b)), em que é possível visualizar as estruturas vórticais capturadas pela solução numérica gerada com o esquema SDPUS-C1. Para complementação, na Figura 7.41, ilustram-se os campos de pressão e velocidade (nas direções $z$ e $r$ ). Os resultados numéricos obtidos com o esquema FDPUS-C1 são desconsiderados, uma vez que apresentam similaridade com os gerados com o esquema SDPUS-C1.

$$
\mathrm{t}=10 \mathrm{~s}
$$

(a)

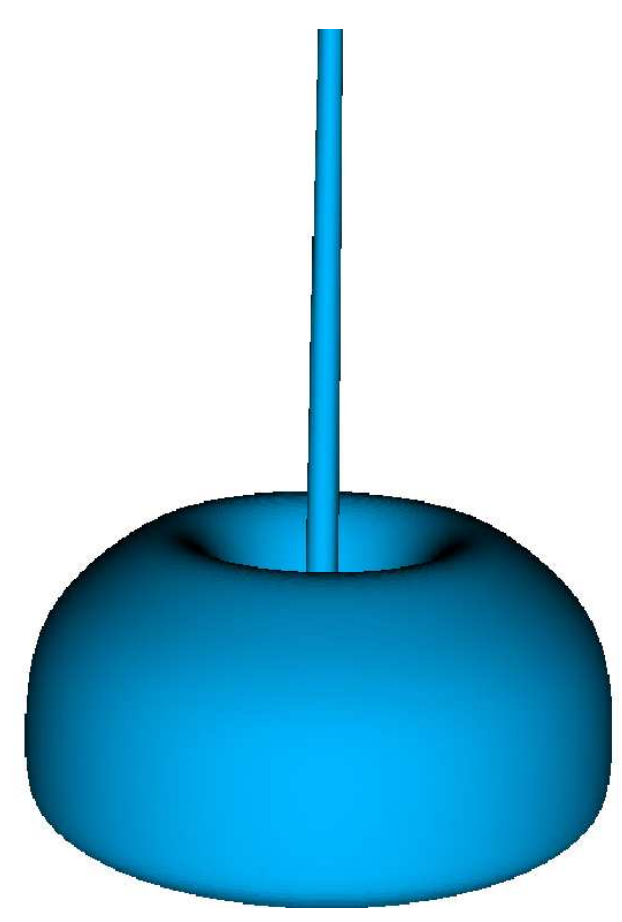

(b)

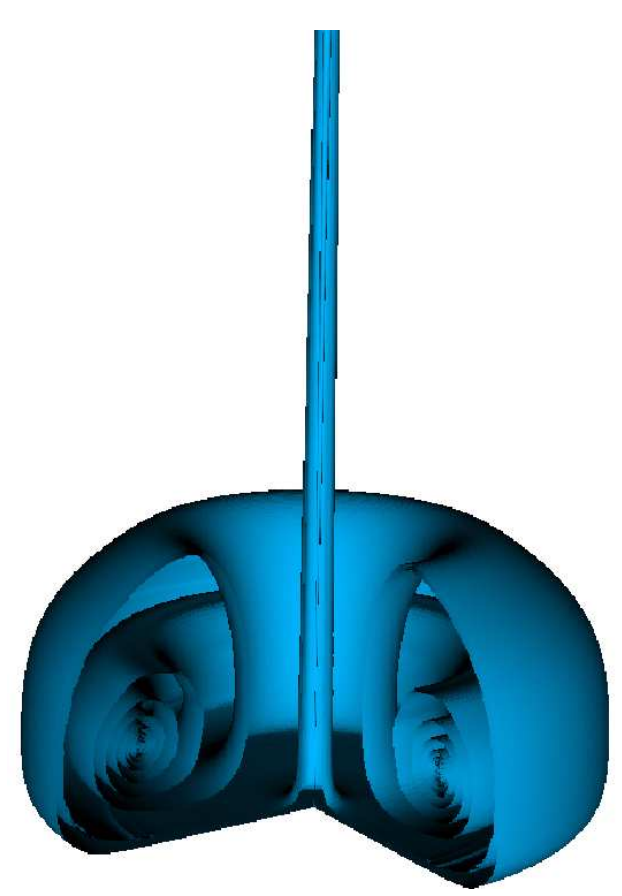

Figura 7.40: Experimento de Taylor $(\mathbf{R e}=\mathbf{2 0 0})$. Estrutura completa (a); estrutura com corte (b) capturada pelo esquema SDPUS-C1, a $R e=200$ e no tempo $t=10 \mathrm{~s}$. 


\section{Campo de pressão}

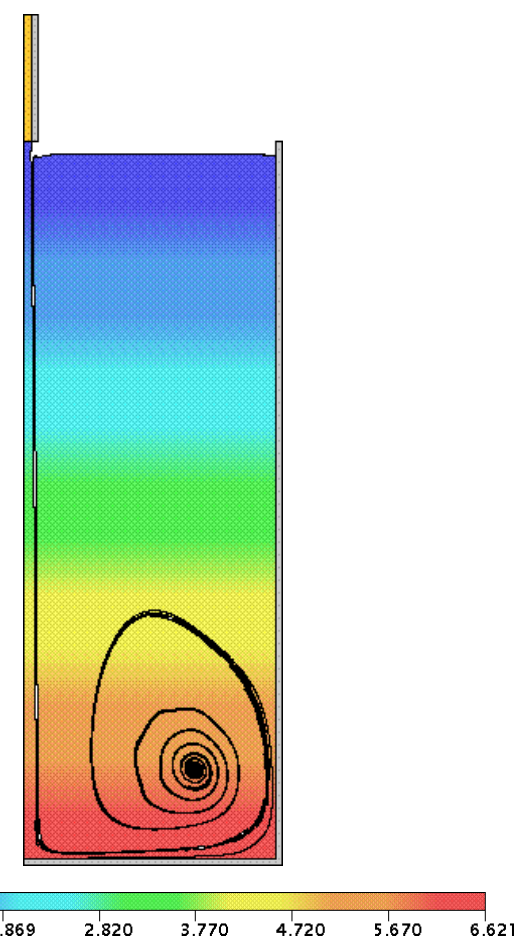

Campo de velocidade na direção $r$ Campo de velocidade na direção y
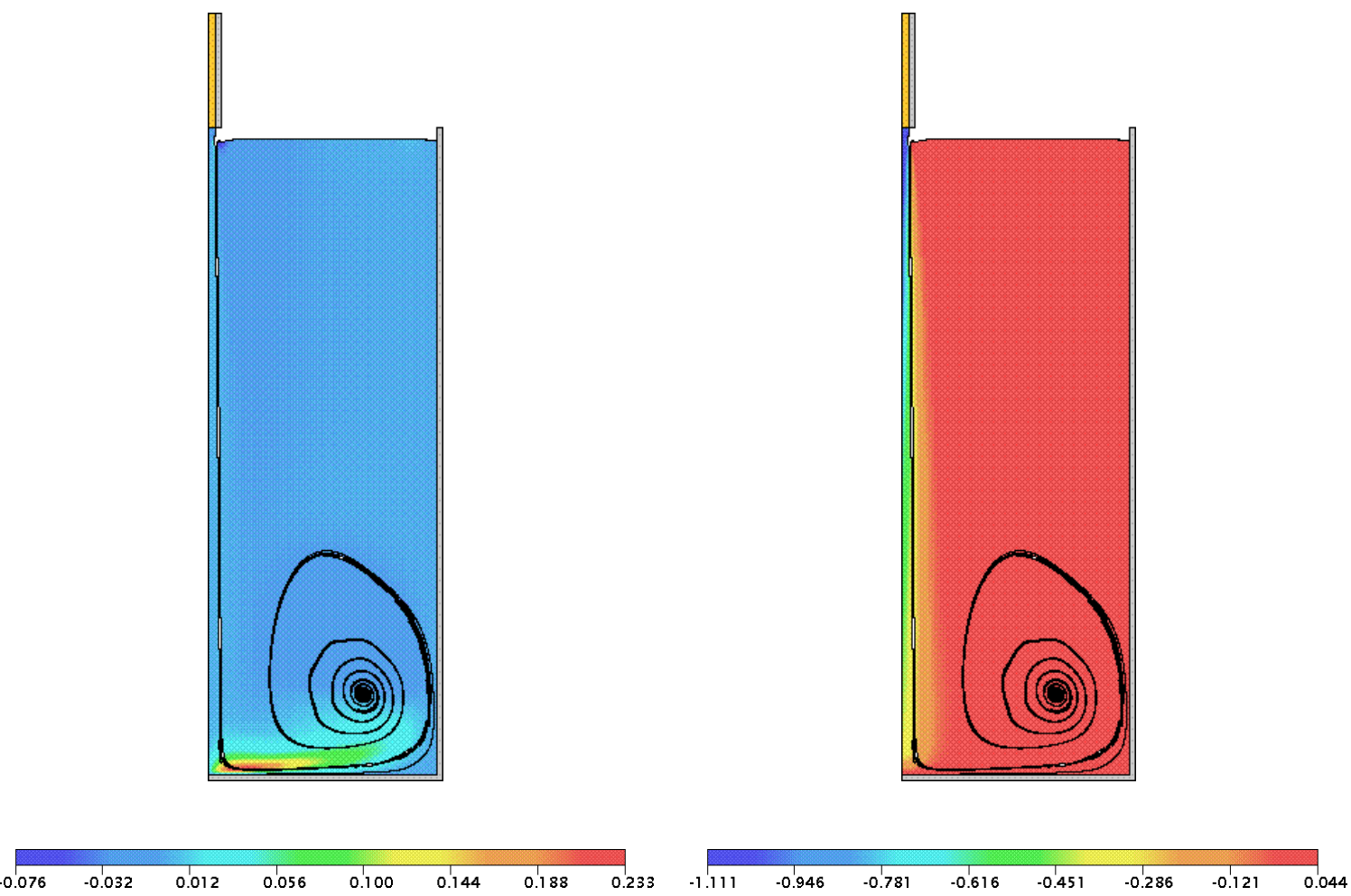

Figura 7.41: Experimento de Taylor $(\mathbf{R e}=\mathbf{2 0 0})$. Resultados numéricos do esquema SDPUS-C1, com respeito a evolução da superfície livre do fluido para os campos de pressão e velocidade (nas direções $x$ e $y$ ), no tempo $t=10 \mathrm{~s}$. 


\subsection{Simulação de Escoamentos Viscoelásticos}

Nesta seção são apresentados os resultados numéricos para simulação de escoamentos incompressíveis viscoelásticos de um fluido Oldroyd-B. Para tanto, os esquemas FDPUS-C1 e SDPUS-C1 são implementados no código Freeflow 2D [12] (versão incrementada com o modelo Oldroyd-B). Os resultados numéricos gerados pelo esquema SDPUS-C1 são comparados com dados teóricos. Os resultados numéricos obtidos com o esquema FDPUS-C1 são omitidos nesse texto pois são similares aos resultados do esquema SDPUS-C1.

\subsubsection{Escoamento em um Canal}

Nesse problema é considerado um canal de placas paralelas, com um injetor na extremidade direita e um ejetor na extremidade esquerda (ver Figura 7.42). Inicialmente, o fluido é injetado na seção de entrada e, após um determinado tempo, ele alcança a região de saída. Esse problema apresenta solução analítica determinada por Bird et al. [8], para o estado estacionário, dadas, para a velocidade e para os tensores de tensão, por

$$
\begin{gathered}
u(y)=-6 y(y-L), \quad v(y)=0, \\
T_{11}(y)=2 W e(1-\beta)\left(\frac{\partial u}{\partial y}\right)^{2}, \quad T_{12}(y)=(1-\beta)\left(\frac{\partial u}{\partial y}\right) \text { e } \quad T_{22}(y)=0 .
\end{gathered}
$$

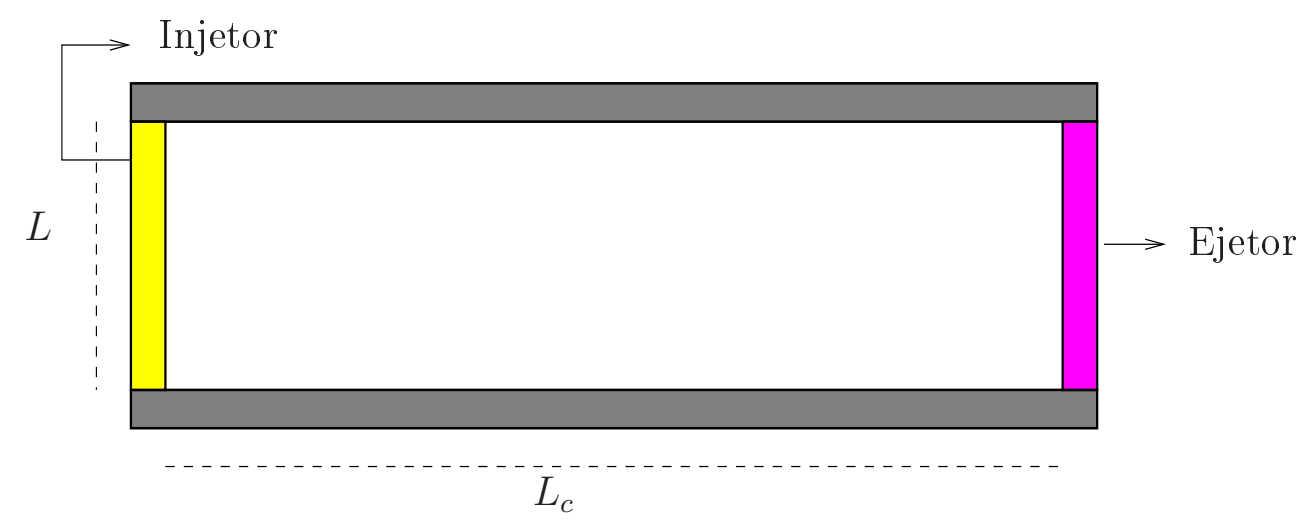

Figura 7.42: Escoamento em um canal. Diagrama esquemático de um canal de placas paralelas.

Caso 1 - Escoamento em um canal de placas paralelas. Para a simulação deste problema são consideradas três malhas com $25 \times 5,50 \times 10$ e $100 \times 20$ células computacionais e os demais dados: 
- Dimensão do domínio: $5 m \times 1 m$;

- Constante gravitacional: $g=9.81 \mathrm{~m} / \mathrm{s}^{2}$;

- Escala de comprimento: $L=1 m$;

- Comprimento do canal: $L_{c}=5 L=5 \mathrm{~m}$;

- Escala de velocidade: $V_{0}=1 \mathrm{~m} / \mathrm{s}$;

- Número de Reynolds: $R e=0.1$;

- Número de Weissenberg: $W e=2$;

- Razão de Retardamento: $\beta=0.5$.

A Figura 7.43 mostra a comparação entre os resultados numéricos gerados pelo esquema SDPUS-C1 e os perfis exatos, para as variáveis $u, T_{11}$ e $T_{12}$, tomadas em $x=2.5 L_{c}$ e no tempo $t=100 \mathrm{~s}$. Por essa figura, constata-se que os resultados numéricos estão em ótima concordância com as soluções dos perfis exatos.

Para completar a validação do método para esse problema, os erros relativos na norma $L_{2}$ $\left(\|E\|_{2}\right)$, cometidos pelo esquema SDPUS-C1 para a aproximação de $u, T_{x x}=T_{11}$ e $T_{x y}=T_{12}$, são calculados para as três malhas e a ordem de convergência do método é estimada. Esses dados estão apresentados na Tabela $7.1 \mathrm{em}$ que pode ser constatado que, na média, o método numérico fornece resultados de segunda ordem.

\begin{tabular}{c|ccc}
\hline \hline Componente & Malha & $\|E\|_{2}$ & Ordem de Convergência \\
\hline \hline$u$ & $25 \times 5$ & $0.54611 \times 10^{-1}$ & - \\
& $50 \times 10$ & $0.14954 \times 10^{-1}$ & 1.8686 \\
& $100 \times 20$ & $0.36004 \times 10^{-2}$ & 2.0543 \\
\hline \hline$T_{11}$ & $25 \times 5$ & $0.94033 \times 10^{-1}$ & - \\
& $50 \times 10$ & $0.15752 \times 10^{-1}$ & 2.5776 \\
& $100 \times 20$ & $0.69010 \times 10^{-2}$ & 1.1907 \\
\hline \hline$T_{12}$ & $25 \times 5$ & $0.58428 \times 10^{-1}$ & - \\
& $50 \times 10$ & $0.51242 \times 10^{-1}$ & 3.5113 \\
& $100 \times 20$ & $0.25099 \times 10^{-2}$ & 1.0297 \\
\hline \hline
\end{tabular}

Tabela 7.1: Escoamento em um canal de placas paralelas. Erros relativos na norma $L_{2}$ e estimativa da ordem de convergência para o esquema SDPUS-C1, para os perfis $u, T_{11}$ e $T_{12}$. 
Componente $u$ da velocidade

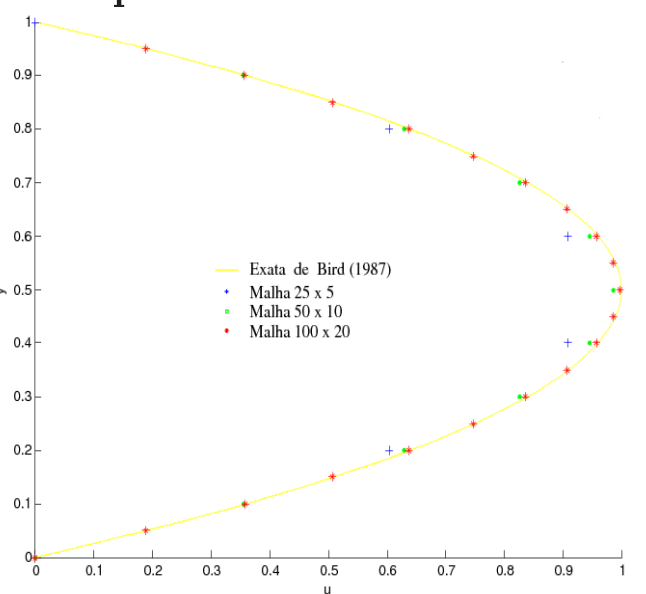

Componente $T_{11}$ do tensor de tensão

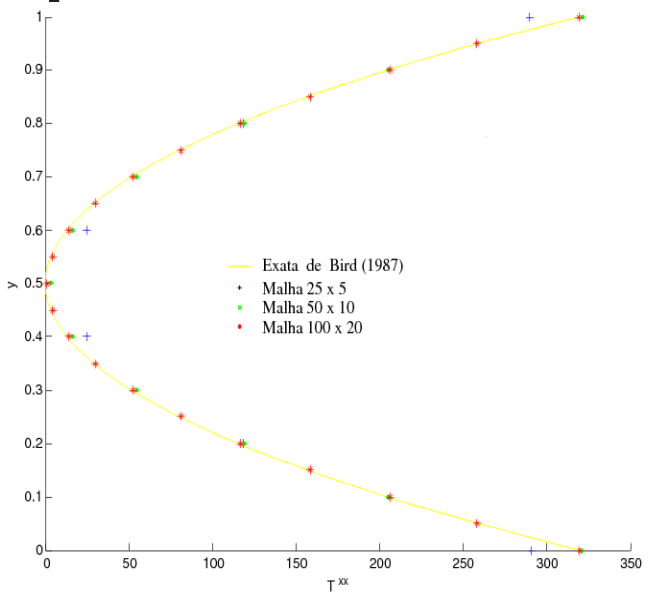

Componente $T_{12}$ do tensor de tensão

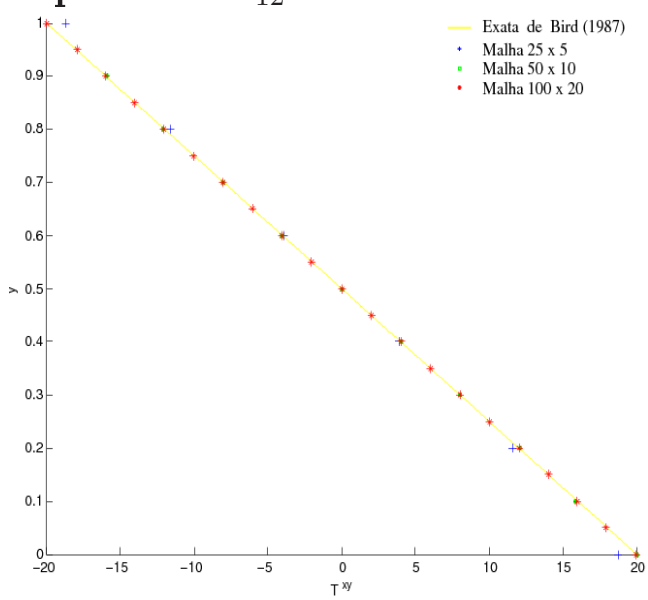

Figura 7.43: Escoamento em um canal de placas paralelas. Comparação entre os perfis exatos e os numéricos (esquema SDPUS-C1) para $u, T_{x x}=T_{11}$ e $T_{x y}=T_{12}$. 


\subsubsection{Inchamento do Extrudado}

Esse problema consiste na extrusão do fluido a partir de um canal inicial de placas paralelas. O fluido é injetado na extremidade esquerda do canal, desenvolve-se e é extrudado na extremidade direita do canal, com uma certa distância do ejetor (ver Figura 7.44).

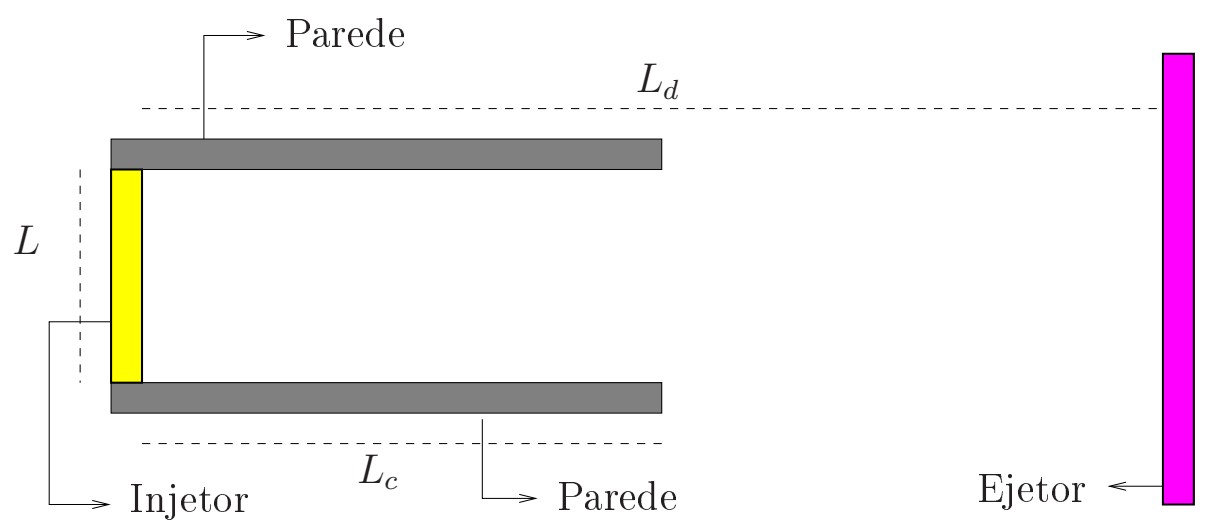

Figura 7.44: Inchamento do Extrudado. Representação esquemática.

Caso 1 - Inchamento do Extrudado. Para a simulação deste caso considera-se uma malha com $80 \times 20$ células computacionais e os seguintes dados:

- Dimensão do domínio: $10 m \times 1 m$;

- Dimensão do canal: $L_{c}=4 m$ e $L=1 m$;

- Constante gravitacional: $g=9.81 \mathrm{~m} / \mathrm{s}^{2}$;

- Escala de comprimento: $L=1 m$;

- Escala de velocidade: $V_{0}=1 \mathrm{~m} / \mathrm{s}$;

- Número de Reynolds: $R e=0.1$;

- Números de Weissenberg: $W e=0.2,0.4, \quad 0.5, \quad 0.6,0.8,1$ e 1.2;

- Razão de Retardamento: $\beta=0.64$.

Os resultados numéricos, obtidos para a taxa de inchamento $S_{r}$ em função de $W e$, são comparados com a solução analítica de Tanner (ver Bird et al. [8] e Tomé et al. [66]), dada por

$$
S_{r}=0.1+\left[1+18(W e)^{2}\right]^{\frac{1}{2}} .
$$

A Figura 7.45 mostra a comparação da solução analítica de Tanner para $S_{r}$ em função de $W e$, com os resultados numéricos obtidos com o esquema SDPUS-C1, no tempos $t=9 \mathrm{~s}$ e $t=13 \mathrm{~s}$ e para o estado estacionário. 


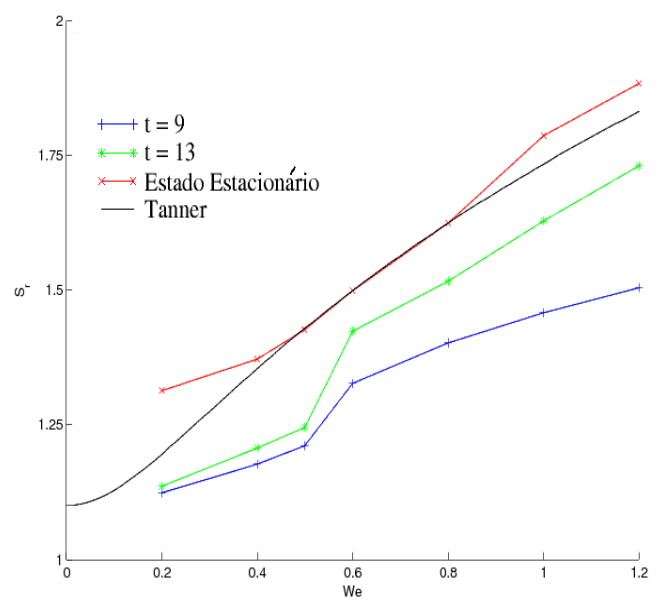

Figura 7.45: Inchamento do Extrudado. Comparação entre a solução analítica (para $S_{r}$ em função de $W e$ ) e o esquema SDPUS-C1 para $t=9 s, t=13 s$ e estado estacionário.

Na Tabela 7.2 apresenta-se a massa numérica (calculada pela vazão no injetor), nos tempos $t=4 s, t=9 s$ e $t=13 s$ (para os valores de $W e=0.2, \quad 0.4,0.5,0.6,0.8,1$ e 1.2). Ainda nessa tabela, apresentam-se o erro relativo dado por

$$
E_{R}=\frac{\mid \text { Massa num. }- \text { Massa exata } \mid}{\mid \text { Massa exata } \mid},
$$

cometidos por esse esquema, uma vez que as massas exatas, calculadas pela vazão no injetor, são dadas por $2.6642(\mathrm{em} t=4 s), 5.9944(\mathrm{em} t=9 \mathrm{~s})$ e $8.6586(\mathrm{em} t=13 \mathrm{~s})$. Para mais detalhes ver Martins [48]. Por essa tabela, ve-se claramente que o esquema SDPUS-C1 apresenta resultados bastantes satisfatórios.

\begin{tabular}{l|c|c|c|c|c|c}
\hline \multirow{2}{*}{$W e$} & \multicolumn{2}{|c|}{$t=4 s$} & \multicolumn{2}{c|}{$t=9 s$} & \multicolumn{2}{c}{$t=13 s$} \\
\cline { 2 - 7 } & Massa Num. & $E_{R}$ & Massa Num. & $E_{R}$ & Massa Num. & $E_{R}$ \\
\hline \hline 0.2 & 2.6639 & $0.126 \times 10^{-3}$ & 5.9914 & $0.497 \times 10^{-3}$ & 8.6515 & $0.823 \times 10^{-3}$ \\
0.4 & 2.6636 & $0.224 \times 10^{-3}$ & 5.9930 & $0.229 \times 10^{-3}$ & 8.6573 & $0.148 \times 10^{-3}$ \\
0.6 & 2.6635 & $0.261 \times 10^{-3}$ & 5.9946 & $0.230 \times 10^{-4}$ & 8.6518 & $0.374 \times 10^{-3}$ \\
0.8 & 2.6632 & $0.358 \times 10^{-3}$ & 5.9923 & $0.350 \times 10^{-3}$ & 8.6521 & $0.752 \times 10^{-3}$ \\
1 & 2.6631 & $0.396 \times 10^{-3}$ & 5.9921 & $0.390 \times 10^{-3}$ & 8.6518 & $0.791 \times 10^{-3}$ \\
1.2 & 2.6630 & $0.435 \times 10^{-3}$ & 5.9921 & $0.386 \times 10^{-3}$ & 8.6571 & $0.171 \times 10^{-3}$ \\
\hline \hline
\end{tabular}

Tabela 7.2: Inchamento do Extrudado. Massa numérica calculada pela vazão do injetor e erros relativos cometidos pelo esquema SDPUS-C1.

Como ilustração, nas Figuras 7.46 e 7.47 apresentam-se as soluções numéricas em $t=0.13 \mathrm{~s}$ e no estado estacionário, respectivamente, obtidas com o esquema SDPUS-C1 para o inchamento do extrudado de um fluido Oldroyd-B, com $W e=0.6$, para $u, v, T_{x x}, T_{x y}$ e $T_{y y}$. 

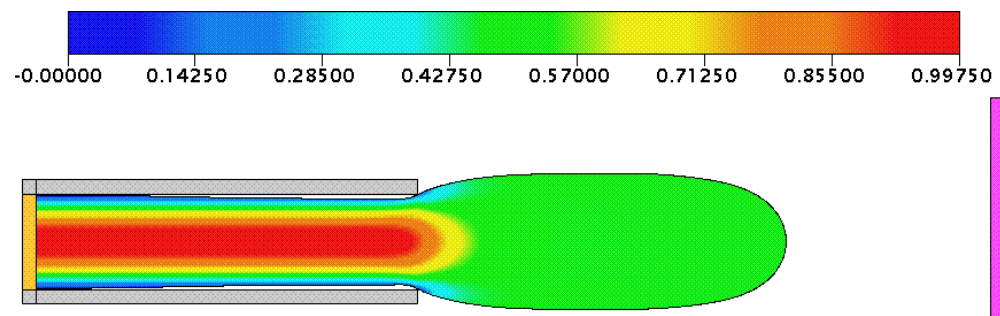

$u$

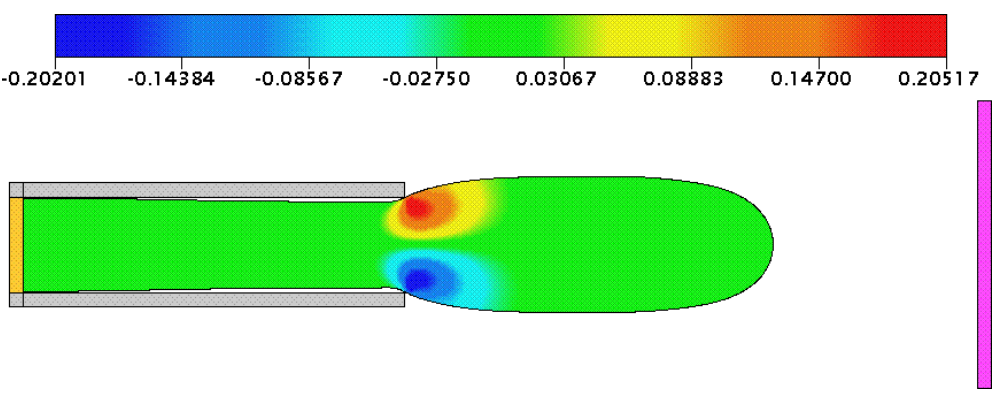

$v$
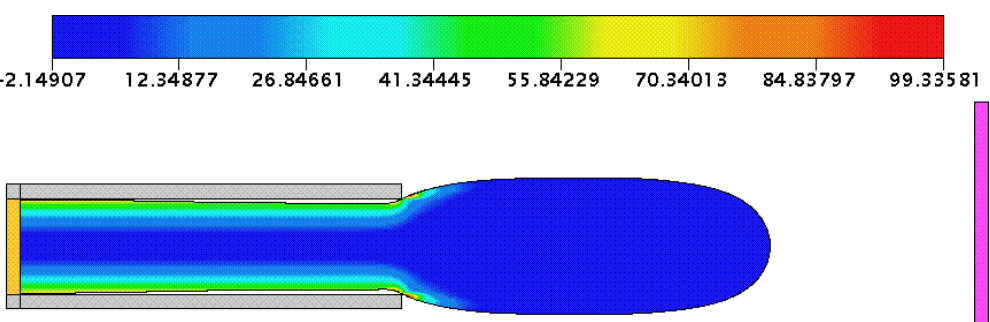

$T_{x x}$
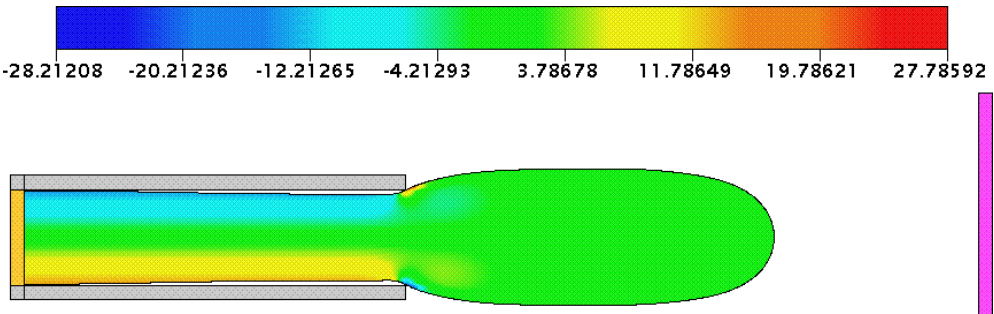

$T_{x y}$
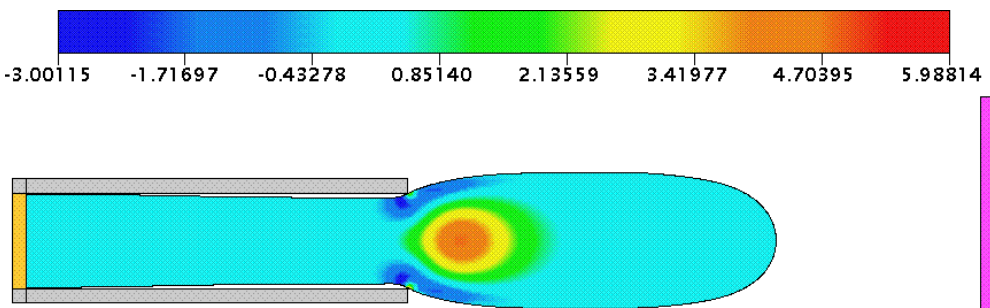

$T_{y y}$

Figura 7.46: Inchamento do Extrudado. Soluções numéricas do esquema SDPUS-C1, para o inchamento do extrudado de um fluido Oldroyd-B, com $W e=0.6$, em $t=0.13 s$, para $u, v, T_{x x}$, $T_{x y}$ e $T_{y y}$. 

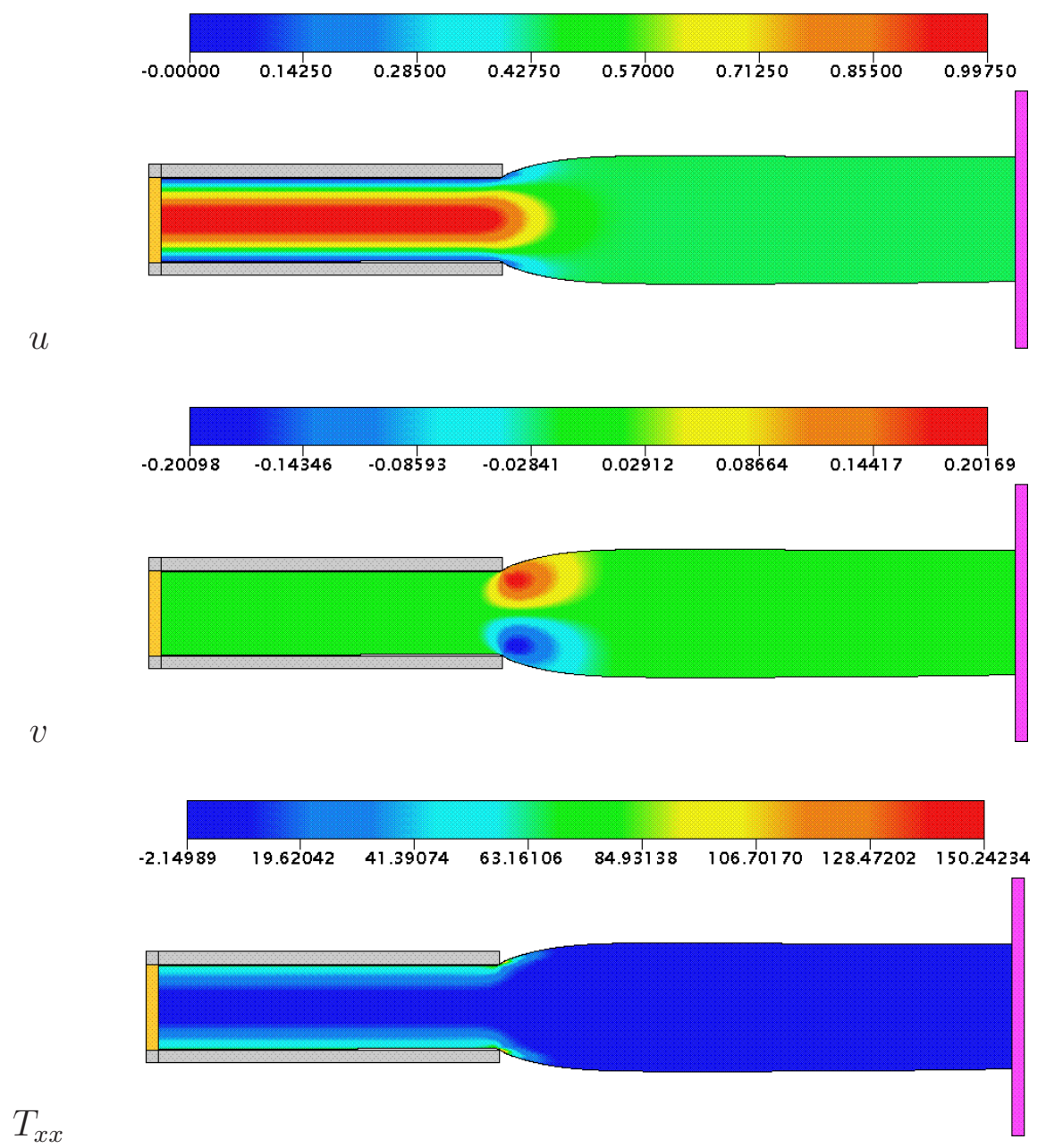

$T_{x x}$
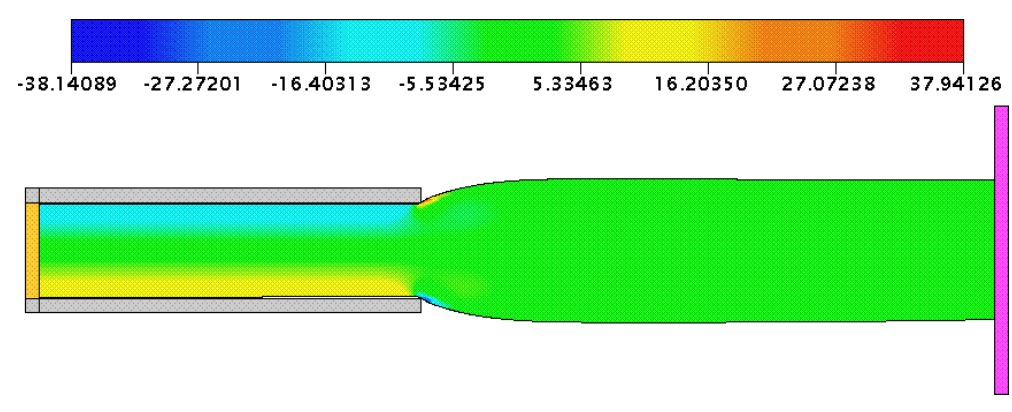

$T_{x y}$
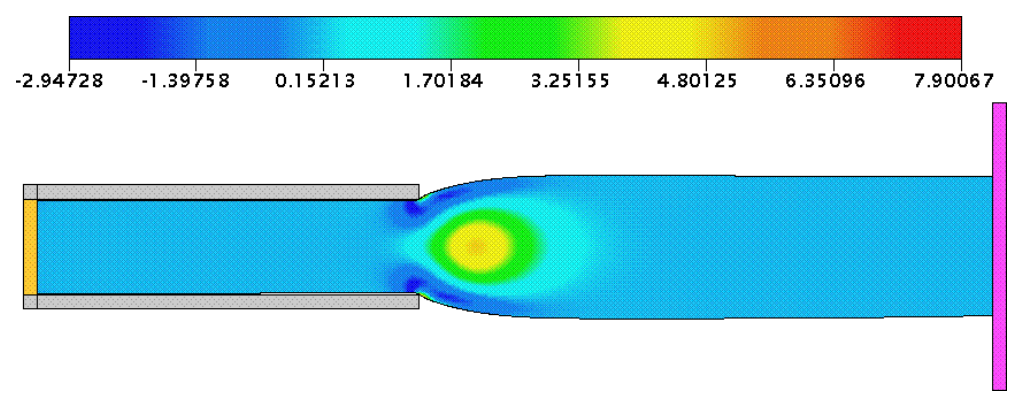

$T_{y y}$

Figura 7.47: Inchamento do Extrudado. Soluções numéricas do esquema SDPUS-C1, para o inchamento do extrudado de um fluido Oldroyd-B, com $W e=0.6$, no estado estacionário, para $u, v$, $T_{x x}, T_{x y}$ e $T_{y y}$. 


\subsection{Escoamento de Fluido Incompressível Turbulento}

Nesta seção são apresentados os resultados numéricos de simulações de escoamentos de fluidos incompressíveis em regime turbulento. Esses resultados são gerados no código Freeflow 2D [12] (incrementado com o modelo de turbulência $\kappa-\varepsilon$ ) em que os esquemas FDPUS-C1 e SDPUS-C1 são implementados. Os resultados obtidos com o esquema SDPUS-C1 não são apresentados, uma vez que são similares aos resultados do esquema FDPUS-C1.

\subsubsection{Colapso de uma Coluna de Fluido}

O esquema FDPUS-C1 é validado na simulação do problema do colapso de uma coluna de fluido, em regime turbulento. Esse problema é descrito na seção 7.2.1. Os resultados obtidos com o esquema FDPUS-C1, para o espalhamento horizontal da coluna de fluido, são comparados com os dados experimentais de Martim e Moyce [47] e Koshizuka e Oka [37].

Caso 1 - Colapso de uma coluna de fluido em regime turbulento. Os dados utilizados para simulação desse caso com turbulência são os mesmo utilizados no Caso1 - Colapso de fluido (Martim e Moyce e Koshizuka e Oka), apresentados na seção 7.2.1.

A Figura 7.48 mostra a comparação entre os dados experimentais, de Martim e Moyce [47] e Koshizuka e Oka [37], e os resultados numéricos gerados com o esquema FDPUS-C1, para a posição frontal do escoamento do fluido $\left(x_{\max }\right)$ em função do tempo, para as três malhas consideradas (com $150 \times 75,300 \times 150$ e $600 \times 300$ células computacionais). Por essa figura, constata-se que os resultados numéricos estão em boa concordância com os dados experimentais.

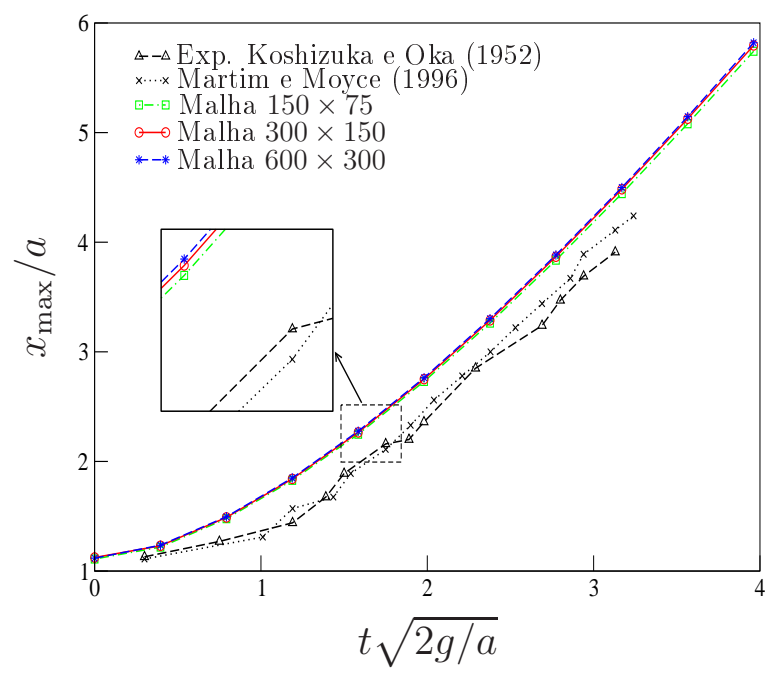

Figura 7.48: Colapso de uma coluna fluido em regime turbulento. Comparação entre os dados experimentais e o esquema FDPUS-C1 para $x_{\max }$ em função do tempo.

Como ilustração, na Figura 7.49 apresenta-se o resultado numérico gerado pelo esquema FDPUS-C1 com respeito a evolução da superfície livre do fluido para o campo de pressão em $t=0.2 s$. 


\section{Campo de pressão}

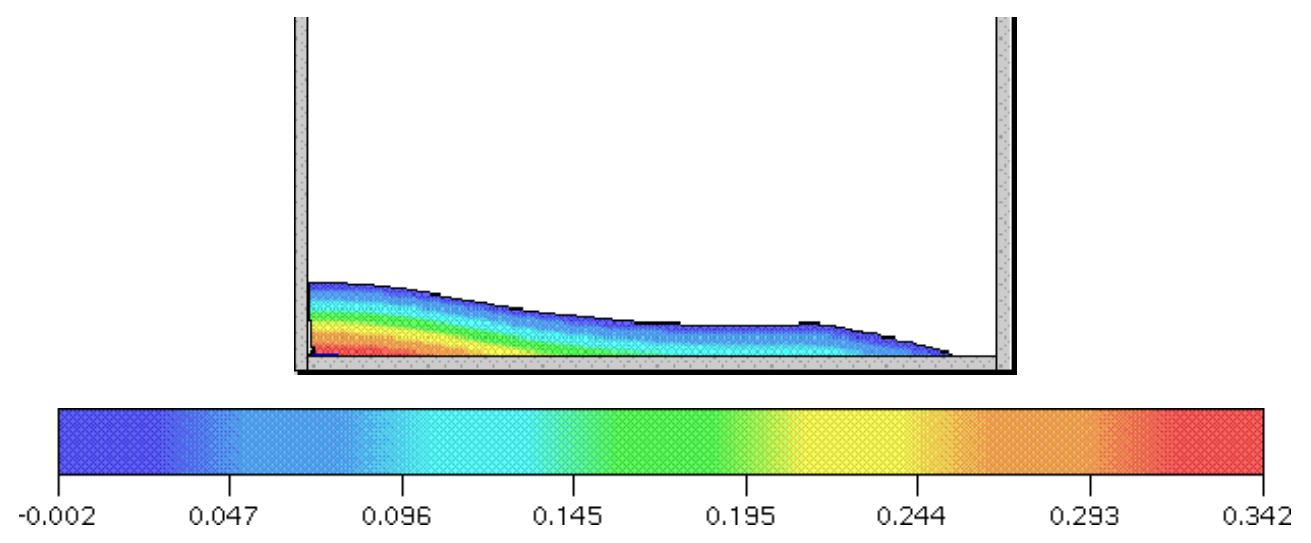

Figura 7.49: Colapso de uma coluna de fluido em regime turbulento. Resultados numéricos do esquema FDPUS-C1 para a evolução da superfície livre do fluido para o campo de pressão em $t=0.2 s$. 


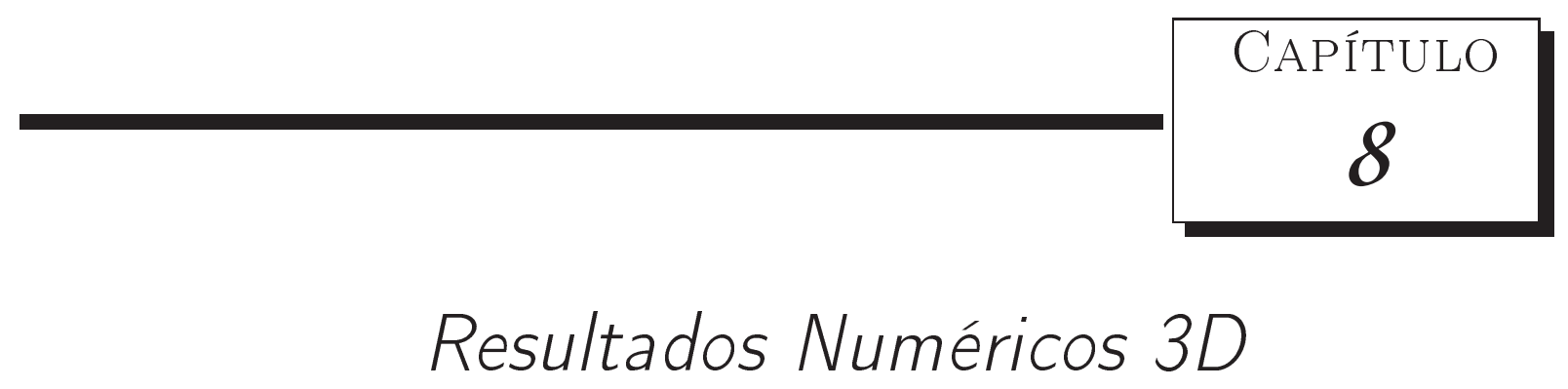

Este capítulo tem por objetivo mostrar que os esquemas FDPUS-C1 e SDPUS-C1 são apropriados para a simulação de problemas 3D complexos de escoamentos de fluidos incompressíveis com superfície livres móveis modelados pelas equações de Navier-Stokes. Os resultados numéricos são gerados por meio do ambiente de simulação Freeflow 3D de Castelo et al. [12], equipado com os esquemas FDPUS-C1 e SDPUS-C1.

\subsection{Ressalto Hidráulico Circular}

O fenômeno salto hidráulico circular ocorre com frequência no dia-a-dia. Por exemplo, ao se abrir a torneira da pia da cozinha, um jato circular de água incide perpendicularmente à cuba (superfície rígida) e espalha radialmente a partir do ponto de impacto. Após um determinado raio, surge uma onda estacionária, como ilustrado na Figura 8.1. Kurihara [39] e Tani [63] afirmam que essa descontinuidade se deve ao fato de que durante o espalhamento, a espessura do fluido diminui, então, uma certa distância cresce repentinamente sob à ação do gradiente de pressão adverso. Esse gradiente de pressão causa separação da película em alguma distância radial, levando à formação de um fenômeno conhecido por ressalto hidráulico circular. Os problemas que exibem o fenômeno de ressalto hidráulico são excelentes testes para a validação de métodos numéricos para resolução de problemas de escoamentos com superfícies livres. Os resultados numéricos obtidos pelos esquemas FDPUS-C1 e SDPUS-C1 são comparados com dado experimental de Ellegard [23].

Caso 1 - Ressalto hidráulico circular. Neste caso, considera-se que o diâmetro do injetor é dado por $D=0.05 \mathrm{~m}$ e altura do injetor por $H=0.001 \mathrm{~m}$. Para simulação são empregados uma malha de $120 \times 120 \times 10$ células computacionais e os demais dados: 


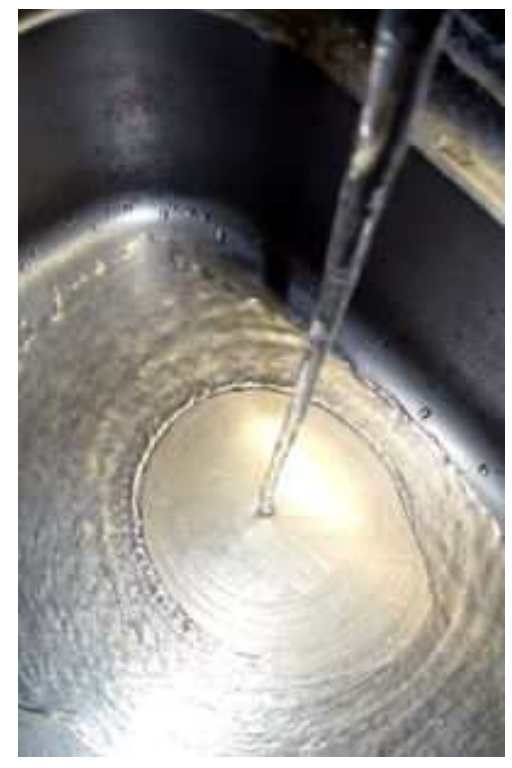

Figura 8.1: Ressalto hidráulico circular. Fenômeno de ressalto hidráulico.
- Dimensão do domínio: $0.6 m \times 0.6 m \times 0.05 m$;
- Constante gravitacional: $g=9.81 \mathrm{~m} / \mathrm{s}^{2}$;
- Escala de comprimento: $L=D=0.05 m$;
- Escala de velocidade: $V_{0}=1 \mathrm{~m} / \mathrm{s}$;
- Coeficiente de viscosidade cinemática: $\nu=5 \times 10^{-5} \mathrm{~m}^{2} / \mathrm{s}$;
- Número de Reynolds: $R e=\frac{V_{0} \cdot L}{\nu}=1000$;
- Número de Froud: $F r=\frac{V_{0}}{\sqrt{g \cdot L}}=1.4278$.

Na Figura 8.2 são comparados qualitativamente o experimento de Ellegard [23] (ver Fig. 8.2-(a)) com os resultados numéricos obtidos com os esquemas FDPUS-C1 (Fig. 8.2-(b)) e SDPUS-C1 (Fig. 8.2-(b)). Por essa figura, constata-se que ambos os esquemas FDPUS-C1 e SDPUS-C1 capturaram o fenômeno, ou seja, após o salto hidráulico, esses esquemas polinomiais conseguem capturar as irregularidades na superfície livre do fluido.

Como ilustração, na Figura 8.3 apresentam-se os campos de pressão e velocidade, nas direções $x$ e $z$, no plano $x \perp z(y=0.5)$ obtidos pelo esquema SDPUS-C1. Os resultados para o esquema FDPUS-C1 são semelhantes e portanto são omitidos.

\subsection{Jatos Oscilantes}

Um fenômeno bastante interessante e comum em dinâmica dos fluidos e com várias aplicações (por exemplo em indústrias de alimentos e processamento de polímeros) são as instabilidades físicas (flambagem) que aparecem, quando um jato de fluido altamente viscoso é incidido 
(a)

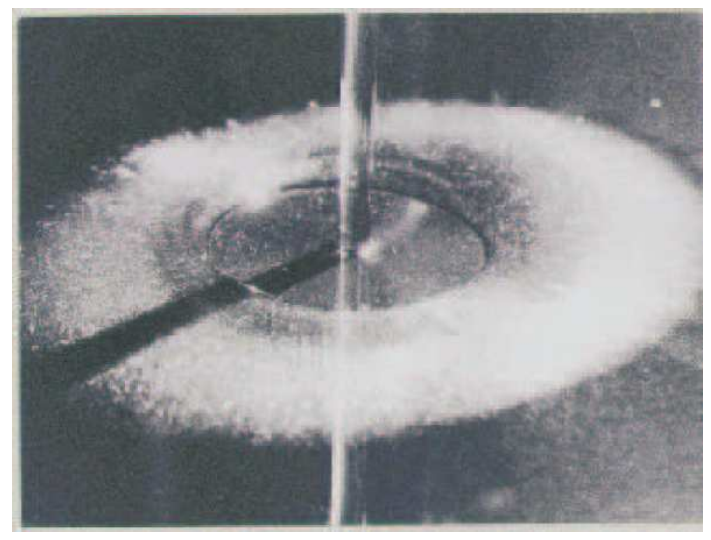

(b)

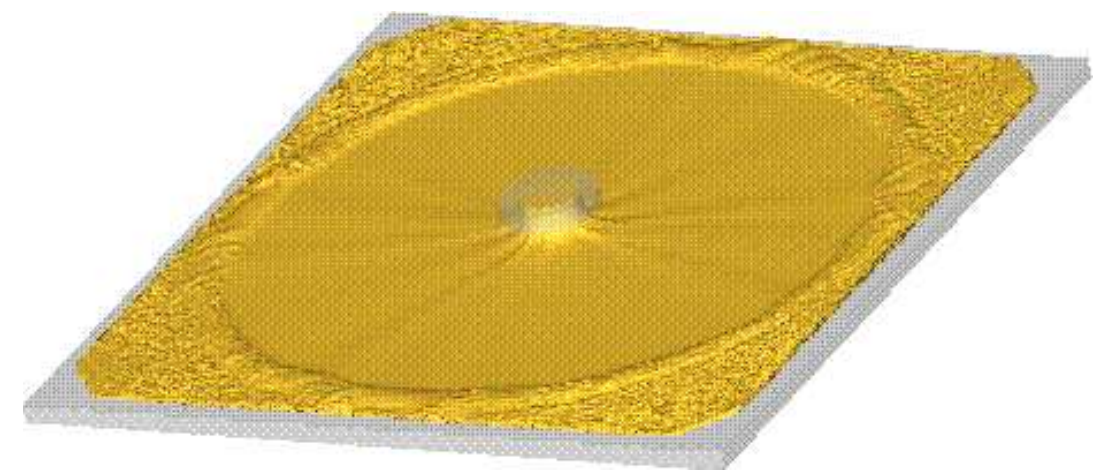

(c)

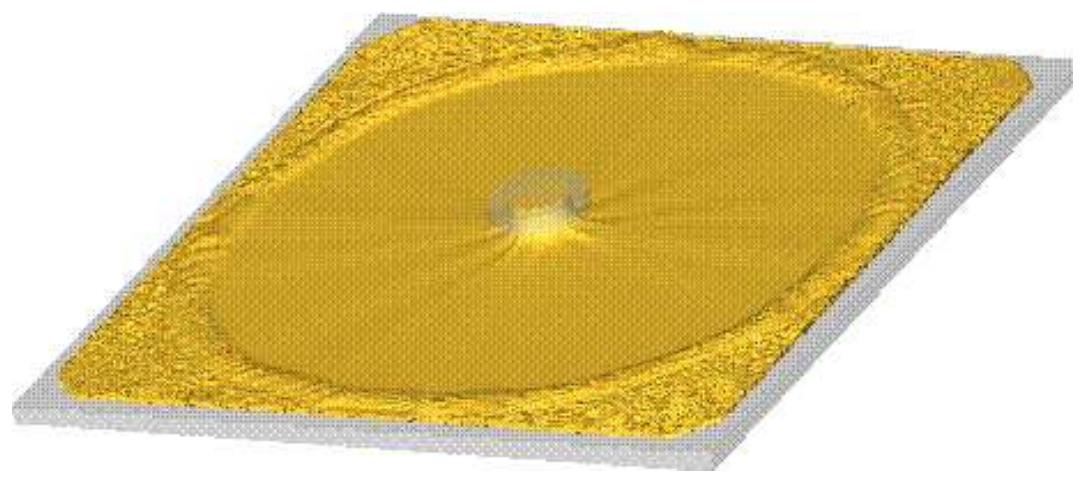

Figura 8.2: Ressalto hidráulico circular. Comparação entre o experimento de Ellegard (a) e os esquemas FDPUS-C1 (b) e SDPUS-C1 (c). 


\section{Pressão}

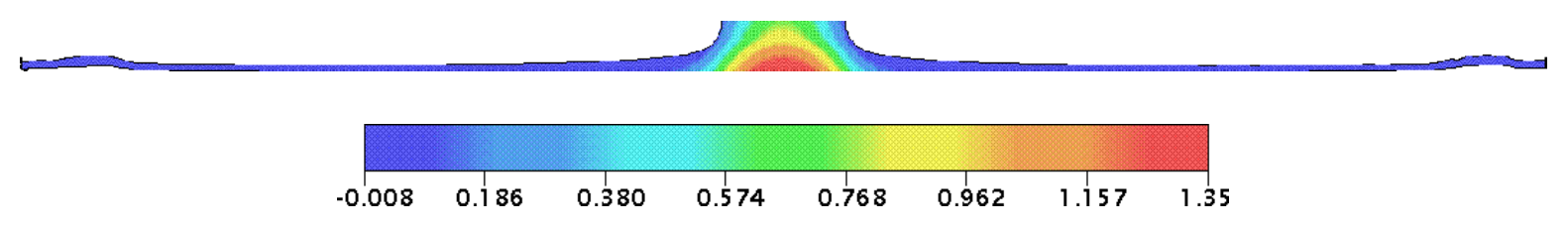

Velocidade na direção $\mathrm{x}$

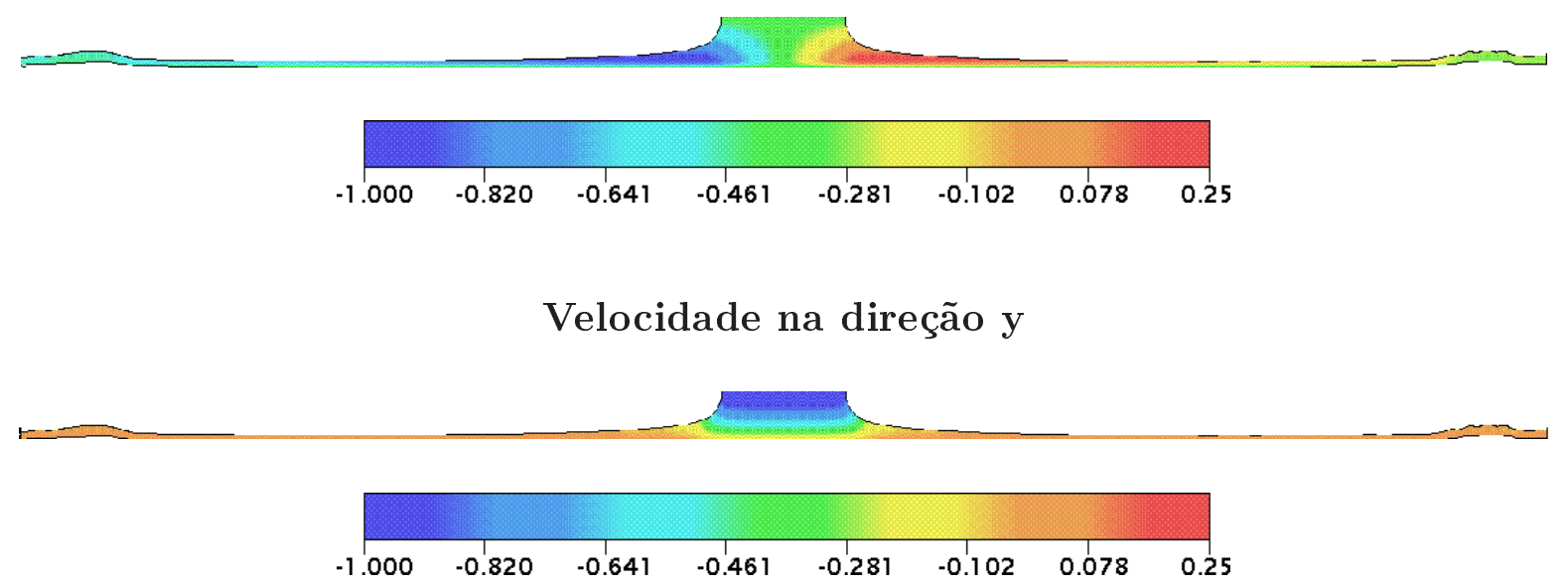

Figura 8.3: Ressalto hidráulico circular. Resultados numéricos do esquema SDPUS-C1 para os campos de pressão e velocidade (nas direções $x$ e $z$ ) no plano $x \perp z(y=0.5)$. 
em uma superfície rígida e impermeável. Esse fenômeno é ilustrado na Figura 8.4. Segundo Cruickshank [17], alguns fluidos com alto coeficiente de viscosidade afinam devido ao efeito da tensão superficial e à presença do campo gravitacional. Ao aderirem a superfície rígida formam dobras ou espirais e por essa razão são denominados jatos oscilantes (ver Figura 8.4).

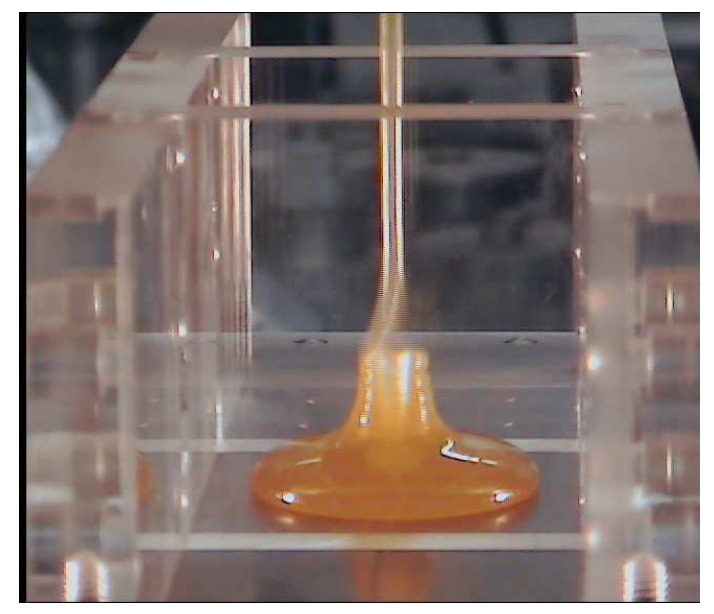

Figura 8.4: Jatos oscilantes. Fenômeno de jato oscilante.

Caso 1 - Jato Oscilante. Para simulação desse problema considera-se que a altura do injetor, com respeito ao fundo da superfície rígida, é $H=0.11 \mathrm{~m}$ e o diâmetro do injetor $D=0.006 \mathrm{~m}$. As soluções numéricas geradas com os dois esquemas FDPUS-C1 e SDPUS-C1 são calculadas em uma malha com $100 \times 100 \times 100$ células computacionais e adotando-se os seguintes dados:

- Dimensão do domínio: $0.06 m \times 0.06 m \times 0.11 m$;

- Constante gravitacional: $g=9.81 \mathrm{~m} / \mathrm{s}^{2}$;

- Escala de comprimento: $L=D=0.006 m$;

- Escala de velocidade: $V_{0}=1 \mathrm{~m} / \mathrm{s}$;

- Coeficiente de viscosidade cinemática: $\nu=1.2 \times 10^{2} \mathrm{~m}^{2} / \mathrm{s}$;

- Número de Reynolds: $R e=\frac{V_{0} \cdot L}{\nu}=0.5$;

- Número de Froud: $F r=\frac{V_{0}}{\sqrt{g \cdot L}}=4.12182$.

Nas Figuras 8.5 e 8.6 apresentam-se os resultados numéricos obtidos com os esquemas FDPUS-C1 e SDPUS-C1, respectivamente (nos tempos $t=0.2 s, t=0.4 s, t=0.6 s$ e $t=0.8 s$ ). Por essas figuras constata-se que os esquemas capturaram, muito bem, o fenômeno de jato oscilante. Como ilustração, na Figura 8.7 apresentam-se os campos de pressão e velocidade (nas direções $x, y$ e $z$ ) para os tempos $t=0.2 s$ e $t=0.6 s$, obtidos com o esquema FDPUS-C1. Os resultados do esquema SDPUS-C1 não são mostrados devido a semelhança com os dados apresentados. 

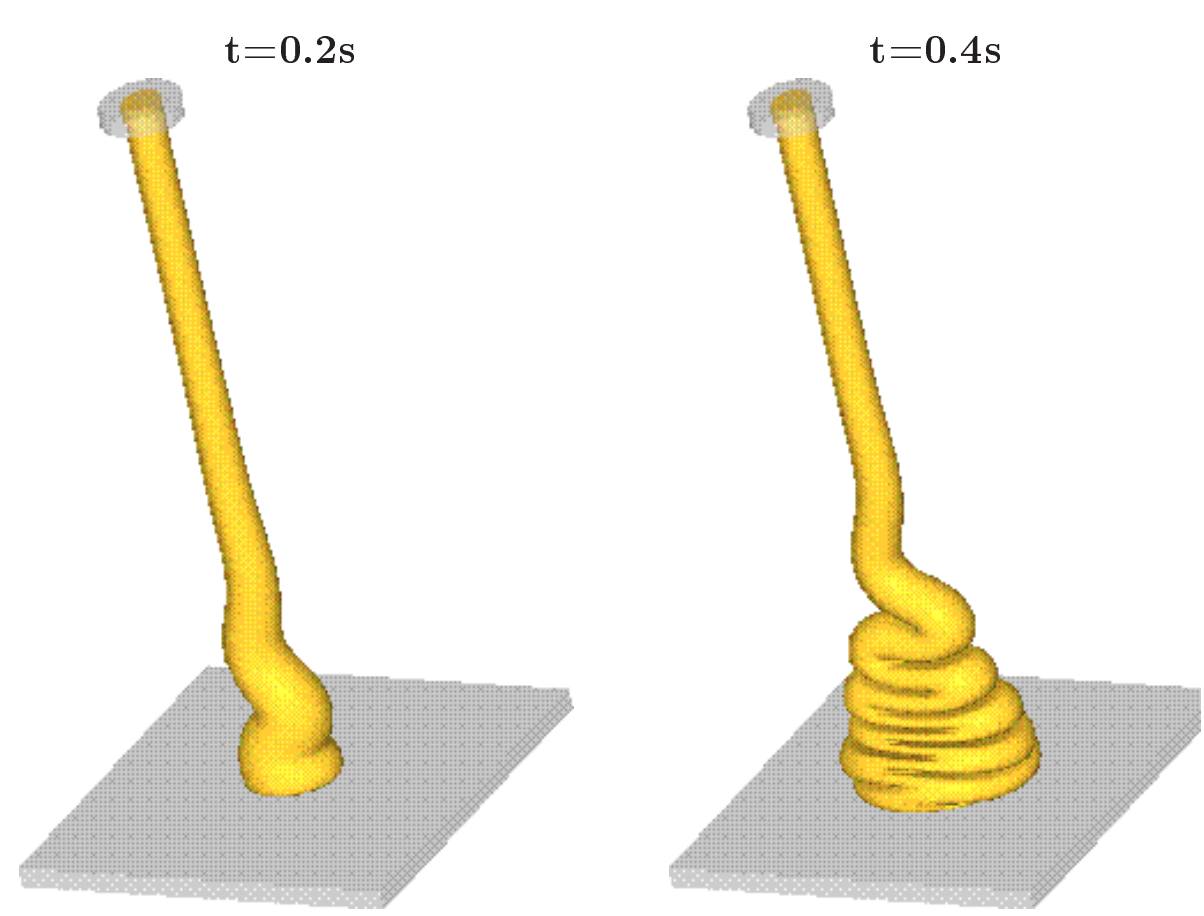

$\mathrm{t}=0.6 \mathrm{~s}$

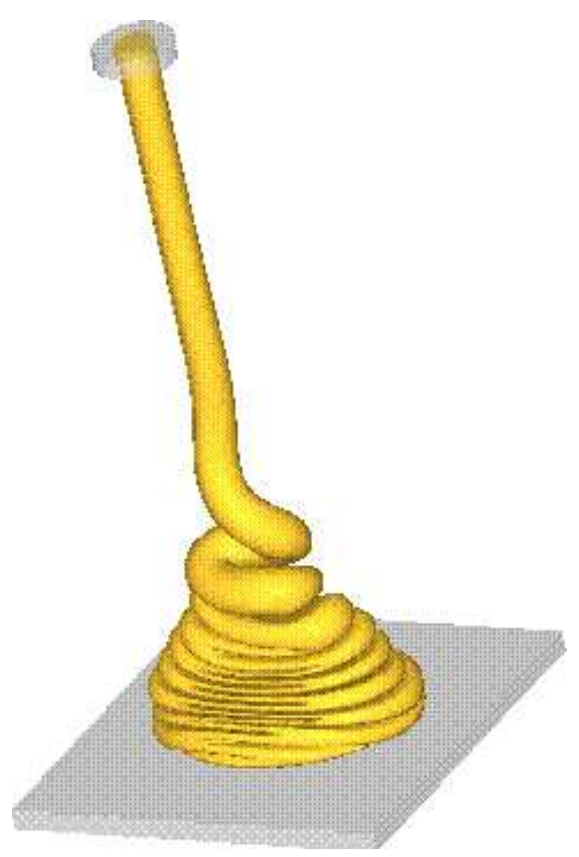

$\mathrm{t}=0.8 \mathrm{~s}$

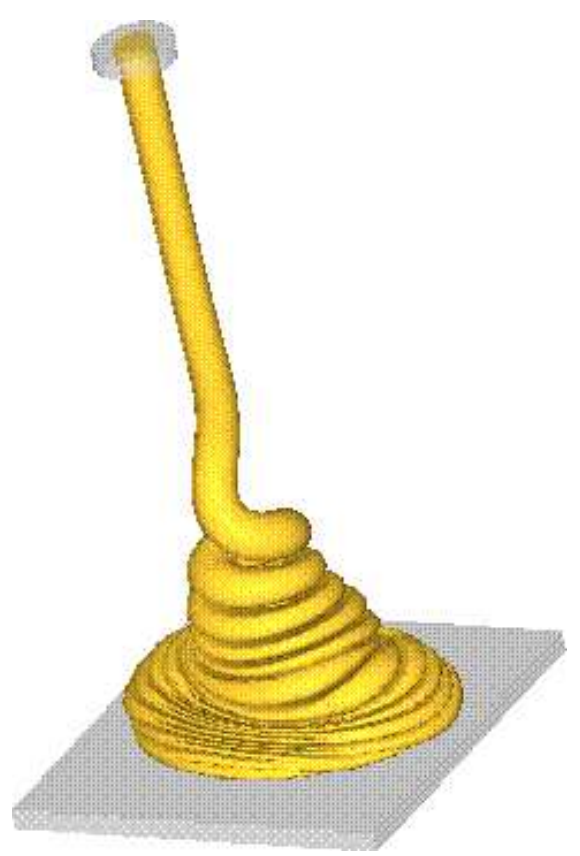

Figura 8.5: Jatos oscilantes. Resultados numéricos do esquema FDPUS-C1, nos tempos $t=0.2 s$, $t=0.4 s, t=0.6 s$ e $t=0.8 s$. 


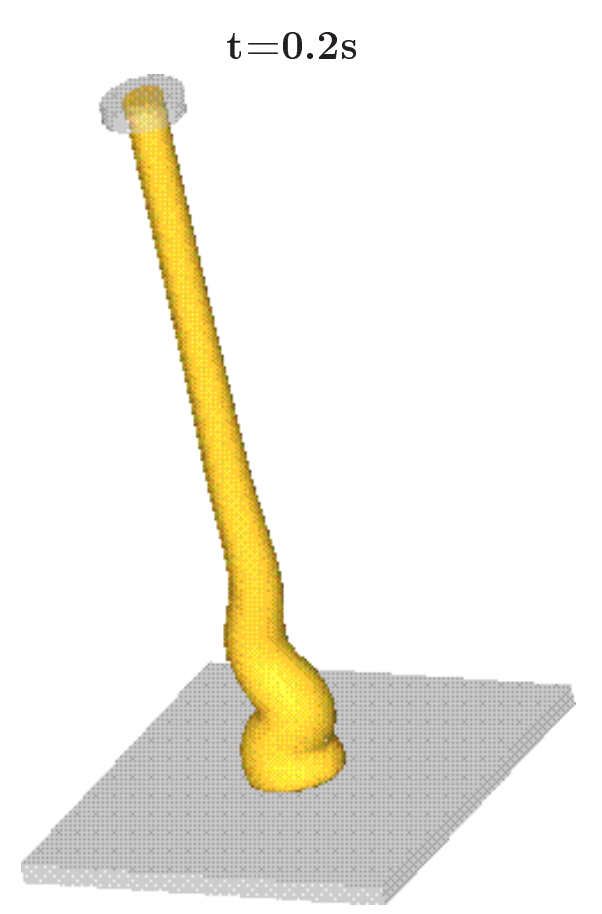

$\mathrm{t}=\mathbf{0 . 6 \mathrm { s }}$

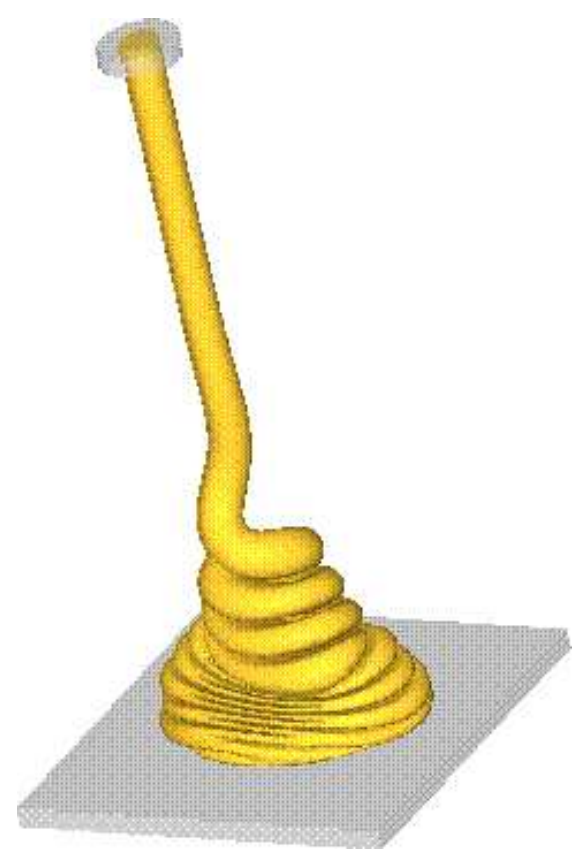

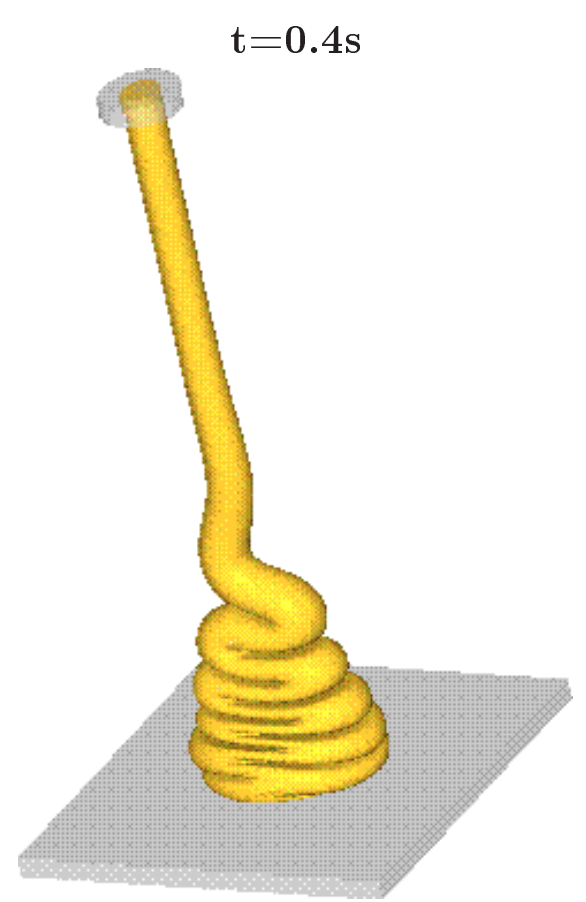

$\mathrm{t}=0.8 \mathrm{~s}$

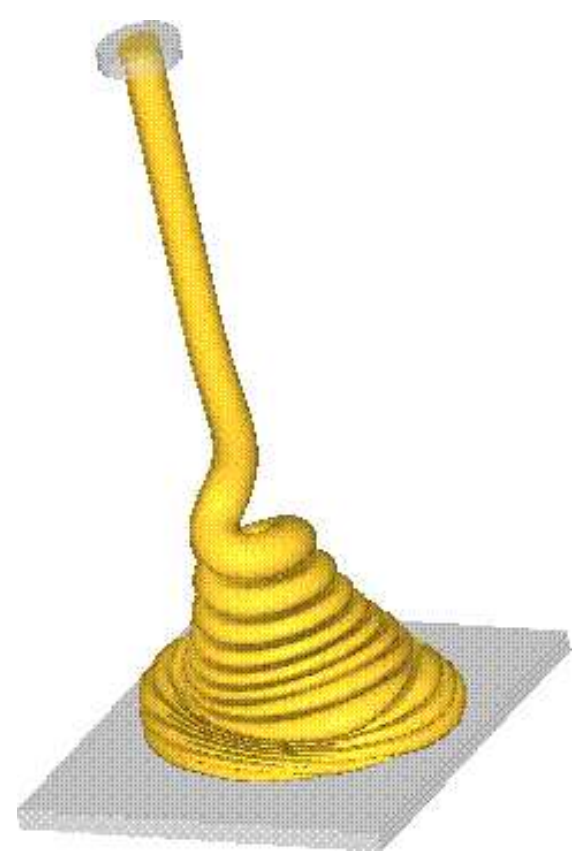

Figura 8.6: Jatos oscilantes. Resultados numéricos do esquema SDPUS-C1, nos tempos $t=0.2 s$, $t=0.4 s, t=0.6 s$ e $t=0.8 s$. 


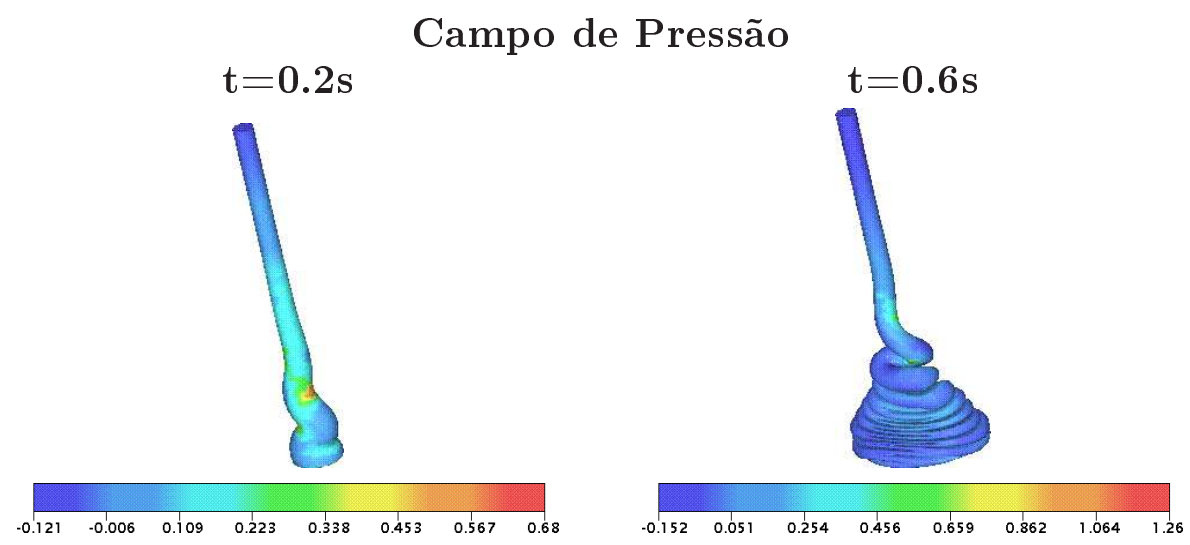

Velocidade na direção $\mathrm{x}$
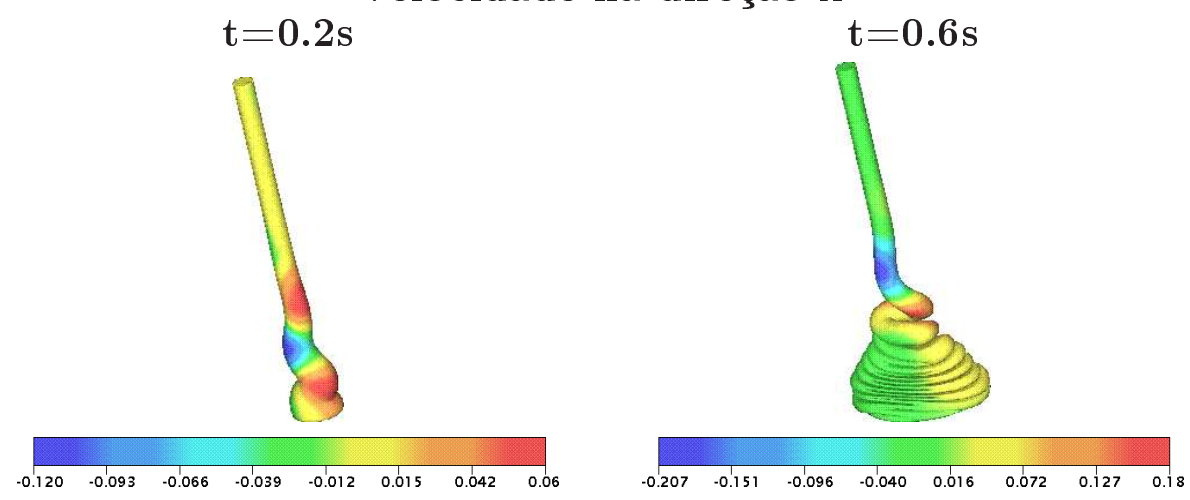

Velocidade na direção y $t=0.2 s$ $t=0.6 s$
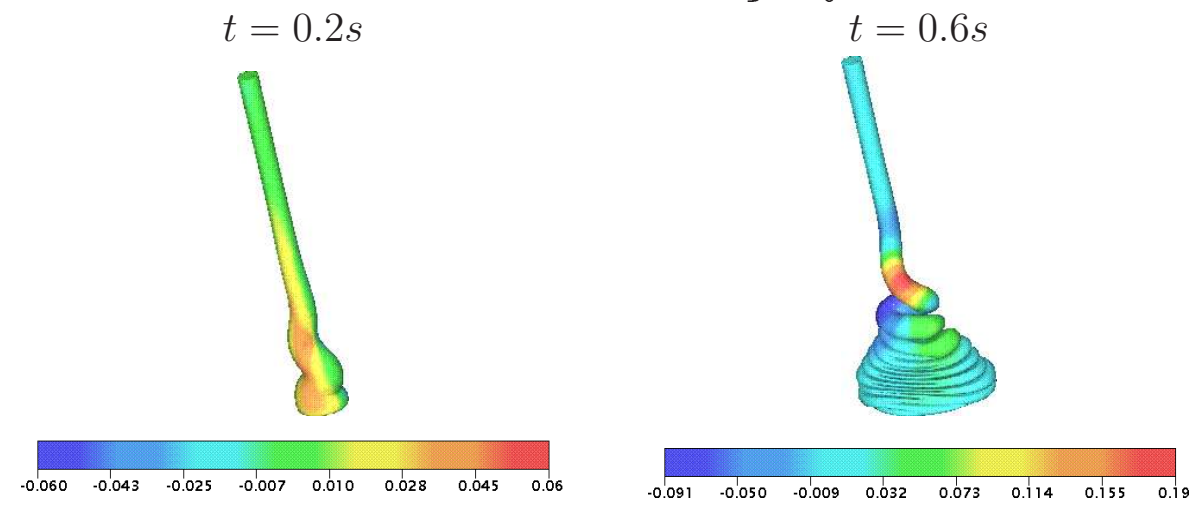

Velocidade na direção $z$
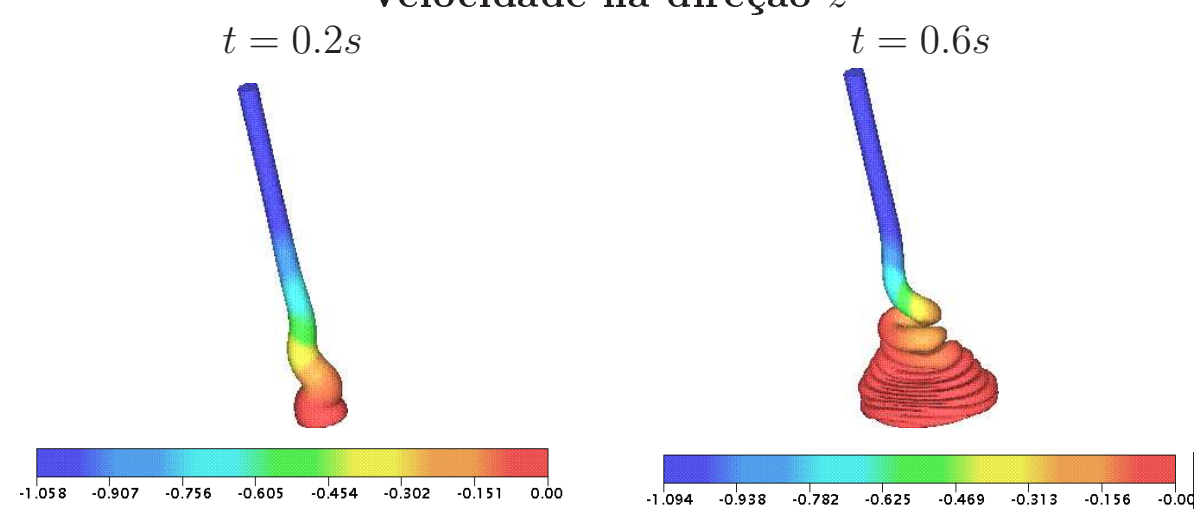

Figura 8.7: Jatos oscilantes. Resultados numéricos do esquema SDPUS-C1, nos tempos $t=0.2 \mathrm{~s}$ e e $t=0.6 s$, para os campos de pressão e velocidade (nas direções $x, y$ e $z$.). 


\section{Contribuições da Autora}

A seguir são listados os artigos completos publicados/aceitos/submetidos originados dessa dissertação. Também, são apresentados os resumos desses trabalhos.

\subsection{Artigos Submetidos em Periódicos}

- Valdemir G. Ferreira, Rafael A. Queiroz, Giseli A. B. Lima, Rafael G. Cuenca, Cassio M. Oishi e Sean McKee, An upwind differencing scheme for conservation laws and related fluid dynamics problems, submetido ao Computers $\&$ Fluids, 2009.

Abstract: A high resolution upwind differencing scheme for the numerical solution of time dependent hyperbolic conservation laws and related fluid dynamics problems is presented. The scheme is based on TVD and CBC stability criteria and is implemented in the context of the finite difference methodology. The performance of the scheme is investigated by solving the $1 D$ advection equation with discontinuous initial data and $1 D$ Riemann problems for Burgers and Euler equations. The scheme is then applied to the simulation of $2 D$ aerodynamic flows and $2 D / 3 D$ incompressible viscous flows with free surfaces. The numerical results displayed good agreement with other existing numerical and experimental data.

- Valdemir G. Ferreira, Magda K. Kaibara, Giseli A. B. Lima, Juliana M. Silva, Marcelo H. Sabatine, Paulo F. A. Mancera e Sean McKee, A robust TVD-NV-basesd upwinding a scheme for solving complex fluid flow problems, submetido ao Internacional Jornal for Numerical Methodoss in Fluids, 2009.

Abstract: The development and further applications of a recently introduced high resolution upwind differencing scheme, namely ADBQUICKEST (Int. J. Numer. Meth. Fluids 2009; 60: 1-26), will be carried out. The ADBQUICKEST scheme is a new TVD version of the QUICKEST (Comput. Method. Appl. Mech. Engineering 1979; 19: 59-98) for solving nonlinear balance laws. The scheme 
is based on the concept of NV and TVD formalisms and satisfies a convective boundedness criterion. The accuracy of the scheme is compared with other popularly used convective upwinding schemes for solving nonlinear conservation laws (for example, Burgers, Buckley-Leverett, shallow water and Euler equations). The ADBQUICKEST scheme is then used to solve four types of fluid flow problems of increasing complexity: namely, 2D aerosol filtration by fibrous filters; axisymmetric flow in a tubular membrane; 2D two-phase flow in a fluidized bed; and 3D incompressible flow involving free surfaces. The numerical tests indicate that this convective upwinding scheme is a good alternative for solving complex fluid dynamics problems.

- Giseli A. B. Lima, Laís Corrêa, Miguel A.C. Candezano, Patrícia Sartori e Valdemir G. Ferreira, Avaliação computacional de esquemas de alta reesolução em problemas de dinâmica dos fluidos, submetido a TEMA, 2009.

Resumo: Este artigo fornece uma avaliação computacional dos esquemas upwind de alta resolução populares WACEB, CUBISTA e ADBQUICKEST para resolver problemas não lineares em dinâmica dos fluidos. Os esquemas são analisados e implementados em variáveis normalizadas de Leonard e no contexto do método de diferenças finitas. O desempenho dos esquemas é avaliado em problemas de Riemann para as equações de Burgers, Euler e águas rasas. Dos resultados numéricos, os esquemas são classificados segundo seus desempenhos em resolver essas equações não-lineares unidimensionais. O esquema selecionado é então aplicado na simulção numérica de escoamentos incompressívies tridimensionais com superfícies livres móveis.

\subsection{Artigos Publicados em Anais de Congresso}

- Giseli A. B. Lima, Miguel A. C. Candezano e Valdemir G. Ferreira, Uma avaliação computacional de três esquemas de discretização upwind para leis de conservação não lineares, DINCON'09 - 8th Brazilian Conference on Dynamics, Control and Aplications, 2009, Bauru/SP.

Resumo: Neste trabalho de pesquisa é apresentada uma avaliação computacional dos esquemas de alta resolução WACEB (Int. J. Numer. Meth. Fluids 2000; 32: 881-897), CUBISTA (Int. J. Numer. Meth. Fluids 2003; 41:47-75) e ADBQUICKEST (Int. J. Numer. Meth. Fluids 2009:In Press) para resolver leis de conservação lineares e não lineares. Utilizando-se a metodologia de diferenças finitas, esses esquemas são analisados e implementados no contexto de variáveis normalizadas de Leonard. Para acessar o desempenho dos esquemas, quatro problemas (um linear e três não lineares) são considerados, a saber: problema de advecção de um escalar; e problemas de Riemann para as equações de Burgers, Euler e águas rasas.

- Giseli A. B. Lima e Valdemir G. Ferreira, Sobre variação total e convergência de três esquemas upwind para leis de conservação, CNMAC2009 - XXXII Congresso Nacional de Matemática aplicada e Computacional, 2009, Cuiabá/MT. 
Resumo: Três esquemas upwind de alta resolução originais para solução computacional de leis de conservação desenvolvidos no LCAD-ICMC-USP (ADBQUICKEST, TOPUS e o novo Esquema I) são analisados com respeito a variação total e a ordem de convergência. Em particular, os esquemas são aplicados aos casos de convecção de escalares e problemas de Riemann para as equações não lineares de Burgers e Euler. A partir dos resultados numéricos, constata-se que os esquemas apresentam resultados bastantes interessantes em todos os casos investigados.

- Giseli A. B. Lima, Valdemir G. Ferreira, Rafael A. B. Queiroz, Miguel A. C. Candezano e Laís Corrêa, Development and evaluation of upwind schemes for conservation laws, COBEM2009 - International Congress of Mechanical Engineering, 2009, Gramado/RS.

Abstract: This work deals with a computational evaluation of two new high resolution upwind schemes, namely ADBQUICKEST ( Int. J. Numer. Meth. Fluids 2009; 60:1-26) and TOPUS (Comput. Fluids - Submitted), for solving general hyperbolic conservation laws. By using the finite difference methodology, the schemes are analyzed and implemented in the context of normalized variables of Leonard (1988). In order to access the performance of these schemes, a series of one-dimensional test problems are examined beginning with classical linear advection of scalars and ending with Riemann problems for Burgers, Buckley-Leverett, shallow water, and Euler equations. And, as application, the ADBQUICKEST and TOPUS schemes are used for the numerical simulation of $3 D$ incompressible Navier-Stokes equations involving free surface.

- Rafael A. B. Queiroz, Giseli A. B. de Lima e Valdemir G. Ferreira, Comparison of two new upwind schemes for hiperbolic conservation laws with respect to symmetric property, BCSciComp2009 - First Brazil-China Conference on Scientific Computing, 2009, Petrópolis/RJ.

Abstract: The modelling of convection terms is a key point in reproducing complex physical phenomena in conservation laws and related fluid dynamics problems. The objective of this work is to evaluate the performance of two new high order bounded upwind schemes, namely TOPUS and FSFL, for solving linear and nonlinear hiperbolic conservation laws. These schemes satisfy TVD principle of Harten and are based on NVD formulation of Leonard,. Inicially, the symmetric property is emphasized when one solves advection of scalars and Riemann problems for Burgers, Buckley-Leverett, shallow water and Euler equations. The, as an application, the schemes are employed for solving incompressible flows involving free sufraces. From numercial tests, and depending of the problem in hand, both the schemes provide satisfatory results.

- Giseli A. B. Lima, Rafael A. B. Queiros e Valdemir G. Ferreira, A TVD upwinding scheme for compressible and incompressible flows, 30th CILAMCE - Iberian-Latin-American Congress on Computational Methods in Engineering, 2009, Búzios/RJ.

Abstract: The modelling of convection terms is a key point in reproducing complex physical phenomena 
in fluid flow problems. The objective of this study is to evaluate the performance of a new TVD upwind scheme (FDPUS-C1) for solving both compressible and incompressible fluid flows. In particular, results for 1D/2D Euler of the gas dynamics and 2D Navier Stokes equations with free surfaces are presented. The scheme satisfy TVD principle of Harten and is based on NVD formulation of Leonard. From numerical tests, one can see that the present TVD upwinding scheme gives satisfatory results.

\subsection{Artigos Submetidos para Publicação em Anais de Con- gresso}

- Giseli A. B. Lima, Laís Corrêa, Miguel A.C. Candezano, Patrícia Sartori e Valdemir G. Ferreira, A simple NVD/TVD-based upwinding scheme for convection term discretization, submetido ao ECCOMAS CFD 2010-Fifth European Conference on Computational Fluid Dynamics, 2010, Lisboa/Portugal.

Abstract: The correct modeling for processes involving convection, without introducing excessive artificial damping while retaining high accuracy, stability, boundedness and simplicity of implementation continues being nowadays a challenging task for the cientific CFD community (see [1,2,5]). In this context, the objective of this study is to present and to evaluate the performance of a new TVD-based upwinding scheme, namely Six Degree Polynomial Upwind Scheme of C1 Class (SDPUS-C1), for convection term discretization. SDPUS-C1 satisfies the TVD principle of Harten [3] and is based on the NVD formulation of Leonard [4]. Firstly, a description of the scheme is done and then numerical results are presented for three-dimensional hyperbolic conservation laws, such as acoustics, Burgers and Euler equations. Finally, as application, the SDPUS-C1 scheme is used for the computational simulation of three-dimensional incompressible fluid flows involving moving free surfaces.

- Patrícia Sartori, Giseli A. B. Lima, Laís Corrêa, Miguel A.C. Candezano e Valdemir G. Ferreira, Avaliação computacional de três esquemas upwind originais, submetido ao SIMMEC $20109^{\circ}$ Simpósio de Mecânica Computacional, 2010, São João Del Rei/MG.

Resumo: Resolver numericamente problemas em dinâmica dos fluidos é uma tarefa difícil e desafiadora, principalmente quando tais problemas são dominados por convecção. Isso requer o desenvolvimento de esquemas numéricos tipo upwind que sejam precisos, monotônicos e robustos. O presente trabalho é destinado à avaliação computacional de três novos esquemas upwind de alta resolução, desenvolvidos no LCAD-ICMC/USP, denominados ADBQUICKEST (ADaptative Quickest), TOPUS (Third-Order Polynomial Upwind Scheme) e SDPUS-C1 (Six-Degree Polynomial Upwind Scheme of C1 Class). O desempenho desses esquemas é investigado a partir da simulação computacional de problemas de leis de conservação hiperbólicas, a saber, advecção e problemas de Riemann para acústica e Euler da dinâmica dos gases. E então, como aplicação, esses esquemas são utilizados na simulação de escoamentos incompressíveis com superfícies livres móveis modelados pelas equações de Navier-Stokes $3 \mathrm{D}$. 
- Laís Corrêa, Giseli A. B. Lima, Patrícia Sartori, Miguel A.C. Candezano e Valdemir G. Ferreira, A new polynomial upwind convection scheme for fluid flow simulations, submetido ao CONEM 2010 - VI Congresso Nacional de Engenharia Mecânica, 2010, Campina Grande/PB.

Abstract: The simulation of fluid flow problems involving strong convective character is a difficult problem to solve and has atracted many researchers in the CFD community. In this scenario, we present in this work a new polynomial upwind scheme, called SDPUS ("Six Degree Polynomial Upwind Scheme of C1 Class"), for numerical solution of conservation laws and related fluid dynamics problems. The scheme is developed in the context of normalized variables of Leonard and satisfies the CBC and TVD stability criteria of Gaskell and Lau, and Harten, respectively. The numerical solutions obtained with this scheme can achieve second/third order of accuracy in smooth regions and first order near to discontinuities (shocks). The performance of the SDPUS is assessed in the solution of nonlinear hyperbolic systems, such as shallow water, acoustics, and Euler equations of gas dynamics. As application, the scheme is then used in the solution of incompressible Navier-Stokes equations in cylindrical coordinates. From numerical results, one can clearly see that the SDPUS-C1 scheme is a robust tool for resolving both compressible and incompressible complex flow problems.

\subsection{Material Publicado em Curso de Curta Duração}

- Valdemir G. Ferreira, Giseli A. B. Lima e Hélio A. Navarro, Solução numérica de leis de conservação e problemas de dinâmica dos fluidos, DINCON'09 - 8th Brazilian Conference on Dynamics, Control and Aplications, 2009, Bauru/SP.

Resumo: O minicurso é direcionado, principalmente, a alunos de graduação que tenham alguma familiaridade com cálculo diferencial, cálculo numérico e física elementar. O seu objetivo principal é mostrar ao aluno como resolver, usando o método das diferenças finitas, as equações da dinâmica dos fluidos. A introdução ao tema é feita de maneira gradativa por meio da apresentação de aspectos teóricos e práticos de simulações numéricas 1D/2D/3D. Além de exercícios envolvendo problemas 1D apresentados nesse texto, os aspectos práticos são contemplados em outros materiais complementares sobre simulações numéricas 2D/3D de escoamentos de fluidos. Assim, além de contribuir na formação acadêmica do aluno, pretende-se despertar o interesse do mesmo pela área de dinâmica dos fluidos computacional.

\subsection{Material Submetido para Curso de Curta Duração}

- Valdemir G. Ferreira e Giseli A. B. Lima, Simulação computacional de alguns problemas em dinâmica dos fluidos, DINCOM'10-9th Brazilian Conference on Dynamics, Control and Their Applications, 2010, Serra Negra/SP.

Resumo: No presente mini-curso será apresentada uma revisão breve do estado da arte em simulação computacional de alguns problemas em dinâmica dos fluidos. A maior parte do mini-curso será destinada a exemplos e ilustrações de simulações numéricas de escoamentos incompressíveis. A introdução 
ao tema será feita gradativamente por meio da resolução numérica de equações modelo $1 \mathrm{D}$, tais como equação da condução de calor (parabólica), equação de advecção (hiperbólica) e equação de Poisson (elíptica).

A proposta do mini-curso é mostrar que dinâmica dos fluidos computacional é a ciência de construir soluções numéricas para equações de conservação, avançando a solução no espaço e no tempo para obter uma descrição numérica do escoamento de interesse.

Os tópicos a serem abordados no mini-curso são resumidos como seguem: descrever técnicas para a marcha no tempo e o método de diferenças fnitas; discretizar as equações modelo 1D; apresentar o modelo fundamental em dinâmica dos fluidos (as equações de Navier-Stokes); compreender o significado físico de cada termo das equações de Navier-Stokes; introduzir um algoritmo de cálculo para as equações de Navier-Stokes; mostrar uma variedade de simulações de escoamentos incompressíveis; discutir alguns problemas práticos usando filmes ilustrativos.

O presente mini-curso é direcionado, principalmente, a alunos de graduação que tenham alguma familiaridade com cálculo avançado, cálculo numérico e física elementar. E o seu objetivo principal é mostrar ao aluno como resolver, no contexto de diferenças fnitas, as equações de Navier-Stokes para o caso incompressível.

\subsection{Relatório Técnico Científico}

- Valdemir G. Ferreira, Rafael A. Queiroz, Rafael G. Cuenca, Giseli A. B. Lima, Cassio M. Oishi, e Sean McKee, An upwind differencing scheme for conservation laws and related fluid dynamics problems,2009, Departamento de Matemática da Universidade de Strathclyde Glasgows/Escócia.

Abstract: A high resolution upwind differencing scheme for the numerical solution of time dependent hyperbolic conservation laws and related fluid dynamics problems is presented. The scheme is based on TVD and CBC stability criteria and is implemented in the context of the finite difference methodology. The performance of the scheme is investigated by solving the $1 D$ advection equation with discontinuous initial data and 1D Riemann problems for Burgers, Euler and shallow water equations. The scheme is then applied to the simulation of $2 D$ aerodynamic transonic flows and $2 D / 3 D$ complex incompressible viscous flows with free surfaces. The numerical results displayed good agreement with other existing numerical and experimental data. 
CAPÍTULO

\section{Conclusões e Trabalhos Futuros}

Neste trabalho de mestrado, dois novos esquemas upwind de alta resolução, denominados FDPUS-C1 (Five-Degree Polynomial Upwind Scheme of $C^{1}$ Class) e SDPUS-C1 (Six-Degree Polynomial Upwind Scheme of $C^{1}$ Class), para a discretização dos termos convectivos lineares e não-lineares de leis de conservação e problemas relacionados em dinâmica dos fluidos, foram desenvolvidos, analisados e implementados. Os esquemas são baseados nos critérios de estabilidade TVD e CBC e foram implementados, nos contextos das metodologias diferenças finitas e volume finitos, no ambiente de simulação Freeflow para escoamentos incompressíveis e no código bem conhecido CLAWPACK para problemas compressíveis.

O desempenho dos esquemas FDPUS-C1 e SDPUS-C1 foi, primeiramente, avaliado com respeito a vários experimentos numéricos 1D, a saber: i) advecção de escalares; ii) equações não lineares de Burgers e Buckley-Leverett; e iii) sistemas hiperbólicos de águas rasas de Euler da dinâmica dos gases. Esses testes numéricos mostraram que os esquemas FDPUS-C1 e SDPUS-C1 são comparáveis (com desempenho igual ou superior) com os esquemas presentes na literatura, tais como, van Leer, Superbee, CUBISTA, VONOS, TOPUS, ADBQUICKEST e WENO de terceira ordem. Esses esquemas foram, então, estendidos para resolução de uma variedade de leis de conservação $2 \mathrm{D}$ e escoamentos incompressíveis $2 \mathrm{D}$ altamente complexos, tais como escoamentos turbulentos e viscoelásticos com superfícies livres. Por fim, os esquemas foram aplicados na resolução de escoamentos 3D laminares com superfícies livres.

Os resultados numéricos obtidos com os esquemas FDPUS-C1 e SDPUS-C1 mostraram claramente que esses esquemas upwind de alta resolução podem ser usados com confiança na simulação de sistemas hiperbólicos e problemas complexos de escoamentos de fluidos. Essas aplicações evidenciaram também que as principais vantagens desses novos esquemas são suas habilidades em resolver uma gama ampla de problemas complexos - desde o tubo de choque de Wood e Collella até escoamentos 3D com superfícies livres móveis - relativamente rápido. É 
importante salientar que, em geral, os esquemas FDPUS-C1 e SDPUS fornecem bons resultados a números de CFL moderados e podem ser prontamente aplicados para resolver corretamente escoamentos transientes 3D onde os termos advectivos não lineares requerem atenção especial. Em síntese, este trabalho, além de fornecer dois novos esquemas upwind de alta resolução, dispõe de uma gama bastante significativa de dados numéricos precisos de simulações. Isto permitirá, no futuro, pesquisadores comparar seus dados com os aqui apresentados.

A pesquisa científica descrita neste texto pode ser incrementada de várias maneiras, sobretudo no que diz respeito à combinação dos dois novos esquemas FDPUS-C1 e SDPUS com modelagem da turbulência tipo URANS de duas equações para a simulação de escoamentos turbulentos de fluidos não newtonianos 3D com superfícies livres móveis. Esta frente de trabalhos futuros reside a principal contribuição do plano de doutorado da autora, uma vez que o estado da arte atual na área não contempla ainda a simulação computacional desses escoamentos altamente complexos na presença de superfícies livres móveis. 
APÊNDICE

Demonstração da Propriedade TVD: Esquema FDPUS-C1

Neste apêndice demonstra-se que o esquema FDPUS-C1 dado por

$$
\hat{\phi}_{f}= \begin{cases}-4 \hat{\phi}_{U}^{5}+14 \hat{\phi}_{U}^{4}-16 \hat{\phi}_{U}^{3}+6 \hat{\phi}_{U}^{2}+\hat{\phi}_{U}, & \hat{\phi}_{U} \in[0,1], \\ \hat{\phi}_{U}, & \hat{\phi}_{U} \notin[0,1],\end{cases}
$$

satisfaz a propriedade TVD de Harten [30].

Relembrando que, no contexto de variáveis normalizadas, um esquema convectivo é TVD se ele satisfaz as seguintes condições (ver Figura 3.4):

$$
\begin{cases}\hat{\phi}_{f} \in\left[\hat{\phi}_{U}, 2 \hat{\phi}_{U}\right] \text { e } \hat{\phi}_{f} \leq 1, & \hat{\phi}_{U} \in[0,1], \\ \hat{\phi}_{f}=\hat{\phi}_{U}, & \hat{\phi}_{U} \notin[0,1],\end{cases}
$$

Demonstração: Constata-se claramente por (A.1) que $\hat{\phi}_{f}=\hat{\phi}_{U}$ para $\hat{\phi}_{U} \notin[0,1]$. Resta então, monstrar que, para $\hat{\phi}_{U} \in[0,1]$, o esquema (A.1) satisfaz $\hat{\phi}_{f} \in\left[\hat{\phi}_{U}, 2 \hat{\phi}_{U}\right]$ e $\hat{\phi}_{f} \leq 1$. Seguem as demonstrações das três implicações:

i) $\hat{\phi}_{f} \geq \hat{\phi}_{U}$ para $0 \leq \hat{\phi}_{U} \leq 1$.

Seja $h=\hat{\phi}_{f}-\hat{\phi}_{U}$. Substituindo (A.1) em $h$, obtém-se

$$
h=-4 \hat{\phi}_{U}^{5}+14 \hat{\phi}_{U}^{4}-16 \hat{\phi}_{U}^{3}+6 \hat{\phi}_{U}^{2}
$$

Então, deve ser provado que $h \geq 0$ para $0 \leq \hat{\phi}_{U} \leq 1$. De fato, as raízes de $h$ são 1 (de multiplicidade 2), 0 (de multiplicidade 2) e $\frac{3}{2}$. Do estudo de sinal da função $h$, conclui-se que 
$h \geq 0$ para $0 \leq \hat{\phi}_{U} \leq 1$. Para ilustrar essa implicação, a Figura A.1 mostra o gráfico da função $h$ em função de $\hat{\phi}_{U}$.

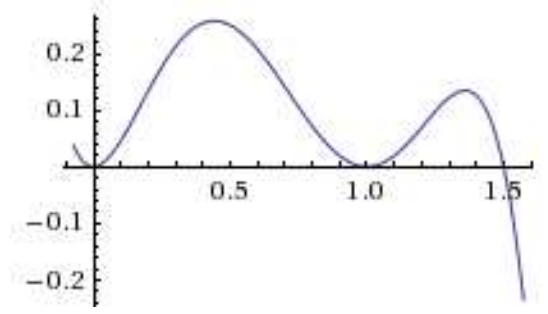

Figura A.1: Esquema FDPUS-C1 - Análise TVD. Função $h=\hat{\phi}_{f}-\hat{\phi}_{U}$.

ii) $\hat{\phi}_{f} \leq 2 \hat{\phi}_{U}$ para $0 \leq \hat{\phi}_{U} \leq 0.5$.

Seja $g=\hat{\phi}_{f}-2 \hat{\phi}_{U}$. Substituindo (A.1) em $g$, obtém-se

$$
g=-4 \hat{\phi}_{U}^{5}+14 \hat{\phi}_{U}^{4}-16 \hat{\phi}_{U}^{3}+6 \hat{\phi}_{U}^{2}-\hat{\phi}_{U}
$$

Então, deve ser provado que $g \leq 0$ para $0 \leq \hat{\phi}_{U} \leq 0.5$. De fato a raiz real de $g$ é 0 e as raízes imaginárias são $0.24 \pm 0.20 i$ e $1.50 \pm 0.39 i$. Com estudo do sinal da função $g$ segue-se que $g \leq 0$ para $0 \leq \hat{\phi}_{U} \leq 0.5$. Para ilustrar essa implicação, a Figura A.2 esboça o gráfico da função $g$.

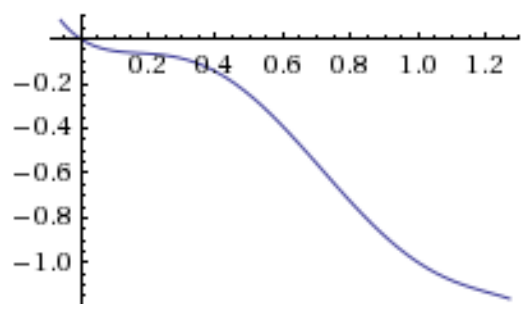

Figura A.2: Esquema FDPUS-C1 - Análise TVD. Função $g=\hat{\phi}_{f}-2 \hat{\phi}_{U}$.

iii) $\hat{\phi}_{f} \leq 1$ para $0.5 \leq \hat{\phi}_{U} \leq 1$.

Seja $s=\hat{\phi}_{f}-1$. Substituindo (A.1) em $g$, obtém-se

$$
s=-4 \hat{\phi}_{U}^{5}+14 \hat{\phi}_{U}^{4}-16 \hat{\phi}_{U}^{3}+6 \hat{\phi}_{U}^{2}+\hat{\phi}_{U}-1 .
$$

Então, deve ser provado que $s \leq 0$ para $0.5 \leq \hat{\phi}_{U} \leq 1$. De fato as raízes reais de $s$ são dadas por $-0.32,1$ e 1.64 e as raízes imaginárias por $0.58 \pm 0.35 i$. Novamente, com estudo do sinal da função $s$ segue que $s \leq 0$ para $0.5 \leq \hat{\phi}_{U} \leq 1$. Para ilustrar essa implicação, na Figura A.3 apresenta-se o gráfico da função $s$.

Em suma, das demonstrações acima, conclui-se que o esquema FDPUS-C1 (A.1) é TVD. 


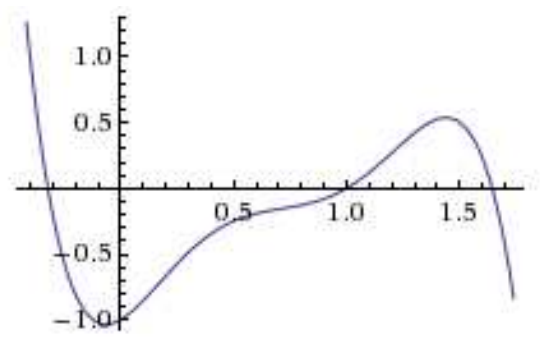

Figura A.3: Esquema FDPUS-C1 - Análise TVD. Função $s=\hat{\phi}_{f}-1$. 


\section{Demonstração da Propriedade TVD: Esquema}

$$
\text { SDPUS-C1 }(\gamma=12)
$$

Neste apêndice demonstra-se que o esquema SDPUS-C1 dado, para $\gamma=12$, por

$$
\hat{\phi}_{f}= \begin{cases}24 \hat{\phi}_{U}^{6}-76 \hat{\phi}_{U}^{5}+92 \hat{\phi}_{U}^{4}-52 \hat{\phi}_{U}^{3}+12 \hat{\phi}_{U}^{2}+\hat{\phi}_{U}, & \hat{\phi}_{U} \in[0,1], \\ \hat{\phi}_{U}, & \hat{\phi}_{U} \notin[0,1],\end{cases}
$$

satisfaz a propriedade TVD de Harten [30].

Demonstração: Considerando as restrições (A.2) demonstra-se que o esquema SDPUS-C1, com $\gamma=12$, dado por (B.1) é TVD. Constata-se claramente por (B.1) que $\hat{\phi}_{f}=\hat{\phi}_{U}$ para $\hat{\phi}_{U} \notin[0,1]$. Resta então, demonstrar que (B.1), para $\hat{\phi}_{U} \in[0,1]$, satisfaz $\hat{\phi}_{f} \in\left[\hat{\phi}_{U}, 2 \hat{\phi}_{U}\right]$ e $\hat{\phi}_{f} \leq 1$. Seguem as demonstrações das três implicações:

i) $\hat{\phi}_{f} \geq \hat{\phi}_{U}$ para $0 \leq \hat{\phi}_{U} \leq 1$.

Seja $h=\hat{\phi}_{f}-\hat{\phi}_{U}$. Substituindo (B.1) em $h$, obtém-se

$$
h=24 \hat{\phi}_{U}^{6}-76 \hat{\phi}_{U}^{5}+92 \hat{\phi}_{U}^{4}-52 \hat{\phi}_{U}^{3}+12 \hat{\phi}_{U}^{2} .
$$

Então, deve ser provado que $h \geq 0$ para $0 \leq \hat{\phi}_{U} \leq 1$. De fato as raízes reais de $h$ são 0 (multiplicidade 2) e 1 (multiplicidade 2), e as raízes imaginárias são dadas por $\frac{1}{12}(7 \pm i \sqrt{23})$. Com o estudo do sinal da função $h$ conclui-se que $h \geq 0$ para $0 \leq \hat{\phi}_{U} \leq 1$. A Figura B.1, esboça essa implicação, uma vez que traz o gráfico da função $h$.

ii) $\hat{\phi}_{f} \leq 2 \hat{\phi}_{U}$ para $0 \leq \hat{\phi}_{U} \leq 0.5$. 


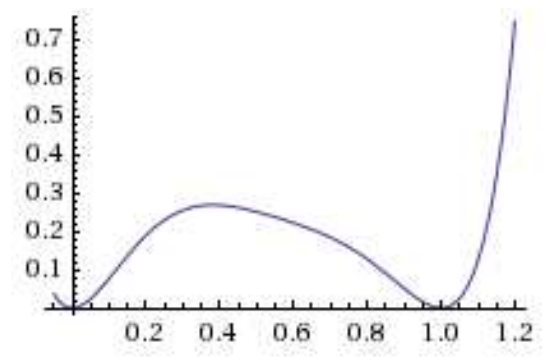

Figura B.1: Esquema SDPUS-C1 - Análise TVD. Função $h=\hat{\phi}_{f}-\hat{\phi}_{U}$.

Seja $g=\hat{\phi}_{f}-2 \hat{\phi}_{U}$. Substituindo (B.1) em $g$, obtém-se

$$
g=24 \hat{\phi}_{U}^{6}-76 \hat{\phi}_{U}^{5}+92 \hat{\phi}_{U}^{4}-52 \hat{\phi}_{U}^{3}+12 \hat{\phi}_{U}^{2}-\hat{\phi}_{U}
$$

Então, deve ser provado que $g \leq 0$ para $0 \leq \hat{\phi}_{U} \leq 0.5$. De fato a raízes reais de $g$ são dadas por 0 e 1.23, e as raízes imaginárias são $0.19 \pm 0.06 i$ e $0.770 \pm 0.46 i$. Com o estudo do sinal da função $g$ segue que $g \leq 0$ para $0 \leq \hat{\phi}_{U} \leq 0.5$. Para ilustrar essa implicação, a Figura B.2 esboça o gráfico da função $g$.

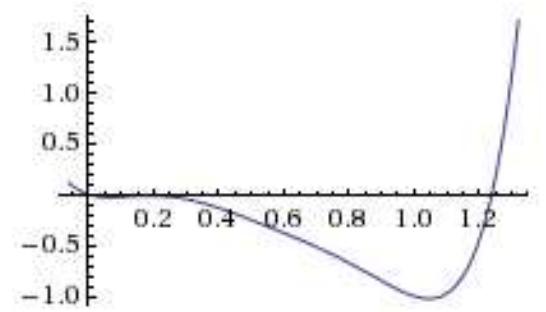

Figura B.2: Esquema SDPUS-C1 - Análise TVD. Função $g=\hat{\phi}_{f}-2 \hat{\phi}_{U}$.

iii) $\hat{\phi}_{f} \leq 1$ para $0.5 \leq \hat{\phi}_{U} \leq 1$.

Seja $s=\hat{\phi}_{f}-1$. Substituindo (A.1) em $g$, obtém-se

$$
s=24 \hat{\phi}_{U}^{6}-76 \hat{\phi}_{U}^{5}+92 \hat{\phi}_{U}^{4}-52 \hat{\phi}_{U}^{3}+12 \hat{\phi}_{U}^{2}+\hat{\phi}_{U}-1
$$

Então, deve ser provado que $s \leq 0$ para $0.5 \leq \hat{\phi}_{U} \leq 1$. De fato as raízes reais de $s$ são dadas por -0.20 e 1 . As raízes imaginárias são dadas por $0.33 \pm 0.38 i$ e $0.85 \pm 0.19 i$. Novamente, com estudo do sinal da função $s$ segue-se que $s \leq 0$ para $0.5 \leq \hat{\phi}_{U} \leq 1$. Para ilustrar essa implicação, a Figura B.3 mostra o gráfico da função $s$.

Em suma, das demonstrações acima, conclui-se que o esquema SDPUS-C1 (B.1), para $\gamma=$ 12, é TVD. 


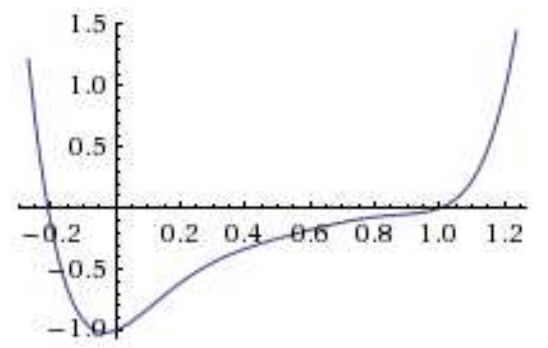

Figura B.3: Esquema SDPUS-C1 - Análise TVD. Função $s=\hat{\phi}_{f}-1$. 
APÊNDICE

\section{Princípio de Sweby e Diferenciabilidade}

Neste apêndice demonstra-se a seguinte implicação:

- A condição $\hat{\phi}_{f}^{\prime}(0)=1$ (uma das condições impostas para que a o esquema $\hat{\phi}_{f}=\hat{\phi}_{f}\left(\hat{\phi}_{U}\right)$ seja de classe $\left.C^{1}\left(\hat{\phi}_{U}\right), \hat{\phi}_{U} \in \mathbb{R}\right)$ não é satisfeita, simultaneamente, com o princípio de Sweby, dado por

$$
\text { se } r \longrightarrow 0 \text {, então } \psi^{\prime}(0) \longrightarrow 2 \text {. }
$$

Demonstração: seja o esquema definido, para $\hat{\phi}_{U} \in[0,1]$, por um polinômio de grau $n+1$, dado por

$$
\hat{\phi}_{f}\left(\hat{\phi}_{U}\right)=\sum_{i=0}^{n+1} a_{i} \hat{\phi}_{U}^{i}
$$

e escrito da seguinte forma (ver Waterson e Deconinck [75])

$$
\hat{\phi}_{f}=\hat{\phi}_{U}+\frac{1}{2} \psi(r)\left(1-\hat{\phi}_{U}\right)
$$

Considerando $\psi(r)$ como $\Psi\left(\hat{\phi}_{U}\right)$, devido a $\hat{\phi}_{U}=\frac{r}{1+r}$, tem-se que o limitador de fluxo é um polinômio de grau $n$ (ver Waterson e Deconinck [75]), dado por

$$
\Psi\left(\hat{\phi}_{U}\right)=\sum_{j=0}^{n} \alpha_{j} \hat{\phi}_{U}^{j}=\alpha_{n} \hat{\phi}_{U}^{n}+\alpha_{n-1} \hat{\phi}_{U}^{n-1}+\cdots+\alpha_{1} \hat{\phi}_{U}+\alpha_{0} .
$$


Reescrevendo-se novamente o limitador de fluxo $\Psi\left(\hat{\phi}_{U}\right)$ em função de $r$ por $\psi(r)$, obtém-se

$$
\Psi\left(\hat{\phi}_{U}\right)=\psi(r)=\alpha_{n}\left(\frac{r}{1+r}\right)^{n}+\alpha_{n-1}\left(\frac{r}{1+r}\right)^{n-1}+\cdots+\alpha_{1}\left(\frac{r}{1+r}\right)+\alpha_{0} .
$$

Primeiramente aplica-se o princípio de Sweby. Para tanto, deriva-se (C.5) para obter

$$
\begin{aligned}
\psi^{\prime}(r)= & \frac{n \alpha_{n} r^{n-1}(1+r)^{n}-\alpha_{n} r^{n} n(1+r)^{n-1}}{(1+r)^{2 n}} \\
& +\frac{(n-1) \alpha_{n-1} r^{n-2}(1+r)^{n-1}-\alpha_{n-1} r^{n-1}(n-1)(1+r)^{n-2}}{(1+r)^{2 n-2}} \\
& \vdots \\
& +\frac{\alpha_{1}(1+r)-\alpha_{1} r}{(1+r)^{2}}
\end{aligned}
$$

e aplica-se a definição desse princípio, dada por (C.1), e conclui-se

$$
\psi^{\prime}(0)=\alpha_{1}=2
$$

Agora, aplica-se a condição $\hat{\phi}_{f}^{\prime}(0)=1$. Para isso, reesecreve-se (C.3) considerando (C.4), ou seja,

$$
\hat{\phi}_{f}=\hat{\phi}_{U}+\frac{1}{2} \Psi\left(\hat{\phi}_{U}\right)\left(1-\hat{\phi}_{U}\right)
$$

e deriva-se (C.7) para obter

$$
\hat{\phi}_{f}^{\prime}\left(\hat{\phi}_{U}\right)=1+\frac{1}{2}\left[\Psi^{\prime}\left(\hat{\phi}_{U}\right)\left(1-\hat{\phi}_{U}\right)-\Psi\left(\hat{\phi}_{U}\right)\right]
$$

aplicando a condição $\hat{\phi}_{f}^{\prime}(0)=1$, obtém-se

$$
\hat{\phi}_{f}^{\prime}(0)=1+\frac{1}{2}\left[\Psi^{\prime}(0)-\Psi(0)\right]=1
$$

o que implica em

$$
\Psi^{\prime}(0)=\Psi(0)
$$

Para avaliar a equivalência (C.10), deriva-se (C.5) e obtém-se

$$
\Psi^{\prime}\left(\hat{\phi}_{U}\right)=\sum_{k=1}^{n} k \alpha_{k} \hat{\phi}_{U}^{k-1}=n \alpha_{n} \hat{\phi}_{U}^{n-1}+(n-1) \alpha_{n-1} \hat{\phi}_{U}^{n-2}+\cdots+2 \alpha_{2} \hat{\phi}_{U}+\alpha_{1}
$$


De (C.11), tem-se

$$
\Psi^{\prime}(0)=\alpha_{1}
$$

e por (C.5), tem-se

$$
\Psi(0)=\alpha_{0}
$$

Portanto, de (C.10), (C.12) e (C.13), obtém-se

$$
\alpha_{1}=\alpha_{0} .
$$

Lembrando que $\hat{\phi}_{f}(0)=0$ (uma condição necessária para que o esquema $\hat{\phi}_{f}=\hat{\phi}_{f}\left(\hat{\phi}_{U}\right)$ seja monotônico e livre de difusão numérica), implica em $\alpha_{0}=0$. Concluindo-se portanto que

$$
\alpha_{1}=0 .
$$

Portanto de (C.6) e (C.15), conclui-se que a condição $\hat{\phi}_{f}^{\prime}(0)=1$ não é satisfeita, simultaneamente, com o princípio de Sweby. 


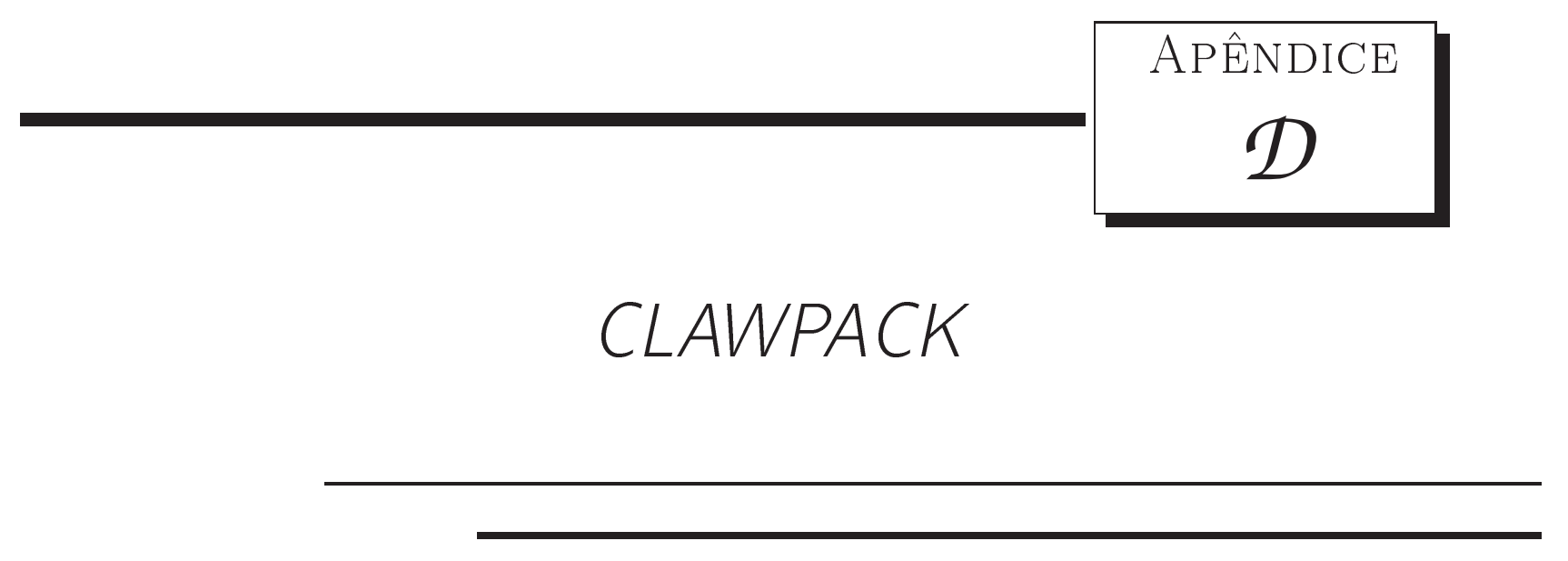

Neste apêndice faz-se uma descrição sintética do software CLAWPACK (Conservation LAW PACKage). Esse software foi desenvolvido por LeVeque et al. [53] e simula, no contexto do método de volumes finitos, leis de conservação hiperbólicas 1D, 2D e 3D. A seguir apresenta-se o algoritmo de resolução empregado no software apenas para o caso 1D. Detalhes da resolução das leis de conservação 2D e 3D podem ser encontrados em LeVeque [44].

Lembrando que a leis de conservação 1D são dadas por:

$$
\phi_{t}+F(\phi)_{x}=0
$$

em que $\phi$ é o vetor das variáveis conservadas e $F(\phi)$ é o vetor da função fluxo. No pacote computacional CLAWPACK, (D.1) é considerada na forma quasi-linear, dada por

$$
\phi_{t}+A(x, t) \phi_{x}=0
$$

em que $A(x, t)$ é a matriz jacobiana $F^{\prime}(\phi(x, t))=A(x, t)$.

O algoritmo de resolução utilizado no CLAWPACK para resolução de leis de conservação é como segue:

Passo 1: Substitui-se a solução pontual por uma distribuição constante por partes;

Passo 2: Resolve-se o problema de Riemann aproximado pelo método de Roe (ver LeVeque [44]). O solucionador do problema de Riemann retorna, para qualquer dois estados $\phi_{i-1}$ e $\phi_{i}$, um conjunto de $M$ ondas definidas como $\sum_{q=1}^{M} \mathcal{W}_{i-\frac{1}{2}}^{q}=\phi_{i}-\phi_{i-1}=\Delta \phi_{i-\frac{1}{2}}$, e as flutuações 


$$
\begin{aligned}
\mathcal{A}^{-} \Delta \phi_{i-\frac{1}{2}} & =\sum_{q}^{M}\left(\hat{s}^{q}\right)^{-} \mathcal{W}_{i-\frac{1}{2}}^{q} \\
\mathcal{A}^{+} \Delta \phi_{i-\frac{1}{2}} & =\sum_{q}^{M}\left(\hat{s}^{q}\right)^{+} \mathcal{W}_{i-\frac{1}{2}}^{q},
\end{aligned}
$$

em que $\left(\hat{s}^{q}\right)^{-}=\min (\hat{\lambda}, 0)$ e $\left(\hat{s}^{q}\right)^{+}=\max (\hat{\lambda}, 0)$, sendo $\hat{\lambda}$ o autovalor da matriz $A$;

Passo 3: Aplica-se a fórmula de atualização. O pacote computacional CLAWPACK dispõe de dois métodos distintos:

- O esquema de primeira ordem de Godunov. Esse método é dado por

$$
\phi_{i}^{n+1}=\phi_{i}^{n}-\frac{\delta_{t}}{\delta_{x}}\left(\mathcal{A}^{+} \Delta \phi_{i-\frac{1}{2}}+\mathcal{A}^{-} \Delta \phi_{i+\frac{1}{2}}\right)
$$

- O esquema de alta resolução, proposto por LeVeque. Esse esquema consiste no método de primeira ordem de Godunov com termo de correção e é dado por

$$
\phi_{i}^{n+1}=\phi_{i}^{n}-\frac{\delta_{t}}{\delta_{x}}\left(\mathcal{A}^{+} \Delta \phi_{i-\frac{1}{2}}+\mathcal{A}^{-} \Delta \phi_{i+\frac{1}{2}}\right)-\frac{\delta_{t}}{\delta_{x}}\left(\widetilde{F}_{i+\frac{1}{2}}-\widetilde{F}_{i-\frac{1}{2}}\right)
$$

em que

$$
\widetilde{F}_{i-\frac{1}{2}}=\mathcal{A}^{+} \phi_{i}+\mathcal{A}^{-} \phi_{i-1}+\frac{1}{2}|A|\left(I-\frac{\delta_{t}}{\delta_{x}}|A|\right)\left(\phi_{i}-\phi_{i-1}\right)
$$

com

$$
\phi_{i}-\phi_{i-1}=\sum_{q}^{M} \widetilde{\alpha}_{i-\frac{1}{2}} \hat{s}^{q}
$$

e

$$
\widetilde{\alpha}_{i-\frac{1}{2}}=\alpha_{i-\frac{1}{2}} \psi\left(r_{i-\frac{1}{2}}^{q}\right)
$$

em que $\alpha=R^{-1} \delta, R$ é a matriz do autovetor, $\delta=\phi_{i}-\phi_{i-1}, \psi$ são as funções limitadores de fluxo e $r_{i-\frac{1}{2}}^{q}$ é a razão dos gradientes consecutivos dado por

$$
r_{i-\frac{1}{2}}^{q}=\frac{\alpha_{I-\frac{1}{2}}^{q}}{\alpha_{i-\frac{1}{2}}^{q}} \text { em que } I= \begin{cases}i-1 & \lambda^{q} \leq 0 \\ i+1 & \lambda^{q}<0\end{cases}
$$

$\operatorname{com} \lambda^{q}$ autovalores. 
Vale observar que o pacote CLAWPACK disponibiliza os limitadores de fluxo Minmod, Superbee, MC, van Leer e Bean-Warming e ainda possibilita a inclusão de novos limitadores de fluxo. Para o estudo neste trabalho de mestrado, o pacote computacional CLAWPACK foi equipado com os limitadores de fluxo, genuinamente brasileiros, ADBQUICKEST, TOPUS, FDPUS-C1 e SDPUS-C1. 


\section{Referências Bibliográficas}

[1] R. Ahmed. Numerical schemes applied to the Burgers and Buckley-Leverett equations. Master's thesis, University of Reading-Departament of Mathematics, 2004.

[2] M. A. Alves, P. J. Oliveira, and F. T. Pinho. A convergent and universally bounded interpolation scheme for the treatment of advection. International Journal for Numerical Methods in Fluids, 41:47-75, 2003.

[3] S. W. Armfield. Finite difference solutions of the Navier-Stokes equations on staggered and non-staggered grids. Computers \&f Fluids, 20:1-17, 1991.

[4] M. Arora and P. L. Roe. A well-behaved TVD limiter for high-resolution calculation of unsteady flow. Journal Computational Physics, 132:3-11, 1997.

[5] U. M. Ascher and L. R. Petzold. Computer methods for ordinary differential equations and differential-algebraic equations. SIAM, 1998.

[6] D. S Balsara and C. W. Shu. Monotonicity preserving weighted essentially non-oscilatory scheme with increasingly high order of accuracy. Journal of Computational Physics, 160:405-452, 2000.

[7] C. Berthon. Stability of the MUSCL schemes for the euler equations. Communications in Mathematical Sciences, 3:133-157, 2005.

[8] R. B. Bird, R. C. Armstrong, and O. Hassager. Dynamics of polymeric liquids. John Willey $\mathcal{G}$ Sons, 2nd edition, 1987.

[9] J. Boussinesq. Memoires presentes par divers savants sciences mathematique at physiques theorie de l'ecoulemente tourbillant. Master's thesis, Academie des Scienses, Paris, 1877.

[10] A. C. Brandi. Estratégias upwind e modelagem k-epson para simulação numérica e escoamentos com superfícies livres em altos números de reynolds. Master's thesis, Dissertação de Mestrado - Instituto de Ciências Matemáticas e de Computação (ICMC-USP), 2005. 
[11] A. F. Castelo, N. Mangiavacchi, M. F. Tomé, J. A. Cuminato, V. G. Ferreira, and S. McKee. Surface tension implementation for GENSMAC 2D. Journal of the Brazilian Society of Mechanical Sciences, XXIII:523-532, 2001.

[12] A. F. Castelo, M. F. Tomé, C. N. L. César, S. McKee, and J. A. Culminato. Freeflow: An integrated simulation system for three-dimensional free-surface flows. Journal of Computers and Visualization in Science, 2:199-210, 2000.

[13] A. J. Chorin. Numerical solution of the Navier-Stokes equations. Mathematics Computational, 22:745-762, 1968.

[14] A. Colagrossi and M. Landrini. Numerical simulation of interfacial flows by smoothed particle hydrodynamics. Journal of Computational Physics, 191:448-475, 2003.

[15] G. Colicchio, M. Landrini, and L. C. Chaplin. Level-set modelling of the air-water flow generated buy a surface piercing body. Proc 8th Int. Conference on Numerical Ship Hydrodynamics, Korea, 2003.

[16] R. Courant, E. Isaacson, and M. Rees. On the solution of nonlinear hyperbolic differential equations by finite differences. Community Pure Applied Mathematics, 5:243-255, 1952.

[17] J. O. Cruickshank. Low-Reynolds-number instabilities in stagnating jet flows. Journal of Fluid Mechanic, 193:111-127, 1987.

[18] J. O. Cruickshank and B. R. Munson. The viscou-gravity jet in stagnatio flow. Journal of Fluids Engineering, 104:360-362, 1982.

[19] C. Cunha. Métodos numéricos. Campinas, 2 edition, 2000.

[20] F. M. Denaro. On the applications of the helmoltz-hodge decomposition in projection method for incompressible flows with general boundary conditions. International Journal for Numerical Methods in Fluids, 43:43-69, 2003.

[21] P. A. Durbin. On the k-epson stagnation point anormaly. Journal Heat and Fluid Flow, $17: 89-90,1996$.

[22] J. A. Ekaterinaris. Performance of high-order-accurate low diffusion numerical schemes for compressible flow. AIAA Journal, 42:493-500, 2004.

[23] C. Ellegaard, A. E. Hansen, A. Haaning, K. Hansen, A. Marcussen, and T. Bohr. Poligonal hydraulic jumps. Nonlinearity, 12:1-7, 1999.

[24] I. Fatkullin and J. S. Hesthaven. Adaptative high-order finite-difference method for nonlinear wave problems. Journal of Scientific Computing, 16(1):47-67, 2001. 
[25] V. G. Ferreira. Análise e implementação de esquemas de convecção e modelos de turbulência para simulação de escoamentos incompressíveis envolvendo superfícies livres. Master's thesis, PhD thesis, Instituto de Ciências Matemática e de Computação (ICMC-USP), 2001.

[26] V. G. Ferreira, R. A. B. Queiroz, G. A. B. Lima, R. G. Cuenca, C. M. Oishi, and S. McKee. An upwind differencing scheme for conservation laws and related fluid dynamics problems. Computers 8 Fluids, page Submitted, 2009.

[27] V.G. Ferreira, F.A. Kurokawa, R.A.B. Queiroz, M.K. Kaibara, C.M. Oishi, J.A. Cuminato, A. Castelo, M.F. Tomé, and S. Mckee. Assessment of a high-order finite difference upwind scheme for the simulation of convection-diffusion problems. International Journal for Numerical Methods in Fluids, 60:1-26, 2009.

[28] P. H. Gaskell. and A. K. C. Lau. Curvature-compensated convective transport: Smart, a new boundedness preserving transport algorithm. International Journal for Numerical Methods in Fluids, 8:617-641, 1988.

[29] M. Greco, M. Landrini, and O. M. Faltinsen. Impact flows and loads on shi-deck structures. Journal Fluids Structures, page submitted, 2003.

[30] A. Harten. High resolution schemes for hyperbolic conservation laws. Journal of Computational Physics, 49:357-393, 1983.

[31] A. Harten. Eno scheme with subcell resolution. Journal of Computational Physics, 83:148-184, 1989.

[32] A. Harten and P. D. Lax. On a class of high resolution total-variation-stable finite-difference schemes. SIAM Journal on Numerical Analysis, 21:1-23, 1984.

[33] M. Herrmann, G. Blanquart, and V. Raman. Flux corrected finite volume scheme for preserving scalar boundedness in reacting large-eddy simulations. AIAA Journal, 44:2879-2886, 2006.

[34] M. R. Hestenes and E. Stiefel. Methods of conjugate gradients for solving linear systems. Journal of Research of the National Bureau of Standads, 49:409-436, 1952.

[35] G. S. Jiang and C. W. C. W. Shu. Efficient implementation of weighted eno shemes. Journal Computation Physics, 126:202-212, 1996.

[36] V. I. Karpman. Non-linear waves in dispersive media. Fluid Mechanic, Pergamon, Elmsford, 1975.

[37] S. Koshizuka and S. Oka. Moving-particle semi-implicit method for fragmentation of incompressible fluids. Nuclear Science and Engineering, 123:421-434, 1996. 
[38] K. B. Kuan and C. A. Lin. Adaptative QUICK-based scheme to approximate convective transport. AIAA Journal, 38:2233-2237, 2000.

[39] M. Kurihara. A consideration about hydraulic jump. Technical Report, Kyusyu Imperial University, 3:11-25, 1946.

[40] F. A. Kurokawa. Um esquema upwind para leis de conservação e sua aplicação na simulação de escoamentos incompressíveis $2 \mathrm{~d}$ e 3d laminares e turbulentos com superfícies livres. Master's thesis, Universidade de São Paulo-Instituto de Ciências Matemáticas e de Computação, 2009.

[41] B. P. Leonard. The QUICK algorithm: a uniformly third-order finite difference method for highly convective flows. Computational Methods Applicate Mechanic Engineering, 19:59-98, 1979.

[42] B. P. Leonard. Simple high-accuracy resolution program for convective modelling of discontinuities. International Journal for Numerical Methods in Fluids, 8:1291-1318, 1988.

[43] B. P. Leonard. Universal limiter for transiente interpolation modeling of the advective transport equations: The ULTIMATE conservation difference sheme. NASA Technical Memorandum 100916, 1988.

[44] R. J. LeVeque. Finite volumes methods for hyperbolic problems. Press Syndicate of the University of Cambridge, 2004.

[45] C. H. Lin and C. A. Lin. Simple high-order bounded convection scheme to model discontinuities. AIAA Journal, 35:563-565, 1997.

[46] H. Lin and C.C. Chieng. Characteristic-based flux limiters of an essentially third-order flux-splitting method for hyperbolic conservation laws. International Journal for Numerical Methods in Fluids, 13:287-307, 1991.

[47] J. C. Martin and W. J. Moyce. An experimental study of the collapse of liquid columns on a rigid horizontal plate. Philosophical transactions of royal society of ondon, series. A mathematical, Phisical and Engineering Sciences, 244:312-324, 1952.

[48] F. P. Martins. Desenvolvimento de um método numérico implícito para a simulação de escoamentos viscoelásticos com superfícies livres. Master's thesis, Instituto de Ciências Matemáticas e Computação - ICMC-USP, 2009.

[49] M. L. B. Oliveira. Freeflow-axi: Um ambiente de simulação de escoamentos axissimétricos com superfícies livres. Master's thesis, Universidade de São Paulo - Instituto de Ciências Matemáticas e de Computação, 2002. 
[50] S. Osher. Convergence of generalized MUSCL schemes. SIAM Journal of Numerical Analysis, 22:947-961, 1985.

[51] G. W. Platzman. An exact integral of complect spectral equation for unsteady one-dimensional flow. Master's thesis, Manuscript received - The University of Chicago, 1964.

[52] R. A. B. Queiroz. Desenvolvimento e teste de esquemas upwind de alta resolução e suas aplicações em escoamentos incompressíveis com superfícies livres. Master's thesis, Universidade de São Paulo - Instituto de Ciências Matemáticas e de Computação, 2009.

[53] J. LeVeque R, J. O. Langhset, M. Berger, D. McQueen, D. Calhoun, P. Blossey, and S. Mitran. Clawpack 5.0: software package designed to compute numerical solution to hyperbolic partial differential equations using a wave propagation approach. http://www.amath.washington.edu/claw, 2006.

[54] D. Rajagopalan, R. Amstrong, and R. Brown. Finite element method for calculation of steady viscoelastic flow using constitutive equations with newtonian viscosity. Journal of Non-Newtonian Fluid Mechanics, 36:159-192, 1990.

[55] M. Ricchiuto, A. Csik, and H. Deconinck. Residual distribution for general time-dependent conservation laws. Journal of Computational Phisycs, 209:249-289, 2005.

[56] A. Ritter. Die fortpflanzung der wassserwellen. Z. Ver Deut. Ing., 36:1982.

[57] C. W. Shu and S. Osher. Efficient implementation of essentially non-oscilatory shock capturing schemes. Journal of Computational Physics, 83:32, 1989.

[58] G. Sod. A survey of several finite difference methods for systems of nonlinear hyperbolic conservation laws. Journal of Computation Physics, 27:1, 1978.

[59] D. L. Sondak and R. H. Pletcher. Application of wall functions to generalized nonorthogonal curvilinear coordinate systems. AIAA Journal, 33:33-41, 1995.

[60] B. Song, G. R. Liu, K. Y. Lam, and R. S. Amano. On a higher-order bounded discretization sheme. International Journal for Numerical Methods in Fluids, 32:881-897, 2000.

[61] D. B. Spalding. A novel finite difference formulation for differential expressions involving both first and second derivatives. International Journal for Numerical Methods in Fluids, 4:551-559, 1972.

[62] P. K. Sweby. High resolution schemes using flux limiters for hyperbolic conservation laws. SIAM Journal on Numerical Analysis, 21:995-1011, 1884. 
[63] I. Tani. Flow separation in thin liquid layers. Journal of the Physics Society of Japan, 4:212-215, 1948.

[64] G. I. Taylor. Low-reynolds number flows. National Commitee for Fluid Mechanics Films. Illustrated experiments in fluid mechanics., 1974.

[65] J. W. Thomas. Numerical partial differential equations: finite difference methods. 1995.

[66] M. F. Tomé, N. Mangiavacchi, J. Culminato, A. Castelo, and S. Mckee. A finite difference technique for simulating unsteady viscoelastic free surface flow. Journal Non-Newtonian Fluid Mechanic, 106:61-106, 2002.

[67] M. F. Tomé and S. Mckee. Gensmac: A computational marker-and-cell method for free surface flows in general domains. Journal of Computational Physics, 110:171-186, 1994.

[68] E. F. Toro. Rieman solvers and numerical methods for fluids dynamics. New York, 1999.

[69] G. D. van Albada, B. van Leer, and W. W. Roberts. A comparative study of computational methods in cosmic gas dynamics. Astronomy \& Astrophysics, 108:76-84, 1982.

[70] B. van Leer. Towards the ultimate conservative difference scheme II monotonicity and conservation combined in a second-order scheme. Journal Computational Physics, 14:361-370, 1974 .

[71] B. van Leer. Towards the ultimate conservation difference scheme IV. a new approach to numerical convection. Journal Computational Physics, 23:263-275, 1977.

[72] B. VanLeer. Towards the ultimate conservative difference scheme. V. a second-order sequel to godunov's method. Journal of Computational Physics, 33:101-136, 1977.

[73] A. Varonos and G. Bergeles. Development and assessament of a variable-order non-oscilatory scheme for convection term discretization. International Journal for Numerical Methods in Fluids, 26:1-16, 1988.

[74] R. F. Warming and R. M. Beam. Upwind second-order difference schemes and application in aerodynamics flows. AIAA Journal, 14:1241-1249, 1976.

[75] N. P. Waterson and H. Deconinck. Design principles for bounded higher-order convection schemes - a unified approach. Journal of Computational Physics, 224:182-207, 2007.

[76] E. J. Watson. The radial spread of a liquid jet over a horizontal plane. Journal of Fluid Mechanics, 20:481-499, 1964.

[77] G. W. Wei and Y. Gu. Conjugated filter approach for solving burger's equation. Journal of Computational and Applied Mathematics, 149:439-456, 2002. 
[78] P. Woodward and P. Collela. The numerical simulation of two-dimensional fluid flow with strong shocks. Journal Computation Physics, 149:439-456, 2002.

[79] S. C. Xue, N. Phan-Thien, and R. I. Tanner. Upwinding with deferred correction (UPPC): and effective implementation of high-order convection schemes for implicit finite volume methods. Journal of Non-Newtonian Fluid Mechanics, 108:1-24, 2002.

[80] M. Zijlema. On the construction of a third-order accurate monotone convection scheme with application to turbulent flows in general domains. International Journal for Numerical Methods in Fluids, 22:619-641, 1996. 